LAWRENCE LIVERMORE NATIONAL LABORATORY

\title{
Electron Transport Workshop, September 9-11, 2002
}

J. Edwards, S. Glenzar, E. Alley, R. Town, D. Braun, S. Kruer, B. Lasinski, A. Mackinnon, M. Haines, R. Kingham, N. Nicolai, E. Valco, S. Krasheninnikov

June 17, 2003 
This document was prepared as an account of work sponsored by an agency of the United States Government. Neither the United States Government nor the University of California nor any of their employees, makes any warranty, express or implied, or assumes any legal liability or responsibility for the accuracy, completeness, or usefulness of any information, apparatus, product, or process disclosed, or represents that its use would not infringe privately owned rights. Reference herein to any specific commercial product, process, or service by trade name, trademark, manufacturer, or otherwise, does not necessarily constitute or imply its endorsement, recommendation, or favoring by the United States Government or the University of California. The views and opinions of authors expressed herein do not necessarily state or reflect those of the United States Government or the University of California, and shall not be used for advertising or product endorsement purposes.

This work was performed under the auspices of the U.S. Department of Energy by University of California, Lawrence Livermore National Laboratory under Contract W-7405-Eng-48. 


\title{
Electron Transport Workshop
}

\section{Purple Orchid (www.purpleorchid.com) 9256068855}

\section{9-11 September 2002}

\section{Agenda}

\author{
Monday $9^{\text {th }}$ September \\ 8:30 Introduction \& Welcome \\ (Edwards) \\ 9:00 Overview of issues for NIF \\ 9:45 Physics of magnetic fields \& \\ (Glenzer) \\ Criterion for validity of linear transport \\ (Haines) \\ 10:30 Break \\ 11:00 Hybrid electron transport package \\ (Alley) \\ 11:45 Hohlraums according to lasnex \\ (Edwards) \\ 12:30 Lunch \\ 1:30 Non-Local heat flow in FCI2 \\ (Schurtz) \\ Coupled MHD non-local model \\ (Schurtz) \\ 2:30 Application of FCI2 to laser expts \\ (Nicolai) \\ 3:30 Break \\ 4:00 Omega direct drive implosions \\ (Town) \\ 4:30 Omega \& NIF slab experiments \\ (Braun) \\ 5:00 High intensity slab modeling with FCI2 \\ (Nicolai)

\section{5:30 BBQ}


Tuesday $10^{\text {th }}$ September

8:30 Tidman and Shanny instability

(Haines)

- viscosity or f2?

9:30 Filamentary structures

10:00 Electro-thermal instability

(Mackinnon)

(Haines)

10:30 Break

11:00 Interface with LPI

(Kruer)

11:15 B-fields around a laser speckle

(Lasinski)

11:30 Detuning SBS

(Haines)

11:45 I/c driven fields in lasnex

(Edwards)

12:00 Collisional $\delta$ model

(Valeo)

12:30 Lunch

1:30 2-D Fokker Planck with B

(Kingham)

2:30 2-D FP without B

(Town)

2:50 1-D FP with B \& $v^{-2}$

(Haines)

3:30 Break

4:00 An improved model of non-local heat flow in laser heated plasmas

4:45 A 3D model for non-local heat flow

(Matte)

(Krasheninnikov)

\section{Wednesday $11^{\text {th }}$ Septenber}

Discussion sessions on future plans. This will shape up during the first two days

\section{8:00 Future theory/computation}

topics include:

Is $2 \mathrm{D}$ good enough?

Micro-turbulence

Simulations \& code development (Fokker-Planck vs non-local)

Numerical benchmarks

11:00 Thomson scattering measurements of heat flow (Hawreliak)

\section{2:00 lunch}

\section{1:00 Wash up discussions}




\title{
Heat Transport in 2D Simulations of High Intensity, Direct Drive Ablation
}

\author{
David Braun, David Bradley, Gilbert Collins,
} John Edwards and Larry Suter

LLNL 


\section{High pressure shock experiments are required to verify equation of state models}

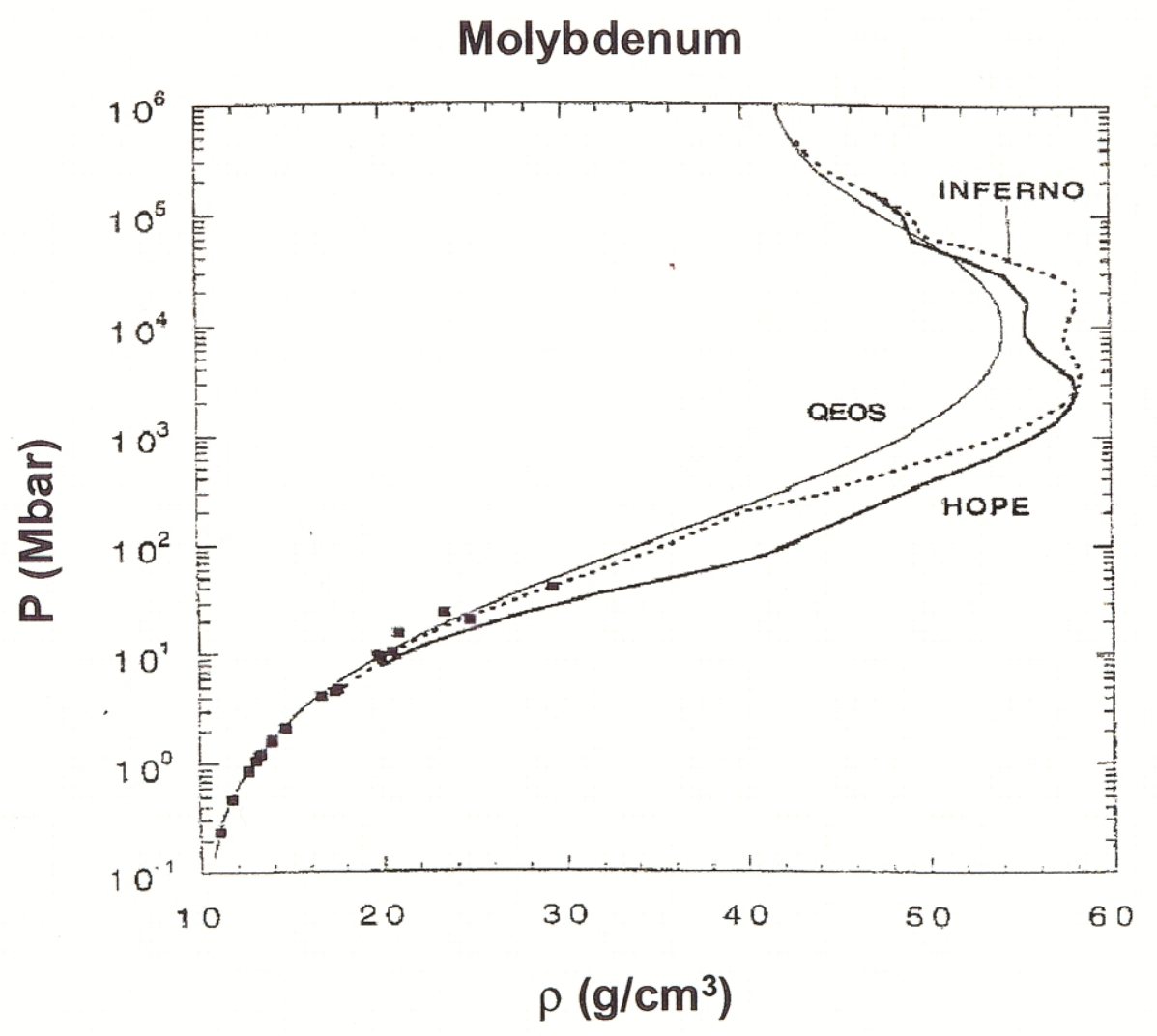

- Existing compression data from gas gun experiments is limited to pressures of less than 100 Mbar

- At 1 Gbar, ionization effects dominate materials' equation of state

- Current eos models diverge in this regime

- High intensity, direct drive ablation experiments on the NIF will access the 1 Gbar regime 


\section{Initial experiments were performed last July at Omega, using a moderate laser intensity}

relative eos experiment, compares the shock speed in the sample to that in Mo

- 6 beams from $22^{\circ}$, 6 from $48^{\circ}$

- $\quad$ spot radius of $412 \mu \mathrm{m}$, spreading to an ellipse on target

- intensity on target of $3.8 \mathrm{e} 14 \mathrm{~W} / \mathrm{cm} 2$

- $\quad$ shock breakout times were recorded using a reflectivity-based VISAR diagnostic

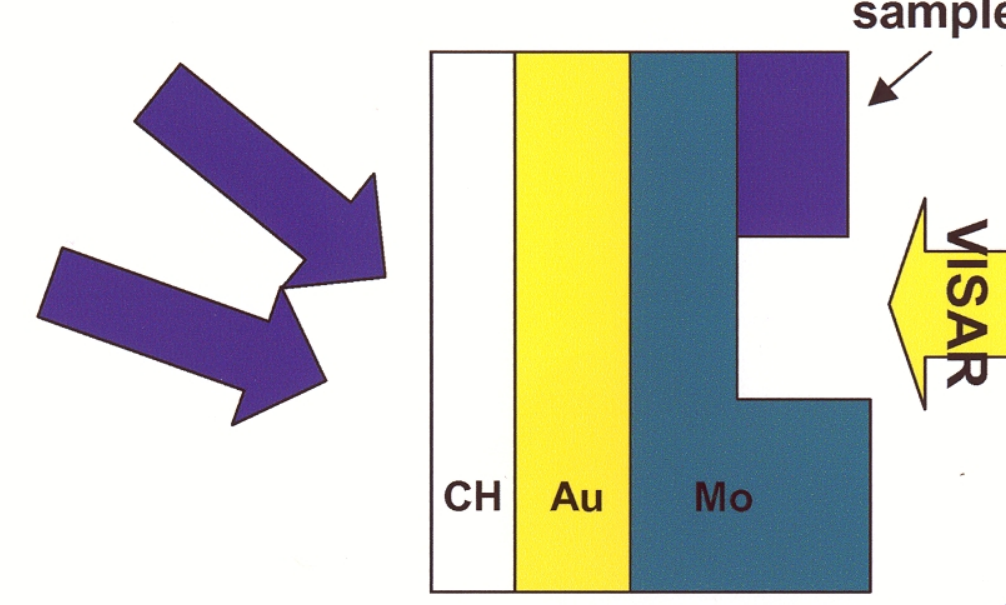

fiducial

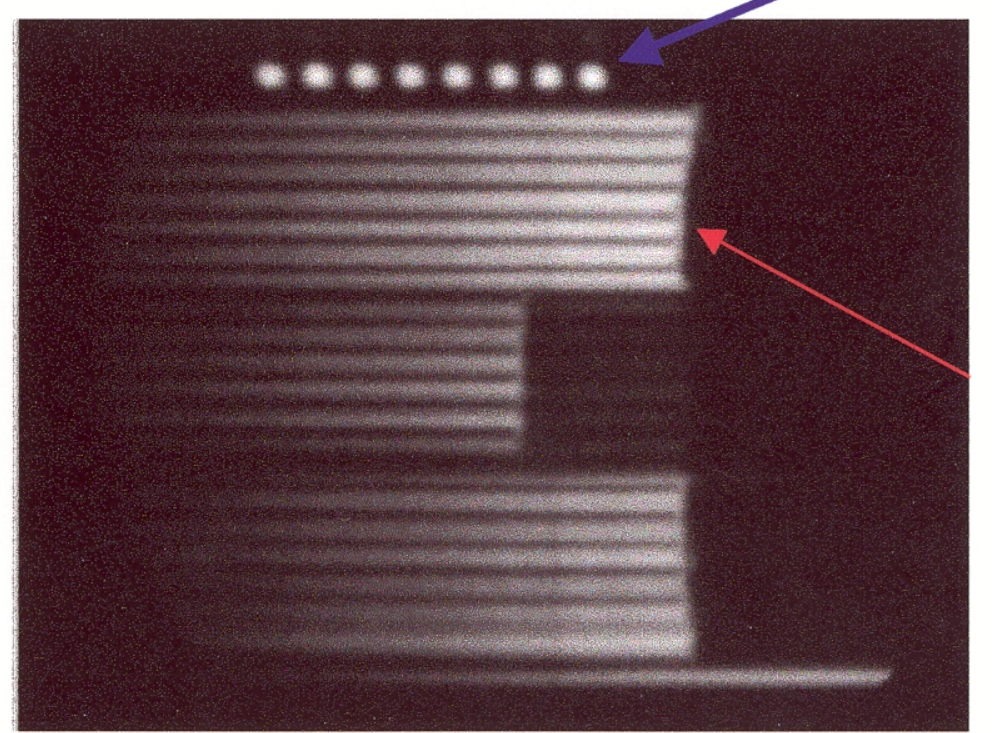

breakout 


\section{Measured shock breakout times were consistently longer than those predicted by 1D simulations}

1D lasnex results vs measured

shock breakout times

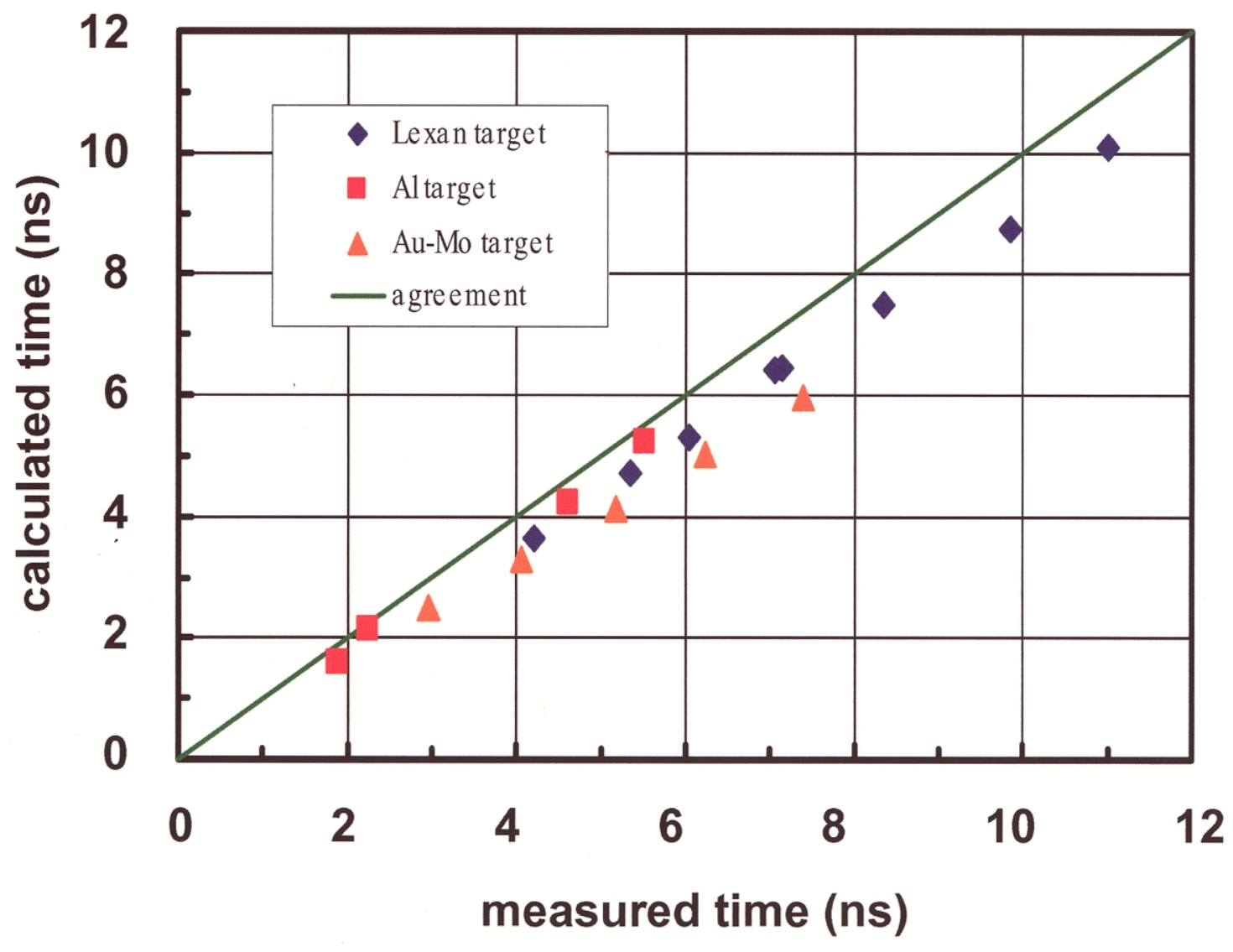

- results on Mo were consistent with a pattern obtained using various targets

- data includes shots with intensities from $2 * 10^{13}$ to $4{ }^{*} 10^{14} \mathrm{~W} / \mathrm{cm}^{2}$

- simulations used measured laser pulses

- spot size based on measured beam profile

- agreement requires intensity reduction of 30 $50 \%$, which is beyond the experimental uncertainty 


\section{Good agreement has been obtained between 2D calculations and the measured shock speed}

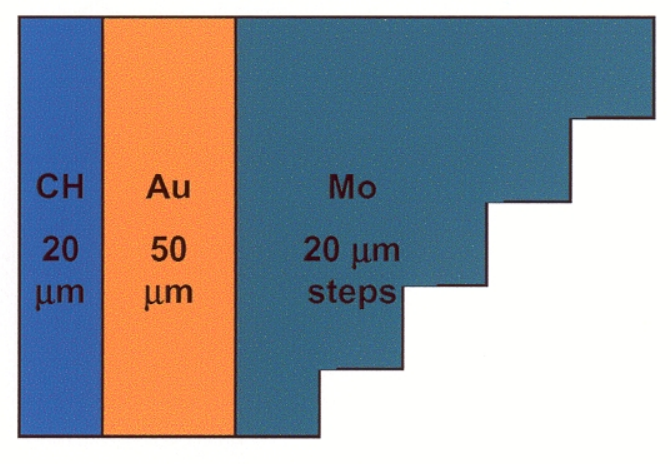

visar image

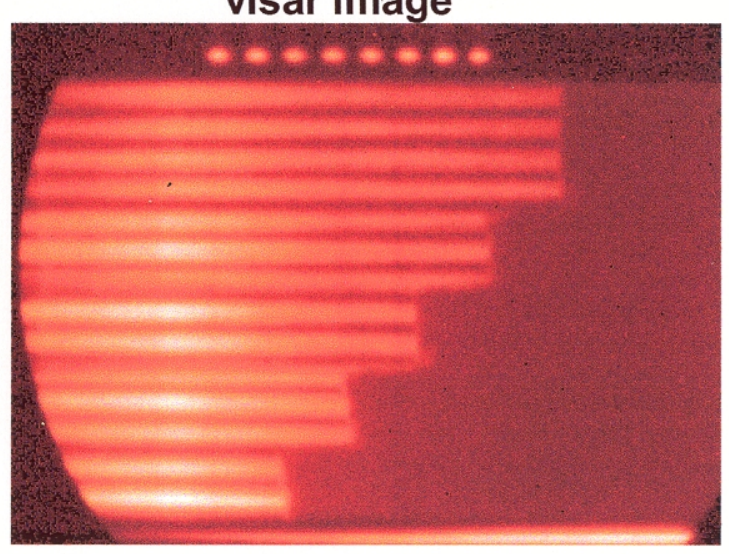

$\rightarrow$ time (ns) calculated vs measured shock breakout times in Mo

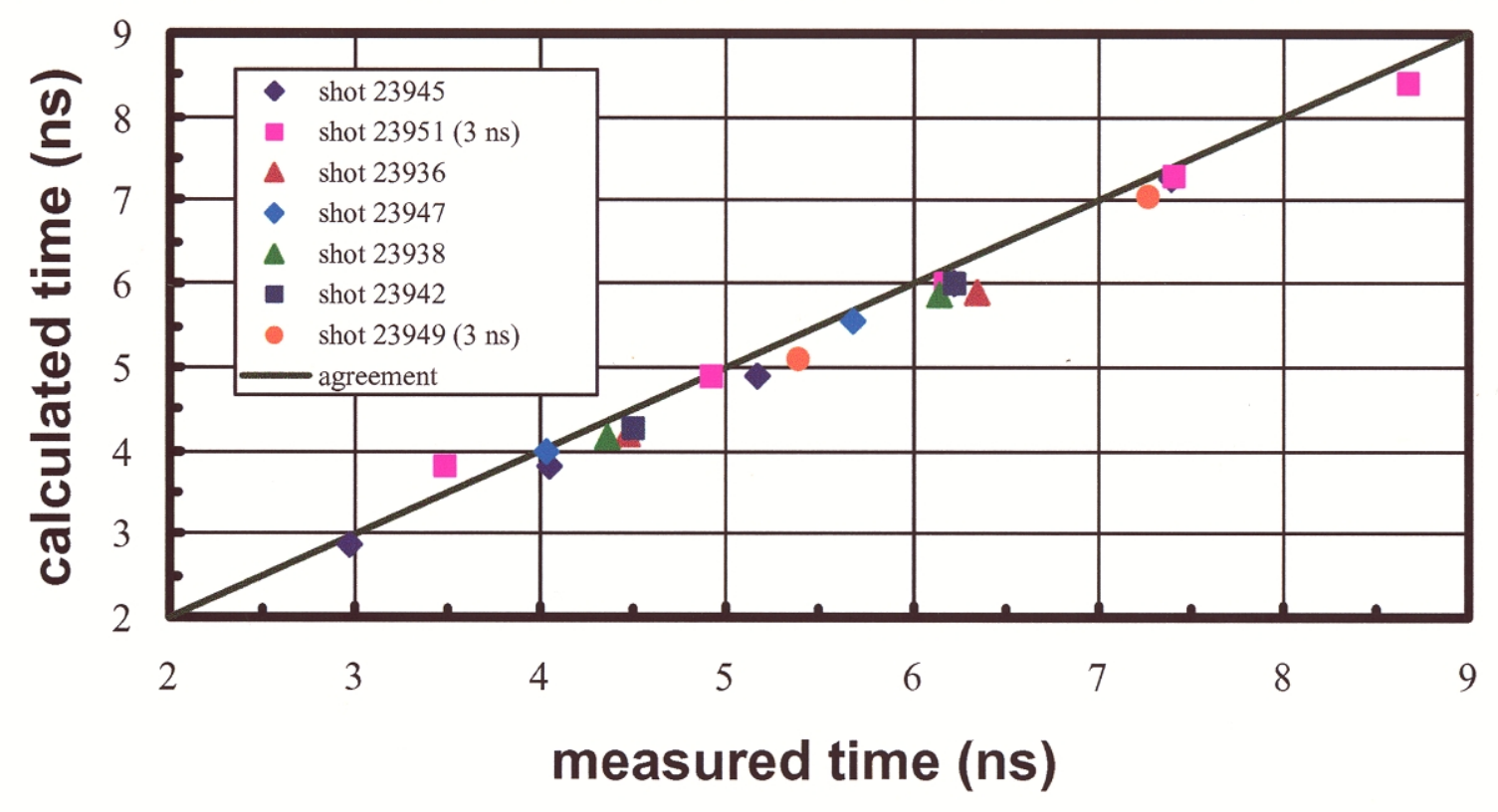

- 2D lasnex simulations, fully Lagrangian

- simulations use the measured laser pulse and beam spot size

- electron flux limiter $=0.1$ 


\section{D simulations of the experiments show that radial energy loss dominates in the coronal plasma}

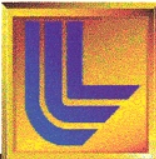

Contours of Te (keV) at the end of a 2 ns pulse on a Au-Mo target
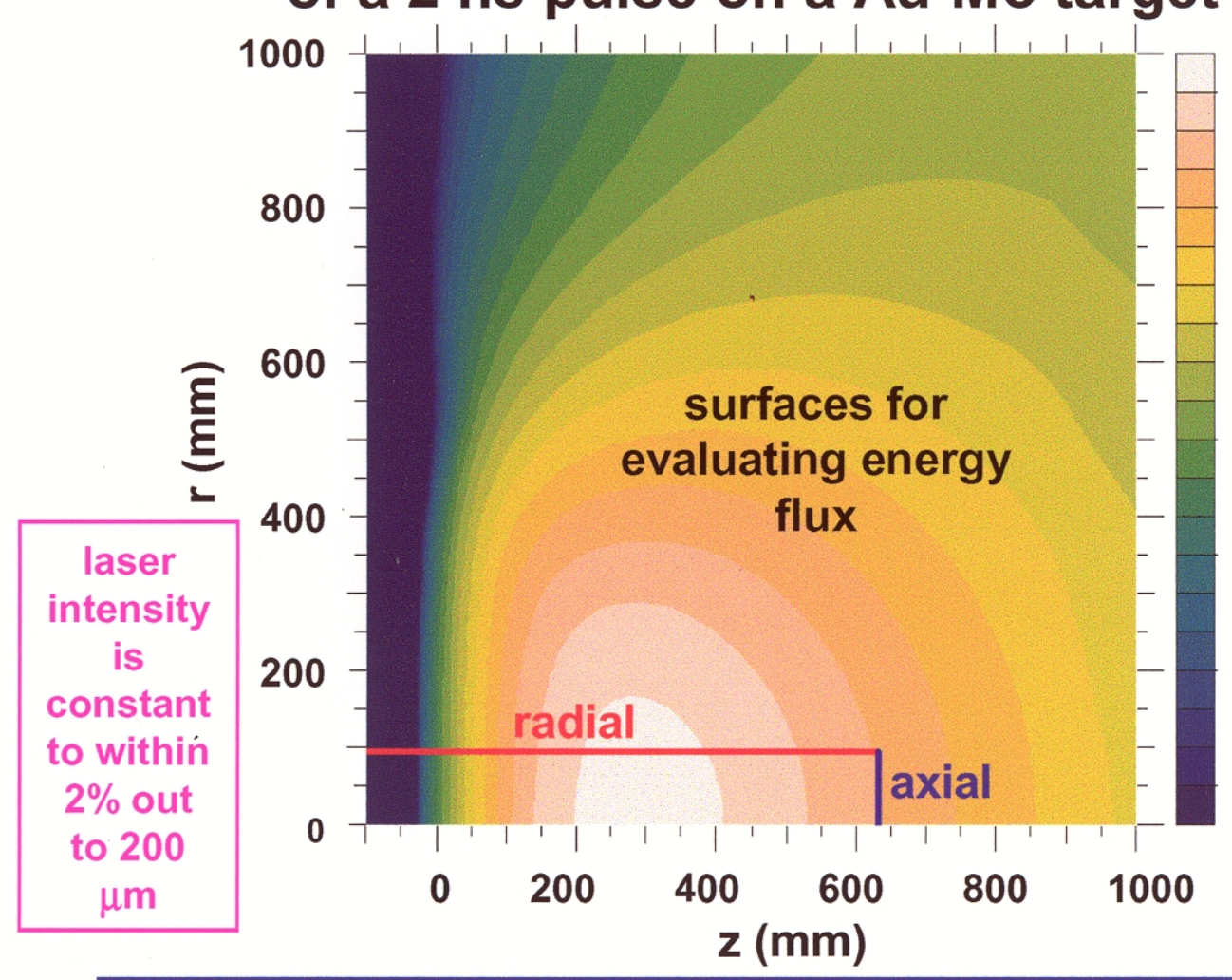

Energy flux crossing the radial and axial surfaces vs time 1.9

1.6 1.3

1.0

0.7

0.4

0.1

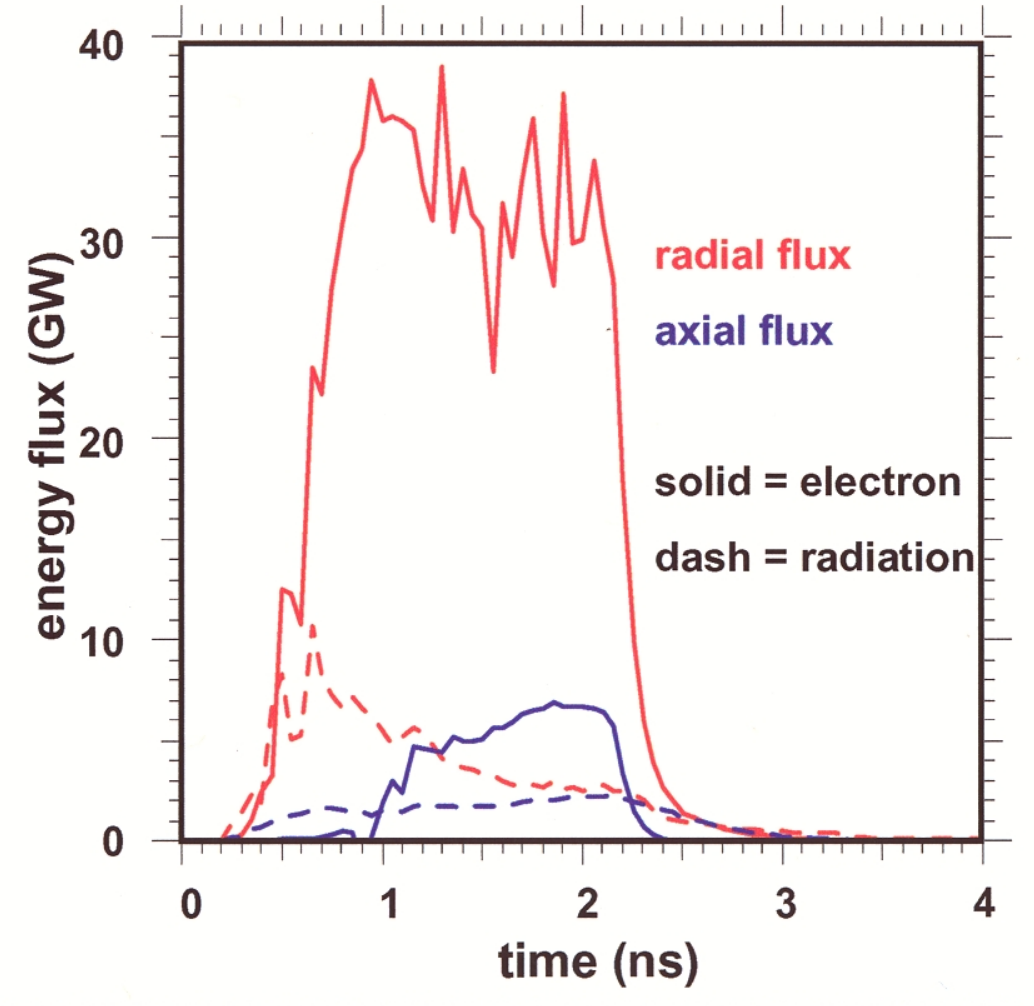

- Even at small radii, the gradients are sufficiently large so that, with the large surface area, radial energy loss dominates

- In a 1D simulation, the energy builds up until the gradient can drive an axial flux 


\section{Radial energy loss in the 2D simulation yields a lower plasma pressure for the same on-axis laser intensity}

Contours of pressure (Mbar) at the end of a 2 ns pulse on a Au-Mo target

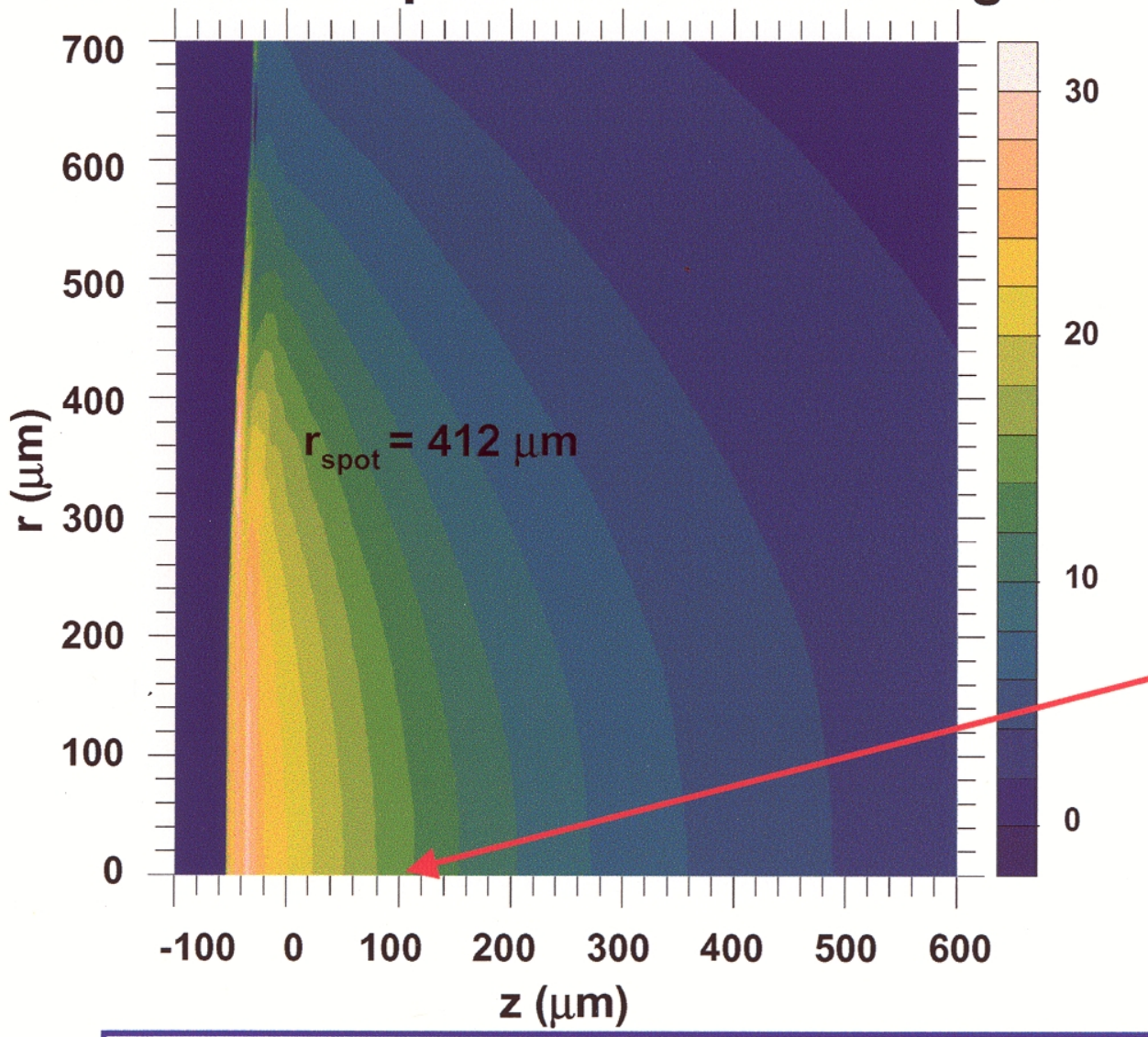

Pressure vs. time at a point on beam axis, $z=100$ um

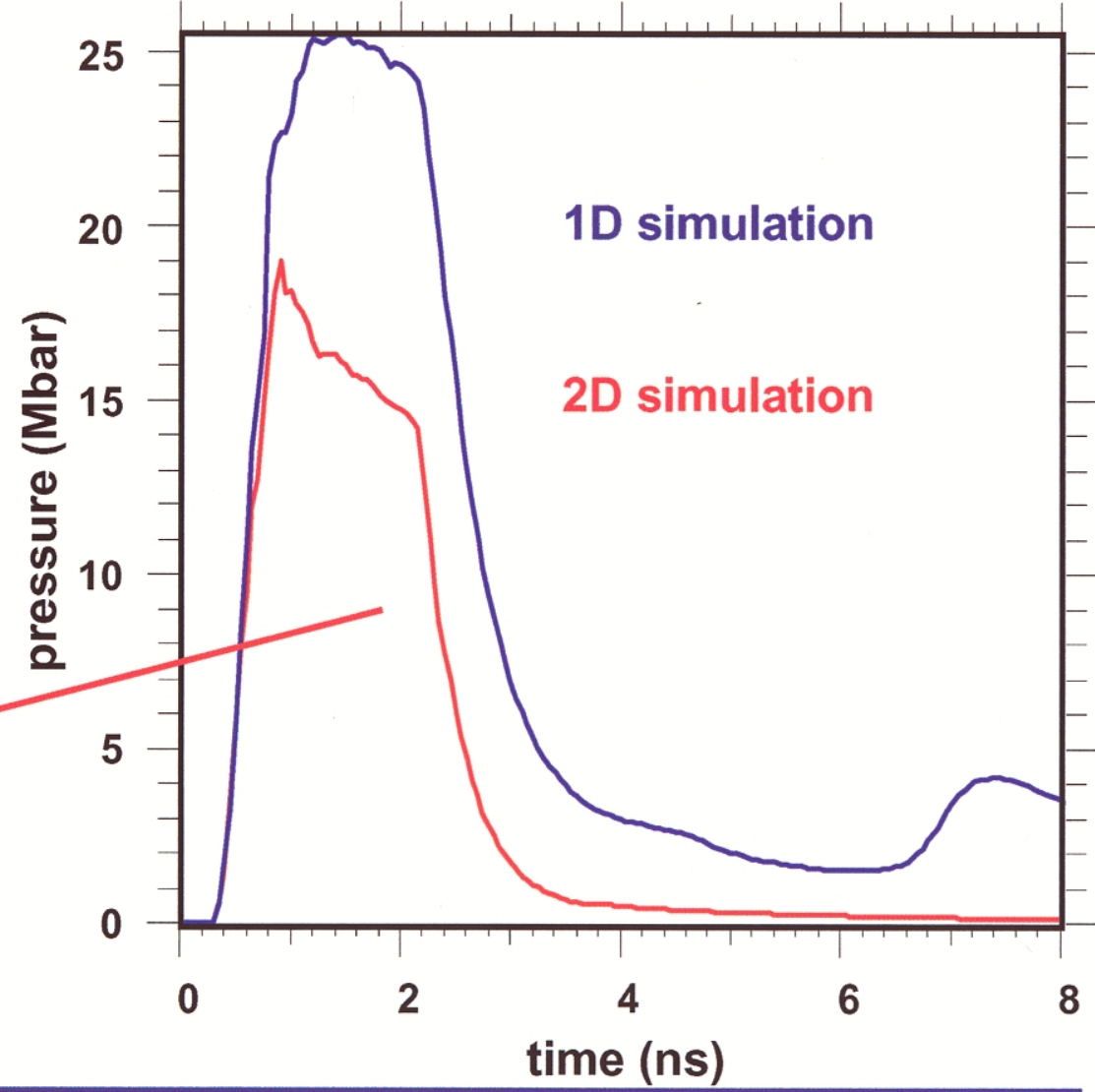

The 2D simulation pressure is $70 \%$ of the $1 \mathrm{D}$ calculation, producing the slower shock speeds consistent with the experiments 


\section{High-Z eos experiments proposed for NIF were designed to use laser intensities of $10^{16} \mathrm{~W} / \mathrm{cm}^{2}$}
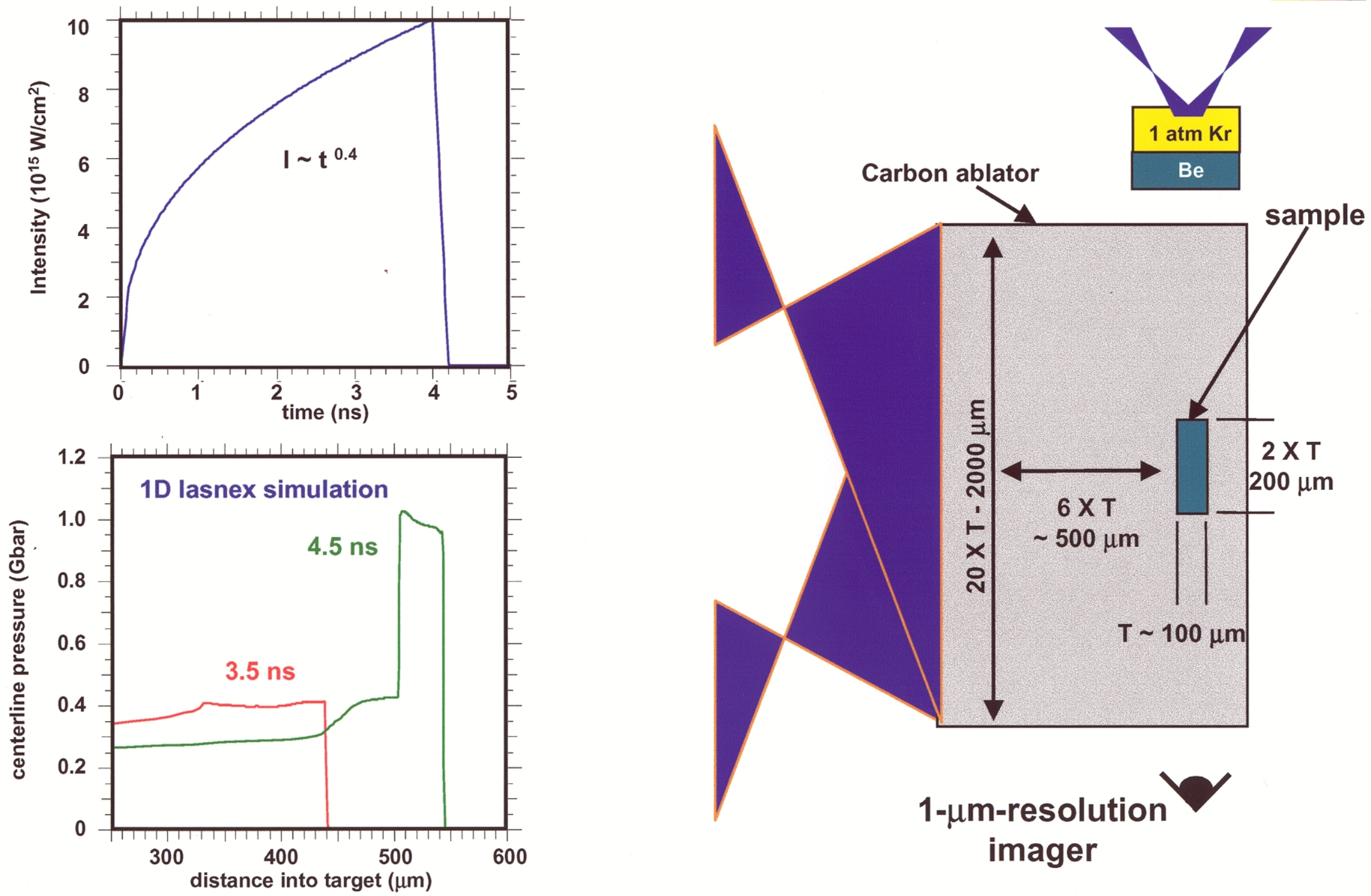


\section{The difference between the 2D and 1D simulations is even greater in the proposed NIF experiment}

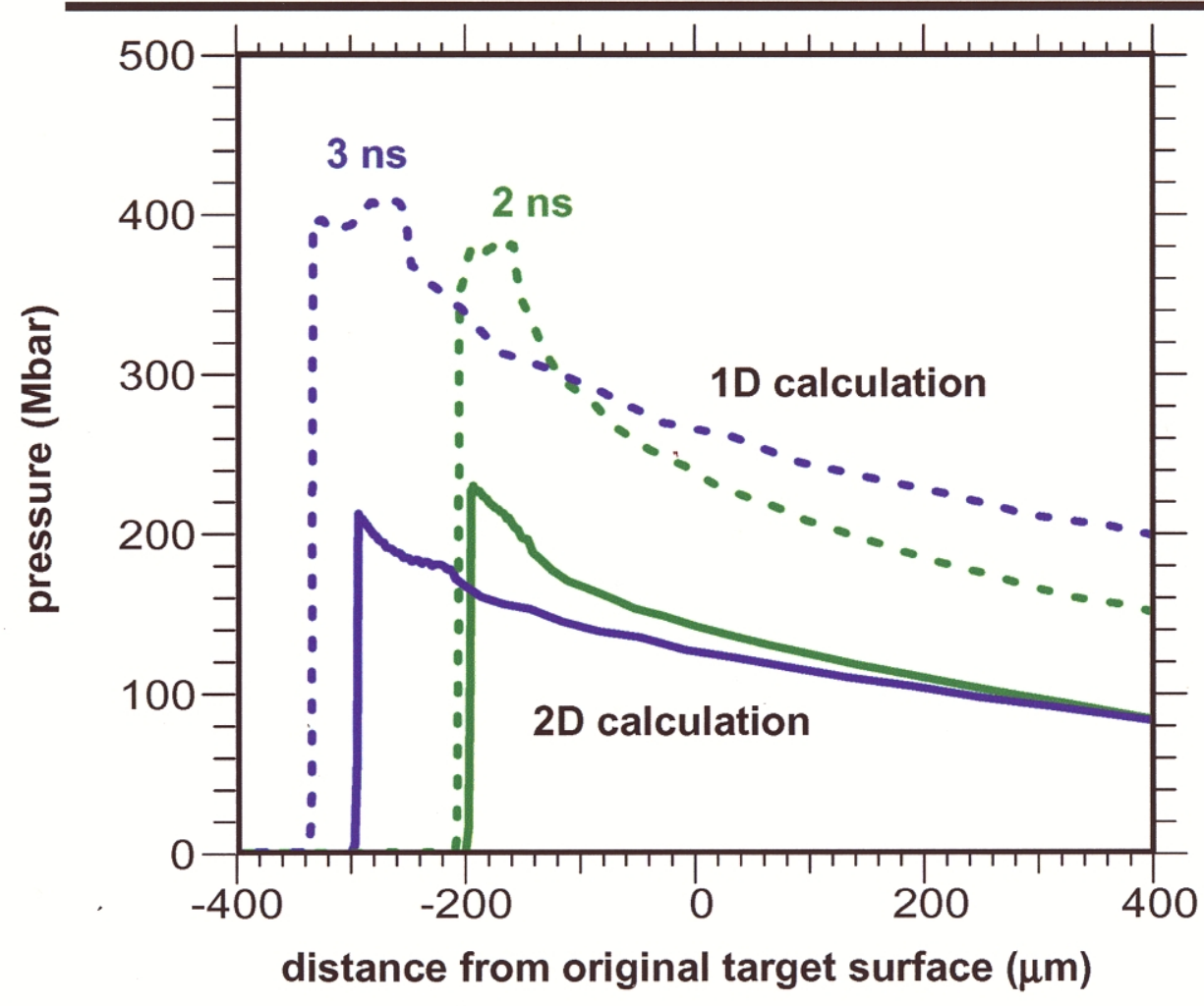

- shock pressure in the 2D simulation is a factor of 2 lower than predicted in the 1D calculation

- $T_{e}$ is reduced from $12 \mathrm{keV}$ in the 1D simulation to $7 \mathrm{keV}$

- both 1D and 2D simulations use a 4 ns ramped laser pulse to an intensity $10^{16} \mathrm{~W} / \mathrm{cm}^{2}$

- 2D calculation uses $r_{\text {spot }}=0.1 \mathrm{~cm}$

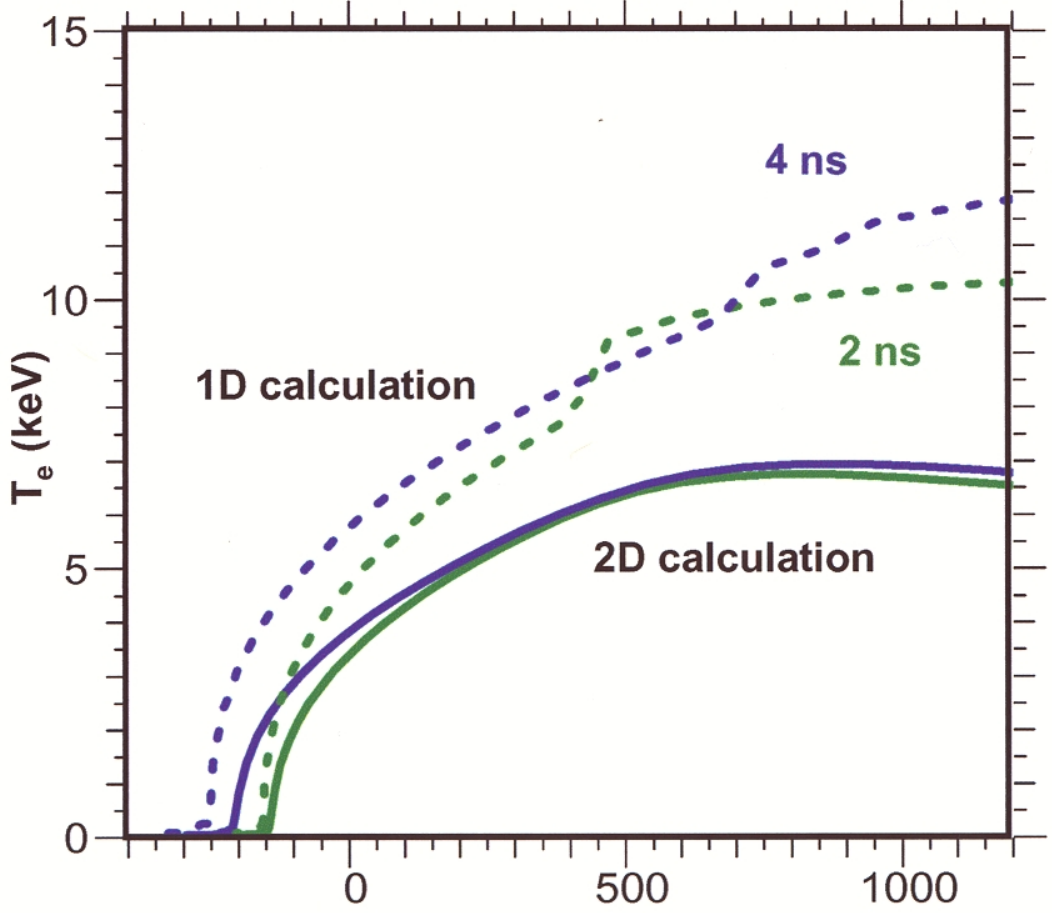

distance from original target surface $(\mu \mathrm{m})$ 


\section{In the NIF experiment, the absorption region has moved out a distance comparable to the spot size}

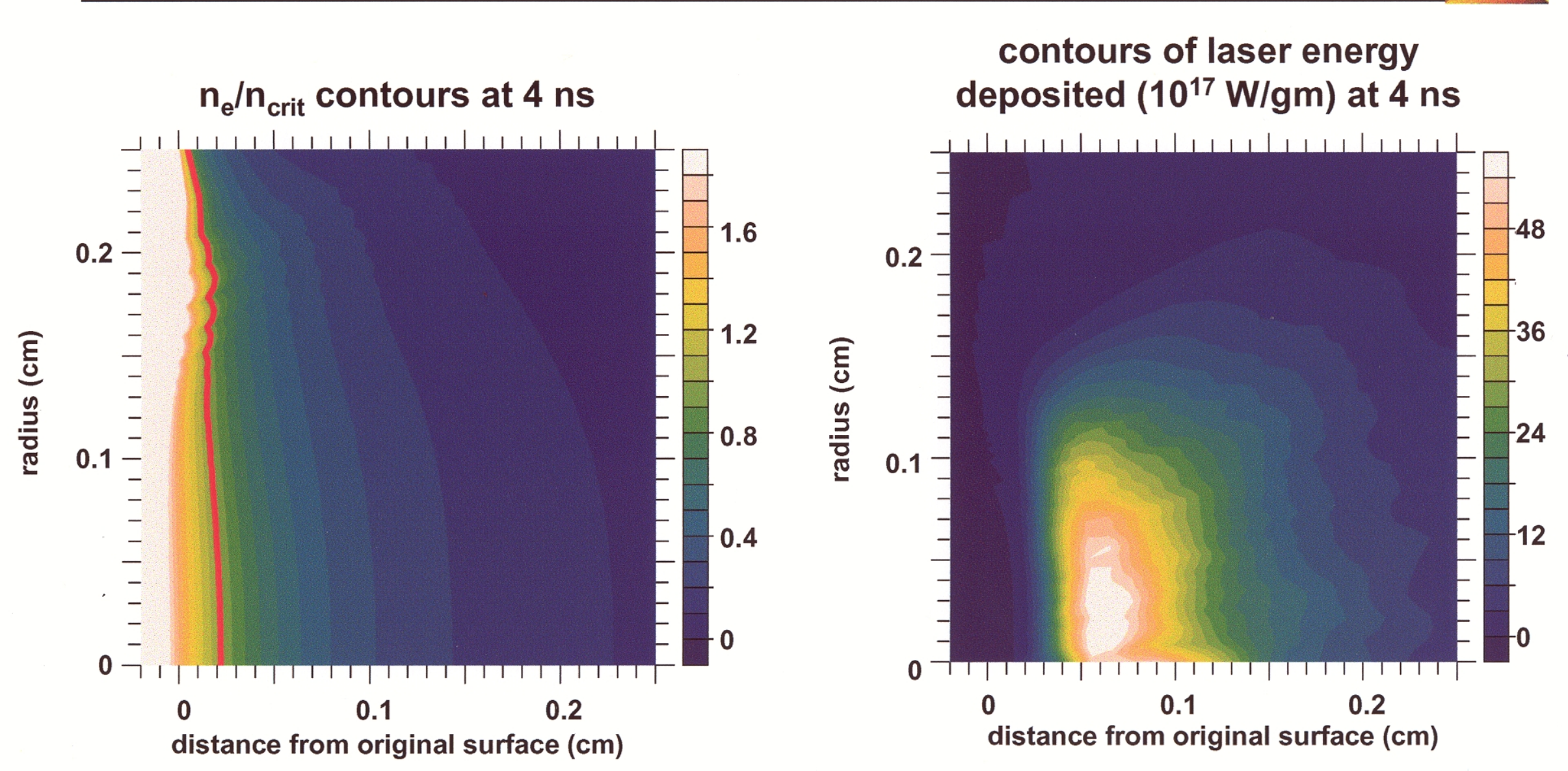

the region of laser absorption is comparable to the $0.1 \mathrm{~cm}$ radius spot, so that a $2 \mathrm{D}$ simulation is required 


\section{The deviation of the results from the 1D simulation scales with laser intensity}

ratio of shock pressure in graphite, 2D / 1D

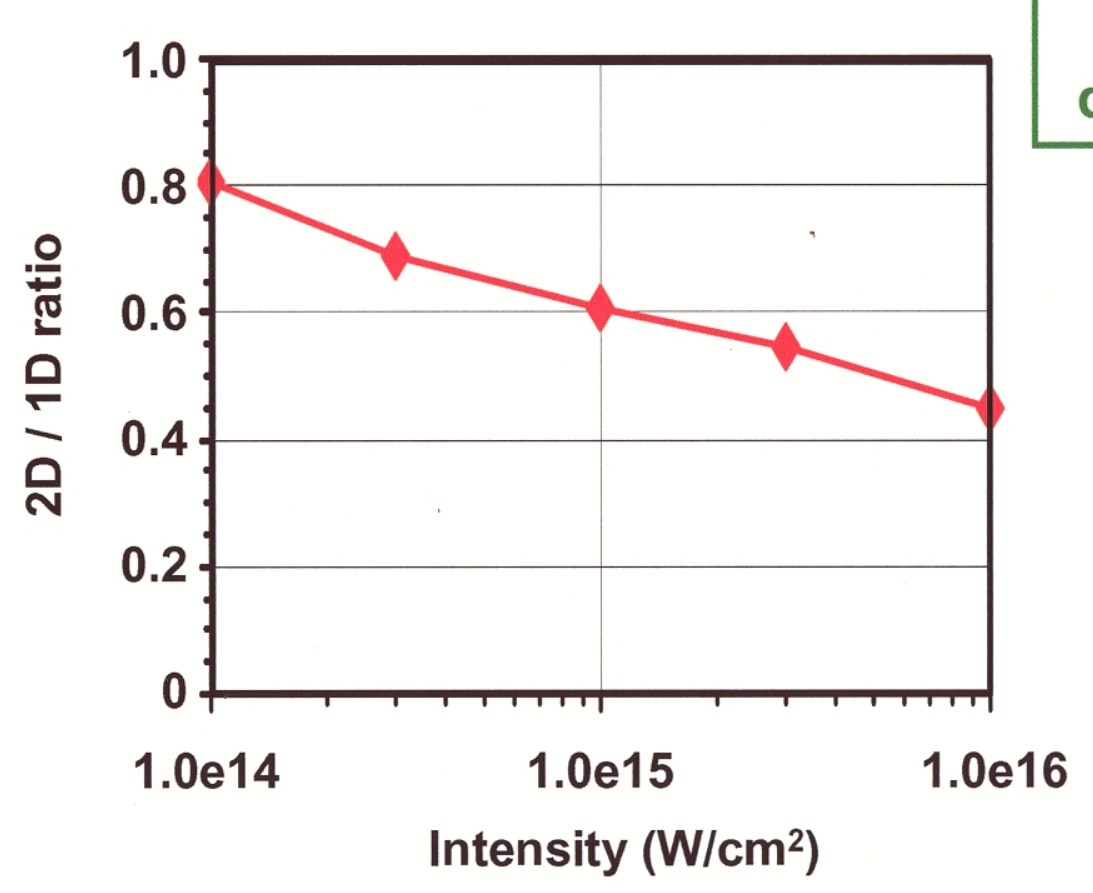

absorption axial location of critical density in 2D simulations $\mu \mathrm{m}$ spot, data at 4 ns

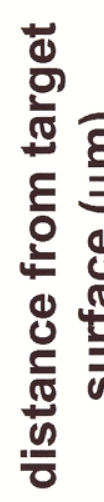

$1.0 \mathrm{e} 14$

Intensity $\left(\mathbf{W} / \mathrm{cm}^{2}\right)$

$\mathrm{n}_{\mathrm{e}} / \mathrm{n}_{\text {crit }}=0.5$

$\mathrm{n}_{\mathrm{e}} / \mathrm{n}_{\text {crit }}=1.0$

(1.0

$1.0 \mathrm{e} 15 \quad 1.0 \mathrm{e} 16$

\section{increased laser intensity ablation rate}

$\Rightarrow$ further from surface geometry more 2dimensional 


\section{An order of magnitude increase of the spot size is required to recover the $1 \mathrm{D}$ results}

2D simulations with laser ramp to $I=1.0 \mathrm{e} 16 \mathrm{~W} / \mathrm{cm} 2$ and variable spot size

shock pressure (Mbar) in doped graphite

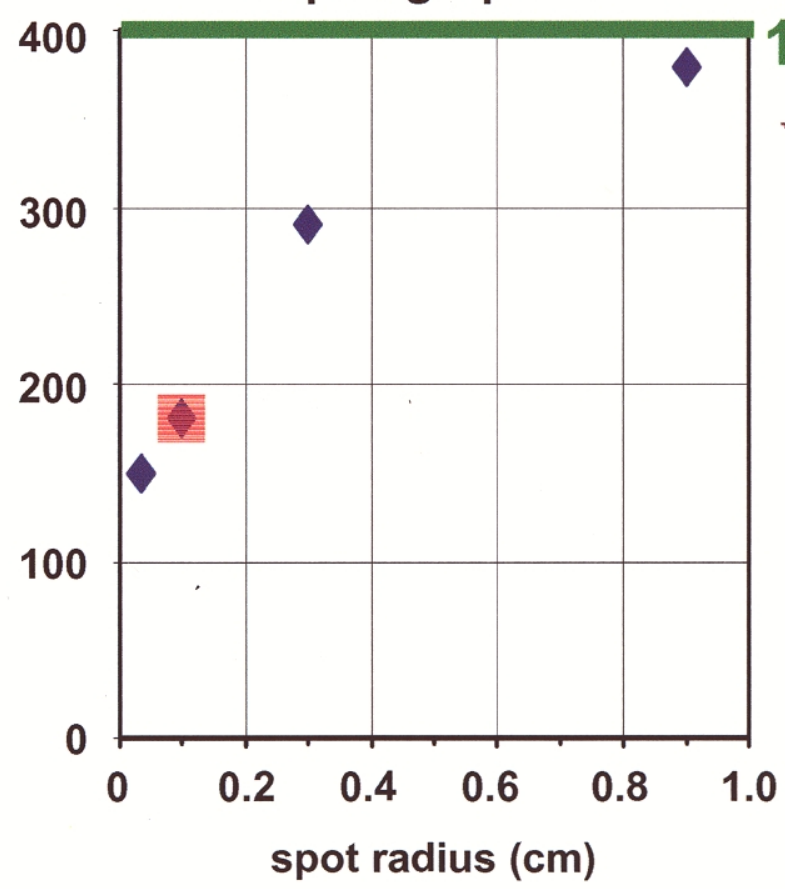

peak Te (kev) in corona

$1 \mathrm{D}$

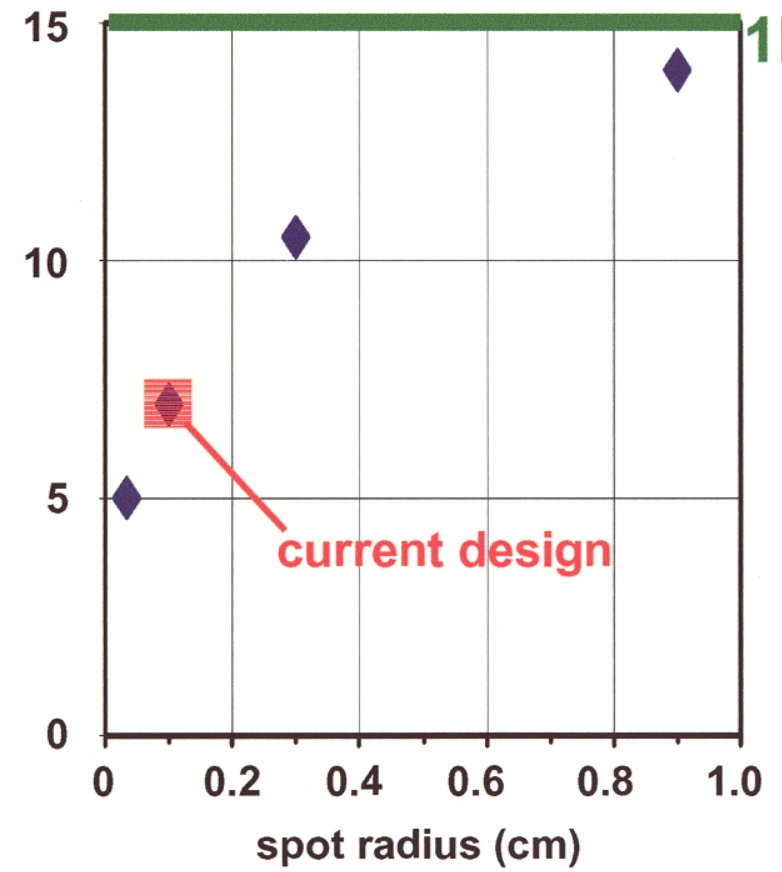

axial position $(\mathrm{cm})$ of $\mathrm{n}_{\text {crit }}$ at $4 \mathrm{~ns}$

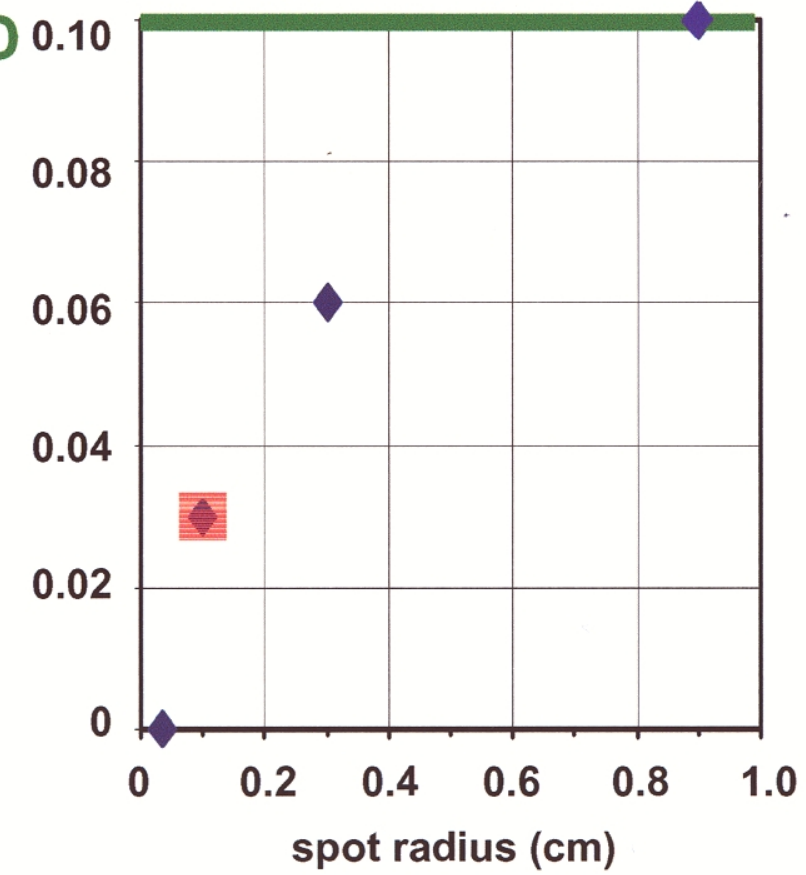

- at high intensity, power limitations prohibit even $2 x$ increases in spot size - the experiments are inherently 2D, their performance limited by radial transport 


\section{Dominated by radial transport, coronal plasma conditions}

depend on the value chosen for the flux limiter

- Lasnex limits the maximum electron energy flux to a fraction of its free-streaming value, $\Gamma^{\max }=f^{*} n_{e} v_{e}{ }^{\text {th }}$

- Decreasing the flux limiter from 0.1 to 0.03 in the NIF experiment;

increases $T_{e}$ in the corona,

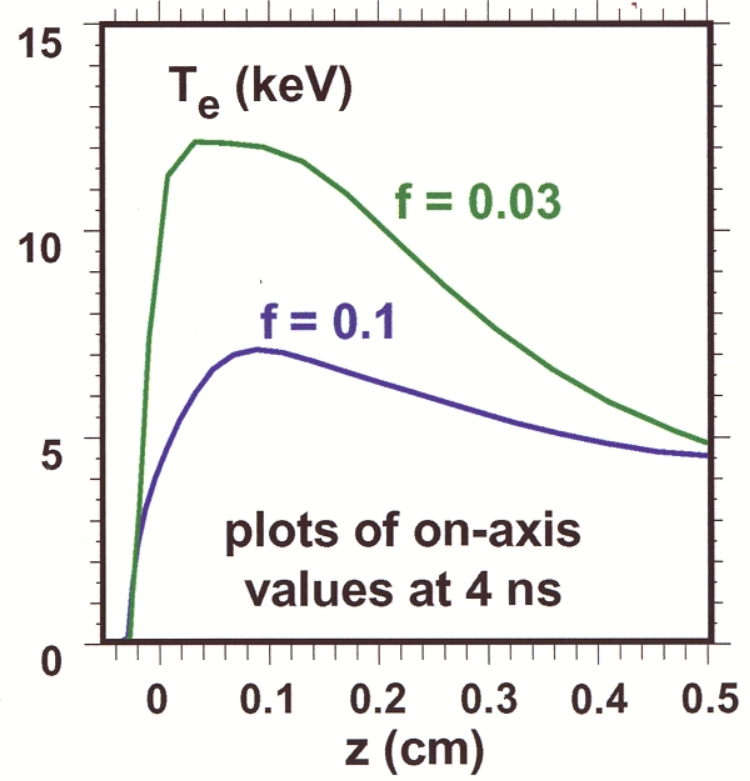

which causes the density to decreases,

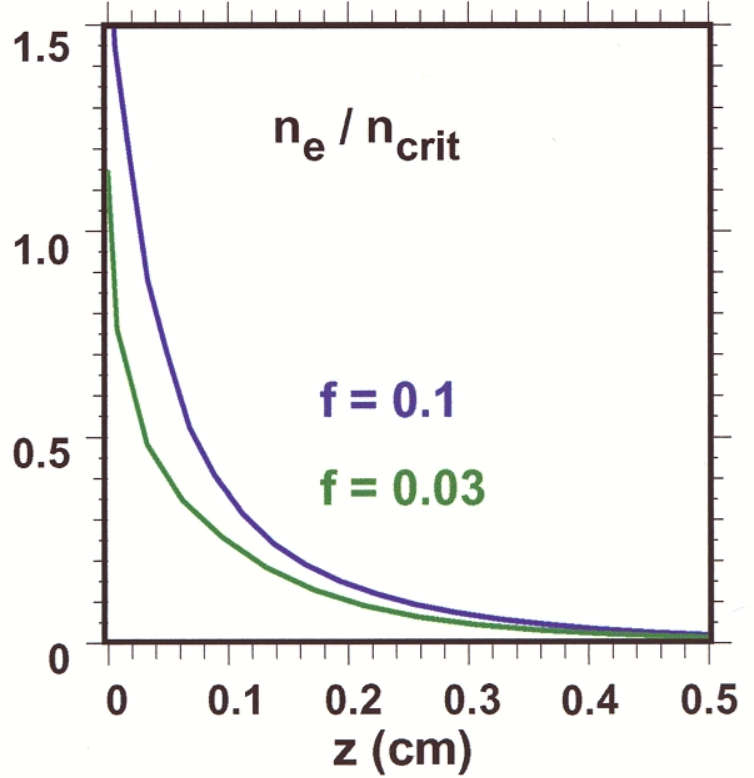

so that the pressure does not significantly increase

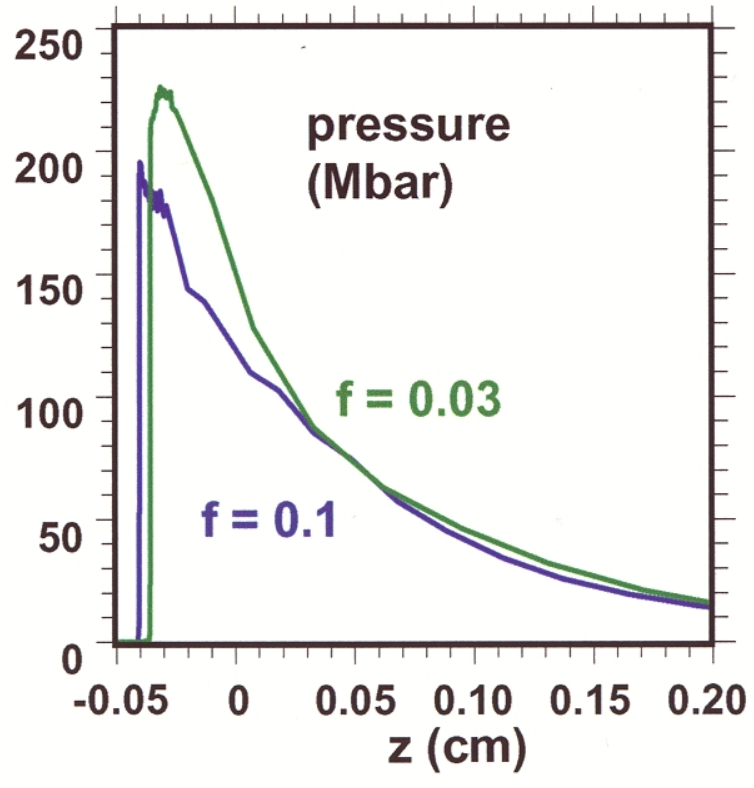

- Initial experiments at moderate intensity $\left(4^{*} 10^{14} \mathrm{~W} / \mathrm{cm}^{2}\right)$ are well modeled by $f=0.1$

- A different flux limiter may be appropriate at the high intensity NIF experiments 


\section{Self-generated magnetic fields in the corona may restrict electron energy transport}

- Ohm's law, balancing the ambipolar field and the pressure,

$$
e n_{e} \vec{E}=-\vec{\nabla} p_{e}
$$

combined with Faraday's law,

$$
\frac{\partial \vec{B}}{\partial t}=-\vec{\nabla} \times \vec{E}
$$

predicts magnetic field generation

$$
\frac{\partial \vec{B}}{\partial t}=\frac{k \vec{\nabla} n_{e} \times \vec{\nabla} T_{e}}{e n_{e}}
$$

- Magnetic field generation is greatest at the edge of the corona, where the gradients are not parallel calculated magnetic field density (MG) at 4 ns for the NIF target

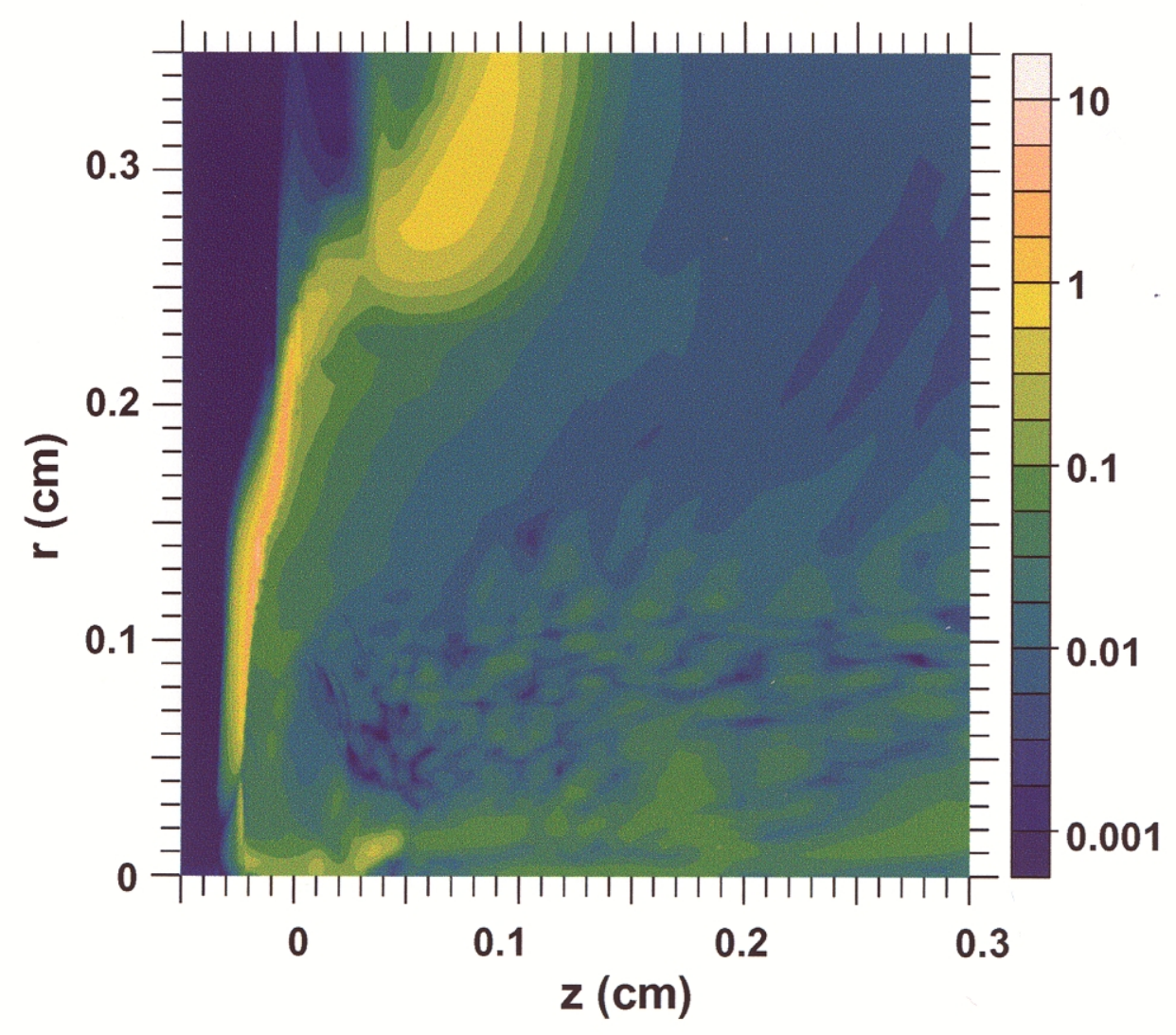




\section{The generation of magnetic fields can also result in increased values of $T_{e}$ in the corona}
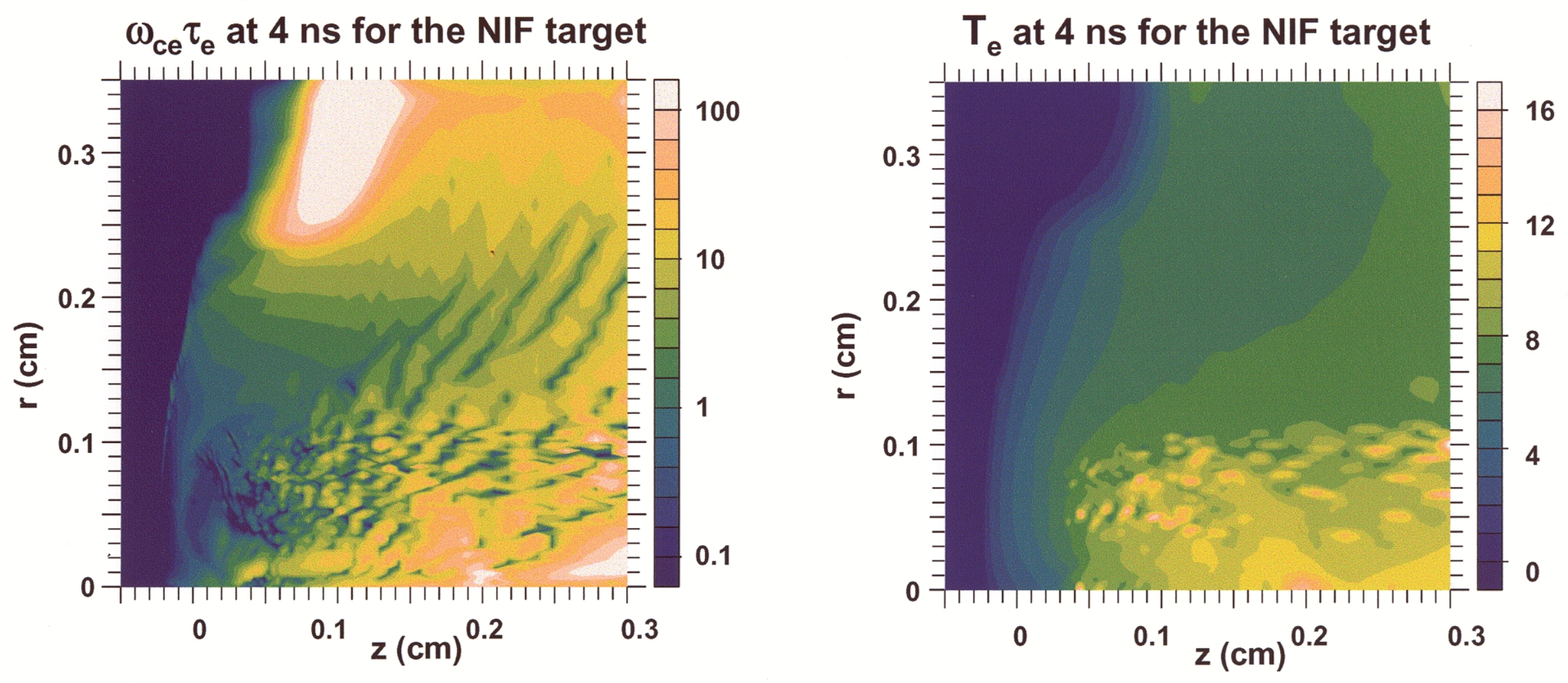

- $\mathrm{T}_{\mathrm{e}}$ is increased from 7 to $\sim 12 \mathrm{keV}$ with this magnetic field model

- predicted increase in $T_{e}$ is model dependent

- experiments are needed to verify the magnetic field model 


\section{$X$-rays generated in the corona degrade the experiment by preheating the high-Z sample}

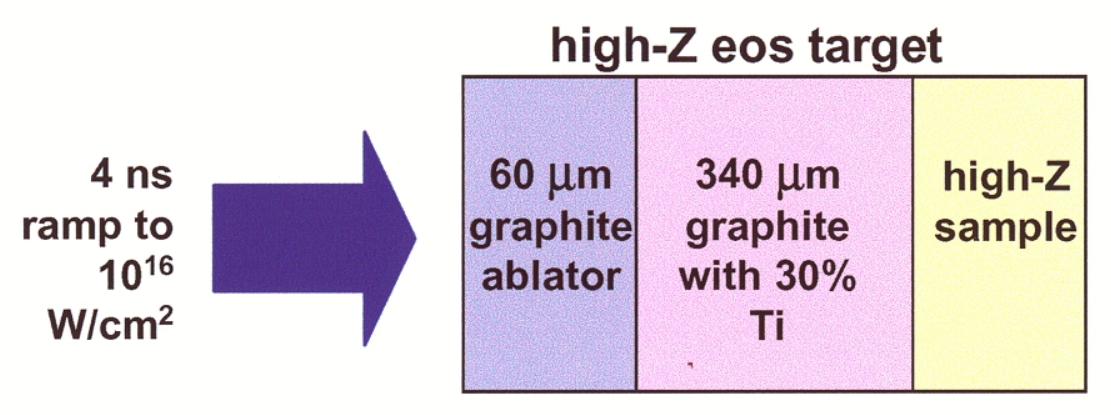

- dopant added to the graphite shields still permits penetration of high energy $x$-rays ( $>10 \mathrm{keV}$ )

- desired preheat level is $<0.1 \mathrm{eV}$

- too much dopant can obstruct the backlighter image
$T_{e}$ profiles on the centerline of

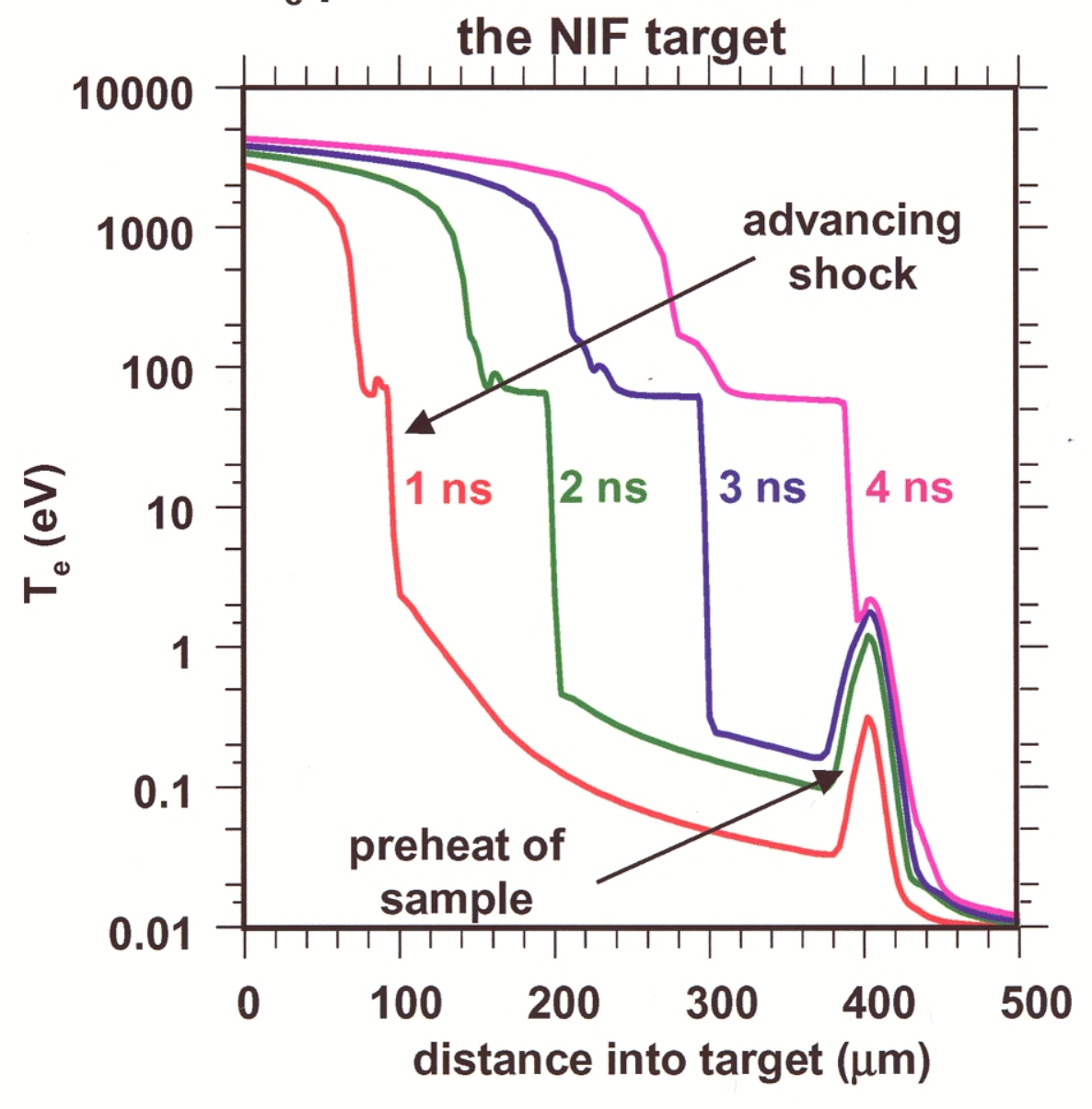

success of the experiment depends on what value of the coronal $\mathrm{T}_{\mathrm{e}}$ is produced at a given pressure 


\section{Summary}

- Direct drive ablation experiments on the NIF will generate shock pressures above $100 \mathrm{Mbar}$ for high-Z equation of state measurements

- Initial experiments at moderate laser intensities have been successfully modeled using LASNEX

- 2D simulations are required as radial transport dominates coronal energy losses, especially in the high intensity NIF experiments

- The value of $T_{e}$ in the corona may vary by a factor of 2 depending on the transport model

- The experiment is very sensitive to the coronal $T_{e}$, as preheat of the high-Z sample by high energy $X$-rays degrades the experiment

- Validation of physics models at high intensity is needed for effective target design

- electron heat transport

- magnetic field generation 


\title{
Magnetic fields \& electron transport In high energy density hohlraums
}

\section{Is our capability good enough?}

\author{
L \\ Lawrence Livermore \\ National Laboratory \\ 1952-2002 \\ John Edwards \\ Jim Hammer, Judy Harte, Ed Alley, George Zimmerman \\ Lawrence Livermore National Laboratory
}

\author{
Malcolm Haines \\ Imperial College, London, UK
}

This work was performed under the auspices of the U.S. Department of Energy by the University of California, Lawrence Livermore National Laboratory under contract No. W-7405-Eng-48. 


\section{Introduction}

Electron transport workshop

9-11th Sepetmber

Purple Orchid Inn, Livermore 2002 


\section{So what's changed?}

Despite all that, we have strong evidence B-fields are important in at least some aspects of hohlraums
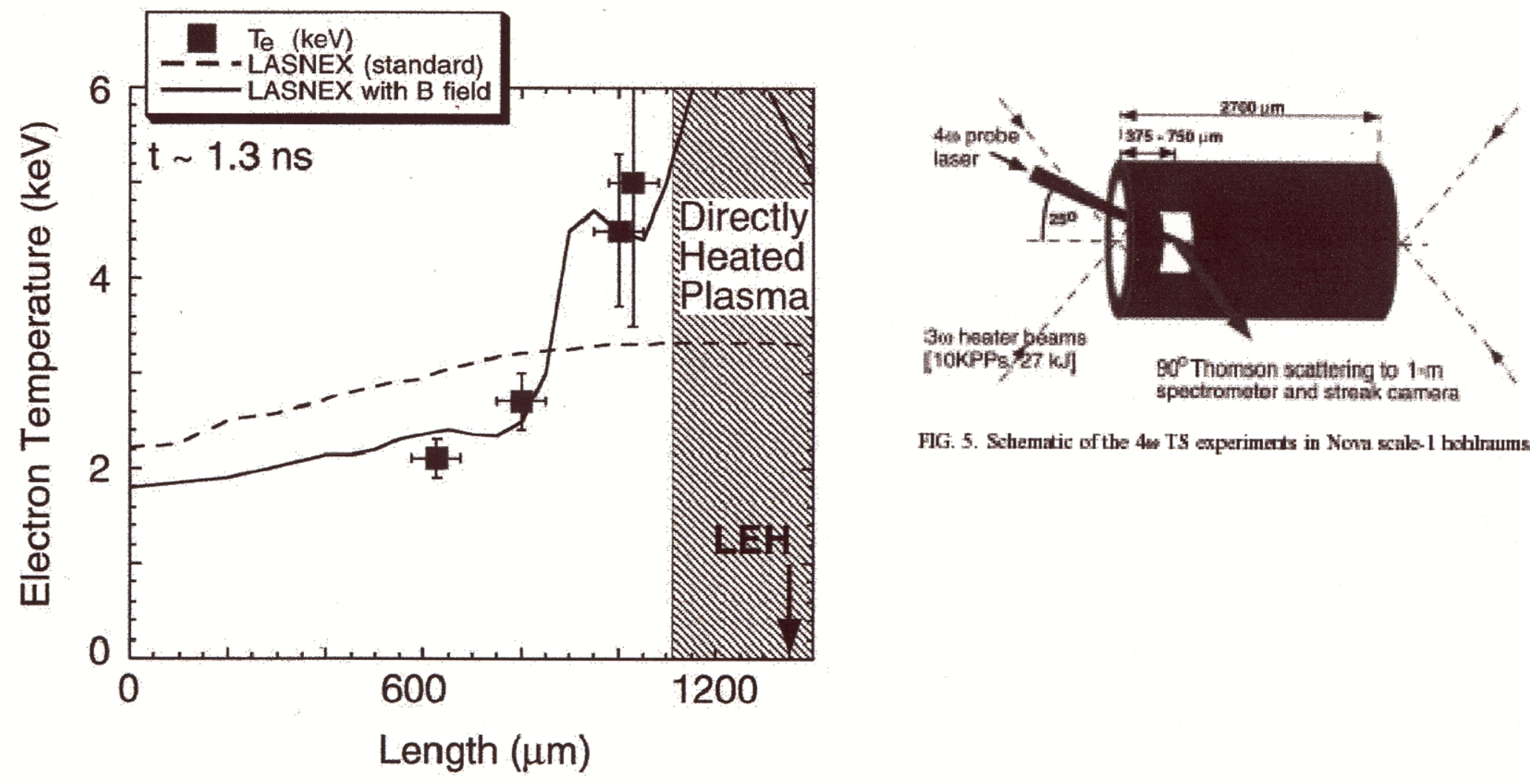

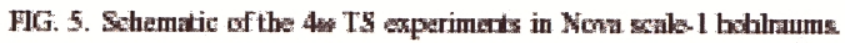




\section{Is there really a problem?}

In the early days of laser fusion:

Spitzer-Harm inapplicable

$B$ via $\nabla \mathbf{n} X \nabla \mathbf{T}$ recognized

At high intensities bad stuff began to happen with direct drive $\Rightarrow$ so for this \& other reasons we went to indirect drive

Nova was a spectacular success \& led to NIF

There was no indication that non-local transport or B-fields were a big issue. Hohlraums worked just fine (with a flux limiter $\sim 0.1$ ) (exceptions are small hohlraums - we have no idea why yet!)

In the mean time the rest of the world plugged away with direct drive Both this and LPI in hohlraums drove a very aggressive \& Very successful beam smoothing effort 


\section{So what's changed......?}

NIF is very much closer now!

When we do something different there's often a surprise

We're planning to do much more with NIF than ignition and go into regimes that are far from our current experiences

The beam smoothing success has seduced us into thinking that maybe we can take advantage of better over all coupling into targets compared to indirect drive

Ultra-high intensity lasers => proton probing - electro-thermal-instability?

Short pulse lasers for physics studies 


\section{Workshop objectives}

Our focus this time round is "long pulse" regime especially for high energy density hohlraums \& direct drive

What physics do we need to include?

What is a sensible way forward computationally?

Fokker-Planck something else (eg Monte Carlo) reduced model

What developments \& benchmarks do we need?

What should we do to test our ideas \& models? 


\section{Theory \& computation}

Fokker-Planck for laser plasmas in 1D (Bell, 1981)

Then in 2D (Epperlein, 1988)

Now 2D with $B$ to $f_{1}$ keeping $\mathrm{df}_{1} / \mathrm{dt}$ (Kingham, 2002)

At the same time, driven by extreme cost of FP People got busy making reduced non-local models to use in hydrocodes (1D, Luciani, 1983; .... 2D, Schurtz, 2000)

We're now 20 years on is it time to try FP in our design codes? and if not, what? 


\section{The failure of Spitzer in laser plasmas is not news}

We use $q \sim \kappa \nabla T$ which we know breaks down

To fix it we set $\quad q \leq f q_{f s} \quad$ where typically $\mathbf{f} \sim 0.1$

Some results from 2D Fokker-Planck coupled to hydro are:

$$
\frac{q}{q_{f s}}=\mathrm{g}(\overrightarrow{\mathrm{r}}, \mathrm{t}) \quad \frac{q}{q_{S H}}=h(\overrightarrow{\mathrm{r}}, \mathrm{t}) \quad q \nVdash \nabla T
$$

And then there's magnetic fields....... 


\section{A simple estimate indicates we need to worry about non-local effects in all laser plasmas}

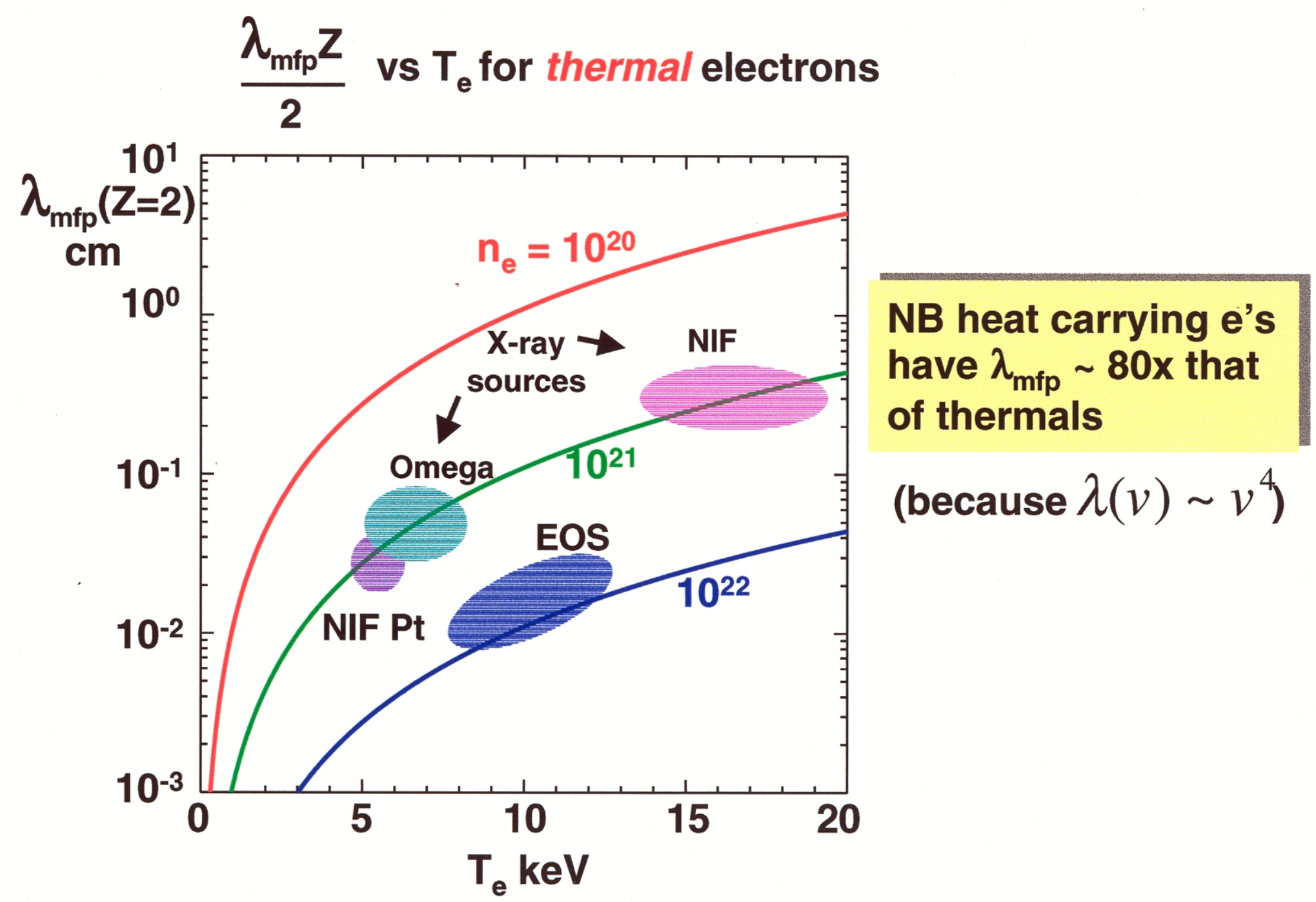




\section{Even a small field can effectively localize electrons}

Lamour radius $a_{e}=\frac{m_{e} v}{e B}$

For thermals $v \sim\left(2 \mathrm{kT} / \mathrm{m}_{\mathrm{e}}\right)^{1 / 2} \Rightarrow \mathrm{a}_{\mathrm{e}}=\frac{\mathrm{T}_{\mathrm{e} \text { keV }}^{1 / 2}}{\mathrm{~B}_{\mathrm{MG}}} \mu \mathrm{m}$

Fields of $\sim 1 \mathrm{MG}$ are adequate to localize magnetized electrons for nearly all typical NIF plasmas 
The magnetic field is generated (mainly) via $\nabla n \times \nabla T$ at the hohlraum wall

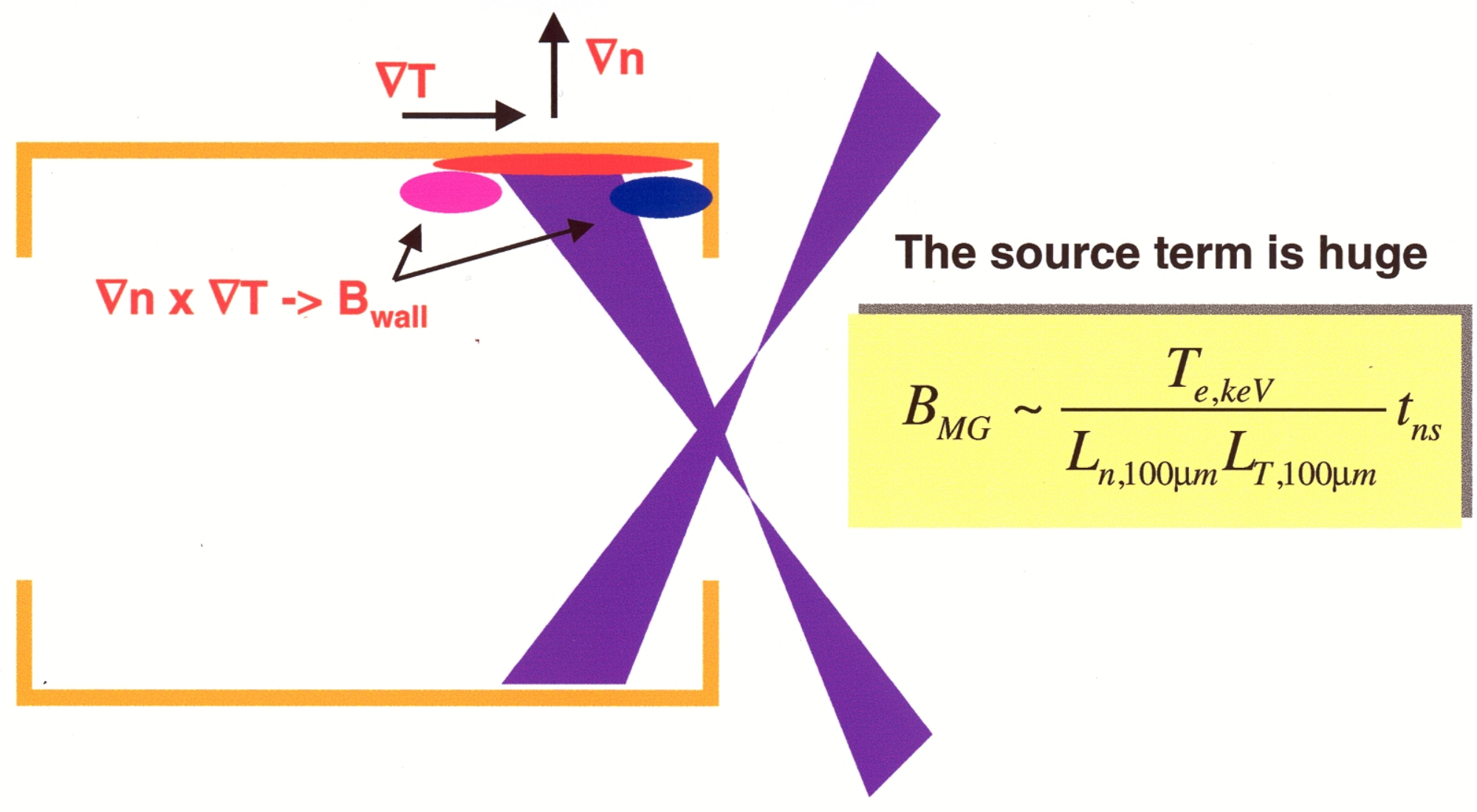




\section{But convection rapidly limits the}

\section{field near the wall to $\sim 1 \mathrm{MG}$}

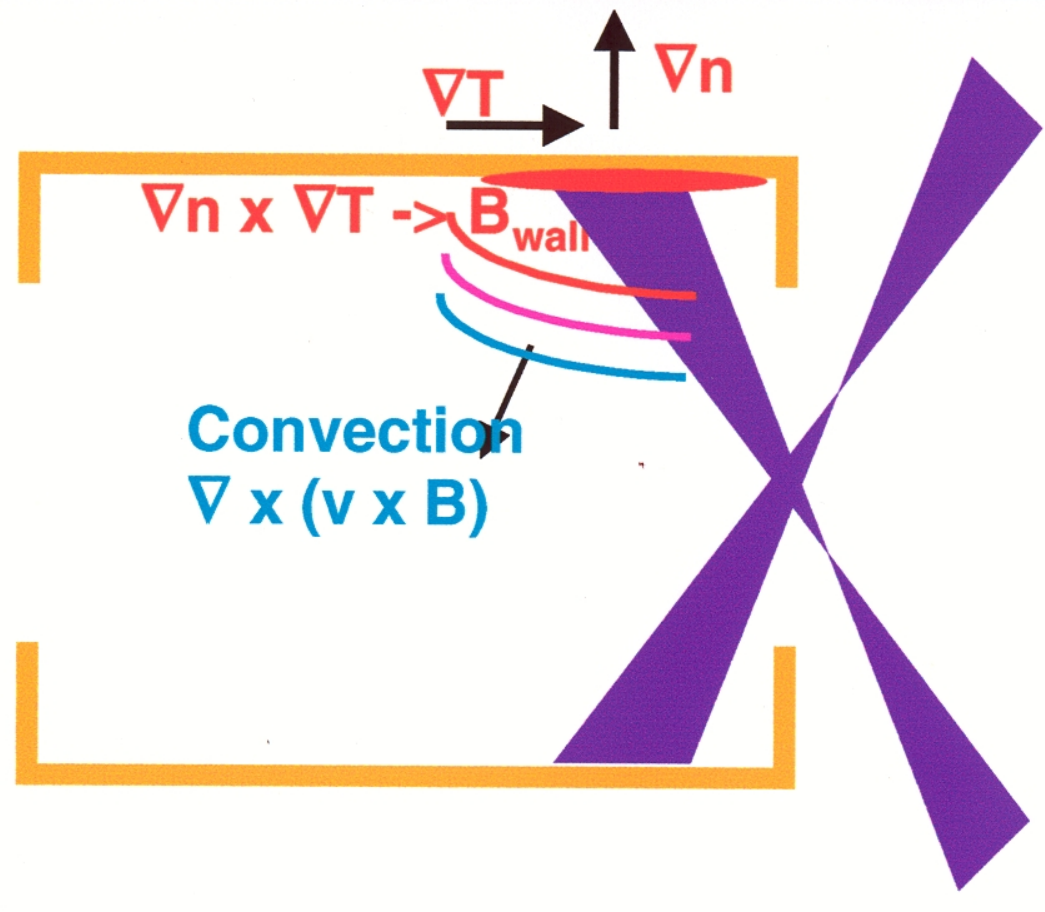

Balancing hydro \& source for $B$ near the wall

$$
\begin{aligned}
& B_{M G} \sim \frac{T_{e}}{c_{s} L_{T}} \sim \frac{1}{L_{T, 100 \mu m}} \frac{T_{e, k e V}^{1 / 2}}{T_{R, h e V}^{1 / 4}} \\
& \Rightarrow \mathbf{B}_{\mathrm{MG}} \sim \mathbf{1} \mathbf{M G}
\end{aligned}
$$

This happens on a time scale

$$
\begin{aligned}
t_{n s} \sim \frac{L_{n}}{c_{s}} \sim \frac{L_{n, 100 \mu m}}{T_{R, h e V}^{1 / 4} T_{e, k e V}^{1 / 2}} & =>\mathbf{t}<1 \mathrm{~ns}
\end{aligned}
$$




\section{Indirect evidence of B fields in hohlraums was seen on Nova}
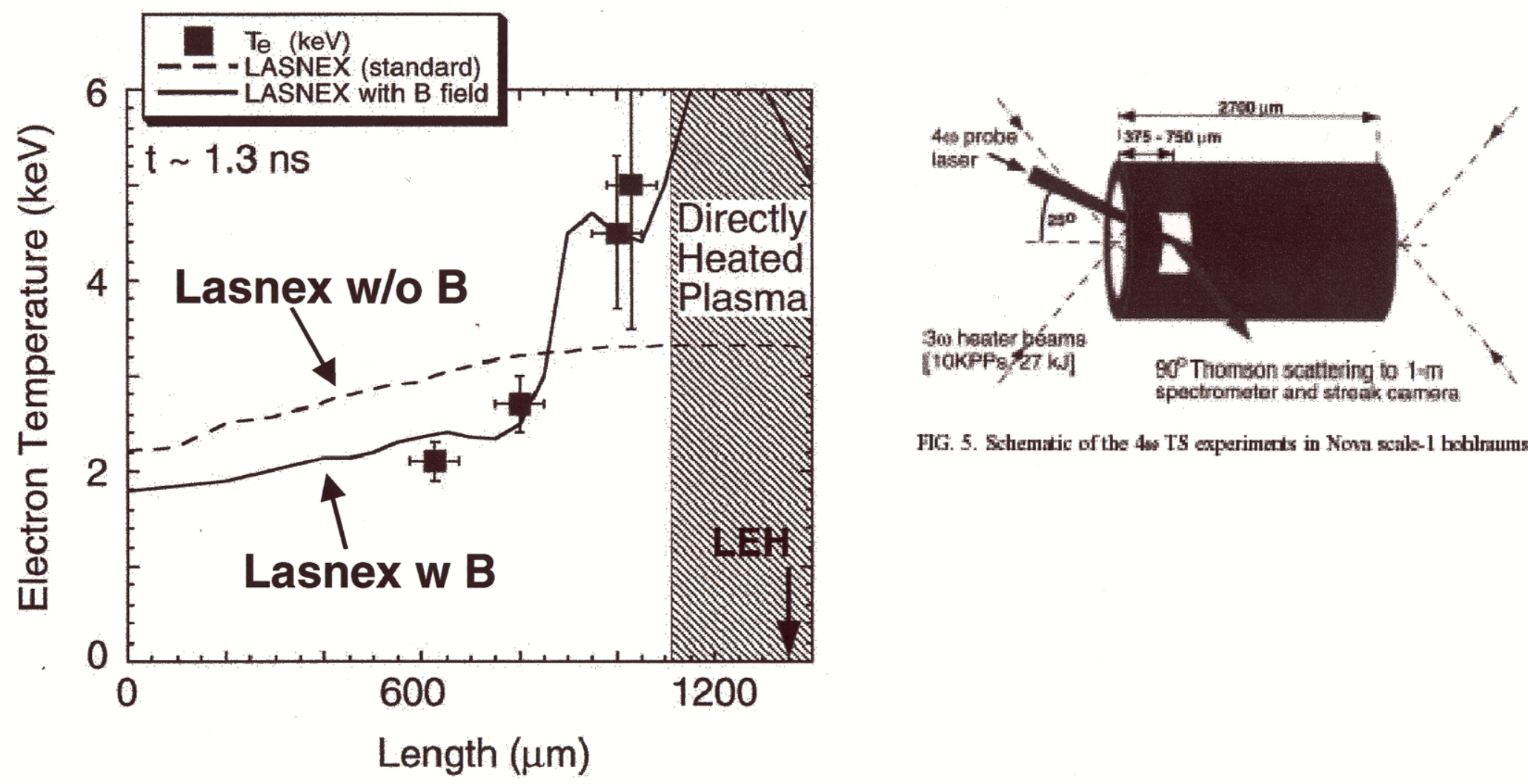

FIG. 5. Schematic of the 4s TS experiments in Nonn scals-1 bohlmums

Siegfried Glenzer Nova measurement Lasnex's from Jim Hammer \& Kent Estabrook 


\section{This is what we expected/saw in studies based around this experiment}

Relatively slow scaling of B in hot spots No saturation in the volume (time scale too short)

$\omega \tau<<1$ in the gold $\omega \tau \gg 1$ in the gas

local heat flow approximation in general marginal

The major consequences of this for ICF are most likely to be related to eg beam pointing, LPI, hard X-ray asymmetry in double shell capsules 


\section{Based on a wide variety of recent hohlraum calculations from 10-500 TW we find}

The major impact of including B

(rather independent of power or size):

$$
\begin{aligned}
& \mathrm{T}_{\mathrm{e}, \text { LEH }} \text { (with B) } \sim 2 \times \mathrm{T}_{\mathrm{e}, \text { LEH }} \text { (without B) } \\
& \mathrm{T}_{\mathrm{R}} \text { NOT affected }
\end{aligned}
$$

Possible knock on consequences need to be investigated LPI, LEH beam energy transfer, hard X-ray production, beam pointing, drive symmetry, preheating

Local heat flow approximation appears marginal need better heat flow - difficult problem!

We need good experiments that measure and correlate $T_{e} \& B$ 


\section{We'll consider just 2 gas filled examples to illustrate this}

1)

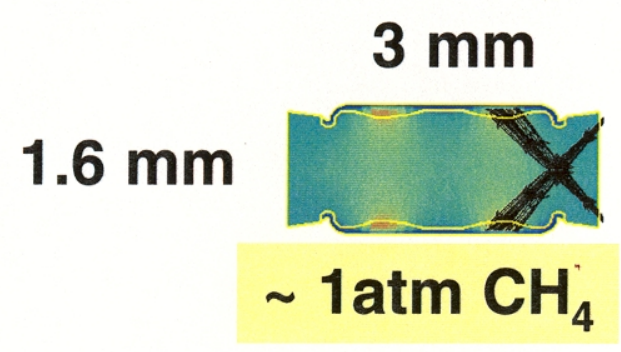

Nova scale 1 - 75\% LEH 200 TW

(calculations

spanning 10-500 TW produce very similar conclusions with non-local effects becoming gradually worse as power is increased)

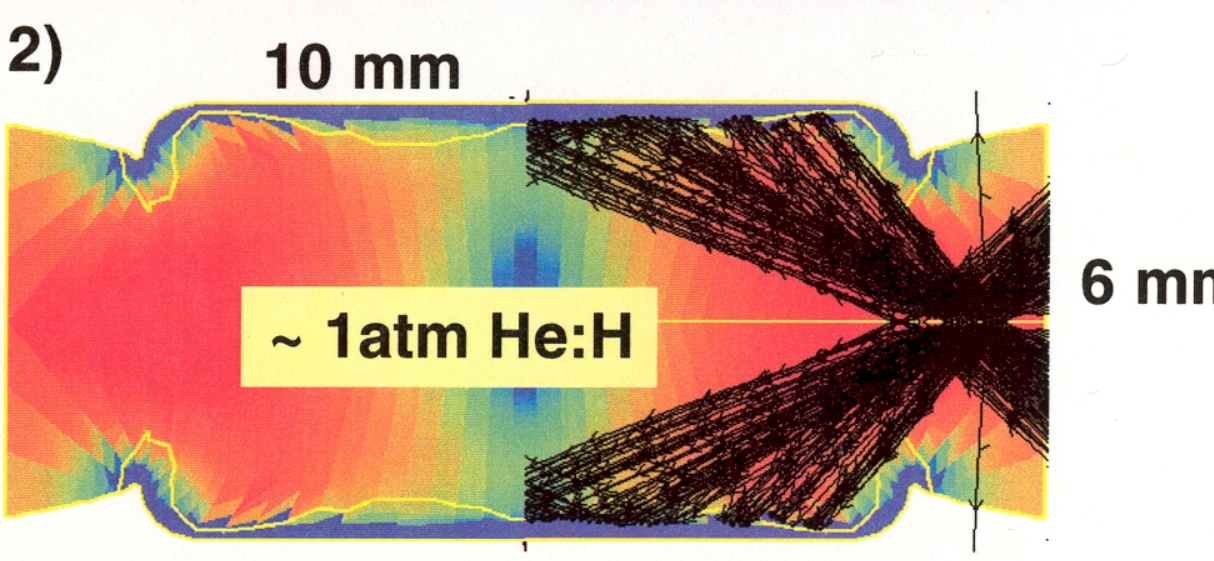

NIF ignition scale - $75 \%$ LEH $\sim 400$ TW 


\section{B field is formed at the wall \& gradually convected into the hohlraum}

\section{TW: magnetic field evolution}

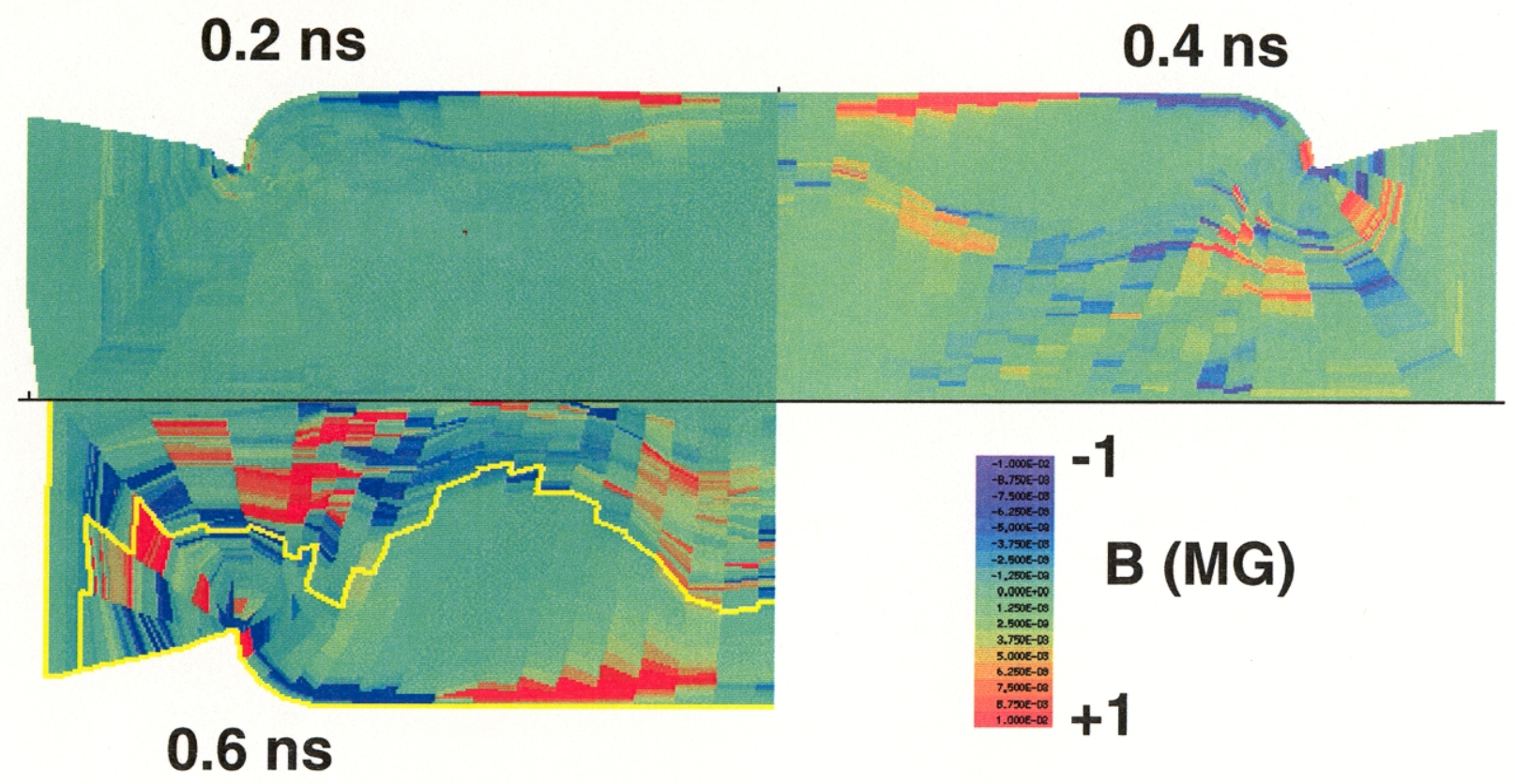

fields rapidly approach $\sim 1 M G$ 


\section{The main difference when $B$ fields are included is a much higher $\mathrm{T}_{e}(\sim \times 2)$ in the LEH}

$0.6 \mathrm{~ns} ; 200 \mathrm{TW}$

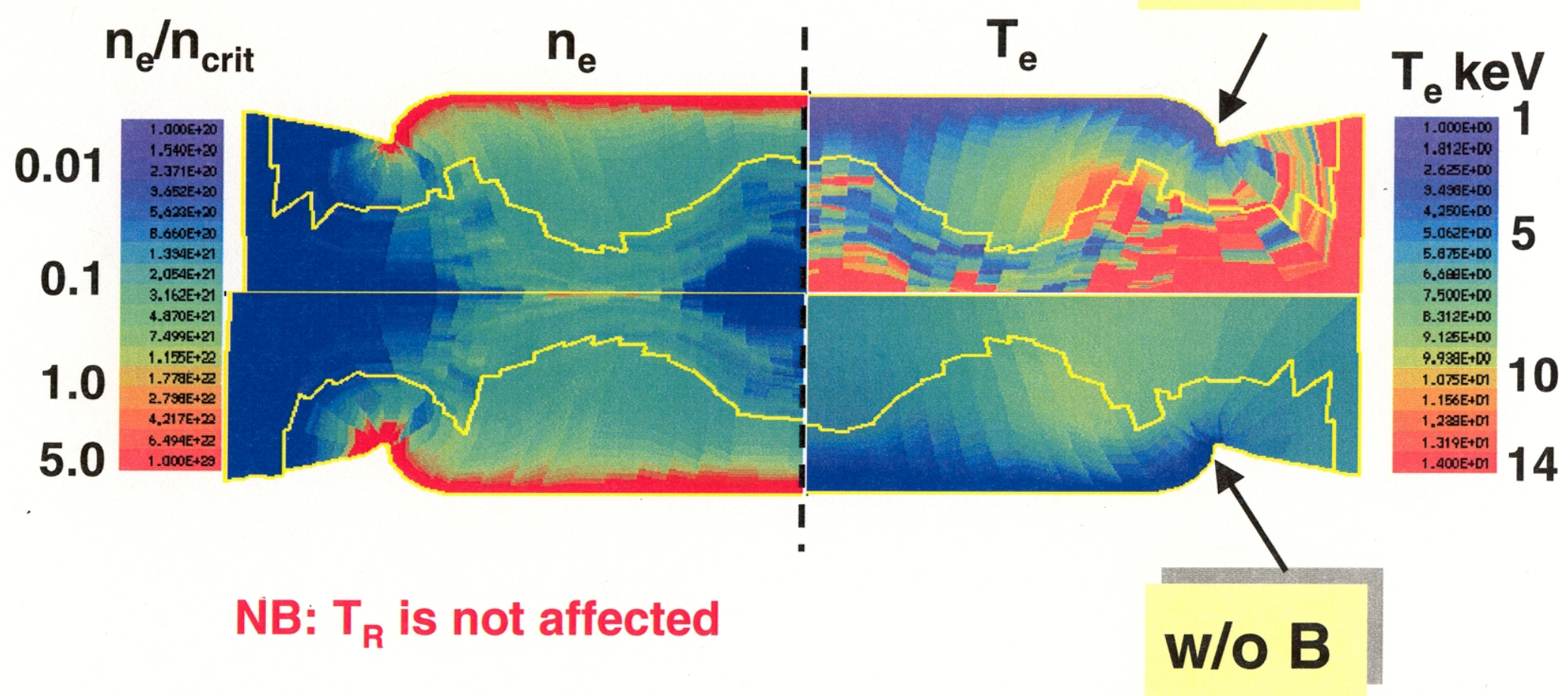

We find this to be very typical of a wide variety of hohlraums 
Righi-Leduc heat flow is responsible for this

\section{6 ns; 200 TW - Te}

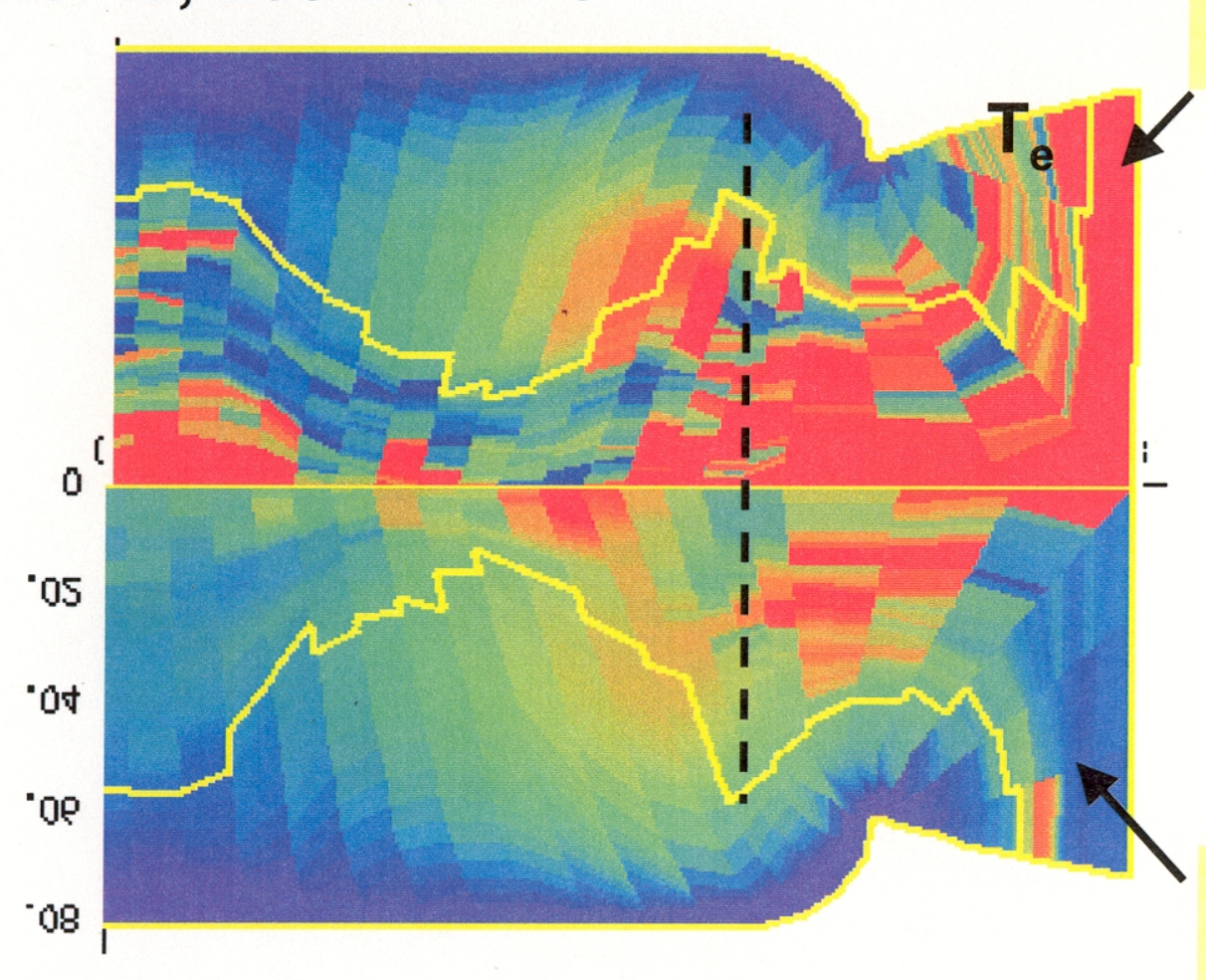

\section{RL X1.0}

$\mathrm{T}_{\mathrm{e}} \mathrm{keV}$

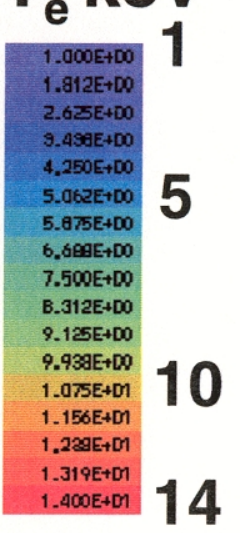

RL X0.1 


\section{Despite significant B-fields local transport is questionable}

0.6 ns; 200 TW

$$
\frac{\lambda_{\text {thermal }}}{L_{T}}
$$

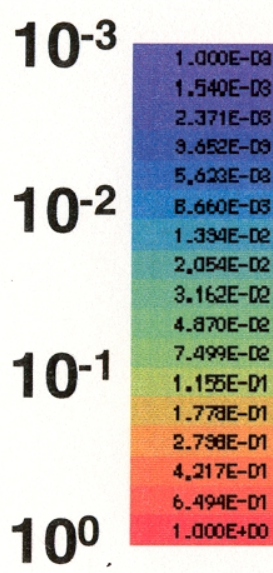

$$
V_{c}^{6}=\frac{L_{T}^{2}}{2 a_{e}^{2}}+\left[\frac{L_{T}^{4}}{4 a_{e}^{4}}+\frac{L_{T}^{2}}{\lambda_{e}^{2}}\right]^{\frac{1}{2}}
$$

$1.0005+\infty 001$ $1.250 E+L O$ $1.750 E+D 0$ $2,000 E+D 0$ 2. $250 E+D 0$ $2.500 E+D 0$ $3.000 E+\infty 0$ $3.000 E+D 0$
$3.250 E+D 0$ $3.500 E+\infty 0$
$3.750 E+\infty$

$4.000 \mathrm{E}+\mathrm{D}$ $4.250 E+\infty$ 4. $250 E+D 0$ non-local 3 local $\checkmark$ 5

\section{$a_{\text {thermal }}$ $L_{T}$}




\section{It slowly gets worse (more non-local) as the power goes up}

\section{$\mathbf{V}_{\text {crit }}$}

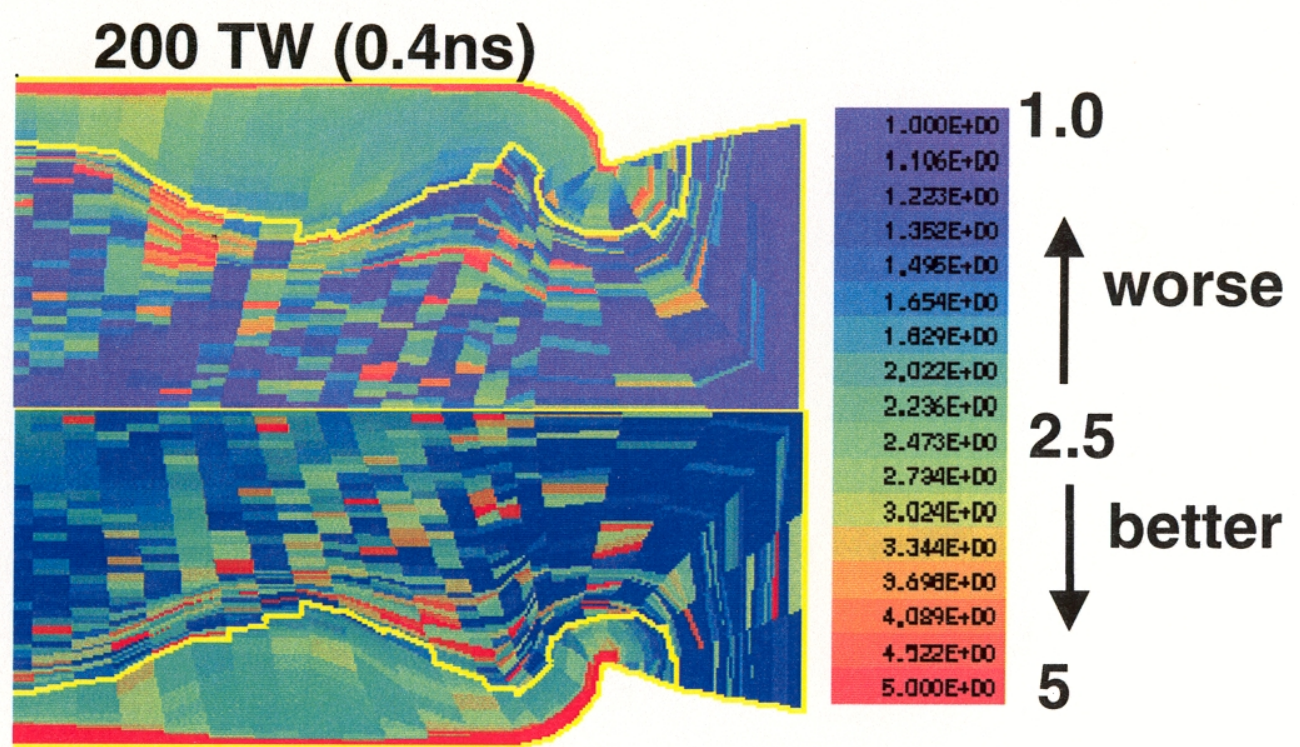

50 TW (0.5ns) 


\section{Righi-Leduc makes the calculation "noisy"}

$\&$ is often responsible for crashes, but IS needed

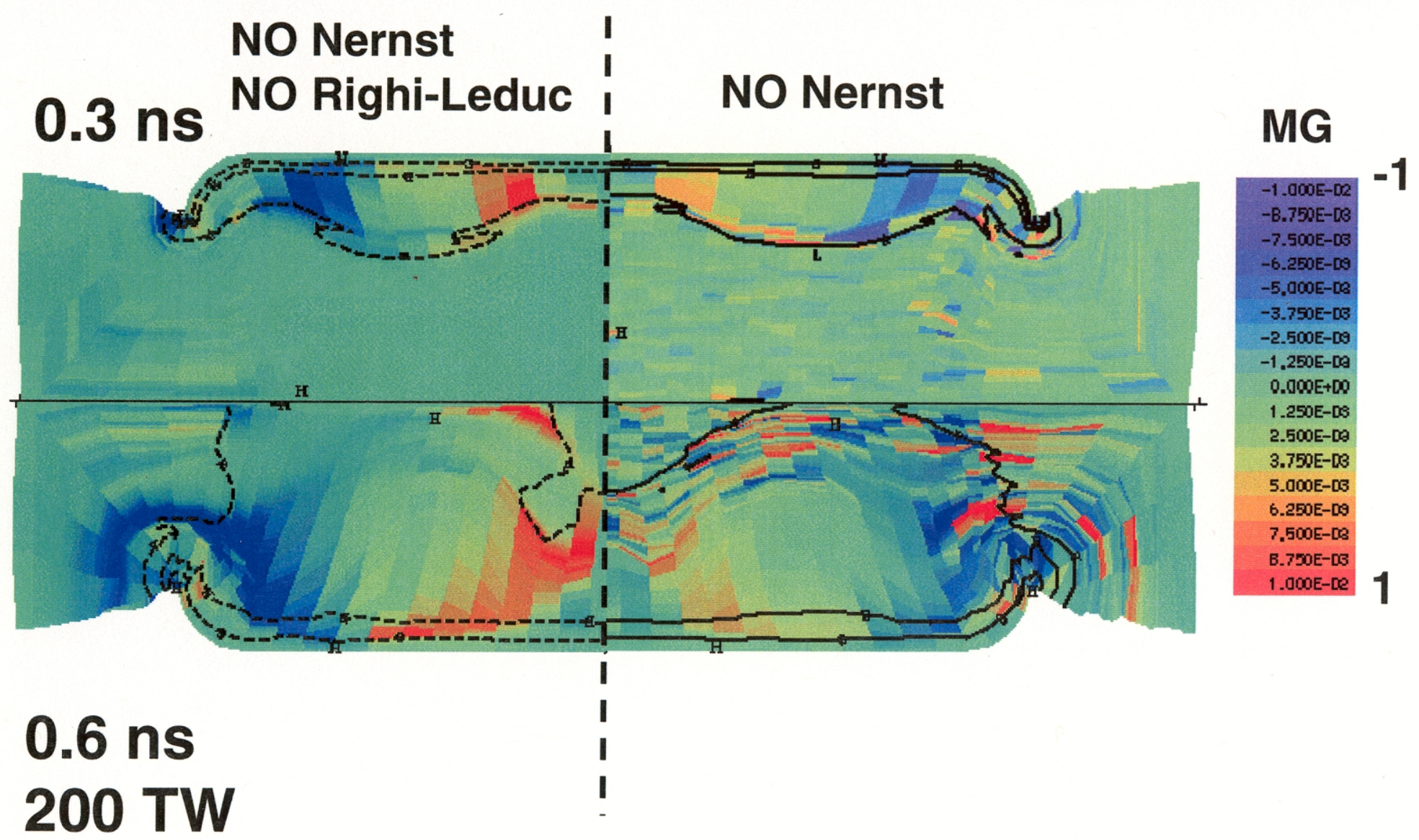




\section{"Righi-Leduc" can also drive a thermomagnetic instability}

A key point is that $\nabla T \& \nabla n$ are parallel: $\nabla T . \nabla n>0$

B field generation from $T$ perturbation
Heat flow

reinforces $\mathrm{T}$ perturbation

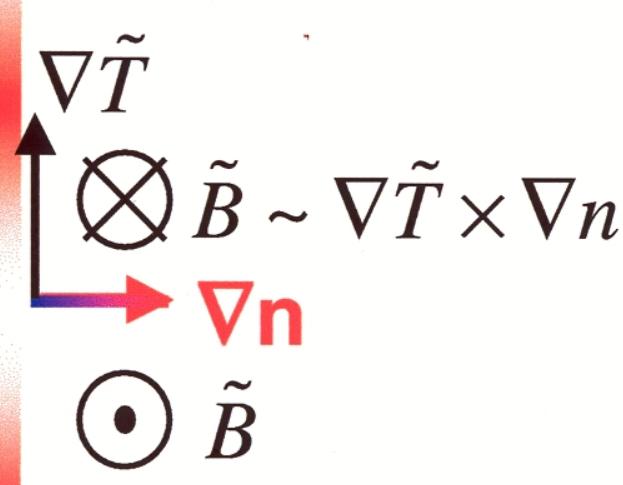

$$
\begin{aligned}
& q_{R L} \sim-\kappa_{\wedge} \tilde{B} \times \nabla T \\
& \otimes \\
& \longrightarrow \nabla T \\
& \odot \tilde{B} \\
& q_{R L} \sim-\kappa_{\wedge} \tilde{B} \times \nabla T
\end{aligned}
$$

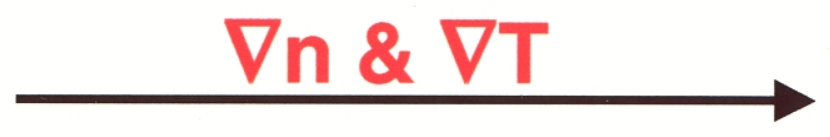

Tidman \& Shanny 1974 


\section{Conditions in the gas are perfect for thermomagnetic instability!}

cos (angle between $\nabla T \& \nabla n_{e}$ )

\section{TW}

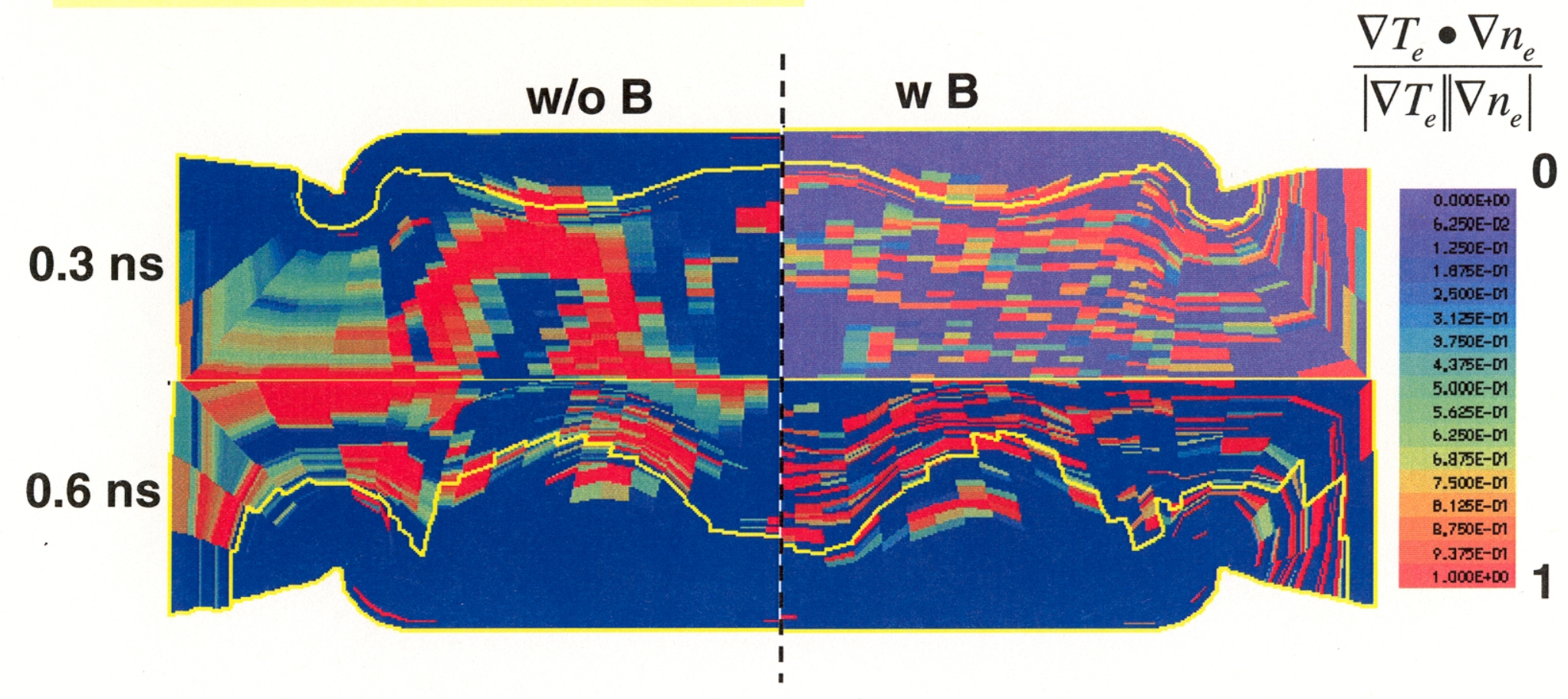




\section{Thermomagnetic instability results 1 (Tidman \& Shanny, 1974)}

$\nabla T \& \nabla n$ parallel: $\nabla T$. $\nabla n>0$

High wavenumber cutoff driven by field diffusion

$$
\lambda>\lambda_{e} \quad \text { collisions }
$$

Theory needs: $\quad \lambda<<L_{n}, L_{T} \quad$ gradient supports wave

$$
\frac{\omega}{k}>>c_{s} \quad \text { static ions }
$$

$$
\begin{aligned}
& \lambda_{M} \sim \frac{1}{2} \phi^{1 / 2}\left(L_{n} L_{T}\right)^{1 / 2} \quad \gamma \sim \text { const } \frac{T^{5 / 2}}{n_{i} Z^{2} \ln \Lambda} \frac{1}{L_{n} L_{T}} \\
& \phi=\frac{c \ln \Lambda}{v_{e}} \frac{Z}{n_{e} \lambda_{D}^{3}}
\end{aligned}
$$




\section{Thermomagnetic instability results 2 (Tidman \& Shanny, 1974)}

$$
\begin{aligned}
& T \sim 10 \mathrm{keV} ; \quad Z \sim 3.5 ; \quad n_{\mathrm{e}} \sim \mathrm{n}_{\text {ecrit }} / 4 ; \quad(\operatorname{In} \Lambda \sim 8.5) ; \mathrm{L}_{\mathrm{n}} \sim \mathrm{L}_{\mathrm{T}} \sim 0.02-0.2 \mathrm{~cm} \\
& \lambda_{M} \sim \frac{1}{7}\left(L_{n} L_{T}\right)^{1 / 2} \quad \gamma \sim \frac{7 \times 10^{8}}{\left(L_{n} L_{T}\right)_{c m^{2}}} s^{-1} \\
& \lambda_{M} \sim 280 \mu m \quad 1 / \gamma \sim 50 p s \\
& \lambda_{e} \sim 200 \mu m
\end{aligned}
$$




\section{We see the same $\sim \times 2 T_{e}$ elevation in ignition hohlraums}

ignition hohlraum @ 16.2ns near peak drive ( $280 \mathrm{eV}$ in this case)

with B fields

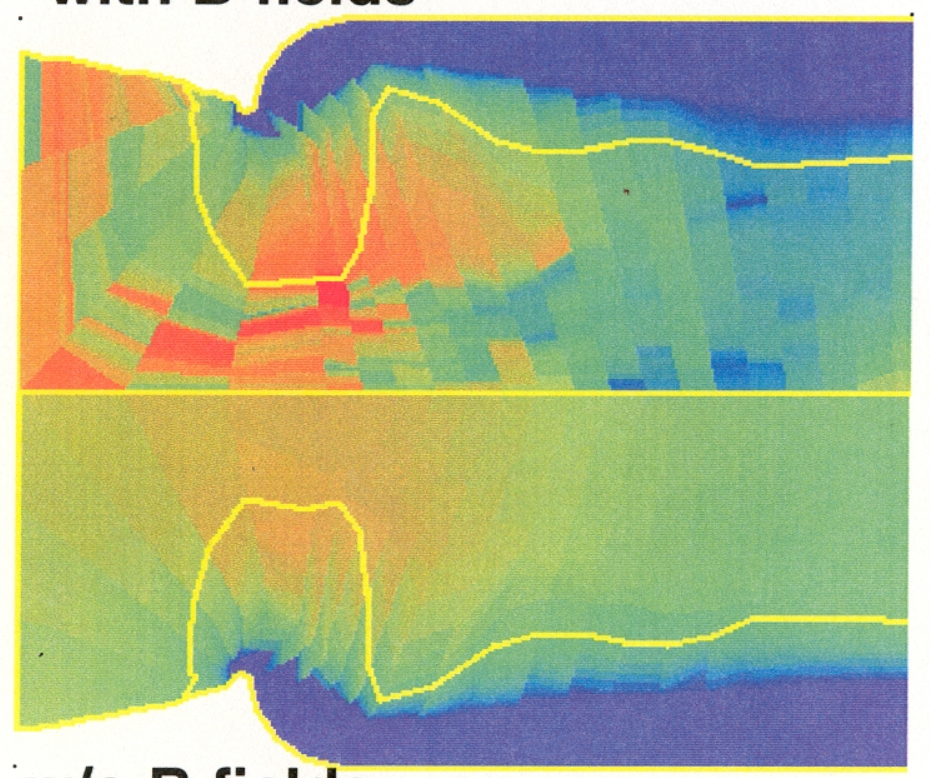

w/o B fields
$\mathrm{T}_{\mathrm{e}} \mathrm{keV}$

$1.000 E+\infty 0$ $1.206 E+\infty 0$

$1.459 E+\infty D$

$1.754 E+\infty 0$

$2,115 E+\infty 0$

$2.550 \mathrm{E}+\mathrm{DO}$

$9.05 E+\infty 0$

a. $709 E+\infty 0$

$4.472 E+D$

$5.393 E+\infty 0$

$6.500 E+\infty 0$

$7.943 E+D$

$9.457 E+\infty 0$

$1.140 \mathrm{E}+\mathrm{OM}$

1,3 BEE+M

$1.659 \mathrm{E}+\mathrm{OM}$

$2.000+1017$
$1-T_{R}$ is not affected

- Knock on consequences will most likely relate to

- LPI

- beam pointing

- energy transfer

- hard X-ray production

local transport appears equally questionable 


\section{Our general conclusions are rather independent of hohlraum or laser power}

As expected the scaling is rather slow with

$B_{\mathrm{VOL}}$ shows no sign of saturation (expected)

If this is right $B$ helps localize the electrons

BUT the local heat flow approximation appears marginal

The major impact of this appears to be in $\mathrm{T}_{\mathrm{e}}$ distribution ( $\sim$ X 2 at LEH when B is included); $T_{R}$ NOT affected

This may affect:

LPI, symmetry, beam pointing, hard X-ray generation.....

For all this to be right we have to be modeling B correctly \& this is linked to the heat flow model which in our case is local

Righi-Leduc is our main problem in running calculations

We need data! (correlate $T_{e}$ with $B$ ) 


\title{
Introduction
}

\author{
John Edwards \\ Electron transport workshop \\ 9-11th Sepetmber \\ Purple Orchid Inn, Livermore 2002
}




\section{Is there really a problem?}

In the early days of laser fusion:

Spitzer-Harm inapplicable

$B$ via $\nabla n \times \nabla T$ recognized

At high intensities bad stuff began to happen with direct drive

$=>$ so for this \& other reasons we went to indirect drive

Nova was a spectacular success \& led to NIF

There was no indication that non-local transport or B-fields were a big issue. Hohlraums worked just fine (with a flux limiter $~ 0.1$ ) (exceptions are small hohlraums - we have no idea why yet!)

In the mean time the rest of the world plugged away with direct drive Both this and LPI in hohlraums drove a very aggressive \& Very successful beam smoothing effort 


\section{So what's changed?}

Despite all that, we have strong evidence B-fields are important in at least some aspects of hohlraums
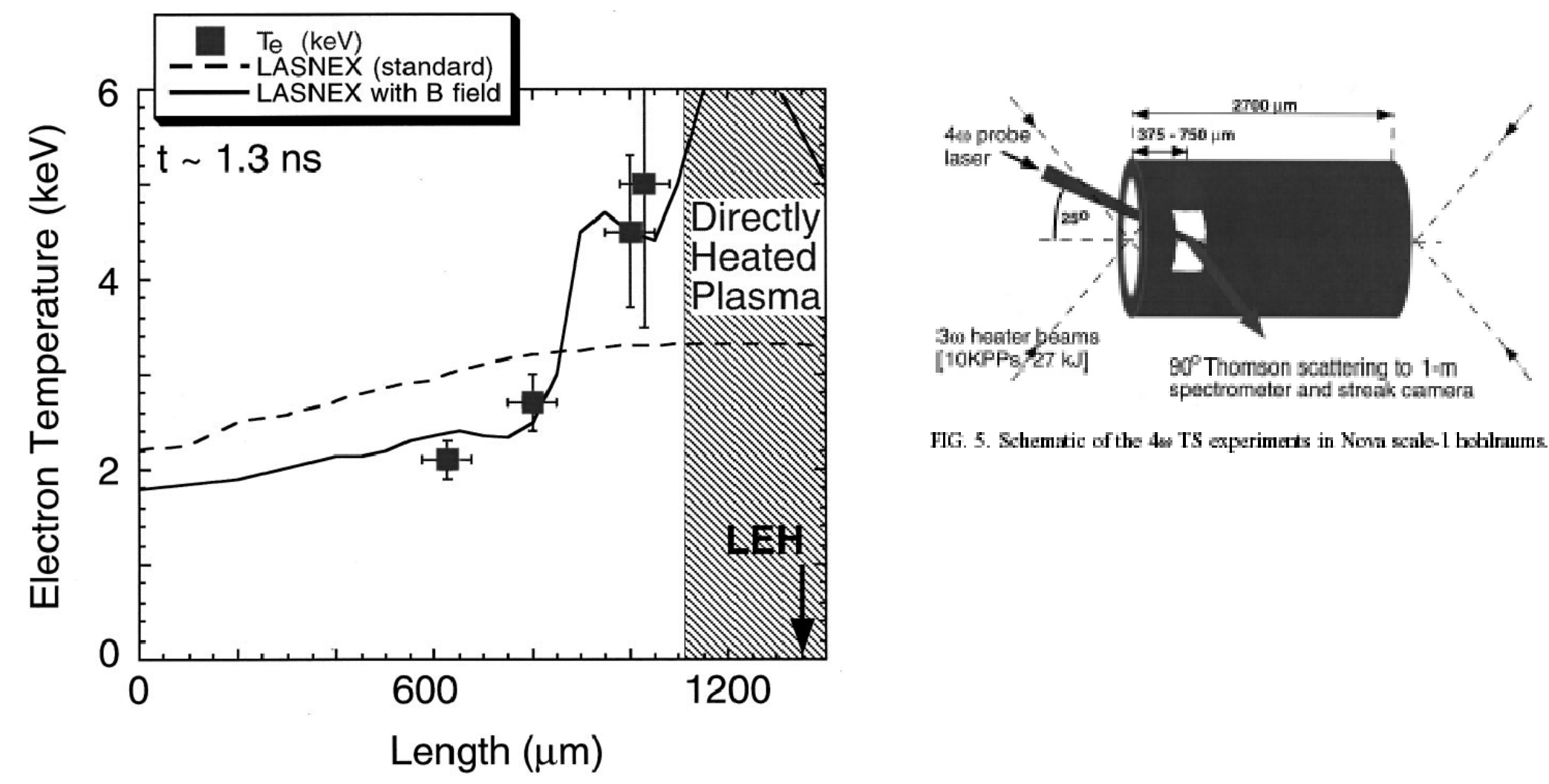

FIG. 5. Schematic of the 4m TS experimerts in Nova scale-1 bohlrams. 


\section{So what's changed......?}

NIF is very much closer now!

When we do something different there's often a surprise

We're planning to do much more with NIF than ignition and go into regimes that are far from our current experiences

The beam smoothing success has seduced us into thinking that maybe we can take advantage of better over all coupling into targets compared to indirect drive

Ultra-high intensity lasers => proton probing - electro-thermal-instability?

Short pulse lasers for physics studies 


\section{Theory \& computation}

Fokker-Planck for laser plasmas in 1D (Bell, 1981) Then in 2D (Epperlein, 1988)

Now 2D with $B$ to $f_{1}$ keeping $\mathrm{df}_{1} / \mathrm{dt}$ (Kingham, 2002)

At the same time, driven by extreme cost of FP People got busy making reduced non-local models to use in hydrocodes (1D, Luciani, 1983; .... 2D, Schurtz, 2000)

We're now 20 years on

is it time to try FP in our design codes? and if not, what? 


\section{Workshop objectives}

Our focus this time round is "long pulse" regime especially for high energy density hohlraums \& direct drive

What physics do we need to include?

What is a sensible way forward computationally?

Fokker-Planck something else (eg Monte Carlo) reduced model

What developments \& benchmarks do we need?

What should we do to test our ideas \& models? 
Physics of $\underline{B}$ generation

Ohm's law

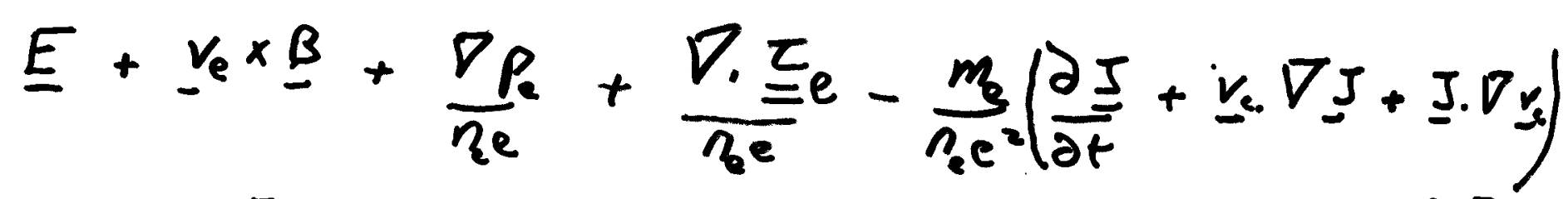

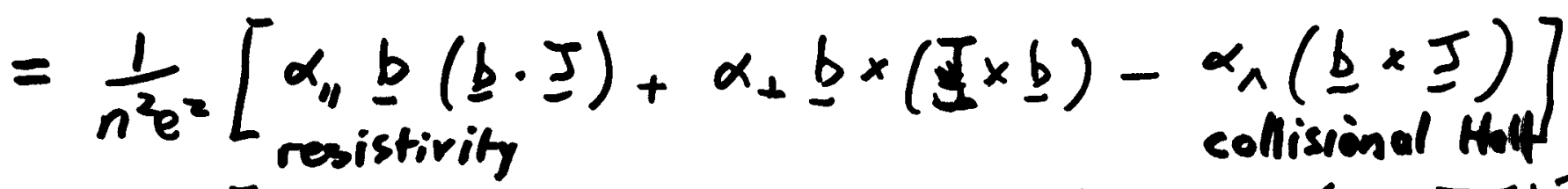

$$
\begin{aligned}
& -\frac{1}{e}\left[\beta_{\| \underline{b}} \underline{b}\left(\underline{\underline{b}} \cdot \nabla T_{e}\right)+\beta_{\perp} \underline{b} \times\left(\nabla T_{e} \times \underline{b}\right)+\beta_{\Lambda}\left(\underline{b} \times \nabla T_{e}\right)\right]
\end{aligned}
$$

Terms in green are extra to Braginskii

Faraday's law

$$
\frac{\partial B}{\partial t}=-\nabla \times E
$$

Include laser effects (see Can. I. Phys. 64, 912 (1989)

$$
v_{e} \rightarrow \underline{v}_{e}+\frac{\dot{\dot{\xi}}}{\text { quiver velocity }}
$$

Extra terms $\langle\dot{\xi} \times \tilde{B}\rangle=$ radiation pressure $\langle>$ time averaged due to absorption or reflection

$$
\frac{\nabla}{n_{e}}\left\langle n_{e} m_{e} \dot{\xi} \dot{\xi}\right\rangle=\begin{gathered}
\text { ponderomotive } \\
\text { force }
\end{gathered}
$$


Source terms

(i)

$$
\nabla \times\left(\frac{\nabla p_{e}}{n_{e}^{e}}\right)=\frac{\nabla T_{e} \times \nabla r}{n_{e} e}
$$

known as a dynamo in astrophysics

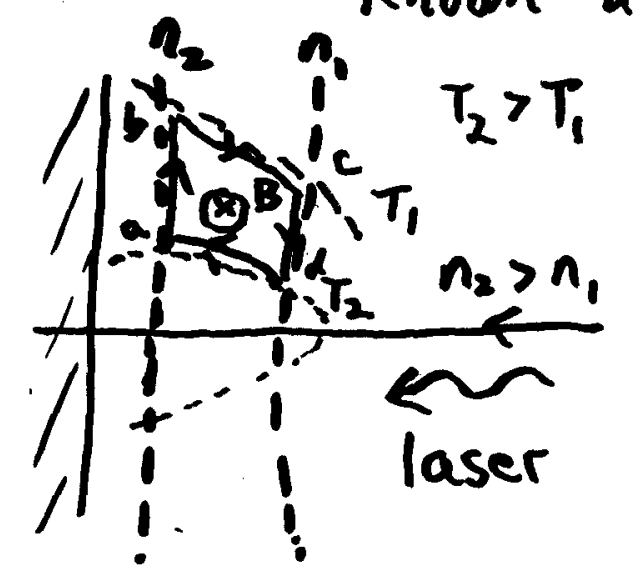

Consider line integral abode

$$
\begin{aligned}
& \int \frac{\nabla p}{n} \cdot d l=\int_{a}^{b} \nabla T \cdot \underline{d l}+\int_{b}^{c} T \frac{\nabla_{n}}{n} d \underline{d l} \\
& +\int_{c}^{d} \nabla T \underline{d}+\int_{d}^{a} T \frac{\nabla_{n}}{n} d l . \\
& =-\left(T_{2}-T_{1}\right)-T_{1} \ln \frac{n_{2}}{n_{1}}+\left(T_{2}-T_{1}\right)+T_{2} \ln \frac{n_{2}}{n_{1}} \\
& =\left(T_{2}-T_{1}\right) \ln \frac{n_{2}}{n_{1}}=\frac{\partial B_{0}}{\partial t} \times \text { area. }
\end{aligned}
$$

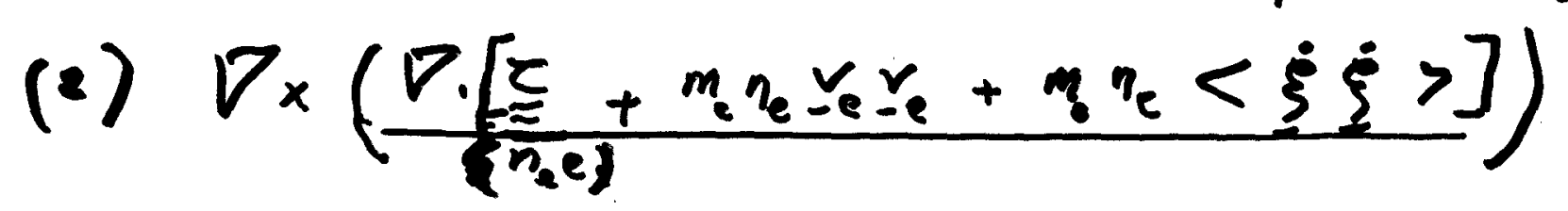

weill

instability

egg. resonance absorption 
Convection and amplification of $\underline{B}$ A good approximation to Ohm's law which holds for any $f_{0}$, including when non linear heat flow and other transport drives $t_{0}$ non-Maxmellian, is

$$
\begin{aligned}
& E+\left(V_{e}+\underline{V}_{T}\right) \times \underline{B}+\frac{\nabla_{p}}{\eta_{e}}+\underline{\beta} \nabla T_{e}=\eta \underline{I} \\
& \underline{V}_{T}=\frac{q_{e}}{5 / 2 P_{e}}=\frac{\text { electron heat flux }}{5 / 2 \text { electron preserve }} \text { scalar }
\end{aligned}
$$

We still need a good calculation of qu, but

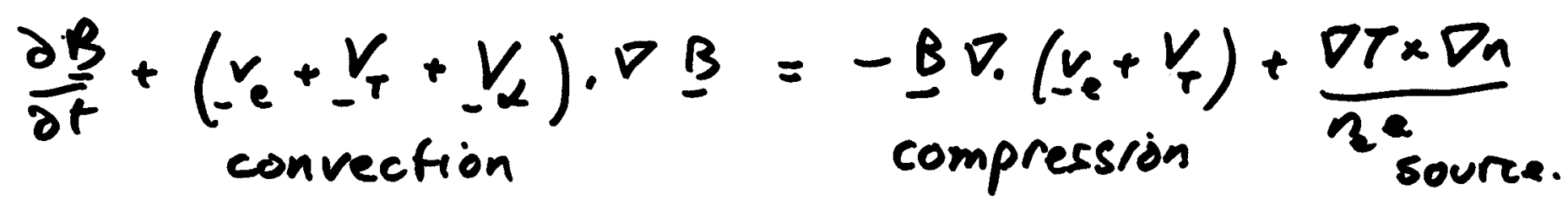

$$
\begin{aligned}
& V_{\alpha}=-\frac{\nabla_{\eta}}{\mu_{0}} \\
& +p_{0}^{Z \nabla^{2} \delta}+(\underbrace{(\underline{B} \cdot \nabla)\left(\underline{V}_{e}+\underline{V}_{-}-V_{\alpha}\right)+\nabla\left(\underline{V}_{\alpha}, \underline{B}\right)}
\end{aligned}
$$

Convection by $V_{T}$ is because the magnetic field is more frozen to hot electrons than cold If $\nabla \cdot V_{T}<0$, eng. heat flow up a pressure gradient, amplification of $\underline{B}$ occurs. (Nishiguelietal) - also true nonlinearity (Rho + Mains) ID F.P. 
2 distinct cases

(1) $\nabla \times E=0$ at $t=0$

Then there is still the possibility that $B$ can be generated as a result of an instability

- Weibel instability - collissontess (Pic coders)

- collissoinar (Epperlecin ital)

- Thermomagnetic (Tidman + Shanny) $\left.\lambda_{m_{p}}\right) c / w_{n}$

- Electrothermal; . driven by hest flow

$$
\begin{aligned}
& \lambda_{\text {map }}<c / \omega_{p e} \\
& E_{2}=\eta J_{c} .
\end{aligned}
$$

(Macias)

(2) $\nabla \times E \neq 0$ at $t=0$ is $2-D$ state

- $\nabla T \times \nabla n$ generating $+B_{0}$ around a laser focal spot $(+Z$ is direction of laser)

- In overdense plasma, especially in fast igniter generating $-B_{0}$ due to

- $E_{z}(r)=\eta J_{\text {cola }}$ (need collisions) in contrast to - Weibel instability $\Rightarrow$ filaments $\Rightarrow$ coalescence, seen in PIC codes. 
Validity of linear transport

For a tensor expansion of $f$

$$
\begin{aligned}
& f(r, \underline{r}, t)=f_{0}(r, v, t)+\underline{f}_{1}(r, v, t) \cdot \frac{v}{v} \\
& \text { scalar }+E_{2}(E, r, t): \frac{\underline{\underline{v}}}{v^{2}}+\cdots
\end{aligned}
$$

it has been shown that $f_{0}$ becomes non-Maxwellian for increasing $f$, For a Lorentz plasma t linear transport

$$
F_{1}=\frac{F_{m}}{1+\Omega^{2} V^{6}}\left(-V^{4} \underline{e}-\Omega V^{7} \underline{b} \times \underline{e}-V^{6} \underline{t}-\Omega^{9} \underline{b} \times \underline{t}\right)
$$

where $F=\frac{4 \pi}{n} r_{T}^{3} f, V=\frac{r}{V_{T}}, V_{T}=\sqrt{\frac{2 \pi}{m}}, \nu_{T}=\frac{3 \sqrt{\pi}}{4 \tau}$

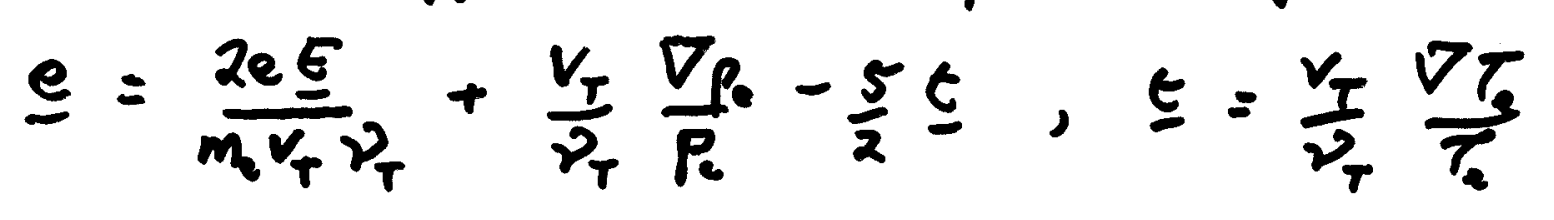

Taking $E$ terms only, and $L=(|\nabla T| / T)^{-1}$ there is a critical velocity $V_{c}$ at which

$$
F_{1}=F_{m}
$$




$$
V_{c}^{6}=\frac{L^{2}}{2 a_{0}^{2}}+\int\left[\frac{L^{4}}{4 a_{0}^{4}}+\frac{L^{2}}{\lambda_{\text {mp }}^{2}}\right]
$$

If $V_{c} \gg 1$ then linear transport holds. Consider 1-D heat flow

$$
\begin{aligned}
-1.5 \sqrt{\pi} \underline{Q}_{.}= & t \int_{0}^{\infty} V^{\prime \prime} \exp \left(-V^{2}\right) d V \\
& +\underline{e} \int_{0}^{\infty} V^{9} \exp \left(-V^{2}\right) d V
\end{aligned}
$$

High powers of $V$. High energy tail important. For $\underline{I}=0$

$$
\underline{e}=-4 \underline{t}
$$

thermoelectric field is set up

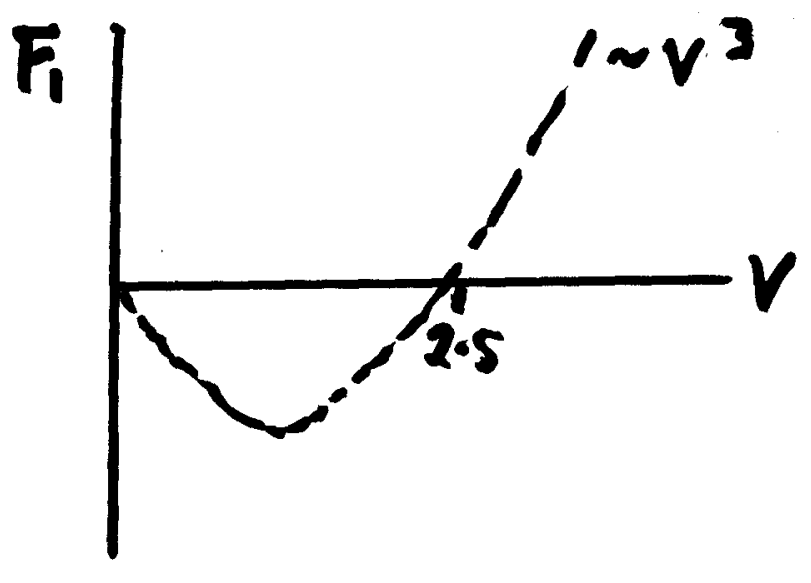

Reversal of $F_{1}$ (and heat flow ) up to $V=2.5$. To capture $60 \%$ of $q$ meed $V=3$ $80 \%, V=3.5$ 
Thermomagnetic instability

D.A.Tidman and R.A. Shanny MaP hs. Fluids 17 , 1207.

Could be occurring in LASNEX with linear transport and magnetic fields.

Equilibrium

$$
\uparrow_{\nabla n_{e 0}} \uparrow_{\nabla T_{e 0}} \stackrel{z}{\prod_{z y}}
$$

Perturbation $e^{j t-i k x}$

$$
\begin{aligned}
& n_{e} \Rightarrow n_{e 0}+n_{1} \\
& B_{1}=\left(0, B_{1}, 0\right)
\end{aligned}
$$$$
T_{e}=T_{e_{0}}+T_{1}
$$

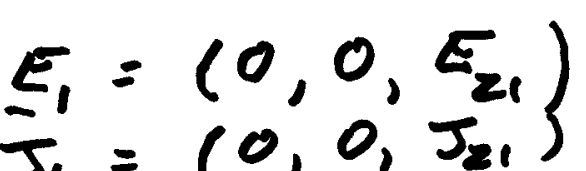$$
\bar{J}_{1}=\left(0,0, J_{21}\right)
$$

Basic physics. Source of $B$ is $\nabla_{n} \times D_{T}$

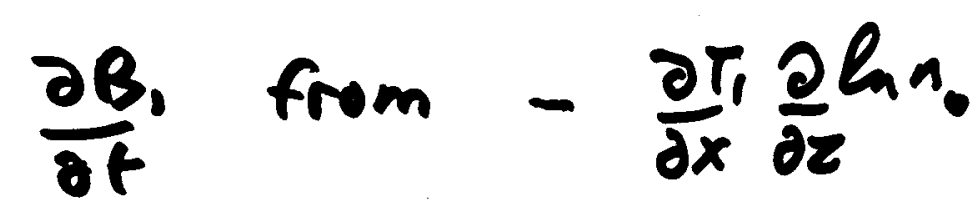

$$
\frac{3}{2} n_{0} \frac{\partial T_{1}}{\partial f} \text { from }-\frac{\partial}{\partial x} q_{x} \text { and } q_{x}=-k_{n_{1}} \underline{b} \times \nabla T_{0}
$$

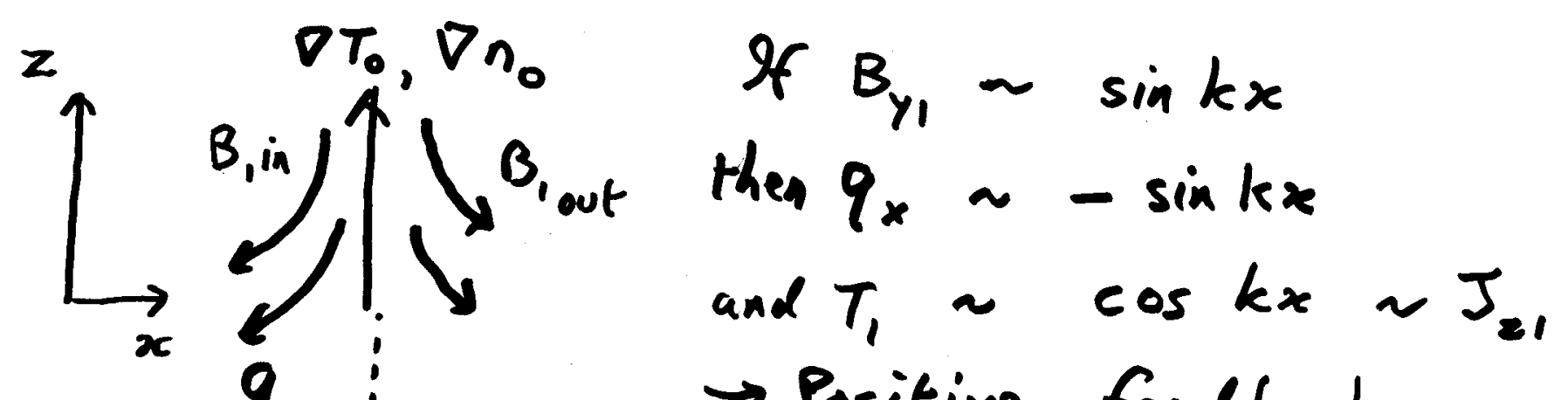

$$
\begin{aligned}
& \rightarrow \text { Positive feedback }
\end{aligned}
$$

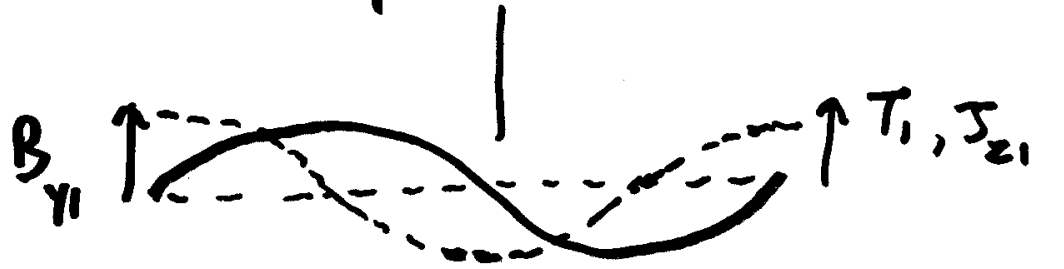

$$
\begin{aligned}
& \rightarrow \frac{\partial B}{\partial f^{\prime}} \sim \sin k x
\end{aligned}
$$


Tidmen + Shanny included resistive diffusion and thermal diffusion

$$
\begin{aligned}
& \frac{\partial B_{1}}{\partial t}=\frac{\partial E_{z_{1}}}{\partial x}-\frac{\partial E_{x_{1}}}{\partial z} \\
& =\frac{\partial}{\partial x}\left(-\frac{T_{1}}{n_{1}} \frac{\partial n_{0}}{\partial z}+\eta_{0} J_{z 1}\right)-\frac{\partial}{\partial z}\left(-\frac{T_{0}}{n_{0} 0} \frac{\partial n_{1}}{\partial x}-\frac{\partial \beta_{1} \theta_{0}}{\partial z} \frac{\partial T_{0}}{\partial z}\right) \\
& \begin{array}{l}
r \text { resistive } \\
\text { diffusion } \\
\text { ignore } n, ~ N e r a s t \\
\text { changes. }
\end{array}
\end{aligned}
$$

$$
\begin{aligned}
& \frac{3}{2} n \cdot \frac{\partial T_{1}}{\partial t}=-\frac{\partial q_{x}}{\partial x}-\frac{\partial q_{z}}{\partial z} \\
& q_{e}=-\frac{\eta_{c} T_{c} \tau}{m_{e}}\left[k_{\perp}^{c} \nabla_{\perp} T+k_{\Lambda}^{c} \underline{b} \times \nabla T\right]-\frac{T_{e}}{e} \beta_{\perp}^{c} J_{\perp}-T_{e} \beta_{\Lambda}^{c} \underline{b}
\end{aligned}
$$

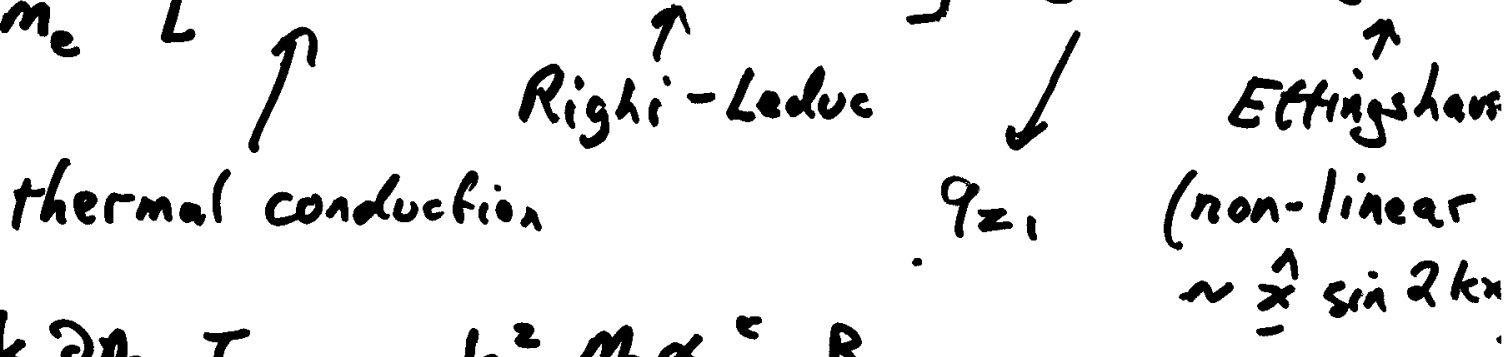

$$
\begin{aligned}
\therefore \gamma B_{1} & =\frac{i k}{n_{0} e} \frac{\partial \eta_{0}}{\partial z} T_{1}-k^{2} \frac{\eta_{\alpha} \alpha_{s}^{c}}{3 e^{2} c \mu_{0}} B_{1} \\
\gamma T_{1} & =-\frac{2}{3} \frac{T_{0} \tau_{0}}{\eta_{0}} k_{L_{0}}^{c} k^{2} T_{1}-i k \gamma_{0} \frac{e \tau^{2} T_{0} \frac{\partial T_{0}}{m_{0}^{2}} B_{1}}{\partial z}
\end{aligned}
$$

with assumptions : (none of which are probably true.!!,

) Local approximation, $k \gg \frac{1}{L_{n}}, \frac{1}{L_{T}}$

2) No ion motion: $n_{1}=0$

3) Linear transport holds ; $\lambda_{\text {map }} \ll \frac{1}{K}$

4) $f_{2}$ - anisotropy negligible. 
Dispersion relation

$$
\left(f+d_{1} k^{2}\right)\left(j+d_{2} k^{2}\right)=d_{1} d_{2} k_{c}^{2} k^{2}
$$

where $\quad \alpha_{1}=\frac{m_{0} \alpha_{2}^{c}}{m^{2} \theta \mu_{0}}, \quad \alpha_{2}=\frac{2}{3} \frac{T_{0} \tau_{0}}{m_{0}} K_{\perp_{0}}^{c}$

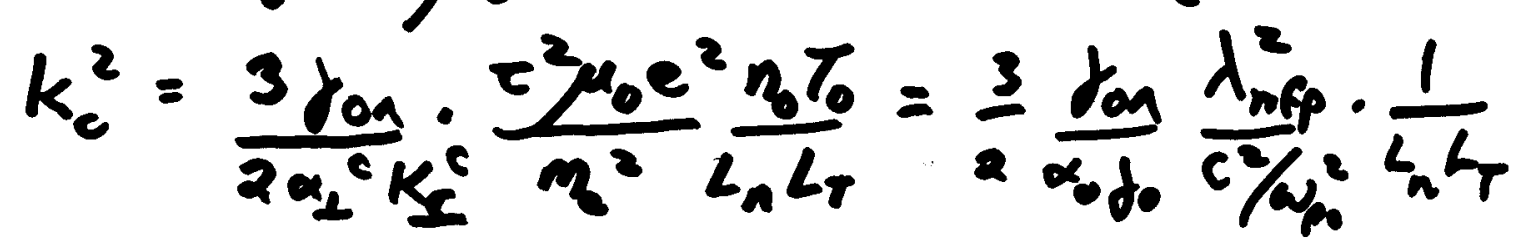

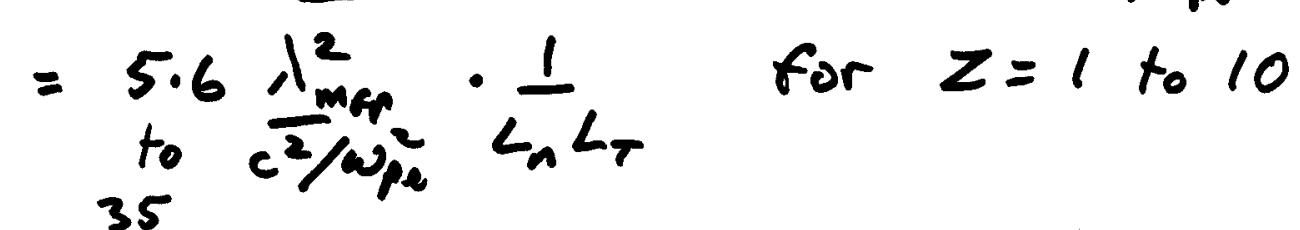

Marginal stability is at $k=k_{c}$-critical wave number $($ at $f=0)$. Unstable for

$$
0 \leqslant k \leqslant k_{c} \text { and } \nabla n_{0} \cdot \nabla T_{0}>0
$$

If follows that $\lambda_{m_{G p}} \gg \frac{c}{u_{p e}}$ for $k^{2} \sim k_{c}^{2} \gg \frac{1}{L_{n} 4}$

This is the opposite of condition for heat flux driven electrothermal instabilities $\left(\lambda_{\text {mp p }}<\frac{c}{w_{p a}}\right)$

Growth rate

$$
j=-\left(\frac{d_{1}+d_{2}}{2}\right) k^{2}+\int\left[\left(\frac{d_{1}-d_{2}}{2}\right)^{2} k^{4}+d_{1} d_{2} k^{2} k_{0}^{2}\right]
$$

This has a maximum at $k^{2}=k_{0}^{2} \frac{\sqrt{\alpha_{1} \alpha_{2}}}{\left(\sqrt{\left.\alpha_{1}+\sqrt{\alpha_{2}}\right)^{2}}\right.}$ 
At small $k, j \rightarrow\left(\alpha_{1} \alpha_{2}\right)^{k_{2}} k_{c} k$

In experiments, resistive diffusion is usually much less than thermal diffusion ie. $\frac{d_{1}}{d_{2}}=x \ll 1$

Example:

$$
\begin{aligned}
& \eta_{e}=3.5 \times 10^{20} \mathrm{~cm}^{-3} \\
& T_{E}=5 \mathrm{keV} \\
& z=4
\end{aligned}
$$

$$
\begin{aligned}
\ln 1=7.256, \quad \tau & =1.2 \times 10^{-11} \\
\gamma_{0}=6.995 \quad \alpha_{0} & =0.3750 \quad \gamma_{01}=34.95 \\
c / \omega_{n_{0}}=2.84 \times 10^{-7} \mathrm{~m} \quad \lambda_{m \in p} & =356 \mathrm{~mm} \\
\lambda_{\text {mp p }}\left(v=3 v_{6}\right) & =288 \mathrm{~mm}
\end{aligned}
$$

$\therefore$ need non-local transport

$$
\begin{aligned}
& \begin{array}{ll}
d_{1}=.0282 \mathrm{~m} / \mathrm{s} & d_{1}=3.2 \times 10^{-5} \\
d_{2}=880.8 \mathrm{~m}=/ \mathrm{s} & d_{2}
\end{array} \\
& \left(\frac{\lambda_{m+p}}{c / \omega_{n}}\right)^{2}=1.57 \times 10^{6} \gg 1 \quad k_{c}=\frac{5601}{\left(L_{n} L_{T}\right)^{1 / 2}} \\
& \frac{\gamma_{\text {max }}}{d_{2} k_{c}^{2}}=\frac{x^{1 / 2}}{(1+\sqrt{x})^{2}}\left\{\int \left[\begin{array}{c}
\left.\frac{1}{4}-\frac{1}{2} x+x^{2}+x^{1 / 2}(1+2 \sqrt{x}+x)\right] \\
\left.-\frac{1}{2}-\frac{1}{2} x\right\}
\end{array}\right.\right.
\end{aligned}
$$

$\rightarrow x$ for $x \ll 1$

$\therefore \gamma_{\max } \approx d_{1} k_{0}^{2} \quad$ at $k=k_{c}\left(\frac{d_{1}}{d_{0}}\right)^{1 / 4}$ 
For $L_{n}=L_{T}=100 \mu \mathrm{m}$

$$
\begin{aligned}
\partial_{\text {max }} & =8.85 \times 10^{13} \mathrm{~s}^{-1} \\
\text { at } \lambda & =\frac{2 \pi}{k}=\frac{2 \pi\left(L_{n} L_{r}\right)^{1 / 2}}{421.3}=1.49 \mu \mathrm{m} .
\end{aligned}
$$

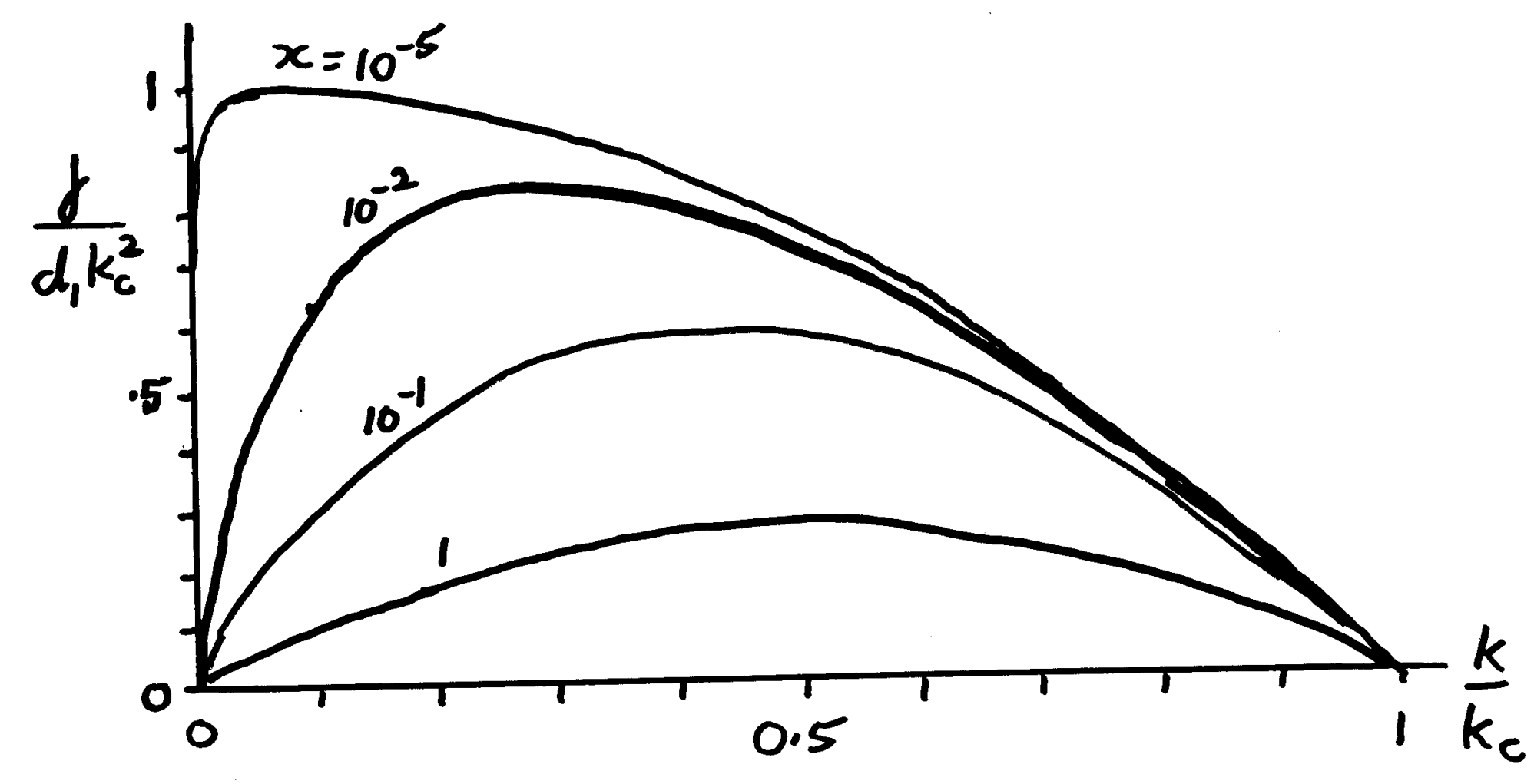

Conditions for validity

$$
\begin{aligned}
& L_{n}, L_{T} \gg \lambda=\frac{2 \pi}{k} \gg \lambda_{m p p} \gg c / \omega_{p e} \\
& { }_{1000 \mu \mathrm{m}}^{100 \mu \mathrm{m}} \underbrace{1.49 \mu \mathrm{m} \text { still bad. }}_{14.9 \mu 4} 356 \mu \mathrm{m} \gg 0.28 \mu \mathrm{m}
\end{aligned}
$$


Include electron viscosity in Ohm's law

$$
\begin{aligned}
E_{21} & =\eta_{0} j_{21}-\frac{T_{1}}{\partial e} \frac{\partial n_{0}}{\partial z}-\frac{1}{n_{e}} \frac{\partial}{\partial x}\left[\frac{\left.n_{0} \tau \tau_{\text {le }} \frac{\partial j_{21}}{\partial x}\right]}{\eta_{0}}\right] \\
& =\frac{\eta_{0}}{\mu_{0}}\left(1+k^{2} \lambda_{\text {np }}^{2}\right) \frac{\partial B_{1}}{\partial x}-\frac{T_{1}}{e L_{n}}
\end{aligned}
$$

ie. replace $d_{1}$ by $d_{1}\left(1+k^{2} d_{m \neq p}^{2}\right)$ and $k_{c}^{2} b_{y} \frac{k_{c}^{2}}{1+k^{2} d_{m p p}^{2}}$

Growth rate

$$
\begin{aligned}
f= & -\frac{1}{2}\left[\left(d_{1}+d_{2}\right) k^{2}+d_{1} k^{4} \lambda_{\text {map }}^{2}\right] \\
& +\sqrt{\xi} \frac{1}{2}\left[\left(d_{1}+d_{2}\right) k^{2}+d_{1} k^{4} \lambda_{\text {m ep }}^{2}\right]^{2}+d_{1} d_{2} k^{2}\left[k_{0}^{2}-k^{2}-k^{4} !\right.
\end{aligned}
$$

Example $T_{e}=10 \mathrm{keV}$

$$
\begin{aligned}
& T_{e}=10 \mathrm{keV} \\
& n=\frac{1}{n_{c}}=0.25 \times 10^{22} \mathrm{~cm}-3 \\
& L_{n}=L_{T}=100 \mu \mathrm{m} . \quad Z=3.5
\end{aligned}
$$

$\Rightarrow \gamma_{\text {max }}=5.3 \times 10^{-12} \mathrm{~s}^{-1}$ (only slightly less) at $\lambda=42.4 \mu \mathrm{man}$ for $\lambda_{\text {mp }}=183 \mu \mathrm{m}$.

But de localisation by $\left[1+\left(30 k \lambda_{\text {map }}\right)^{8 / 3}\right]$ (Epperlein + Short PF B6, 2211 (1992))

should perhaps be applied to viscosity, thermal conduction and (maybe modified) to Righi-Leduc. 
Electrothermal instability associated with nonlinear heat flow from the corona to the molten/vapour core of wire (or capsule)

References. M.G.Haines, J. Plasma Phys 12, 1 (1976)

$\left(K / / B_{0}\right)$ (for current driven ET instable. in $\theta$ peach)

M.6. Hones, Phys. Rev. Lett. 47, 917 (1981)

(for nonlinear heat Alow driven instability in (CF)

For ET instability in magnetised plasm with $\underline{k}+B$

A. Tomimora and M.G. Maine, I. Plasma Phys.

M.G. Haring and F. Marsh, J. Plasma Phys.

+ IAEA

ET instability driven by heat Alow

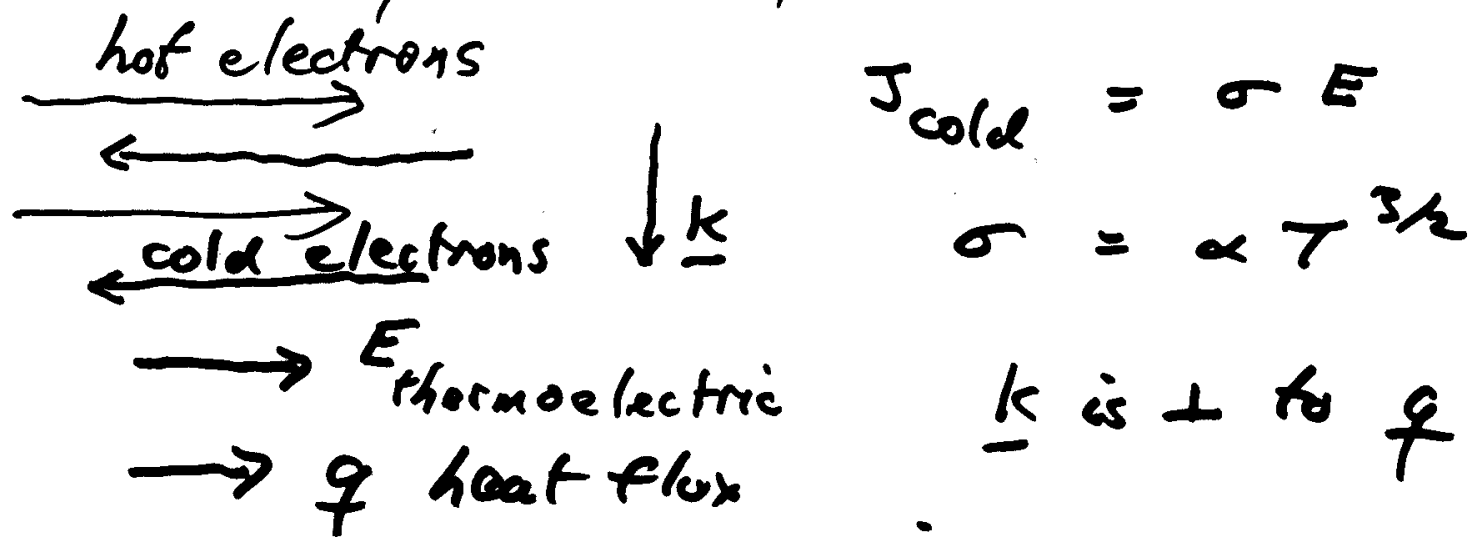

Basic instability is Joule heating of cold electrons by the return (cold) currant. Where $T^{\prime} \sim e^{i k x}$ is higher, $J$ +heating increases, so raising $T^{\prime}$. Include dripping by transererse thermal conduction and $\nabla \times E^{\prime}=-\frac{\partial B^{\prime}}{\partial F}+$ ion motion 
Then found to be unstable if

$$
\lambda_{\text {map }}<c / \omega_{p}
$$

and if Joule heating is sufficiently strong eng. mould create $T_{e}>1.32 T_{i}$

$$
T_{e}\left({ }^{0} \mathrm{~K}\right) \leqslant 0.1 \mathrm{z}^{1 / 4}\left(\mathrm{~m}^{-3}\right) Z^{1 / 2}\left(\frac{\ln 1}{5}\right)^{1 / 2}
$$

Growth rate $\alpha=2.8 \times 10^{-P} \frac{n_{0} Z}{A T_{e 0}^{3 / 2}}$

at wavelength $\lambda=2.4 \times 10^{10} \frac{T^{2}(\%) 4^{1 / 2}}{3\left(\mathrm{~m}^{-3}\right)^{2}}(\mathrm{~m})$

Perturbed magnetic fields, surrounding each filament reduce thermal conduction, relaxes $\lambda_{\text {mop }}<c / \omega_{\text {pe }}$, and filaments get frozen in.

A more rigorous theory requires a F.P. model including $F_{0}^{\prime}$ and the E.F, terms (heating of colds and cooling of hots, and possibly $F_{2}$. 
In hybrid model the hot electrons have perturbed orbits in the $E^{\prime}$ and $B^{\prime}$ fields

The parameter $h$ describing the hot electrons is zero if $J_{\text {hot }}=n_{n} \in\left(k T_{A} / m_{c}\right)^{1 / 2}$

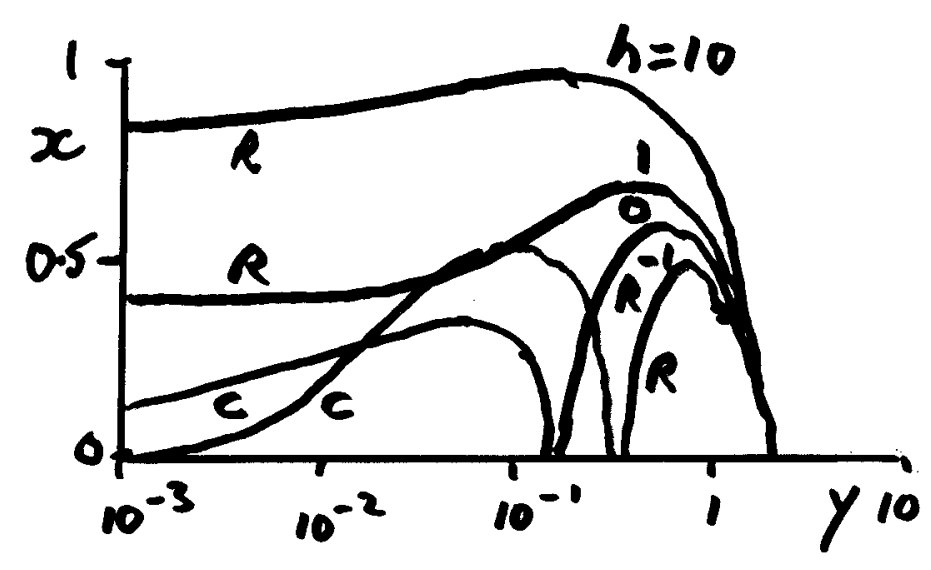

Unstable roots of the dispersion equation $x \propto$ growth,$y \propto k^{2}$

$R=$ real $\quad C=$ complex root 
Curtailment of SBS by localised magnetic field generation M.G.Haines, Imperial College.

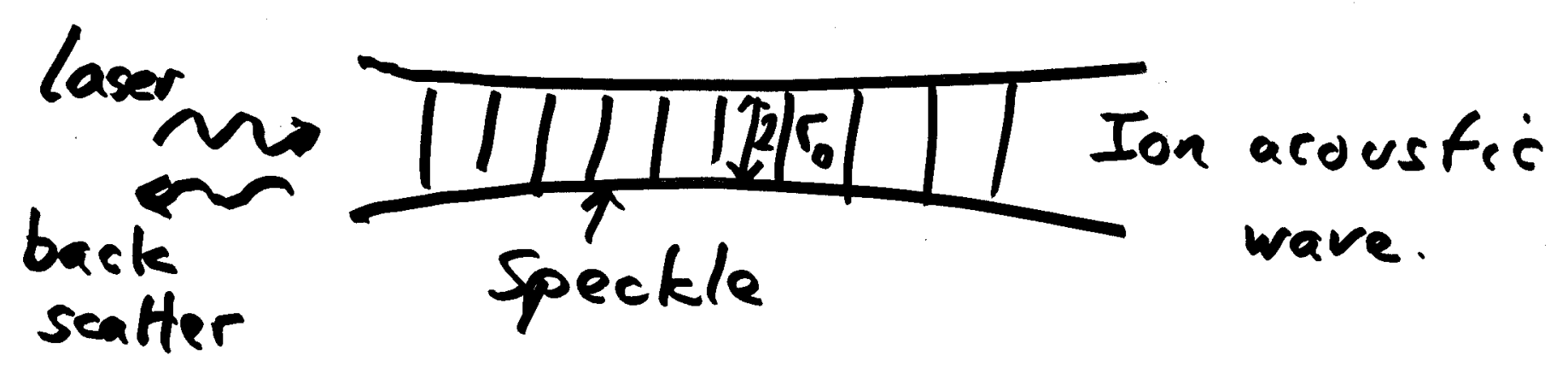

Ponder-
motive
force on electrons

from photon momentum (absorbed + reflected)

$\nabla \times E \neq 0$ $E=-\nabla \xi$ in $\mathrm{AD}$

$E=-\nabla \Phi-\frac{\partial A}{\partial t}$ in gen $=-\frac{\partial p}{\partial f}$

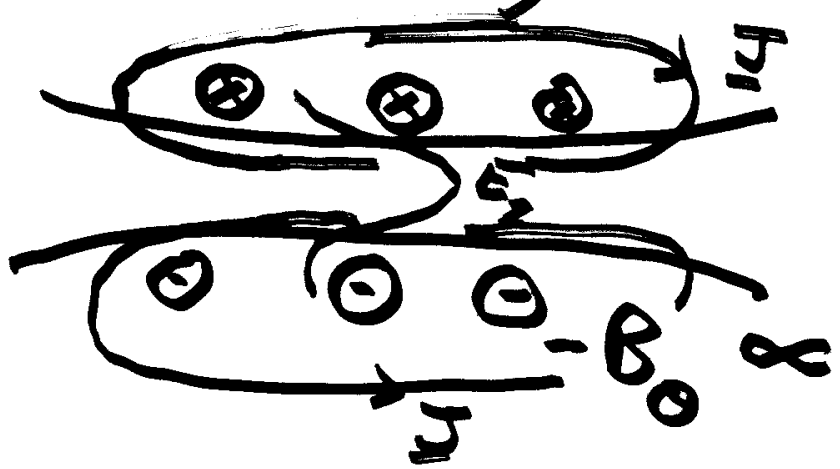

Ion sound waves replaced

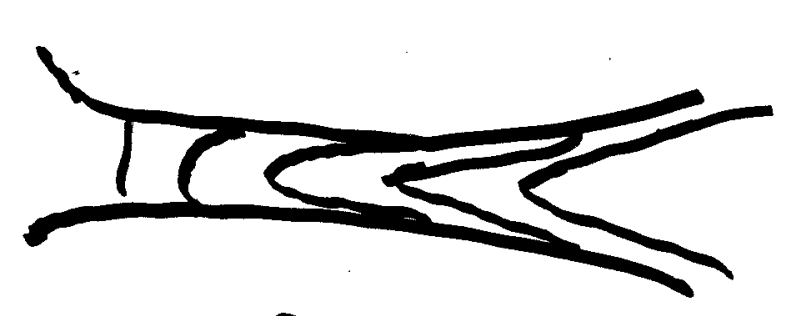

$$
C_{A}^{2}=\frac{B^{2}}{M_{C}}
$$

by magnetosonic fast wave $\sqrt{S^{2}+C_{A}^{2}} \approx c_{3}\left(1+f\left(y r^{2}\right)\right.$ Coherent plane wave of IAW broken up 
Driving currents and azimuthal Magnetic fields from photon momentum

Effective Ohm's law

$$
\begin{gathered}
n_{z} m_{z} \frac{\partial v_{e z}}{\partial E}=-n_{e} e E_{z}-\beta_{e} e\left(v_{e} \times B\right) \\
-\nabla_{z} P_{\alpha z}
\end{gathered}
$$

where $I_{z}=$ radiation intensity in $z$ direction $\alpha=\alpha_{\text {abs }}+2 \alpha_{\text {refl }}$ over length $L$

$$
\frac{\alpha I}{C L}=\langle\tilde{J} \times \tilde{B}\rangle_{2} \quad \begin{aligned}
& \text { average radiation } \\
& \text { pressure }
\end{aligned}
$$

Bot $\frac{\partial E_{z}}{\partial r}=\frac{\partial B_{\theta}}{\partial t}$ and $\frac{1}{r} \frac{\partial}{\partial r}\left(r B_{\theta}\right)=-\mu_{0}$ ?lv $_{e_{2}}$

For a laser heater pulse in a gas-bag or gas-filled hoh/raum at LLNL Neglect electron inertia and $e-i$ collisions

$$
E_{2}=\frac{\alpha I}{n_{p} e c L} \text { and } B_{\theta}=-\frac{z}{r_{0}} \frac{\alpha I}{n_{p} e c L}
$$

$$
\text { For } \begin{aligned}
L & =5 \mathrm{~mm}, I=3.10^{15} \mathrm{W.cm}^{-2}, n_{e}=10^{20} \mathrm{~cm}^{-3} \\
r_{0} & =30 \mathrm{\mu m}, C=5 \mathrm{~ns}, \alpha=0.5 \Rightarrow B_{\theta}=104 \\
\alpha & =\alpha_{\text {absorbed }}+2 \alpha_{\text {reflected }}
\end{aligned}
$$


Siegfried Glenzer's experiment

For a parabolic profile $I=I_{0}\left(1-r / r_{0}\right)$ average intensity $\bar{I}=\frac{1}{2} I_{0}$

$$
\text { If } \begin{aligned}
r_{0} & =125 \mu \mathrm{m} \quad I_{0}=2 \bar{I}=3.10^{15} \mathrm{Wcm}^{-} \\
L & =1.6 \mathrm{~mm} \quad \epsilon=1.5 \mathrm{~ns} \\
\alpha & =\alpha_{\text {abs }}+2 \alpha_{\text {refl }}=0.3+2 \times 0.2=0.7 \\
n_{e} & =5 \times 10^{20} \mathrm{~cm}^{-3} \\
\Rightarrow B_{\theta} & =13.1 \text { tesla (131 KG) }
\end{aligned}
$$

Cavitation time for $\mathrm{CH}$

$$
\begin{aligned}
\bar{Z} & =3.5 \quad \bar{m}_{i}=6.5 m_{p} \\
\epsilon_{c} & =\left(\frac{9}{2} \frac{n_{e} m_{i} r_{0}^{2}}{Z \alpha_{a b s} I_{0}}\right)^{1 / 3} \\
& =2.3 \times 10^{-9} \mathrm{~s}
\end{aligned}
$$

$n_{e}$ is reduced by $\sim \exp \left(-\frac{t_{-}}{\epsilon_{c}}\right)$

$$
\sim e^{-1.5 / 2.3}=0.52
$$

Then $B_{\theta} \simeq 26$ tesla $(260 \mathrm{kG})$

But larger $r_{0}$, smaller $\alpha$ leads to much reduced $B_{0}$. Instead consider speckles and self focusing. 
Speckle

Harvey Rose (Phys. Plasmas 4, 437 (1987) proposed saturation of SBS by ion flows driven by photon momentum deposition. But $\lambda_{\text {map }}$ of ions $\gg$ spot radius $10-40 \mathrm{pm}$ \D $2 \mu \mathrm{m}$.

and will receive a kick from $E_{2}$ as they more through speckle

$\partial E_{2} / \partial r$ will generate $B_{\theta}$ and will convert $c_{s}$ to $\left(c_{s}^{2}+c_{A}^{2}\right)^{1 / 2}$ where $\frac{c_{a}^{2}}{c_{s}^{2}}=\frac{b^{2}}{\mu p_{\theta}}=\frac{2}{\beta}$ which will vary strongly over the speckle radix $r_{0}$ and cause sound waves to break up.

Use $B_{0}=-\frac{2 r}{r_{0}^{2}} \frac{\alpha I t}{n_{e} e c L}$ and $V_{2}=\frac{\alpha I t}{n_{i} m_{i} \subset L}$

$$
\begin{aligned}
& I=6 \times 10^{15} \mathrm{Wem}^{-2}, L=50 \mu \mathrm{m}, r_{0}=1.8 \mu \mathrm{mm}, r_{2} 100 \mathrm{ps} \\
& n_{e}=10^{21} \mathrm{~cm}^{-3} \text { for } z=1, m_{i}=m_{p}, T_{e}=2.10^{3} \mathrm{er} \\
& B_{\theta}=1.4 \times 10^{3} \mathrm{tes} / \mathrm{a} \quad V_{\varepsilon}=1.2 \times 10^{5} \mathrm{~m} / \mathrm{s} \\
& \frac{V_{t}}{C_{s}}=2.42 \\
& \left(C_{5}=4.4 \times 10^{5} \mathrm{~m} / \mathrm{A}\right)
\end{aligned}
$$


Because $B_{\theta}<0$, the $v_{r} B_{\theta}$ and $\frac{q r}{3 / 2 P_{\theta}}$ electric field terms will amplify the magnetic field.

But the 'Nernst' term is only valid

for $\lambda_{\text {map }} / L$ small as it represents the velocity dependence of the collision frequency.

qr however exists and is known in gas bag experiment. $\lambda_{\text {mop }} \sim 44 \mu \mathrm{mm}$ and for $V \sim 3, \lambda_{\text {mes }} \sim 4 \mathrm{~mm}$ Use results of diode problem with plasma between plates at $T_{2}$ and $T_{R}$ with variable $\lambda_{\text {mes }} / L$.

Tho + Bond, J.P lye D kg, L 117 (1991) Bond, Rho + Haynes, Plasma Phys. 24, 1133 (1988)

$$
q_{\text {collisionkss }}=\frac{3}{\pi k_{2}}\left(\frac{2 k_{A}}{\left.m_{R}\right)^{3 / 2}} T^{1 / 2} T_{R}^{1 / 2}\left(T_{R}^{1 / 2}-T_{R}^{K}\right)\right.
$$

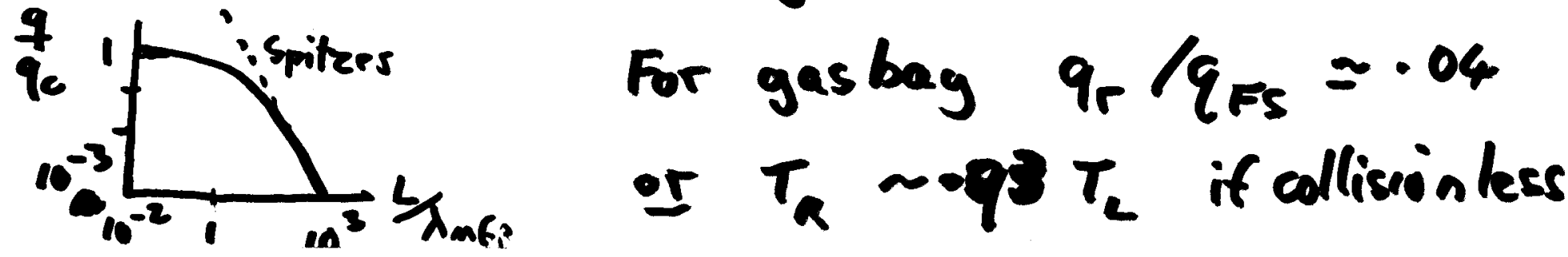


Nonlinear electron transfort in magnetized laser plasmas

T.H. Kho and M.G. Haines Phys Ferids 29,2665 (1866) (Also PRL 55, 825 (1985))

1-D model with transverse $B_{y}$ applied constant at $z=0$

$$
\begin{aligned}
& F_{1}=\left(F_{1}^{x}, 0, F_{1}^{x}\right) \\
& \text { (hanglapa) } \\
& r_{0}=\text { quivir } \\
& \partial_{t} f_{0}+\frac{1}{3 v^{2}} \partial_{2}\left(v^{3} f_{1}^{2}\right)=\frac{1}{v^{2}} \partial\left\{\frac{v^{3}}{3} a \cdot f_{1}+\gamma\left[c f_{0}+\left(D+\frac{n z v^{2}}{6 v}\right) \partial f_{y}\right.\right. \\
& f^{*}=-\frac{\tau}{1+x^{2}}\left[\nu x \partial_{z} f_{0}-\left(a_{x}+k a_{z}\right) \partial_{\nu} f_{0}\right] \\
& f^{2}=-\frac{e}{1+x^{2}}\left[r \partial_{2} f_{0}+\left(x a_{x}-a_{2}\right) \partial_{v} f_{0}\right]
\end{aligned}
$$

where $a=\frac{e E}{m}, \quad \psi(v)=\frac{e B}{m} c(v), \quad c=\frac{v^{3}}{(2+1) q v}$

$$
y=\frac{4+e^{4} \ln \Lambda}{\left.\left(4+\sigma_{0}\right)_{0}^{0}\right)^{0}}, \quad C=f_{0} I_{0}^{0}, \alpha D=\frac{r}{3}\left(I_{2}^{0}+J_{-1}^{0}\right)
$$

$I_{j}^{i}=\frac{4 \pi}{v j} \int_{0}^{v} f^{i} v^{j+2} d u$ are $J_{j}^{i}=\frac{4 \pi}{v j} \int_{j}^{0} f^{i} v^{j+2} d u$ $a_{z}$ adjusted for $J_{z}=0, \quad \nabla T=\frac{\partial T}{\partial z}, J_{x}, q_{x}, q$ 


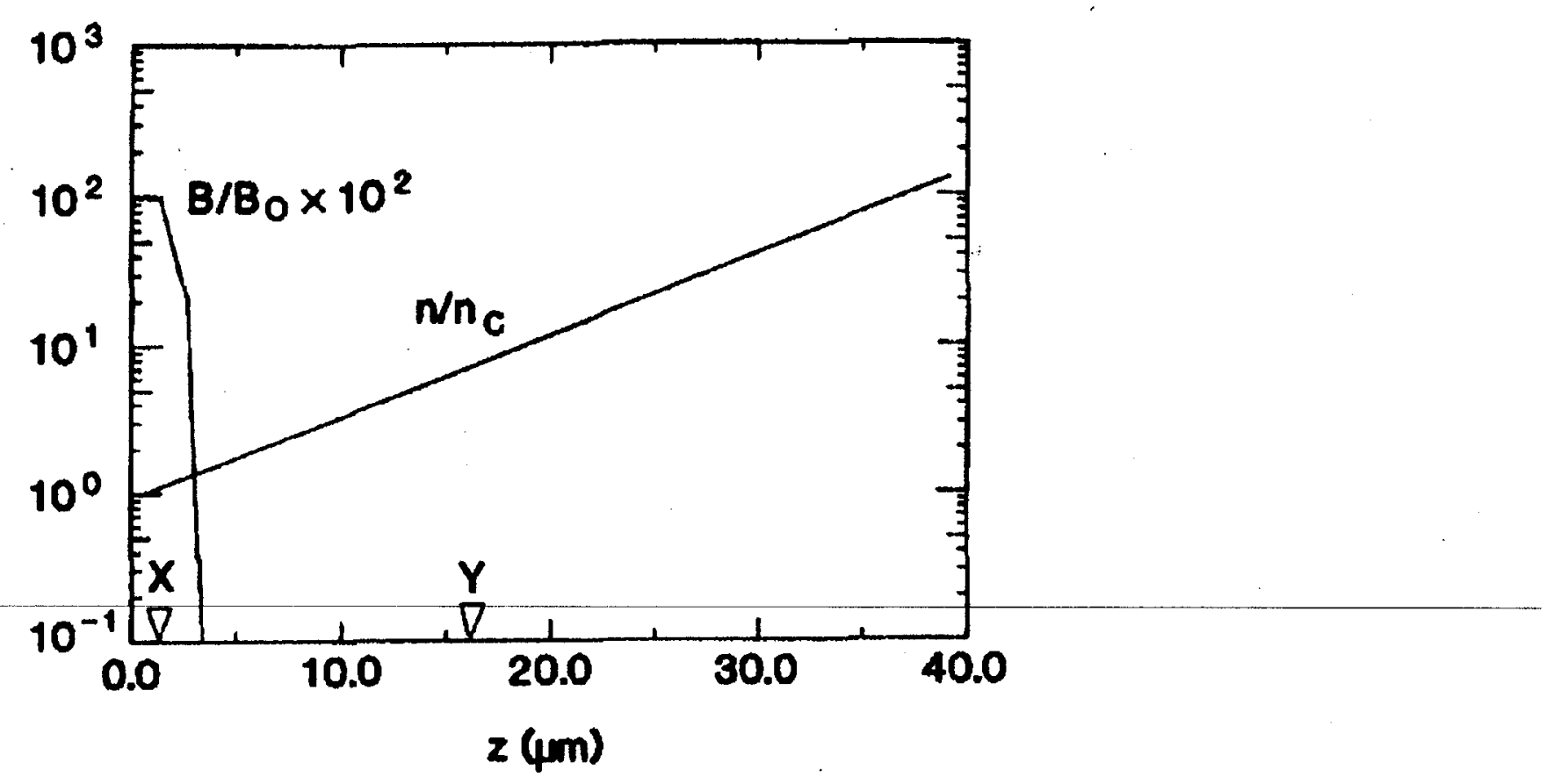

FIG. 1. Fixed exponential density, normalized to critical density $n_{c}$, and initial (normalized) magnetic field $B / B_{0}$, which vanishes for $z>4 \mu \mathrm{m}$. Inverse bremsstrahlung absorption takes place from $z=0$ to $z=X$. Over the same region, the "source" magnetic field $B_{0}$ is kept constant in time. 


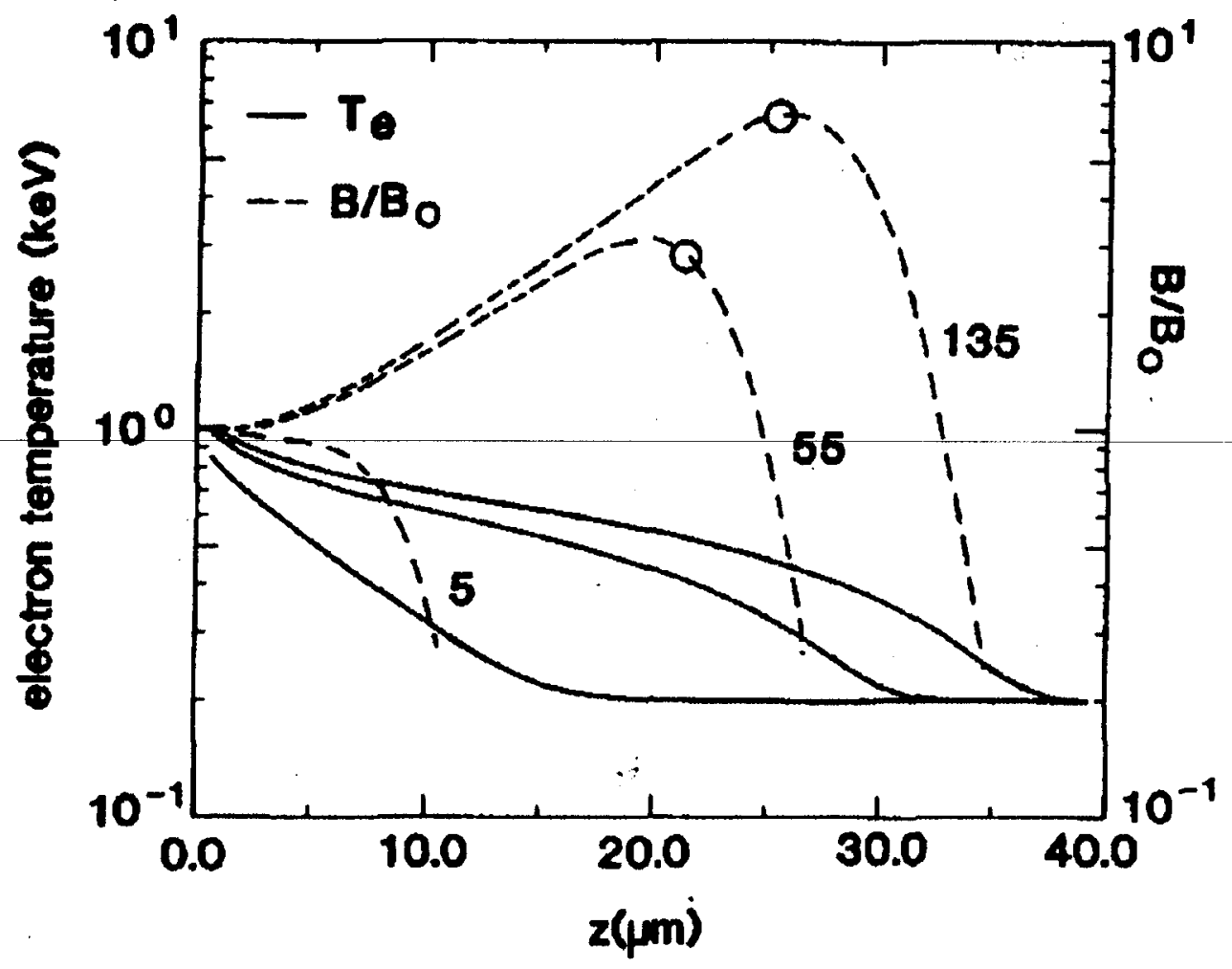

FIG. 2. Temperature $T_{c}$ (solid lines) and magnetic field $B / B_{0}$ (dotted lines) at 5, 55, and $135 \mathrm{psec}$ (from left to right). Circle on the $B / B_{0}$ curves indicate positions where $\left(\lambda_{e} / \lambda_{s}\right)=1$. 

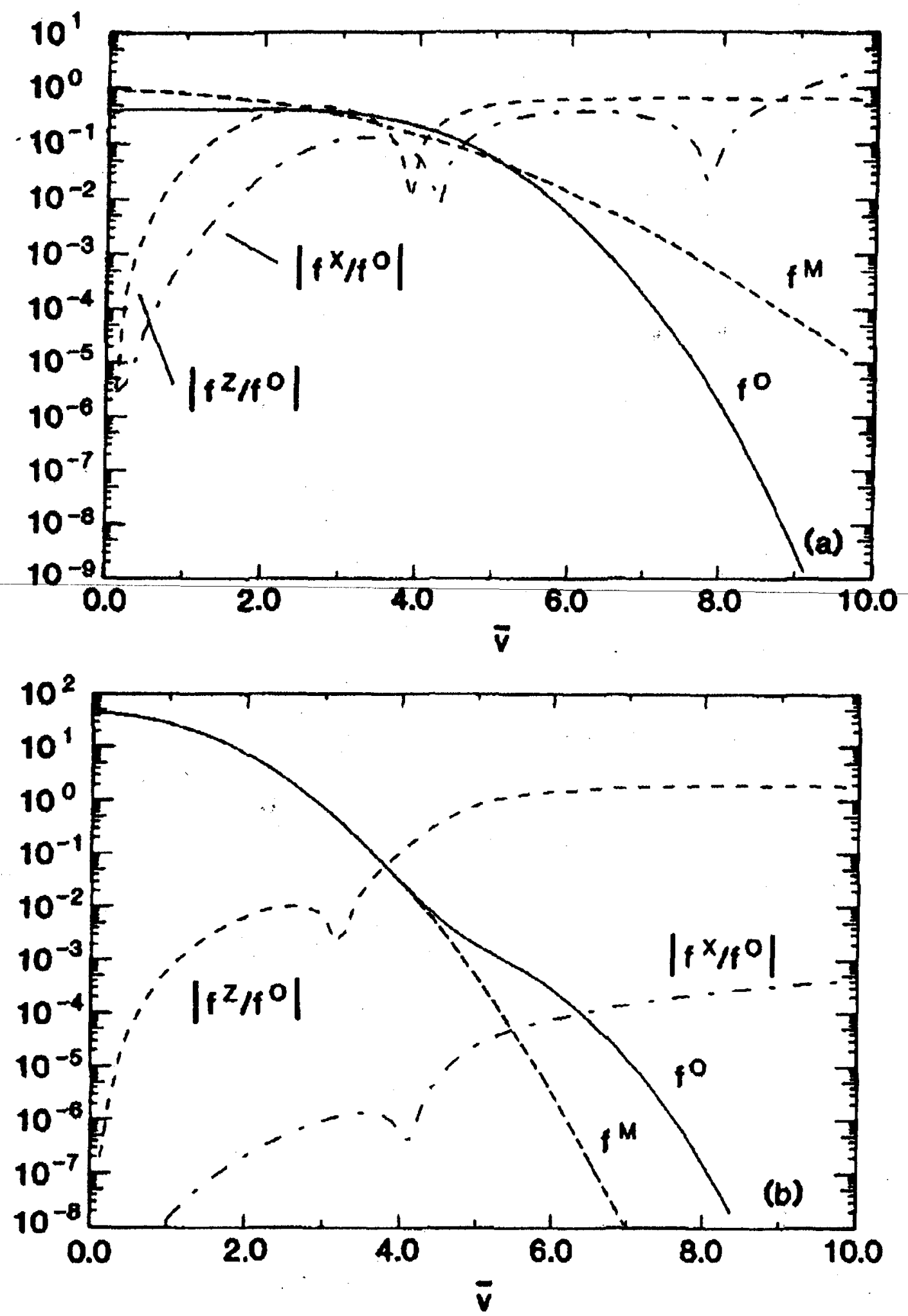

FIG. 4. The distribution functions at positions $X$ and $Y$ as indicated in Fig. 1 , at 5 psec. Here $f^{m}$ is the local Maxwellian and $\bar{v}=10$ corresponds to a 10 keV electron. 


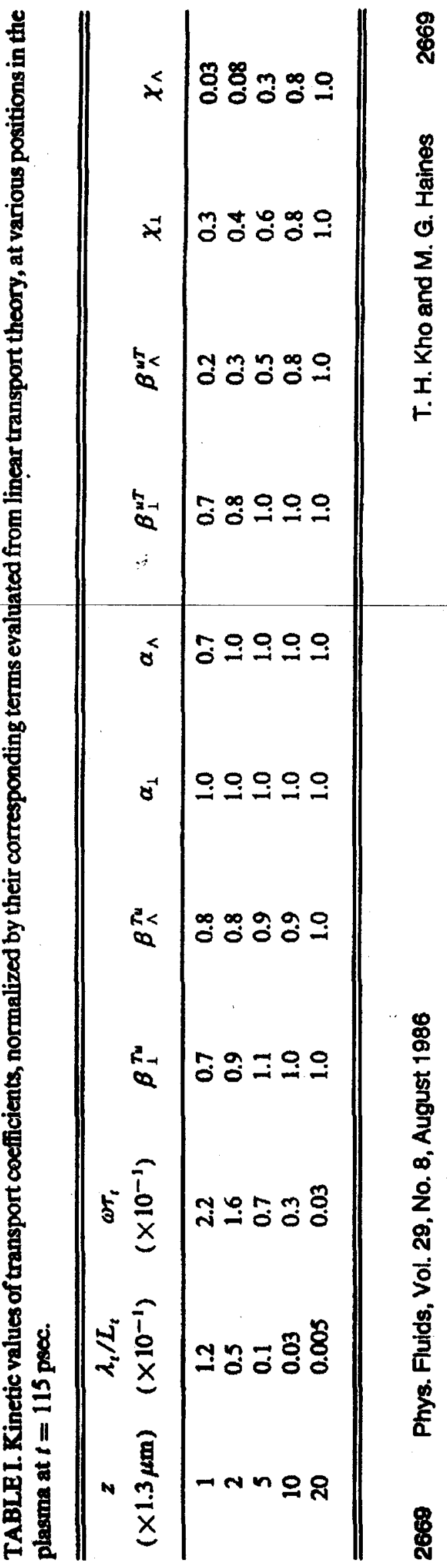


Results and conclusions

1. Nernst convection and amplification of $B$

2. Nernst velocity $\simeq \frac{q}{s / 2 p}=V_{T} \quad\left(\right.$ see $\nu \propto \frac{1}{r^{2}}$ model $)$

3. Righi-Leduc heat flux severely limit ( $3 \%)$ close to critical surface due to depleted tail and we $\sim 0.2<1$

This is partly a result of the Langton $f_{0} \rightarrow e^{-r^{5}}$ tendency 
The collision frequency $\propto v^{-2}$ model

$$
\frac{\partial f_{1}}{\partial t}+v \nabla f_{0}-\frac{e E}{m} \partial_{v} f_{0}-\frac{e \underline{B}}{m_{c}} \times \underline{f}_{1}=-\nu f_{1}
$$

Moments: $\underline{j}=-\frac{4 \pi}{3} e \int^{\infty} d v r^{3} f_{1} \quad$ current density

$$
q_{T}=\frac{2 \pi}{3} m_{0} \int_{0}^{\infty} d r r^{5} f_{1} \quad \text { total heat flux }
$$

For $\nu=\nu_{r} \frac{v_{T}^{2}}{v^{2}}, \times \frac{4 \pi}{3} v^{5}$ and integrate

$$
\frac{2 m_{e}}{5 e p_{e}} \frac{\partial q_{T}}{\partial t}+\frac{m}{e p_{e}} \nabla R+\underline{E}+\frac{2}{5} \frac{q_{T} \times \underline{B}}{p_{c} c}=\frac{2 v_{T} m_{e} j}{5 e n_{e}}
$$

At $t=0,-\nabla \times \underline{E}=\frac{\partial \underline{B}}{\partial r}=\nabla \times\left[\frac{m_{e} \nabla R}{\beta_{e}}\right]$

similar to King ham and Bell, but here

$$
R=\frac{4 \pi m_{0}}{15} \int_{0}^{\infty} f_{0} r^{6} d v \text { and } P_{0}=\frac{4 \pi}{3} m_{0} \int f_{0} v^{4} d r
$$

Note the Nernst convection of $\underline{B}$ by $\frac{q_{1}}{s / 2} P_{R}$ and the equation is true for any $f_{0}$.

If $\nu=$ constant

$$
\text { If } \nu=\frac{m_{e}}{n e^{2}} \frac{\partial \dot{j}}{\partial t}+\frac{1}{n_{e}} \nabla_{p_{e}}+E-\frac{\dot{y} \times B}{n_{e} c c}=\frac{\dot{j}}{\sigma}
$$

Compare term by term. 
For a Maxwellion $R=\frac{p_{e}^{2}}{n m_{c}}$

Also $q_{r}=q_{0}+\frac{5}{4} \frac{m r_{r}}{2} j$

$\frac{m_{e}}{e p_{0}} \nabla R$ term $\rightarrow \frac{\nabla p_{e}}{n e}+\frac{\nabla T}{e} \quad$ Note scalar $\beta+\eta$.

Comparison with Epperlein and Harries, $\nu \propto \frac{1}{r^{3}}$ and ere collisions shows that new Ohm's law is good, especially for $z=3$

M.G. Haines, Plasma Phys. Controll. Fusion 28 (1986)/170s 


\title{
Thomson Scattering Measurements of Heat Flow in a Laser-Produced Plasma
}

\author{
J. Hawreliak
}

Department of Physics, Clarendon Laboratory

Univerisity of Oxford

Oxford, OX1 3PU, UK 


\section{D.M. Chambers \$2 $^{2}$, S.H. Glenzer ${ }^{3}$, A. Gouveia ${ }^{1}$,}

R.J. Kingham ${ }^{4}$, R.S. Marjoribanks ${ }^{5}$, P.A. Pinto ${ }^{2}$,

O. Renner6 ${ }^{6}$, P. Sondhauss ${ }^{1}$, S. Topping 7 ,

E. Wolfrum ${ }^{1} \uparrow$, P.E. Young ${ }^{3}$, and J.S. Wark ${ }^{1}$

1. Department of Physics, Clarendon

Laboratory, University of Oxford, Parks

Road, Oxford, OX1 3PU, UK

2. Steward Observatory, University of Arizona, Tucson, AZ 85721, USA

3. Lawrence Livermore National Laboratory, Livermore, CA 94550, USA

4. Department of Physics, Blackett Laboratory, Imperial College of Science Technology and Medicine, London SW7 2BZ, UK
5. Department of Physics, McLennan Physical Laboratories, University of Toronto, Toronto, Ontario, M5S 1A7, Canada

6. Institute of Physics, Czech Academy of Sciences, 18221 Prague, Czech Republic

7. School of Mathematics and Physics, Queens University Belfast, Belfast, BT7 $1 \mathrm{NN}, \mathrm{UK}$

Current Addreses : †Institut fuer Plasmaphysik, Max-Planck-Gesellschaft, D-85748, Garching, Munich,DE $\ddagger$ Department of Physics, University of York, Heslington, york, YO10 5DD, UK 


\section{Outline of Talk}

- Background

- Brief introduction to Thomson scattering

- Outline of experiment

- Experimental measurements

- Interpretation of results 


\section{History of thermal transport (in three lines or less)}

- Spitzer-Harm / Diffusive theory used to predict thermal transport - fails to model mass ablation rate experiments - introduction of a flux limiter $\dagger$

- Fokker-Planck modeling shows that non-local effects are important in steep temperature gradients *

- No direct experimental confirmation of FP techniques

$\dagger$ D.R. Gray and J.D. Kilkenny, Plasma Phys. 22, 81 (1980)

*A.R. Bell, R.G. Evans, and D.J. Nicholas, Phys. Rev. Let. 51, 1664 (1983) 


\section{Thomson Scattering}

- The scattering of electromagnetic radiation from free electrons

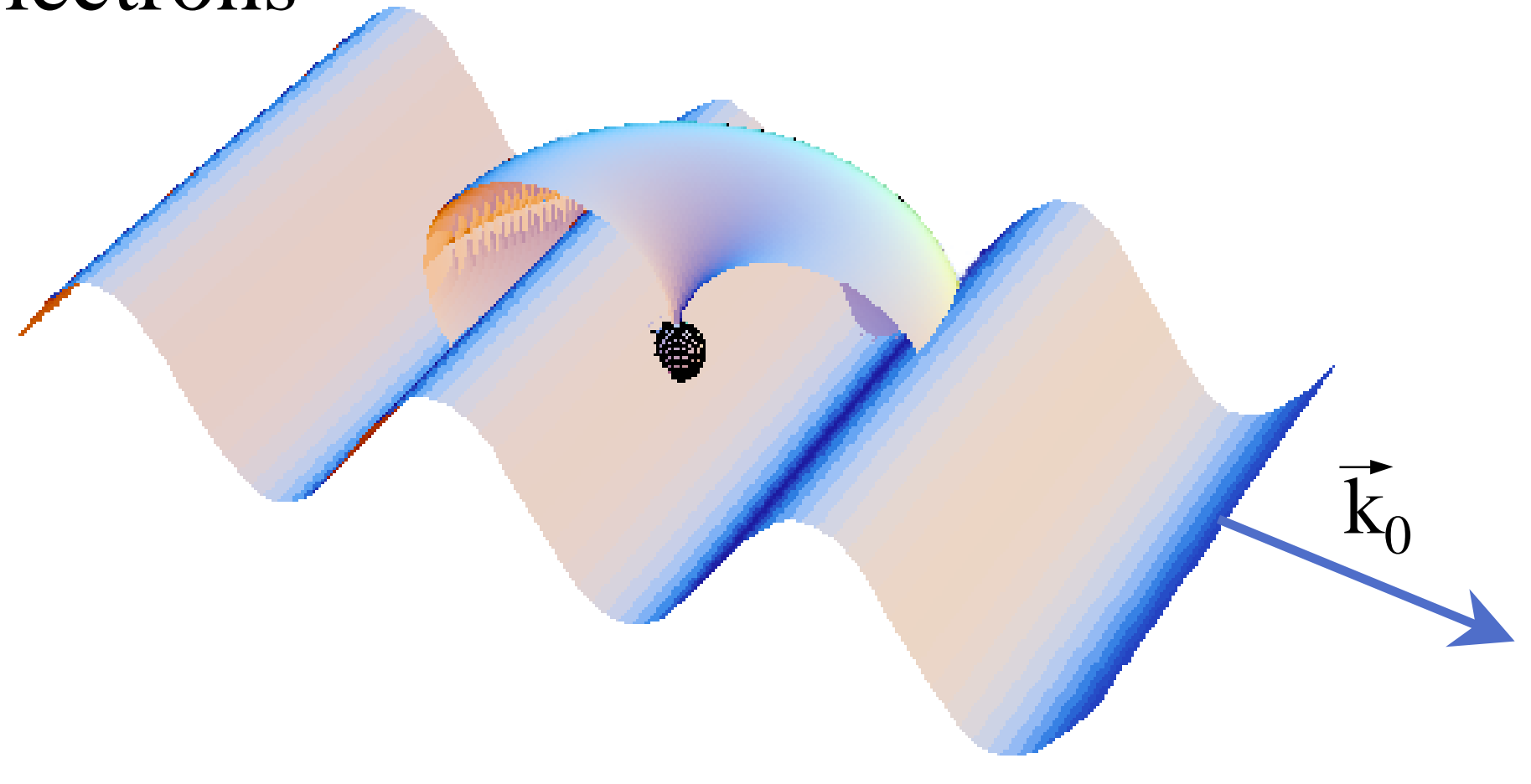




\section{Thomson Scattering from Plasmas (Non-Collective)}

- If the plasma density is low or the wavelength is short $\left(\lambda_{0}<\lambda_{\mathrm{D}}\right)$ the scattering will be the sum of the Doppler shifted frequencies of the electrons
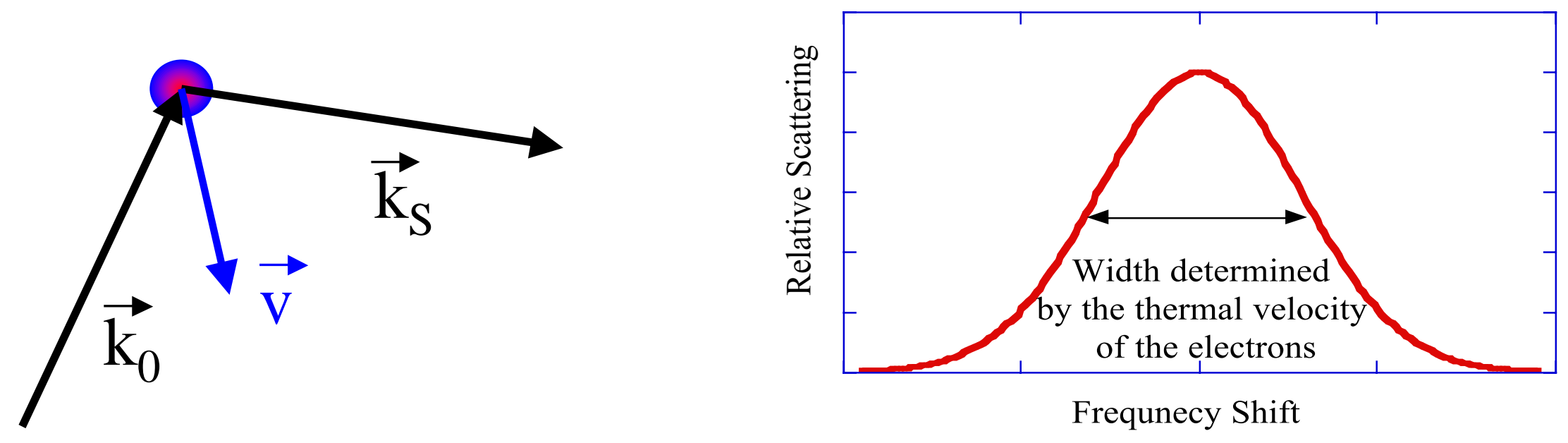


\section{Thomson Scattering from a Laser-Produced Plasma (Collective scattering)}

- For long wavelength probes and higher densities plasmas the correlation of the electron motion will cause enhanced scattering from plasma wave fluctuations

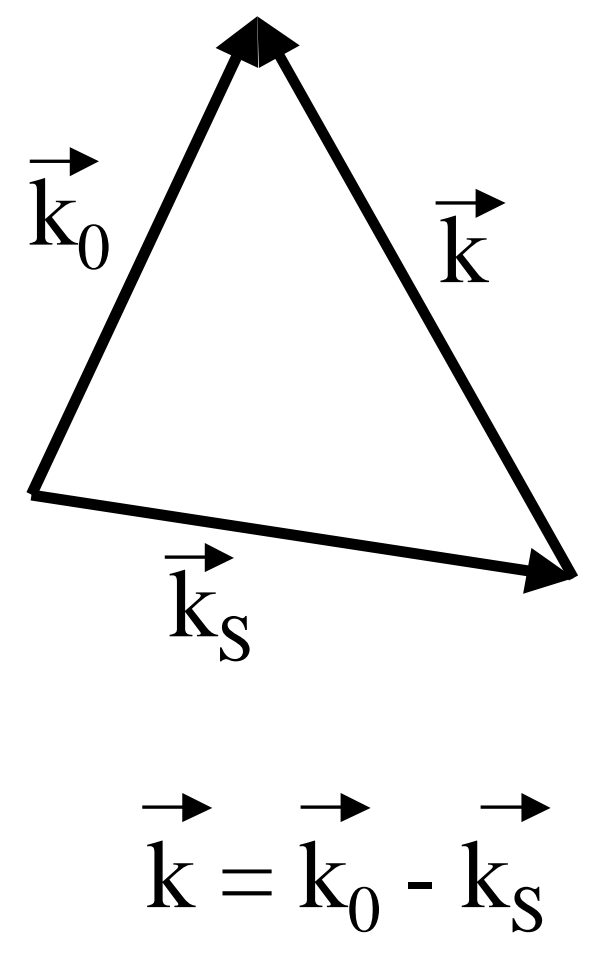




\section{Thomson Scattering from Plasma Wave Fluctuations}

Electron Feature:

Scattering from Langmir waves

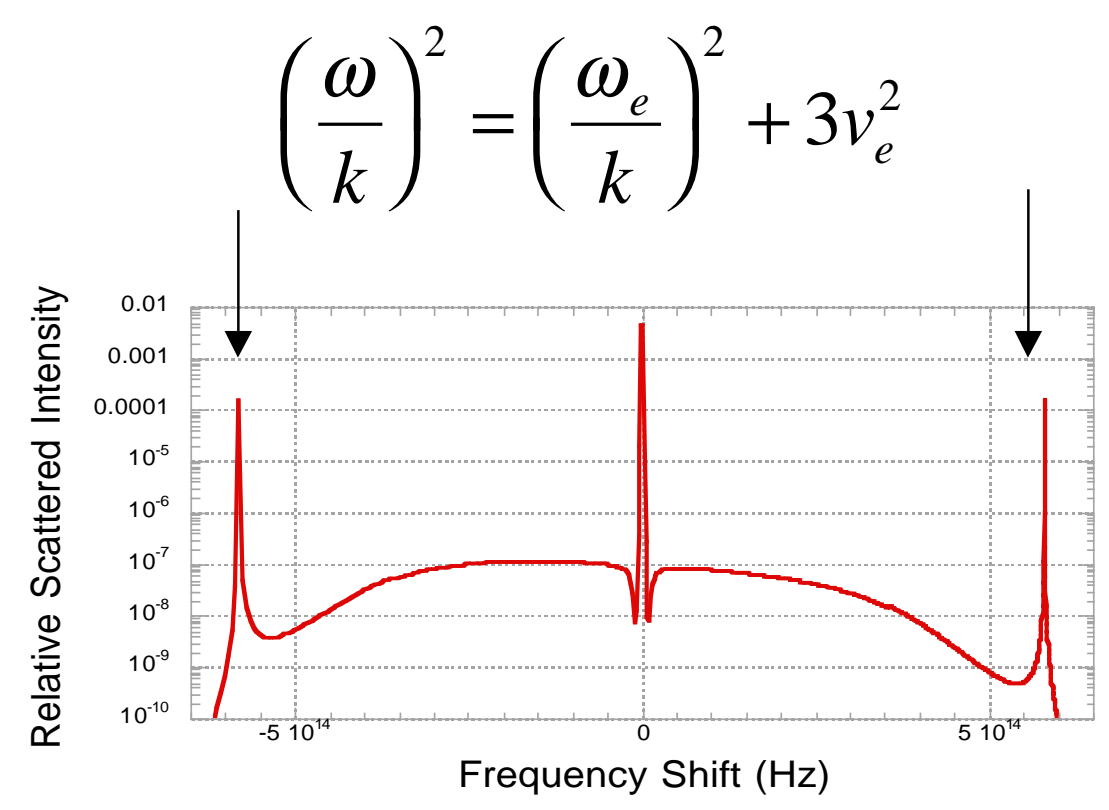

Ion Feature:

Scattering from Ion acoustic waves

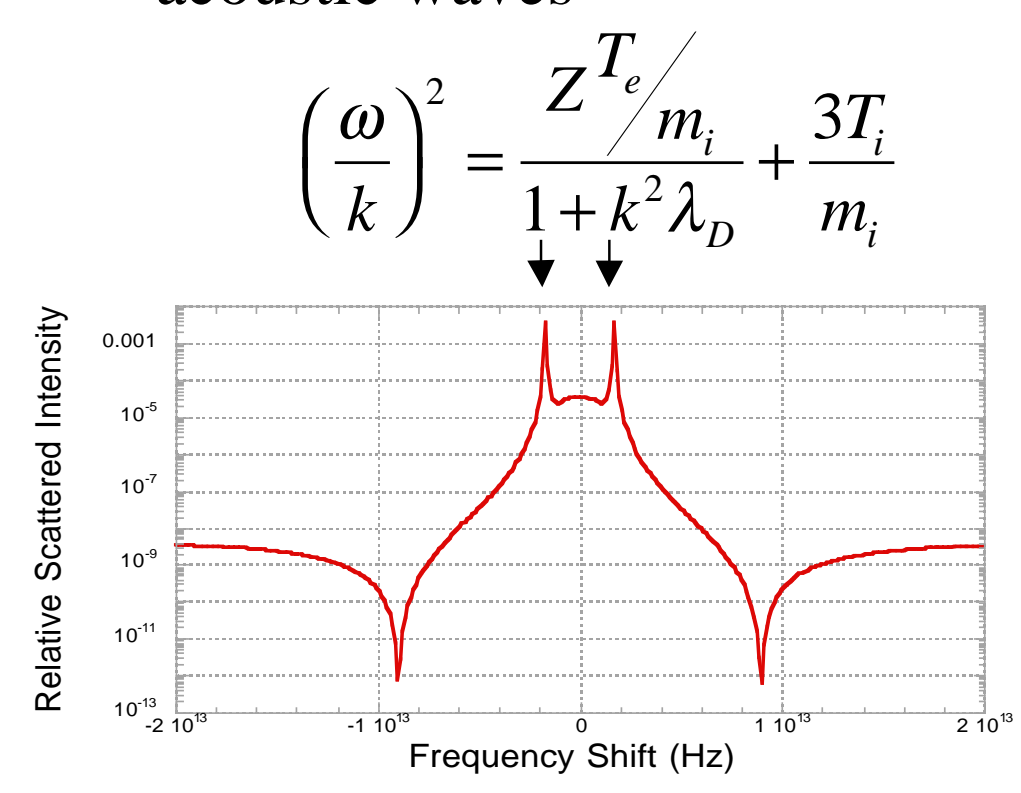

NOTE: We scattered from the ion feature 


\section{Experimental Setup}

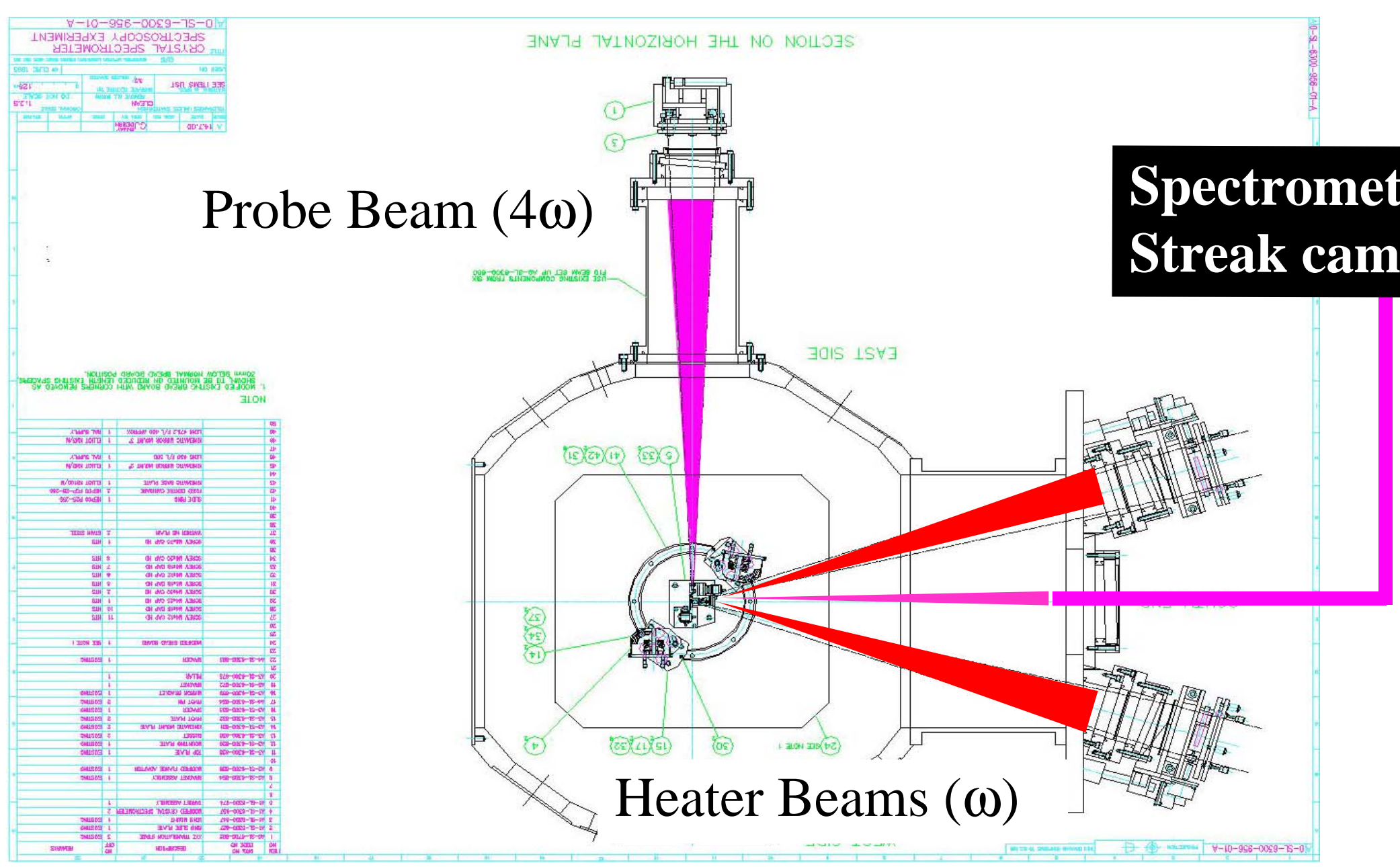




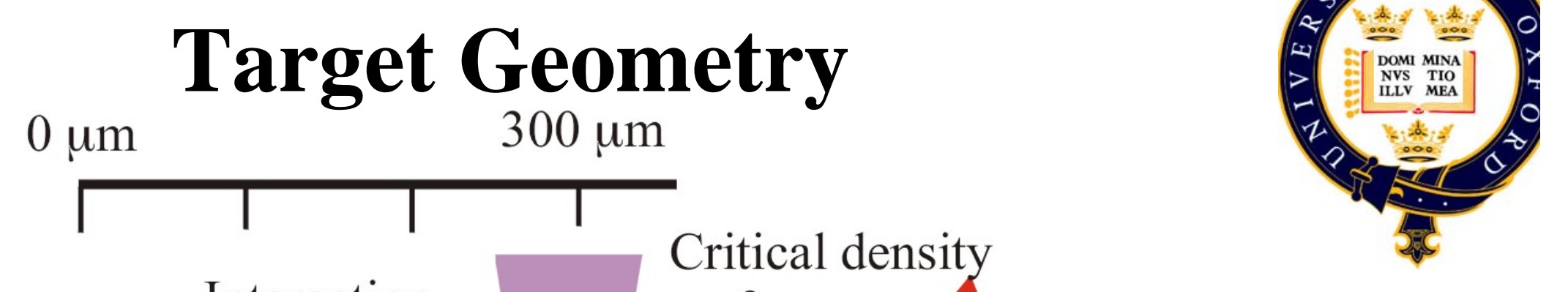
Al Foil
$4 \omega$ probe beam
$1 \omega$ heating beams 


\section{Why $\mathbf{4} \omega$ Probe Beam?}
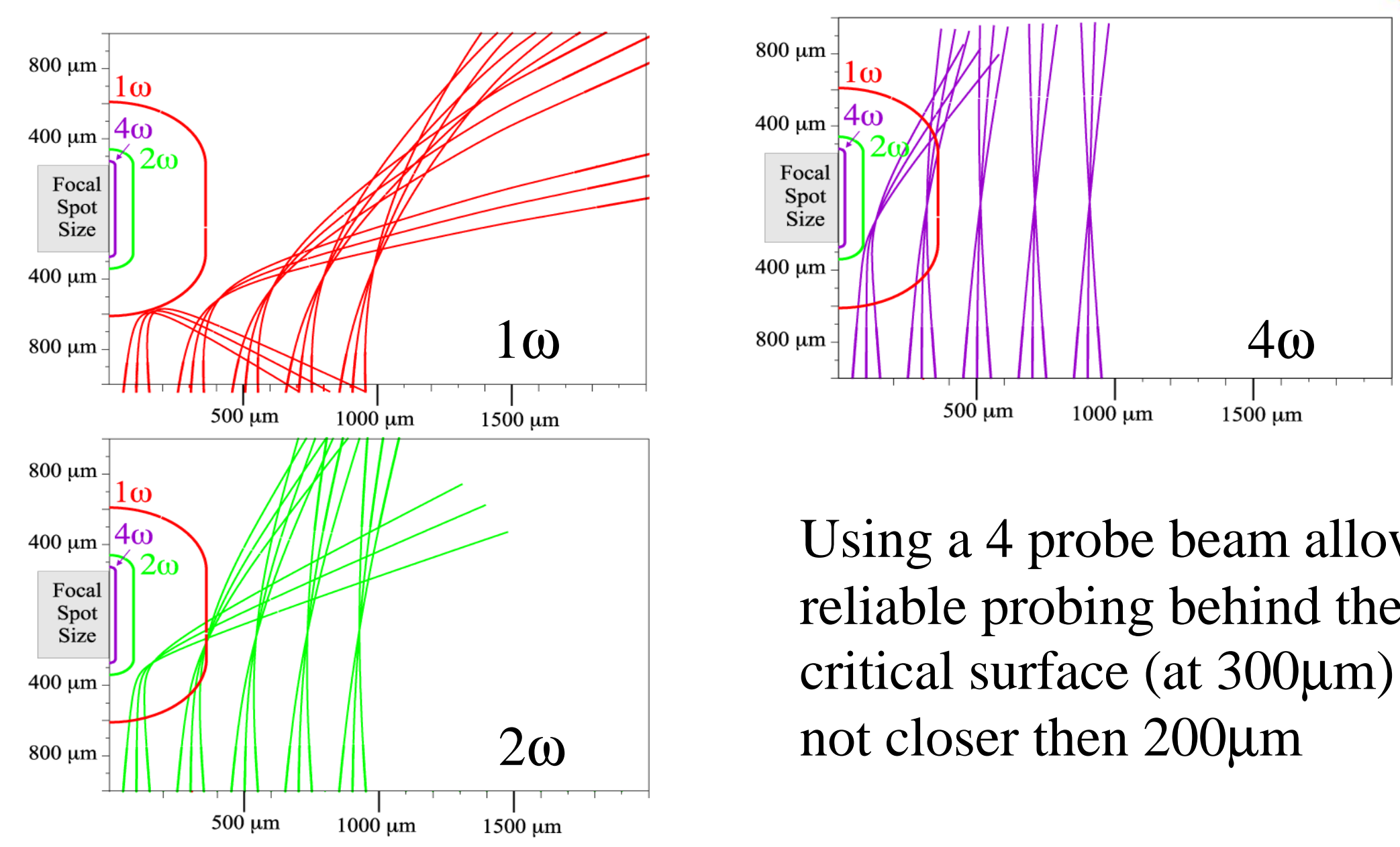

Using a 4 probe beam allows reliable probing behind the critical surface (at $300 \mu \mathrm{m}$ ) but not closer then $200 \mu \mathrm{m}$ 


\section{Experimental Measurement}

Separation of peaks give

Doppler shifted centre of

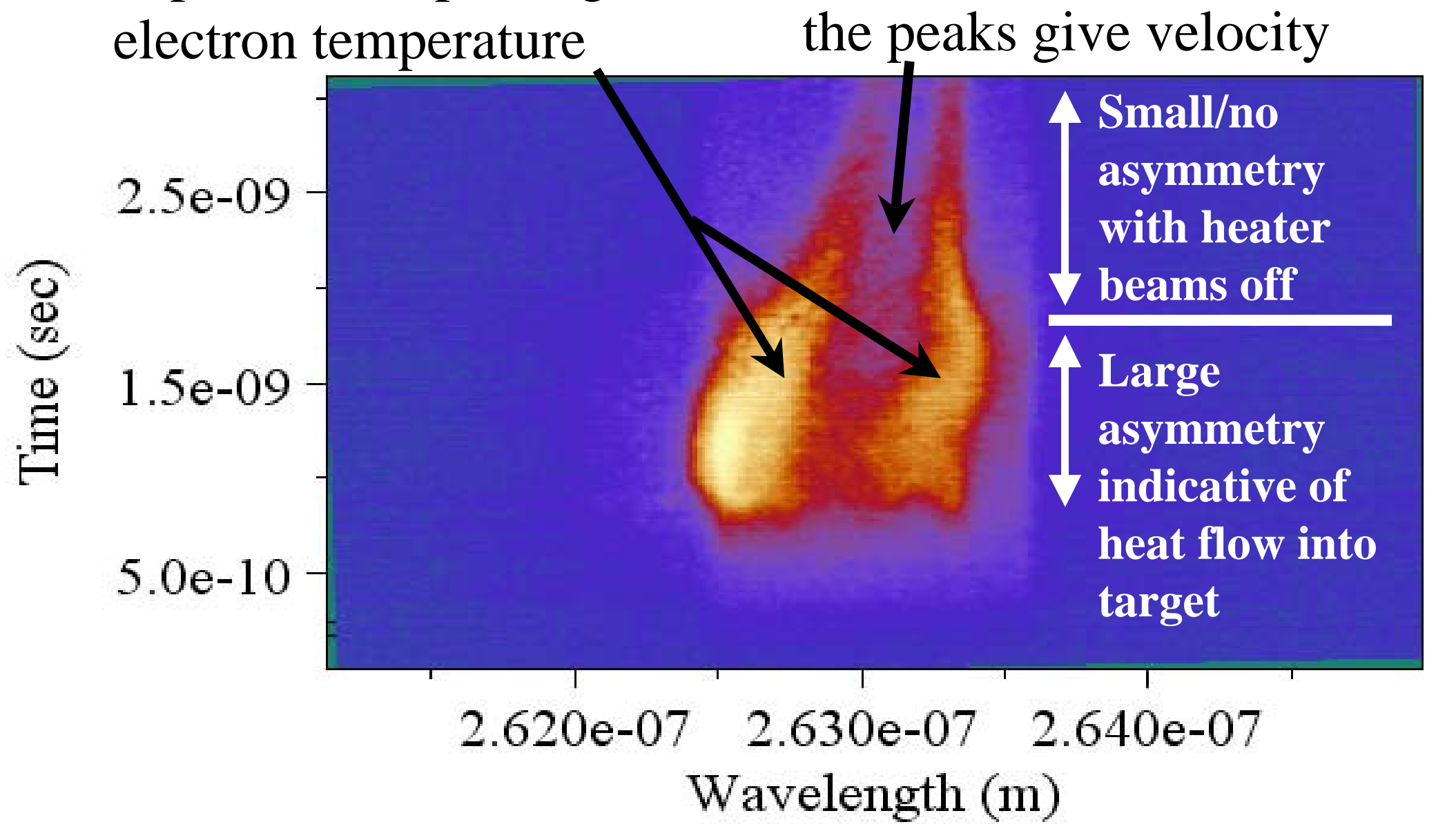




\section{Density Measurement}

- Without measuring the electron feature the mass ablation rate will give a measurement of density

$$
\rho_{M} v=(0.87 \pm 0.06) \times 10^{5}\left(\frac{I_{0}}{10^{13} \frac{\mathrm{W}}{\mathrm{cm}^{2}}}\right)^{0.21 \pm 0.02} \frac{g}{\mathrm{~cm}^{2} \mathrm{sec}}
$$

- With a velocity measurement of $\pm 4 \%$, a beam intensity of $\pm 10 \%$, using the above equation at $300 \mu \mathrm{m}$ we get a density of $10^{21} \mathrm{~cm}^{-3} \pm 20 \%$

* M.H. Key, W.T. Toner, T.J. Goldsack, J.D. Kilkenny, S.A. Veats, P.F. Cunningham, and C.L.S.Lewis, Phys. Fluids 26,2011,(1983) 


\section{Heat Flux Measurement}

- Heat flux can be measured behind the critical density surface by using a basic energy balance, energy can only be carried through thermal transport to the target surface.

$$
Q=\rho v\left[\frac{5}{2}\left(\frac{Z+1}{A_{m p}}\right) k T+\frac{1}{2} v^{2}\right]
$$

- Where the temperature and velocity are taken from the Thomson scattering data. Using the above equation at $300 \mu \mathrm{m}$ (which has been determined to be behind the critical density surface) we get a heat flux of $6 \times 10^{13} \pm 20 \% \mathrm{~W} / \mathrm{cm}^{2}$

- Good agreement with energy balance equations ( 40\% absorbed $1 / 2$ which is transported to target, $1 / 2$ needed to maintain plasma corona)

*R. Fabbro, C. Max, and E. Fabre, Phys. Fluids 28,1463 (1985) 


\section{Thomson Scattering Cross Section}

- The scattering cross section for an electron in a plasma $\sigma(k, \omega)=\sigma_{\mathrm{T}} \mathrm{S}(\mathrm{k}, \omega)$

$$
\begin{aligned}
S(\vec{k}, \omega) & =\left|\frac{1-G_{i}(\omega / k)}{1-G_{e}(\omega / k)-G_{i}(\omega / k)}\right|^{2} f_{0 e}(\omega / k) \\
& +Z\left|\frac{G_{e}(\omega / k)}{1-G_{e}(\omega / k)-G_{i}(\omega / k)}\right|^{2} f_{0 i}(\omega / k)
\end{aligned}
$$

- $\mathrm{S}(\mathrm{k}, \omega)$ is the dynamic form factor, which incorporates the motion and collective affects of the plasma

* D.E. Evans and J. Katzenstein, Reports On Progress Phys. 32, 207 (1969) 


\section{Asymmetric Scattering Peaks}

$$
G_{s}\left(\vec{v}_{0}\right)=\frac{4 \pi Z_{s}^{2} e^{2}}{m_{s} k^{2}} \int \frac{\vec{k} \cdot \vec{\nabla}_{v} f_{0 s}(\vec{v})}{\vec{k} \cdot\left(\vec{v}-\vec{v}_{0}\right)} d \vec{v}
$$

- $\mathrm{G}_{\mathrm{s}}\left(\mathrm{v}_{0}\right)$ is the screening integral in the plasma

- Scattering resonances when $\Re\left\{1-\mathrm{G}_{\mathrm{e}}-\mathrm{G}_{\mathrm{i}}\right\}=0$, so $\mathrm{S}(\mathrm{k}$, ) will depend on the velocity gradient of the distribution function along the direction of the scattering vector, $\mathrm{k}$, at the phase velocity of the wave.

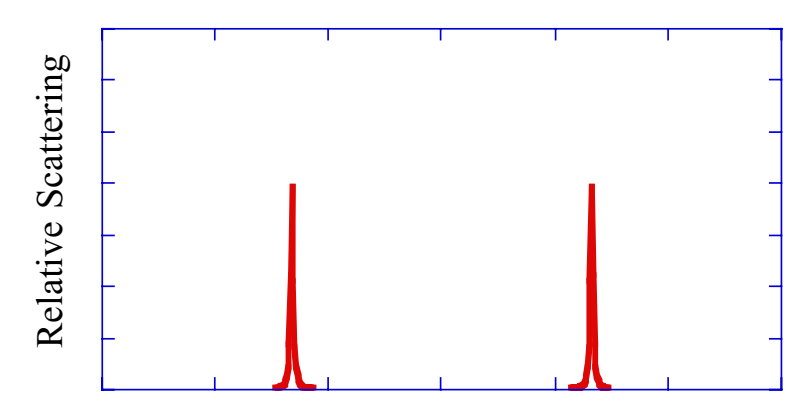

Frequency Shift $(\mathrm{Hz})$

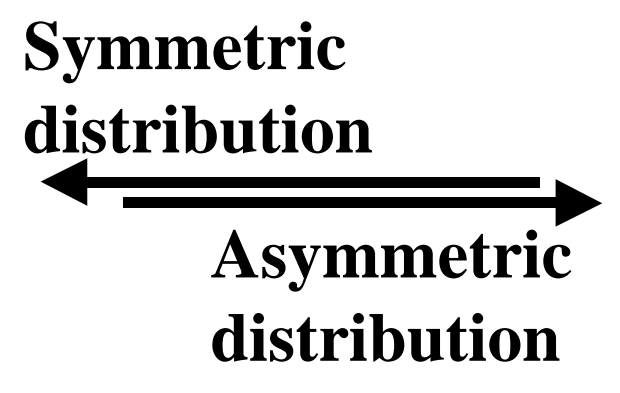

distribution

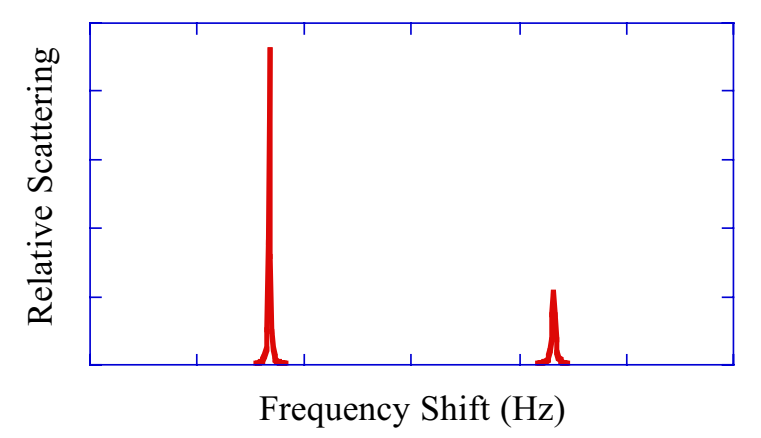

* D.E. Evans and J. Katzenstein, Reports On Progress Phys. 32, 207 (1969) 


\section{A measurement of the distribution function}

- The asymmetry in the Thomson scattering peaks gives information about the difference in the reduced distribution function* at the phase velocity of the ion acoustic waves

$$
{ }^{*} f(v)=\int f(\vec{v}) d \vec{v}_{\perp}
$$




\section{Generating simulated Thomson scattering images}

- Developed Thomson scattering program that numerically solves the screening integrals

- To model experimental geometry where the probe is $45^{\circ}$ to the direction of heat flow transform cylindrically symmetric results

$$
\begin{aligned}
f\left(v_{z}\right) & =\int_{0}^{\pi / 2+\psi} d \theta\left\{2 \int_{0}^{\pi} d \phi\left[f\left(v_{r}, \theta, \phi\right) \frac{v_{z}{ }^{2} \sin (\theta)}{(\cos \psi \cos \theta+\sin \psi \sin \theta \cos \phi)^{3}}\right]\right\} \\
v_{r} & =\frac{v_{z}(\cot \psi \cot \theta)}{\cos \psi \cos \theta+\sin \psi \sin \theta \cos \phi}
\end{aligned}
$$

where $\psi$ is the angle between the direction of heat flow and the scattering vector $\mathbf{k}$ 


\section{Heat Transport Models}

- "Classical" Spitzer-Harm heat transport model

- Transport depends on local quantities

- Conductivity $\propto(\mathrm{kT})^{5 / 2}$

- Distribution taken from numerical results in L.J. Spitzer and R. Harm, Phys. Rev. 89, 977 (1953)

- Fokker-Planck

- IMPACT as outlined in

R.J. Kingham and A.R. Bell, Phys. Rev. Let. 88, 045004/1 (2002) 


\section{Using experimentally measured}

parameters to generate simulated

\section{scattered spectrum}

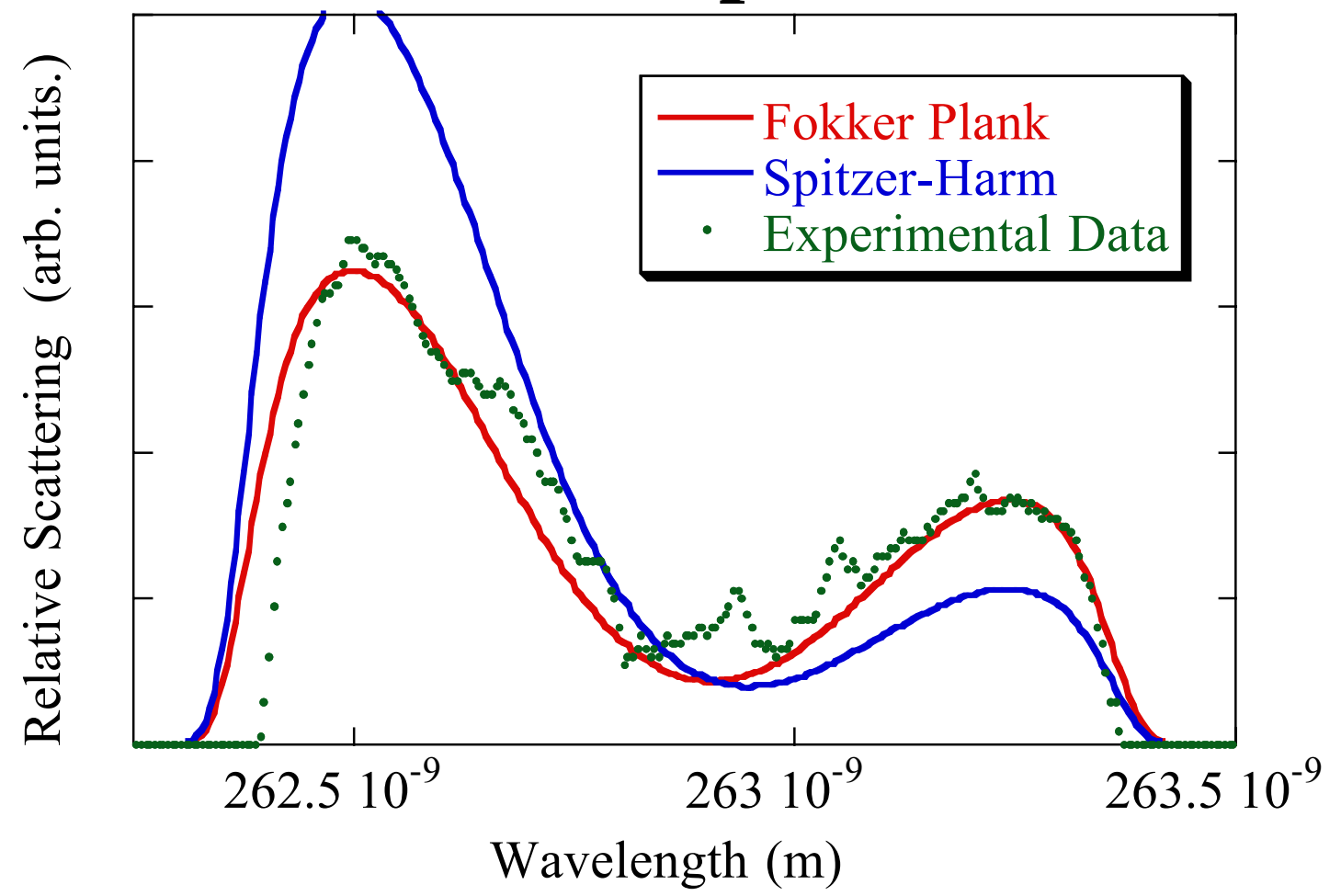

From experimentally measured parameters

$$
\frac{L}{\lambda_{e}}=\frac{T}{\lambda_{e}}\left(\frac{d T}{d x}\right)^{-1}=10 \rightarrow 100 \begin{aligned}
& \text { Within the error bars of } \\
& \text { the measured } \\
& \text { temperature gradient }
\end{aligned}
$$

Fokker Plank

- $\mathrm{T}_{\mathrm{e}}=1850 \mathrm{eV}$

- $\mathrm{T}_{\mathrm{i}}=960 \mathrm{eV}$

- $\mathrm{n}_{\mathrm{e}}=1 \times 10^{21} \mathrm{~cm}^{-3}$

- $\mathrm{Z}=13.0$

- $\mathrm{Q}=5.5 \times 10^{17} \mathrm{~W} / \mathrm{m}^{2}$

(:) $\nabla \mathrm{T}_{\mathrm{e}}=7 \mathrm{eV} / \mu \mathrm{m}$

(i) $L / \lambda_{e}=17$

(:) Asymmetry $=2$

Spitzer-Harm

- $\mathrm{T}_{\mathrm{e}}=1850 \mathrm{eV}$

- $\mathrm{T}_{\mathrm{i}}=960 \mathrm{eV}$

- $\mathrm{n}_{\mathrm{e}}=1 \times 10^{21} \mathrm{~cm}^{-3}$

- $\mathrm{Z}=13.0$

- $\mathrm{Q}=5.5 \times 10^{17} \mathrm{~W} / \mathrm{m}^{2}$

(:) $\nabla \mathrm{T}_{\mathrm{e}}=3 \mathrm{eV} / \mu \mathrm{m}$

;) $\mathrm{L} / \lambda_{\mathrm{e}}=40$

: Asymmetry $=4$ 


\section{Can Spitzer-Harm fit?}

- All the of parameters used to derive the distribution function for both thermal transport models are consistent with experimental measurements ( the temperature gradient was calculated from the models for the experimentally measured heat flux).

- To get a SH spectrum to match the experiment requires a temperature gradient of less then $\mathbf{2} \mathrm{eV} / \mu \mathrm{m}$ and $\mathbf{Q}=\mathbf{3}$ $\mathbf{x 1 0}{ }^{17} \mathrm{~W} / \mathbf{m}^{2}$ which cannot maintain the energy balance. 


\section{Difference between Fokker-Plank and Spitzer-Harm}

Distributions for experimental

conditions at $300 \mu \mathrm{m}$

$\mathrm{f}_{\text {total }}$ showing why noncollective scattering would not resolve difference

$\mathrm{f}_{1}$ terms of the reduced distribution functions

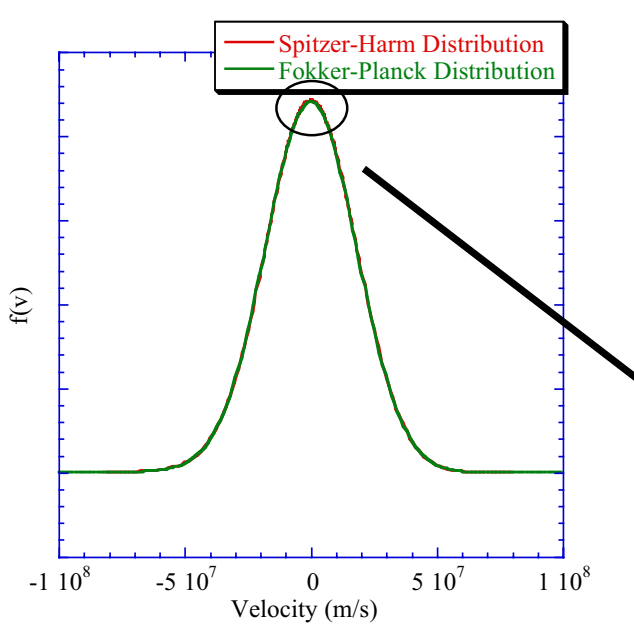
both carrying the same heat flux

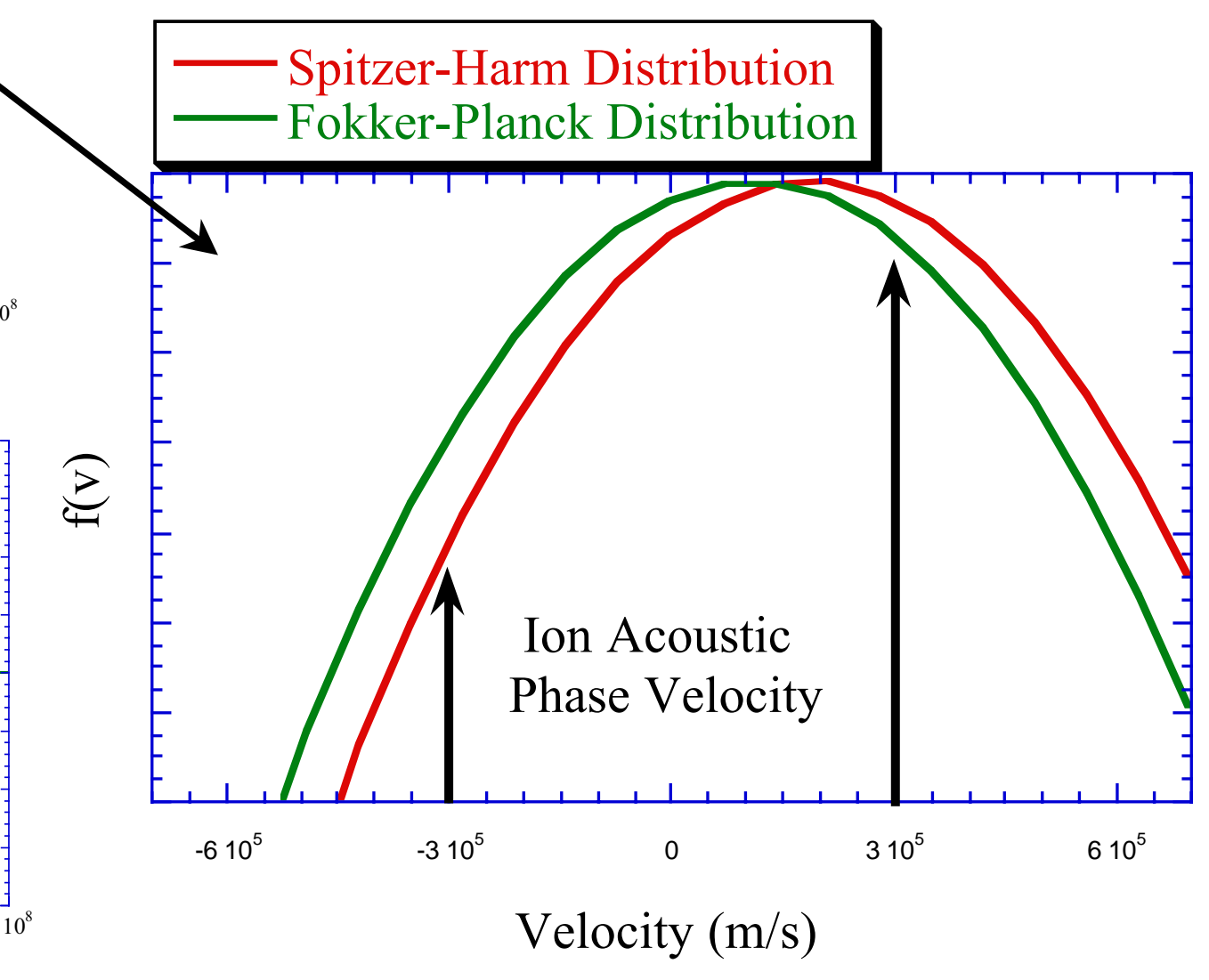




\section{Definition: Asymmetry Measurement}

- The asymmetry in the ion features give us a tool for studying the distribution function
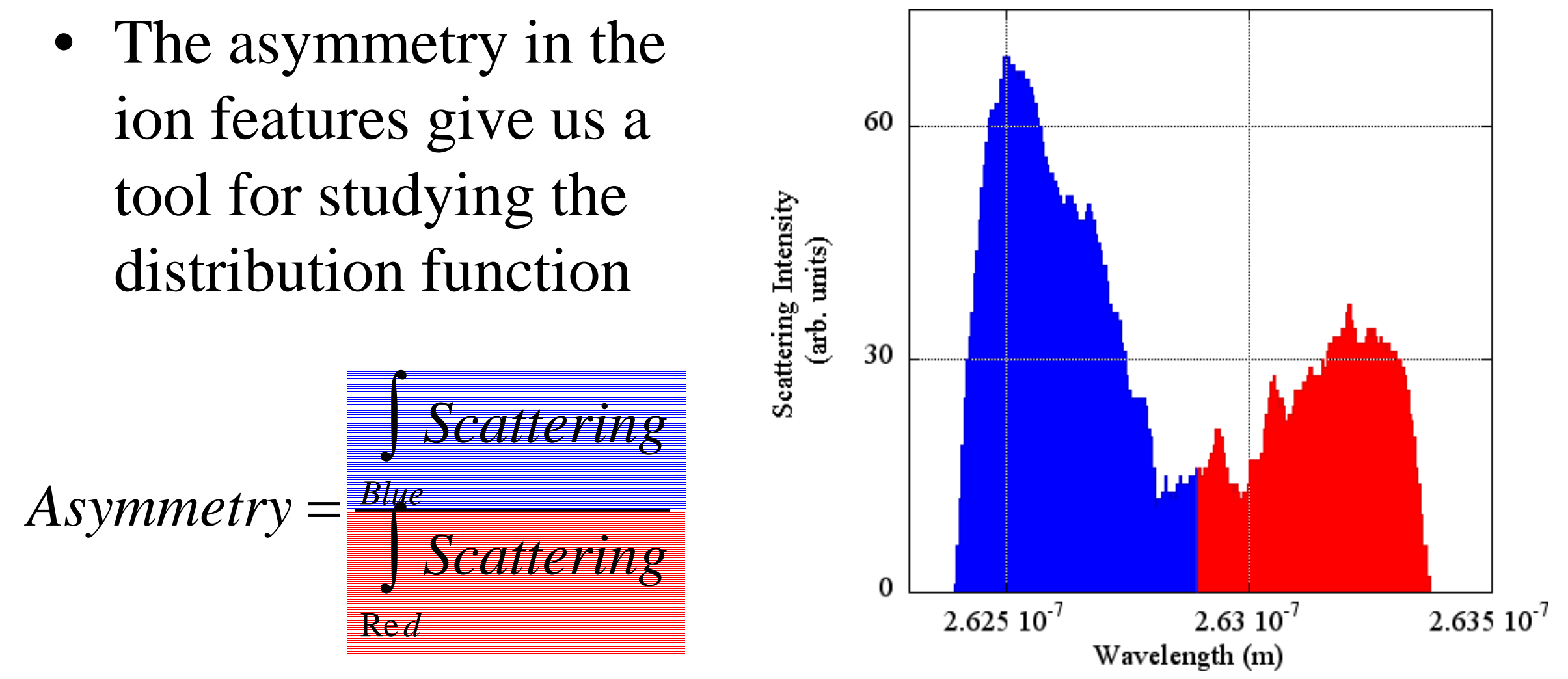


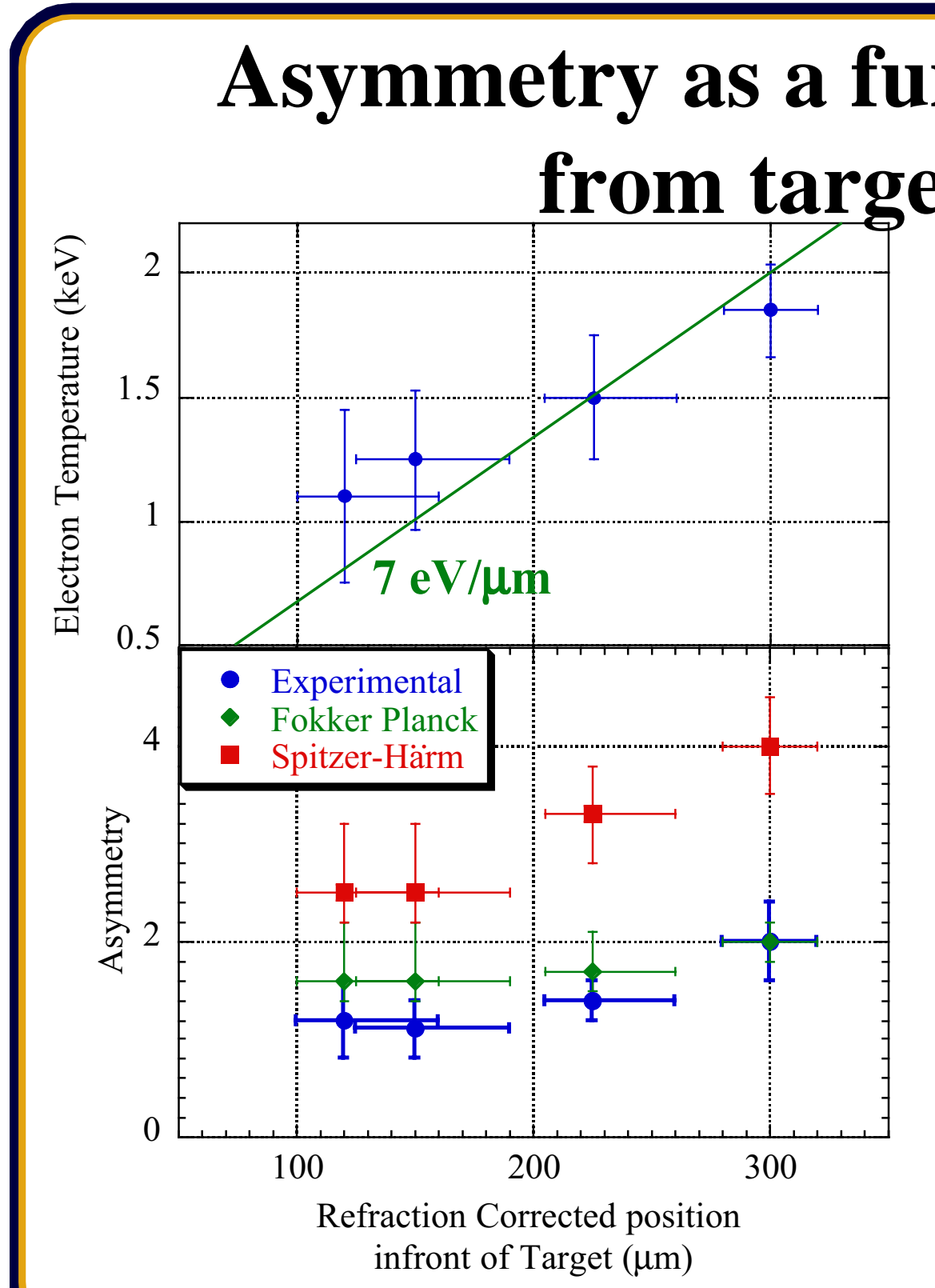

- The Fokker-Planck matches experimental measurement of the asymmetry in the Thomson scattering peaks and is consistent with the measured temperature gradient.

- Spitzer-Harm does not 


\section{Conclusions}

$\checkmark$ The first direct measurement of heat flux behind the critical density surface

$\checkmark$ Confirmation of Fokker-Planck modeling techniques

$\checkmark$ Diagnostic tool for looking at the changes in the electron distribution function due to heat flux 


\title{
Fokker-Planck Modelling of Non-local Magnetic Field Generation in Collisional Plasmas
}

\author{
R. J. Kingham and A. R. Bell
}

Plasma Physics Group, Imperial College, London

LLNL Electron Transport Workshop, Purple Orchid Inn, Livermore $9^{\text {th }}-11^{\text {th }}$ September 2002 


\section{Outline of Talk}

- Goal:

- Know importance of non-local effects for heat flow...

- What role do non-local effects play in B-field generation?

- B-field generation in regime where Braginskii \& Spitzer not valid

圈 Overview of B-field gen. in collisional plasmas

㽟 Description of IMPACT: 2D electron Fokker-Planck code with self-consistent B-field

逥 FP sim. $\rightarrow$ non-local B-field generation when $\nabla n_{e}=0 \&$ non-uniform heating

[1] Basic explanation of non-local B-field mechanism

FP sim. $\rightarrow$ non-local B-field generation when $\nabla Z \neq 0$ \& uniform heating

Analytical formula for non-local, seed B-field from $\mathrm{T}_{\mathrm{e}}$ perturbation

FP sim. $\rightarrow$ heating in a density gradient \& comparison with classical case 


\section{Standard B-field Sources Need $\nabla n \neq 0$ or $\Pi \neq 0$}

- Standard collisional B-field source...

$$
\dot{B}=-\frac{1}{n}\{\nabla n \times \nabla T\}
$$

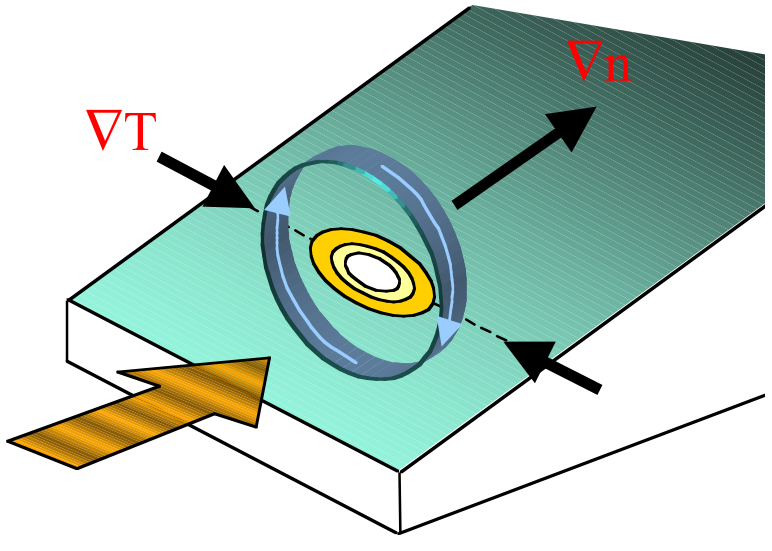

- Originates from "generalized Ohms law" and "Faradays law"

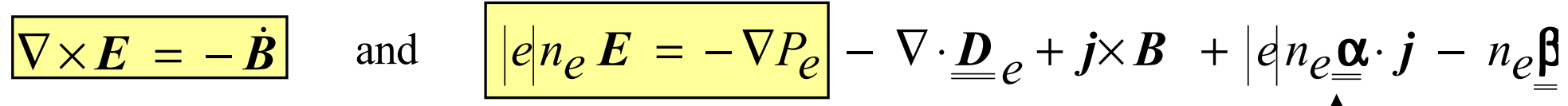

$$
\begin{aligned}
& \text { Scalar pressure }
\end{aligned}
$$

- Ohms' Law, itself, is obtained from the Fokker-Planck equation...

$$
\left[\frac{\partial}{\partial t}+\mathrm{v} \cdot \nabla_{r}+\frac{q}{m_{e}}(\boldsymbol{E}+\mathrm{v} \times \boldsymbol{B}) \cdot \nabla_{\mathrm{v}}\right] f(\boldsymbol{r}, \mathrm{v}, t)=-\nabla_{\mathrm{v}} \cdot\{f\langle\Delta \mathrm{v}\rangle\}+\nabla_{\mathrm{v}} \nabla_{\mathrm{v}}: \underset{\text { Collisional }}{\operatorname{drag}} \quad \begin{gathered}
\text { Collisional } \\
\text { diffusion }
\end{gathered}
$$




\section{Ohm's Law $\leftarrow$ Moment of FP Equation}

- Collisions smooth out fine detail in velocity space...

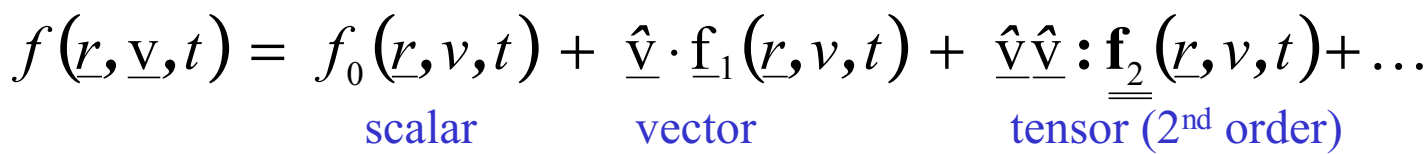

$$
\begin{aligned}
& \mathrm{n}, \mathrm{T} \quad \mathrm{j}, \mathrm{q} \quad \stackrel{\mathrm{P}, \text { viscosity }}{=}
\end{aligned}
$$

- Ohm's law: moment of $\underline{f}_{1}$ eqn. + Say $\mathrm{f}_{0}=\mathrm{f}_{\mathrm{m}} \quad$ (local transport theory)

' Local transport theory: valid $\lambda_{\mathrm{ei}}<<\mathrm{L}, \tau_{\mathrm{ee}}<<\tau$ (also $\mathrm{r}_{\mathrm{g}}<<\mathrm{L}_{\perp}$ if $\omega \tau>>1$ )

- The $\int \mathrm{d}^{3} \mathrm{v}$ moment of the FP equation...

$$
\begin{aligned}
& \frac{\partial f_{0}}{\partial t}+\frac{\mathrm{v}}{3} \nabla \cdot \boldsymbol{f}_{1}-\frac{e}{m_{e}} \frac{1}{3 \mathrm{v}^{2}} \frac{\partial\left(\mathrm{v}^{2} \boldsymbol{E} \cdot \boldsymbol{f}_{1}\right)}{\partial \mathrm{v}}=\underset{\text { Ohmic heating }}{\mathrm{v}^{2}} \frac{v_{\mathrm{ee}}}{\partial \mathrm{v}}\left[\underset{\text { e-e collisions }}{\left.C f_{0}+D \frac{\partial f_{0}}{\partial \mathrm{v}}\right]}\right. \\
& \text { relax. to Maxwellian }
\end{aligned}
$$

- The $\int \underline{\underline{v}} \mathrm{~d}^{3} \mathrm{v}$ moment of the FP equation... (momentum balance)

$$
\frac{\partial \mathrm{f}_{1}}{\partial t}+\mathrm{v} \nabla f_{0}+\frac{2}{5} \nabla \cdot \underline{\underline{\mathrm{f}_{2}}}-\frac{e \mathrm{E}}{m_{e}} \frac{\partial f_{0}}{\partial \mathrm{v}}-\frac{2 e}{5 m_{e} \mathrm{v}^{3}} \frac{\partial}{\partial \mathrm{v}}\left(\underline{\mathrm{E} \cdot \mathrm{f}_{2}}\right)-\frac{e}{m_{e}} \mathrm{~B} \times \mathrm{f}_{1}=-v_{e i} \mathrm{f}_{1}+\left(\frac{\delta \mathrm{f}_{1}}{\delta t}\right)_{e e}
$$


- IMPACT $\rightarrow$ Implicit Magnetised Plasma And Collisional Transport

- Solves $f_{o} \& \mathbf{f}_{1} \mathbf{F P}$ equations for $\mathbf{e}^{-}$self consistently with Maxwell's equations in 2D

- Obtain evolution of ...

$\rightarrow f_{o}(x, y, v) \quad$ Isotropic part of $e^{-}$dist. (defines $T, n$, scalar $p$ )

$\rightarrow f_{x}(x, y, v), f_{y}(x, y, v) \quad$ vector part of $e^{-}$dist. (defines $\mathbf{j}, \mathbf{q}$ )

$\rightarrow E_{x}(x, y), E_{y}(x, y), B_{z}(x, y) \quad$ macroscopic fields

- Geometry: 2D Cartesian grid (x \& y)

- Periodic, reflective and fixed BC's independently in $\mathrm{x}$ and $\mathrm{y}$

- e-e collisions $\rightarrow$ relax. of $f_{o}$ to Maxwellian

- e-i collisions $\rightarrow$ angular scattering of $\mathrm{e}^{-}$

- Ignore e-e collisions in $\mathrm{f}_{1}$ eqn. $\rightarrow$ Lorentz approx. (valid at high Z)

- Keep $\partial \mathbf{f}_{1} / \partial t$ term in $\mathbf{f}_{1}$ eqn. (electron inertia term)

- Configurable ion and $\mathrm{Z}$ profiles (non-evolving)

- No displacement current $\rightarrow \nabla \times \mathbf{B}=\mu_{0} \mathbf{j}$

$\rightarrow$ Valid for overdense plasmas

$\rightarrow$ Plasma can maintain quasineutrality

- $\mathrm{E}, f_{o}, \mathrm{f}_{1}$ are solved implicitly

$\rightarrow$ numerical stability + large "dt" possible

- Change-Cooper differencing scheme used for e-e collision term

$\rightarrow$ Ensures exact relaxation to a Maxwellian 

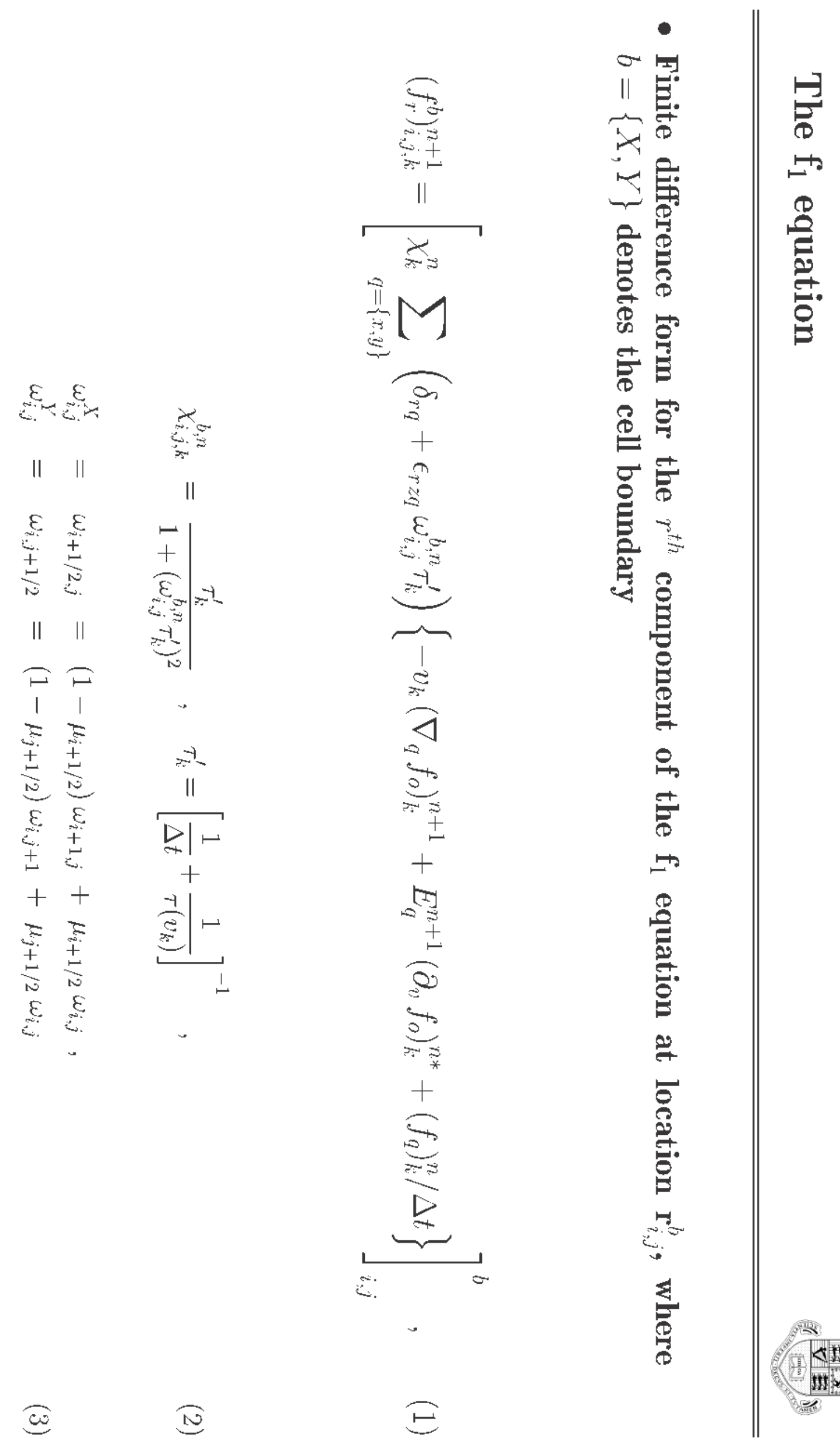


\section{D-FP Simulations With Heating and $\nabla n=0$}

- System \& heating profile...

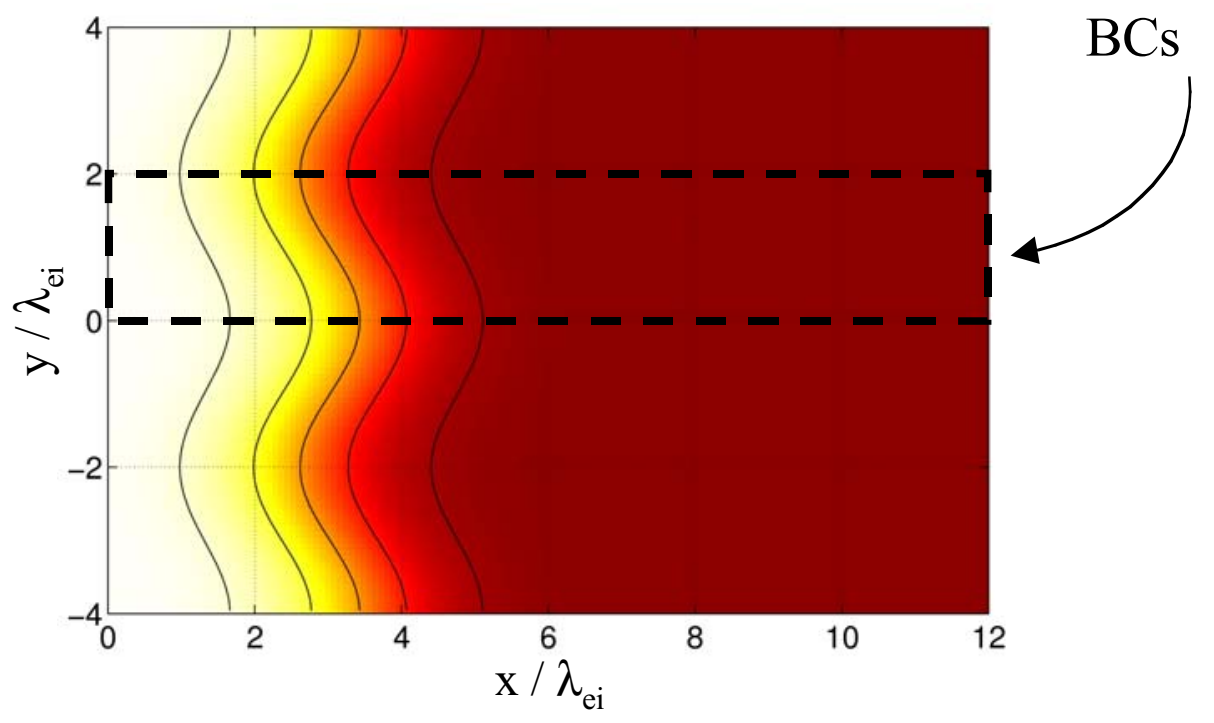

- Parameters...
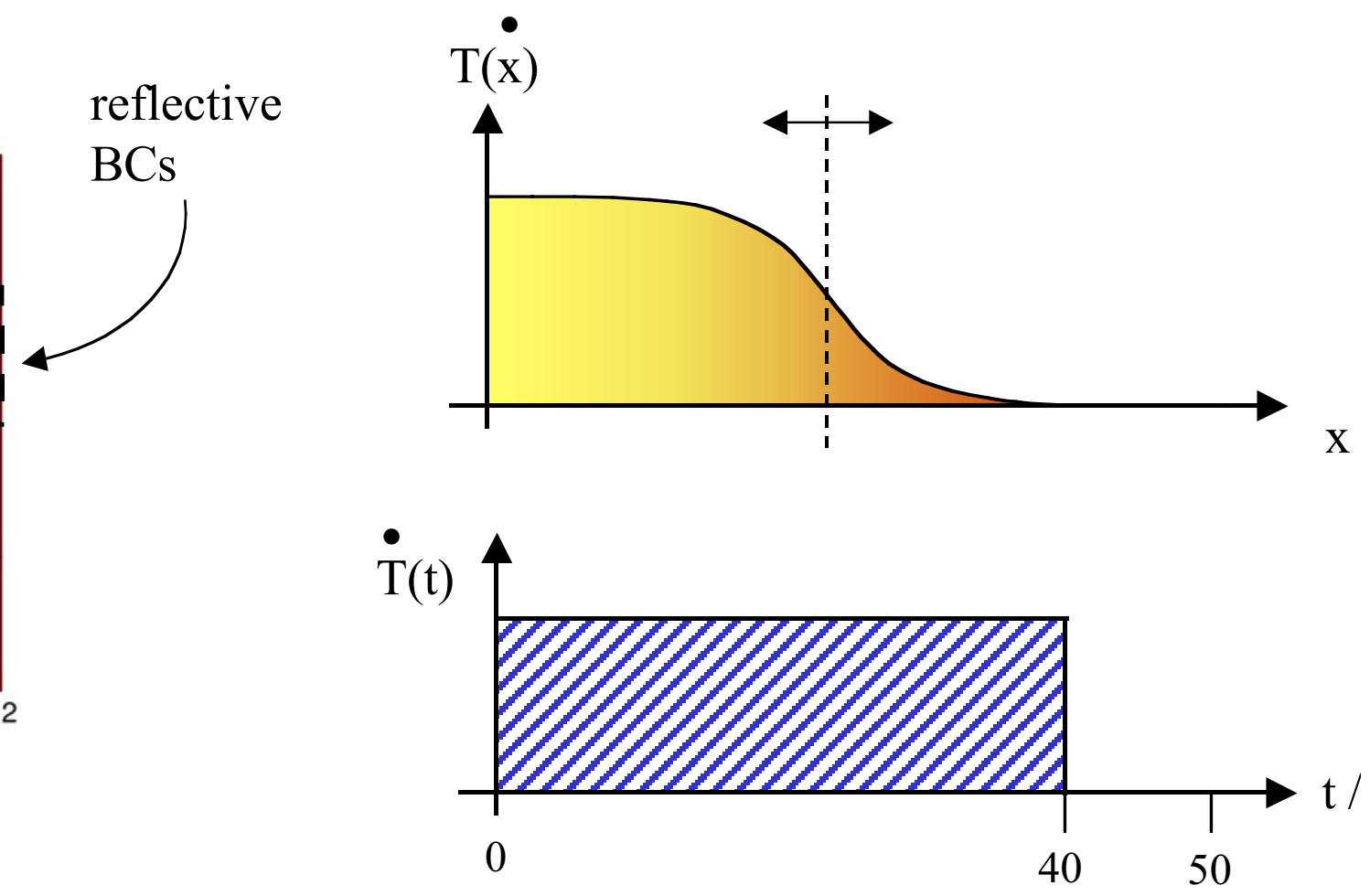

$$
\begin{aligned}
& Z=10 \\
& \frac{\lambda_{\text {ie }}}{\delta_{c}}=100
\end{aligned}
$$

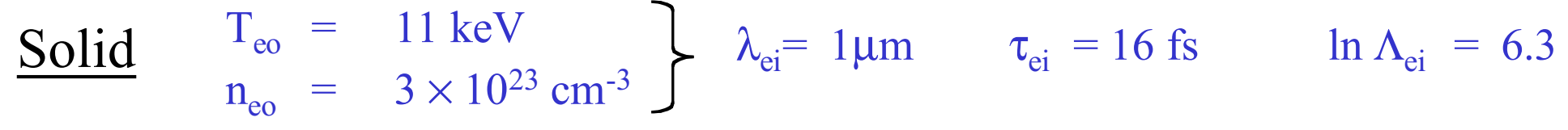

$$
\begin{aligned}
& \left.\underline{10 \times \mathrm{n}_{\mathrm{cr}}} \begin{array}{l}
\mathrm{T}_{\mathrm{eo}}=5 \mathrm{keV} \\
\mathrm{n}_{\mathrm{eo}}=11 \times 10^{21} \mathrm{~cm}^{-3}
\end{array}\right\} \lambda_{\mathrm{ei}}=5 \mu \mathrm{m} \quad \tau_{\mathrm{ei}}=0.12 \mathrm{ps} \quad \ln \Lambda_{\mathrm{ei}}=7.2
\end{aligned}
$$




\section{B-field grows to $\omega \tau>1$ even though $\nabla n=0$}
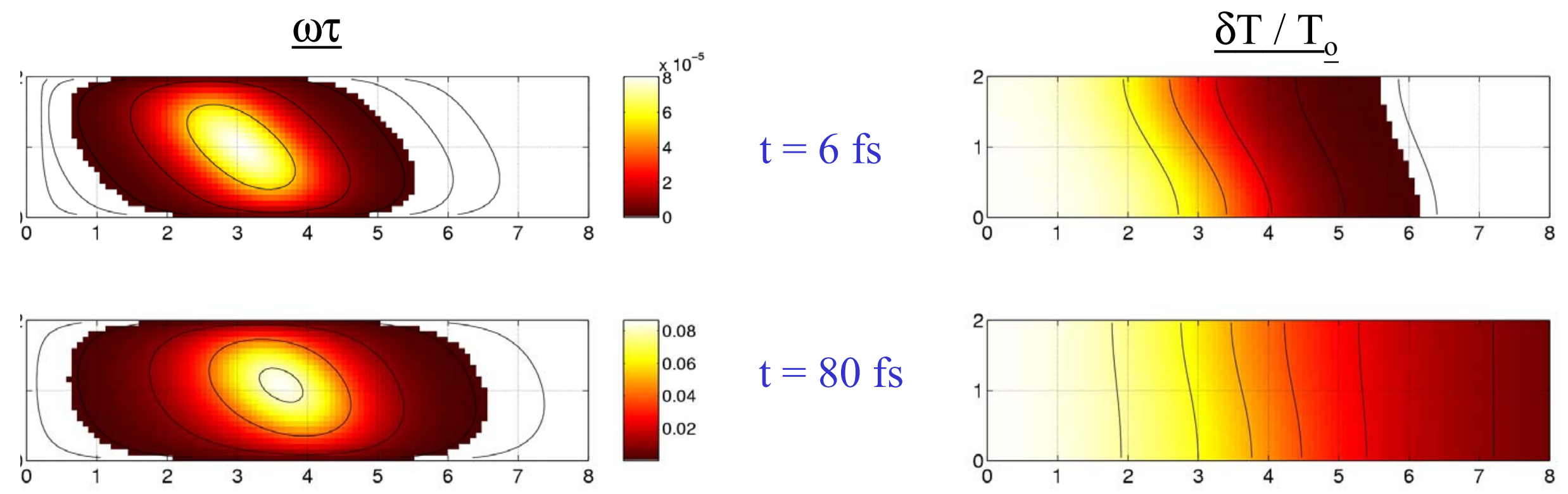

$\mathrm{t}=80 \mathrm{fs}$
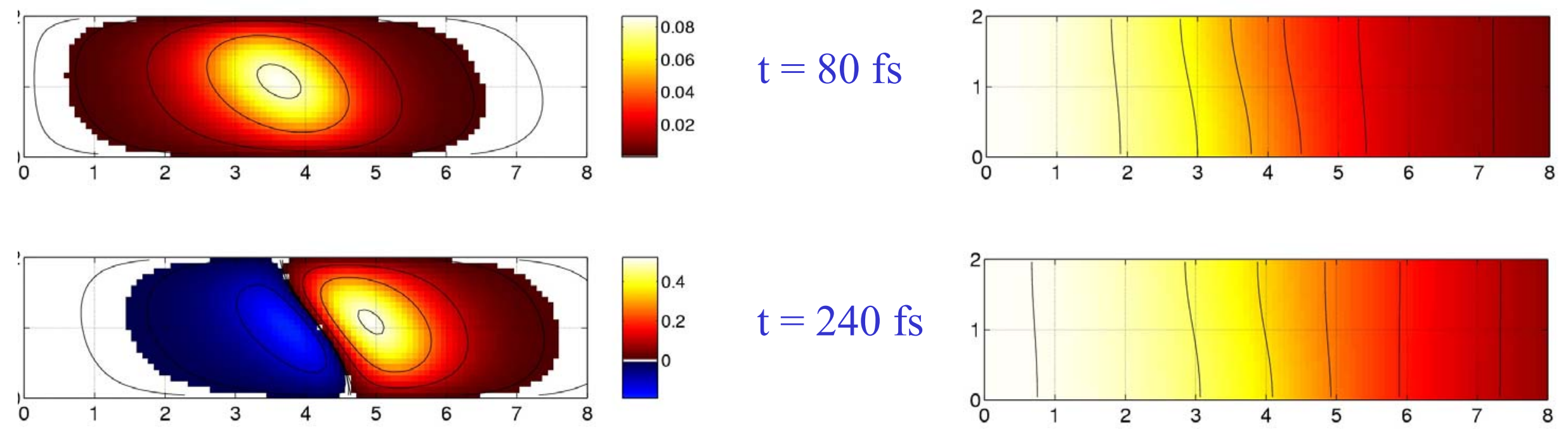

$t=240 \mathrm{fs}$
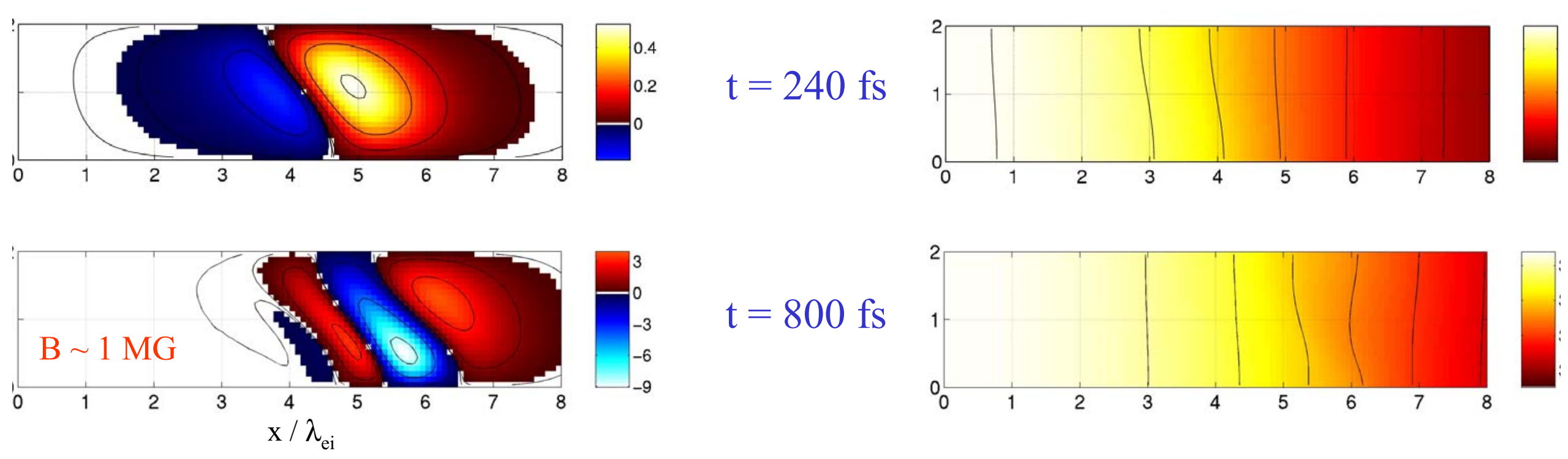


\section{Heat Flow Direction Affected by B-field}
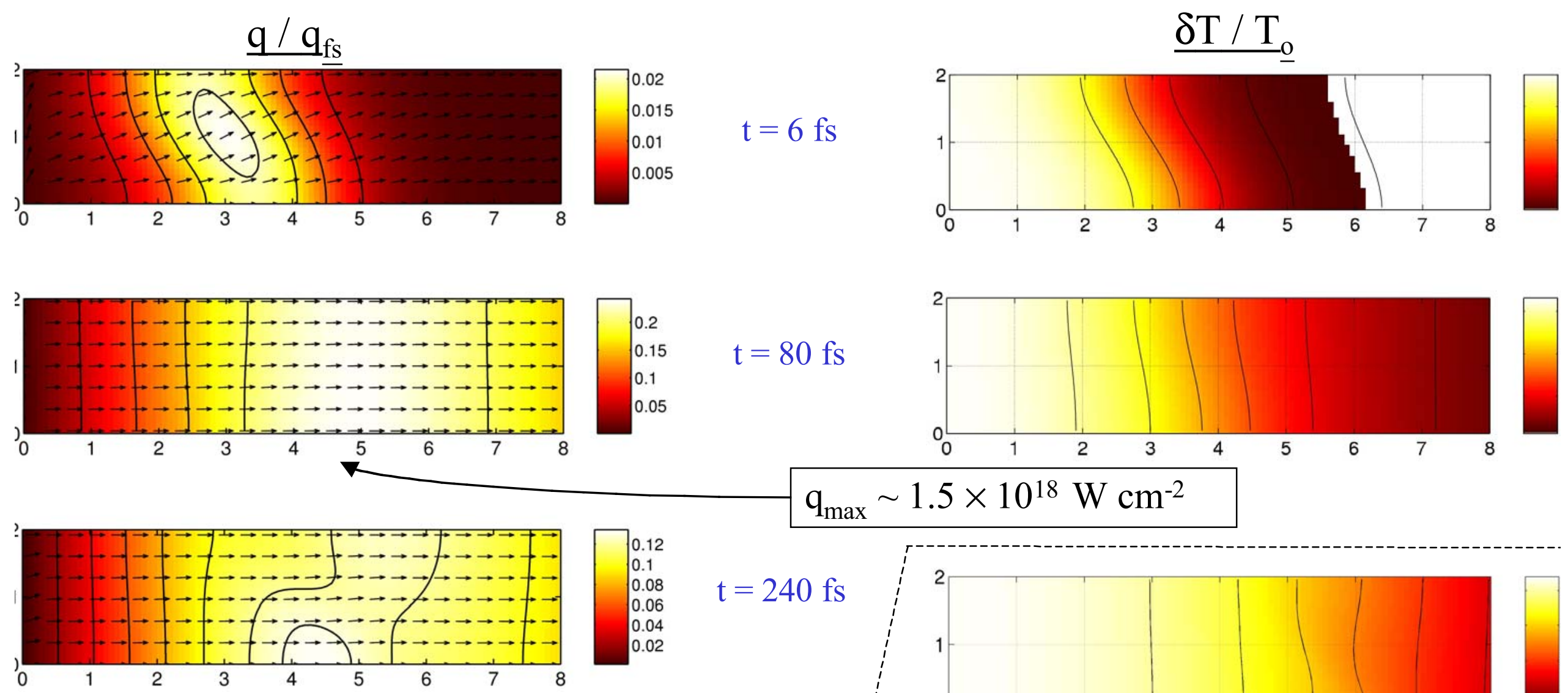

$\mathrm{t}=240 \mathrm{fs}$

$\mathrm{q}_{\max } \sim 1.5 \times 10^{18} \mathrm{~W} \mathrm{~cm}^{-2}$
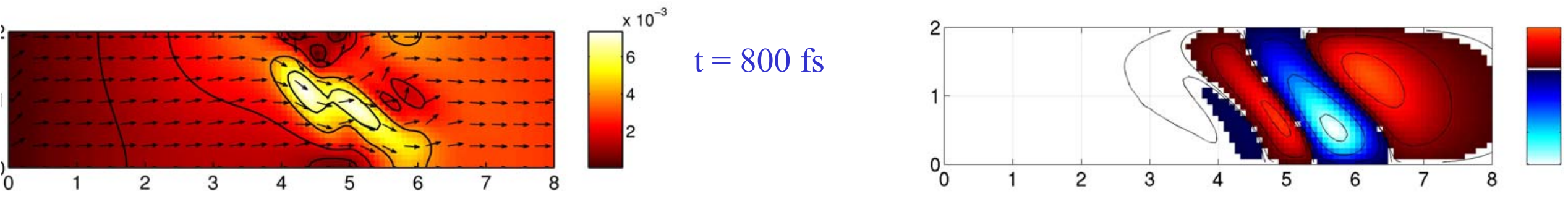


\section{Zoom of Heat Flow After 800fs}

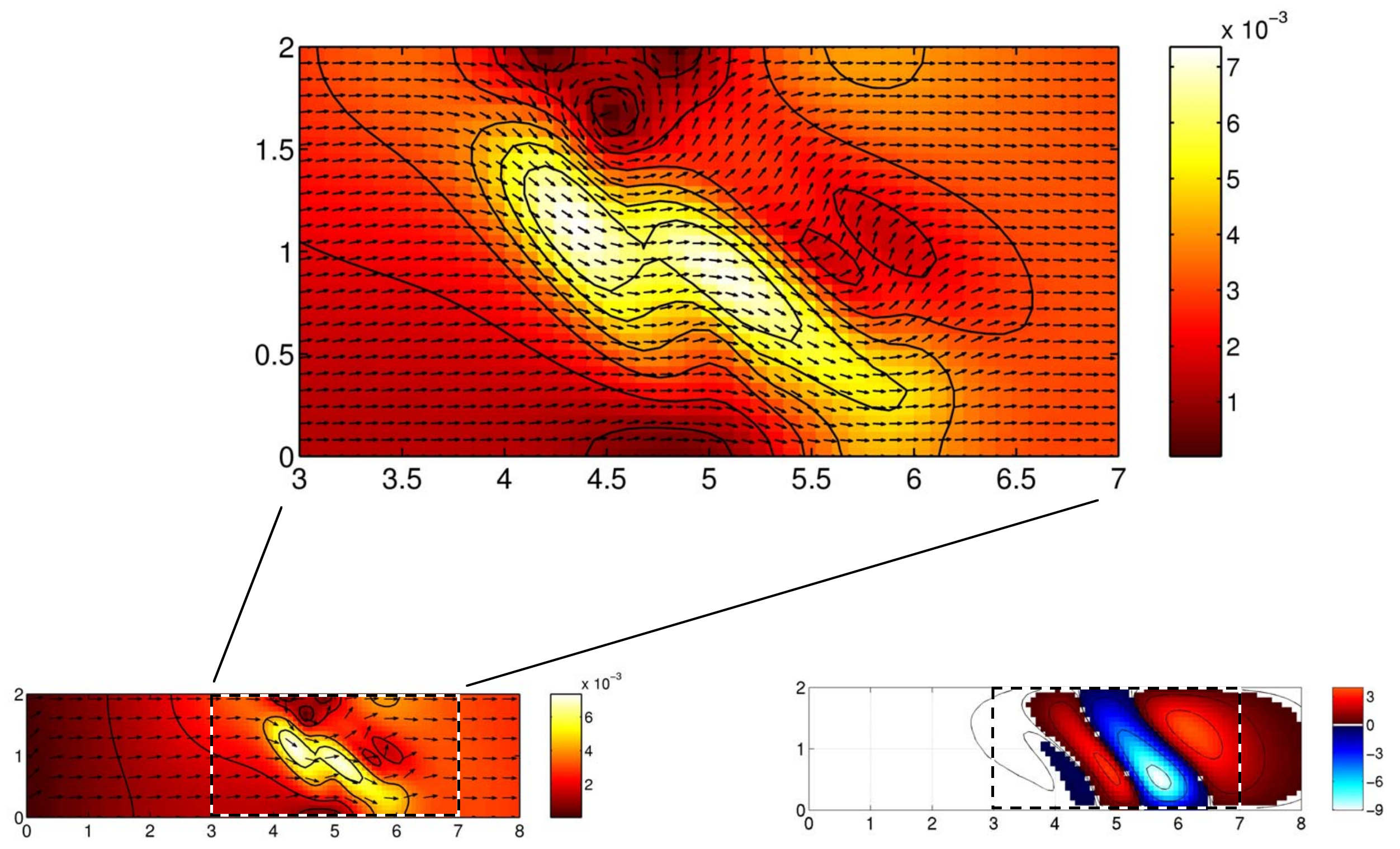




\section{Sign of B-field Alternates Along Ripple}

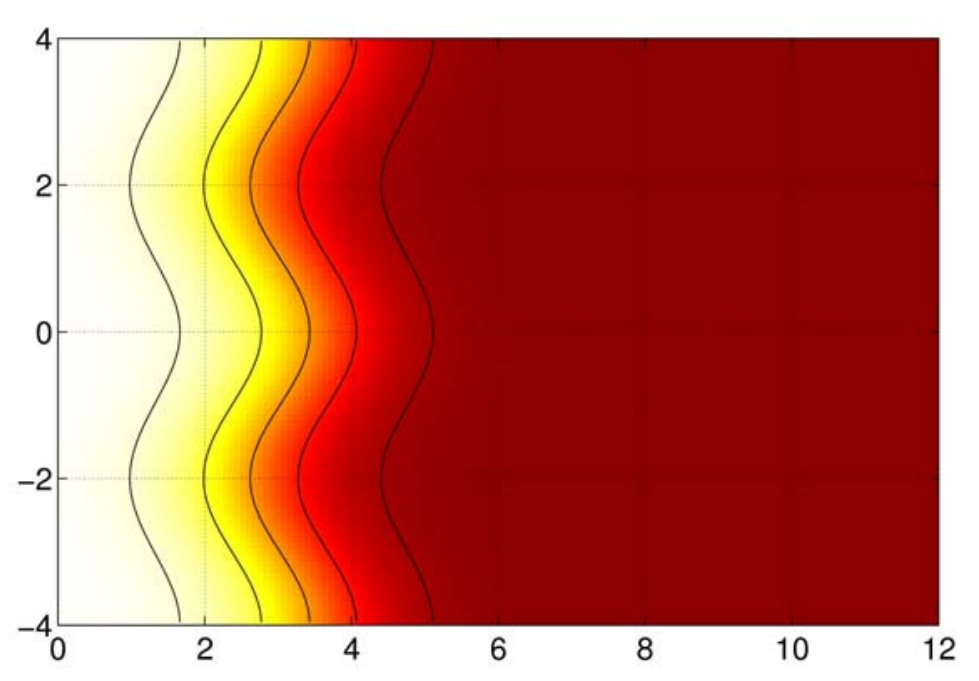

$$
\mathrm{t}=240 \mathrm{fs}
$$

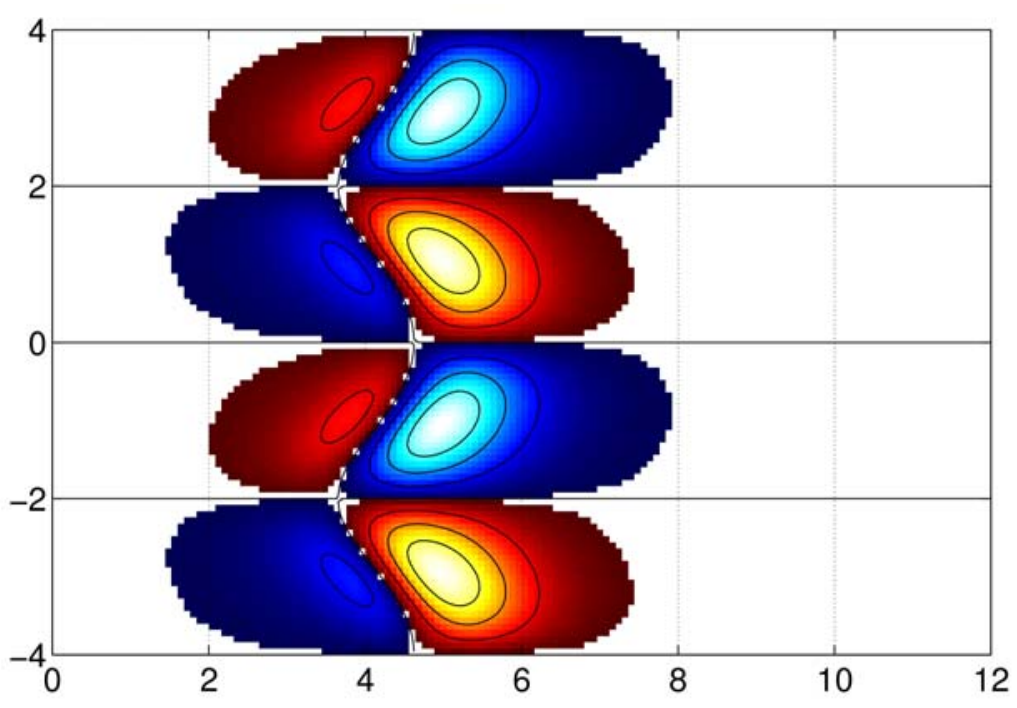




\section{General Expression for $\nabla \times E$}

- Object: derive an expression for $\nabla \times \underline{E}$ when $\underline{B}$ and $j$ are zero

- General expression for $\underline{E}$, valid arbitrary $f_{0} \ldots$

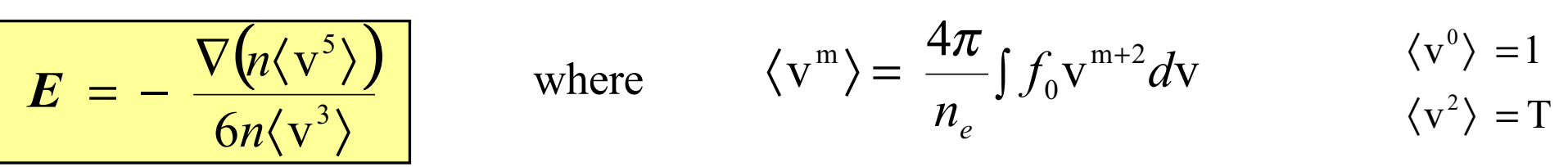

- This originates from the $\int \mathrm{v}^{6} \mathrm{dv}$ moment of the $\underline{\mathrm{f}}_{1}$ equation

$$
\mathrm{v} \nabla f_{0}-\boldsymbol{E} \frac{\partial f_{0}}{\partial \mathrm{v}}=-v_{e i}^{\prime} \frac{Z^{2} n_{i}}{\mathrm{v}^{3}} \boldsymbol{f}_{1} \quad \text { where } \quad \boldsymbol{j} \propto-\int \boldsymbol{f}_{1} \mathrm{v}^{3} d \mathrm{v} \rightarrow 0
$$

Hence in general...

$$
\dot{\boldsymbol{B}}=-\nabla \times \boldsymbol{E}=\frac{-1}{6 n^{2}\left\langle\mathrm{v}^{3}\right\rangle^{2}} \nabla\left(n\left\langle\mathrm{v}^{3}\right\rangle\right) \times \nabla\left(n\left\langle\mathrm{v}^{5}\right\rangle\right)
$$




\section{Non-local B Caused by Non-parallel Moments}

$$
\dot{\boldsymbol{B}}=-\frac{1}{6 n^{2}\left\langle\mathrm{v}^{3}\right\rangle^{2}} \nabla\left(n\left\langle\mathrm{v}^{3}\right\rangle\right) \times \nabla\left(n\left\langle\mathrm{v}^{5}\right\rangle\right)
$$

For a Maxwellian (local approximation) $\ldots \quad\left\langle\mathrm{v}^{m}\right\rangle \propto \mathrm{T}^{m / 2} \quad \nabla\left\langle\mathrm{v}^{m}\right\rangle \propto \mathrm{T}^{\frac{m}{2-1}} \nabla \mathrm{T}$

Local B ...

$$
f_{0}=f_{m}
$$

$$
\begin{array}{rlll}
\dot{\boldsymbol{B}}_{L} \propto \frac{-1}{n^{2} T^{3}} \nabla\left(n T^{3 / 2}\right) \times \nabla\left(n T^{5 / 2}\right) & \Rightarrow-\frac{1}{n} \nabla n \times \nabla T & \nabla n \neq 0 \\
& \Rightarrow \nabla T \times \nabla T=0 & \nabla n=0
\end{array}
$$

Non-local ...

$$
f_{0} \neq f_{m}
$$

$$
\dot{\boldsymbol{B}}_{N L} \propto \frac{-1}{\left\langle\mathrm{v}^{3}\right\rangle^{2}} \nabla\left\langle\mathrm{v}^{3}\right\rangle \times \nabla\left\langle\mathrm{v}^{5}\right\rangle \quad \nabla n=0
$$

- Local approximation forces parallel moments when $\nabla n=0$. 


\section{Non-local Heat Flow Generates "Angle"}

- Hot electrons diffuse faster than cold electrons

- Diffusion coefficient; $\mathrm{D} \propto \mathrm{v}^{5}$

$\Upsilon$ Ripples in $\left\langle v^{5}\right\rangle$ smooth out faster than in $\left\langle v^{3}\right\rangle$

Conceptual illustration: 2-temp. elec. dist.

$$
\begin{aligned}
\mathrm{f}_{0} & =\left(\mathrm{f}_{0}\right)_{\mathrm{h}}+\left(\mathrm{f}_{0}\right)_{\mathrm{c}} \\
\dot{\boldsymbol{B}}_{N L} & \propto-\nabla\left\langle\mathrm{v}^{3}\right\rangle \times \nabla\left\langle\mathrm{v}^{5}\right\rangle \\
\nabla\left\langle\mathrm{v}^{m}\right\rangle & \propto \mathrm{n}_{\mathrm{c}} \mathrm{T}_{\mathrm{c}}^{\mathrm{m} / 2-1} \nabla \mathrm{T}_{\mathrm{c}}+\mathrm{n}_{\mathrm{h}} \mathrm{T}_{\mathrm{h}}^{\mathrm{m} / 2-1} \nabla \mathrm{T}_{\mathrm{h}} \\
\dot{\boldsymbol{B}}_{N L} & \propto \frac{\mathrm{n}_{\mathrm{h}} \mathrm{n}_{\mathrm{c}}\left(\mathrm{T}_{\mathrm{h}} \mathrm{T}_{\mathrm{c}}\right)^{1 / 2}}{\mathrm{n}_{\mathrm{t}}^{2} \mathrm{~T}^{3}}\left[\mathrm{~T}_{\mathrm{h}}-\mathrm{T}_{\mathrm{c}}\right]\left(\nabla \mathrm{T}_{\mathrm{h}} \times \nabla \mathrm{T}_{\mathrm{c}}\right)
\end{aligned}
$$$$
\dot{\mathrm{T}}_{\mathrm{s}} \propto \kappa_{\mathrm{s}} \nabla^{2} \mathrm{~T}_{\mathrm{s}} \propto \mathrm{T}_{\mathrm{s}}^{5 / 2} \nabla^{2} \mathrm{~T}_{\mathrm{s}}
$$
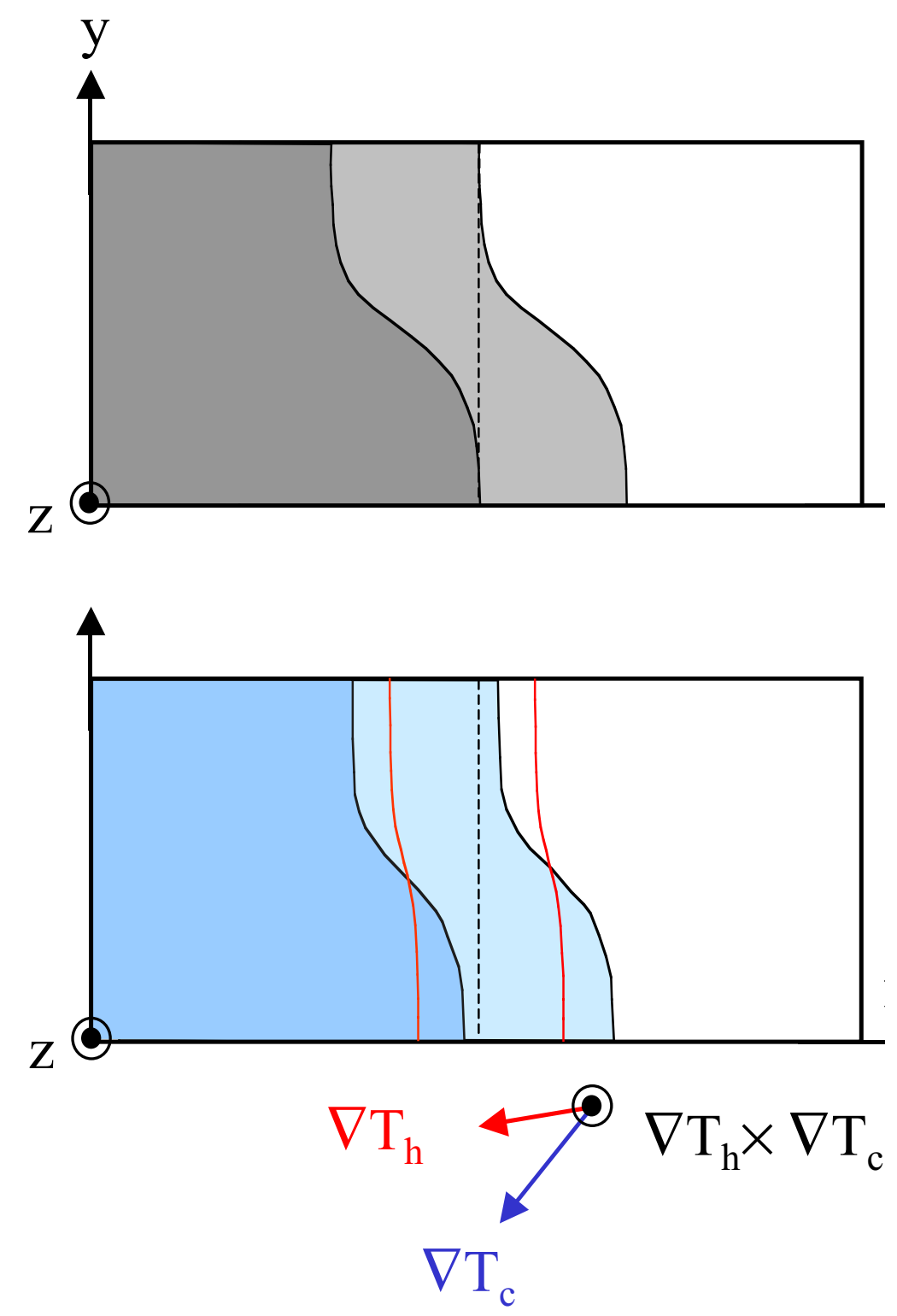


\section{Demonstration of Evolving "Angle"}

-Initial ripple, simulation
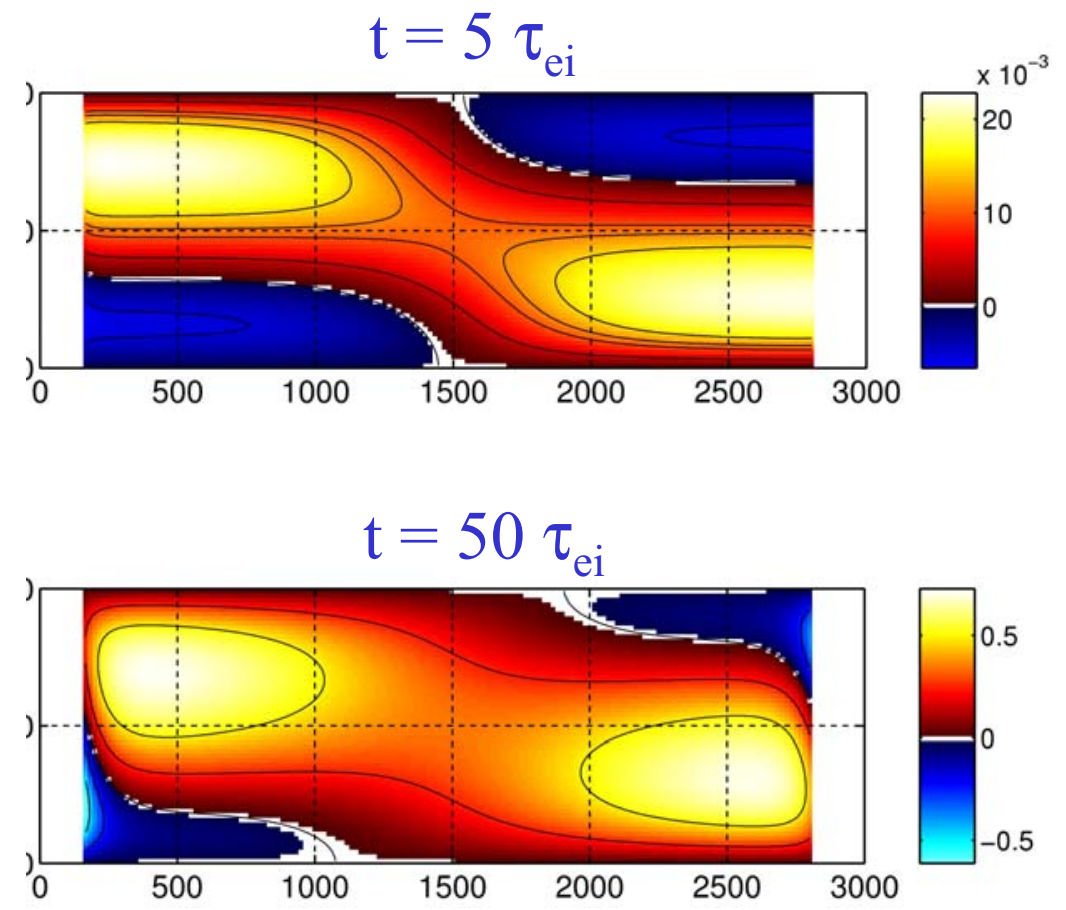

$$
\mathrm{t}=500 \tau_{\mathrm{ei}}
$$

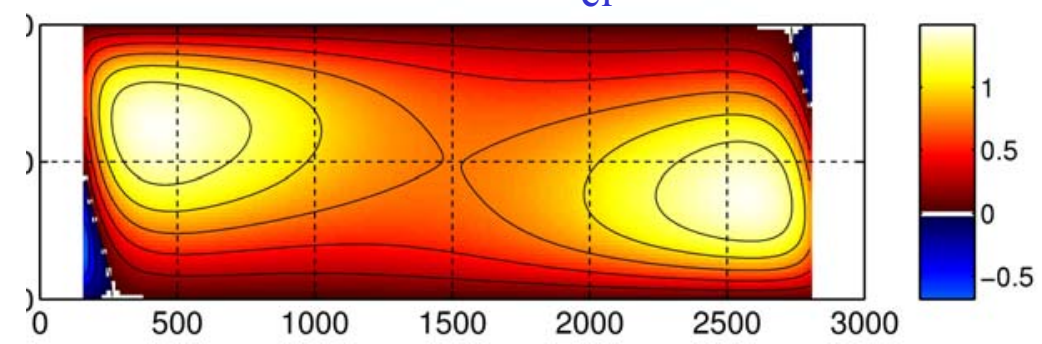

•"Injection", simulation
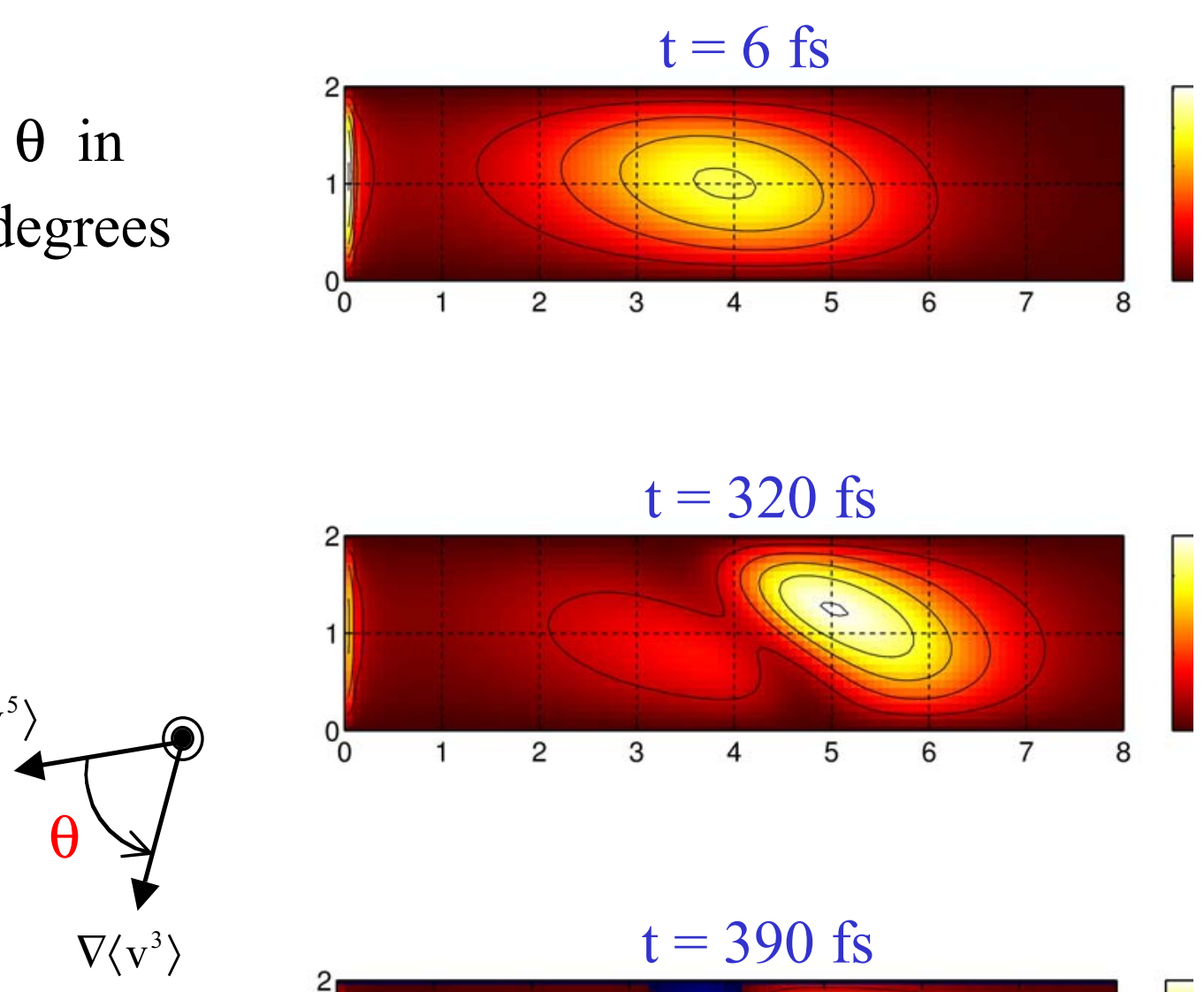

$\theta$ in

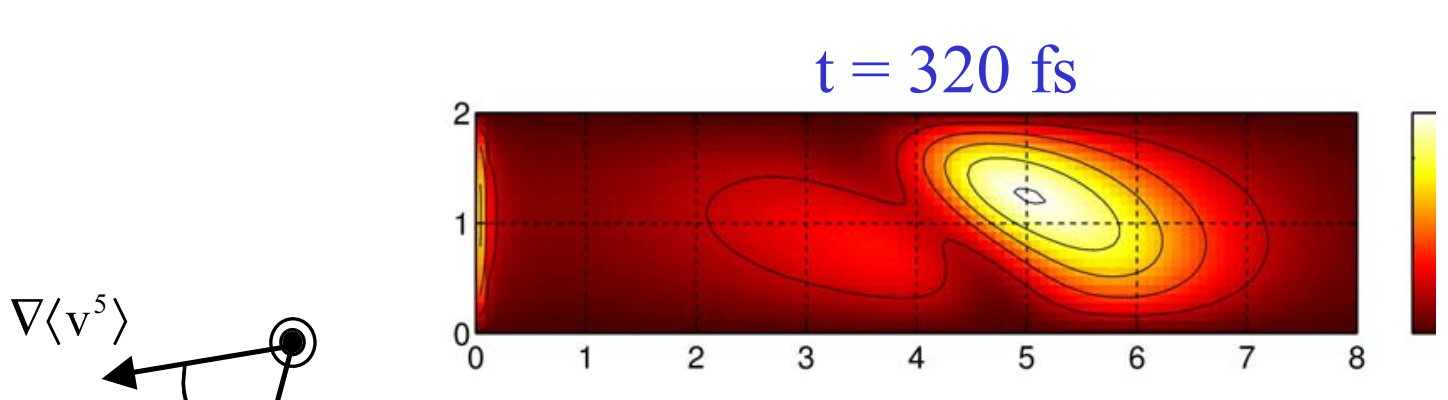
degrees

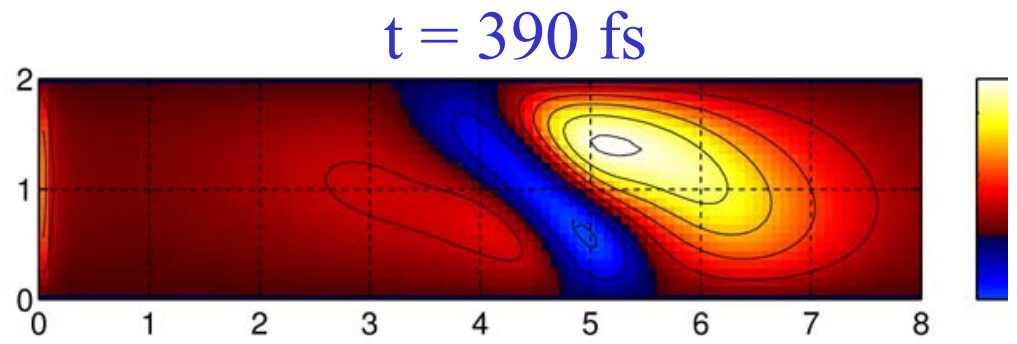




\section{Analytic Formula for $B_{N L}$ Agrees With Sims.}

- Tractable analysis of FP eqn possible when...

$\Upsilon$ e-e collisions neglected

- electron inertia neglected $\left(\partial \mathrm{f}_{1} / \partial \mathrm{t}=0\right)$

$\Upsilon$ effect of $B$ on evolution negligible; early time

- $\mathrm{B}_{\mathrm{NL}}$ from an initial Maxwellian hot spot source...

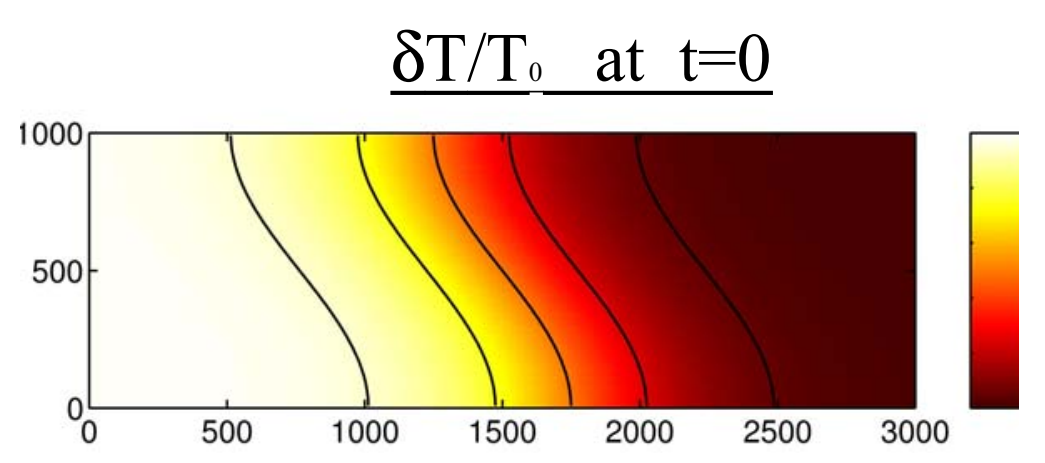

$$
\begin{aligned}
\ddot{\boldsymbol{B}}_{N L} & =C_{N L} \nabla T \times \nabla\left(\nabla^{2} T\right) \\
\left|\frac{\ddot{B}_{N L}}{\overline{\boldsymbol{V}}_{e i}^{3}}\right| & =C_{N L}^{\prime} G\left(\frac{\delta T}{T}\right)^{2}\left(\frac{L}{\bar{\lambda}_{e i}}\right)^{-4} \quad \ldots \text { for } \delta \mathrm{T}<\mathrm{T}_{\mathrm{o}}
\end{aligned}
$$

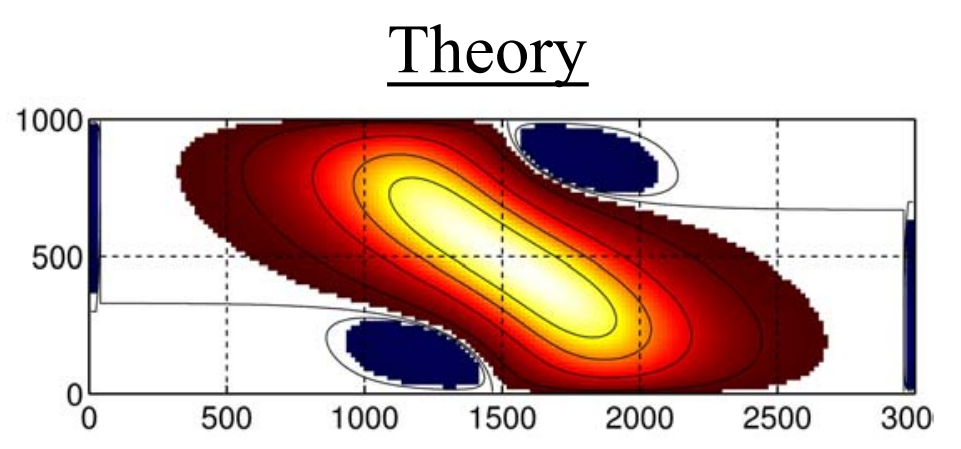

- Compare with $\nabla \mathrm{n} \times \nabla \mathrm{T}$ mechanism...

$$
\left|\frac{\dot{B}_{n \times T}}{\bar{V}_{e i}^{2}}\right|=C_{n \times T}\left|\sin \theta_{n \times T}\right|\left(\frac{\delta T}{T}\right)\left(\frac{\delta n}{n}\right)\left(\frac{L}{\bar{\lambda}_{e i}}\right)^{-2}
$$

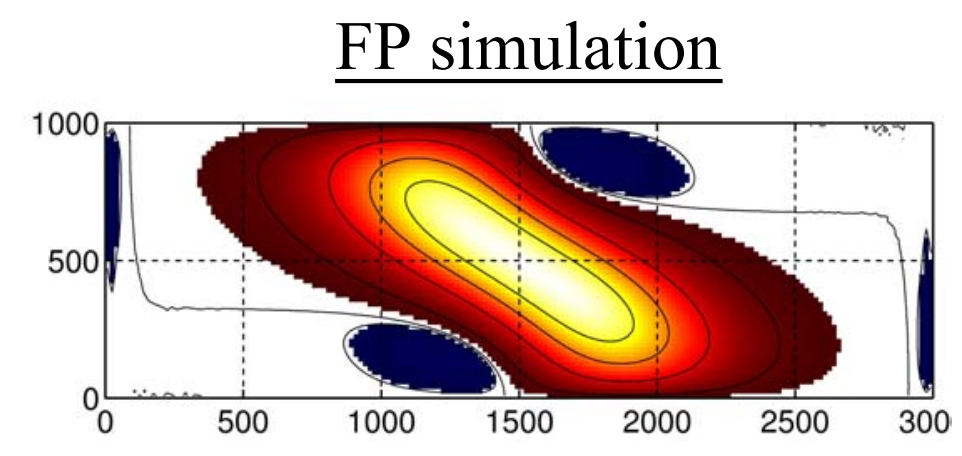




\section{D-FP Sims. With Heating and $\nabla Z$ but $\nabla n=0$}

- System \& Z, heating profiles...

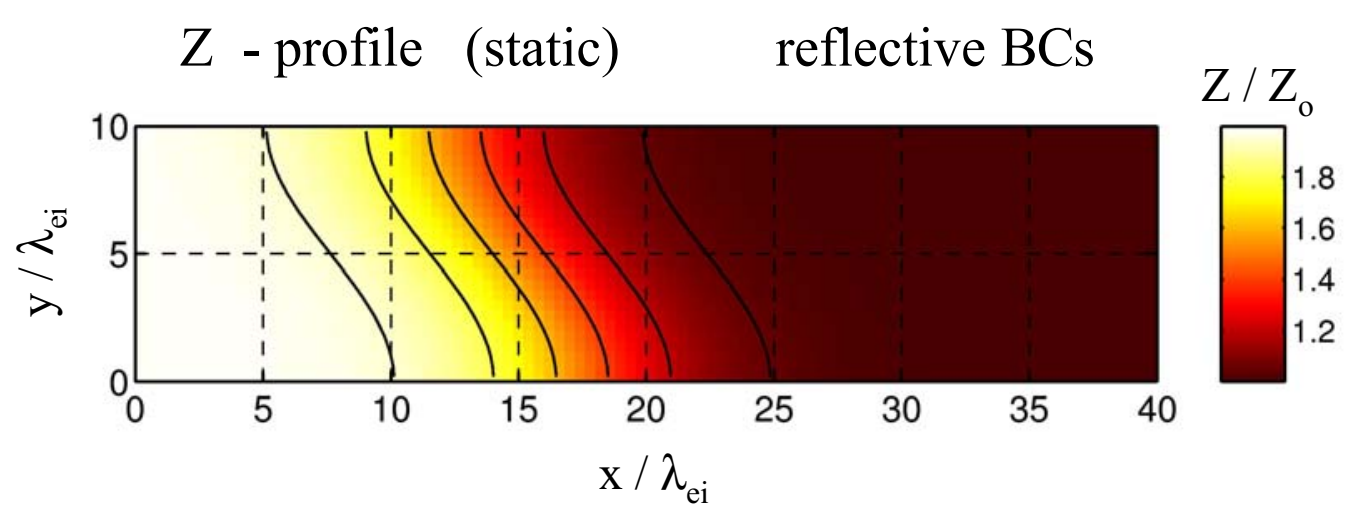

- 1D heating profile

- Parameters...
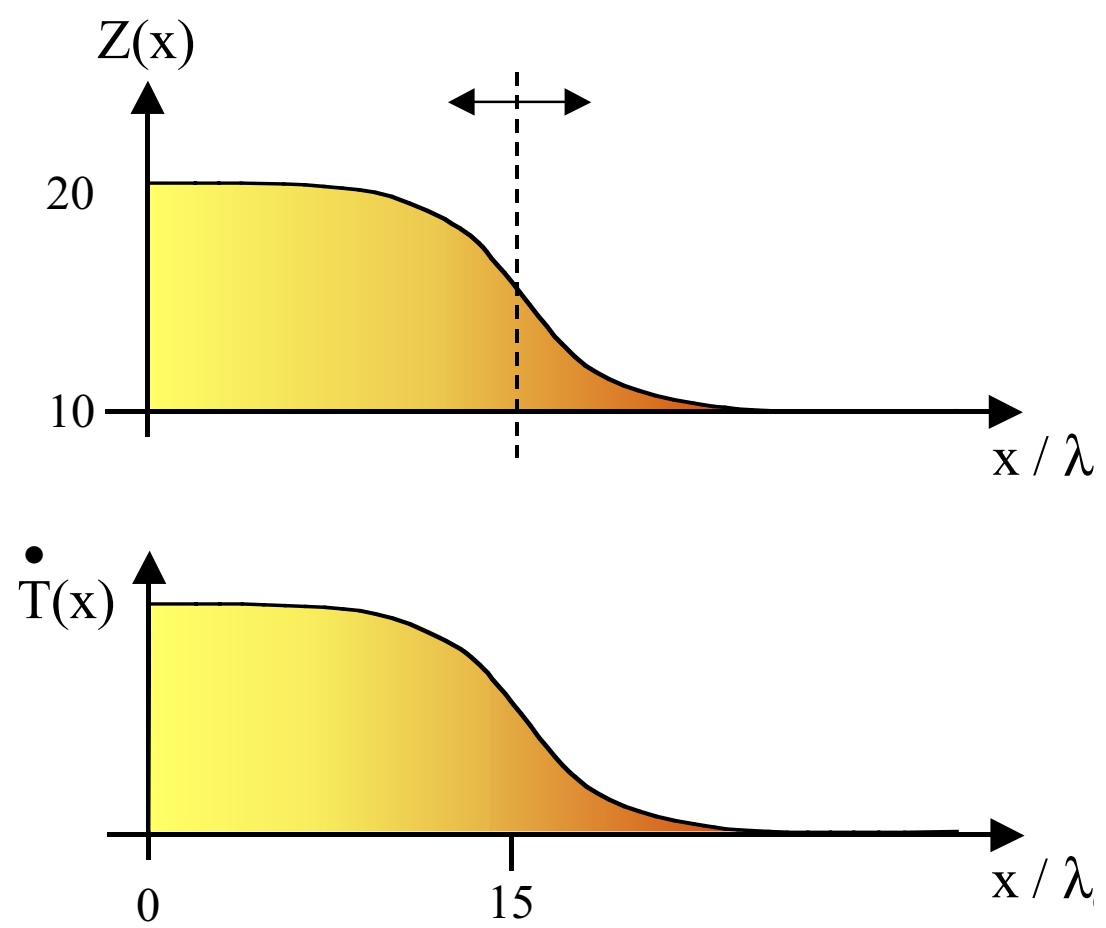

$$
\begin{array}{|ll|}
Z_{\mathrm{o}} & =10 \\
\frac{\lambda_{\text {eio }}}{\delta_{\mathrm{c}}} & =32 \\
\hline
\end{array}
$$

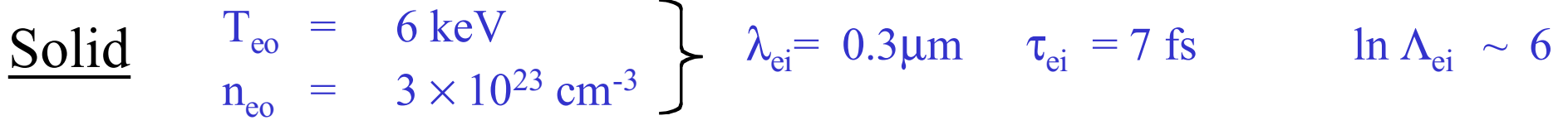

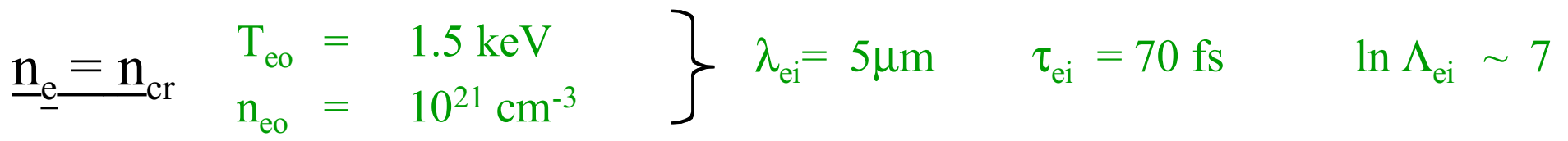




\section{B-field grows to $\omega \tau>1$ even though $\nabla n=0$}
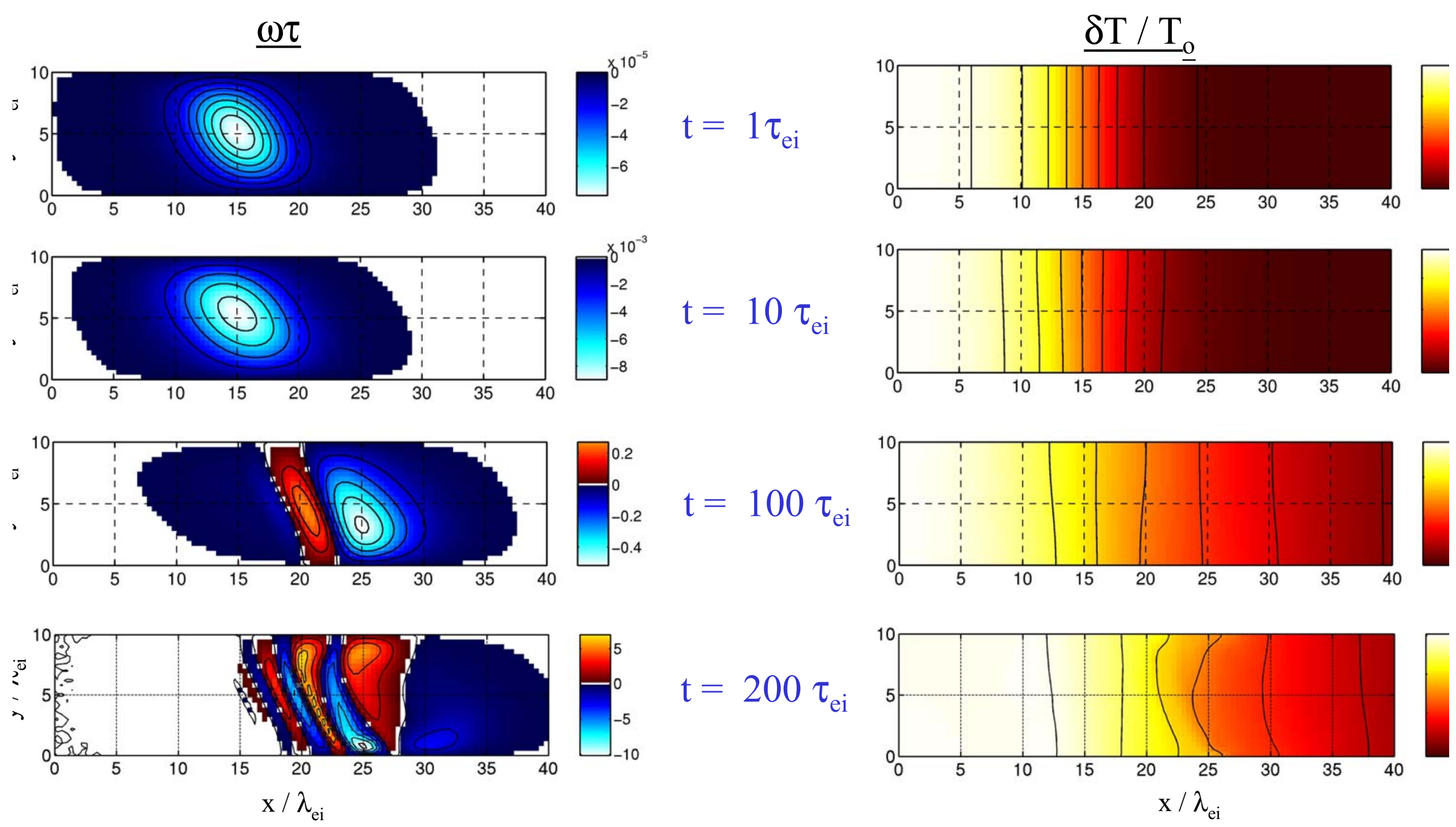

$t=200 \tau_{e i}$

Classical, local theory $\rightarrow \quad \mathrm{B}=0 ! \quad$ (for Lorentz approx.)

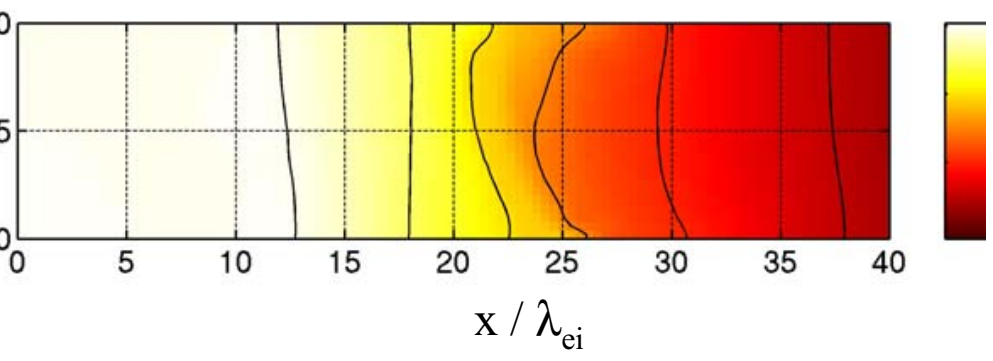




\section{Heat flow direction affected by B-field}

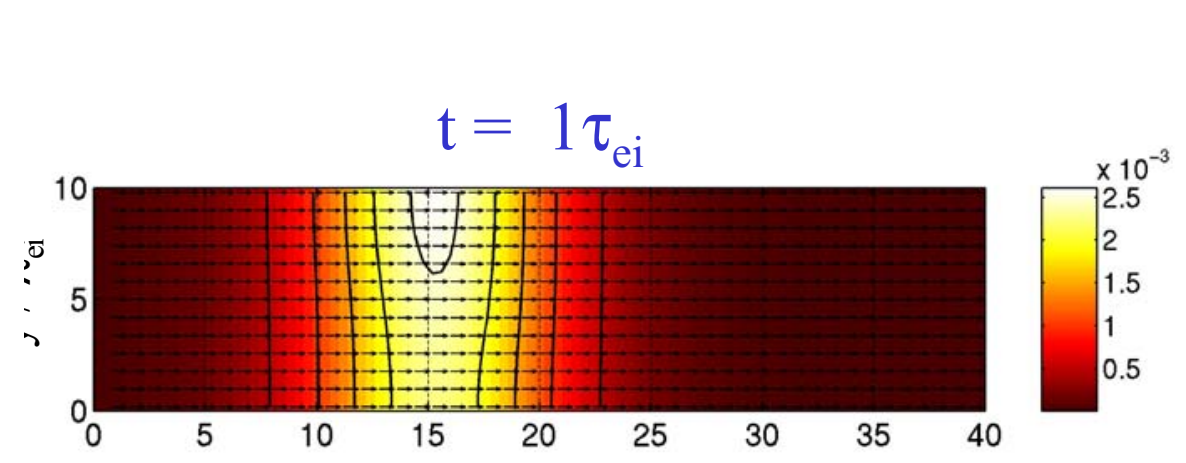

$$
\underline{\mathrm{q} / \mathrm{q}_{\mathrm{fs}}}
$$

$$
\mathrm{t}=10 \tau_{\mathrm{ei}}
$$
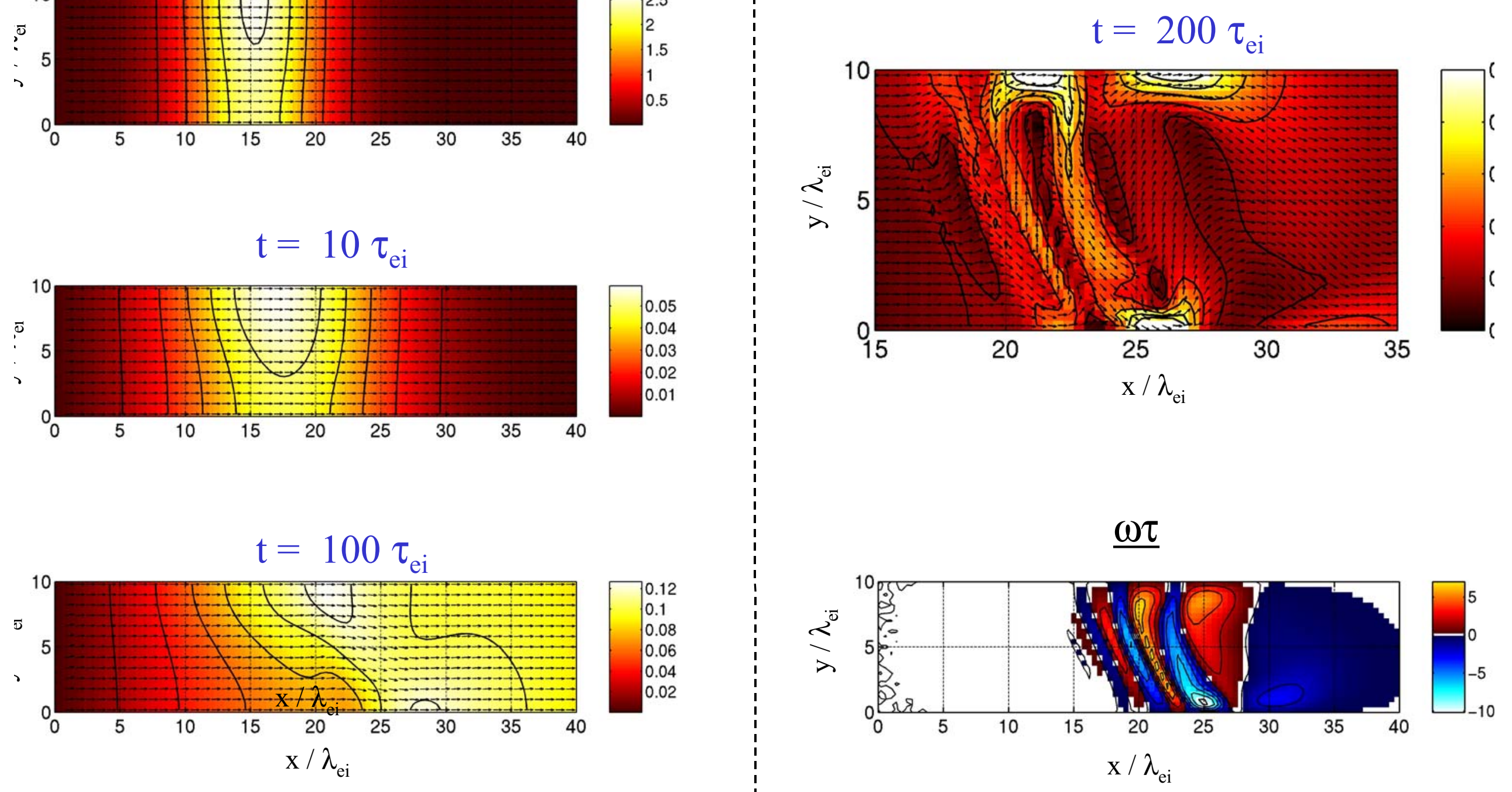


\section{Non-local seed field for $\nabla Z \neq 0$}
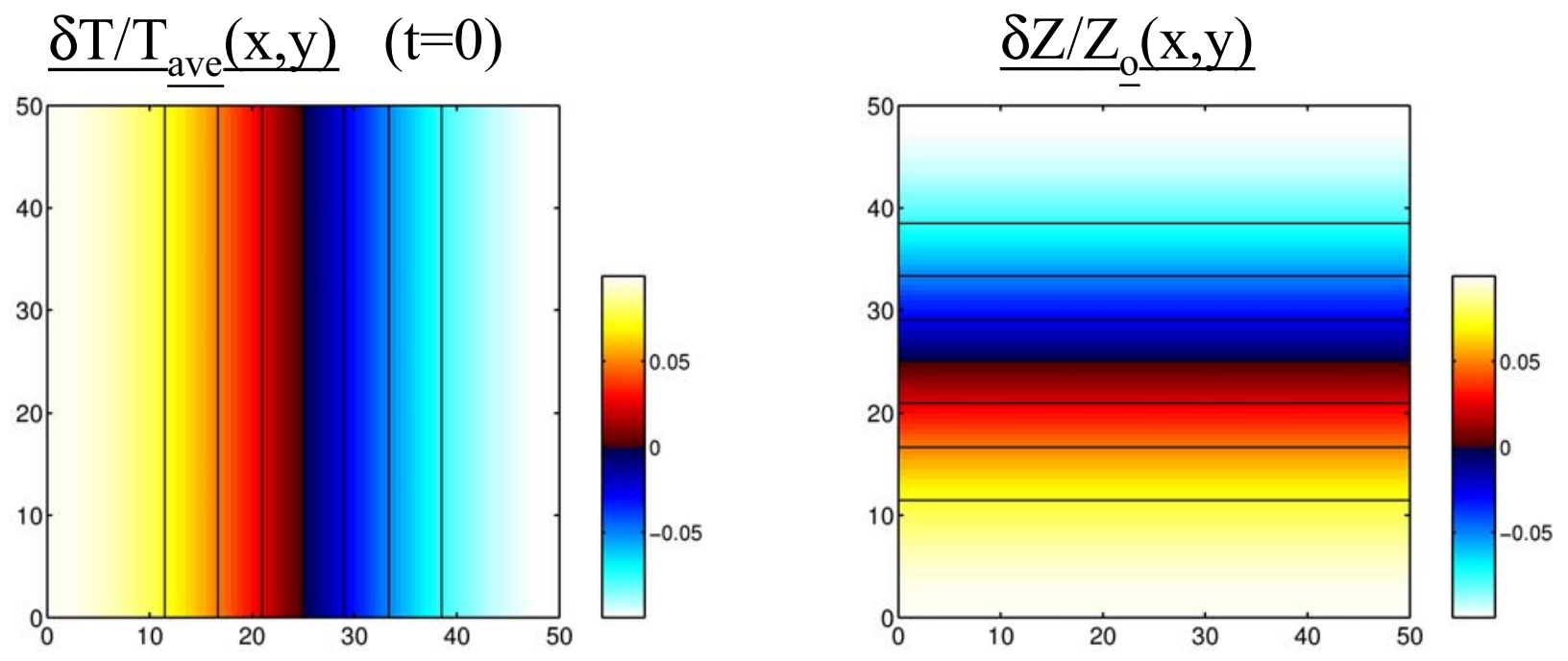

$$
\begin{gathered}
\bullet \mathrm{B} \text { at } \mathrm{t}=\tau_{\mathrm{ei}} \\
-\nabla n=0 \\
B(t)=-t \frac{\nabla n \times \nabla T_{0}}{n}+\frac{t^{2}}{2} \ddot{B}
\end{gathered}
$$
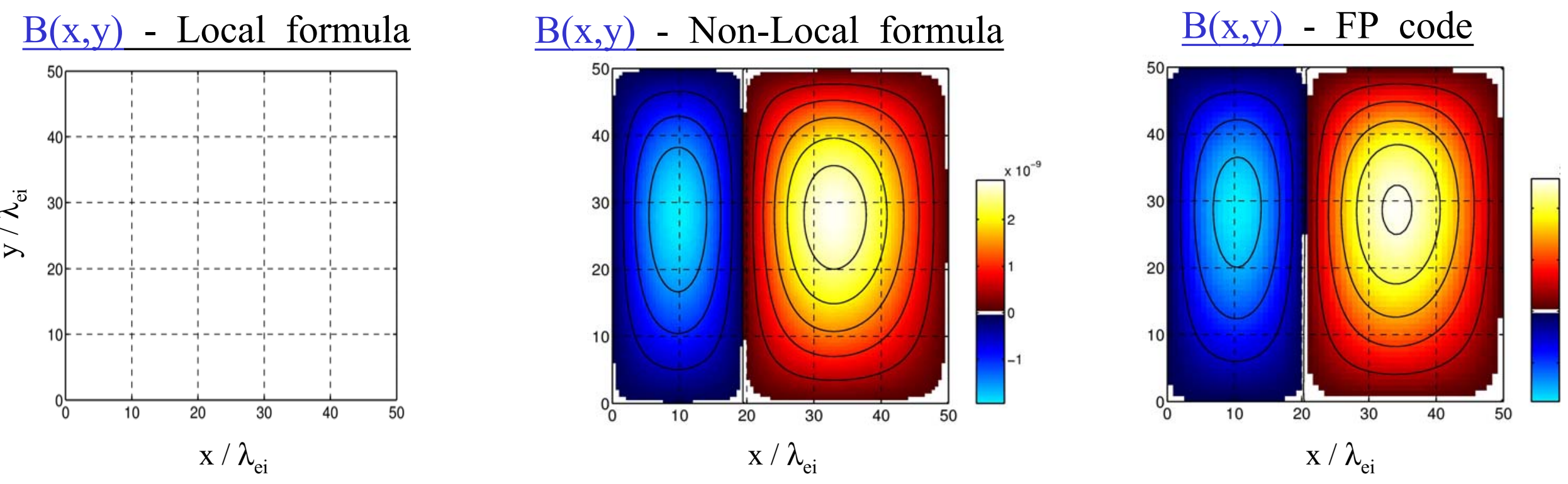


\section{Non-local seed field for $\nabla n \neq 0$}

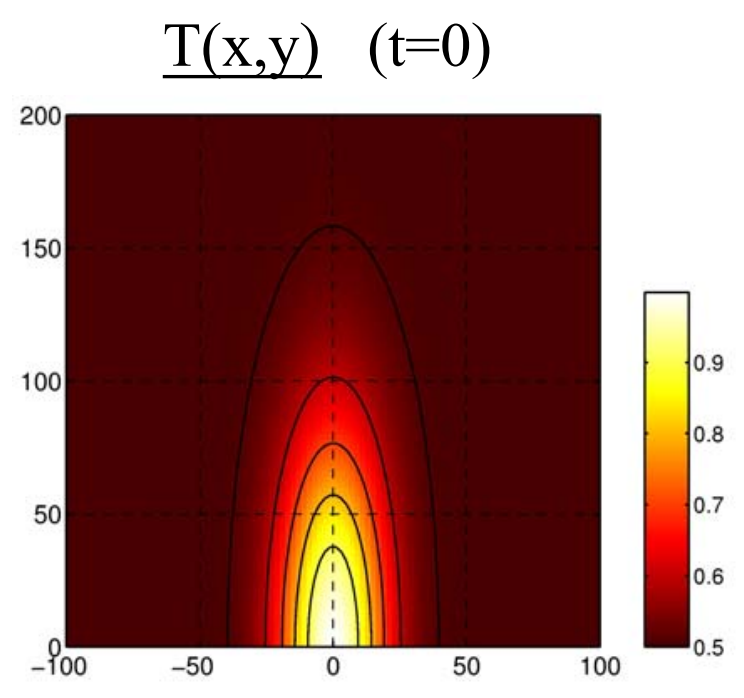

Local formula

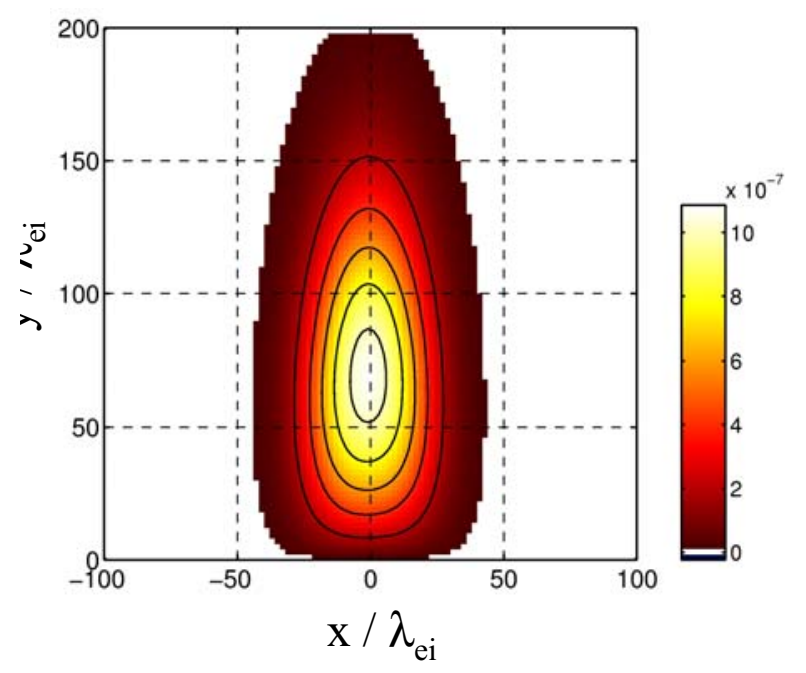

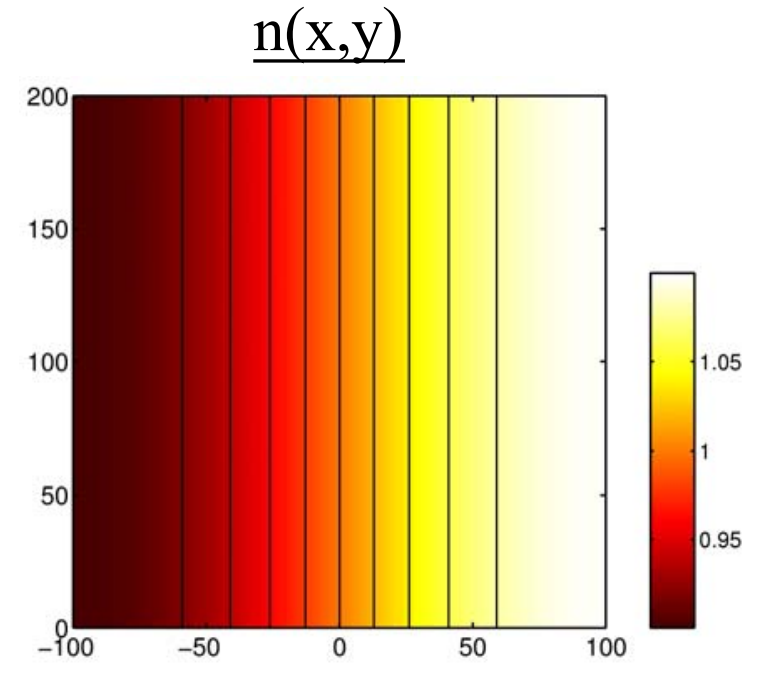

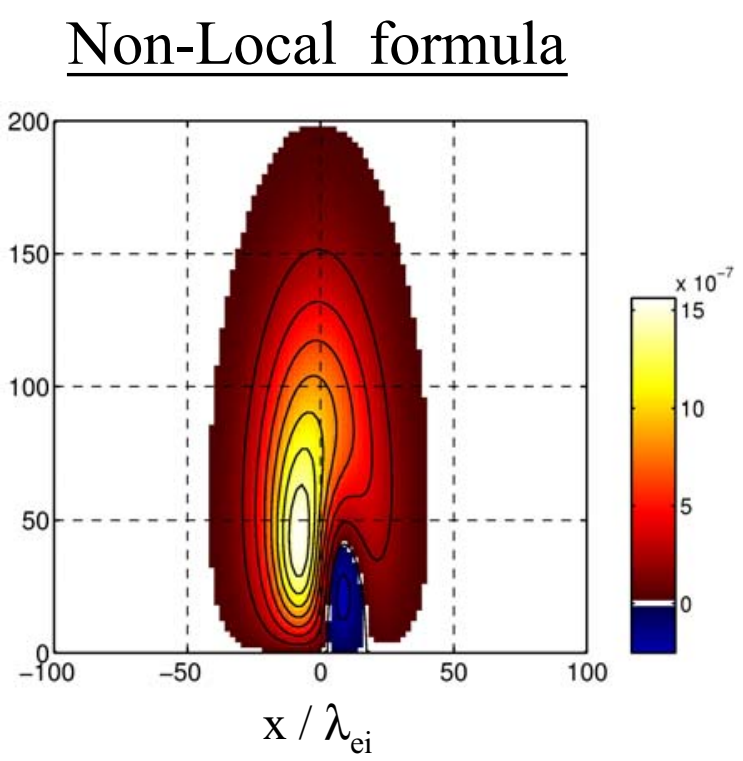

- B at $\mathrm{t}=\tau_{\mathrm{ei}}$

- $\nabla Z=0$

$$
B(t)=-t \frac{\nabla n \times \nabla T_{0}}{n}+\frac{t^{2}}{2} \dot{E}
$$

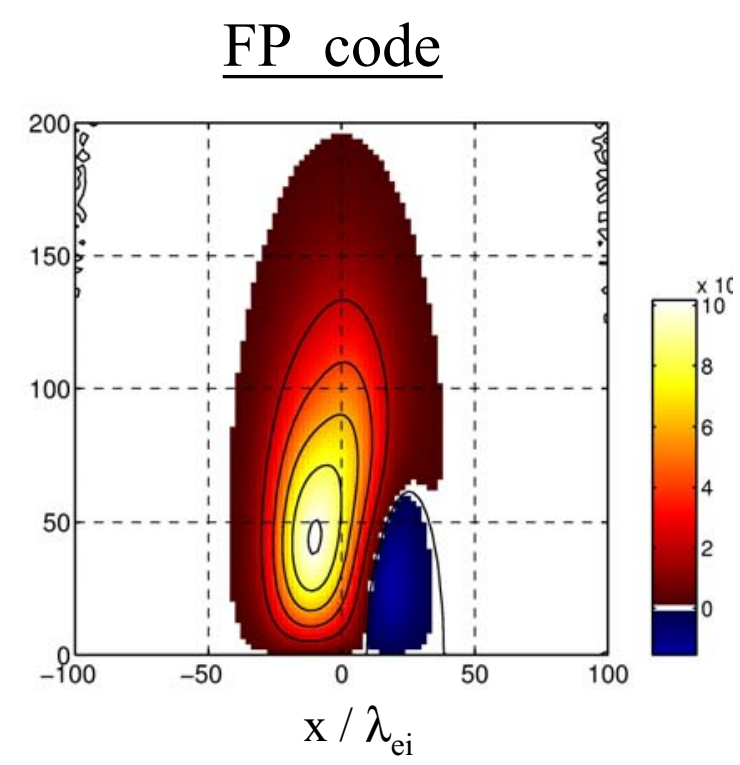




\section{FP sim: heating in a density ramp}
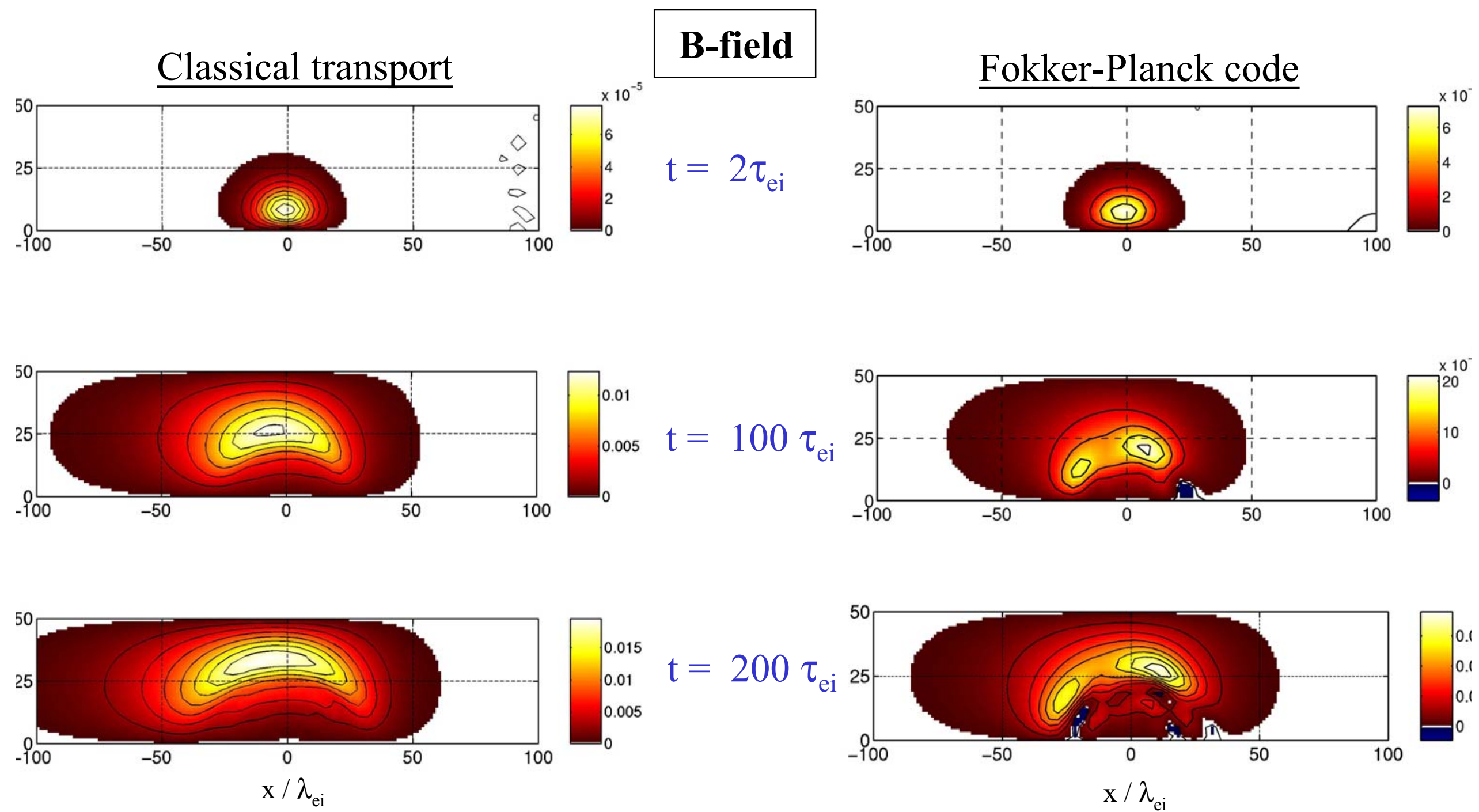

$$
n(x)=1+0.95 \tanh (x / 60)
$$

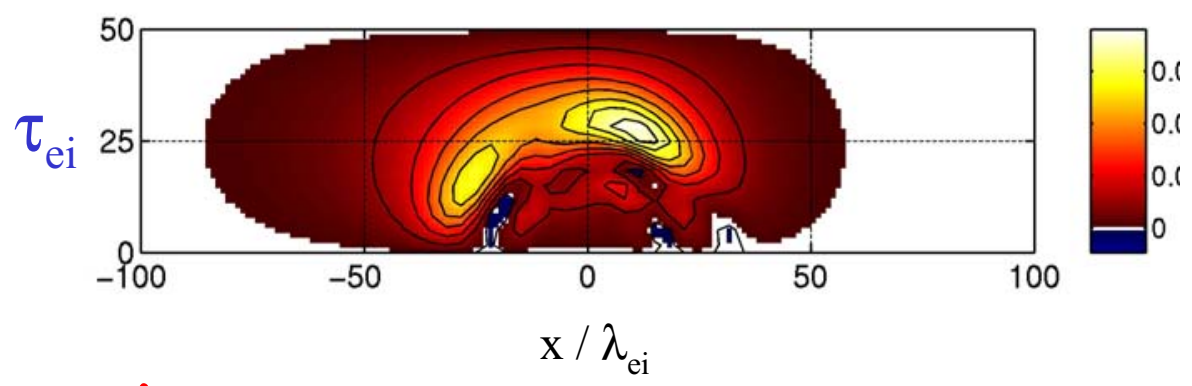

$$
\frac{\lambda_{\text {eio }}}{\delta_{\mathrm{c}}} \sim 1000
$$




\section{FP sim: heating in a density ramp}

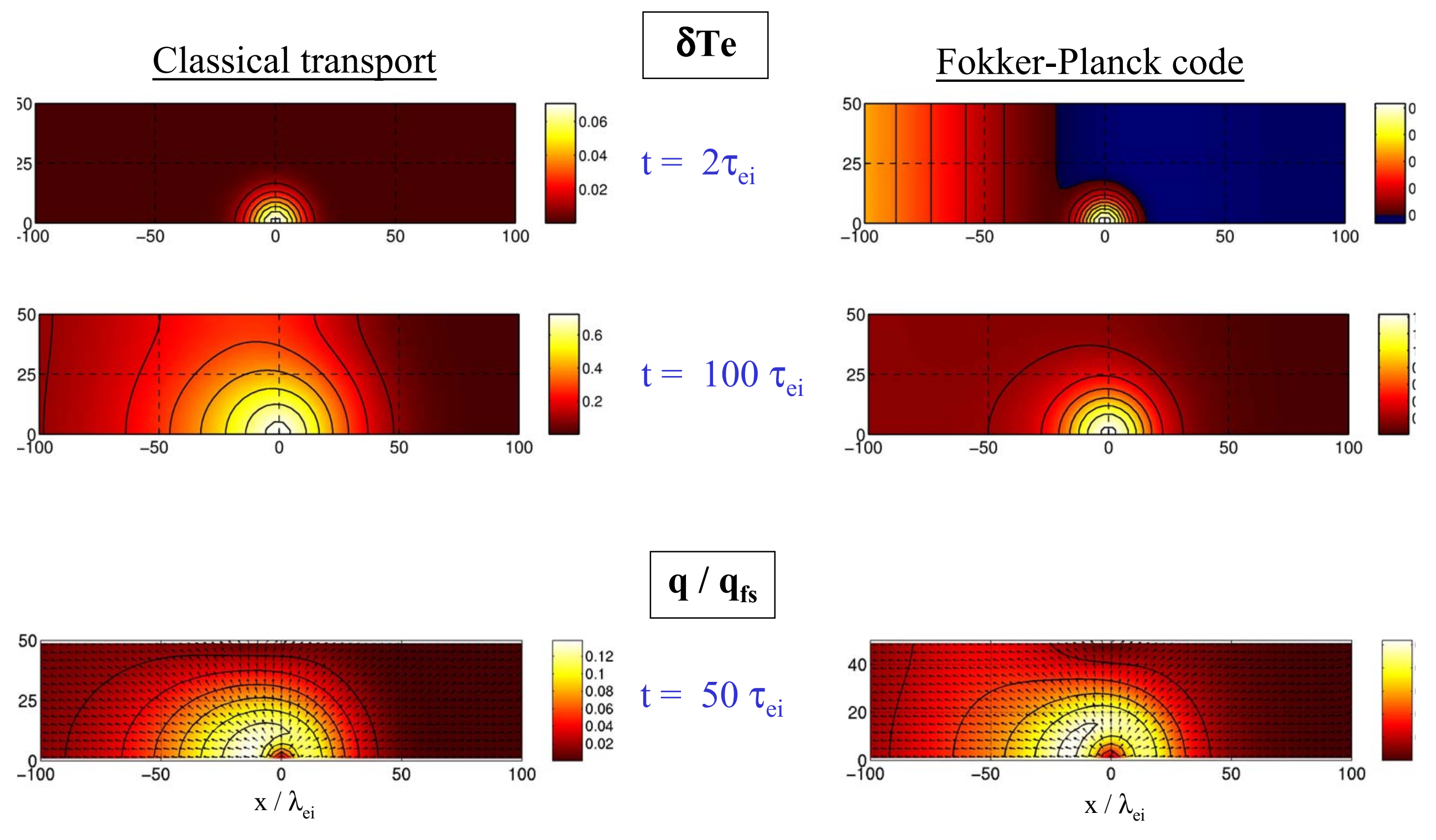




\section{FP sim: heating in a density ramp}

$\underline{\text { Classical transport }}$
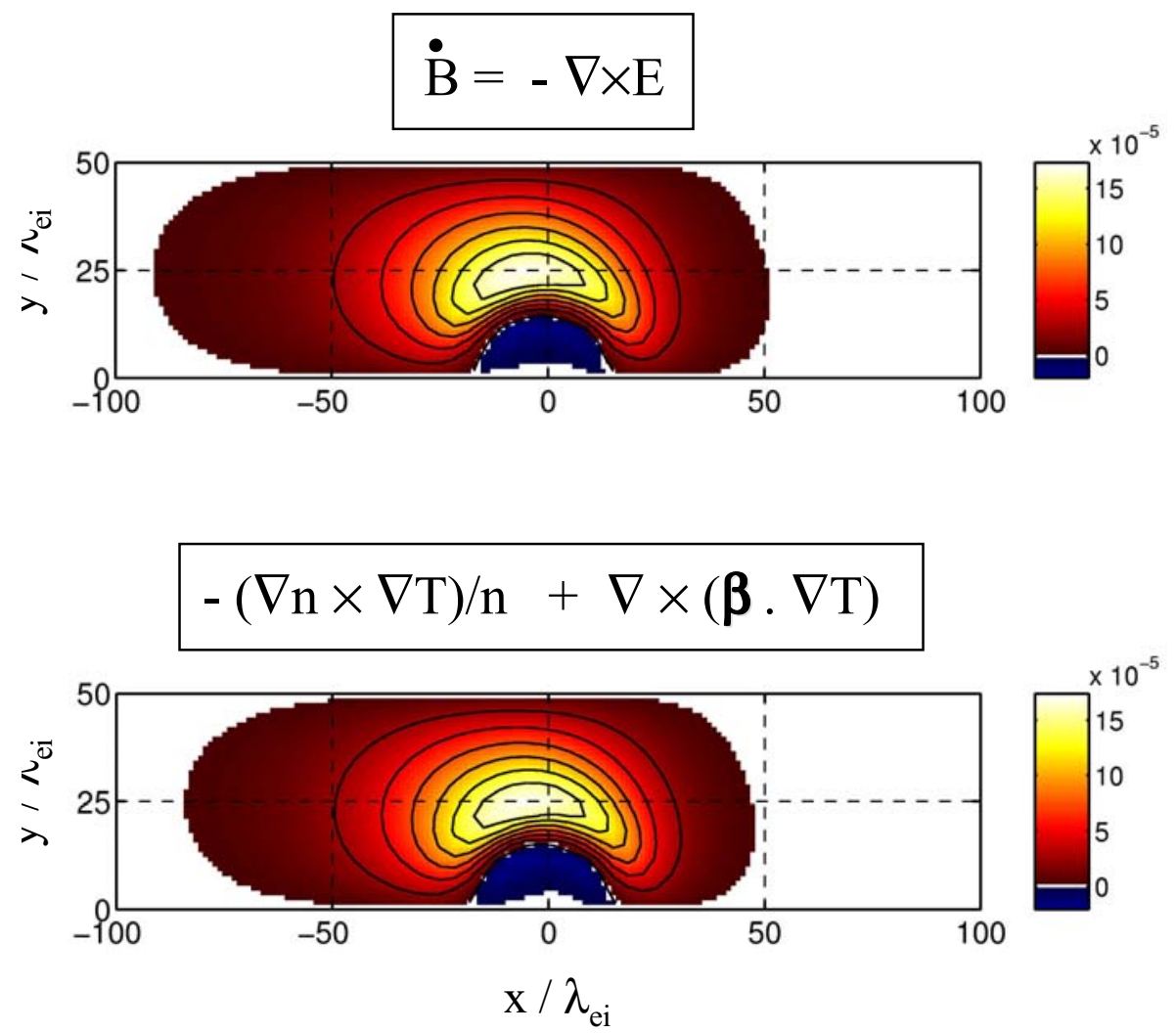

$\underline{\text { Fokker-Planck code }}$
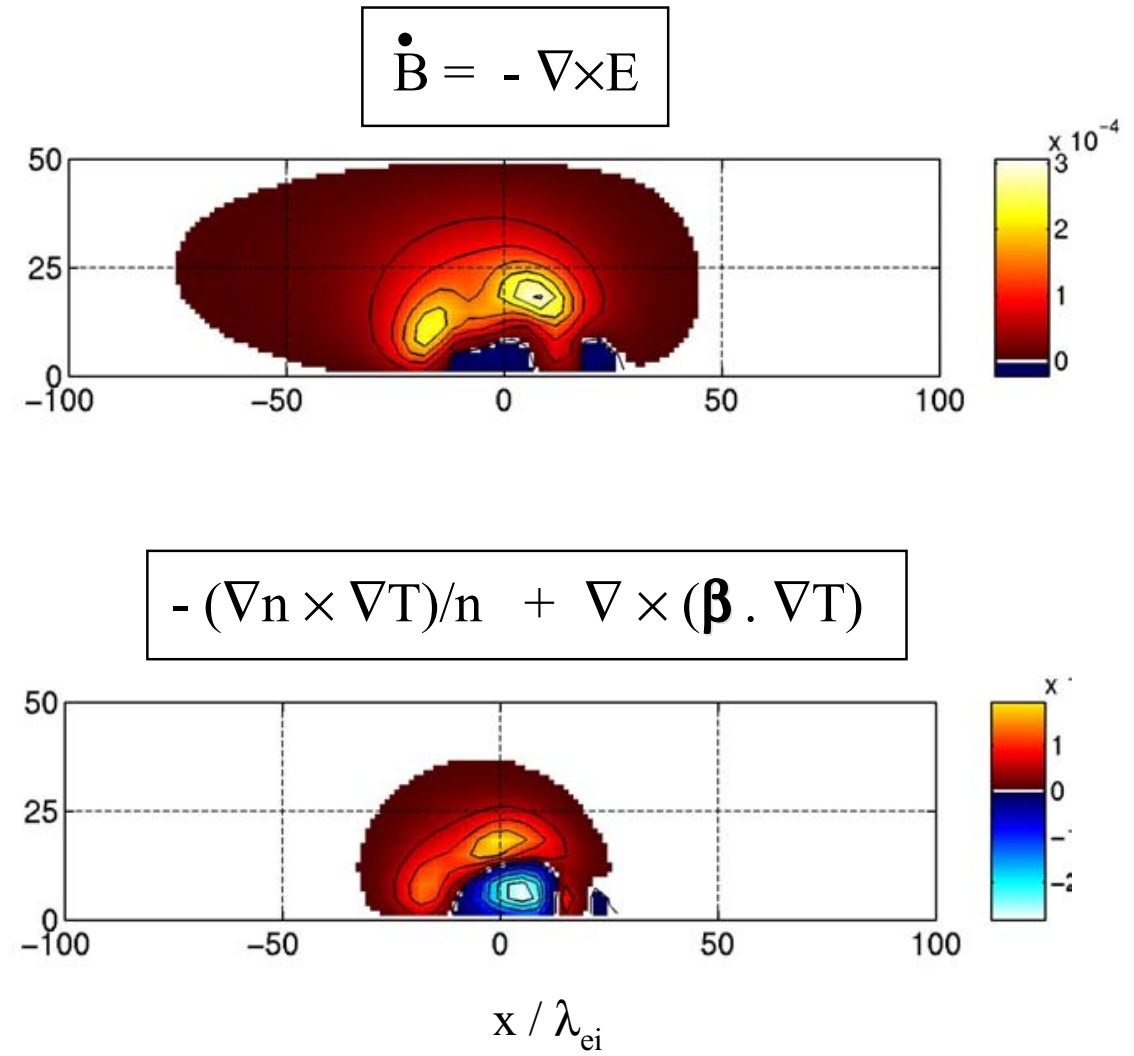


\section{Conclusions}

- New B-field generation mechanism - a non-local effect

- Driven by non-parallel gradients in moments of $\mathrm{f}_{\mathrm{o}}$ $\dot{\boldsymbol{B}} \propto-\nabla\left\langle\mathrm{v}^{3}\right\rangle \times \nabla\left\langle\mathrm{v}^{5}\right\rangle$

- Kingham \& Bell, PRL 88, 045004 (2002)

- Works under conditions where local theory says $B=0$

- Isotropic electron pressure (c.f. anisotropic pressure in PIC \& hybrid code)

- Growth even when $\nabla n=0$ which isn't allowed by $\nabla n \times \nabla T$

- Growth for $\nabla Z \neq 0$ and uniform heating in Lorentz approximation

- FP sims: Get growth of B-field to $\grave{u} \hat{o}>1$ and see its effect on transport

- Anayltical formula for seed B-field from initial Maxwellian $\mathrm{f}_{0}$

- Arbitrary $\mathrm{Z}, \mathrm{n}_{\mathrm{e}}$ and initial $\mathrm{T}_{\mathrm{e}}$ profile

- New terms for $\ddot{B} \longrightarrow$ e.g. $\ddot{B} \propto-\nabla T \times \nabla\left(\nabla^{2} T\right)$

- "Local-like" terms bigger in non-local case

- FP simulation: Density gradient + heating ...

- 2x larger B-field than local equivalent \& less Nerst advection + more structure

- Future: go to $\mathbf{f}_{2}$ (and beyond) + couple to hydro 


\title{
3D models for non-local electron heat flux
}

\author{
Sergei Krasheninnikov \\ University California San Diego
}

Workshop on Electron Transport

Livermore, September 9-11, 2002 


\section{Outline}

I. 3D heat transport in "high Z" approximation

Ia. Conclusions

II. 3D self-similar solution of electron kinetic equation

IIa. Conclusions

III. Alternatives 


\section{3D heat transport in "high Z" approximation}

- Consider kinetic equation for the electron distribution function $f(\mathbf{v}, \mathbf{r})$ neglecting electron-ion energy exchange and assuming zero averaged ion velocity:

$$
\begin{aligned}
\mathbf{v} \cdot \nabla_{\mathbf{r}} \mathrm{f}(\mathbf{v}, \mathbf{r})-\mathrm{e} \nabla_{\mathbf{r}} \varphi(\mathbf{r}) \nabla_{\mathbf{v}} \mathrm{f}(\mathbf{v}, \mathbf{r}) & \\
= & \frac{2 \pi \mathrm{e}^{4}}{\mathrm{~m}^{2}} \Lambda\left\{\mathrm{C}_{\mathrm{ee}}(\mathbf{v}, \mathrm{f})+\mathrm{Z}_{\mathrm{eff}} \mathrm{n}(\mathbf{r}) \frac{\partial}{\partial \mathrm{v}_{\alpha}}\left(\mathrm{v}_{\alpha \beta} \frac{\partial \mathrm{f}}{\partial \mathrm{v}_{\beta}}\right)\right\},
\end{aligned}
$$

where $\Lambda$ is the Coulomb logarithm, e, $\mathrm{m}$, and $\mathrm{n}$ are the electron charge, mass, and density, $Z_{\text {eff }}$ is the effective ion charge

$$
\begin{aligned}
& \mathrm{C}_{\mathrm{ee}}(\mathbf{v}, \mathrm{f})=\frac{\partial}{\partial \mathrm{v}_{\alpha}} \int\left(\mathrm{f}(\mathbf{v}, \mathbf{r}) \frac{\partial \mathrm{f}\left(\mathbf{v}^{\prime}, \mathbf{r}\right)}{\partial \mathrm{v}_{\alpha}^{\prime}}-\mathrm{f}\left(\mathbf{v}^{\prime}, \mathbf{r}\right) \frac{\partial \mathrm{f}(\mathbf{v}, \mathbf{r})}{\partial \mathrm{v}_{\alpha}}\right) \mathrm{U}_{\alpha \beta} \mathrm{d} \mathbf{v}^{\prime}, \\
& \mathrm{U}_{\alpha \beta}=\left(\mathrm{u}^{2} \delta_{\alpha \beta}-\mathrm{u}_{\alpha} \mathrm{u}_{\beta}\right) / \mathrm{u}^{3}, \mathbf{u}=\mathbf{v}-\mathbf{v}^{\prime}, \text { and } \mathrm{v}_{\alpha \beta}=\left(\mathrm{v}^{2} \delta_{\alpha \beta}-\mathrm{v}_{\alpha} \mathrm{v}_{\beta}\right) / \mathrm{v}^{3}
\end{aligned}
$$


- Introducing total energy $\varepsilon=\mathrm{mv}^{2} / 2+\mathrm{e} \varphi(\mathbf{r})$, from (1) we have

$$
\mathbf{v} \cdot \nabla_{\mathbf{r}} \mathrm{f}(\varepsilon, \mathbf{r})=\frac{2 \pi \mathrm{e}^{4}}{\mathrm{~m}^{2}} \Lambda\left\{\mathrm{C}_{\mathrm{ee}}(\mathbf{v}, \mathrm{f})+\mathrm{Z}_{\mathrm{eff}} \mathrm{n}(\mathbf{r}) \frac{\partial}{\partial \mathrm{v}_{\alpha}}\left(\mathrm{v}_{\alpha \beta} \frac{\partial \mathrm{f}}{\partial \mathrm{v}_{\beta}}\right)\right\}
$$

- Next we assume that the distribution function $f(\mathbf{v}, \mathbf{r})$ can be represented by the sum of the symmetric function, $\mathrm{f}_{0}(\mathrm{v}, \mathbf{r})$, and the small asymmetric part, $\mathrm{f}_{1}(\mathbf{v}, \mathbf{r})$. With this approximation for $\mathrm{Z}_{\mathrm{eff}}>>1$ we find

$$
\mathrm{f}_{1}(\mathbf{v}, \mathbf{r})=-\frac{\mathrm{v}^{3} \mathrm{~m}^{2}}{4 \pi \mathrm{e}^{4} \mathrm{Z}_{\mathrm{eff}} \mathrm{n}(\mathbf{r})} \mathbf{v} \cdot \nabla_{\mathbf{r}} \mathrm{f}_{0}(\varepsilon, \mathbf{r})
$$

- Averaging Eq. (2) and using (3) we find

$$
\nabla_{\mathbf{r}} \cdot\left(\frac{\mathrm{v}^{5} \mathrm{~m}^{2}}{12 \pi \mathrm{e}^{4} \mathrm{Z}_{\mathrm{eff}} \mathrm{n}(\mathbf{r})} \nabla_{\mathbf{r}}\right)+\frac{2 \pi \mathrm{e}^{4}}{\mathrm{~m}^{2}} \Lambda \mathrm{C}_{\mathrm{ee}}\left(\mathrm{v}, \mathrm{f}_{0}\right)=0 .
$$


- Since the electrons with the energies higher than thermal, $\mathrm{T}_{\mathrm{e}}$, contribute most to the heat flux, we can use superthermal approximation for e-e collision term

$$
\nabla_{\mathbf{r}} \cdot\left(\frac{\mathrm{m}^{2}}{12 \pi \mathrm{e}^{4} \mathrm{Z}_{\mathrm{eff}} \mathrm{n}_{\mathrm{e}}(\mathbf{r})} \nabla_{\mathbf{r}} \mathrm{f}_{0}\right)+\frac{4 \pi \mathrm{e}^{4} \Lambda \mathrm{n}_{\mathrm{e}}(\mathbf{r})}{\mathrm{m}^{2}} \frac{\partial}{\mathrm{v}^{7} \partial \mathrm{v}}\left(\mathrm{f}_{0}+\frac{\mathrm{T}(\mathbf{r})}{\mathrm{mv}} \frac{\partial \mathrm{f}_{0}}{\partial \mathrm{v}}\right)=0
$$

- Following Albritton et al. PRL, 1986 with the modifications from Krasheninnikov Phys. Fluids B, 1993

$$
\mathrm{f}_{0} \approx \mathrm{f}_{\mathrm{M}}+\mathrm{A}\left(\varepsilon / \mathrm{T}_{\mathrm{e}}\right) \Psi
$$

where $\Psi=\mathrm{f}_{0}+\mathrm{T}_{\mathrm{e}}\left(\partial \mathrm{f}_{0} / \partial \varepsilon\right)$, we find

$$
\begin{aligned}
\left(\begin{array}{l}
\mathbf{q}(\mathbf{r}) \\
\mathbf{j}(\mathbf{r})
\end{array}\right)=-\frac{1}{\pi^{2}}\left(\frac{5 \mathrm{~T}}{2 \mathrm{mZ}_{\mathrm{eff}}}\right)^{1 / 2} \int \mathrm{d} \mathbf{r}^{\prime} \mathrm{n}^{\prime}\left(\mathrm{T}^{\prime}\right)^{5}\left(\begin{array}{l}
1 \\
1 / \mathrm{T}^{\prime}
\end{array}\right) \\
\times\left\{\left(\begin{array}{l}
\mathrm{P}_{0,-3 / 2} \\
\mathrm{P}_{-1 / 5,-5 / 2}
\end{array}\right) \nabla \mathrm{T}^{\prime}-\left(\begin{array}{l}
\mathrm{P}_{0,-5 / 2} \\
\mathrm{P}_{-1 / 5,-7 / 2}
\end{array}\right) \nabla \mathrm{e} \varphi^{\prime}\right\},
\end{aligned}
$$


where

$$
\begin{aligned}
& \mathrm{P}_{\alpha, \beta} \equiv \mathrm{P}_{\alpha, \beta}(\mathrm{g})=\int_{0}^{1} \mathrm{~d} \tau \tau^{\alpha}(1-\tau)^{-3 / 2} \int_{0}^{\infty} \mathrm{d} \sigma \sigma^{\beta} \exp \left(-\sigma-\frac{\mathrm{g}^{2}}{\sigma^{5}(1-\tau)}\right) \\
& \mathrm{g}^{2}=\frac{5}{4} \frac{\left(\mathbf{s}(\mathbf{r})-\mathbf{s}\left(\mathbf{r}^{\prime}\right)\right)^{2}}{\left(\mathrm{~T}^{\prime}\right)^{5}}, \text { and } \mathrm{d} \mathbf{s}=6 \pi \mathrm{e}^{4} \Lambda \mathrm{n}(\mathbf{r})\left[\left(\mathrm{Z}_{\mathrm{eff}}+1\right) \mathrm{T}(\mathbf{r})\right]^{1 / 2} \mathrm{~d} \mathbf{r}
\end{aligned}
$$

\section{Ia. Conclusions}

- The expression for electron heat flux, $\mathbf{q}(\mathbf{r})$, for 3D case is derived

- However, it contains 3D integral and, therefore, it might be rather time consuming to use it. Differential form of $\mathbf{q}(\mathbf{r})$ might be more preferable

- Benchmarking can be an issue. 3D self-similar solutions can be used for benchmarking 


\section{3D self-similar solution of electron kinetic equation}

- Self-similar solutions of electron kinetic equation in 1D2V (azimuthal symmetry) case were introduced in [Krasheninnikov, JETP 1988] (see also numerical solutions in [Krasheninnikov et al, Contr. Plasma Phys., 1992] and high $\mathrm{Z}_{\text {eff }}$ in [Bakunin and Krasheninnikov, Plasma Phys. Reports, 1995])

- In $1 \mathrm{D} 2 \mathrm{~V}$ case electron kinetic equation written in spherical coordinates $(\mathrm{V}, \mu=\cos \vartheta)$ in velocity space ( $\vartheta$ is the angle between the $\mathbf{x}$ and $\mathbf{v}$ ) has the form

$$
\begin{aligned}
\mathrm{v} \mu \frac{\partial \mathrm{f}(\mathrm{v}, \mu, \mathrm{x})}{\partial \mathrm{x}}-\mathrm{eE}(\mathrm{x}) & \left(\mu \frac{\partial \mathrm{f}(\mathrm{v}, \mu, \mathrm{x})}{\partial \mathrm{v}}+\frac{1-\mu^{2}}{\mathrm{v}} \frac{\partial \mathrm{f}(\mathrm{v}, \mu, \mathrm{x})}{\partial \mu}\right) \\
& =\frac{2 \pi \mathrm{e}^{4}}{\mathrm{~m}^{2}} \Lambda\left\{\mathrm{C}_{\mathrm{ee}}(\mathrm{v}, \mathrm{f})+\mathrm{Z}_{\mathrm{eff}} \mathrm{n} \frac{\partial}{\partial \mathrm{v}_{\alpha}}\left(\mathrm{v}_{\alpha \beta} \frac{\partial \mathrm{f}}{\partial \mathrm{v}_{\beta}}\right)\right\},
\end{aligned}
$$

- The trick here is to use self-similar variable $\mathbf{w}=\mathbf{v} \sqrt{\mathrm{m} / 2 \mathrm{~T}(\mathrm{x})}$ and apply the anzatz for electron distribution function

$$
\mathrm{f}(\mathrm{v}, \mathrm{x})=\mathrm{F}(\mathrm{w}, \mu) /(\mathrm{T}(\mathrm{x}))^{\alpha}
$$


- As a result, rather to deal with $1 \mathrm{D} 2 \mathrm{~V}$ equation (II.1) for $\mathrm{f}(\mathbf{v}, \mathrm{x})$ we have $2 \mathrm{~W}$ kunetic equation for $\mathrm{F}(\mathrm{w}, \mu)$

$$
\begin{aligned}
\gamma w \mu\left(\alpha \mathrm{F}+\frac{\mathrm{w}}{2} \frac{\partial \mathrm{F}}{\partial \mathrm{w}}\right)-\frac{\gamma_{\mathrm{E}}}{2}\left(\mu \frac{\partial \mathrm{F}}{\partial \mathrm{w}}+\frac{1-\mu^{2}}{\mathrm{w}} \frac{\partial \mathrm{F}}{\partial \mu}\right) \\
=\frac{1}{4}\left\{\mathrm{C}_{\mathrm{ee}}(\mathbf{w}, \mathrm{F})+\frac{\mathrm{Z}_{\mathrm{eff}}}{\mathrm{w}^{3}} \frac{\partial}{\partial \mu}\left(\left(1-\mu^{2}\right) \frac{\partial \mathrm{F}}{\partial \mu}\right)\right\} .
\end{aligned}
$$

where

$$
\begin{aligned}
& \gamma=-\mathrm{T}^{2}(\mathrm{~d} \ln \mathrm{T} / \mathrm{dx}) /\left(2 \pi \mathrm{e}^{4} \Lambda \mathrm{n}\right) \equiv(\lambda / \mathrm{L})=\text { const., and } \\
& \gamma_{\mathrm{E}}=\mathrm{eET} /\left(2 \pi \mathrm{e}^{4} \Lambda \mathrm{n}\right)=\text { const. }
\end{aligned}
$$

- From (II.2) and (II.4) we have $\mathrm{n} \propto \mathrm{T}^{3 / 2-\alpha}$ and $\mathrm{T}^{\alpha+1 / 2}(\mathrm{~d} \ln \mathrm{T} / \mathrm{dx})=$ const., which gives

$$
\mathrm{T}(\mathrm{x}) \propto \mathrm{x}^{1 /(\alpha+1 / 2)} .
$$


- Conservation of heat flux, $\mathrm{q}$, along $\mathrm{x}$ coordinate with anzatz (II.2) results in

$$
\mathrm{q}=\int \mathrm{f} \mathbf{v}\left(\mathrm{mv}^{2} / 2\right) \mathrm{d} \mathbf{v} \propto(\mathrm{T}(\mathrm{x}))^{3-\alpha} \int \mathrm{Fw}^{2} \mathbf{w} \mathrm{d} \mathbf{w}=\text { const. } \rightarrow \alpha=3 .
$$

- Solution of nonlinear self-similar kinetic equation (II.3) gives the following asymptotic expression for $\mathrm{F}(\mathrm{w}, \mu)$ at $\xi \equiv \mathrm{w}^{2} \rightarrow \infty$

$$
\mathrm{F}(\xi, \mu)=\frac{\Phi(\mu)}{\xi^{\alpha}}
$$

where $\Phi(\mu)$ is asymmetric function which magnitude and asymmetry depends on $Z_{\text {eff }}$

- As one can see, with (II.7) the expression (II.6) for $\alpha=3$ logarithmically diverges at high $\xi$ at any (even arbitrary small) $\gamma \equiv(\lambda / \mathrm{L})$

$$
q=\int f \mathbf{v}\left(m v^{2} / 2\right) d \mathbf{v} \propto e^{-A / \gamma^{1 / 3}} \int \Phi(\mu) \mu d \mu \int \xi^{2-\alpha} d \xi
$$

- It demonstrates effects of non-expandable terms and importance of ENTIRE density and temperature profiles for the problem of non-local heat conduction 
- Here we show that self-similar technique can be extended in a way which allows to study the effects of 3D geometry on non-local electron heat conduction

- We assume spherical symmetry of the problem in $\mathbf{r}$ space and introduce local spherical coordinates $(\mathrm{v}, \mu=\cos \vartheta)$ in velocity space, $\vartheta$ is the angle between the $\mathbf{r}$ and $\mathbf{v}$

$$
\begin{gathered}
\mathrm{v}\left(\mu \frac{\partial \mathrm{f}(\mathrm{v}, \mu, \mathrm{r})}{\partial \mathrm{r}}+\frac{1-\mu^{2}}{\mathrm{r}} \frac{\partial \mathrm{f}(\mathrm{v}, \mu, \mathrm{r})}{\partial \mu}\right)-\mathrm{eE}(\mathrm{r})\left(\mu \frac{\partial \mathrm{f}(\mathrm{v}, \mu, \mathrm{r})}{\partial \mathrm{v}}+\frac{1-\mu^{2}}{\mathrm{v}} \frac{\partial \mathrm{f}(\mathrm{v}, \mu, \mathrm{r})}{\partial \mu}\right) \\
=\frac{2 \pi \mathrm{e}^{4}}{\mathrm{~m}^{2}} \Lambda\left\{\mathrm{C}_{\mathrm{ee}}(\mathrm{v}, \mathrm{f})+\mathrm{Z}_{\mathrm{eff}} \mathrm{n} \frac{\partial}{\partial \mathrm{v}_{\alpha}}\left(\mathrm{v}_{\alpha \beta} \frac{\partial \mathrm{f}}{\partial \mathrm{v}_{\beta}}\right)\right\}, \quad \text { (II.9) }
\end{gathered}
$$

$\mathrm{v}\left(\mu \frac{\partial \mathrm{f}(\mathrm{v}, \mu, \mathrm{r})}{\partial \mathrm{r}}+\frac{1-\mu^{2}}{\mathrm{r}} \frac{\partial \mathrm{f}(\mathrm{v}, \mu, \mathrm{r})}{\partial \mu}\right)-\mathrm{eE}(\mathrm{r})\left(\mu \frac{\partial \mathrm{f}(\mathrm{v}, \mu, \mathrm{r})}{\partial \mathrm{v}}+\frac{1-\mu^{2}}{2 \mathrm{v}} \frac{\partial \mathrm{f}(\mathrm{v}, \mu, \mathrm{r})}{\partial \mu}\right)$

- Following [Krasheninnikov JETP 1988] we introduce self-similar variable $\mathbf{w}=\mathbf{v} \sqrt{\mathrm{m} / 2 \mathrm{~T}(\mathrm{r})}$ and use the anzatz for electron distribution function

$$
f(\mathbf{v}, \mathbf{r})=F(w, \mu) /(T(r))^{\alpha}
$$


- Then from (II.9) we find

$$
\begin{array}{r}
\gamma w\left\{\mu\left(\alpha \mathrm{F}+\frac{\mathrm{w}}{2} \frac{\partial \mathrm{F}}{\partial \mathrm{w}}\right)-\frac{1-\mu^{2}}{(\mathrm{~d} \ln \mathrm{T} / \mathrm{d} \ln \mathrm{r})} \frac{\partial \mathrm{F}}{\partial \mu}\right\}-\frac{\gamma_{\mathrm{E}}}{2}\left(\mu \frac{\partial \mathrm{F}}{\partial \mathrm{w}}+\frac{1-\mu^{2}}{\mathrm{w}} \frac{\partial \mathrm{F}}{\partial \mu}\right) \\
=\frac{1}{4}\left\{\mathrm{C}_{\mathrm{ee}}(\mathbf{w}, \mathrm{F})+\frac{\mathrm{Z}_{\mathrm{eff}}}{\mathrm{w}^{3}} \frac{\partial}{\partial \mu}\left(\left(1-\mu^{2}\right) \frac{\partial \mathrm{F}}{\partial \mu}\right)\right\} .
\end{array}
$$

where $\quad \gamma=-\mathrm{T}^{2}(\mathrm{~d} \ln \mathrm{T} / \mathrm{dr}) /\left(2 \pi \mathrm{e}^{4} \Lambda \mathrm{n}\right) \equiv(\lambda / \mathrm{L})=$ const., and

$$
\gamma_{\mathrm{E}}=\mathrm{eET} /\left(2 \pi \mathrm{e}^{4} \Lambda \mathrm{n}\right)=\text { const. }
$$

- From (II.10) and (II.12) we have $\mathrm{n} \propto \mathrm{T}^{3 / 2-\alpha}$ and $\mathrm{T}^{\alpha-1 / 2}(\mathrm{dT} / \mathrm{dr})=$ const., which gives

$$
\mathrm{T}(\mathrm{r}) \propto \mathrm{r}^{1 /(\alpha+1 / 2)}
$$


- As a result Eq. (II.11) can be written as follows

$$
\begin{gathered}
\gamma w\left\{\mu\left(\alpha \mathrm{F}+\frac{\mathrm{w}}{2} \frac{\partial \mathrm{F}}{\partial \mathrm{w}}\right)-(\alpha+1 / 2)\left(1-\mu^{2}\right) \frac{\partial \mathrm{F}}{\partial \mu}\right\}-\frac{\gamma_{\mathrm{E}}}{2}\left(\mu \frac{\partial \mathrm{F}}{\partial \mathrm{w}}+\frac{1-\mu^{2}}{\mathrm{w}} \frac{\partial \mathrm{F}}{\partial \mu}\right) \\
=\frac{1}{4}\left\{\mathrm{C}_{\mathrm{ee}}(\mathbf{w}, \mathrm{F})+\frac{\mathrm{Z}_{\mathrm{eff}}}{\mathrm{w}^{3}} \frac{\partial}{\partial \mu}\left(\left(1-\mu^{2}\right) \frac{\partial \mathrm{F}}{\partial \mu}\right)\right\} .
\end{gathered}
$$

- Notice an extra (in comparison with 1D2V case considered before) term $(\alpha+1 / 2)\left(1-\mu^{2}\right)(\partial \mathrm{F} / \partial \mu)$ causing, as we will see, picking of $F$ around $\mu=1$

- Conservation of heat flux for spherically symmetric case with anzatz (II.10) results in

$$
4 \pi r^{2} q \propto r^{2} \int f v^{2} \mathbf{v d} \mathbf{v} \propto r^{2} T^{3-\alpha} \int F w^{2} \mathbf{w d} \mathbf{w} \propto r^{2} T^{3-\alpha}=\text { const. }
$$

which is compatible with the self-similar temperature profile (II.13) for

$$
\alpha=-4 \text {. }
$$


- 2D Eq. (II.14) can be treated either numerically or, at small $\gamma$, analytically

- Here we analyze (II.14) for large energies. If only convective term would be important, i. e.

$$
\mu\left(\alpha \mathrm{F}+\frac{\mathrm{w}}{2} \frac{\partial \mathrm{F}}{\partial \mathrm{w}}\right)-(\alpha+1 / 2)\left(1-\mu^{2}\right) \frac{\partial \mathrm{F}}{\partial \mu}=0
$$

then the solution of (II.17) can be written as follows

$$
F(\xi, \mu)=\frac{H\left(\left(1-\mu^{2}\right) \xi^{-(2 \alpha+1)}\right)}{\xi^{\alpha}},
$$

where $\xi=\mathrm{w}^{2}$ and $\mathrm{H}(\mathrm{x})$ is an arbitrary function

- From (II.18) we find that with increasing $\xi$ the function $F(\xi, \mu)$ becomes more and more picked around $\mu=1$ caused by divergence of electron flux due to simple geometrical effects 
- As a result, unlike 1D case, to analyze energetic tail of the distribution function we need to keep Coulomb scattering term

$$
\mu\left(\alpha \mathrm{F}+\xi \frac{\partial \mathrm{F}}{\partial \xi}\right)-(\alpha+1 / 2)\left(1-\mu^{2}\right) \frac{\partial \mathrm{F}}{\partial \mu}=\frac{1+\mathrm{Z}_{\mathrm{eff}}}{4 \xi^{2}} \frac{\partial}{\partial \mu}\left(\left(1-\mu^{2}\right) \frac{\partial \mathrm{F}}{\partial \mu}\right)
$$

- Introducing $\eta=(1-\mu) / \xi^{2 \alpha+1}$ and $F=\tilde{F} / \xi^{\alpha}$, and considering (II.19) at $\mu \approx 1$ we have

$$
\xi^{2 \alpha+4} \frac{\partial \tilde{\mathrm{F}}}{\partial \xi}=\frac{1+\mathrm{Z}_{\mathrm{eff}}}{2 \gamma} \frac{\partial}{\partial \eta}\left(\eta \frac{\partial \tilde{\mathrm{F}}}{\partial \eta}\right)
$$

- Solution of (II.20) can be found easily and as a result in

$$
\mathrm{F}(\xi, \mu)=C \xi^{\alpha+3} \exp \left(-\frac{(1-\mu) \xi^{2}}{\mathrm{D}}\right)
$$

where $\mathrm{D}=-\left(1+\mathrm{Z}_{\text {eff }}\right) /(2 \alpha+3) 2 \gamma$ 
- For $\alpha=-4$, the expression for electron flux

$$
\mathrm{j} \propto \int \mathrm{F}(\xi, \mu) \xi \mathrm{d} \xi \mathrm{d} \mu \propto \int \frac{\exp \left(-(1-\mu) \xi^{2} / \mathrm{D}\right)}{\xi^{-(\alpha+3)}} \xi \mathrm{d} \xi \mathrm{d} \mu \propto \int \xi^{\alpha+2} \mathrm{~d} \xi,
$$

converges at $\xi \rightarrow \infty$, while the heat flux

$$
\mathrm{q} \propto \int \mathrm{F}(\xi, \mu) \xi^{2} \mathrm{~d} \xi \mathrm{d} \mu \propto \int \frac{\exp \left(-(1-\mu) \xi^{2} / \mathrm{D}\right)}{\xi^{-(\alpha+3)}} \xi^{2} \mathrm{~d} \xi \mathrm{d} \mu \propto \int \xi^{\alpha+3} \mathrm{~d} \xi,
$$

diverges logarithmically at $\xi \rightarrow \infty$

\section{IIa. Conclusions}

- Geometry can indeed play an important role in non-local electron heat transport

- 3D self-similar solutions of electron kinetic equation are available and can be used for benchmarking of both reduced models and codes 


\section{Alternatives}

- Finally it does not matter where we are taking our non-local heat flux expressions from

- We may "guess" about the heat flux expression and benchmark it against some "exact" expressions (e. g. Spitzer-Harm and self-similar solutions) and numerics

- "Conventional" wisdom suggests that non-locality of electron heat flux can be written as follows

$$
\mathrm{q}(\mathrm{x})=\int \mathrm{q}_{\mathrm{SH}}\left(\mathrm{x}^{\prime}\right) \mathrm{K}\left(\mathrm{x}, \mathrm{x}^{\prime}\right) \mathrm{dx^{ \prime }}
$$

where $\mathrm{q}_{\mathrm{SH}}(\mathrm{x})$ is the Spitzer-Harm heat flux and the kernel $\mathrm{K}\left(\mathrm{x}, \mathrm{x}^{\prime}\right)$, describing nonlocal effects, obeys the normalization

$$
\int \mathrm{K}\left(\mathrm{x}, \mathrm{x}^{\prime}\right) \mathrm{dx}^{\prime}=1 \text {. }
$$

- However, 1D2V self-similar solution with $\alpha=3$ corresponds to $\mathrm{q}_{\mathrm{SH}}(\mathrm{x})=$ const. (recall $\gamma=(\lambda / \mathrm{L}) \propto \mathrm{T}^{\alpha-1 / 2}(\mathrm{dT} / \mathrm{dx})=$ const. $)$. As a result, logarithmic divergence of self similar heat flux, $\mathrm{q}=\int \mathrm{f} \mathbf{v}\left(\mathrm{mv}^{2} / 2\right) \mathrm{d} \mathbf{v} \propto \int \Phi(\mu) \mu \mathrm{d} \mu \int \xi^{2-\alpha} \mathrm{d} \xi \propto \ln \xi$, at high energies (= large x) CANNOT be recovered with Eq. (III.1) 
- Should we alter normalization/asymptotic of $\mathrm{K}\left(\mathrm{x}, \mathrm{x}^{\prime}\right)$ at $\left|\mathrm{x}^{\prime}\right| \rightarrow \infty$ ?! E. g.

$$
\mathrm{K}\left(\mathrm{x},\left|\mathrm{x}^{\prime}\right| \rightarrow \infty\right) \propto \exp \left(-\mathrm{A} /\left(\gamma_{ \pm \infty}\right)^{\mathrm{p}}\right), \quad \text { with } \quad \gamma=\lambda / \mathrm{L} .
$$

- Differential models for non-local heat flux can also be considered. E. g. diffusive model

$$
\lambda(x) \frac{d}{d x}\left(\lambda(x) \frac{d q(x)}{d x}\right)+q_{S H}(x)-q(x)=0 .
$$

- However, "standard" solution of the equation (III.4) is equivalent to the integral form like (III.1) with the kernel

$$
\mathrm{K}\left(\mathrm{x}, \mathrm{x}^{\prime}\right)=\exp \left(-\int_{\mathrm{x}}^{\mathrm{x}^{\prime}} \mathrm{dx^{ \prime \prime }} / \lambda\left(\mathrm{x}^{\prime \prime}\right) \mid\right) / 2 \lambda(\mathrm{x}) .
$$

- Could it be that "nonstandard" solution of the equation (III.4) with nonzero boundary conditions at $|\mathrm{x}| \rightarrow \infty$ or mixed diffusion/convection model, e. g.

$$
\lambda(x) \frac{d}{d x}\left(\lambda(x) \frac{d q}{d x}+\sigma(x) q\right)+q_{S H}(x)-q=0 \quad \text { with } \quad \sigma(x) \propto(\lambda / L)^{p} .
$$

will work?! 


\section{Magnetic Fields In Laser Light Speckles.}

B. F. Lasinski, C. H. Still, A. B. Langdon,

D. E. Hinkel, and E. A. Williams

for the

Electron Transport Workshop

September 9-11, 2002.

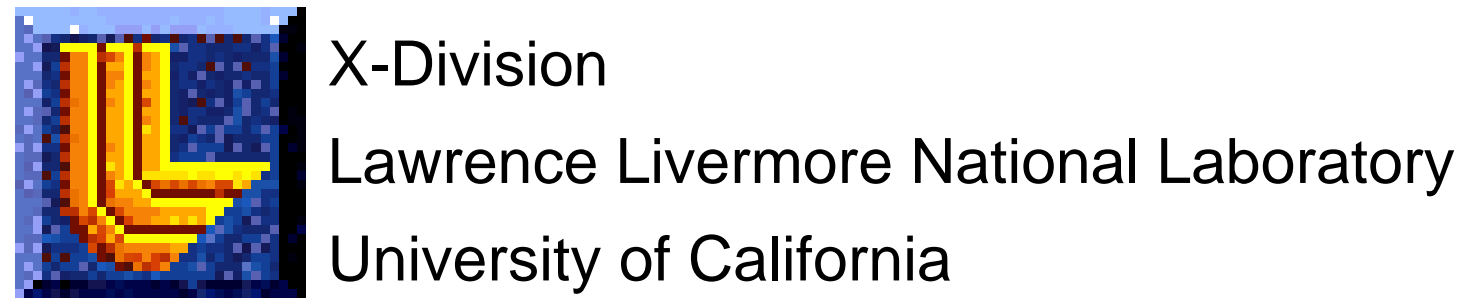

Work performed under the auspices of the US DoE by the Lawrence Livermore National Laboratory under contract W-7405-ENG-48 


\section{Static magnetic field structures due to Raman scatter have been identified in MPP 3D and 2D PIC simulations - a preliminary report.}

- In laser light speckles, Raman scatter, both forward and back, generates localized currents of forward going electrons.

- These currents $\left(J_{z}\right)$ result in surrounding magnetic fields $\left(B_{\theta}\right)$.

- We have identified these magnetic fields in our MPP 3D and 2D PIC simulations with Z3 for parameters associated with NIF high temperature hohlraums and NIF ignition hohlraums.

- Large 2D and 3D PIC simulations with dedicated diagnostics are required for this effort.

- These magnetic fields are MG and are large enough to confine the background electrons and hence affect electron transport in these plasmas.

$\lambda$ Studies are underway to elucidate the complex spatial and time dependence of these magnetic fields. 


\section{These B-fields were initially seen in $\mathbf{Z 3}$ simulations of conditions expected in small, high temperature hohlraums.}

$\mathrm{HTH}$ parameters: flat density profile at $0.2 \mathrm{n}_{\mathrm{c}}, \mathrm{ZT}_{\mathrm{e}} / \mathrm{T}_{\mathrm{i}}=13, \mathrm{Te}=14 \mathrm{keV}$;

Gaussian beams or $(\sin )^{4}$ spatial profile

$\mathrm{I}=7 \times 10^{16} \mathrm{~W} / \mathrm{cm}^{2}$ for blue $(1 / 3 \mu \mathrm{m})$ light; intense speckle

Raman scatter is the decay of the incident light wave into an electron plasma wave and a lower frequency light wave.

At these parameters, find vigorous back and forward scatter (A. B. Langdon and D. E. Hinkel, Phys. Rev. Lett. 89, 2002 (015003)).

Will also describe our preliminary findings on B-fields from SRS for parameters relevant to NIF ignition hohlraums. 


\section{Current Z3 simulation volumes are on the order of an $f / 4$ to $f / 8$ speckle.}

Simulation geometry:

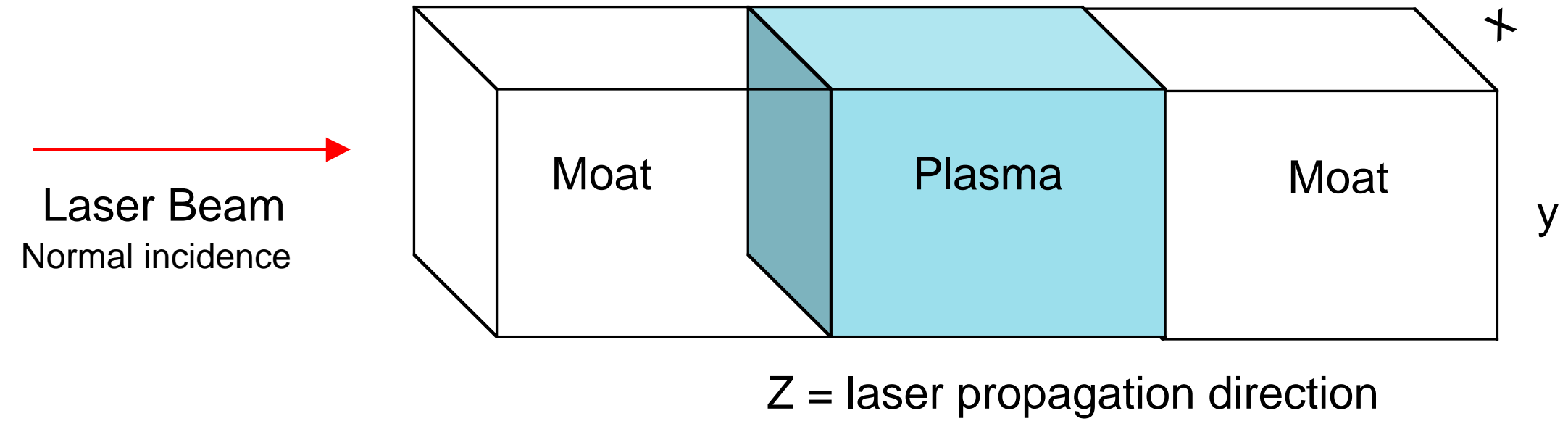

- In these 3D simulations the plasma slab is $24 \lambda_{0} \times 24 \lambda_{0} \times\left(61 \lambda_{0}\right.$ or $\left.138 \lambda_{0}\right)$. The smaller simulation system has $1.4 \times 10^{8}$ cells and $3.4 \times 10^{9}$ particles (electrons and ions).

- 2D simulations are as wide as $98 \lambda_{0}$ in the transverse direction and as long as $300 \lambda_{0}$ in the propagation direction. 


\section{D slices of the Poynting vector, $P_{z}$, show the propagation of this linearly polarized laser $\left(E_{y}, B_{x}\right)$.}

At an early time $(0.14 \mathrm{ps})$, light has not yet penetrated the entire slab.

Plot of $P_{z}$ vs $(x, z)$ in the $y / 2$ plane.

$P Z$ at $Y=76.8$ (iy=193), time 840.03

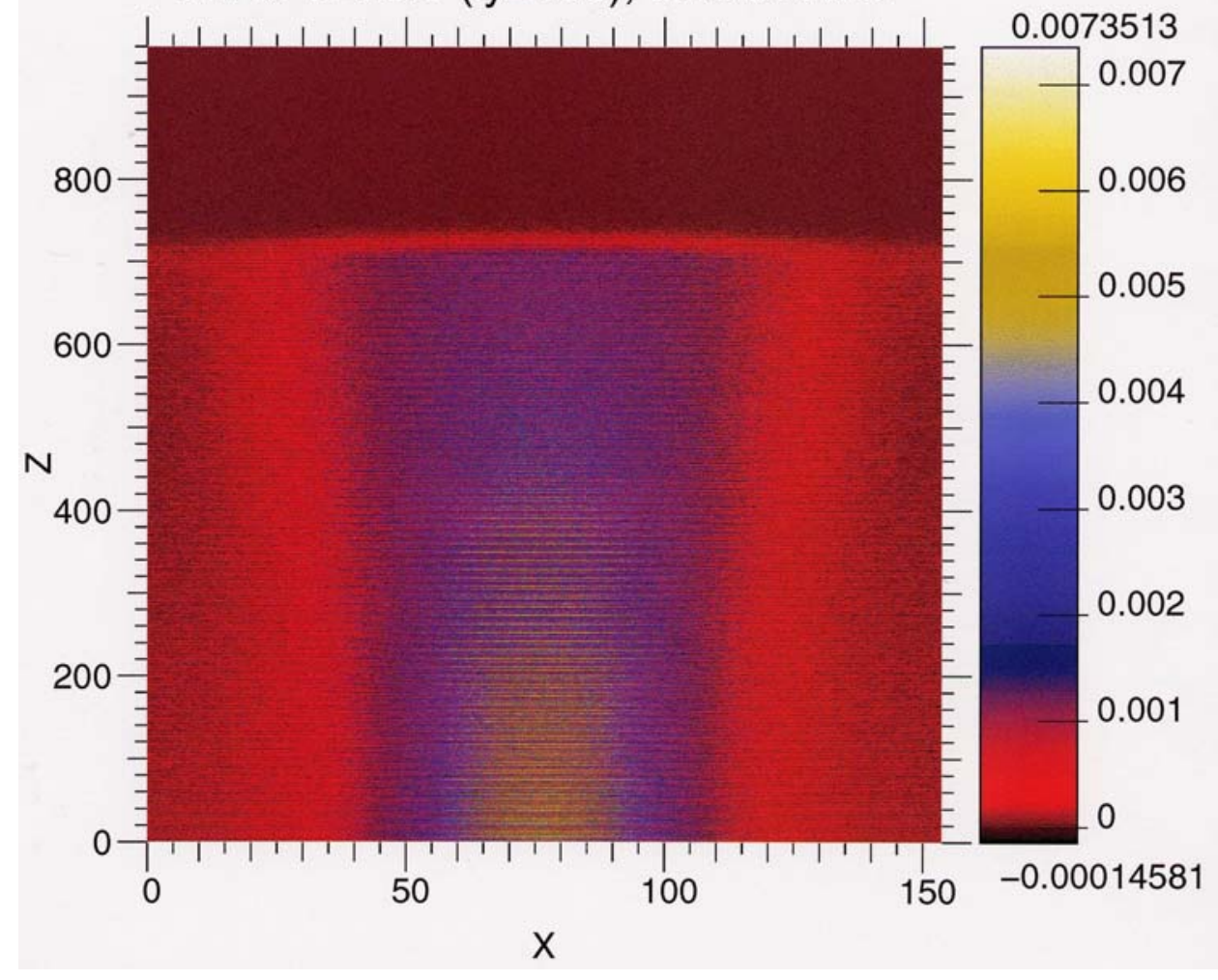

Peak laser in Gaussian beam has $\mathrm{B}_{0}=0.08$ which corresponds to $7 \times 10^{16} \mathrm{~W} / \mathrm{cm}^{2}$ for blue light.

Note different aspect ratios for the two axes.

Spatial scales are in units of $c / \omega_{0}$.

Readily identify $\lambda_{0} / 2$, as expected for snapshot in time of $\mathrm{P}_{\mathrm{z}}$ 


\section{At later times, we identify forward and back Raman scatter in the 2D slice of the Poynting vector.}

As part of the Z3 diagnostics suite, we apply a low pass temporal filter, $\left[\sin \left(\pi \omega / \omega_{0}\right) /\left(\pi \omega / \omega_{0}\right)\right]^{2}$, to fields and fluxes to separate the laser and the low frequency fields and fluxes. We identify these quantities with the subscript $s$

Plot of $\left(P_{z}\right)_{s}$ vs $(x, z)$ in the $y / 2$ plane at $0.27 \mathrm{ps}$

3D; $0.2 \mathrm{nc} ; 14 \mathrm{keV}$; $7 \times 10^{16} \mathrm{~W} / \mathrm{cm}^{2}$

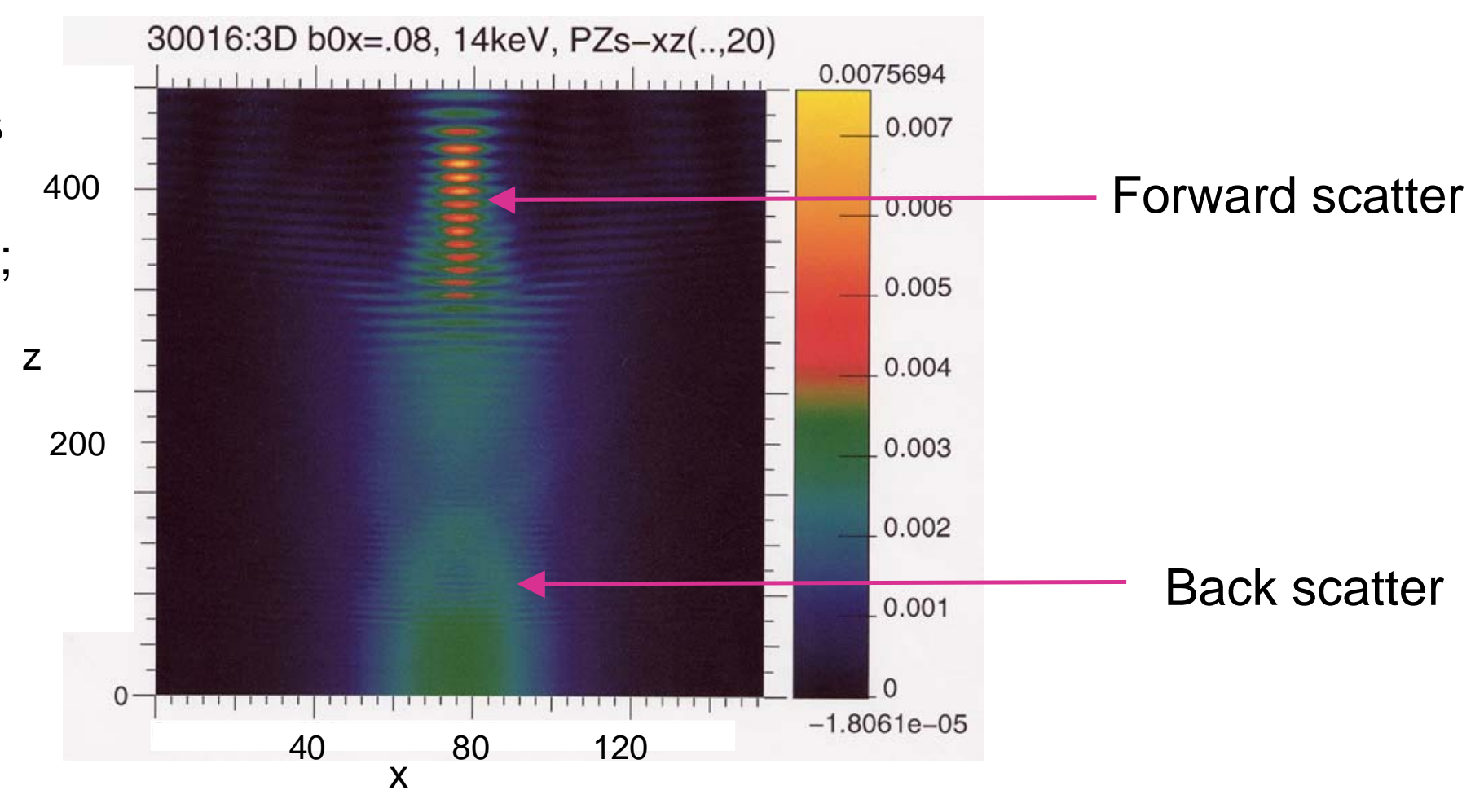




\section{Static magnetic fields associated with back and forward scatter are readily apparent in the 2D slice of $B_{y} v s x$ and $z$}

$3 \mathrm{D}, 0.2 \mathrm{nc}, 14 \mathrm{keV}, 7 \times 10^{16} \mathrm{~W} / \mathrm{cm}^{2}$, linearly polarized $\left(\mathrm{B}_{\mathrm{x}}, \mathrm{E}_{\mathrm{y}}\right)$

Plot of $B_{y} v s(x, z)$ in the $y / 2$ plane at $0.27 p s$.

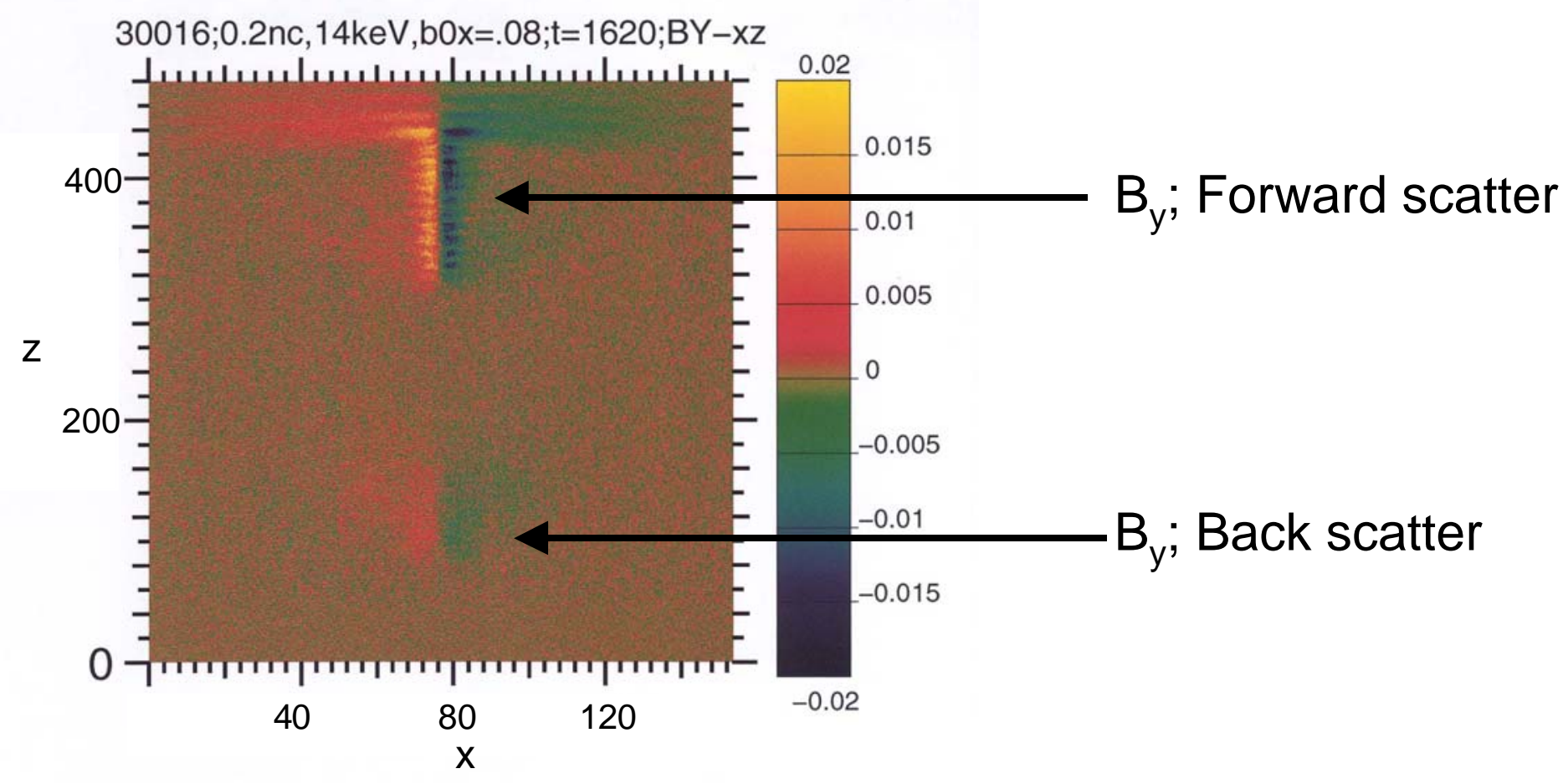




\section{Raman back and forward scatter, as well as their associated magnetic fields, are readily visible in this 2D slice of $(\mathrm{Bx}) \mathrm{s}$.}

$3 \mathrm{D}, 0.2 \mathrm{nc}, 14 \mathrm{keV}, 7 \times 10^{16} \mathrm{~W} / \mathrm{cm}^{2}$, linearly polarized $\left(B_{x}, E_{y}\right)$

Plot of $B_{x} v s(y, z)$ in the $x / 2$ plane at 0.27 ps.

Filtering removes the incident

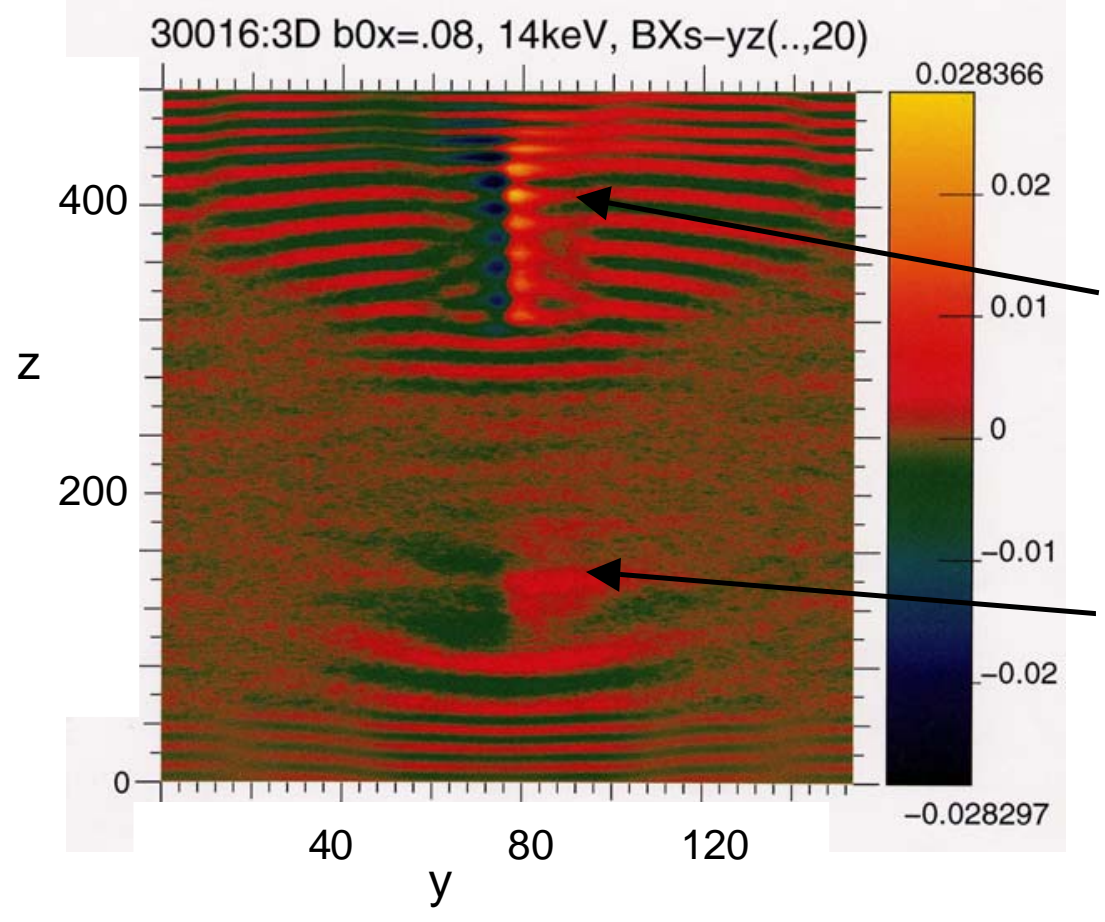
laser and brings out the static magnetic fields and the Raman scatter.

Forward scatter

The long wavelengths of the SRS forward and back scattered light are readily apparent.

Back scatter

The antisymmetric component of $\left(B_{\mathrm{x}}\right)_{\mathrm{s}}$ isolates the static magnetic field. 


\section{In 3D, find the $B_{x}$ and $B_{y}$ components of $B_{\theta}$ consistent with net $\mathrm{J}_{\mathrm{z}}<0$.}

$3 \mathrm{D}, 0.2 \mathrm{nc}, 14 \mathrm{keV}, 7 \times 10^{16} \mathrm{~W} / \mathrm{cm}^{2}$, linearly polarized $\left(\mathrm{B}_{\mathrm{x}}, \mathrm{E}_{\mathrm{y}}\right)$

Components of $B_{\theta}$ at $0.27 \mathrm{ps}$.

Antisymmetric component of $\left(B_{x}\right)_{s}$ vs

$(y, z)$ in the $x / 2$ plane

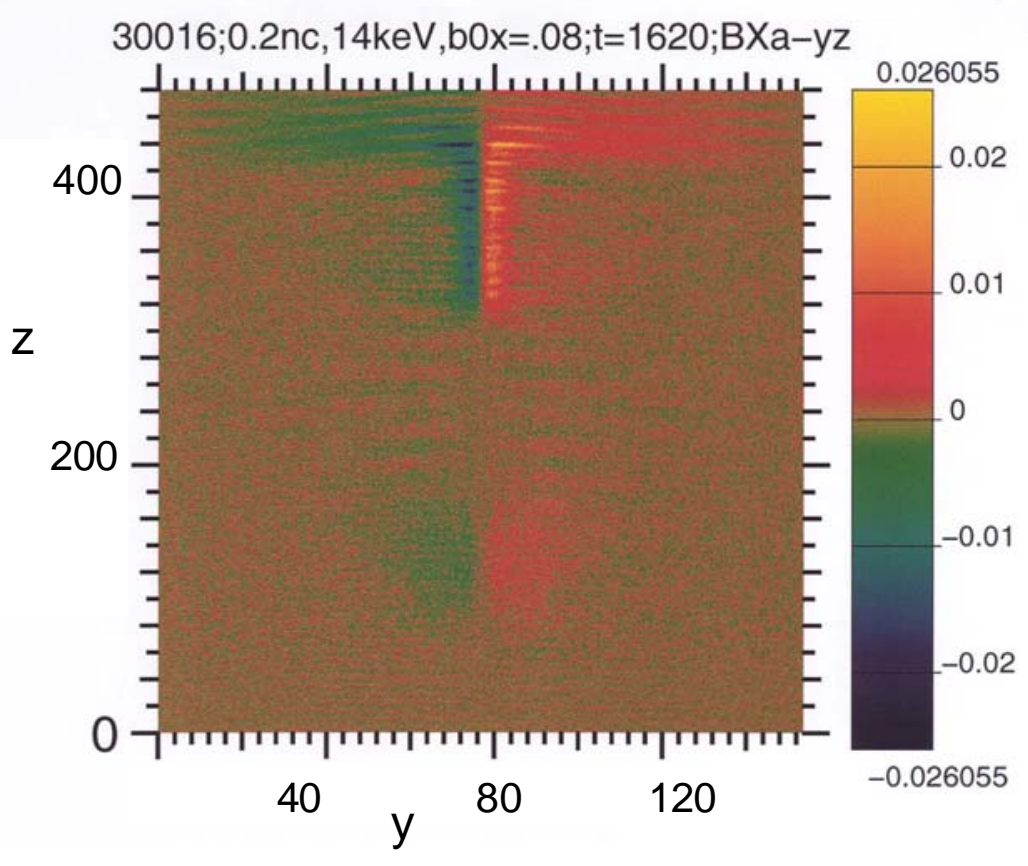

$\left(B_{y}\right)_{s}$ vs $(x, z)$ in the $y / 2$ plane.

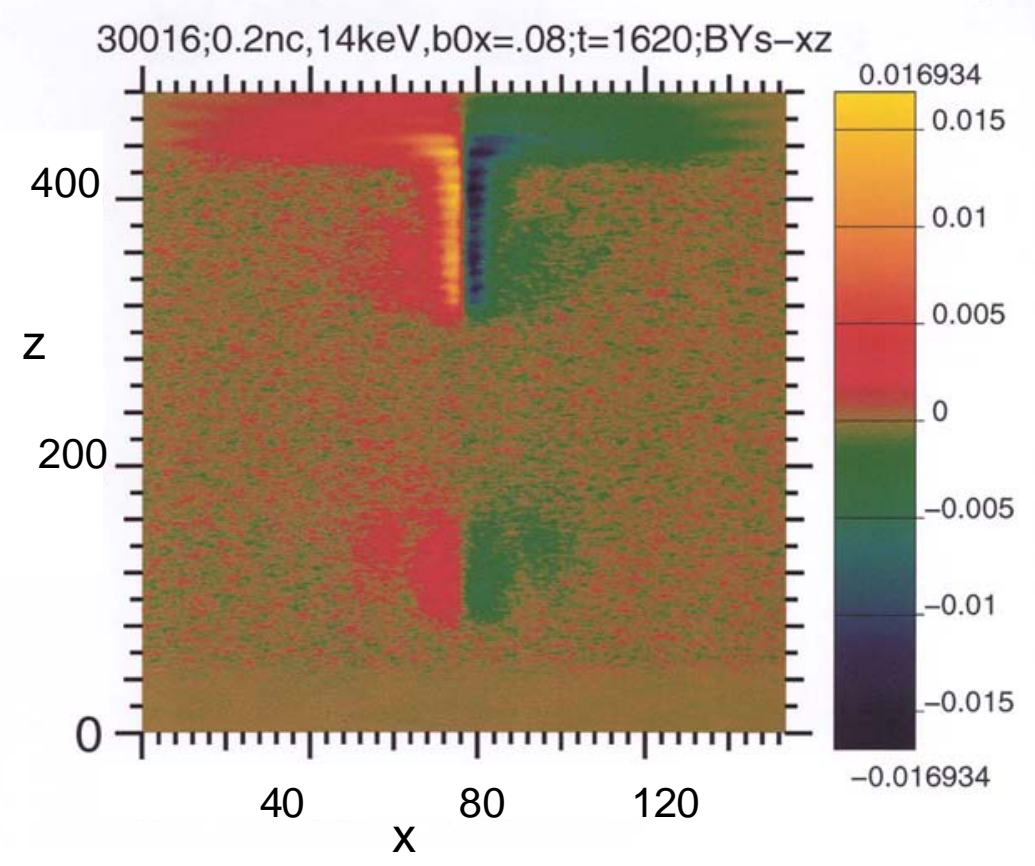

In these units, $\mathrm{B}=0.02$ corresponds to $6 \mathrm{MG}$. 


\section{We also have preliminary results on B-field generation in 2D simulations.}

$2 \mathrm{D}, 0.2 \mathrm{n}_{\mathrm{c}}, 14 \mathrm{keV}, 7 \times 10^{16} \mathrm{~W} / \mathrm{cm}^{2}$, linearly polarized $\left(\mathrm{B}_{\mathrm{y}}, \mathrm{E}_{\mathrm{x}}\right)$; at $0.4 \mathrm{ps}$

$\left(B_{y}\right)_{s}$ vs $(x, z)$

$30034 ; 0.2 \mathrm{nc}, 14 \mathrm{keV}, \mathrm{b} 0 \mathrm{y}=.08, \mathrm{t}=2400 ; \mathrm{BYs}-\mathrm{xz}(\mathrm{x}, 29)$

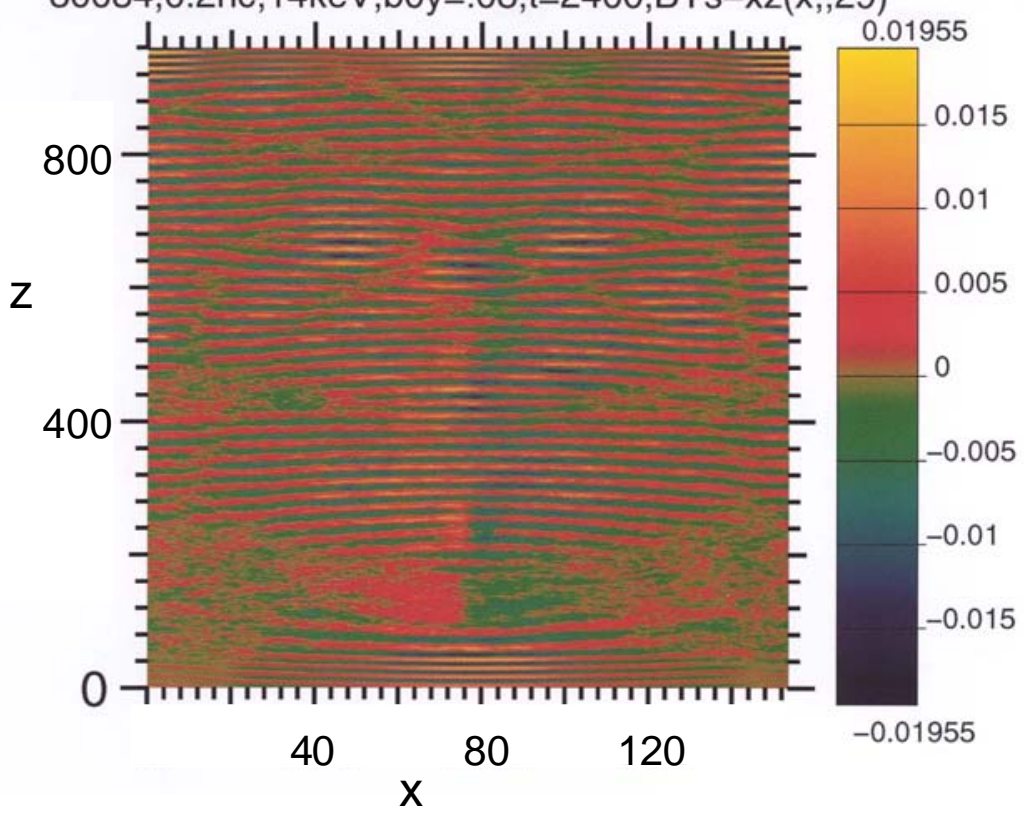

$\left(B_{y}\right)_{a}$ vs $(x, z)$

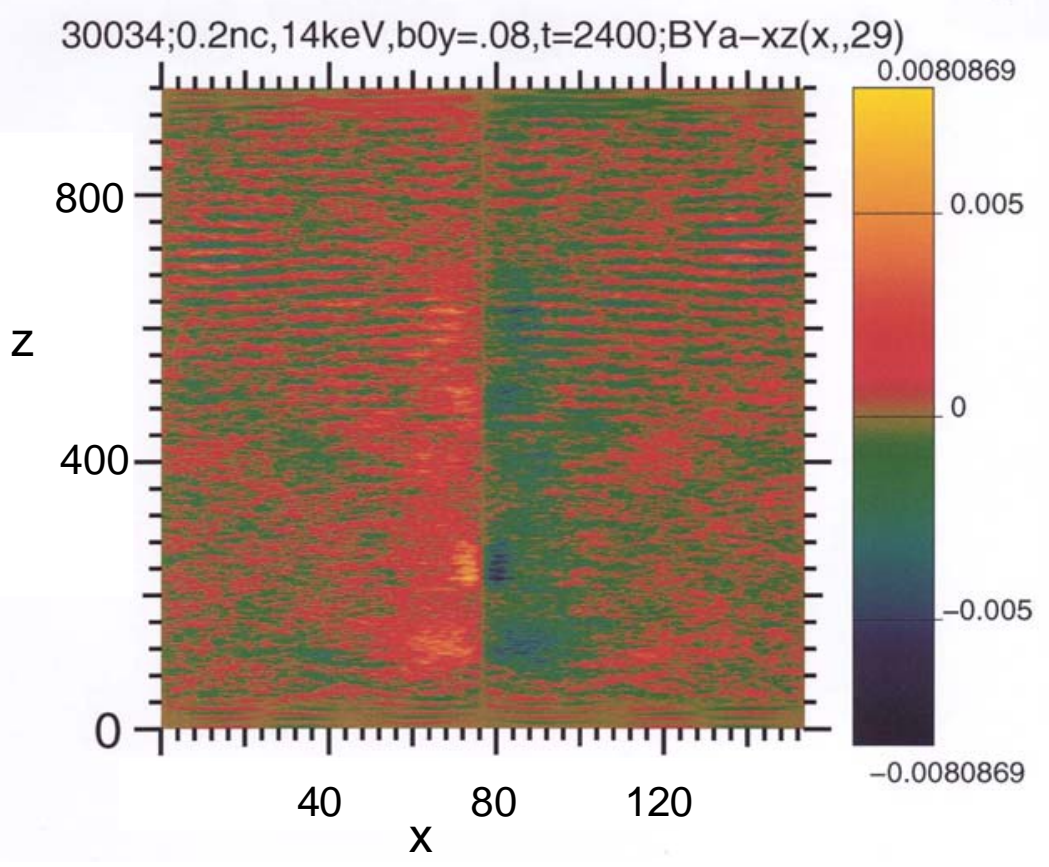

- Find the expected back and forward Raman scatter as well as the $(B)_{s}$

- In 2D, we can readily simulate bigger systems for longer times. 


\section{We find the B-field due to Raman Backscatter in simulations for parameters relevant to NIF ignition hohIraums.}

Parameters; 2D; $0.2 \mathrm{n}_{\mathrm{c}}, 5 \mathrm{keV}, 1 \times 10^{16} \mathrm{~W} / \mathrm{cm}^{2}$ for blue light; an intense speckle

Filtered $B_{y}$ vs $(x, z)$ at $y / 2$ shows

Raman backscatter with static B-field superimposed; $\mathrm{t}=0.37 \mathrm{ps}$

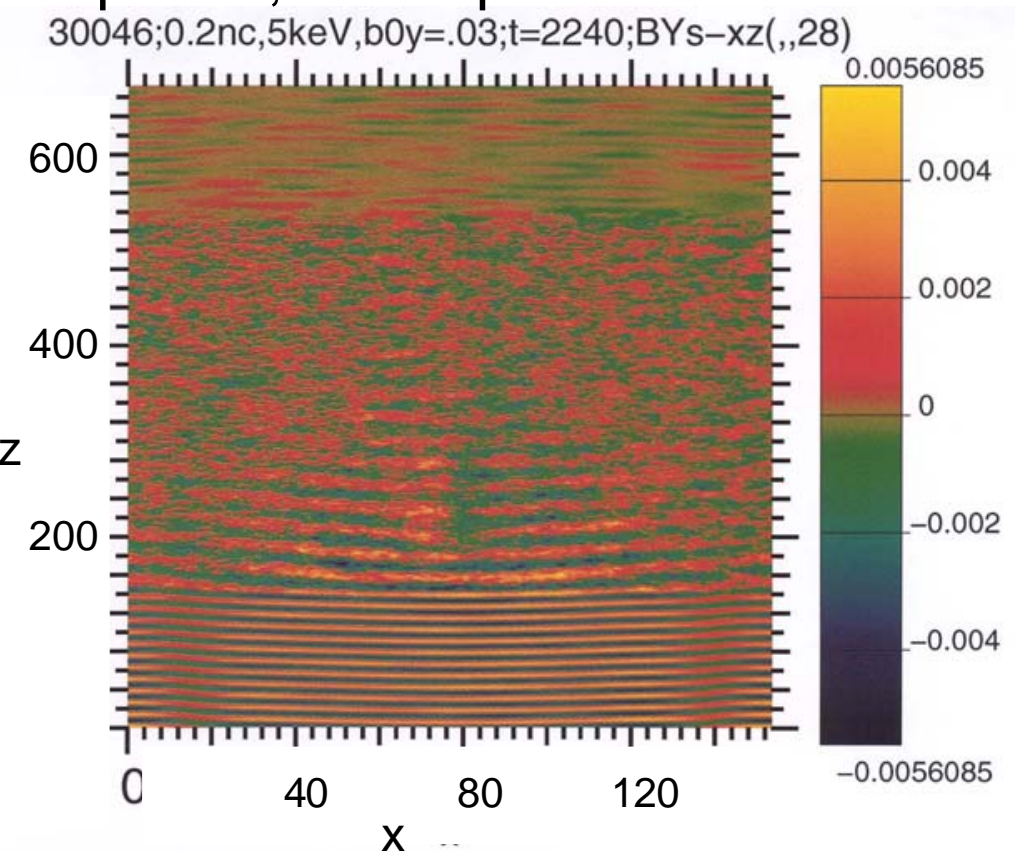

Antisymmetric part of filtered $\mathrm{B}_{\mathrm{y}}$ emphasizes static B-field with peak 1 MG.

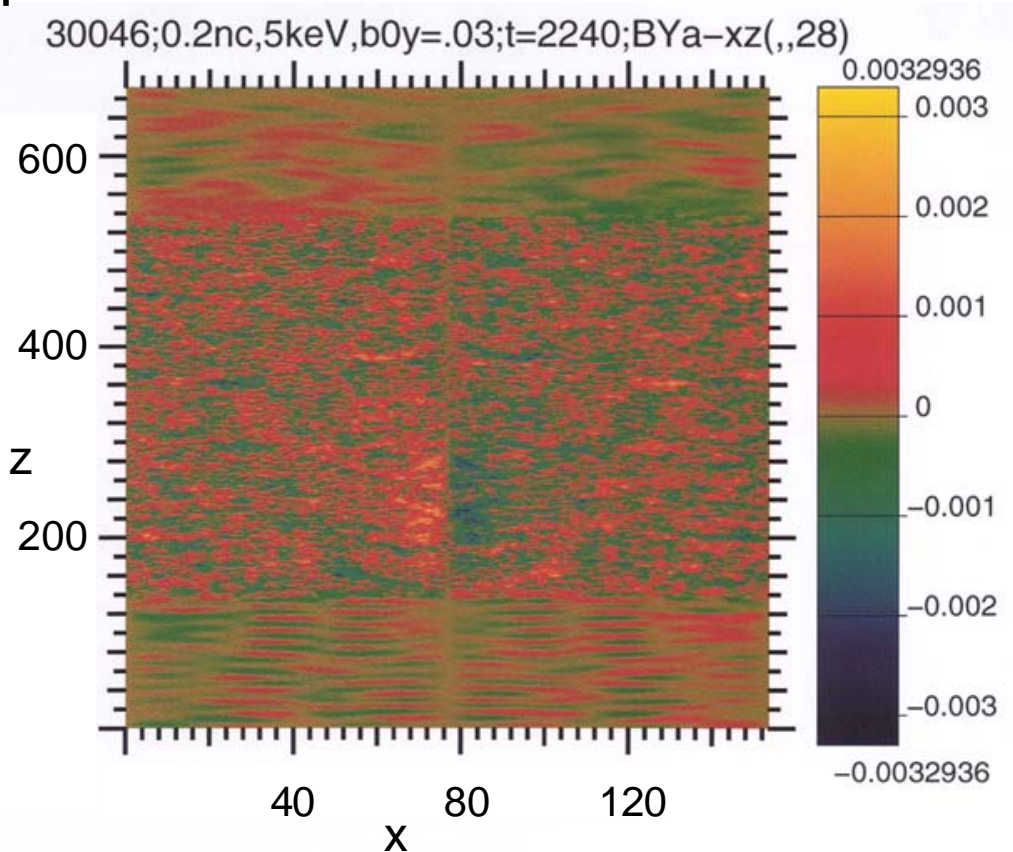

- 3D simulation with these parameters and 2D modeling at lower densities are underway. 


\section{Raman electrons are highly magnetized.}

- The Larmor radius of electrons in MG field is smaller than an $\mathrm{f} / 8$ speckle width. Assume $\mathrm{B}_{\theta}=.02$ or $6 \mathrm{MG}$. Then an $80 \mathrm{keV}$ hot electron has a Larmor radius of $\sim 3 \lambda_{0}$.

- This is less than a speckle width and in rough agreement with the narrow spatial extent of $B_{\theta}$ seen in the simulations.

- We estimate that the net current associated with $B_{\theta}$ is $~$ Alfven current for the Raman forward scatter in the small hohlraum with laserinteractions parameters: $0.2 \mathrm{n}_{\mathrm{c}}, 14 \mathrm{keV}$, and $\mathrm{I}=7 \times 10^{16} \mathrm{~W} / \mathrm{cm}^{2}$ for blue light.

$\lambda$ For NIF ignition hohlraum conditions, we expect mainly Raman backscatter and less energetic hot electrons. Extremely preliminary results indicate $B_{\theta} \sim 1 M G$. 


\section{Static magnetic field structures due to Raman scatter have been identified in MPP 3D and 2D PIC simulations - a preliminary report.}

- In laser light speckles, Raman Scatter, both forward and back, generate localized currents of forward going electrons.

- These currents $\left(J_{z}\right)$ result in surrounding magnetic fields $\left(B_{\theta}\right)$.

- We have identified these magnetic fields in our MPP 3D and 2D PIC simulations with Z3 for parameters associated with NIF high temperature hohlraums and NIF ignition hohlraums.

- Large 2D and 3D PIC simulations with dedicated diagnostics are required for this effort.

- These magnetic fields are MG and are large enough to confine the background electrons and hence affect electron transport in these plasmas.

$\lambda$ Studies are underway to elucidate the complex spatial and time dependence of these magnetic fields. 
If numbers of energetic electrons are the same from forward and back, expect more energetic currents and B-fields of greater magnitude from forward scatter since higher phase velocity of plasma waves leads to more energetic electrons.\{rewrite\} 


\section{Electron Transport Workshop \\ Purple Orchid Inn, Livermore, Ca}

September 9-11, 2002

\section{AN IMPROVED MODEL OF NONLOCAL HEAT FLOW IN LASER HEATED PLASMAS}

Fathallah Alouani Bibi and Jean-Pierre Matte

INRS-Énergie, Matériaux et Télécommunications, Varennes, Québec, Canada J3X-1S2 


\section{OUTLINE}

2 Talks for the price of 1

I) Nonlocal heat flow in plasmas heated by inverse bremsstrhalung.

Work of F. Alouani, Ph. D. student.

How the super-Gaussian deformation of the distribution function by the heating affects nonlocal heat flow.

II) Simulation of high intensity, long pulse, planar experiments.

Collaboration with D. Braun, J. Edwards and L. Suter of LLNL.

Preliminary comparison of an electron kinetic (FPI) simulation to LASNEX. 
INRS- Énergie, Matériaux et Télécommunications.

I) Nonlocal heat flow in plasmas heated by inverse bremsstrhalung

Work of F. Alouani, Ph. D. student.

How the super-Gaussian deformation of the distribution function by the heating affects nonlocal heat flow. 


\section{INTRODUCTION}

- Plasma heating by collisional absorption (IB) leads to super-Gaussian electron distributions.

- Absorption preferably by slow electrons ( because _e-i ${ }^{\times} \mathrm{v}^{-3}$ ) $\Rightarrow$ very superGaussian EVDF: $\exp \left[-(\mathrm{v} / \mathrm{u}(\mathrm{t}))^{5}\right]$ (Langdon, Phys. Rev. Lett. 44, 2717 (1980)).

- Collisional relaxation (e-e) tends to establish a Gaussian (Maxwellian) EVDF: $\exp \left[-(\mathrm{v} / \mathrm{u}(\mathrm{t}))^{2}\right]$

- For finite _ $\left(=\mathrm{Z}\left(\mathrm{V}_{\mathrm{osc}} / \mathrm{V}_{\text {th }}\right)^{2}=\mathrm{IB}\right.$ heating/e-e relaxation rate $)$, the EVDF has shape $\exp \left[-(\mathrm{v} / \mathrm{u}(\mathrm{t}))^{\mathrm{m}}\right]$, where $2<\mathrm{m}<5, \mathrm{~m}$ is an increasing function of _. (Matte, Lamoureux et al., P.P.C.F. 30, 1665 (1988)). 
This causes:

- Reduced absorption (Langdon, Phys. Rev. Lett. 44, 2717 (1980)).

Correction factor to IB absorption R, decrease from 1 to 0.45 as _ increases (ibid).

- Reduced thermal conductivity (Mora and Yahi, Phys. Rev. A 26, 2259 (1982)).

- Increased sound speed (Afeyan et al., Phys. Rev. Lett. 80, 2322 (1998)).

- Reduced Landau damping for Langmuir waves (ibid).

- Reduced ionization and excitation rates (Alaterre et al., Phys. Rev. A 26, 2259 (1986)).

- Needed: accurate study of the resulting non-local effects, and their influence on the plasma macroscopic characteristics (temperature profiles, heat flux, etc...). 


\section{THE FOKKER-PLANCK SIMULATION CODE ("FPI")}

- The "FPI" kinetic code is:

1-D in space; slab geometry.

2-D in $V$-space $\left(V, \mu=V_{x} / V\right)$, Legendre expansion for $\mu$.

- Included physical processes:

- Advection (transport term: $\mathrm{V}_{\mathrm{x}} \partial \mathrm{F} / \partial \mathrm{x}$ )

- Space charge field for quasi-neutrality, and acceleration (-eE/m $\left.\partial \mathrm{F} / \partial \mathrm{V}_{\mathrm{x}}\right)$

- Fokker-Planck term for e-i and e-e collisions

- Heating by Collisional absorption (IB).

Prescribed laser intensity, Gaussian in $\mathrm{x}$ and $\mathrm{t}$. 



\section{PARAMETERS OF THE RUNS AND DIAGNOSTICS}

Laser heated plasma: $\mathrm{N}_{\mathrm{e}}=2 \times 10^{20} \mathrm{~cm}^{-3}\left(\mathrm{~N}_{\mathrm{c}} / 20\right), \mathrm{T}_{\mathrm{e}}=500 \mathrm{eV}$. (Initially uniform)

- Atomic number: $Z=4,11,20$.

- Laser wavelength 0.53 m, intensities from $10^{15}$ to $8 \times 10^{15} \mathrm{~W} / \mathrm{cm}^{2}$, with FWHM's from 38 to 4.75 m. Temporal FWHM was 200 psec in all cases. $\left(\mathrm{I}_{0} * \mathrm{FWHM}=10^{15} \mathrm{~W} / \mathrm{cm}^{2} * 38\right.$ m)

- The Legendre polynomial expansion was to order 3. 
- INRS- Énergie, Matériaux et Télécommunications

\section{COMPARED MODELS}

The FPI results are compared with those obtained by using:

- Flux-limited diffusion with $f$ equal to $0.03,0.05,0.1$ and 0.5 .

- Nonlocal models from literature:

1) Luciani-Mora (LMV) nonlocal heat flow (PRL 51, 1664 (1983))

2) Bendib, Luciani et al. nonlocal heat flow with propagator correction due to the electric field (Phys. F1. 31, 711 (1987))

3) Epperlein-Short (ES) nonlocal heat flow (Phys.Fl. B 4, 2211 (1992))

4) Our new delocalization model including non-Maxwellian heating effects. 


\section{RESULTS AND DISCUSSION}

- Temperature and heat flux profiles:

- Neither of the earlier fluid models correctly reproduces FPI's $T_{e}(X, t)$.

- LMV: Delocalization kernel far too small for high $\mathrm{k} \lambda_{\mathrm{e}}$.

- Even taking into account the effect of the electric field as in Bendib et al., the differences with FPI are still considerable.

- ES: Closer to FPI, but lower near $\mathrm{x}=0$ (center of laser beam).

- Flux limiters: All flat near $\mathrm{x}=0$, sudden drop some distance away. (higher $\mathrm{f}$ : lower $\mathrm{T}_{\mathrm{e}}$ maximum but wider). At $\mathrm{x}=0$ : $\mathrm{f}=0.10$ matches $\mathrm{ES}$ and $\mathrm{f}=0.05$ matches FPI.

- AM: The newly developed model gave a good fit of the temperature profiles for the three cases: immobile ions, mobile ions, mobile ions with the ponderomotive force.

Reason: Our new nonlocal model takes into account the variation of $k \lambda_{\mathrm{e}}$ AND of ${ }_{-}=\mathrm{Z}\left(\mathrm{V}_{\mathrm{osc}} / \mathrm{V}_{\mathrm{th}}\right)^{2}$. 


\section{- $\quad$ Nonlocal kernel:}

The non-local heat flux can be expressed as a convolution over the Spitzer-Harm flux with a non-local kernel:

$$
q(x)=\beta^{-1}(x) \int_{-\infty}^{+\infty} q_{S H}\left(x^{\prime}\right) \frac{w\left(\xi\left(x, x^{\prime}\right)\right)}{\lambda_{e}\left(x^{\prime}\right)} d x^{\prime}
$$

By making a small laser intensity perturbation $(\sim 1 \%)$, we obtained a new propagator:

$$
\tilde{w}\left(k \lambda_{e}, \alpha\left(k \lambda_{e}\right)\right)=\frac{\tilde{q}\left(k \lambda_{e}, \alpha\left(k \lambda_{e}\right)\right)}{\tilde{q_{S H}}\left(k \lambda_{e}, \alpha\left(k \lambda_{e}\right)\right)}
$$

Approach pioneered by Epperlein and Short (Phys. Rev. E, 1994)

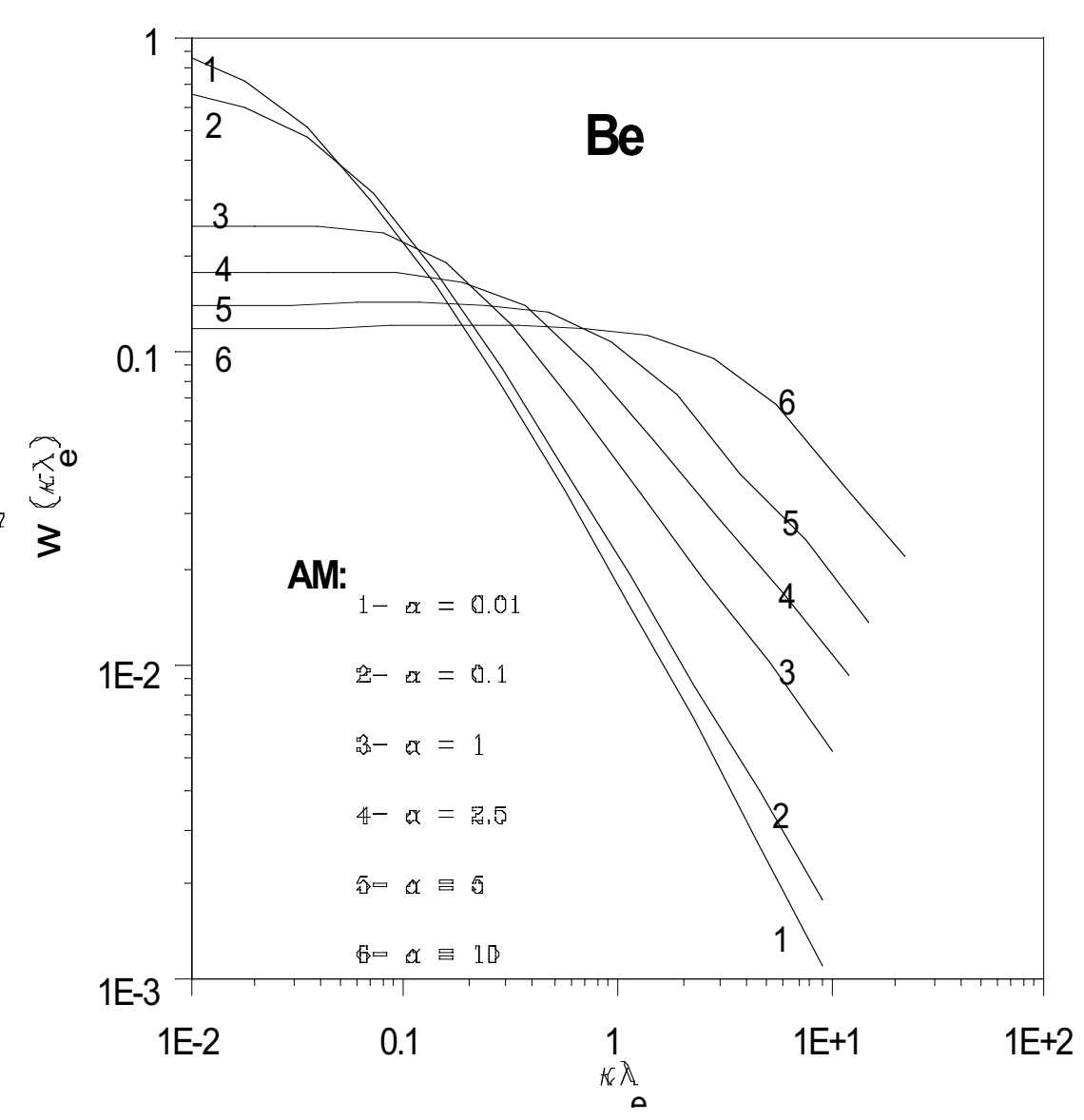

Heath flux propagators for different electron velocity distribution functions, i.e. different . 
Comparison of FPI and flux limiting for $\mathrm{T}_{\mathrm{e}}$ profiles.

$T_{e}\left(x, t_{0}\right)$ temerature profile at the peak of the pulse:

FPI; Flux limiters , in $\mathrm{Al}$ for $T_{e}(x, 0)=0.5 \mathrm{keV}, N_{e}=0.2 \times 10^{21} \mathrm{~cm}^{-3}$.

Laser intensity $=10^{15} \mathrm{~W} / \mathrm{cm}^{2}$, at pulse maximum $\left(t_{0}=200 \mathrm{ps}\right), \mathrm{FWHM}=38 \_\mathrm{m}$.

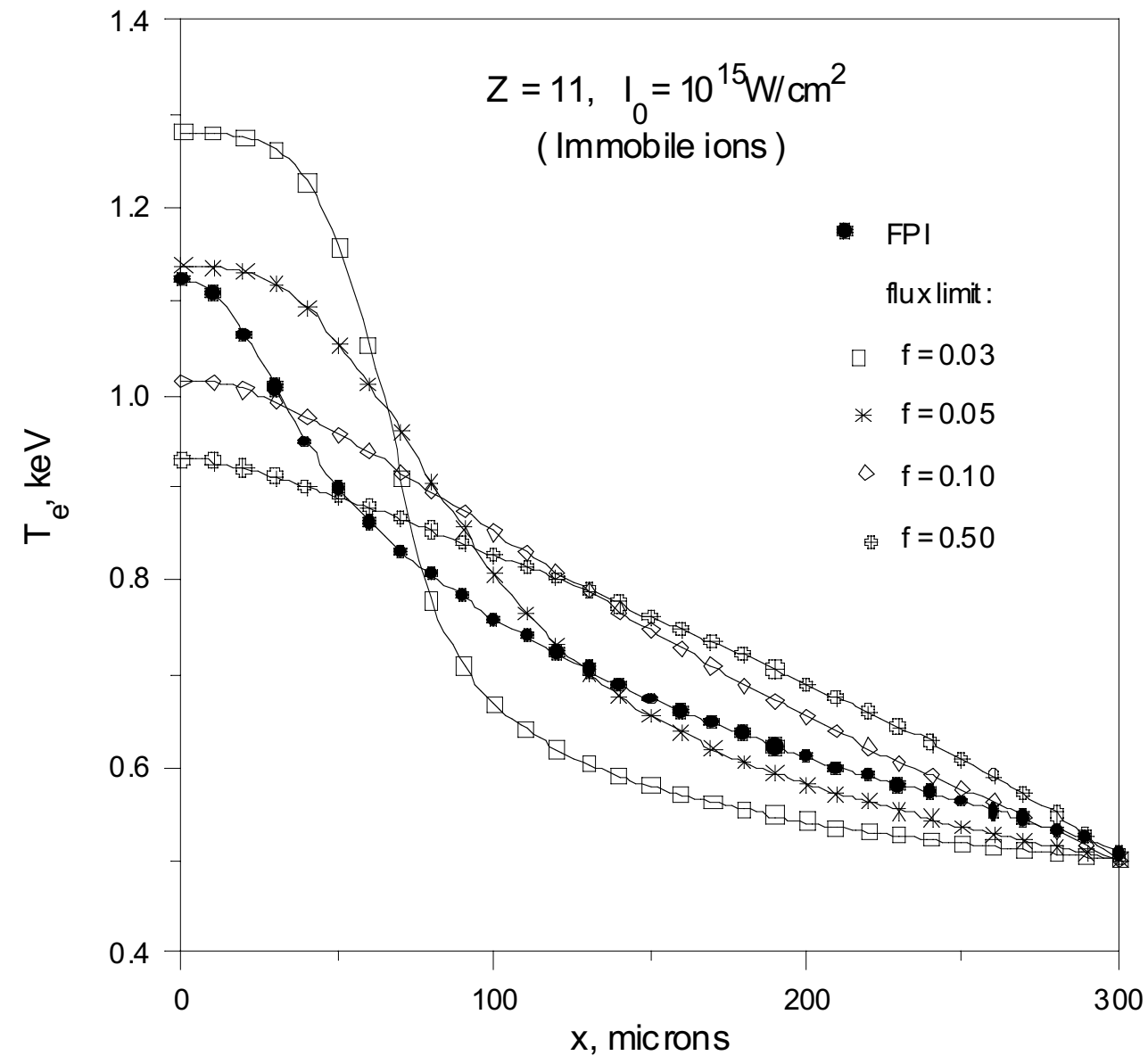


INRS- Énergie, Matériaux et Télécommunications

Temperature and heat flux profiles in plasma irradiated by $10^{15} \mathrm{~W} / \mathrm{cm}^{2}$ laser beam, at the pulse maximum (200ps) and with FWHM equal to 38 _m, the ions are considered immobile:

Comparison of FPI, the our model and some other nonlocal models.
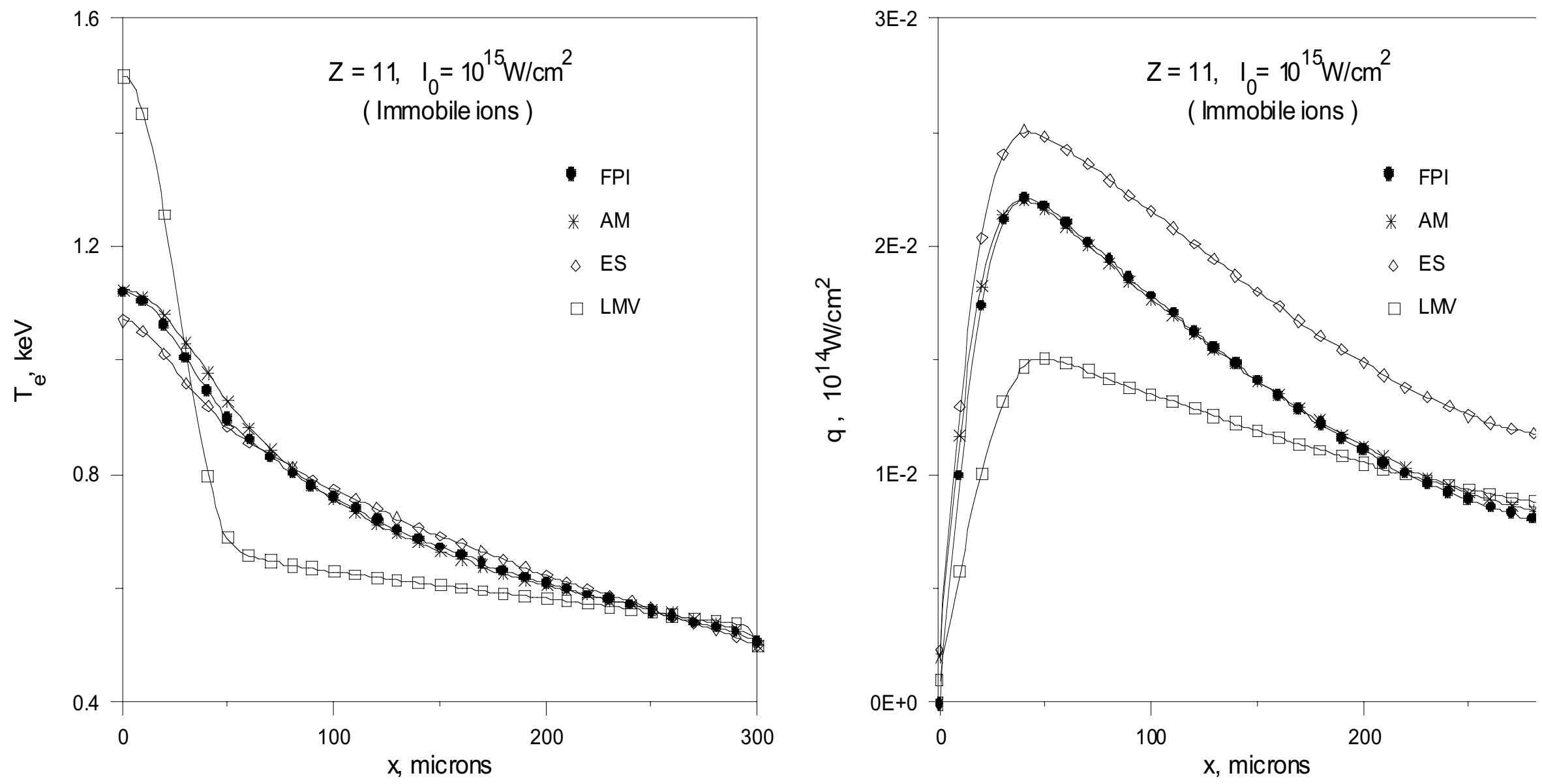


\section{INRS- Énergie, Matériaux et Télécommunications}

Temperature and heat flux profiles in plasma heated by $10^{15} \mathrm{~W} / \mathrm{cm}^{2}$ laser beam, at the pulse maximum (200ps) and with FWHM equal to 38 m, the ions are mobile:

Comparison of FPI, the our model and some other nonlocal models.
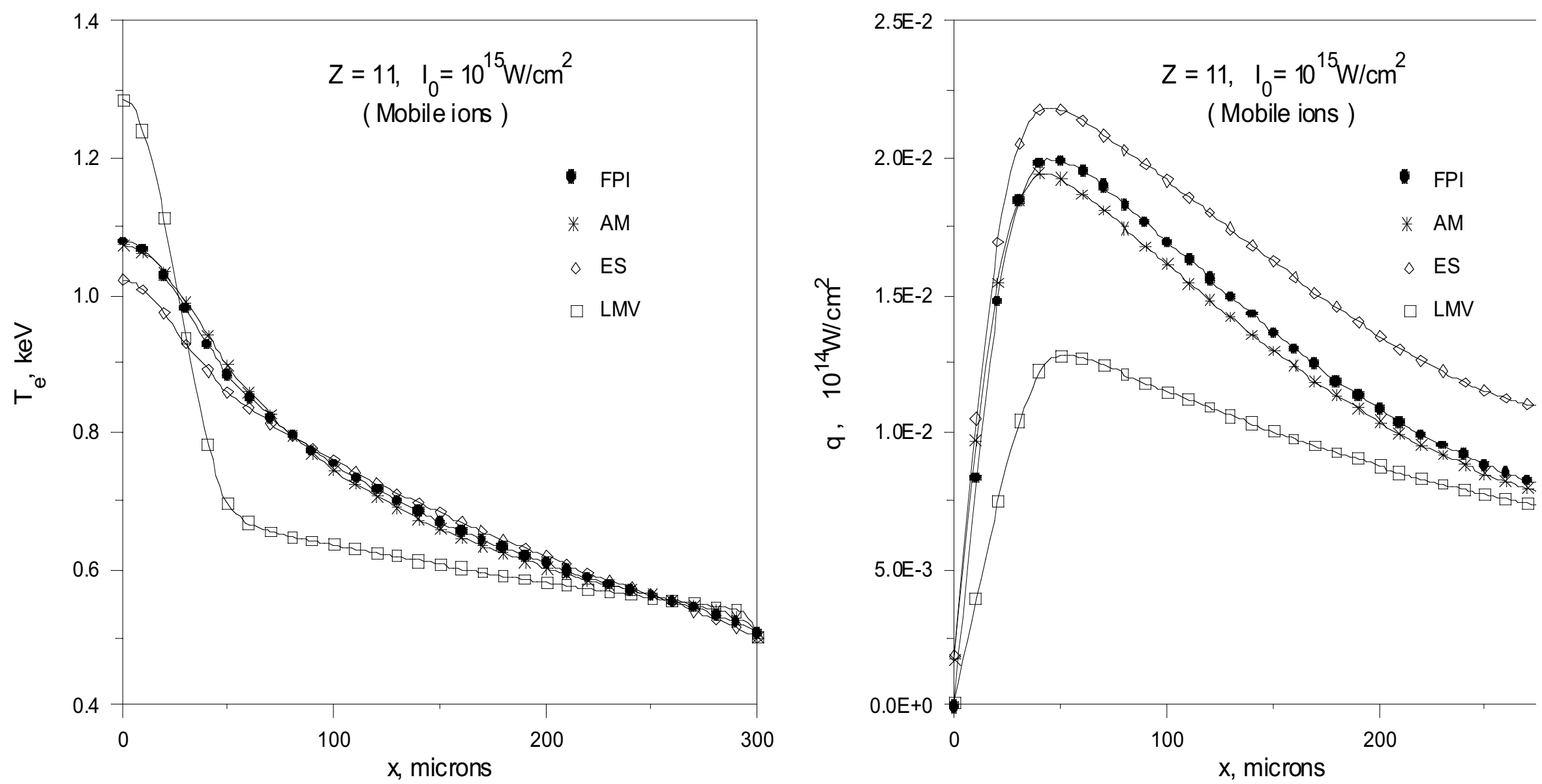


\section{INRS- Énergie, Matériaux et Télécommunications}

Temperature and heat flux profiles in plasma irradiated by $10^{15} \mathrm{~W} / \mathrm{cm}^{2}$ laser beam; at the pulse maximum (200ps) and with FWHM equal to 38 _ $\mathrm{m}$, the ions are mobile and the ponderomotive force is taken into acount: Comparison of FPI, the our model and some other models.
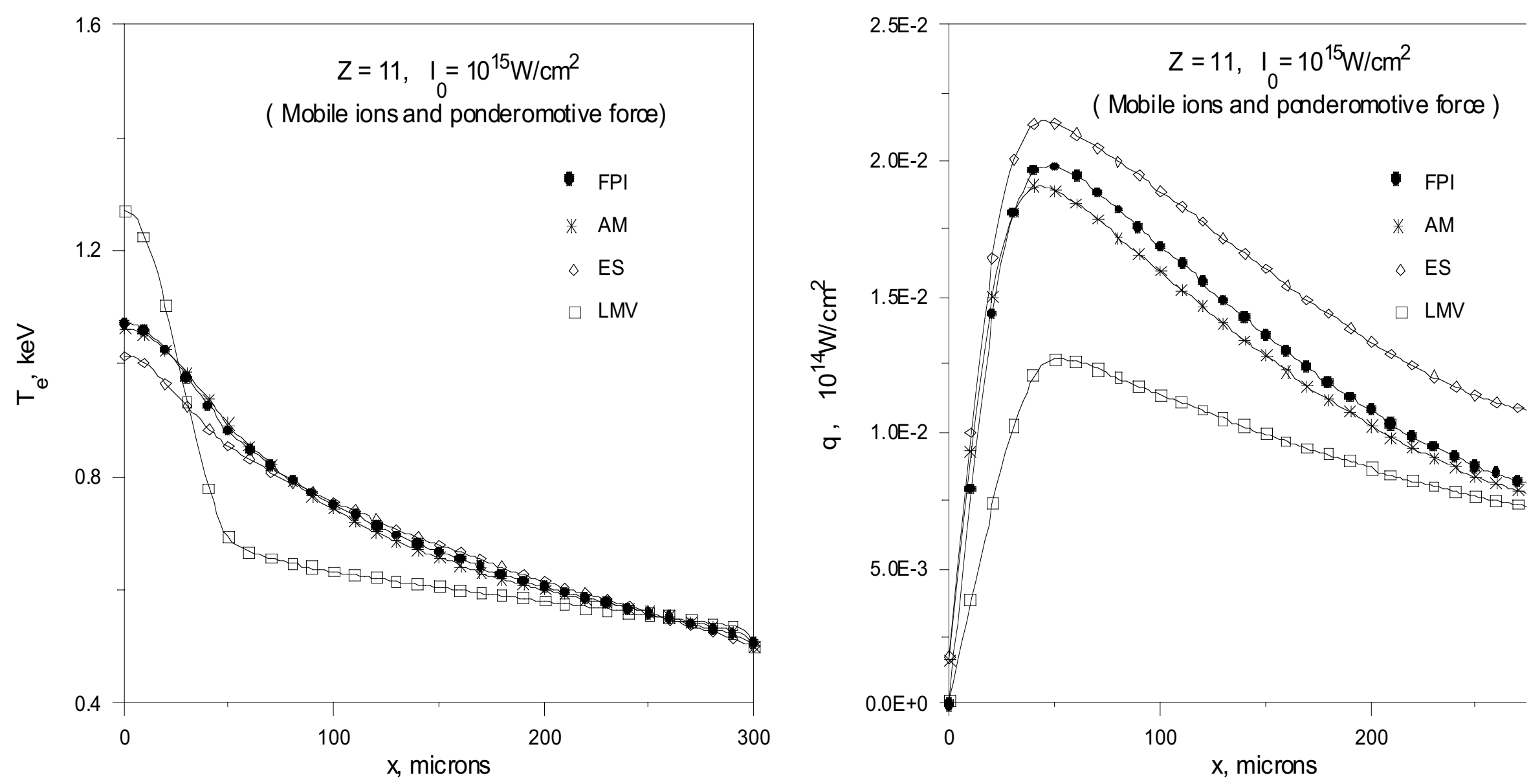


\section{Filamentation growth rate in laser heated plasmas}

Ponderomotive + Thermal

Spatial growth rate $=\quad K=\frac{k_{\perp}}{2 \sqrt{\varepsilon}}\left\{\frac{n}{n_{c}}\left[\gamma_{P}+\gamma_{T}\left(\frac{\kappa_{S H}}{\kappa}\right) \frac{\omega^{2}}{k_{\perp}^{2} c^{2}}\right]-\frac{k_{\perp}^{2} c^{2}}{\omega^{2}}\right\}$

$k_{\perp}$ : perturbation wave number perpendicular to the beam

_ : the laser frequency, _ $=1-n / n_{\mathrm{c}}$,

Ponderomotive filamatation: $\gamma_{P}=\frac{\text { ponderomotive pressure }}{\text { plasma thermal pressure }}$

Thermal filamentation: $\gamma_{T}=\frac{\text { inverse bremsstrahlung heating rate }}{\text { thermal conduction rate across }(c / \omega)}$

Compare effects of two nonlocal conductivities:

Epperlein-Short $\left(\_=\right.$ES $)$

Our new model $\quad\left({ }_{-}=\_\right.$AM $)$ 


\section{INRS- Énergie, Matériaux et Télécommunications}

\section{Results}

1- Low intensity (case 1), i.e. when the EVDF is Maxwellian:

Both models agree and agree with experiments (P.E. Young, Phys. Plasmas (1995))

2- High intensity (case 2), i.e. . when the EVDF is non-Maxwellian, due to I.B.

High k: E-S model predicts large enhancement above ponderomotive growth.

Our model: No enhancement, due higher_ at high k. 


\section{INRS- Énergie, Matériaux et Télécommunications}

Case 1: $I_{0}=10^{13} \mathrm{~W} / \mathrm{cm}^{2}, \lambda_{0}=1.06 \mu \mathrm{m}, T_{\mathrm{e}}=0.8 \mathrm{keV}, n / n_{c}=0.1, Z=5.3$.

Case 2: $I_{0}=2.5 \times 10^{15} \mathrm{~W} / \mathrm{cm}^{2}, \lambda_{0}=1.06 \mu \mathrm{m}, T_{\mathrm{e}}=2 \mathrm{keV}, n / n_{c}=0.1, Z=20$. 


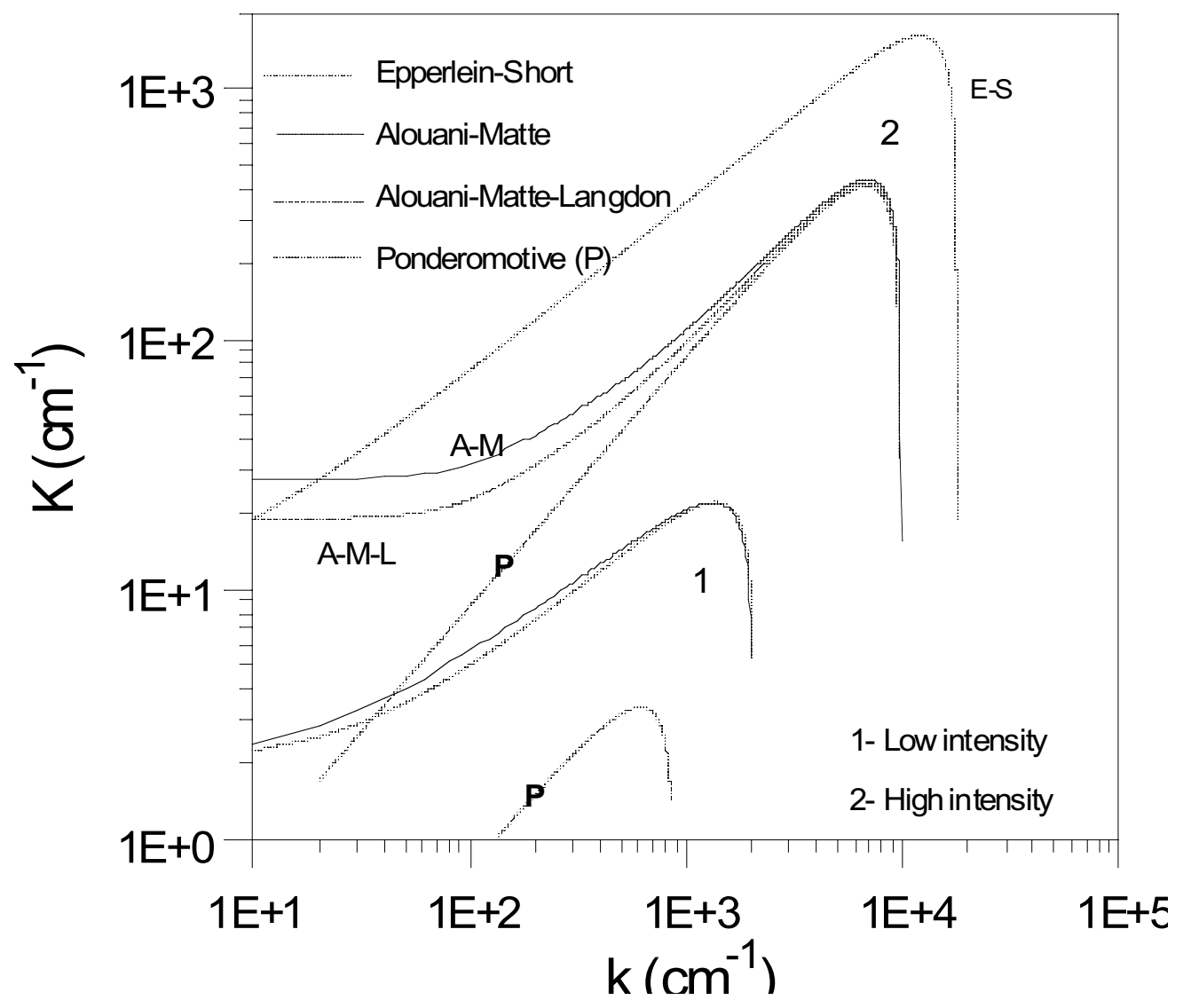

INRS- Énergie, Matériaux et Télécommunications

Distribution functions at maximum laser intensity (in $x$ and $t$ )

$F_{0}$ in He-like $\mathrm{Al}$ for $T_{e}(x, 0)=0.5 \mathrm{keV}, N_{e}=0.2 \cdot 10^{21} \mathrm{~cm}^{-3}\left(N_{c} / 20\right), \mathrm{FWHM}=200 \mathrm{ps}$

A- $\mathrm{I}_{0}=10^{15} \mathrm{~W} / \mathrm{cm}^{2}, \mathrm{FWHM}=38 \_\mathrm{m} ; \quad \mathbf{B}-8 \cdot 10^{15} \mathrm{~W} / \mathrm{cm}^{2}, 4.75 \_\mathrm{m} ;$ 


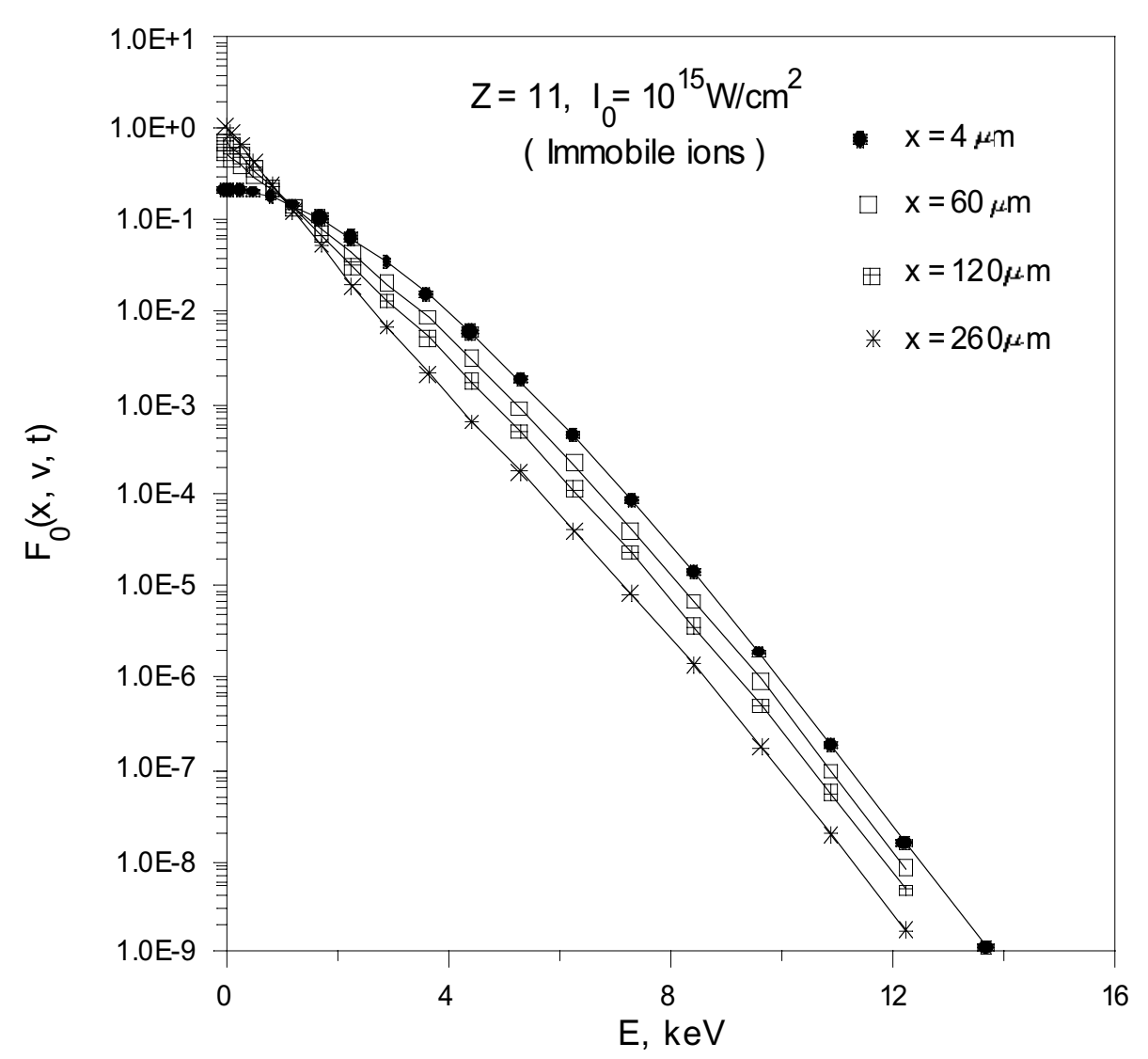

A

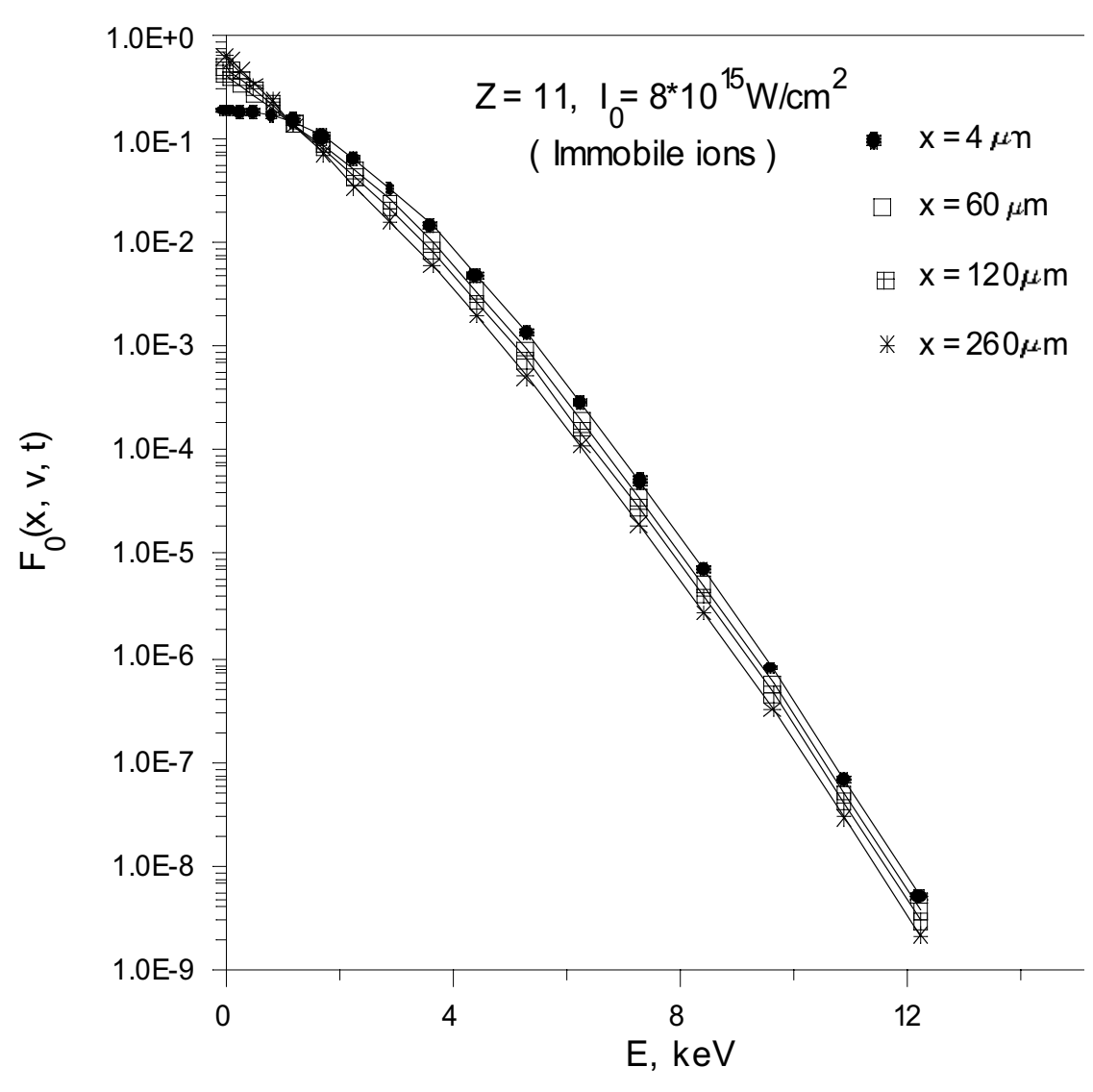

B

INRS-Énergie, Matériaux et télécommunications

$f_{0}(\mathrm{x}, \mathrm{v}, \mathrm{t})$ :

Convolution of local

Estimating the EVDF deformation

Maxwellians.

$f_{0}(x, v)=\int_{-\infty}^{+\infty} g\left(\xi\left(x, x^{\prime}\right), \alpha\left(x^{\prime}\right), v\right) f_{M}\left(x^{\prime}, v\right) d x$

Method analogous to that for heat flux :

Kernel $g(,, v)$ depends on $\_$and $v$ 
Obtained from FPI perturbation runs

$$
\tilde{g}(k, \alpha, v)=\tilde{f}_{0}(k, \alpha, v) / \widetilde{f}_{M}(k, v)
$$

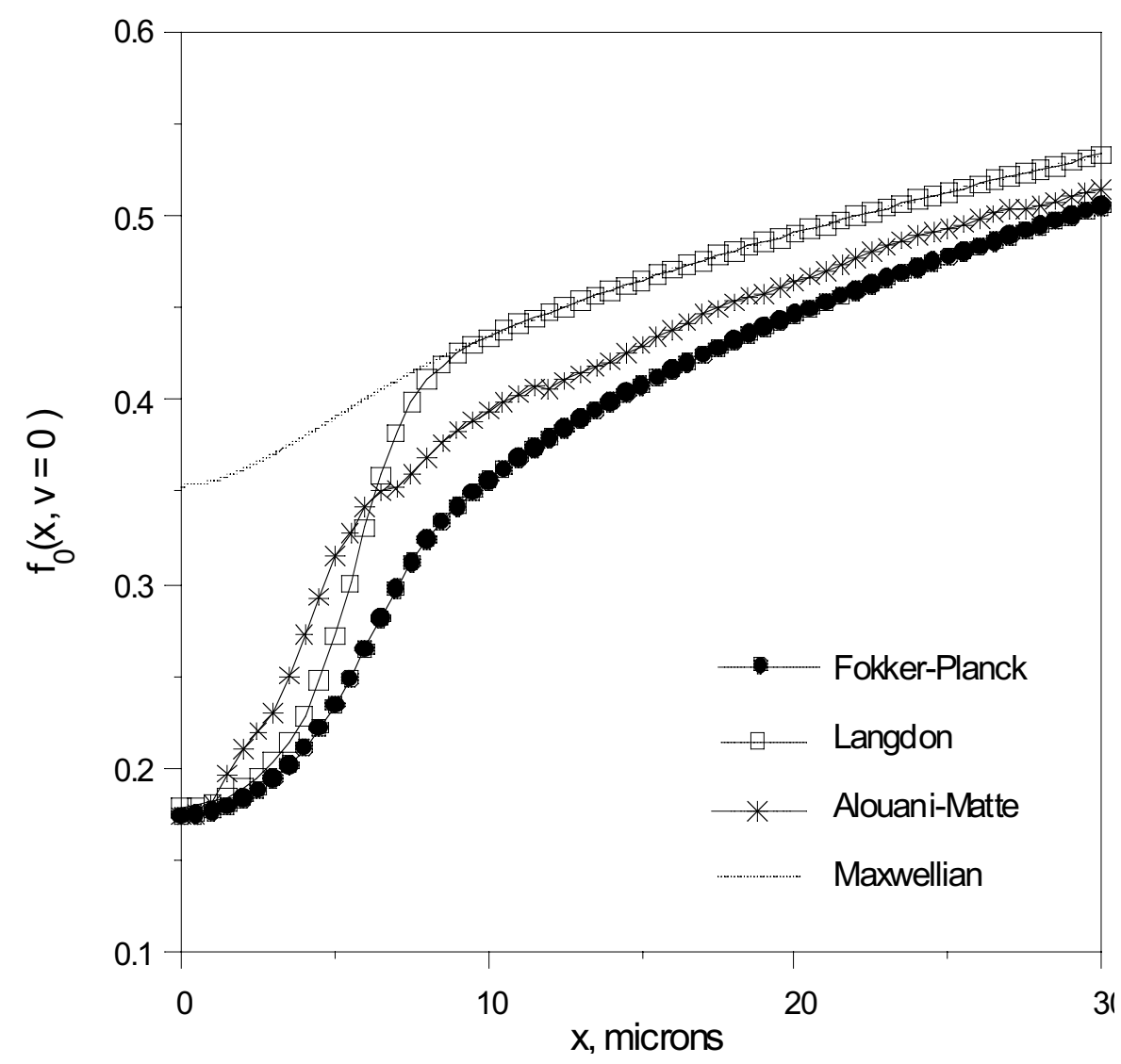

$F_{0}(x=0, v=0)$, in $\mathrm{Al}$ for $T_{e}(x, 0)=0.5 \mathrm{keV}, N_{e}=0.2 \times 10^{21} \mathrm{~cm}^{-3}, \mathrm{I}_{0}=10^{15} \mathrm{~W} / \mathrm{cm}^{2}$, at pulse peak, FWHM's: $38 \_\mathrm{m} ; 200$ ps.

Needs further improvement 
Super-Gaussian indices $m$ from fits to $F P I F_{0}\left(x=0, v, t=t_{0}\right)$ (at pulse peak)

(Fits weighted for thermal electrons)

A- $\mathrm{I}_{0}=10^{15} \mathrm{~W} / \mathrm{cm}^{2}, t_{0}=200$ ps, FWHM's: $38 \_\mathrm{m} ; 200$ ps. B- $8 \times 10^{15} \mathrm{~W} / \mathrm{cm}^{2} ; 4.75 \_\mathrm{m}$.

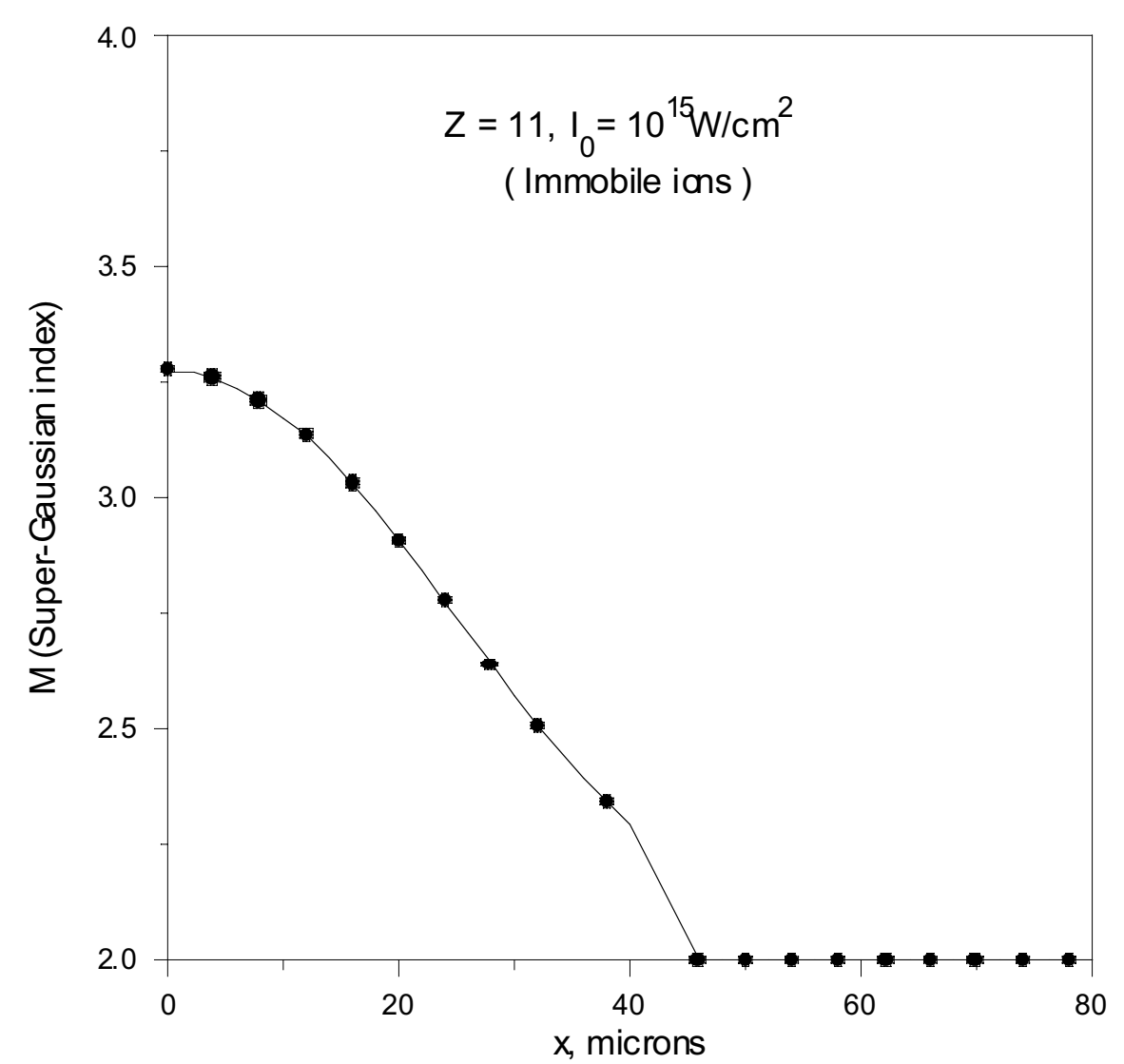

A

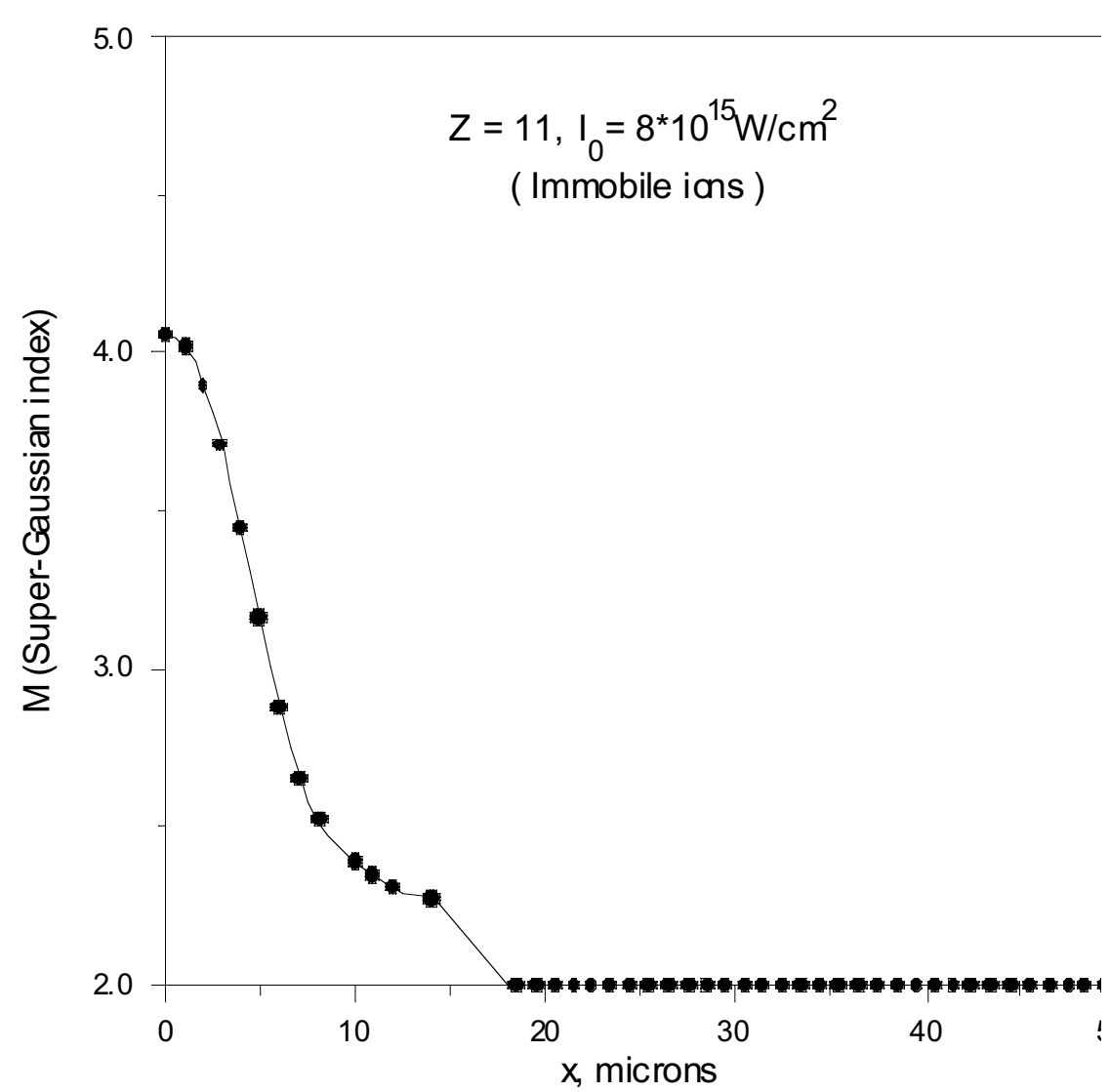

B 
$F_{1} V^{5}$ in Al At pulse peak for $T_{e}(x, 0)=0.5 \mathrm{keV}, N_{e}=0.2 \times 10^{21} \mathrm{~cm}^{-3}, \frac{m_{e}}{6} \int_{0}^{\infty} F_{1}(x, v, t) v^{5} d v=Q_{e}(x, t)$. A- $\mathrm{I}_{0}=10^{15} \mathrm{~W} / \mathrm{cm}^{2}$ FWHM's: 38 m; 200 ps. B- $8 \times 10^{15} \mathrm{~W} / \mathrm{cm}^{2} ; 4.75$ m; 200 ps.

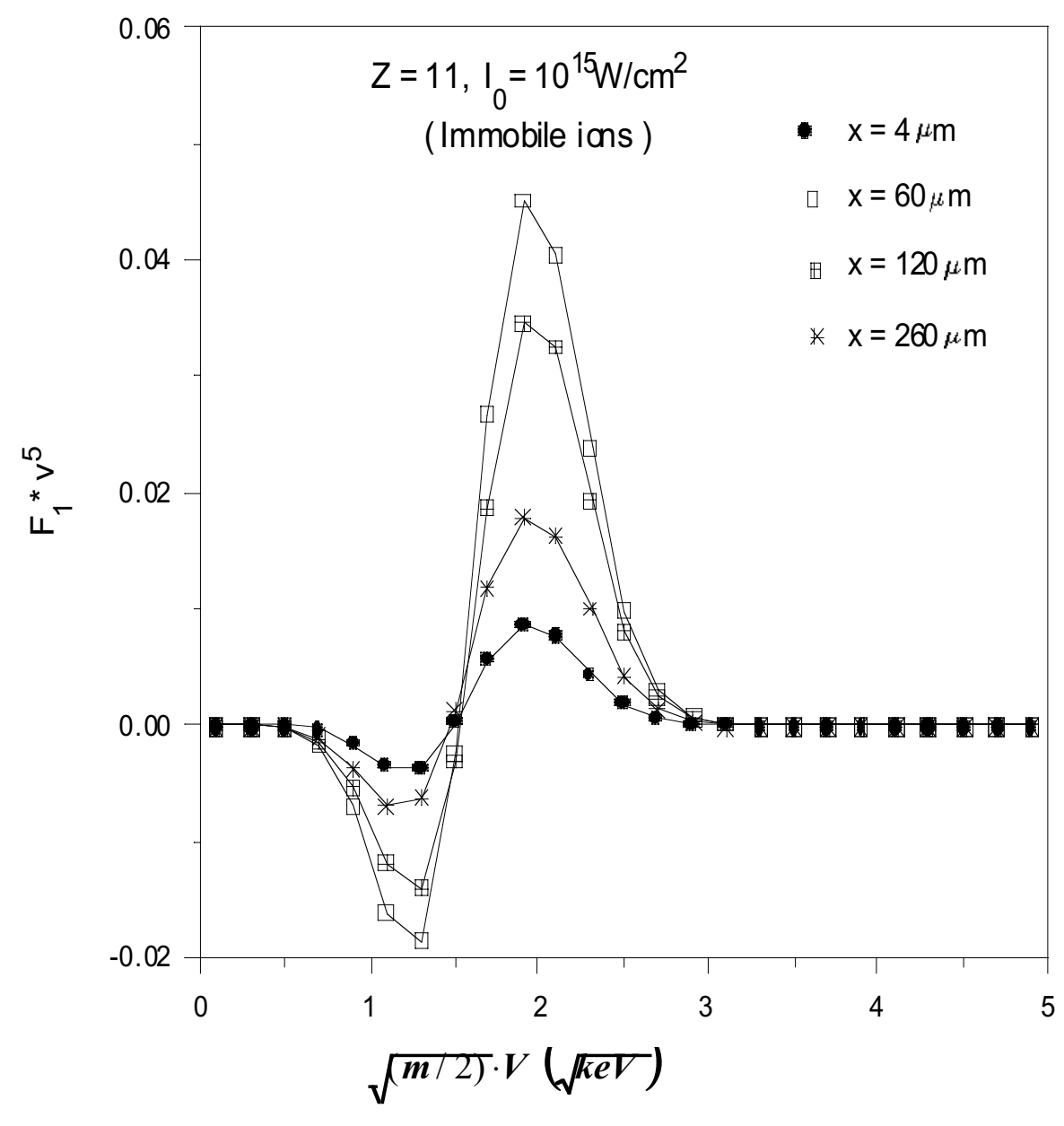

A

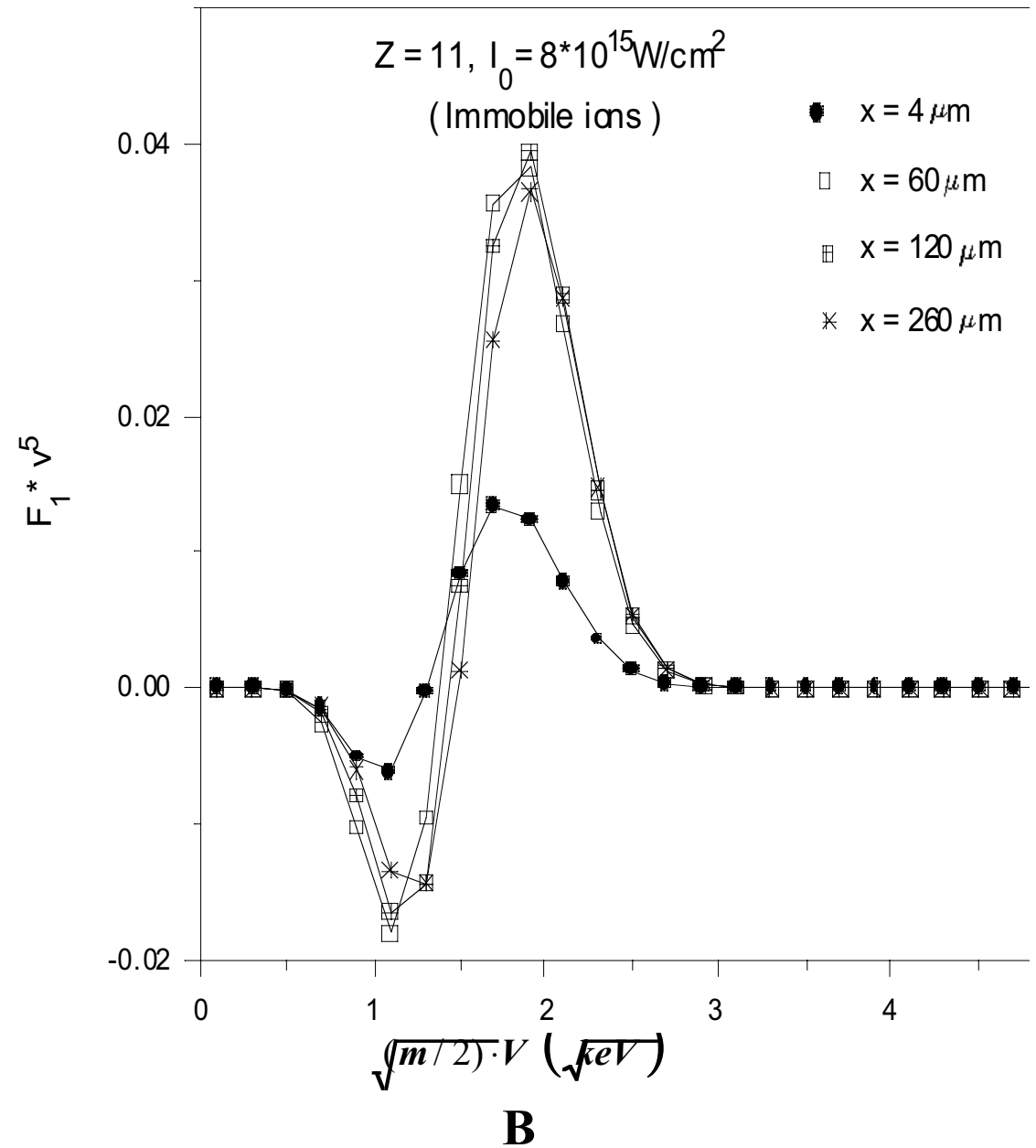




\section{CONCLUSIONS TO PART I}

- Flux limited diffusion temperature profiles do not match FPI's.

- Neither of the earlier delocalization formula reproduces FPI temperature and heat flux profiles well enough.

- $1^{\text {st }}$ Luciani-Mora (LMV) formula inhibits heat flux too much at high $\mathrm{k} \lambda_{\mathrm{e}}$.

- Epperlein-Short (ES) formula does not inhibit it quite enough.

- Thermal filamentation is weaker than calculated by ES.

- Our new nonlocal model shows that in presence of strong collisional heating, i.e. when the electron velocity distribution function super-Gaussian, there are great changes in the filamentation grow rate especially at higher wave vectors $k$, that cannot be predicted by a Maxwellian theory. Another important remark deduced from our model is that the ponderomotive mechanism of filamentation becomes dominant compared to the thermal one. 


\section{INRS- Énergie, Matériaux et Télécommunications}

- The new nonlocal approach reproduces the Fokker-Planck results well in the presence of strong or weak collisional heating in laser created plasma. Our formula for the isotropic component $\left(\mathrm{F}_{0}\right)$ at low velocity of the electron distribution function showed a fair agreement with the Fokker-Planck solution (FPI).

Some improvement still necessary, especially for higher velocities. 
INRS- Énergie, Matériaux et Télécommunications.

II) Simulation of high intensity, long pulse, planar experiments.

Collaboration with D. Braun, J. Edwards and L. Suter of LLNL.

Preliminary comparison of an electron kinetic (FPI) simulation to LASNEX 


\section{INRS- Énergie, Matériaux et Télécommunications.}

\section{Physical situation:}

Initially: Flat, solid carbon target.

Fully stripped (no atomic physics).

$\mathrm{N}_{\mathrm{e} 0}=6.8 \times 10^{23} \mathrm{~cm}^{-3} ; \mathrm{T}_{\mathrm{e} 0}=100 \mathrm{eV}$.

Normal incidence.

WKB approximation for absorption.

Warm, fluid ions.

Laser beam: ${ }_{0}=0.35 \mu \mathrm{m}, 1 \mathrm{nsec}$ "square" pulse, $10^{16} \mathrm{~W} / \mathrm{cm}^{2}$.

0.0-0.1 nsec: linear rise

0.1-0.9 nsec: constant at $10^{16} \mathrm{~W} / \mathrm{cm}^{2}$

0.1-1.0 nsec: linear decrease .

LASNEX profiles of $\mathrm{N}_{\mathrm{e}}, \mathrm{T}_{\mathrm{e}}, \mathrm{T}_{\mathrm{i}}$, and $\mathrm{V}_{\text {hydro }}$ at $\mathrm{t}=0.1$ nsec read by FPI. Interpolated onto FPI's Eulerian grid $(\mathrm{x}=0.25 \mu \mathrm{m})$.

Run FPI for 0.2 nsec, up to $t=0.3$ nsec.

(Simulation box is increased gradually, on the low density side)

Legendre polynomial expansion carried to order 3.

Compare profiles at $\mathrm{t}=0.3$ nsec. 


\section{OBSERVATIONS}

Much more ablation in FPI run: (approx. $9 \mu \mathrm{m}$, vs $5 \mu \mathrm{m}$ ).

Effect of nonlocal heat flow (preheat).

Similar results were obtained at lower intensity by LLE (APS2001) and ILE (PF B, 1992 ; PRL 2002).

$\mathrm{T}_{\mathrm{e}}$ profile: About the same near critical $(6 \mathrm{keV})$

Lesser drop at very low density.

$\mathrm{T}_{\mathrm{i}}$ is lower in the corona, for FPI. 


\section{INRS- Énergie, Matériaux et Télécommunications.}

Initial state for FPI: 0.1 ns output from LASNEX.

( $+\mathrm{V}_{\text {hydro }}$, not shown)

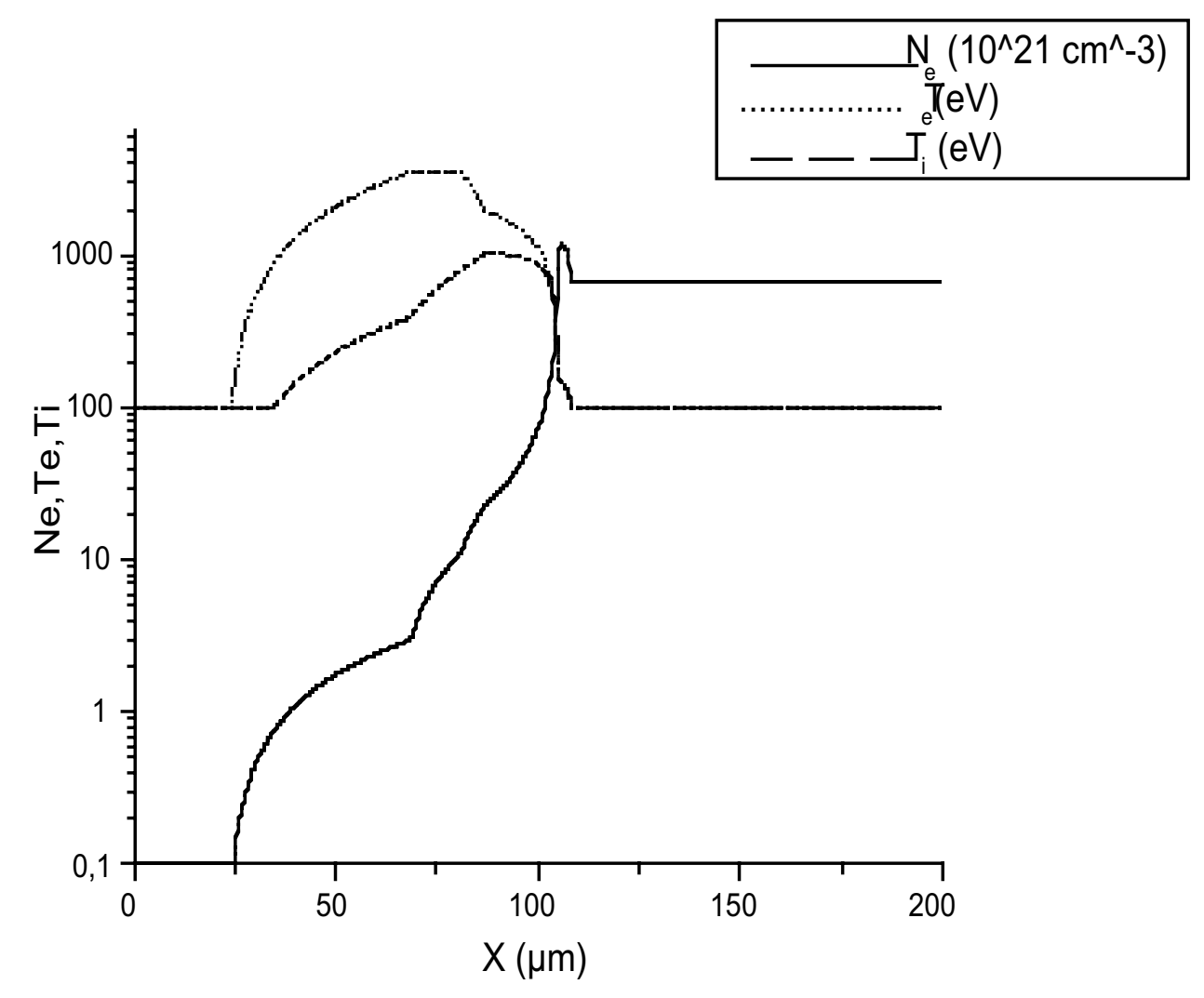




\section{INRS- Énergie, Matériaux et Télécommunications.}

0.3 ns output from LASNEX. $\quad\left(\mathrm{N}_{\mathrm{e}}: 10^{21} \mathrm{~cm}^{-3} ; \mathrm{T}_{\mathrm{e}}, \mathrm{T}_{\mathrm{i}}: \mathrm{eV}\right)$

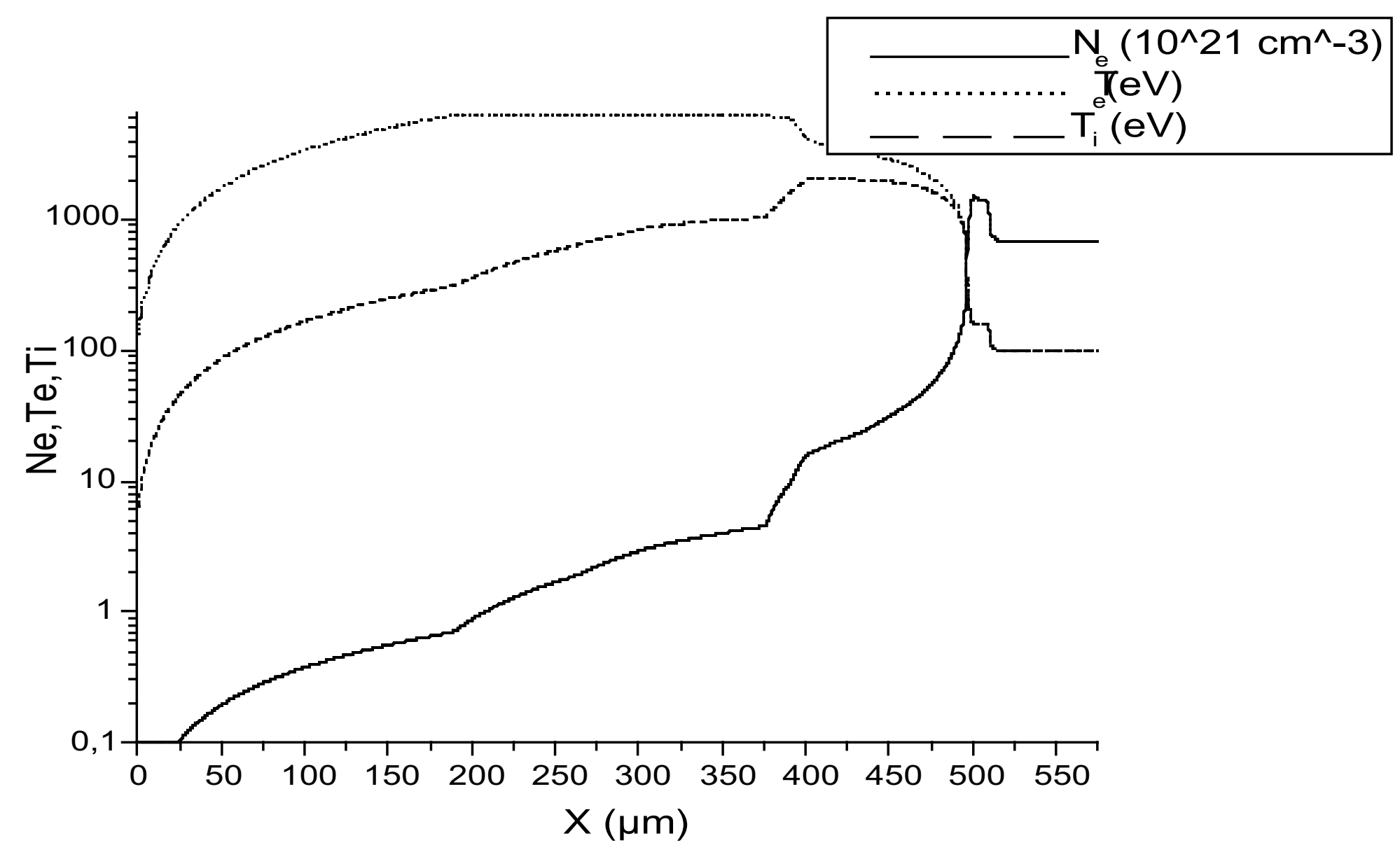




\section{INRS- Énergie, Matériaux et Télécommunications.}

0.3 ns output from FPI. $\quad\left(\mathrm{N}_{\mathrm{e}}: 10^{21} \mathrm{~cm}^{-3} ; \mathrm{T}_{\mathrm{e}}, \mathrm{T}_{\mathrm{i}}: \mathrm{eV}\right)$

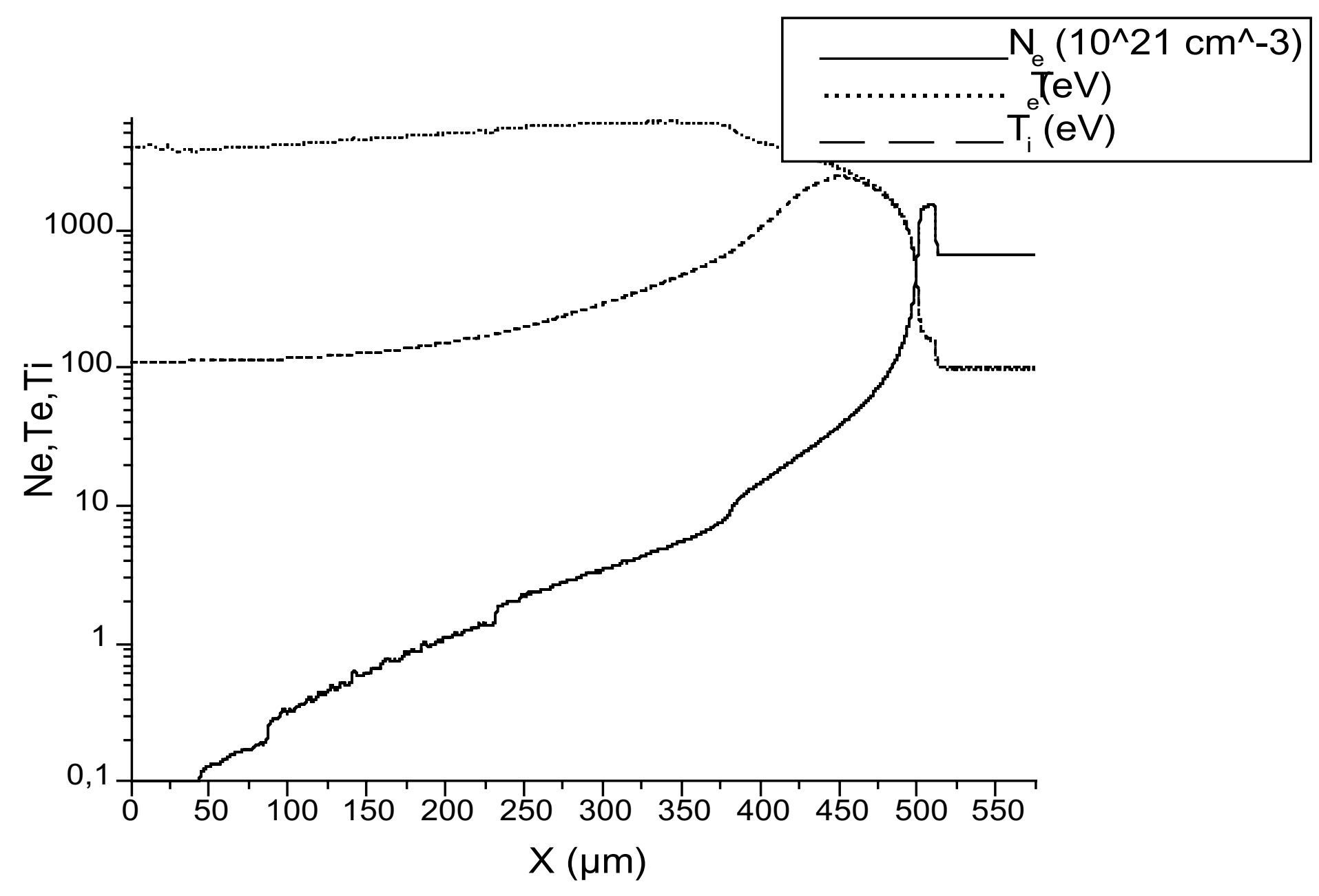




\section{INRS- Énergie, Matériaux et Télécommunications.}

Lagrangian plots: (exclude unperturbed solid) LASNEX, $0.3 \mathrm{nsec}$

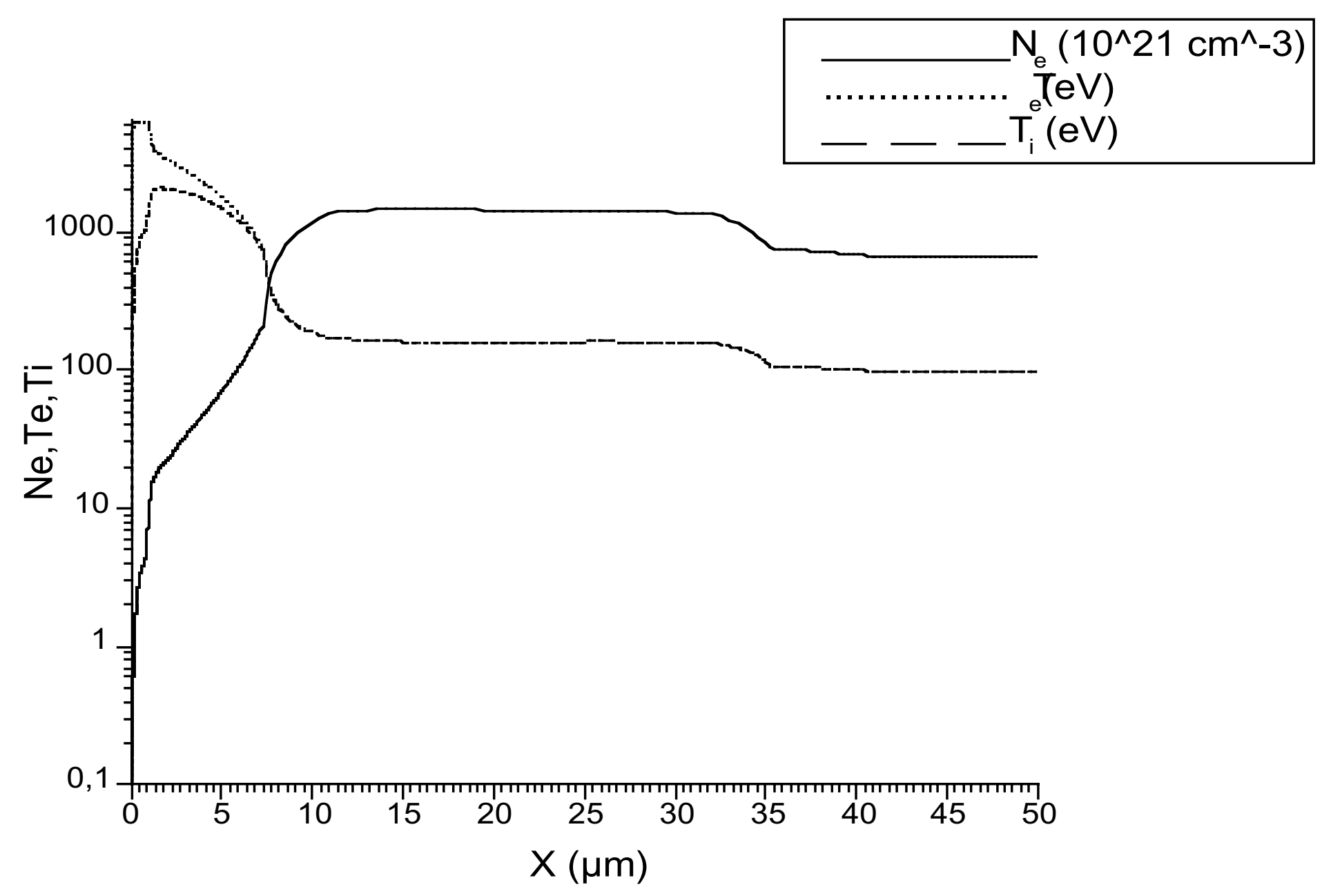




\section{INRS-Énergie, Matériaux et Télécommunications.}

Lagrangian plots: (exclude unperturbed solid) FPI , $0.3 \mathrm{nsec}$

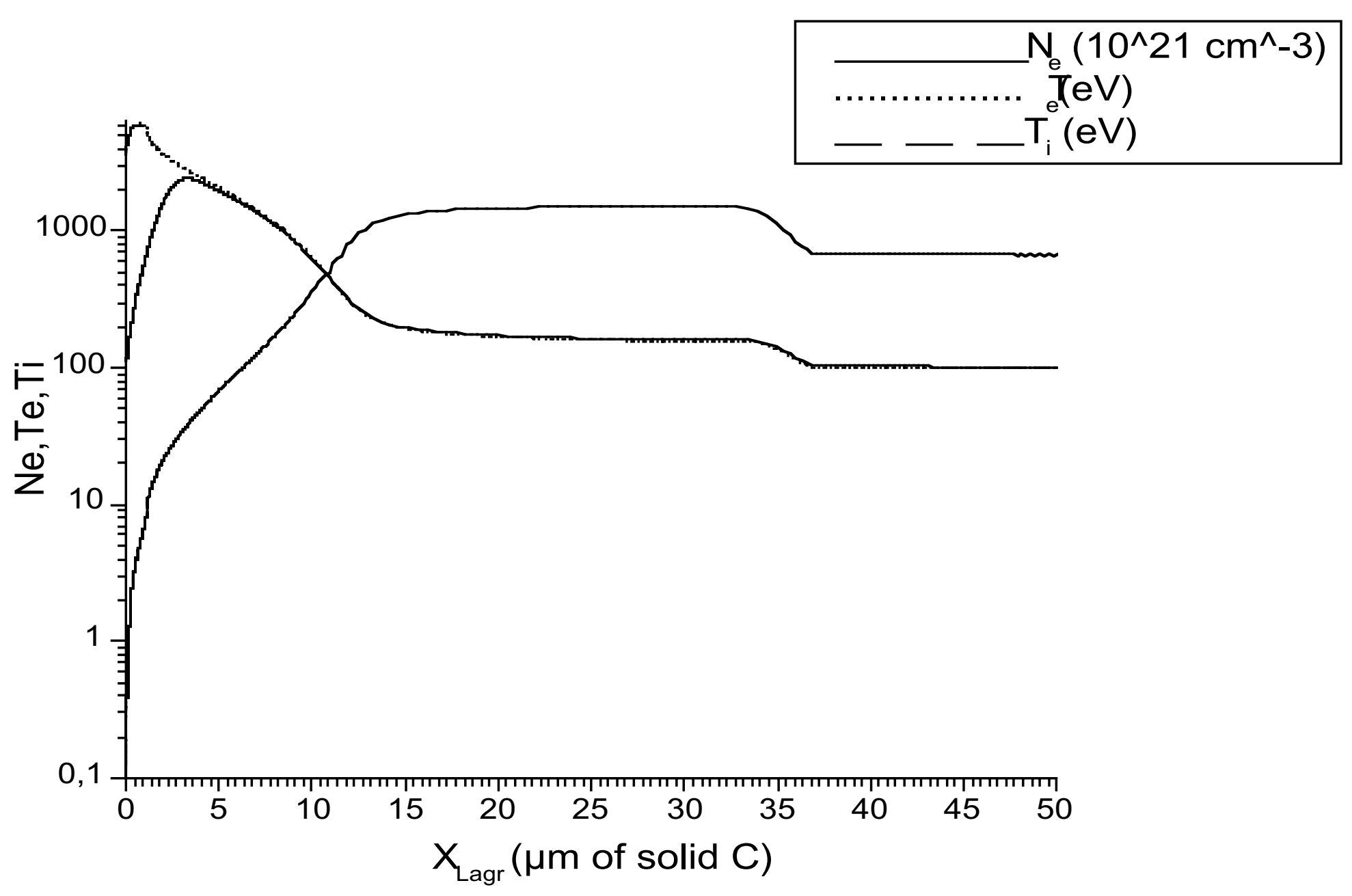




\title{
Distribution function plots $F_{0}(x, v, t)$ vs $\underline{m}_{\underline{e}} \underline{v^{2} / 2}$
}

\author{
$\mathrm{F}_{0}(\mathrm{x}, \mathrm{v}, \mathrm{t})$ : Angle averaged velocity distribution function
}

We will compare $\mathrm{F}_{0}(\mathrm{x}, \mathrm{v}, \mathrm{t})$ to $\mathrm{F}_{\text {Maxw }}(\mathrm{x}, \mathrm{v}, \mathrm{t})$

where $\mathrm{F}_{\text {Maxw }}(\mathrm{x}, \mathrm{v}, \mathrm{t})$ is a Maxwellian of same $\mathrm{N}_{\mathrm{e}}$ and $\mathrm{T}_{\mathrm{e}}=2 / 3<\mathrm{v}^{2}>$ 
INRS- Énergie, Matériaux et Télécommunications.

Cell IX=1379; $\mathrm{N}_{\mathrm{e}}=5.3 \_10^{21} \mathrm{~cm}^{-3}\left(0.59 \mathrm{~N}_{\mathrm{c}}\right) ; \mathrm{T}_{\mathrm{e}}=6141 \mathrm{ev}\left(\right.$ maximum $\mathrm{T}_{\mathrm{e}}$ )

See a SuperGaussian (DLM) distribution up to $50 \mathrm{keV}$.

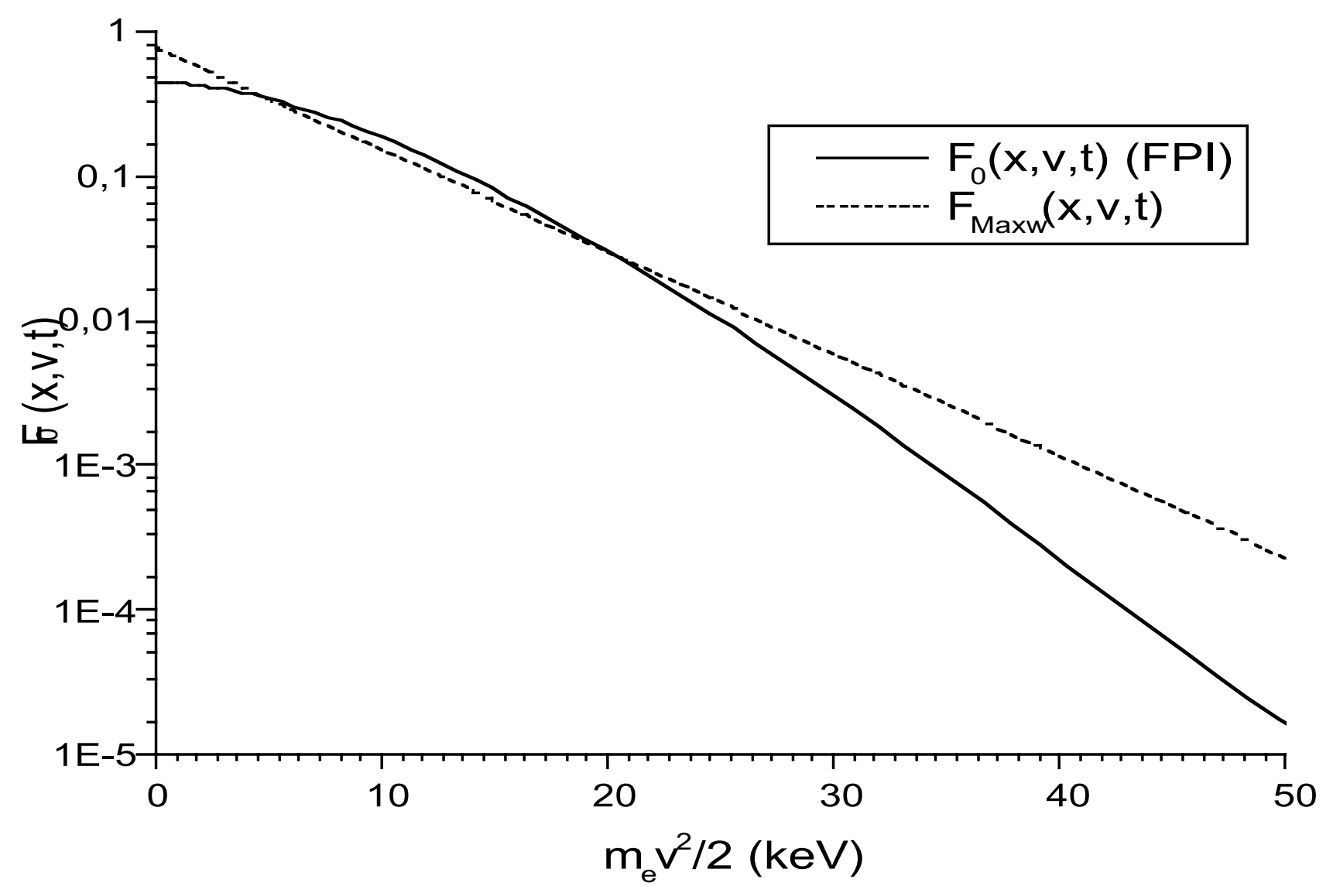




\section{INRS- Énergie, Matériaux et Télécommunications.}

Cell IX=1379; $\mathrm{N}_{\mathrm{e}}=5.3 \_10^{21} \mathrm{~cm}^{-3}\left(0.59 \mathrm{~N}_{\mathrm{c}}\right) ; \mathrm{T}_{\mathrm{e}}=6141 \mathrm{ev}\left(\operatorname{maximum} \mathrm{T}_{\mathrm{e}}\right)$ (cont'd)

Surprise: Beyond $50 \mathrm{keV}$ : Hot tail in the underdense plasma

Reason: _ comparable to $\mathrm{L}_{\mathrm{N}}$, density scale length.

Strong acceleration by ambipolar field, unchecked by collisions.

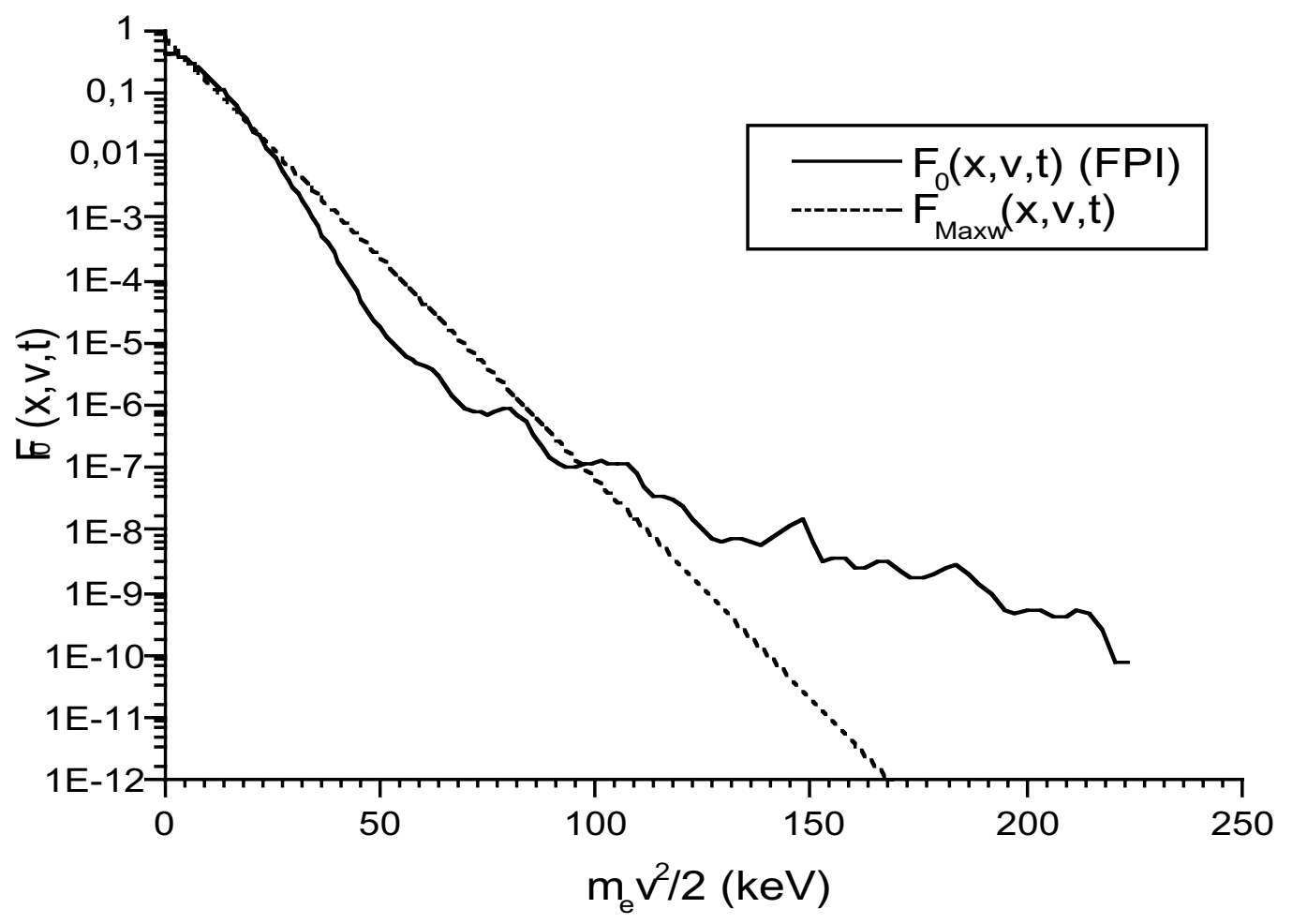


INRS- Énergie, Matériaux et Télécommunications.

Cell IX=1934; $\mathrm{N}_{\mathrm{e}}=101 \_10^{21} \mathrm{~cm}^{-3}\left(11 \mathrm{~N}_{\mathrm{c}}\right) ; \mathrm{T}_{\mathrm{e}}=1678 \mathrm{ev}$

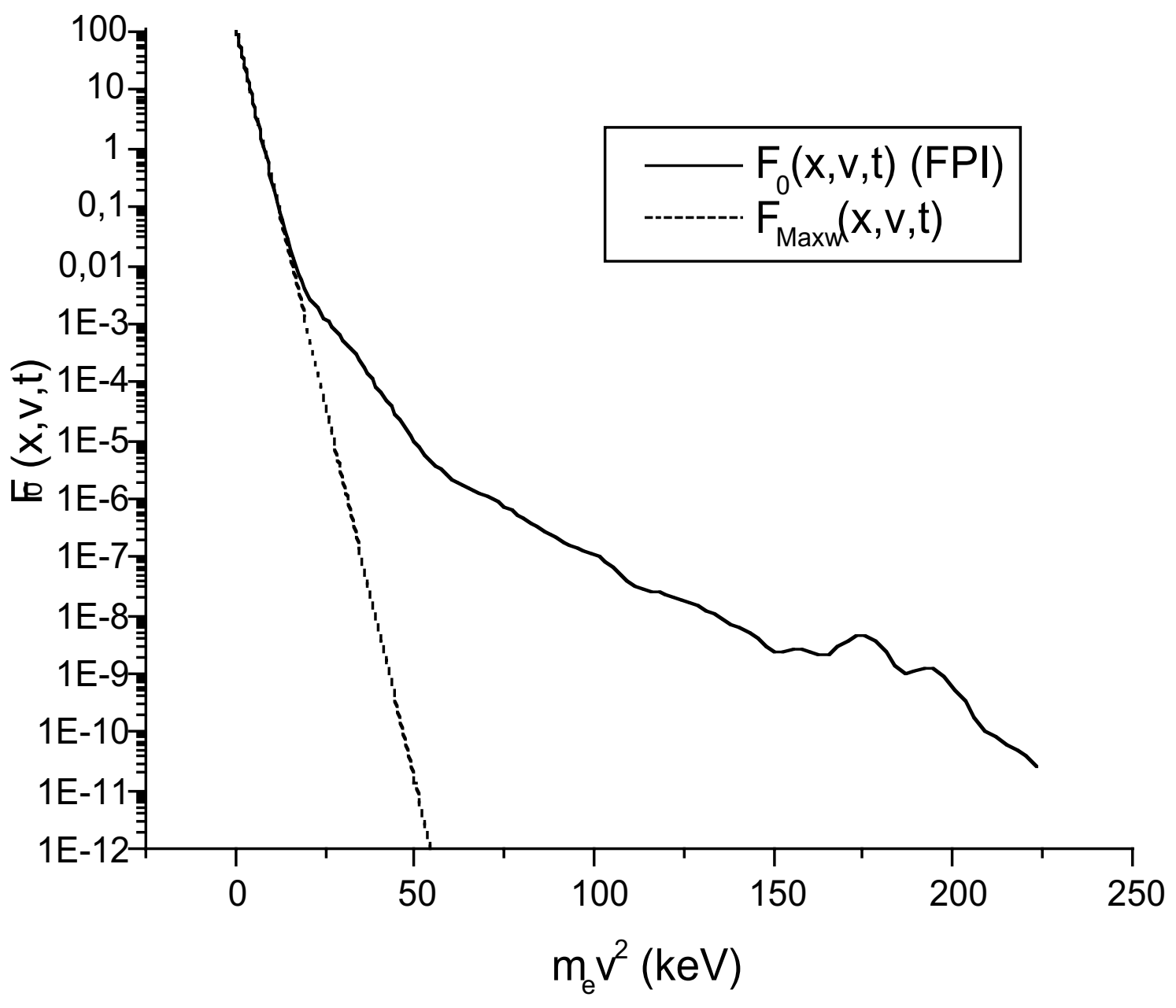


INRS- Énergie, Matériaux et Télécommunications.

Cell IX $=2001 ; \mathrm{N}_{\mathrm{e}}=676 \_10^{21} \mathrm{~cm}^{-3}\left(75 \mathrm{~N}_{\mathrm{c}} \approx \mathrm{N}_{\text {solid }}\right) ; \mathrm{T}_{\mathrm{e}}=362 \mathrm{ev}$ (Ablation surface)

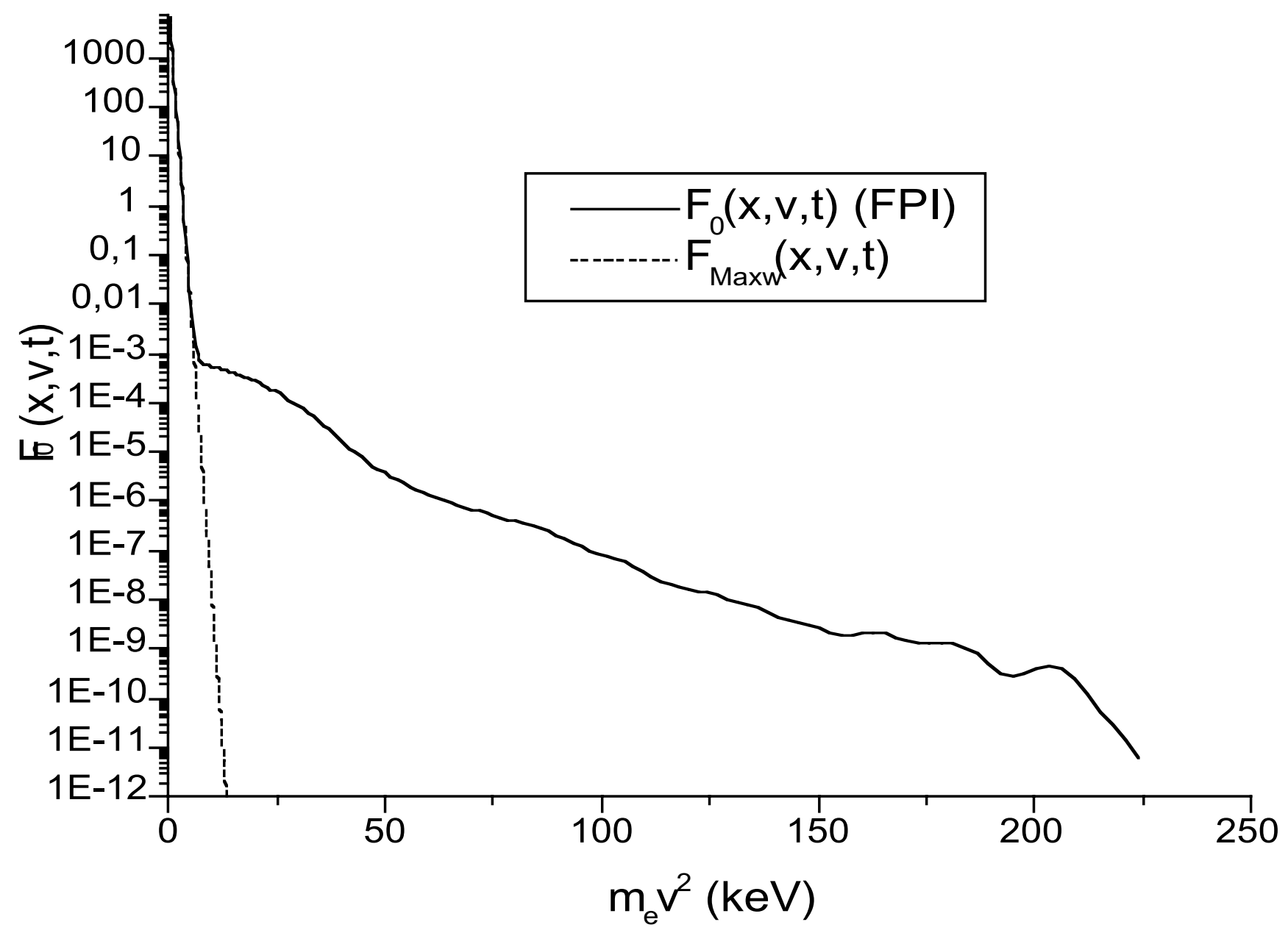


INRS- Énergie, Matériaux et Télécommunications.

Cell IX $=2012 ; \mathrm{N}_{\mathrm{e}}=1400_{-} 10^{21} \mathrm{~cm}^{-3}\left(156 \mathrm{~N}_{\mathrm{c}} \approx 2 \mathrm{~N}_{\text {solid }}\right) ; \mathrm{T}_{\mathrm{e}}=186 \mathrm{ev}$ (compressed)

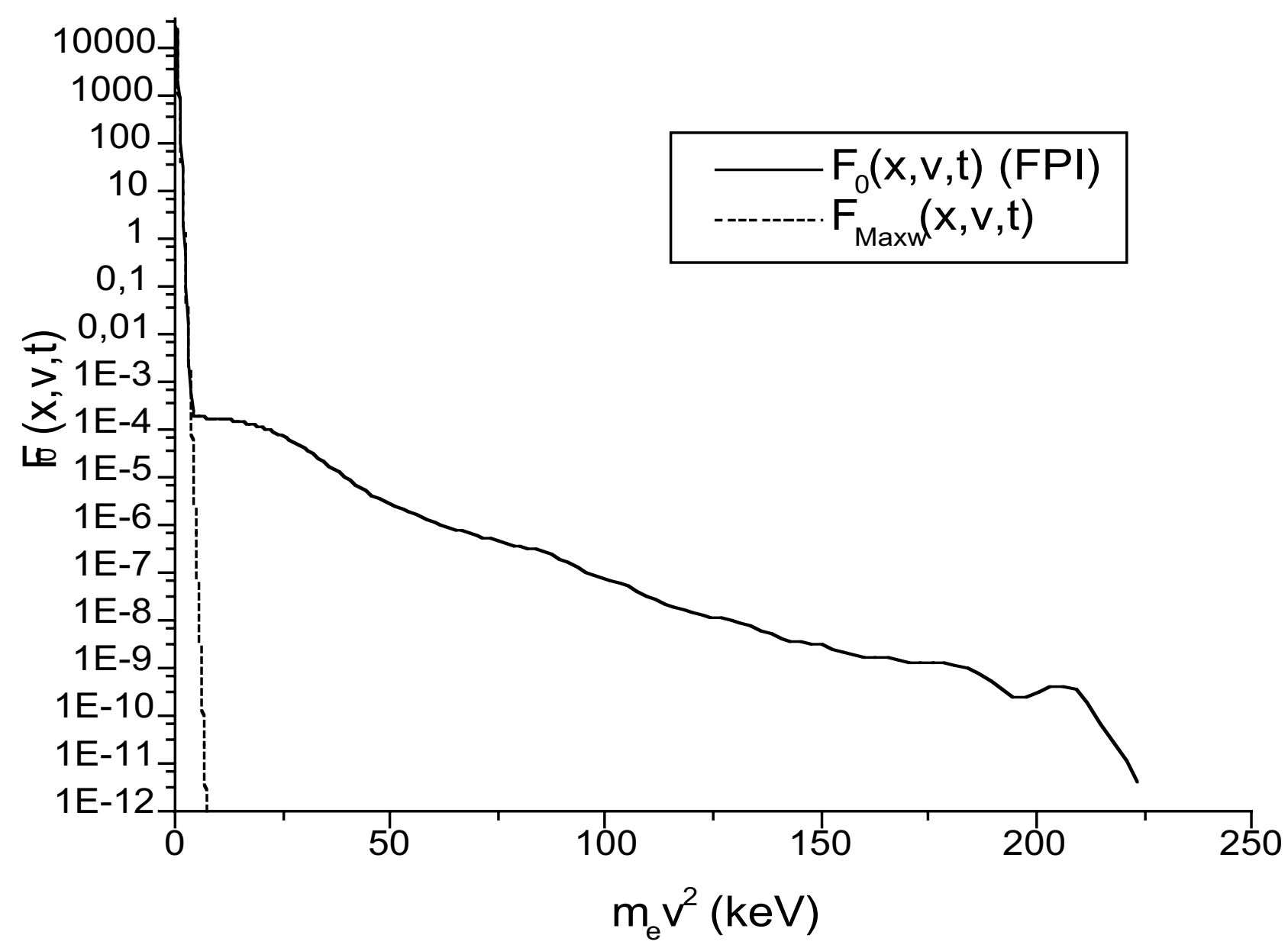




\section{INRS- Énergie, Matériaux et Télécommunications.}

\section{CONCLUSIONS FOR PART 2}

- High intensity ablation has been simulated by both a fluid code (LASNEX) and an electron kinetic code (FPI).

- The maximum electron temperature is about the same for both codes. However, the coronal temperature profile is different.

_ The ion temperature profile in the corona is also different: lower for FPI.

_ Considerably more ablation, due to electron preheat (non local effect).

_ "New" non-Maxwellian effect: acceleration by the ambipolar field in the underdense plasma $\rightarrow$ Hot electron component. 
INRS- Énergie, Matériaux et Télécommunications.

\section{FUTURE WORK}

_ Continue the simulation for longer times ( $1 \mathrm{nsec}$ pulse)

_ Apply our new non-local model to this problem (ongoing). 


\section{Laser expériments : Interpretations and predictions}

Ph. NICOLAÏ, D. BABONNEAU, M. BONNEFILLE, B. CANAUD, F. CHAIGNEAU, E. DATTOLO, C. ESNAULT, J-P. JADAUD, S. LAFFITE, M-C. MONTEIL, G. SCHURTZ, M. VANDENBOOMGAERDE, B. VILLETTE, F. WAGON.
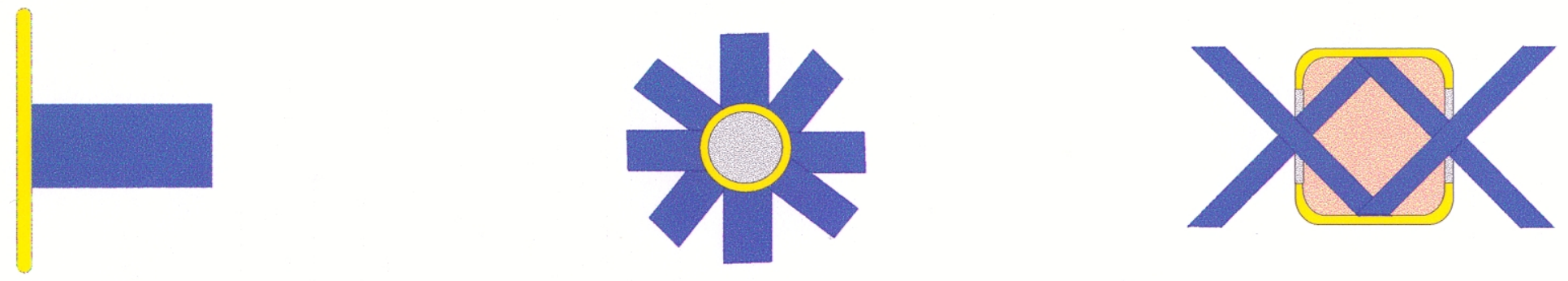


\section{Magnetic fields and nonlocal fluxes}

Effects of each process are less or more large following:

$\square$ experimental geometry [plane-sphérical]

$\square$ observed quantity [Tr, Te, ne, hv ...]

$\square$ plasma zone seen (probed) by diagnostic

The choice of an experiment and diagnostics (can) enables us :

$\square$ to test both effect combined or alone

$\square$ to check our model

$\square$ to improve our understanding of physical processes involved (to improve theory) 


\title{
Planar target (Phebus facility)
}

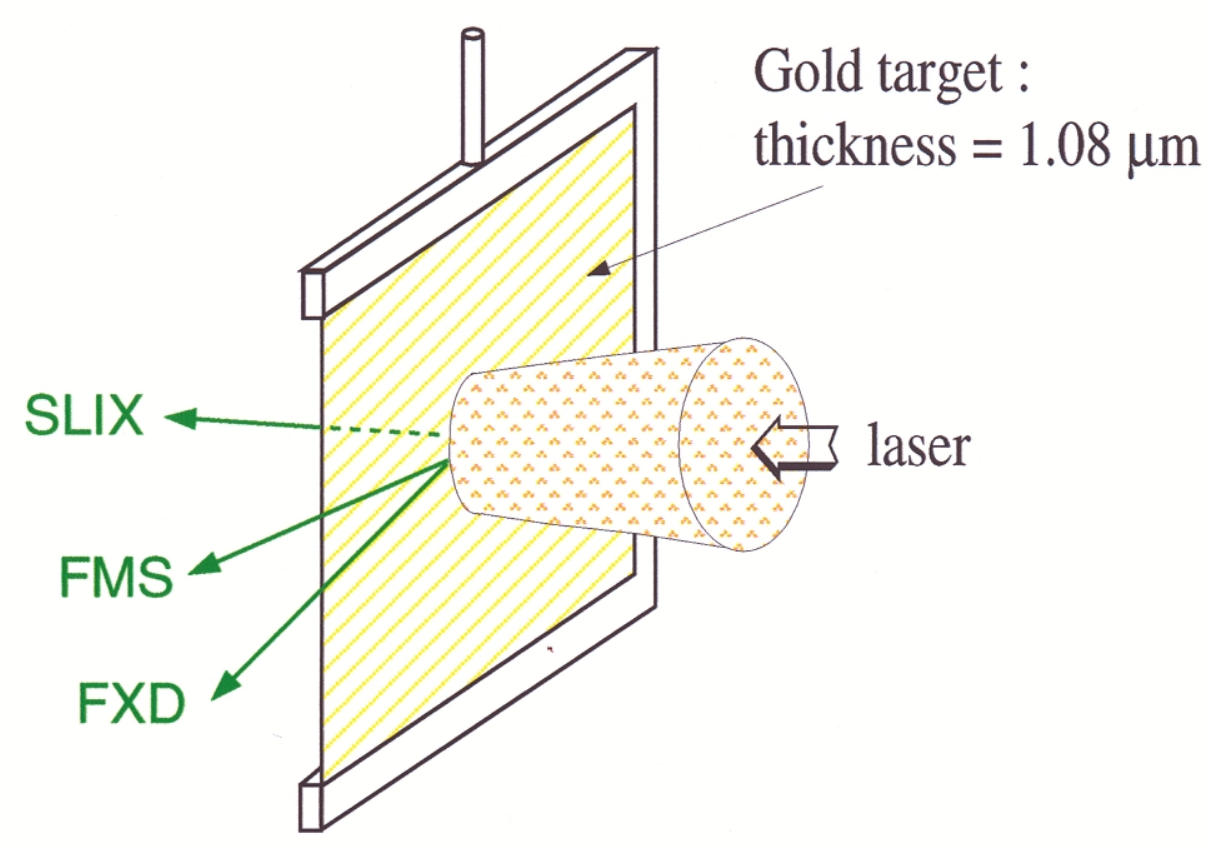

\author{
Laser \\ - smoothed by RPP \\ $-3 \omega$ \\ $-1.5 n s$ square pulse \\ - 3kJ \\ - $\mathrm{FWHM}=340 \mu \mathrm{m}$
}

FMS :streak camera wich images $200 \mathrm{eV}$ X-ray emission perpendicular to laser axis

- provides a time-resolved 1D image

FXD : streak camera wich images above $2 \mathrm{KeV}$ X-ray emission perpendicular to laser axis

- provides a time-resolved 1D image

SLIX : gated microchannel plate detector images $2.5 \mathrm{KeV} \mathrm{X}$-ray (M-shell) from rear side - provides a time-resolved 2D image 


\section{D Simulation of experiment}

target

\section{laser}
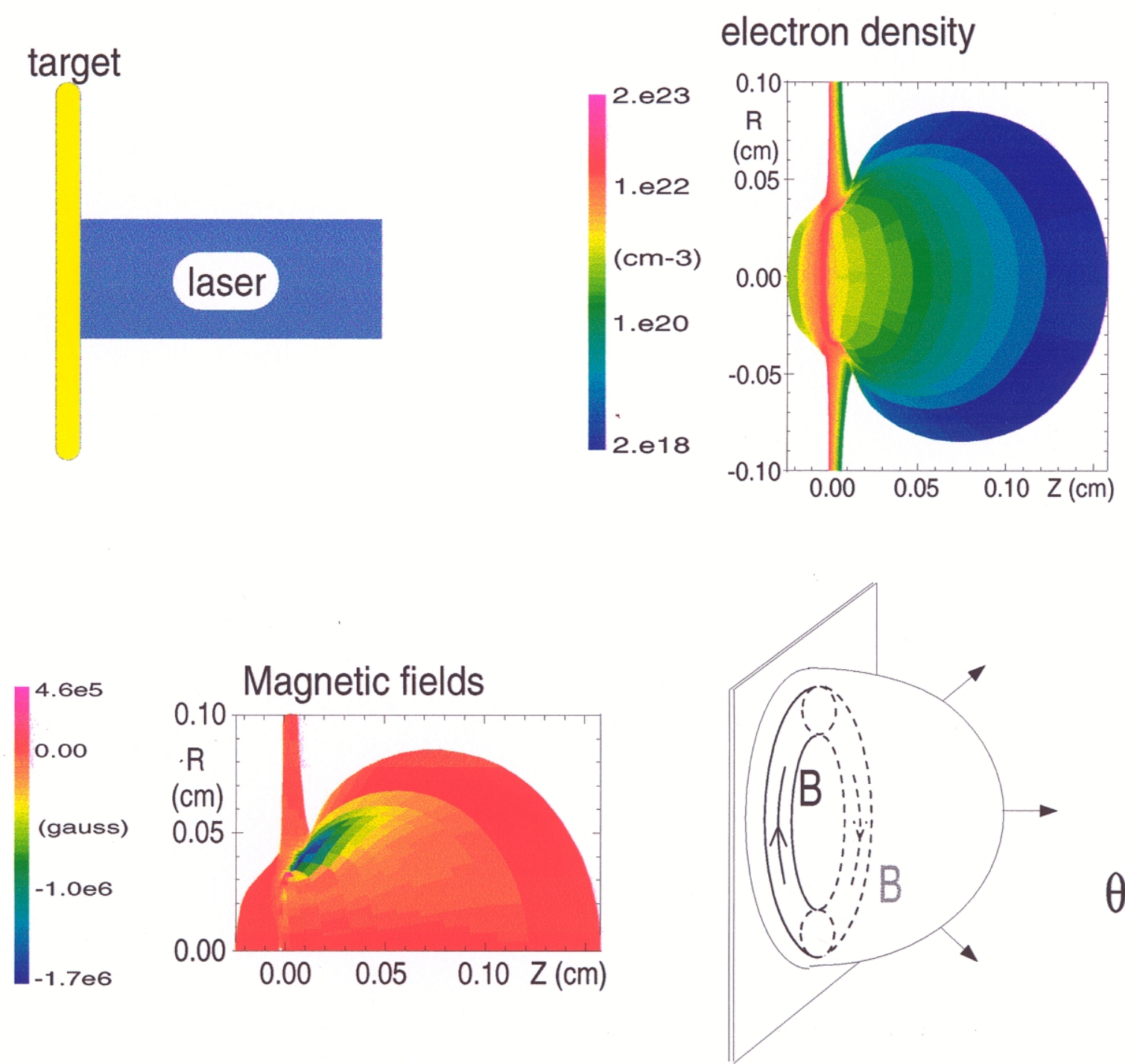

electron température

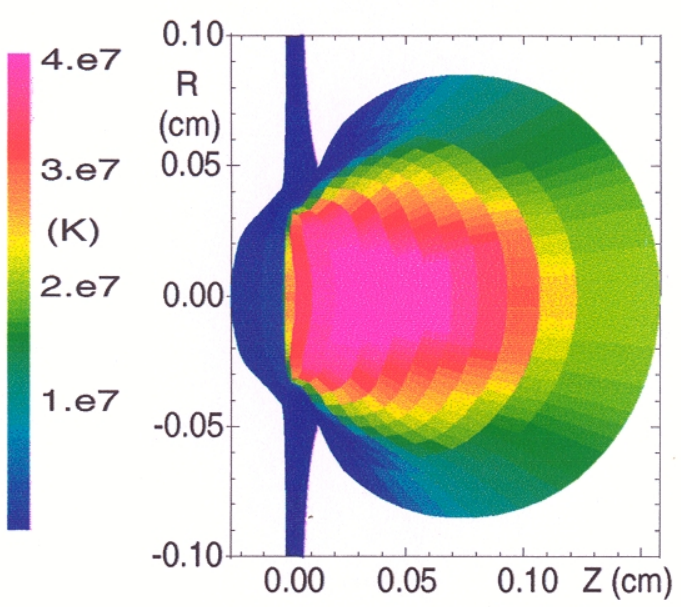

gyrofrequency / collision frequency

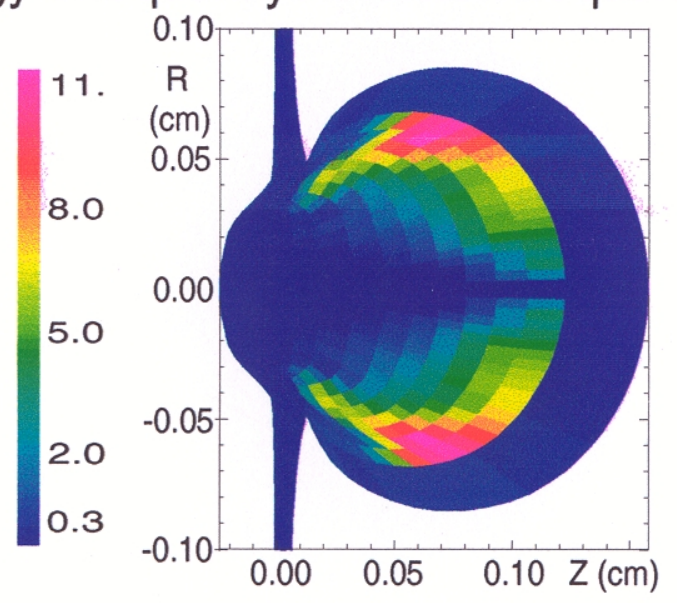




\section{Experiment-simulations comparisons}
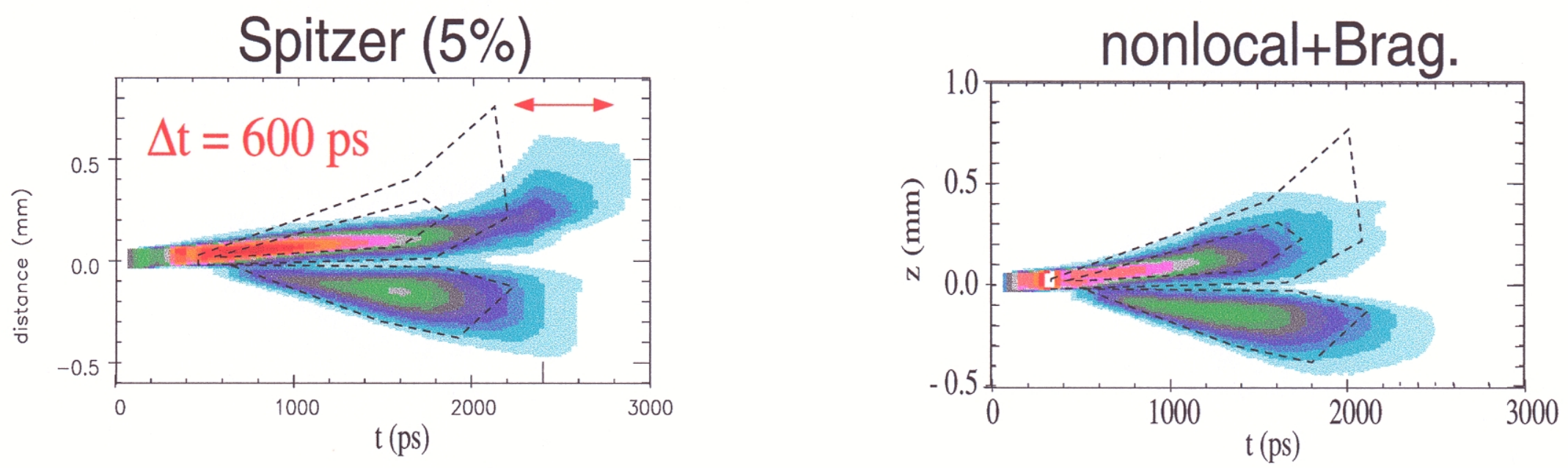

$\checkmark$ Using Spitzer-Harm fluxes, we obtain (front side) an emission too long (600ps).

Second, the slope of the upper part is too small (velocity of the emitting zone).

$\checkmark$ We tried to change limiter value, average between $\mathrm{SH}$ and free streaming, láser parameters, mesh refinement, etc... But no effect on numerical results.

Using B-fields and nonlocal transport, we obtain a emission length shorter and the slope of the upper part is higger and is in good agreement with exp. result . 


\section{Individual effects of each process}
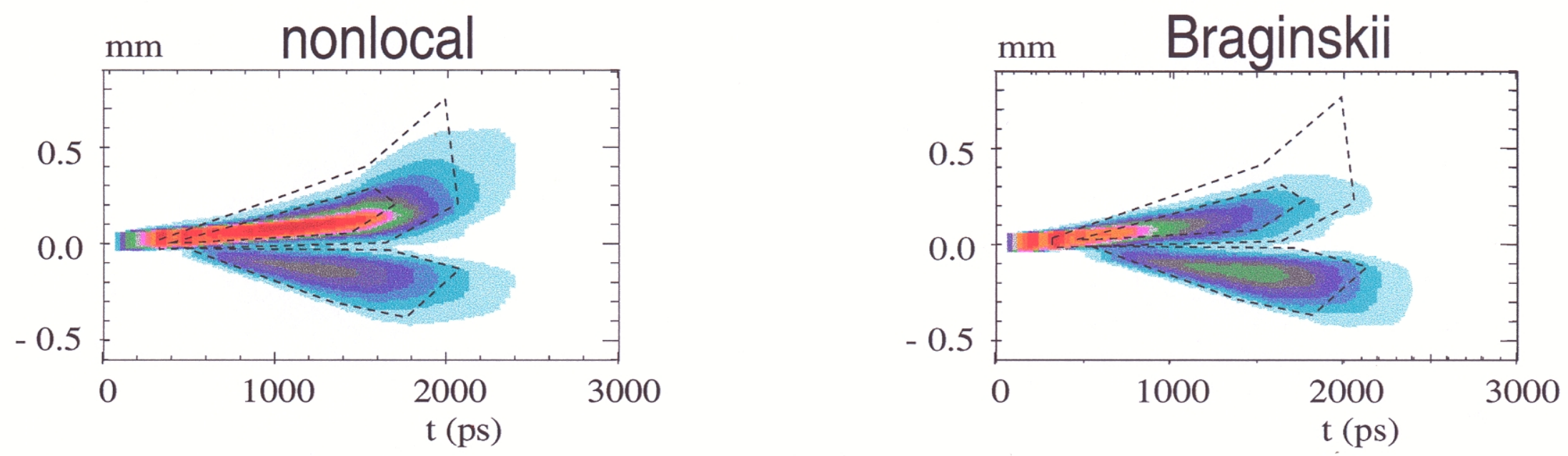

$\checkmark$ Using nonlocal fluxes, we obtain a shortening of emission but not enough to reproduce experiment.The emitting zone shifting (laser side) is correct

$\checkmark$ Using B-fields, we obtain a good length of emision (front side) but the slope of the upper part is too small (slightly)to mach experimental data.

$\checkmark$ So, it seems that combined effects produce the best agreement with experiment 


\section{SLIX Diagnostic}

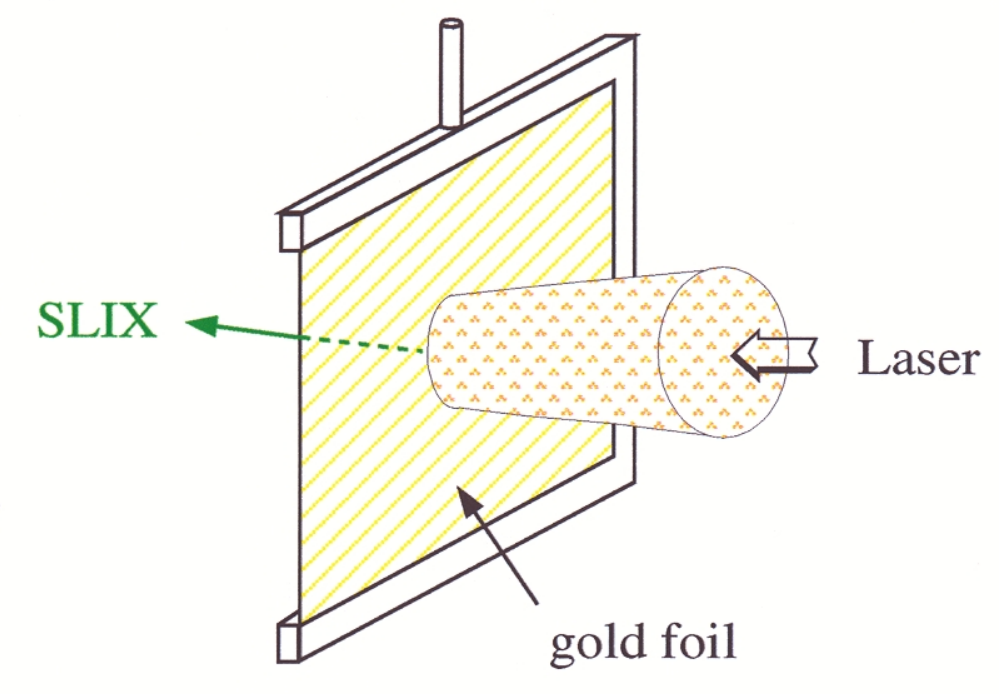

Slix diagnostic takes 'photos' of the rear side in the range about 2.5Kev (M-shell)

Res. $=10 \mu \mathrm{m}$ et $\Delta \mathrm{t}=100 \mathrm{ps}$
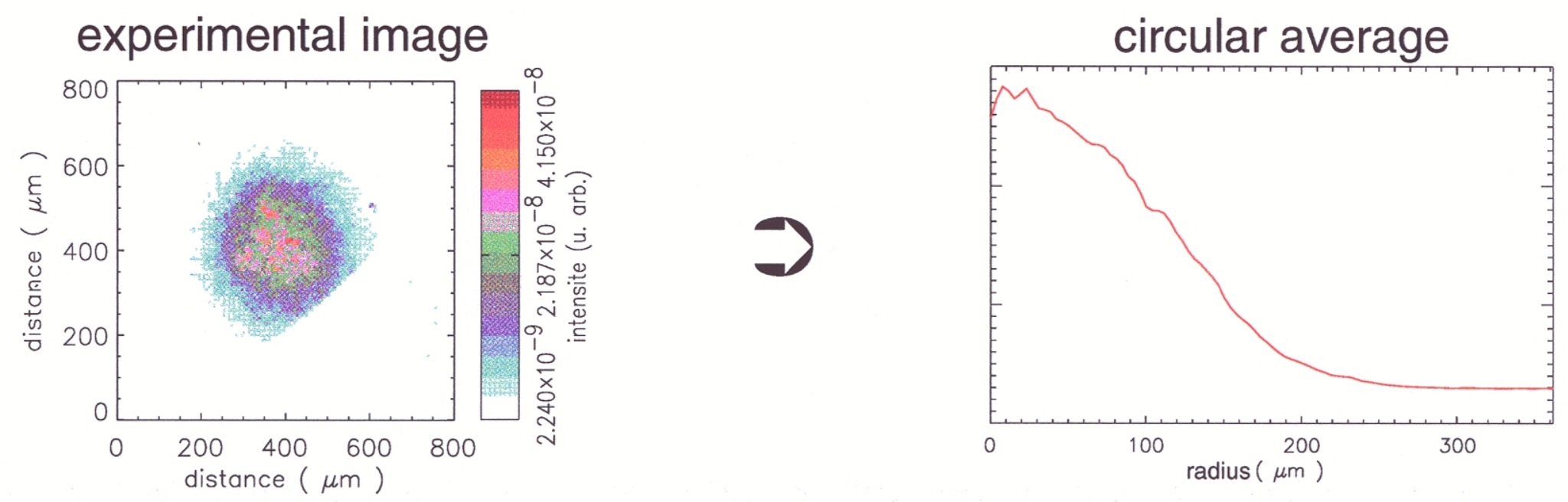

DAM - lle de France 


\section{Expériment-simulation comparisons}

\section{- experiment}
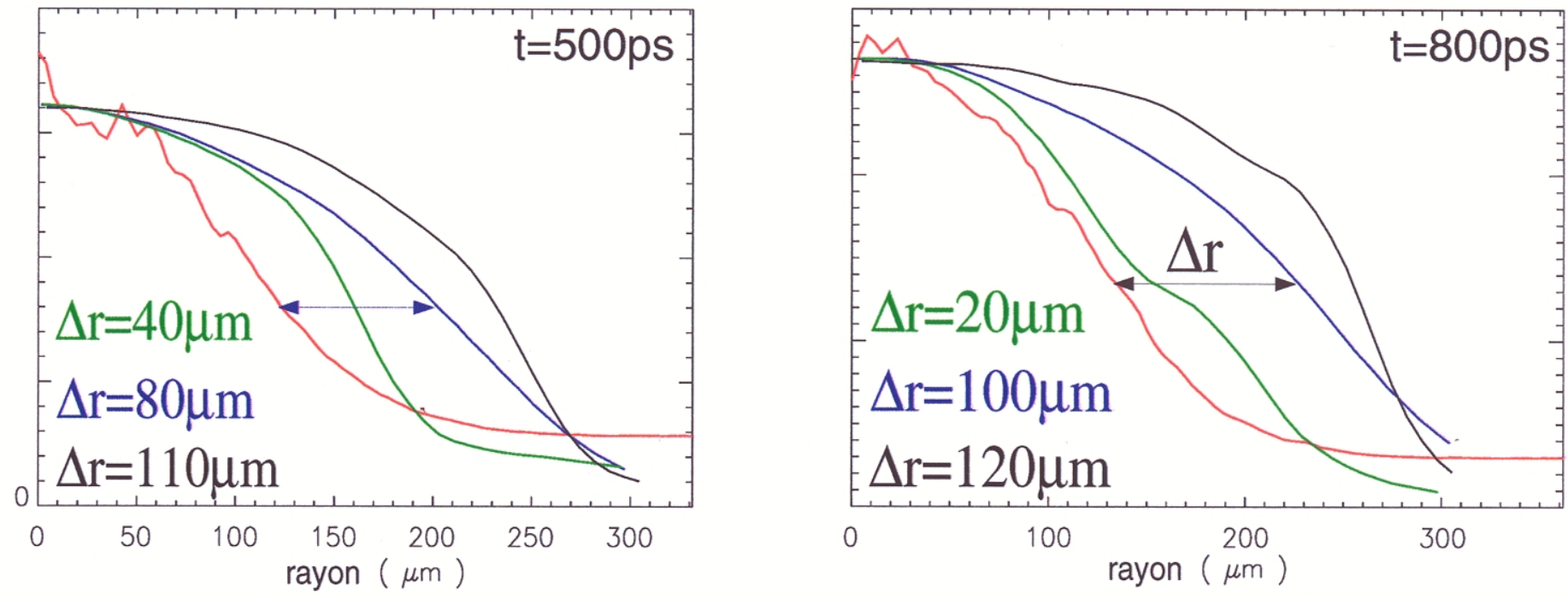

— Spitzer-Harm (5\%) - non local +B - only B

$\checkmark$ Using Spitzer-Harm, we get images too large by about 80 and $100 \mu \mathrm{m}$ at half maximum.

$\checkmark$ B-flieds do not improve results

$\checkmark$ B-flieds and nonlocal fluxes give the best agreement 


\section{Planar target : summary}

$\square$ Using Spitzer-Harm fluxes, simulations are unable to reproduce all diagnostics, whatever variations of flux limiter, laser parameters, zoning,...

$\square$ Both non local and magnetic effects improve simulation results without arbitrary parameter (flux limiter).

$\square$ If we only use one process, we get some improvements but not enough to match experimental data. 


\section{Hohlraum experiments (NOVA / OMEGA facilities)}
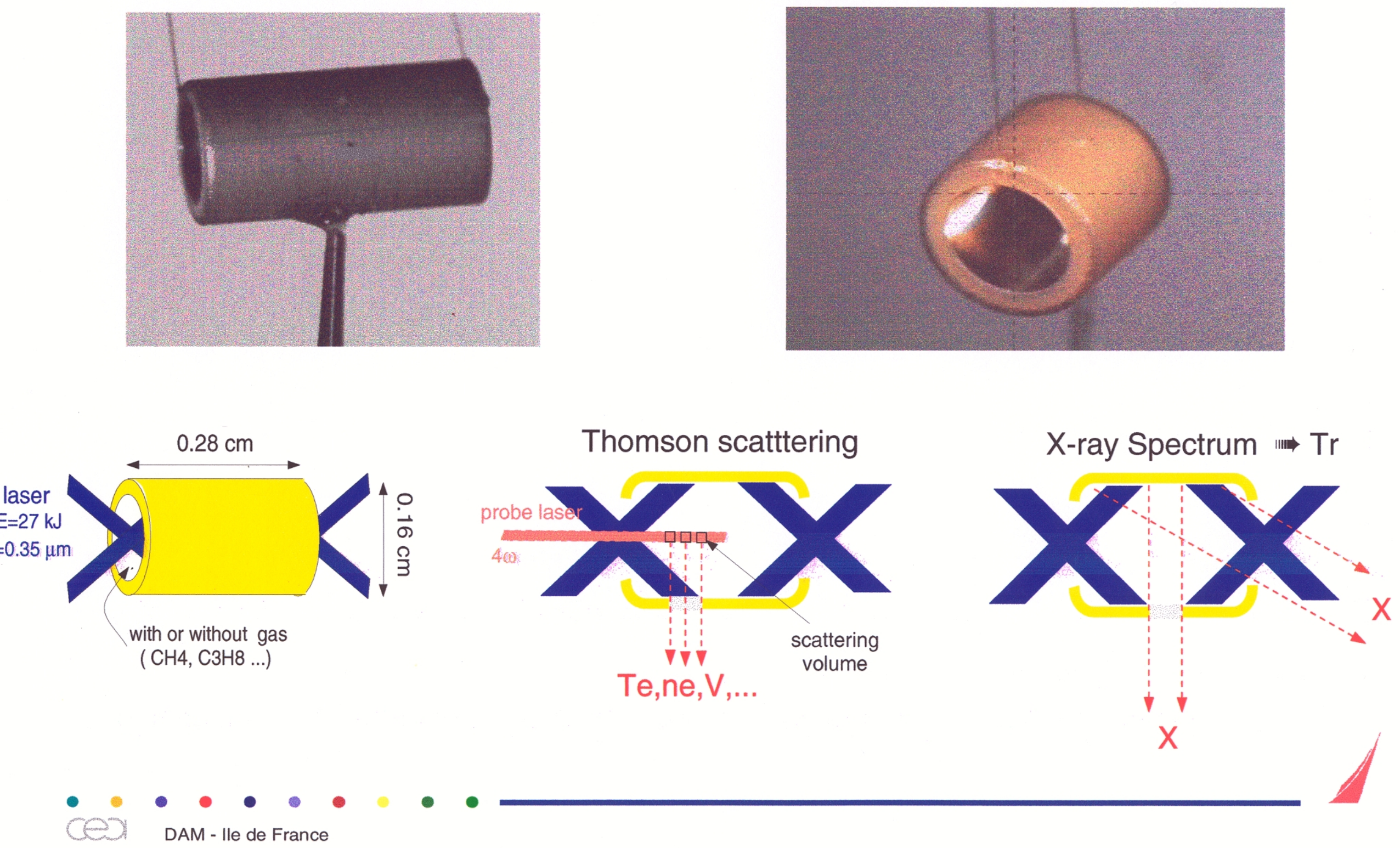


\section{Gas-filled Hohlraum (nova exp.)}

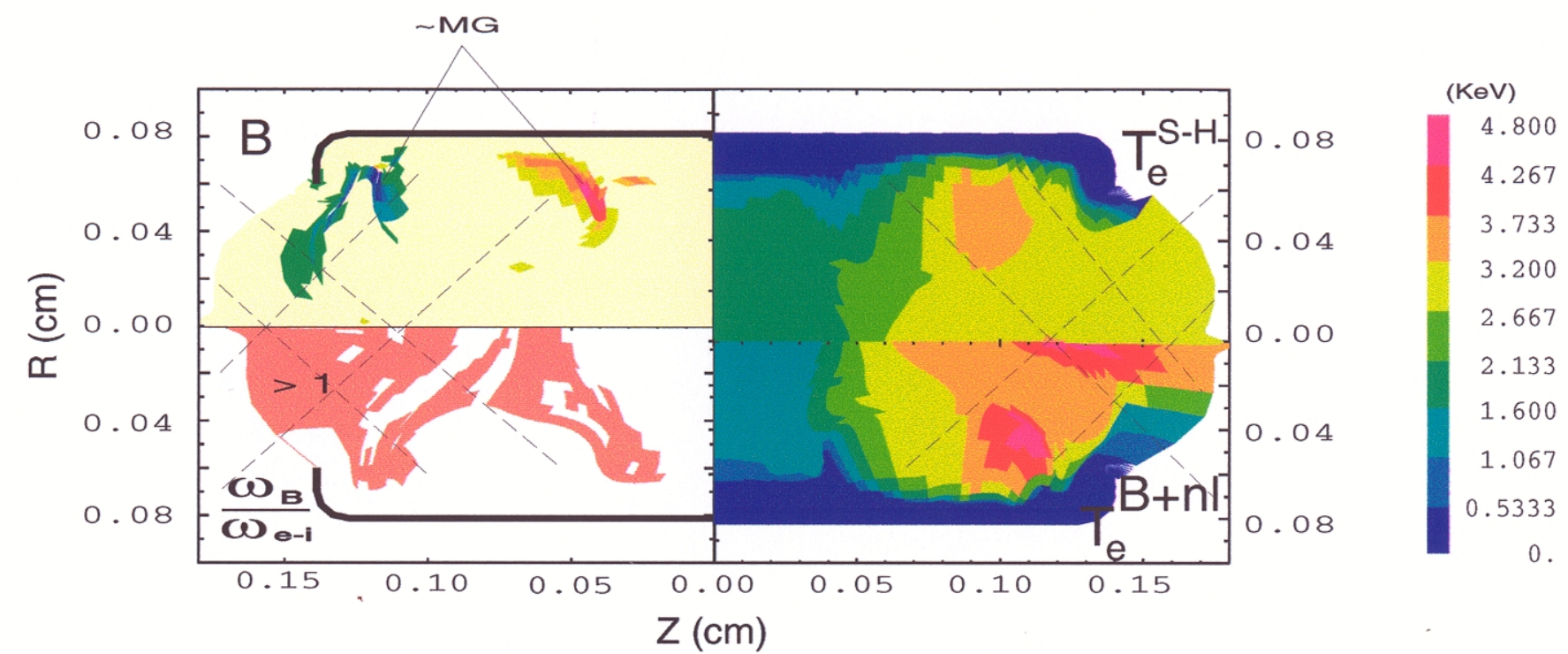

$\checkmark$ Simulations with radiation-hydrodynamic code $(\mathrm{FCl} 2)$ predict $\mathrm{B}$ fields of order of $1 \mathrm{MG}$

$\checkmark$ Hall parameter exceeds unity in a large zone of the hohlraum (B effects)

$\checkmark$ Calculations with SH or with our model (w/o flux limiter) lead to differents results :

- higher electron temperature in off-axis region (from $3.5 \mathrm{keV}$ to $4.8 \mathrm{keV}$ )

- larger temperature gradient along the axis (beams crossing)

experimental data of electron temperature from Thomson scattering seem to confirm large gradients along z-axis and temperature in order of $5 \mathrm{KeV}$ close to LEH 


\section{Hohlraum experiment}

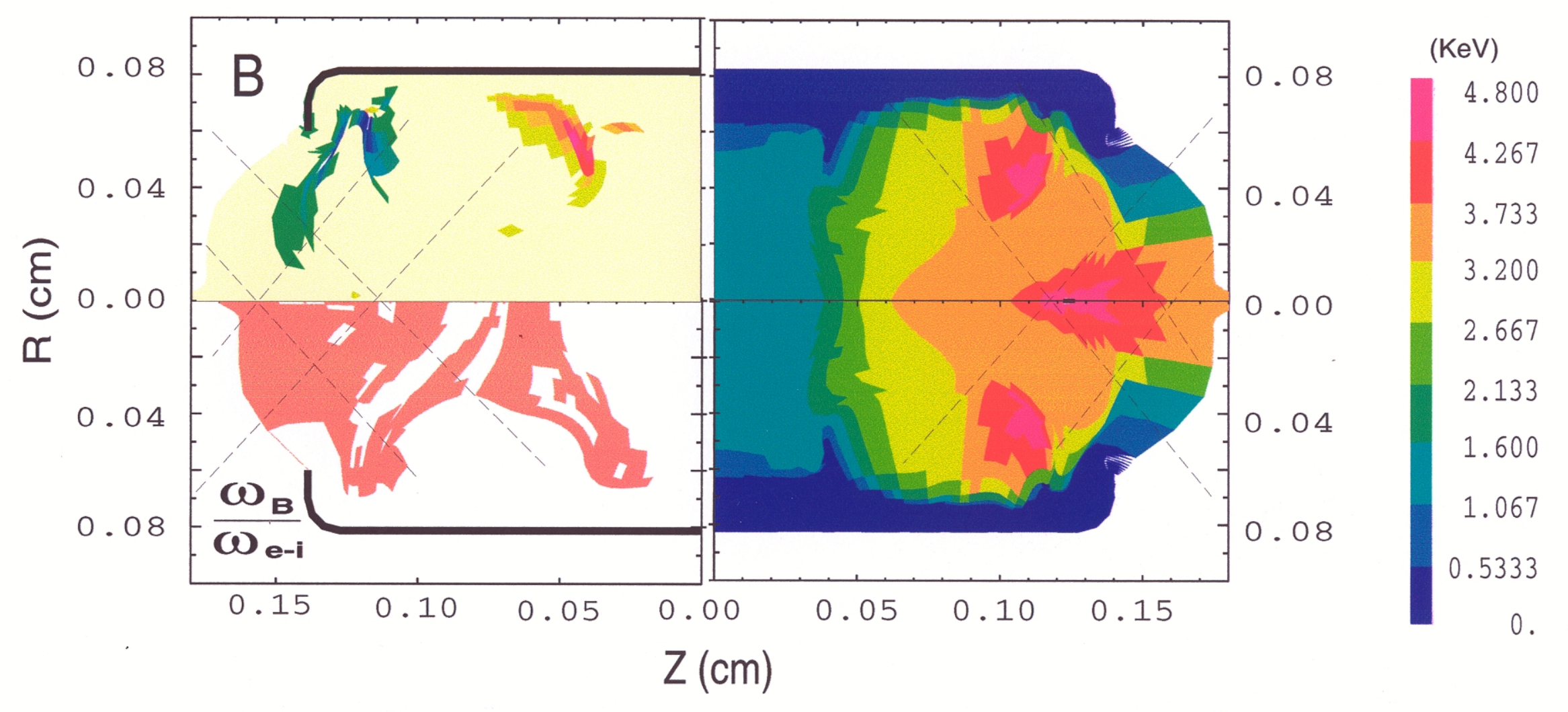




\section{Hohlraum experiments}

$\checkmark$ In a hohlraum, magnetic fields quickly spread in the whole plasma.

$\checkmark$ B-fields reduce or cut nonlocal effects. The deformation of the spherical part of the distribution function due to nonlocal effects may be reduced or cancelled by B-fields.

$\checkmark$ Simulations without nonlocal fluxes but with B effects can correctly reproduce, in this case, experimental results. 


\section{B-fields (nonlocal effects) can modify density}
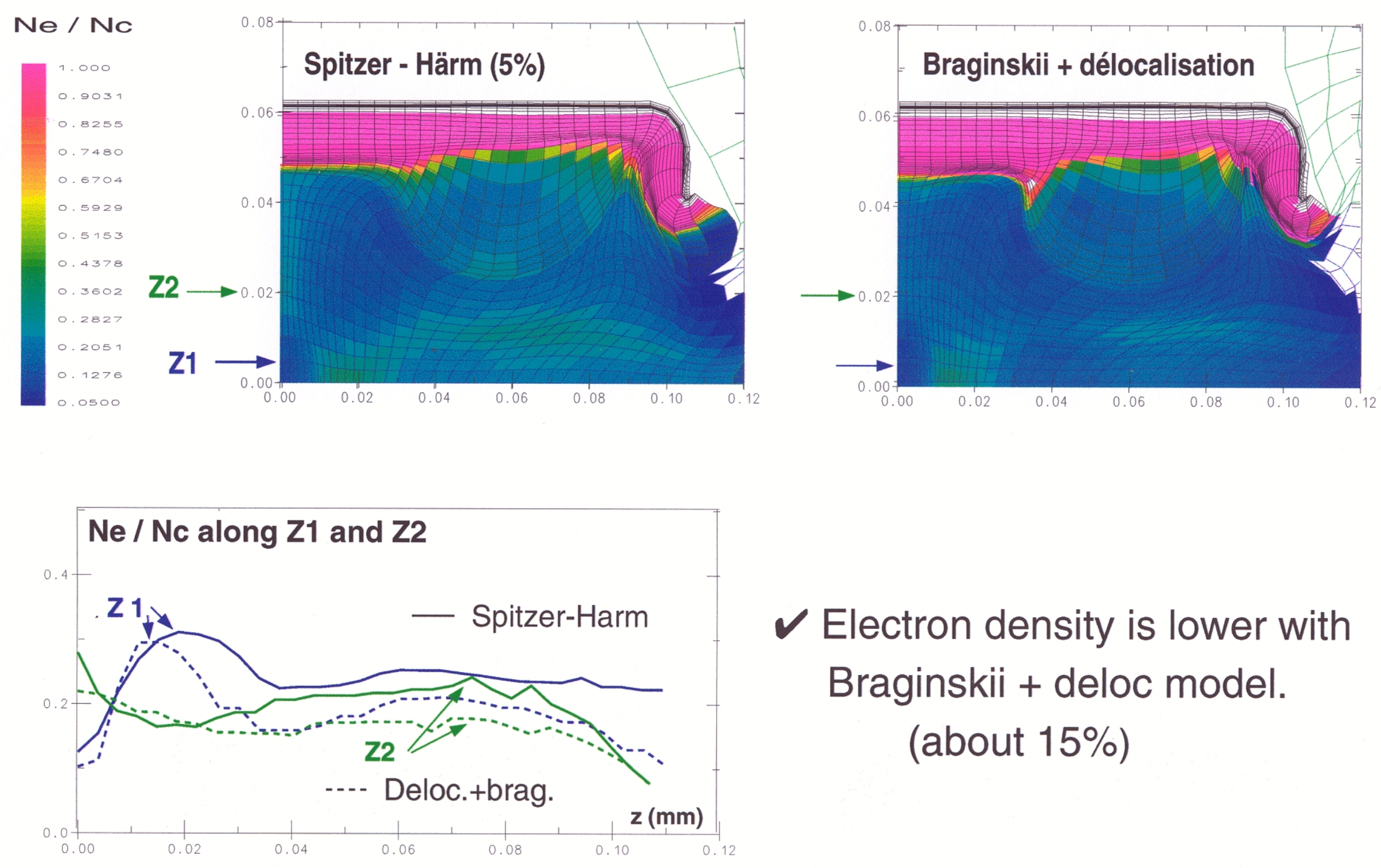

$\checkmark$ Electron density is lower with Braginskii + deloc model.

(about 15\%) 


\section{Same radiation temperature versus formula}

$\mathrm{eV}$
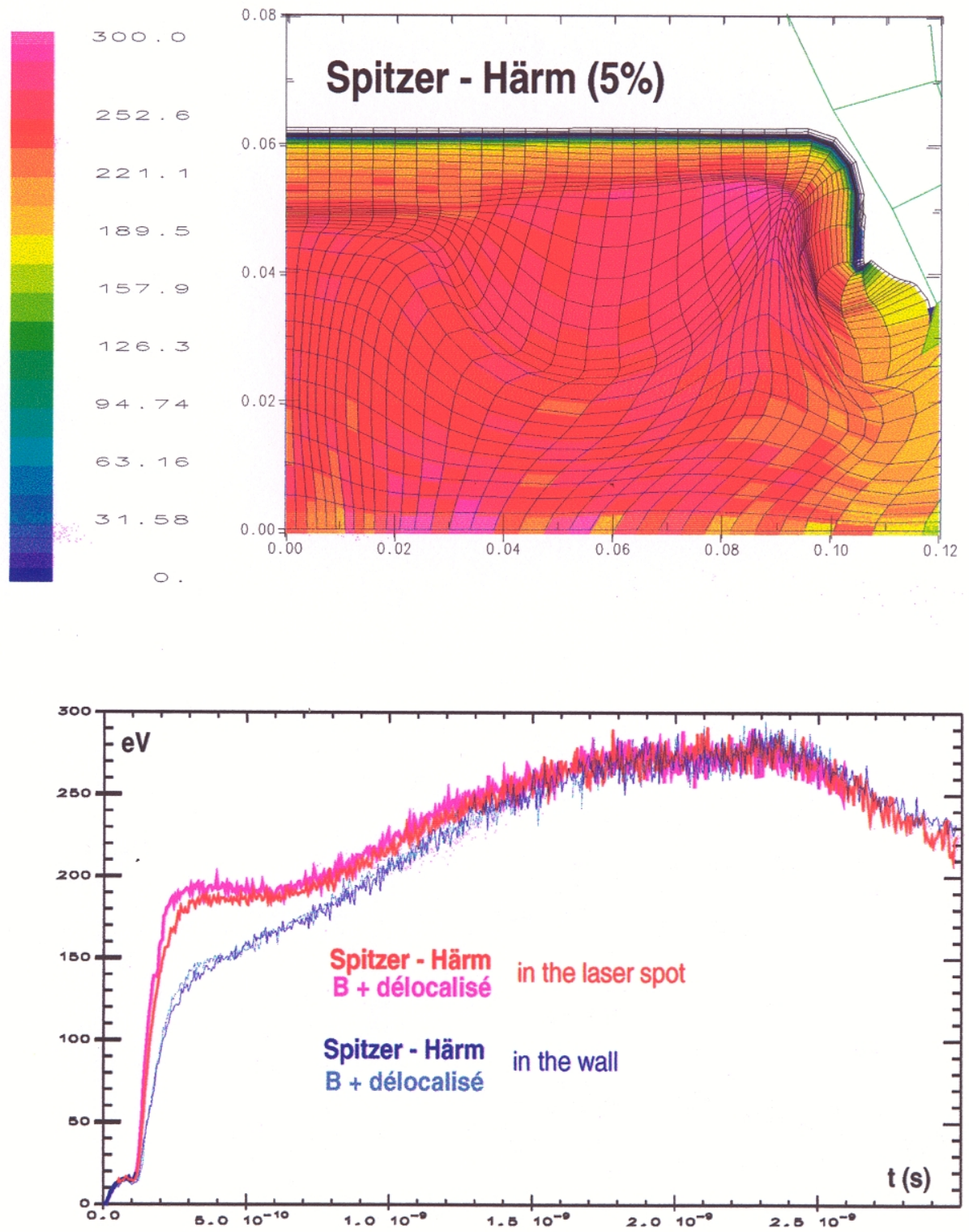

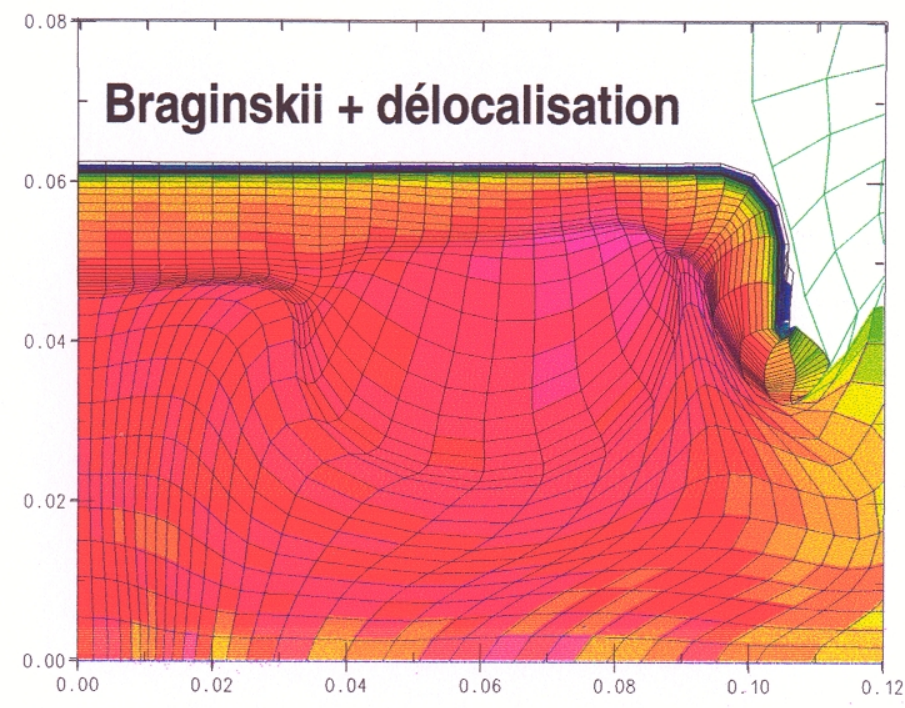

$\checkmark$ same Tr but w/o flux limiter: (good value)

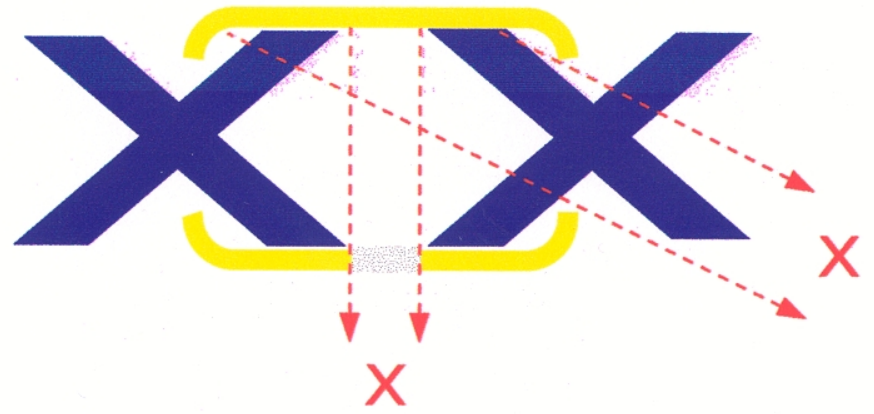

$\checkmark$ So X-ray Diagnostics simulation correct? 


\section{Our electron conduction model can modify hydrodynamic motion}

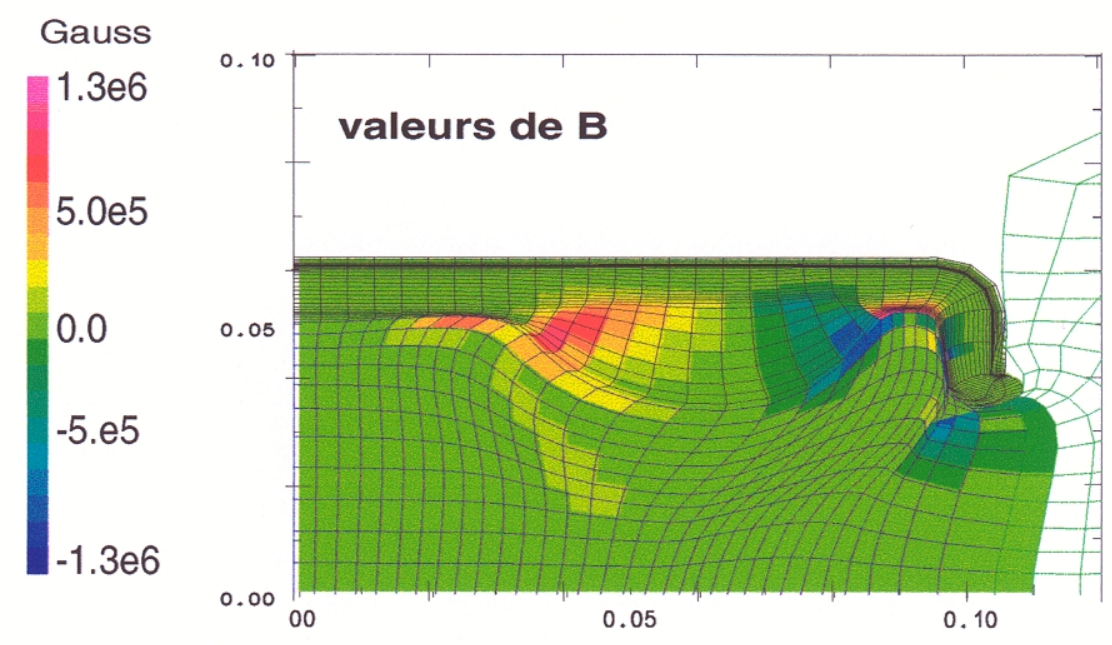

$\checkmark$ heating and expansion of LEH w/o B-fields -> X-ray emission of this zone
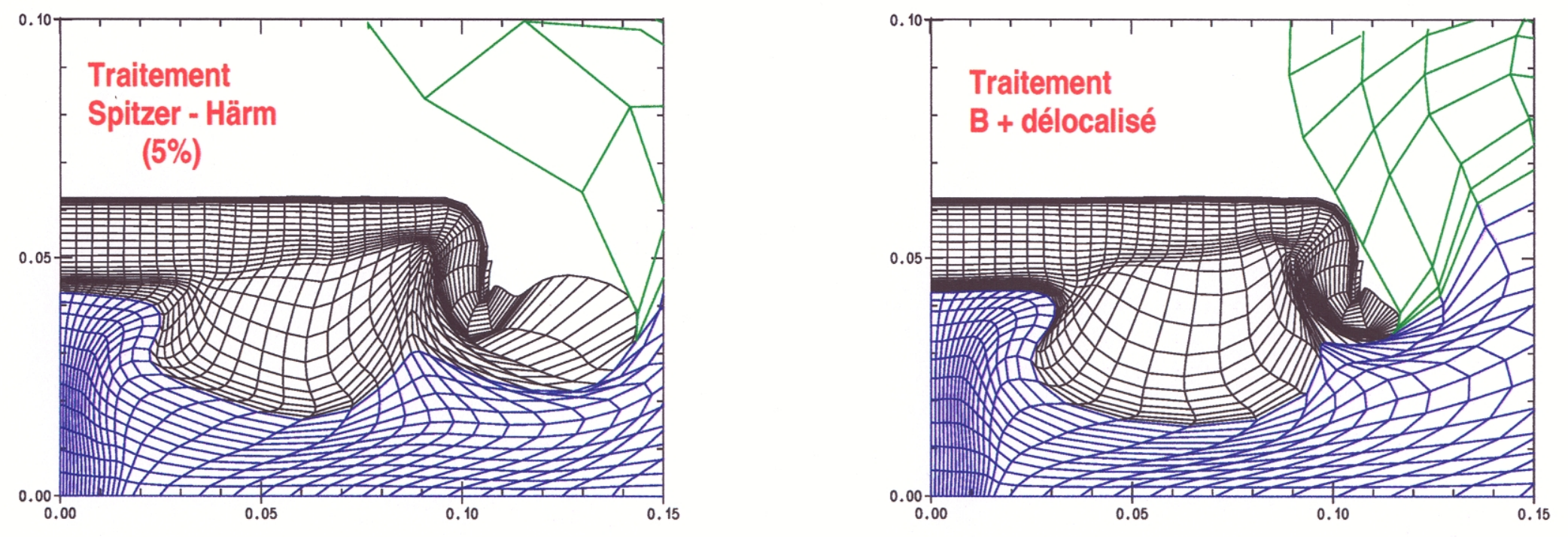

DAM - lle de France 


\section{Hydrodynamic effect on X-ray emission}
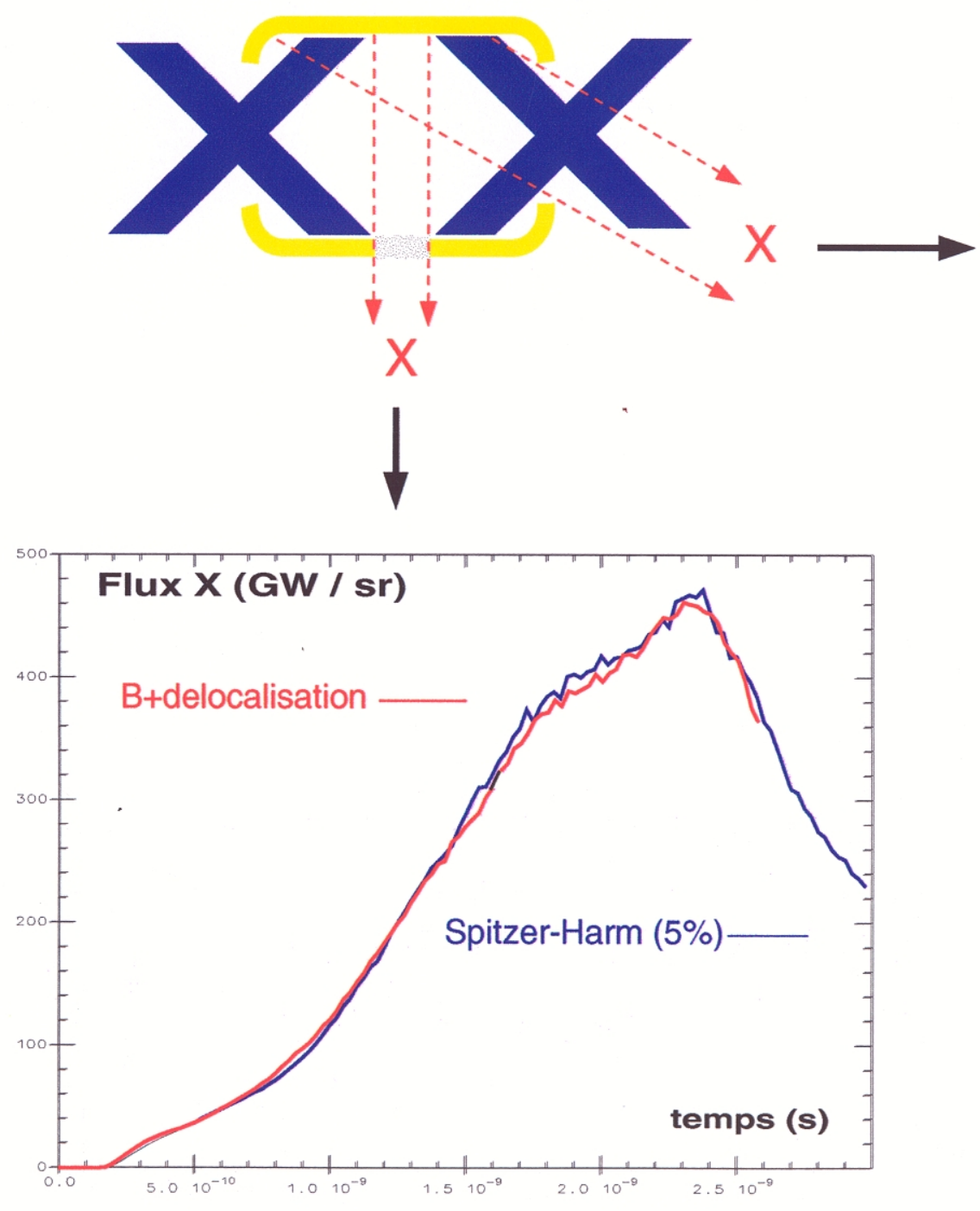

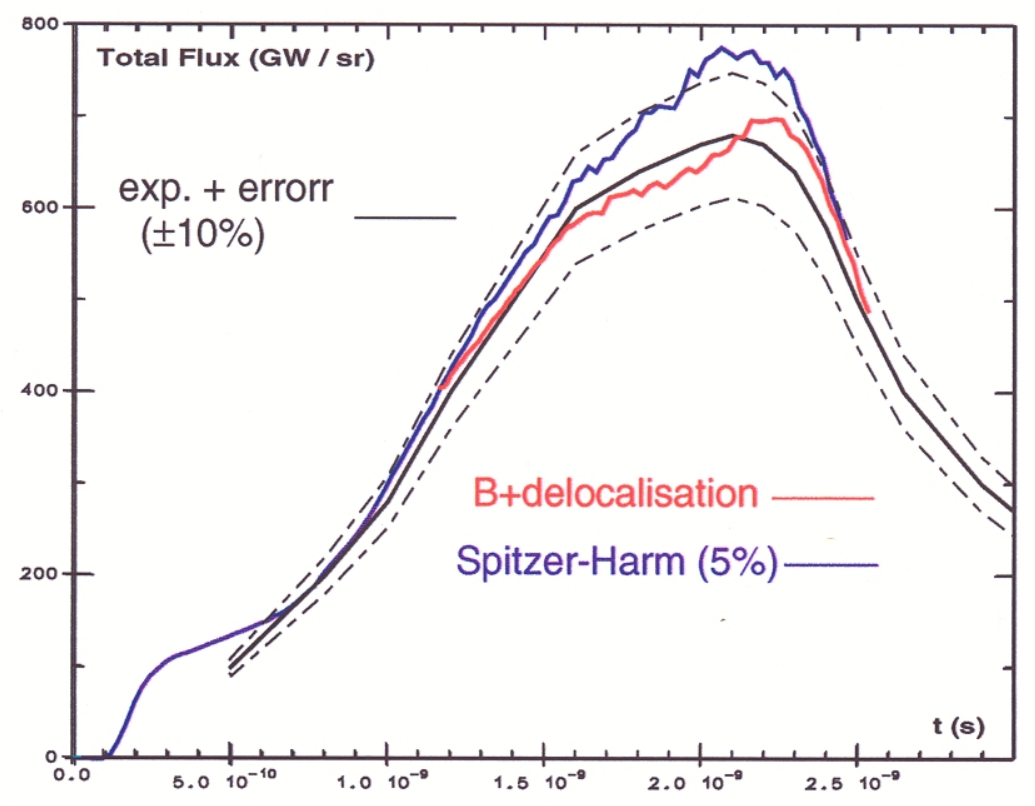

$\checkmark$ For Dante, both heat flux models give similar results

$\checkmark$ B-fields reduce X-ray emission of 'LEH' and enable us to better reproduce exp. data

Both simulations are inside error bars 


\section{Effects of our model on LMJ cavity}

Max. laser power $=400 \mathrm{TW}(16 \mathrm{~ns}) ;$ Laser energy $=1,4 \mathrm{MJ}$

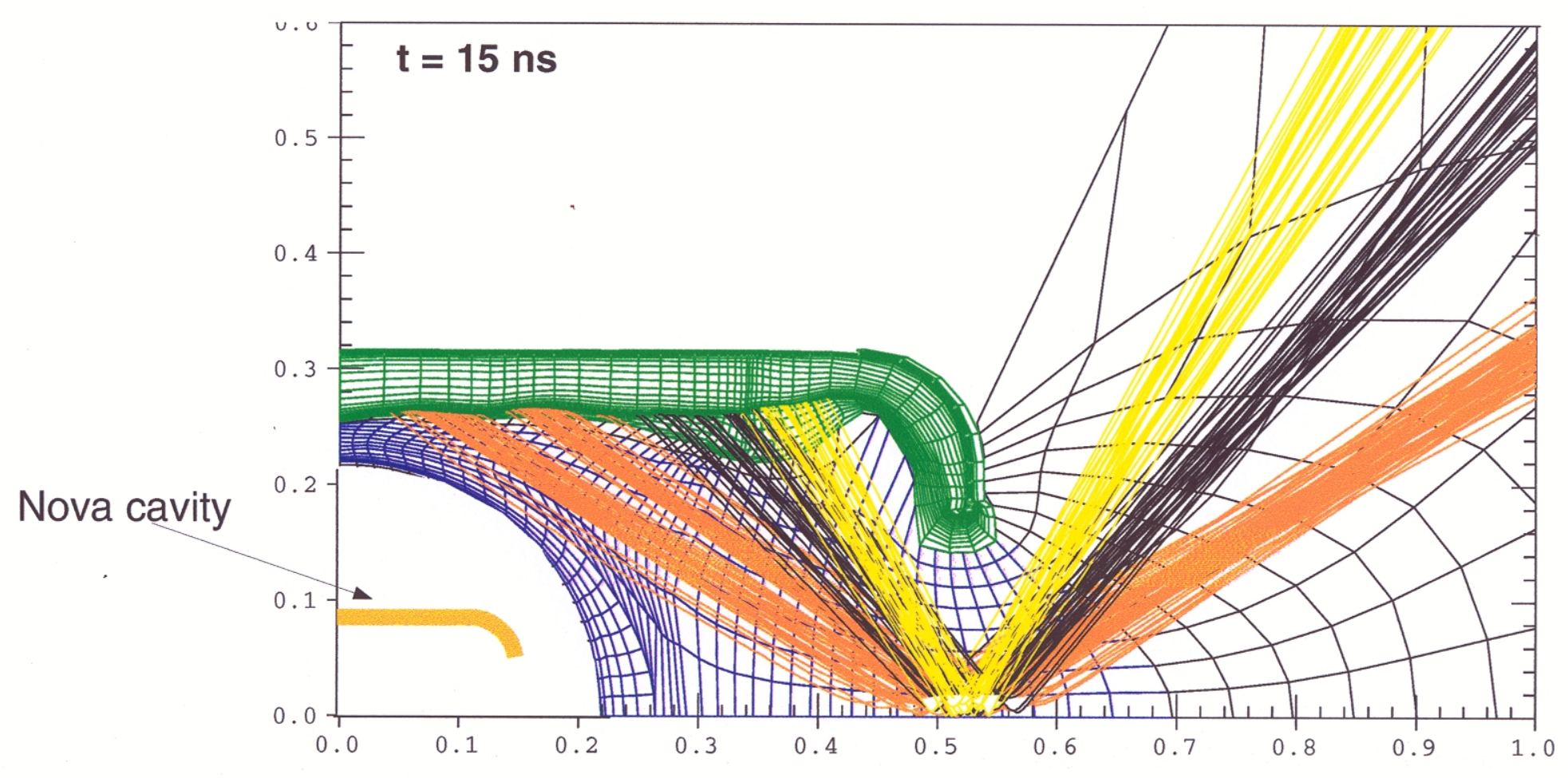




\section{Magnétic fields at 16 ns (max. laser power)}

B effects are important in a large part of the cavity
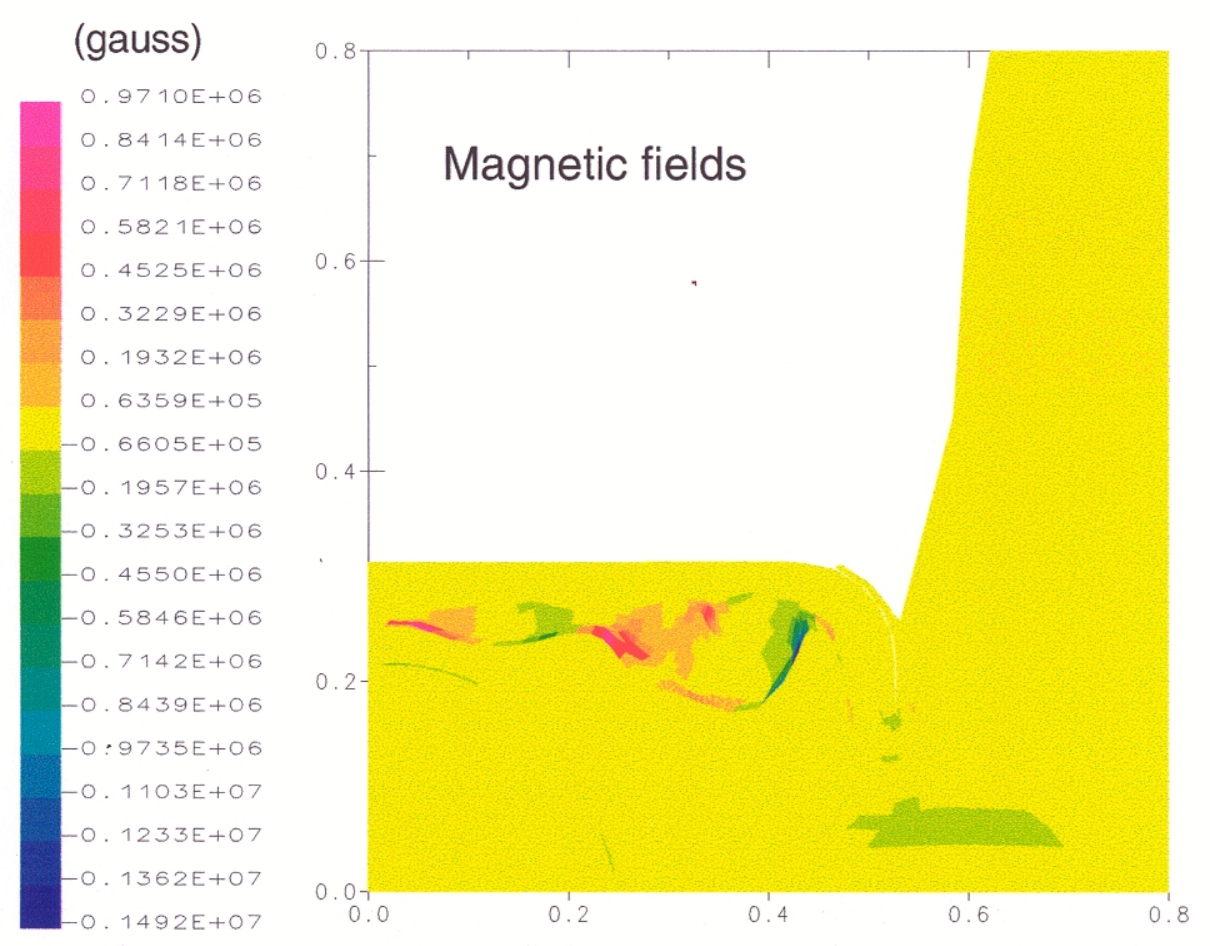

We expect some modifications for

$\checkmark$ electron température

$\checkmark$ ion température

$\checkmark$ density

Jionization

$\checkmark$ hydrodynamic speed

$\checkmark \ldots$ 


\section{Electron temperature color map at $t=16 \mathrm{~ns}$}

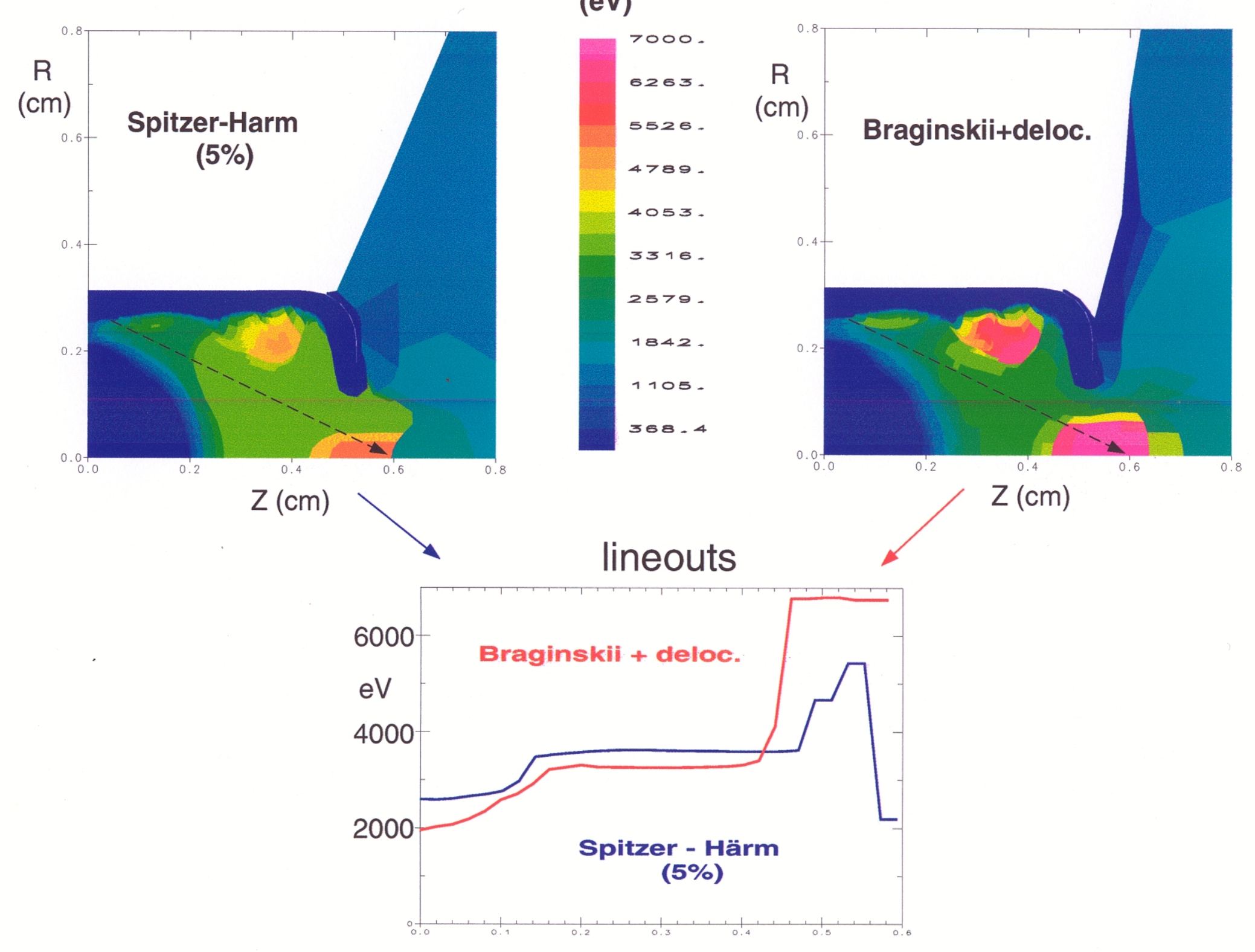

(eV) 


\section{Electron density map at $\mathrm{t}=16 \mathrm{~ns}$}

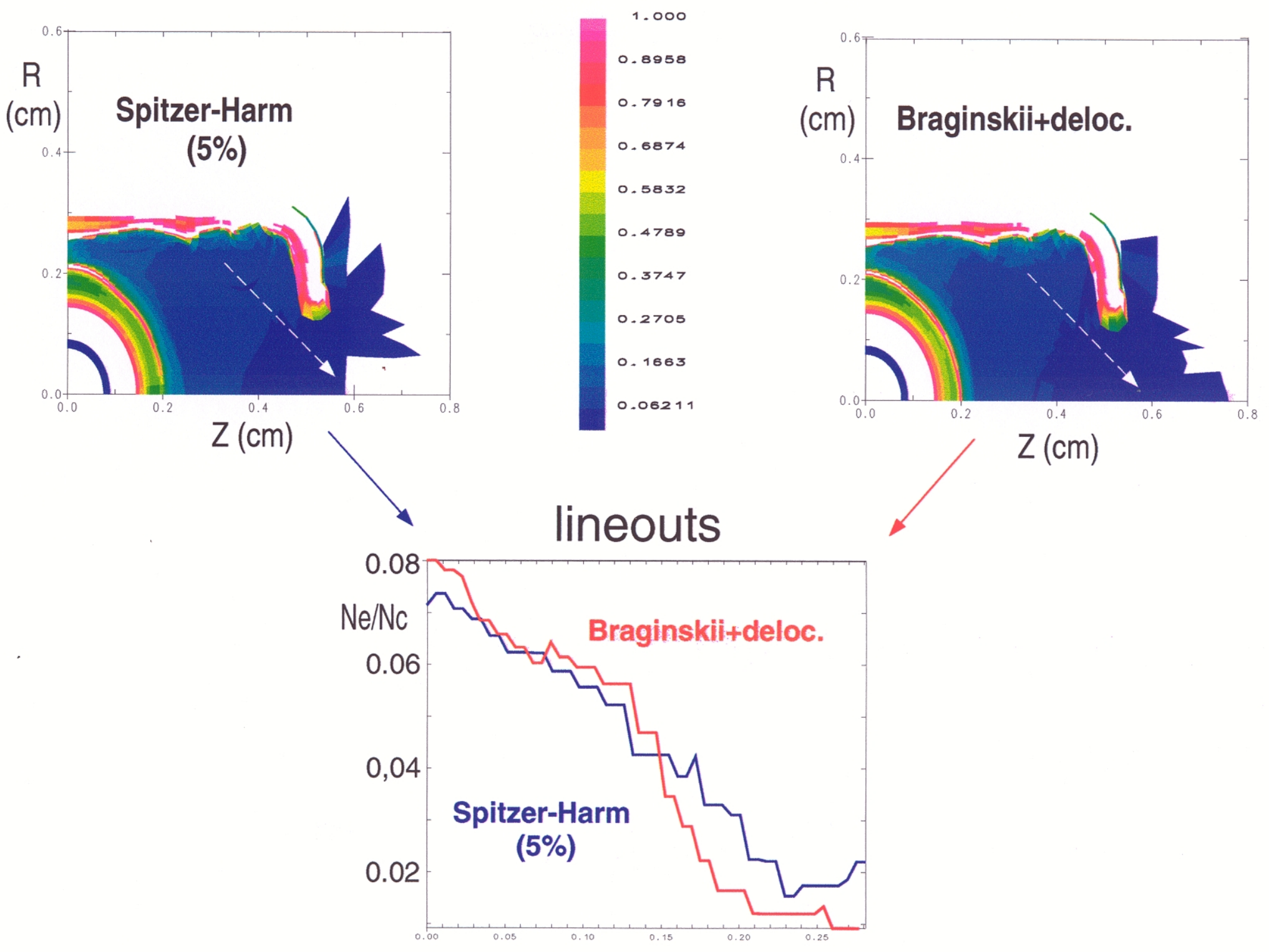




\section{X-rays non uniformities on the ablation front}

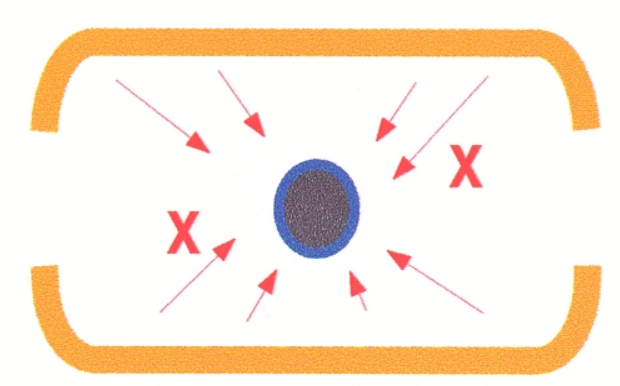

Expansion of the X-ray flux on a Legendre polymomial basis

mode 2

mode 4

mode 6
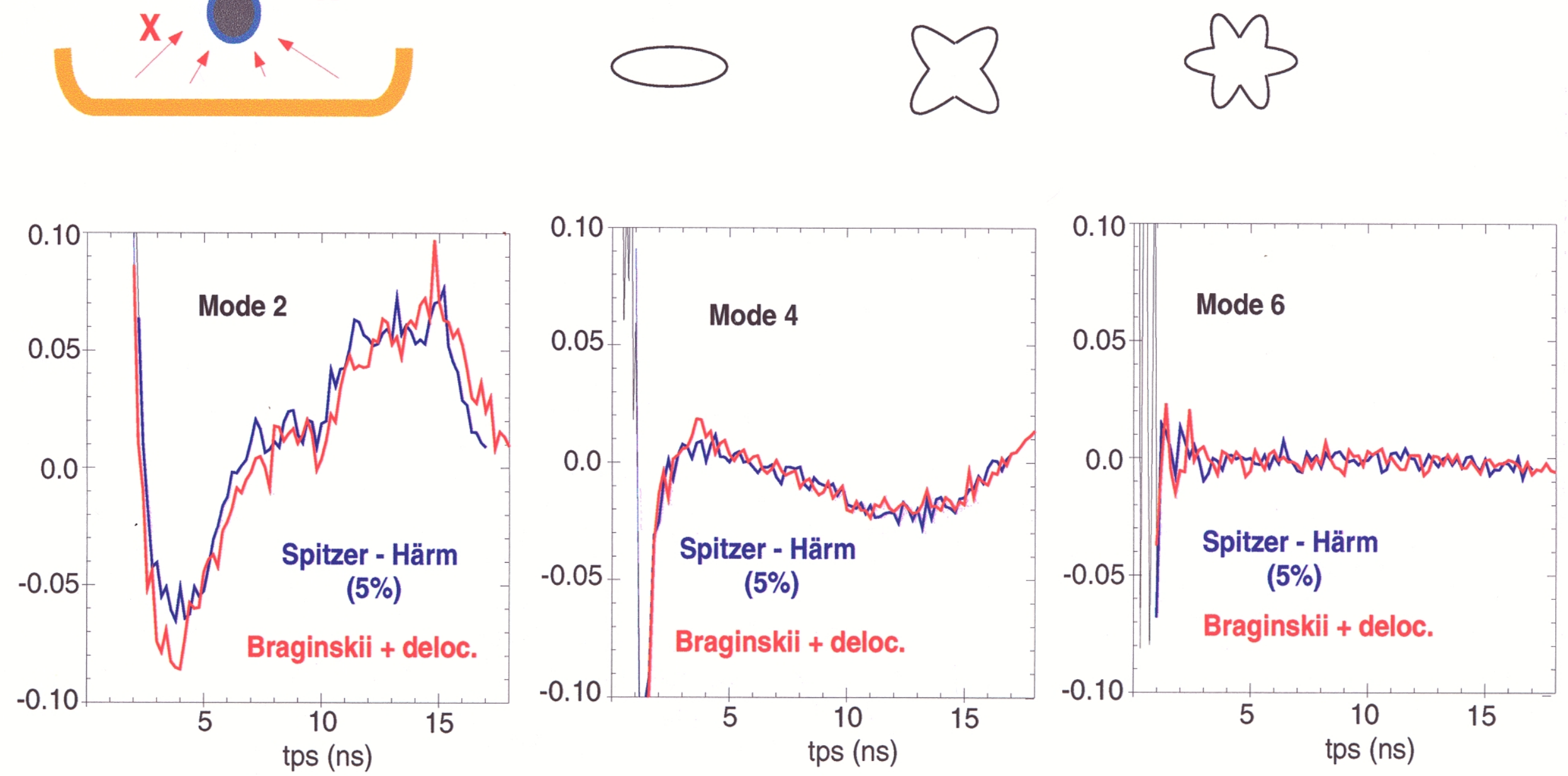

!neither beam-phasing nor pointing optimization ! 


\section{Cavity : summary}

$\square$ Simulations using magnetic fields and nonlocal fluxes match Thomson

scattering results unlike simulations with $\mathrm{S}-\mathrm{H}$ fluxes.

$\square$ The fields diffusion inside cavity reduce non-local effects.

$\Rightarrow$ Using Braginskii fluxes, simulation passes through expérimental error bars

$\square$ Hydrodynamics quantities $(\mathrm{Te}, \mathrm{Ti}, \mathrm{Ne}, \mathrm{V}, \ldots)$ can be hardly modified

by magnétics fields $\Rightarrow$ effects on others processes (Laser Plasma Interaction)

$\square$ The radiation temperature and the irradiation symetry of micro-ballon are not affected by our model $\Leftrightarrow$ limited Spitzer-Harm $(\mathfrak{f} \sim 5 \%)$ 


\section{Spherical target (Omega facility)}

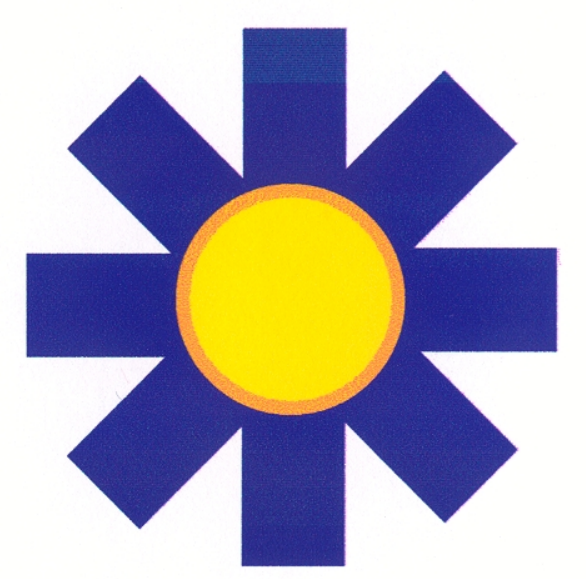

$\checkmark \mathrm{CH}$ targets $(950 \mu \mathrm{m})$ cover with gold $(2.5 \mu \mathrm{m})$

$\checkmark$ laser : $-3 \omega$

- intensities from 1 e13 to $1 \mathrm{e} 15 \mathrm{~W} / \mathrm{cm} 2$

- square pulses for from 1 to 4 ns

- $\mathrm{fwhm} \approx 500 \mu \mathrm{m}$
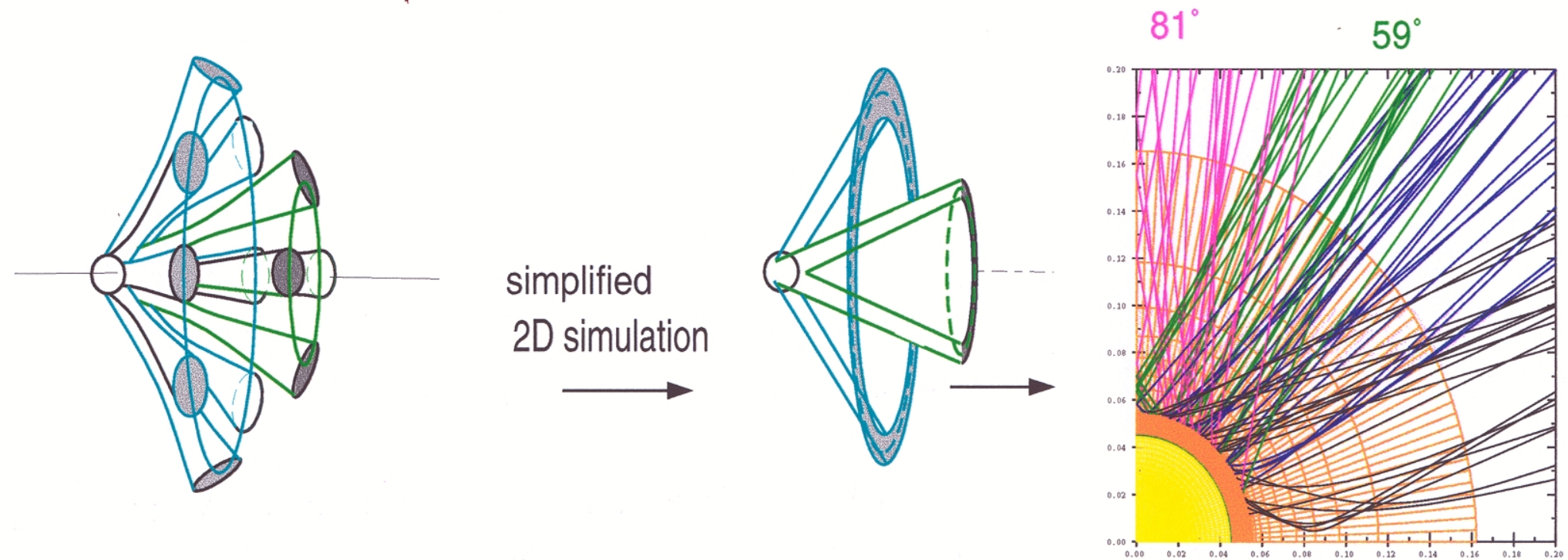

$\checkmark$ X-ray Diagnostics : spectrum, conversion efficiency, imaging with spectral resolution 


\section{Influence of self-generated magnetic fields}

$\boldsymbol{\sim}$ Crossed gradients Te-Ne can create B-fields

$\checkmark$ For this geometry, with an isotropic irradiation, gradients are collinear $->$ no B-fields

$\checkmark$ In experiment, we can have unbalanced laser power between beams (cones)

$$
\mathrm{I}=1.4 \mathrm{e} 14 \text {, at } 21^{\circ}(-14 \%) \text {, at } 42^{\circ}(+2 \%) \text { at } 58^{\circ}(-0.3 \%) \text { at } 81^{\circ}(+6 \%) \text { worst case }
$$
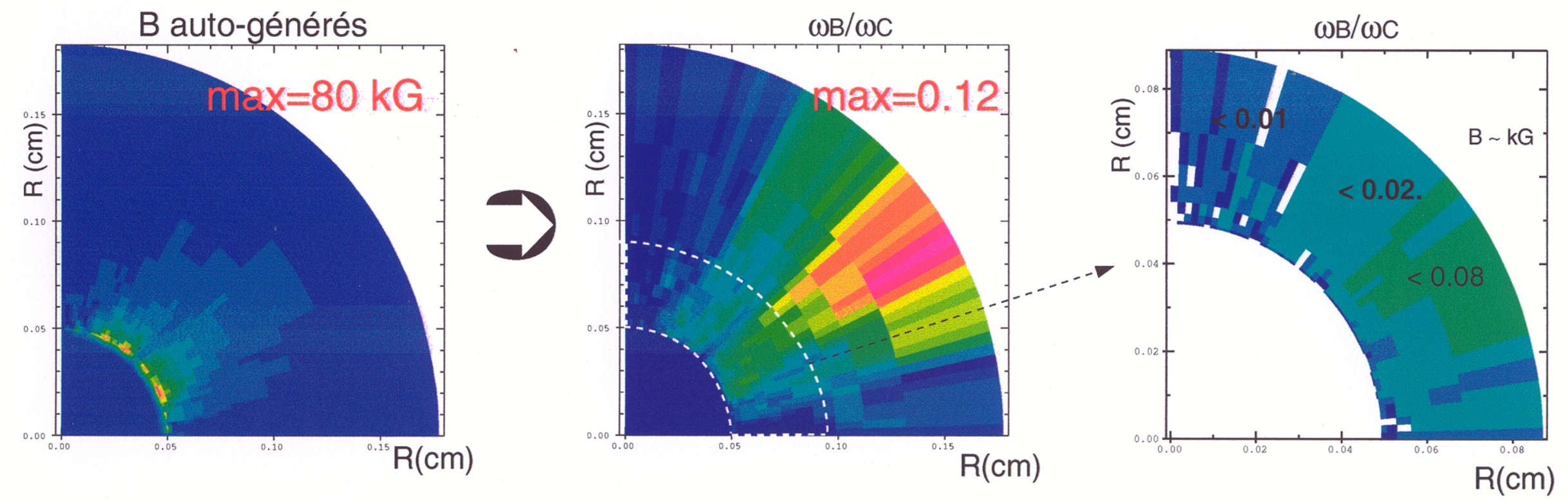

$\rightarrow$ No influence of magnetic fields (same res. w or w/o B)

$\Rightarrow$ Only nonlocal flux acts on plasma in these experiments 


\section{X-ray Conversion}

\section{X-ray Energy / Absorbed laser energy}

$\checkmark$ We test the influence of heat flux on X-ray conversion

$\checkmark$ Expérimental data come from several experiments

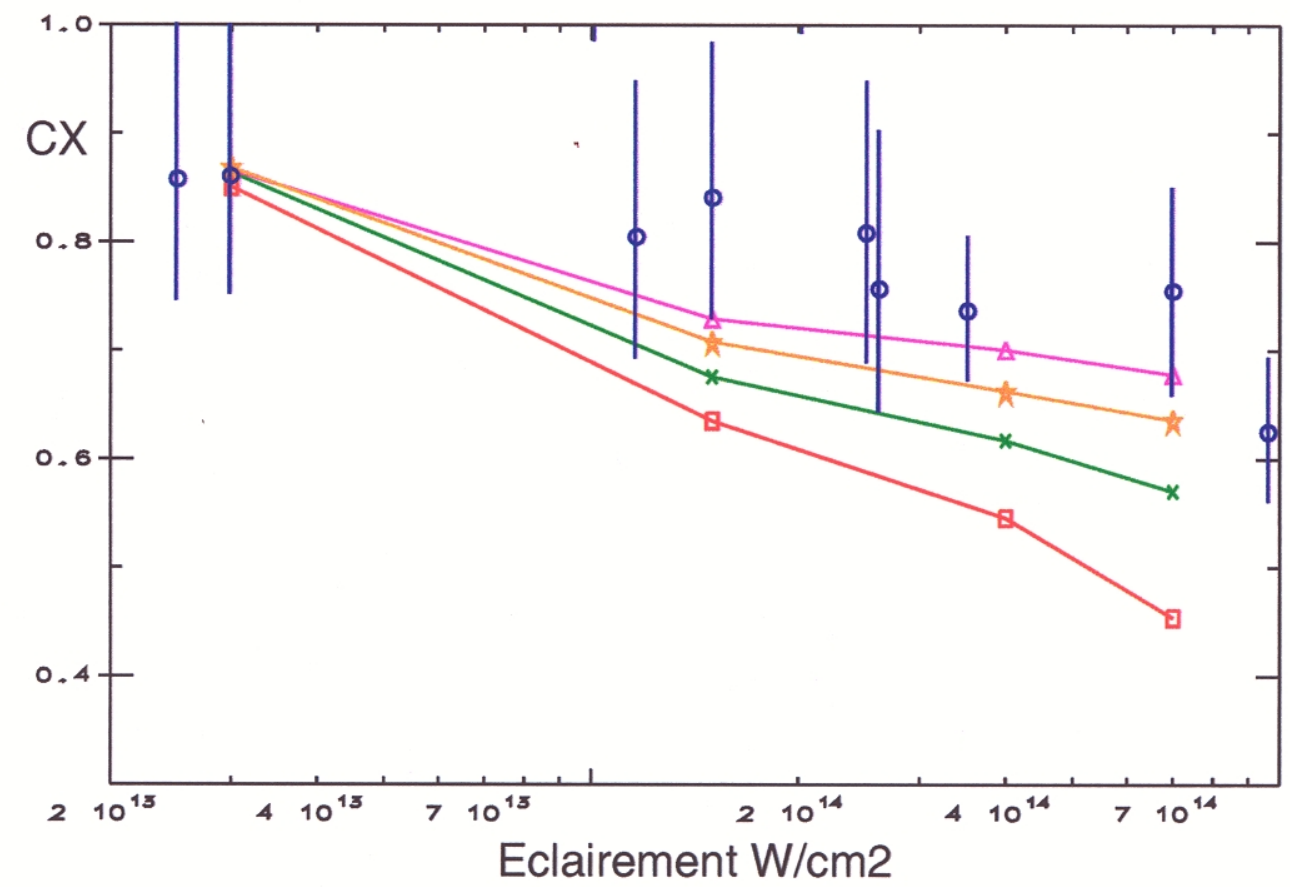

- Experiment

- Spitzer-Harm $f=0.6$

- Spitzer-Harm $\mathbf{f = 0 . 1}$

- Spitzer-Harm $\mathrm{f}=\mathbf{0 . 0 3}$

- non-local

$\checkmark$ The slightly limited $\mathrm{SH}$ flux and the nonlocal flux reproduce experimental data 


\section{Heat fluxes for high and low laser intensities}

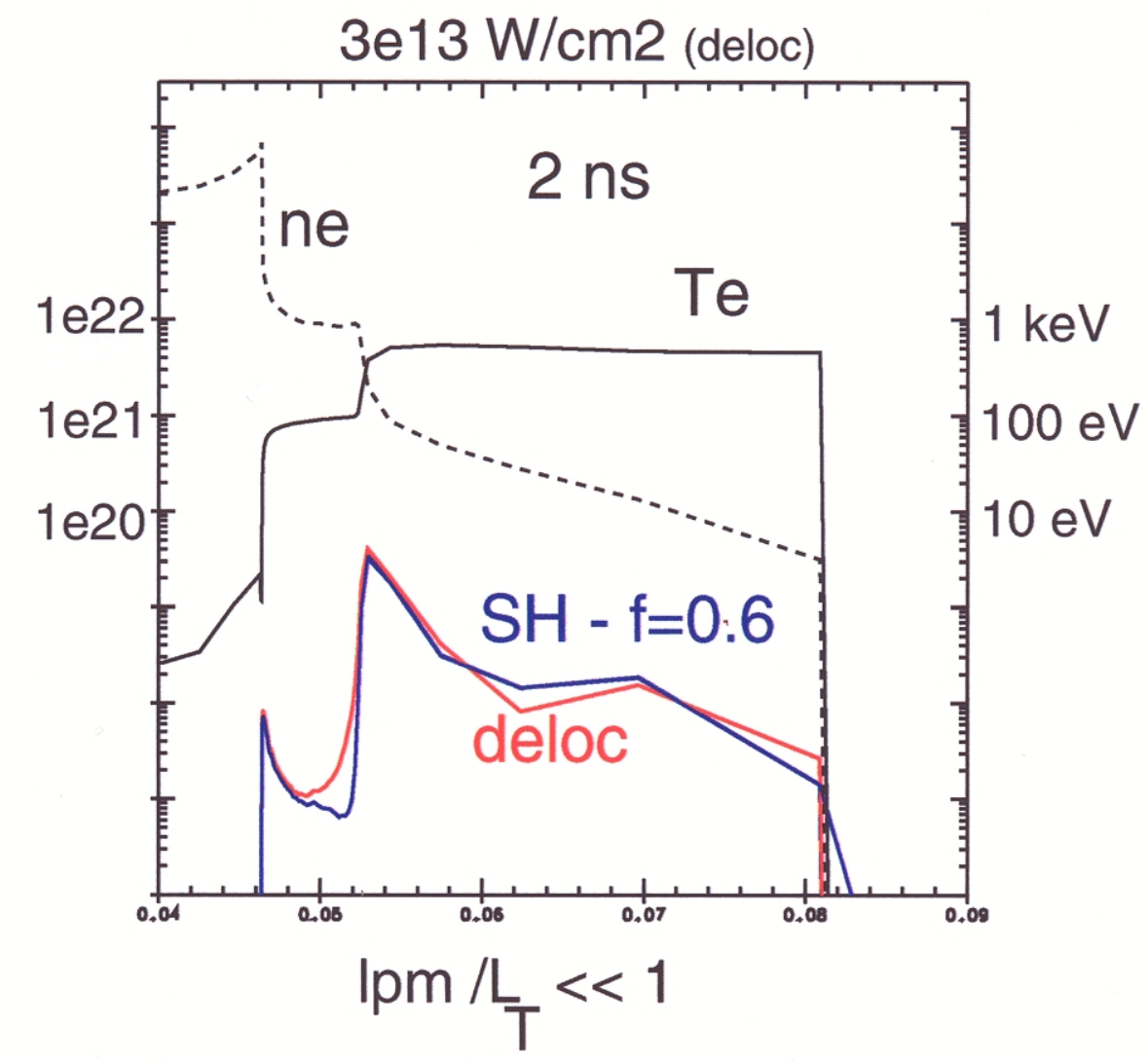

- low temperature, smooth gradient, short

e.m.f.p, the nonlocal flux tends towards

Spitzer-Harm flux.

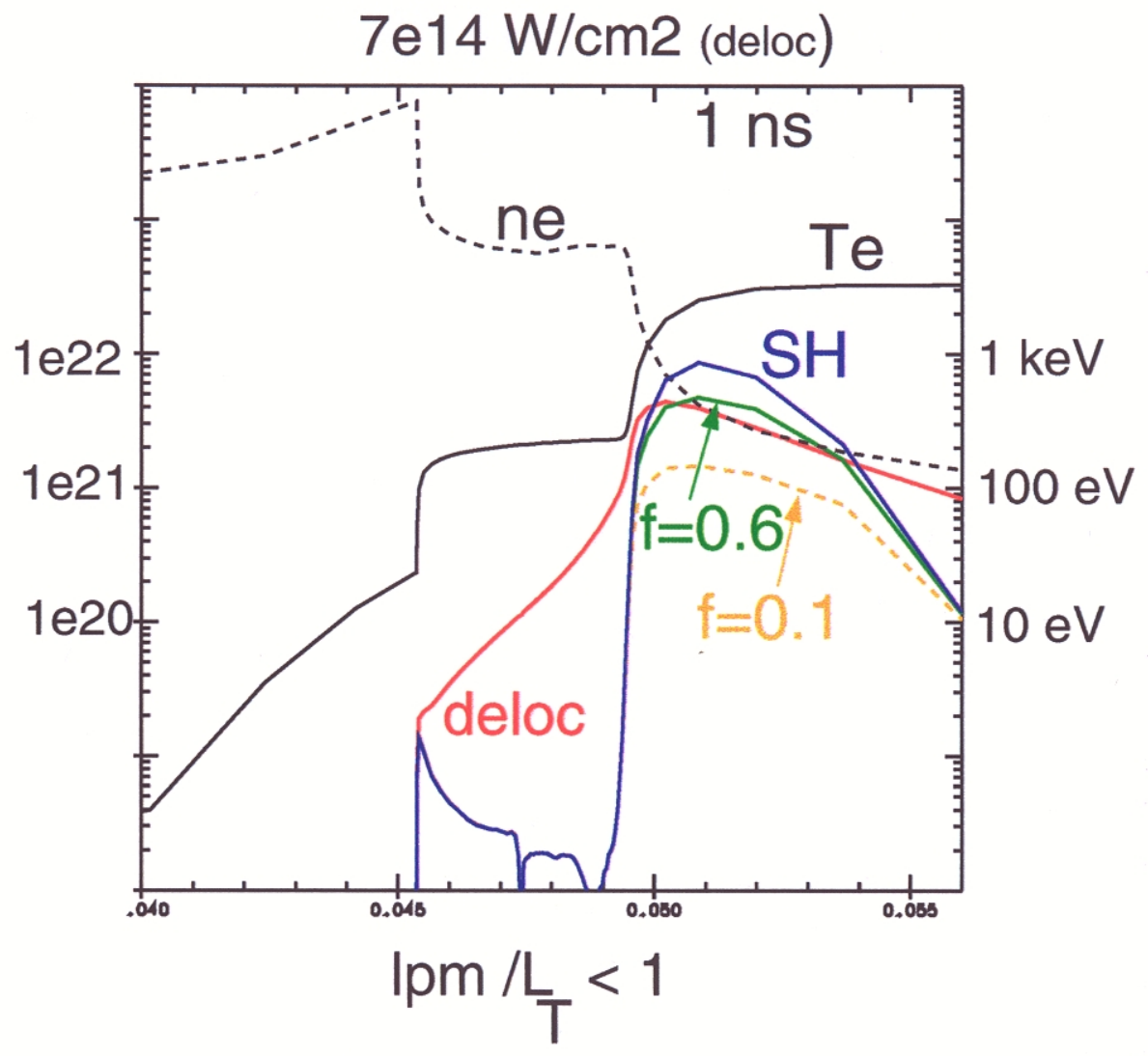

-high température, sharp gradient, long e.m.f.p., the flux is nonlocal and different from Spitzer-Harm flux. 


\section{Simulation of X-ray imaging}

\section{Characterize plasma expansion}

From 2D hydrodynamic computations, we can simulate diagnostic (post-process)

emission at $2 \mathrm{keV}$
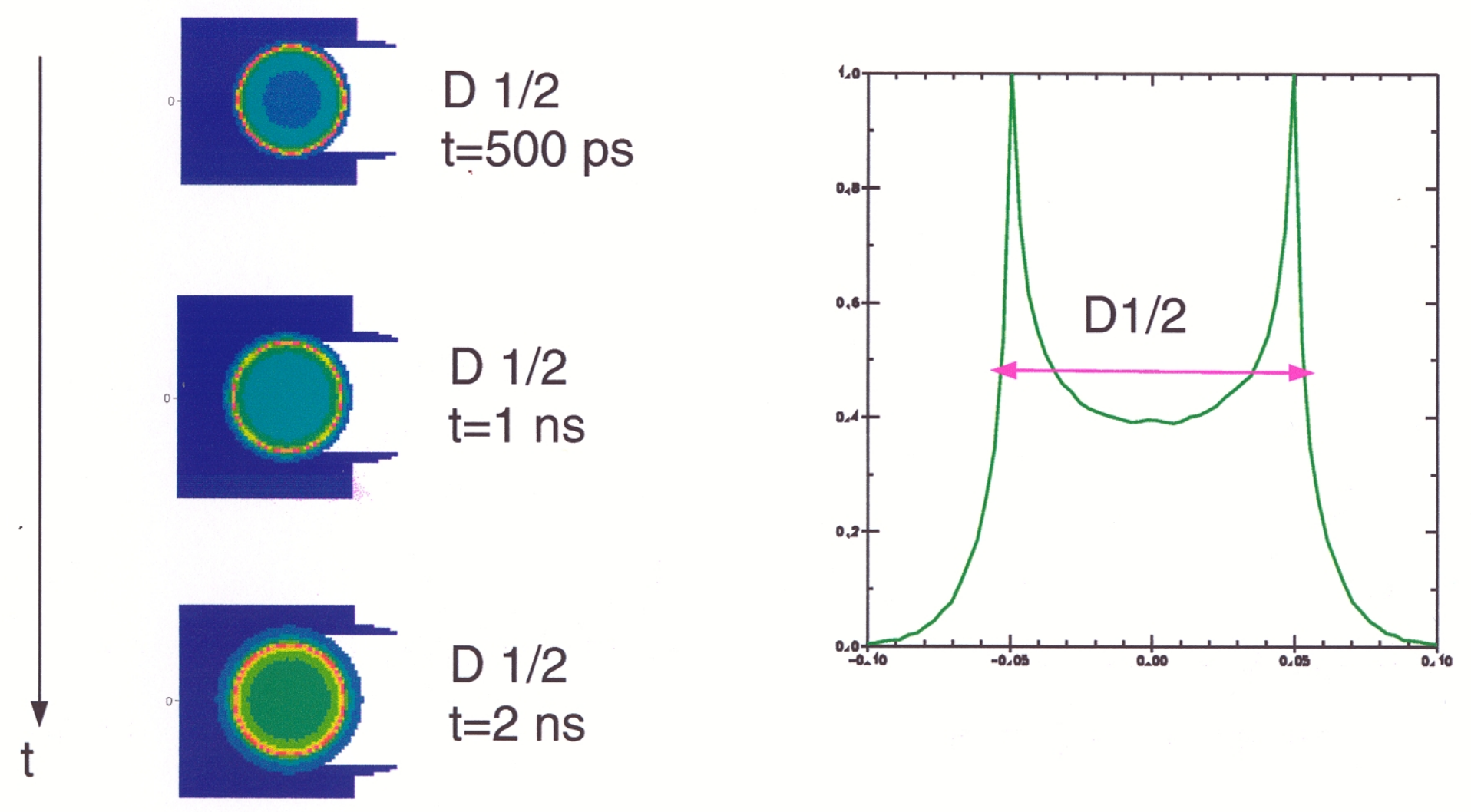


\section{Emitting zones movement for $\mathrm{I}=7 \mathrm{e} 14 \mathrm{~W} / \mathrm{cm} 2$}
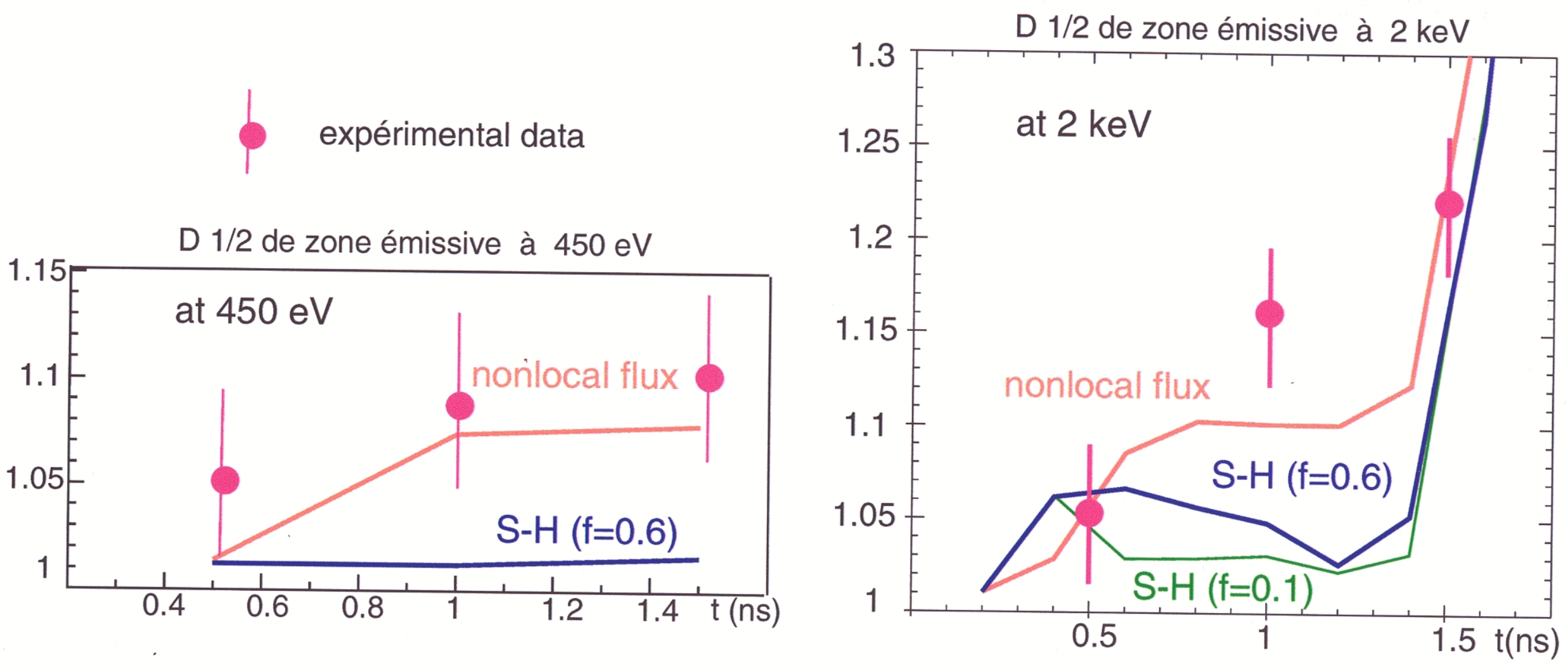

$\checkmark$ Spitzer-Harm fluxes do not be able to reproduce experiment

$\checkmark$ A flux limiter does not improve results

$\checkmark$ Using nonlocal fluxes, we get simuation closer to experimental data 


\section{Emitting zones movement for $\mathrm{I}=4 \mathrm{e} 14 \mathrm{~W} / \mathrm{cm} 2$}

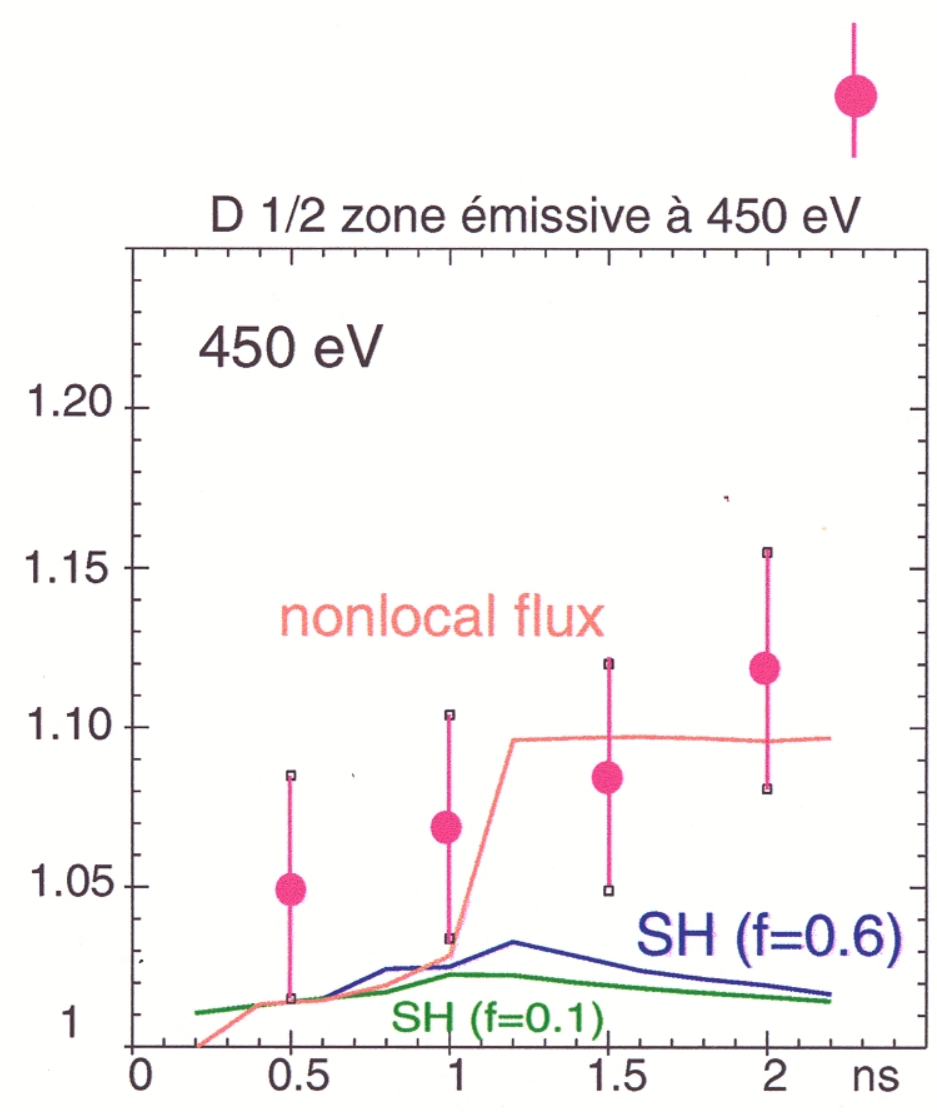

expérimental data

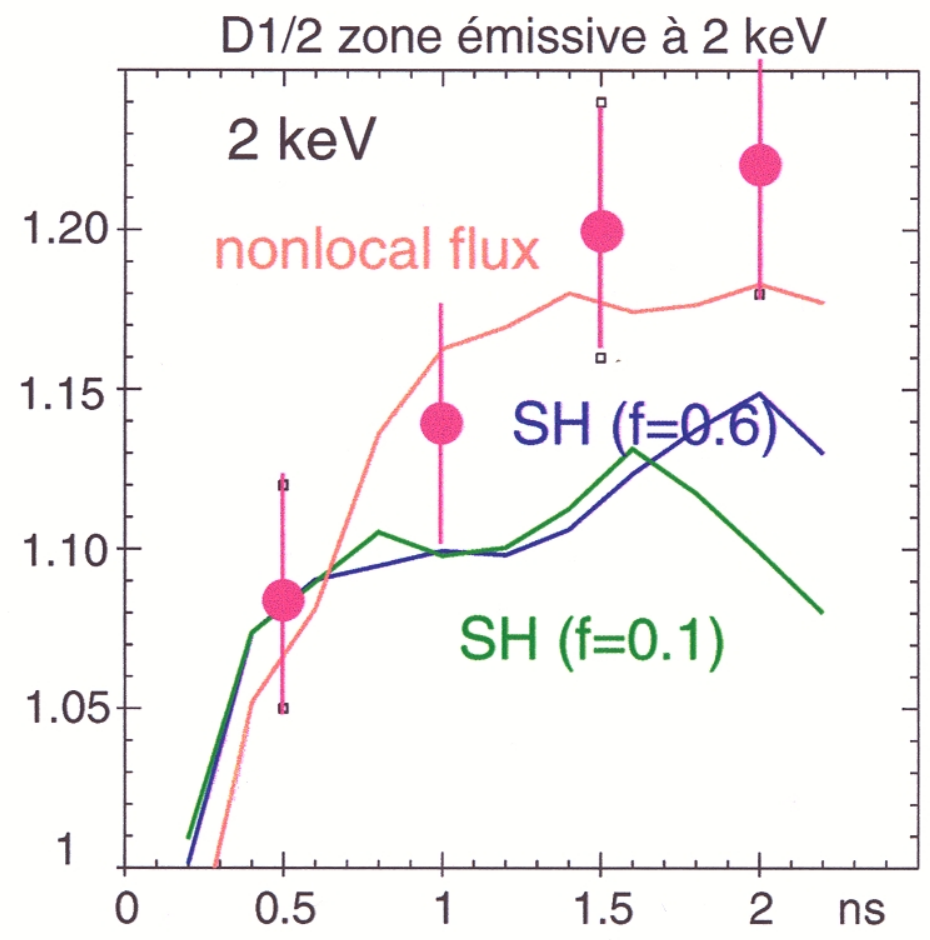

$\checkmark$ Discrepancies between S-H and nonlocal fluxes are reduced but only nonlocal simulation passes through experimental data. 


\section{Emitting zones movement for I = 1e14W/cm2}
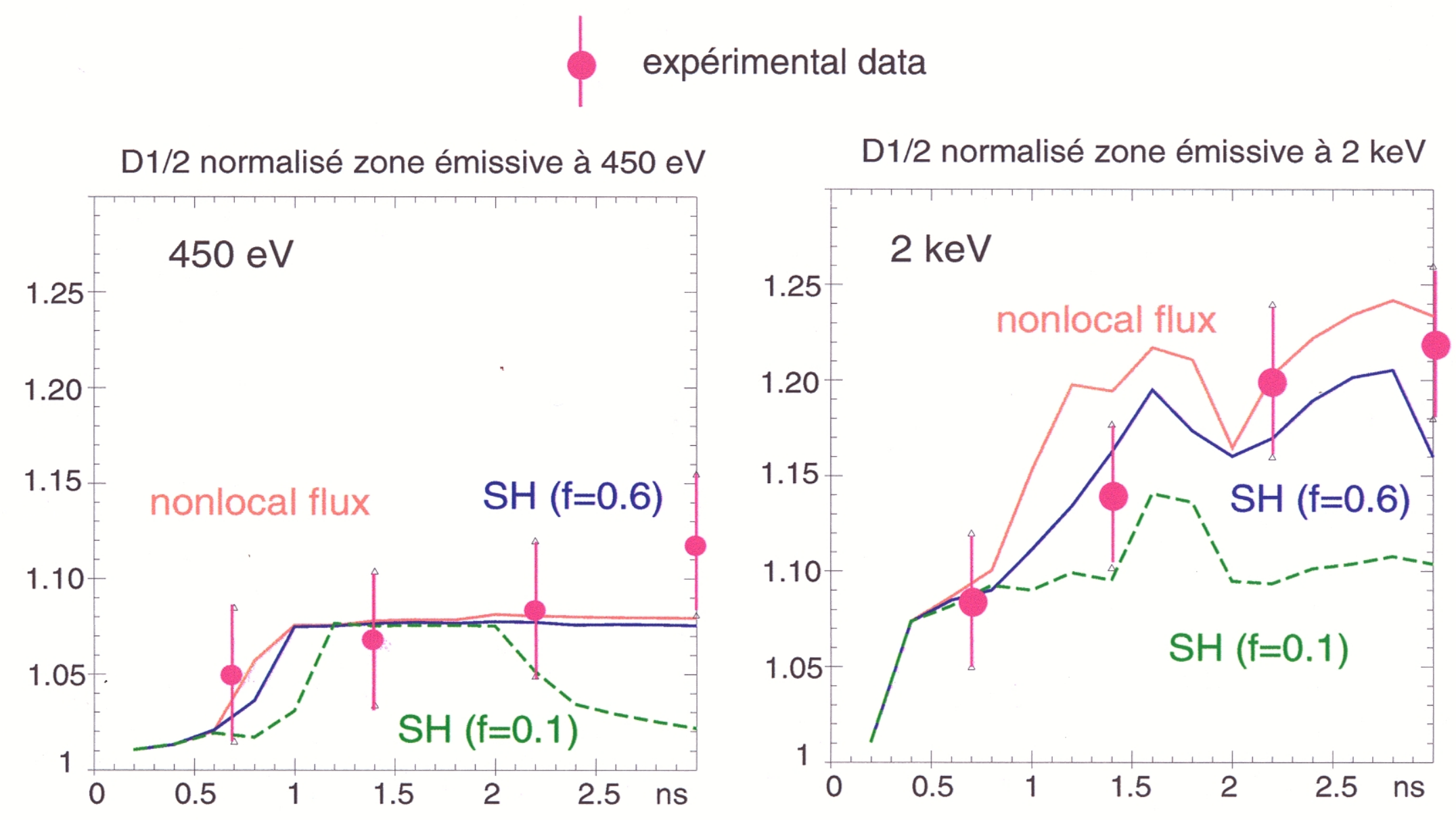

$\checkmark$ Non local flux tends towards $\mathrm{SH}$ flux for low intensity

$\checkmark$ Both models reproduce experiment 


\section{Spherical target : summary}

$\square$ Unlike cavity experiments, only nonlocal effetcs act on heat fluxes

$\square$ If some results like X-ray conversion efficiency can be explain by the use of Spitzer-Harm fluxes, only the nonlocal fluxes reproduce the movement of emitting zones.

$\square$ The variation of laser intensity in experiment allows us to test the convergence of our model to Spitzer-Harm model (low flux). 


\section{Conclusion}

$\square$ The use of Spitzer-Harm fluxes, limited or not, does not allow us to reproduce some experimental results.

$\square$ From one experiment to another, and even from one diagnostic to another, the flux limiter value can be different : interpretation $\Leftarrow \Rightarrow$ prevision

$\square$ Nonlocal fluxes combined with magnetic fields improve simulations and so our understanding of laser plasma experiments (up to now...) 


\section{Laser expériments : Interpretations and predictions}

Ph. NICOLAÏ, D. BABONNEAU, M. BONNEFILLE, B. CANAUD, F. CHAIGNEAU, E. DATTOLO, C. ESNAULT, J-P. JADAUD, S. LAFFITE, M-C. MONTEIL, G. SCHURTZ, M. VANDENBOOMGAERDE, B. VILLETTE, F. WAGON.
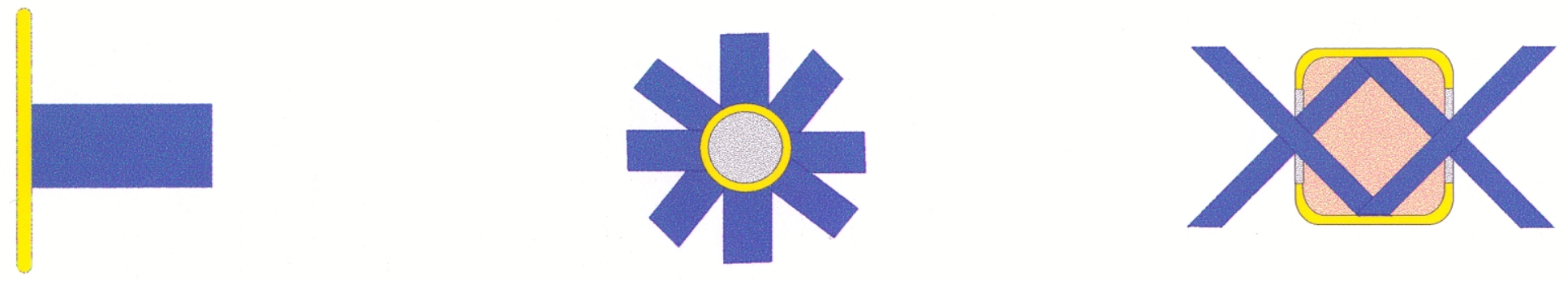


\section{Magnetic fields and nonlocal fluxes}

Effects of each process are less or more large following:

$\square$ experimental geometry [plane-sphérical]

$\square$ observed quantity [Tr, Te, ne, hv ...]

$\square$ plasma zone seen (probed) by diagnostic

The choice of an experiment and diagnostics (can) enables us :

$\square$ to test both effect combined or alone

$\square$ to check our model

$\square$ to improve our understanding of physical processes involved (to improve theory) 


\title{
Planar target (Phebus facility)
}

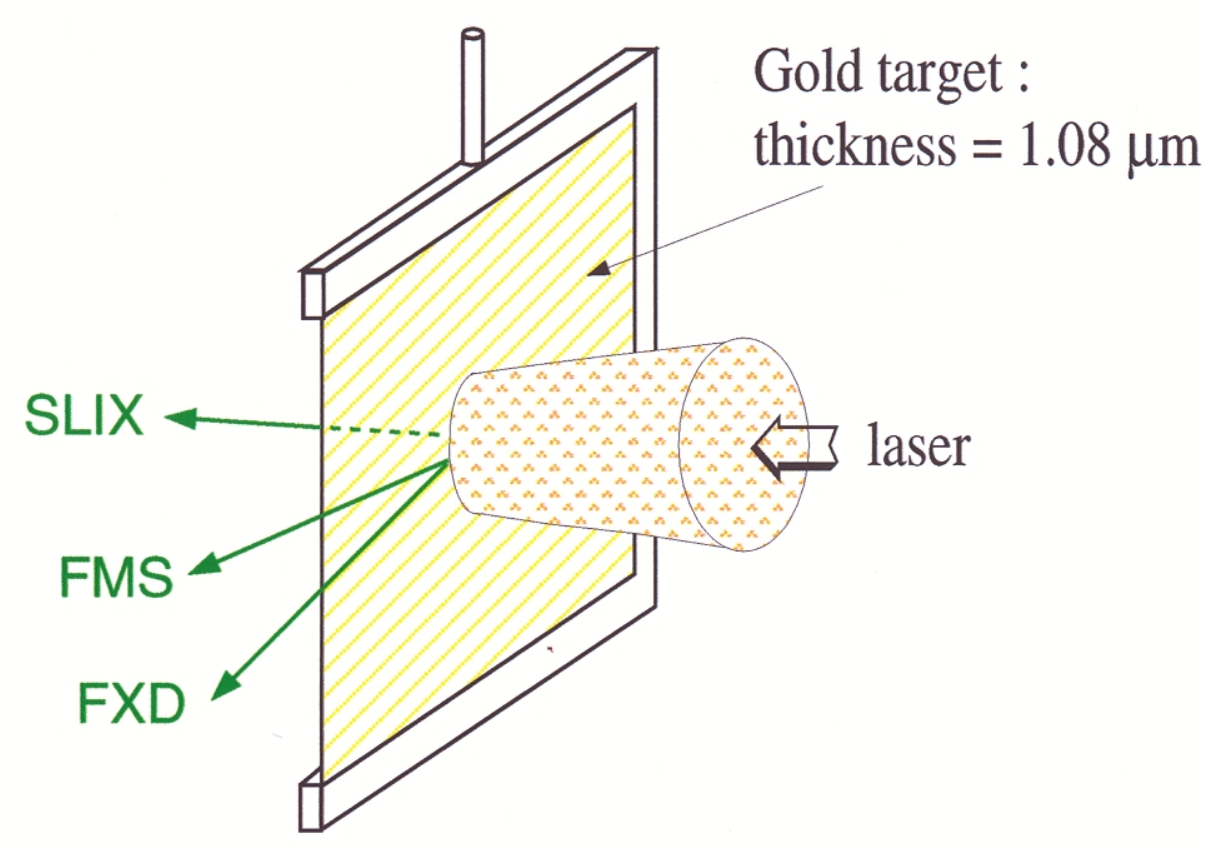

\author{
Laser \\ - smoothed by RPP \\ $-3 \omega$ \\ $-1.5 n s$ square pulse \\ - 3kJ \\ - $\mathrm{FWHM}=340 \mu \mathrm{m}$
}

FMS :streak camera wich images $200 \mathrm{eV}$ X-ray emission perpendicular to laser axis

- provides a time-resolved 1D image

FXD : streak camera wich images above $2 \mathrm{KeV}$ X-ray emission perpendicular to laser axis

- provides a time-resolved 1D image

SLIX : gated microchannel plate detector images $2.5 \mathrm{KeV} \mathrm{X}$-ray (M-shell) from rear side - provides a time-resolved 2D image 


\section{D Simulation of experiment}

target

\section{laser}
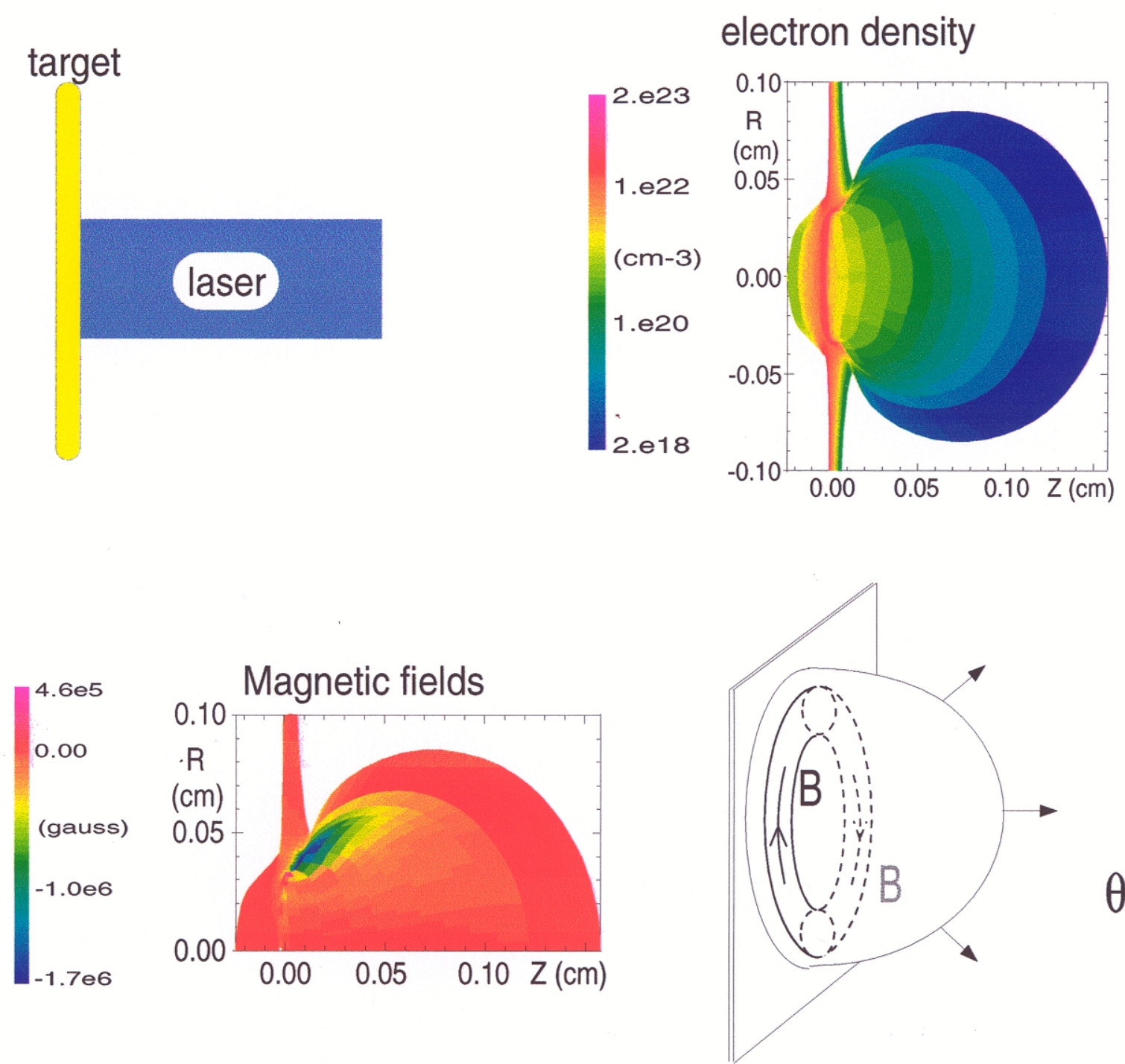

electron température

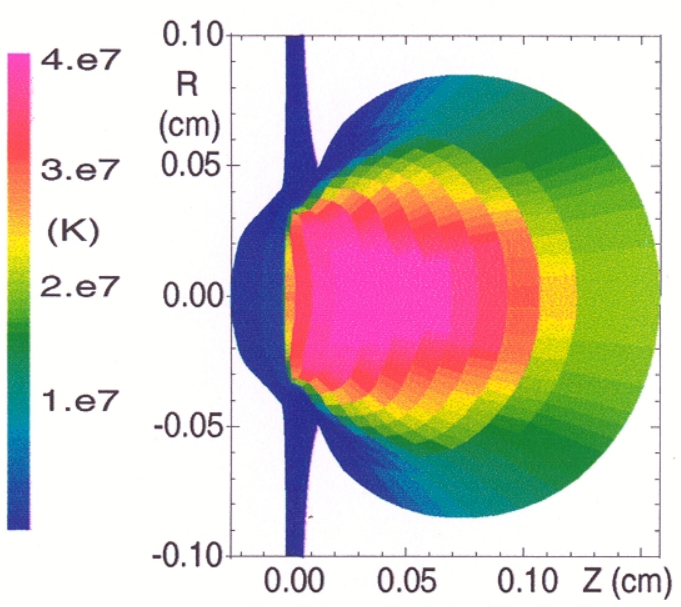

gyrofrequency / collision frequency

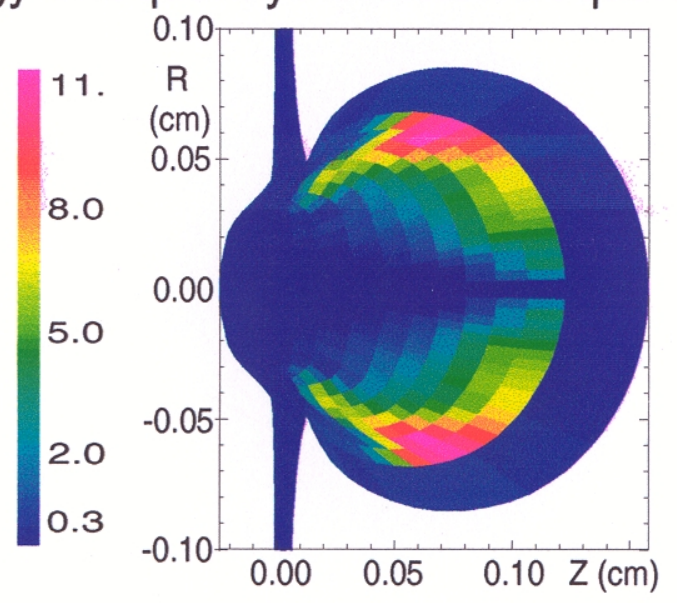




\section{Experiment-simulations comparisons}
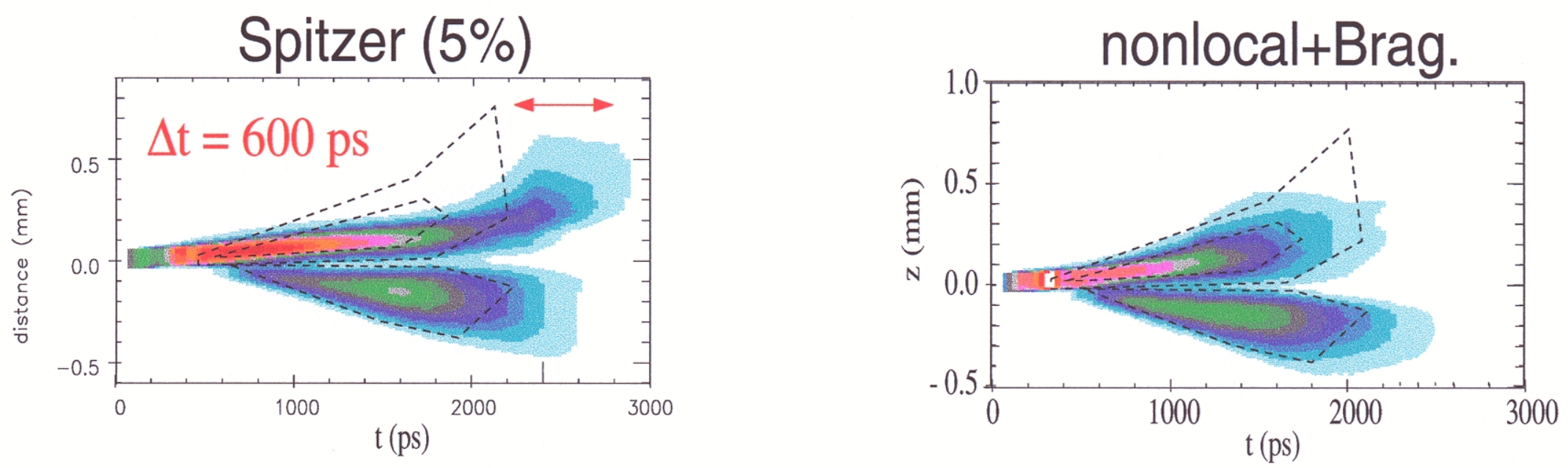

$\checkmark$ Using Spitzer-Harm fluxes, we obtain (front side) an emission too long (600ps).

Second, the slope of the upper part is too small (velocity of the emitting zone).

$\checkmark$ We tried to change limiter value, average between $\mathrm{SH}$ and free streaming, láser parameters, mesh refinement, etc... But no effect on numerical results.

Using B-fields and nonlocal transport, we obtain a emission length shorter and the slope of the upper part is higger and is in good agreement with exp. result . 


\section{Individual effects of each process}
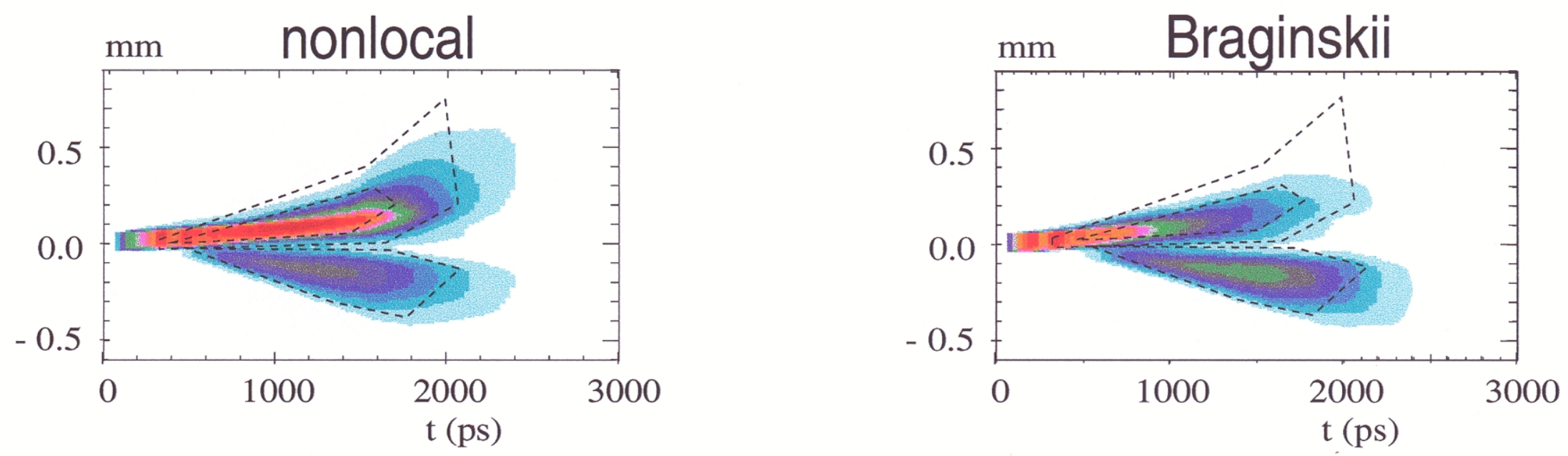

$\checkmark$ Using nonlocal fluxes, we obtain a shortening of emission but not enough to reproduce experiment.The emitting zone shifting (laser side) is correct

$\checkmark$ Using B-fields, we obtain a good length of emision (front side) but the slope of the upper part is too small (slightly)to mach experimental data.

$\checkmark$ So, it seems that combined effects produce the best agreement with experiment 


\section{SLIX Diagnostic}

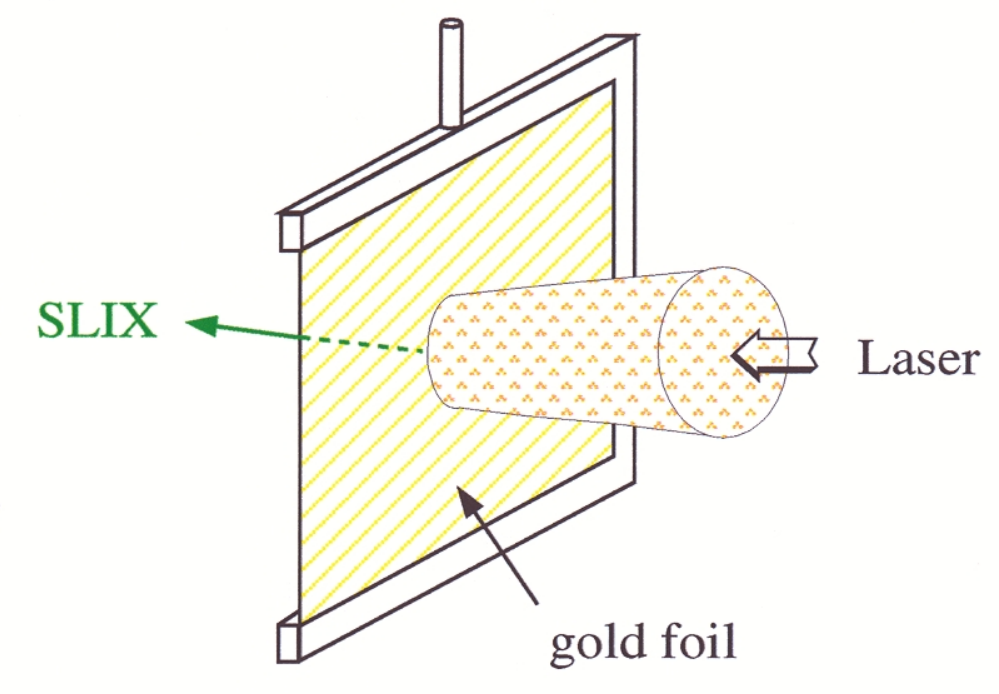

Slix diagnostic takes 'photos' of the rear side in the range about 2.5Kev (M-shell)

Res. $=10 \mu \mathrm{m}$ et $\Delta \mathrm{t}=100 \mathrm{ps}$
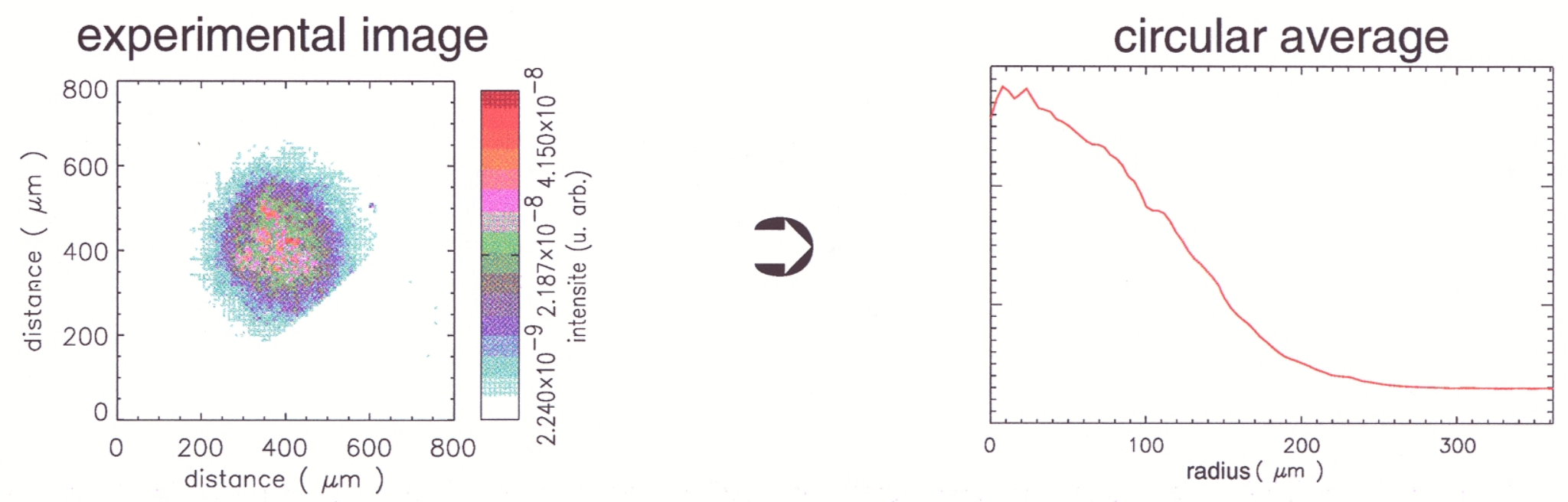

DAM - lle de France 


\section{Expériment-simulation comparisons}

\section{- experiment}
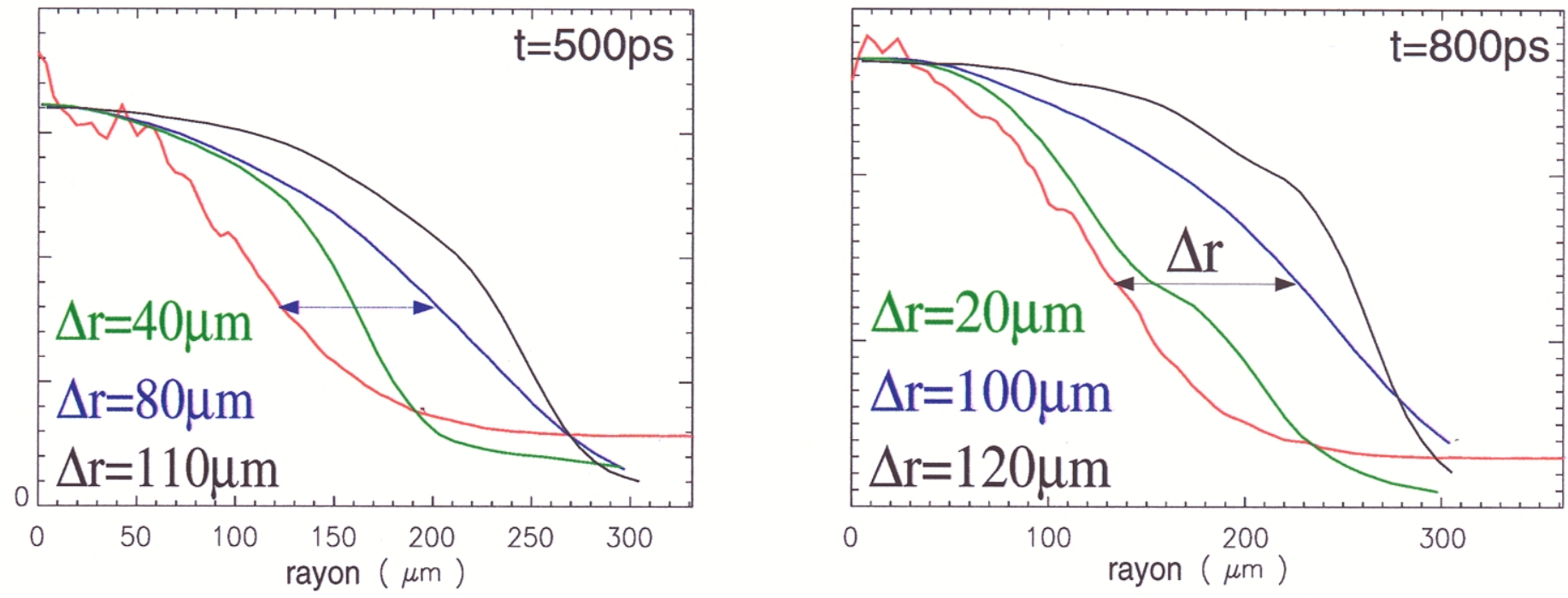

— Spitzer-Harm (5\%) - non local +B - only B

$\checkmark$ Using Spitzer-Harm, we get images too large by about 80 and $100 \mu \mathrm{m}$ at half maximum.

$\checkmark$ B-flieds do not improve results

$\checkmark$ B-flieds and nonlocal fluxes give the best agreement 


\section{Planar target : summary}

$\square$ Using Spitzer-Harm fluxes, simulations are unable to reproduce all diagnostics, whatever variations of flux limiter, laser parameters, zoning,...

$\square$ Both non local and magnetic effects improve simulation results without arbitrary parameter (flux limiter).

$\square$ If we only use one process, we get some improvements but not enough to match experimental data. 


\section{Hohlraum experiments (NOVA / OMEGA facilities)}
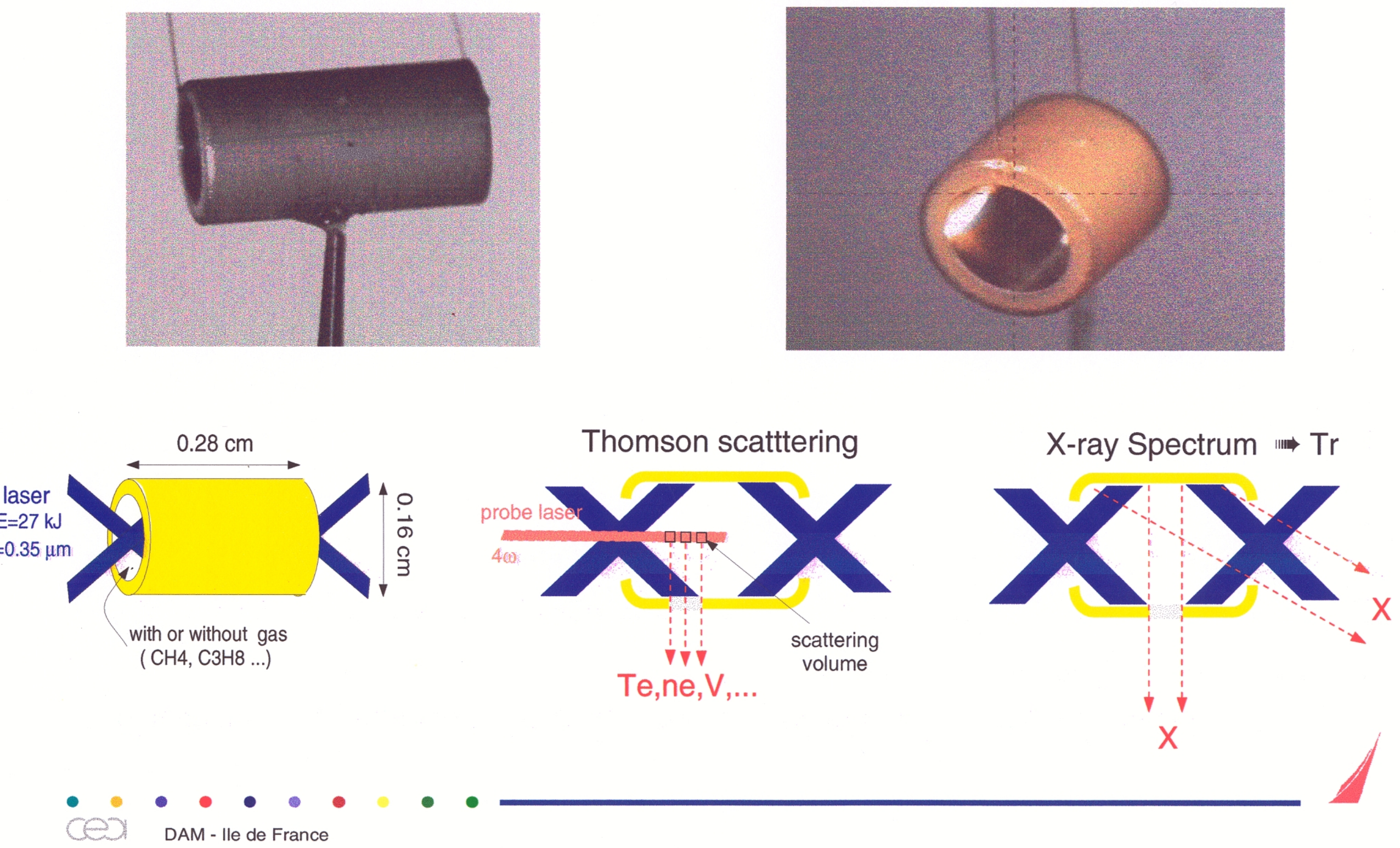


\section{Gas-filled Hohlraum (nova exp.)}

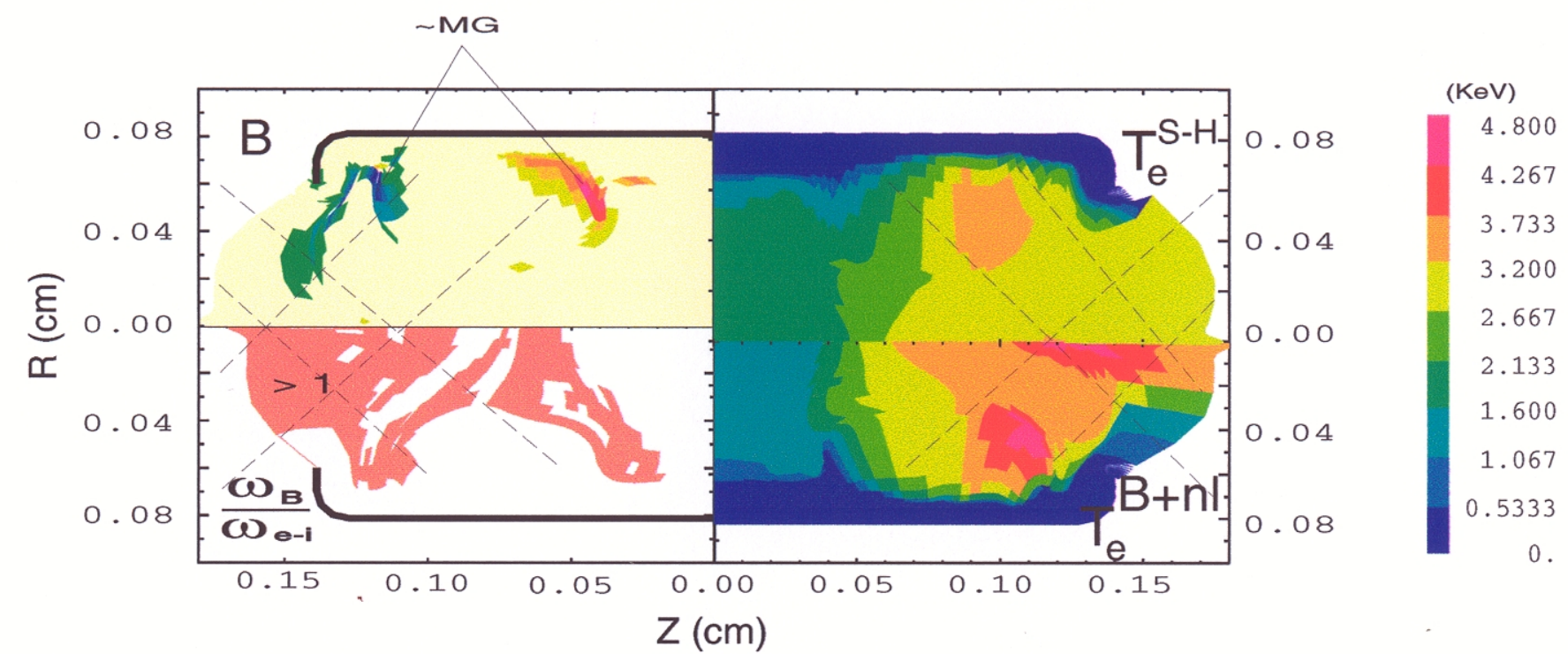

$\checkmark$ Simulations with radiation-hydrodynamic code $(\mathrm{FCl} 2)$ predict $\mathrm{B}$ fields of order of $1 \mathrm{MG}$

$\checkmark$ Hall parameter exceeds unity in a large zone of the hohlraum (B effects)

$\checkmark$ Calculations with SH or with our model (w/o flux limiter) lead to differents results :

- higher electron temperature in off-axis region (from $3.5 \mathrm{keV}$ to $4.8 \mathrm{keV}$ )

- larger temperature gradient along the axis (beams crossing)

experimental data of electron temperature from Thomson scattering seem to confirm large gradients along z-axis and temperature in order of $5 \mathrm{KeV}$ close to LEH 


\section{Hohlraum experiment}

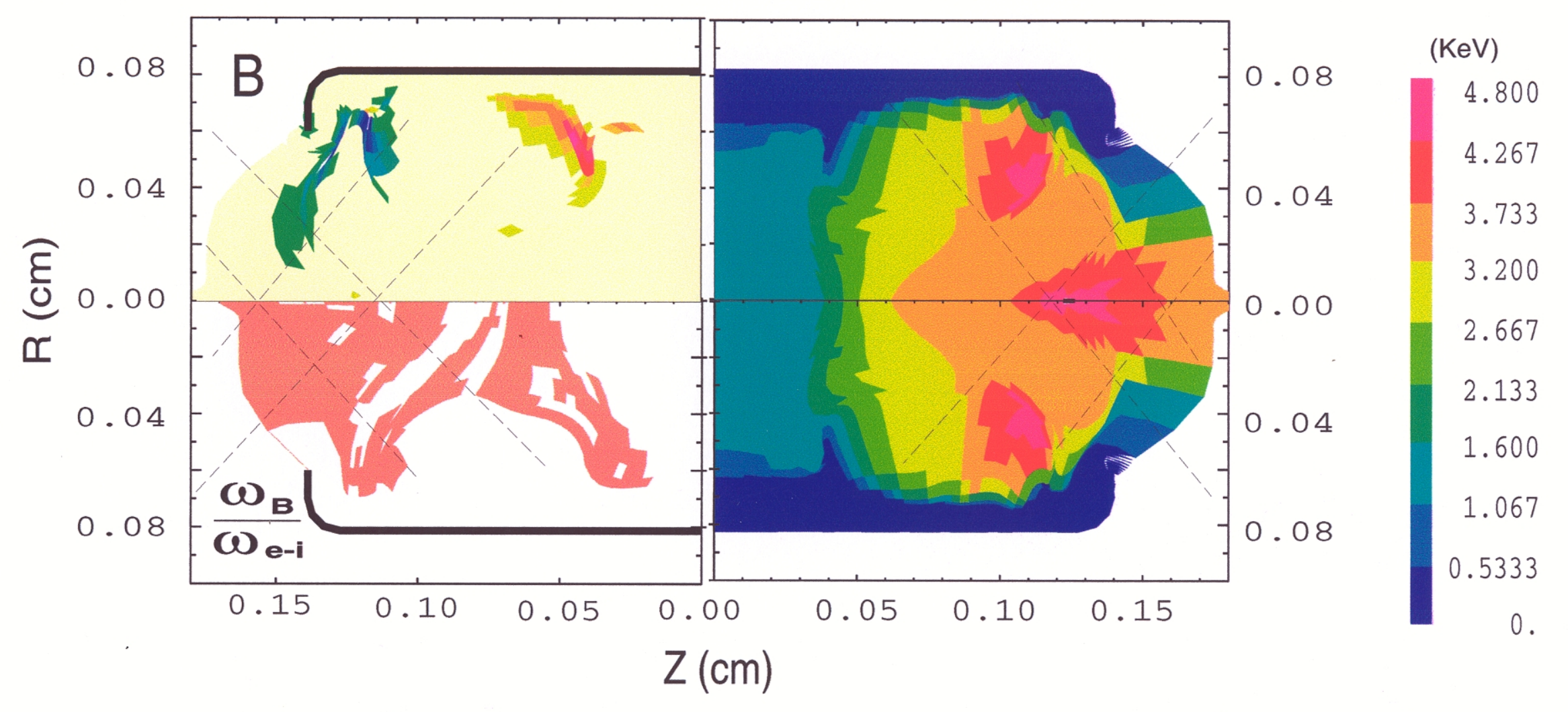




\section{Hohlraum experiments}

$\checkmark$ In a hohlraum, magnetic fields quickly spread in the whole plasma.

$\checkmark$ B-fields reduce or cut nonlocal effects. The deformation of the spherical part of the distribution function due to nonlocal effects may be reduced or cancelled by B-fields.

$\checkmark$ Simulations without nonlocal fluxes but with B effects can correctly reproduce, in this case, experimental results. 


\section{B-fields (nonlocal effects) can modify density}
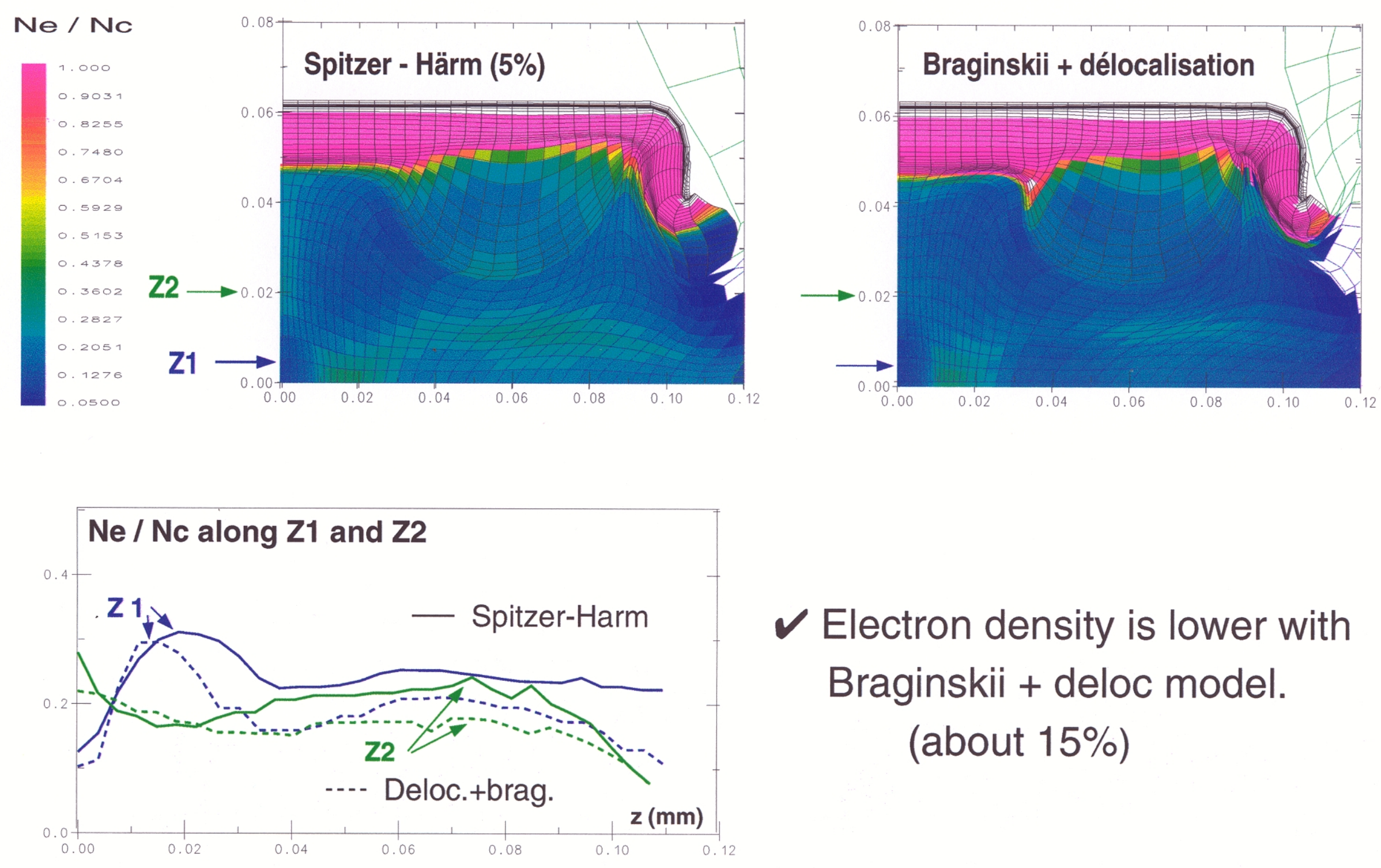

$\checkmark$ Electron density is lower with Braginskii + deloc model.

(about 15\%) 


\section{Same radiation temperature versus formula}

$\mathrm{eV}$
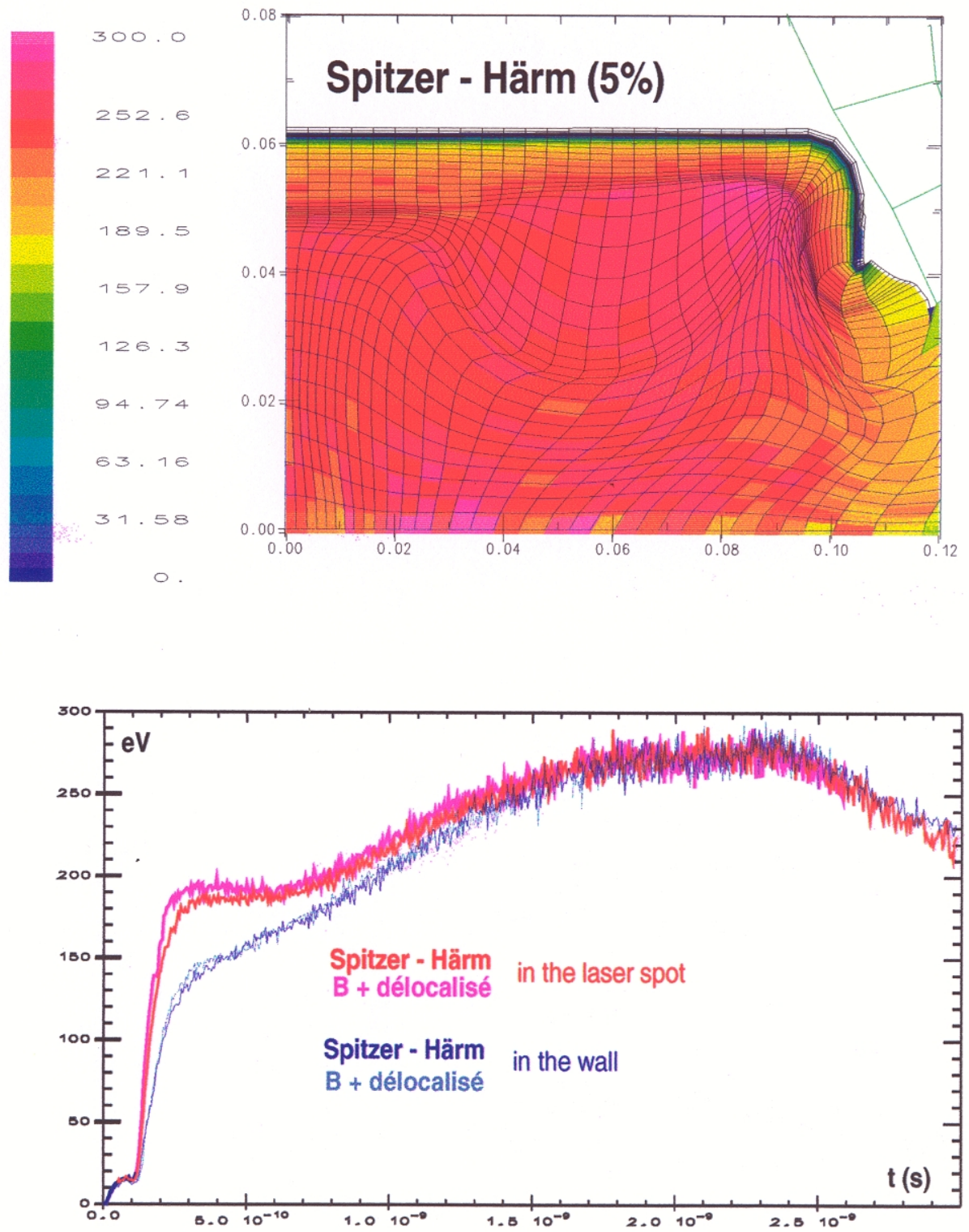

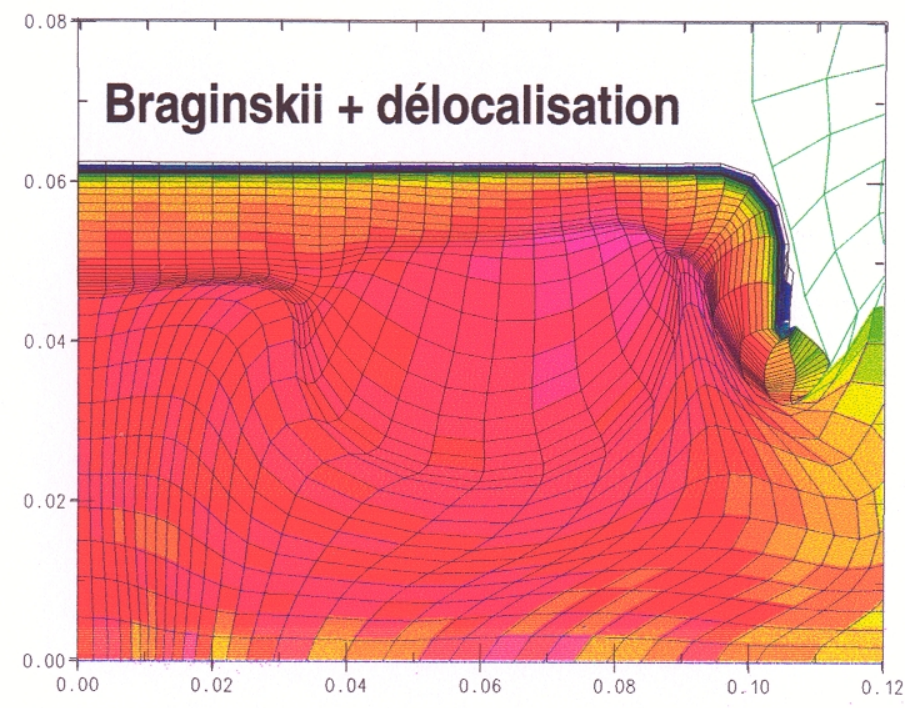

$\checkmark$ same Tr but w/o flux limiter: (good value)

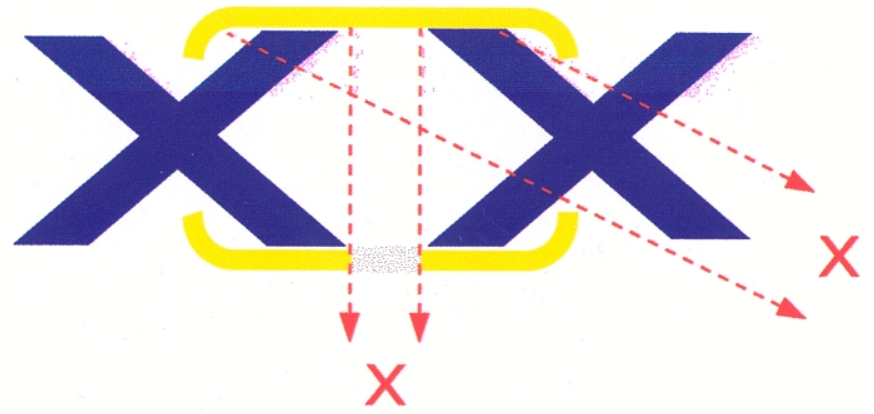

$\checkmark$ So X-ray Diagnostics simulation correct? 


\section{Our electron conduction model can modify hydrodynamic motion}

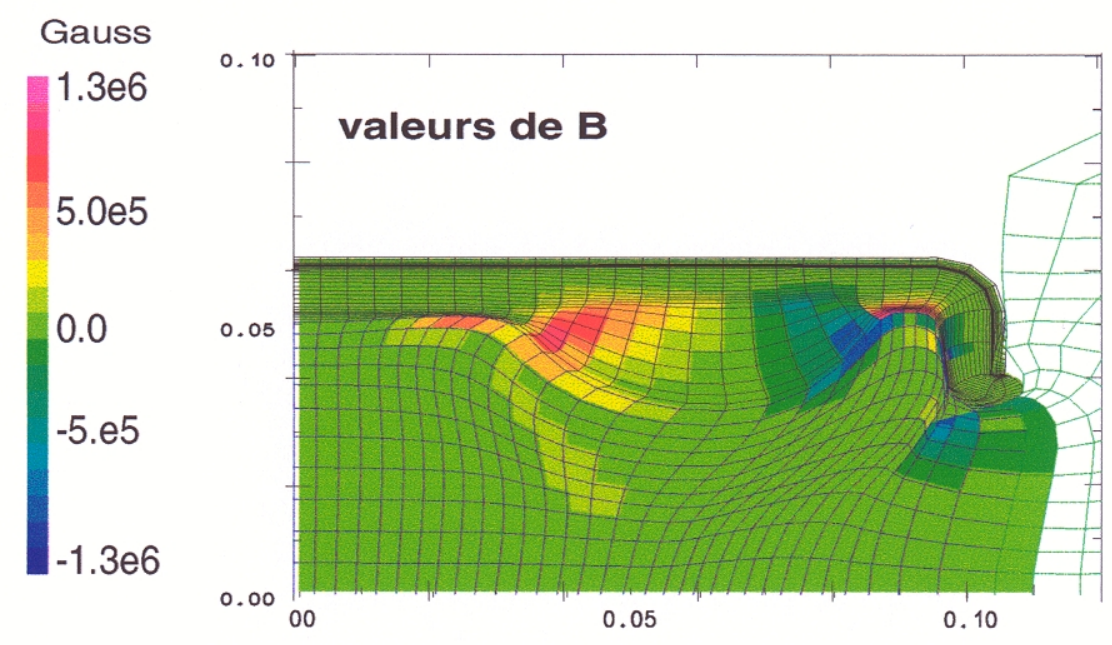

$\checkmark$ heating and expansion of LEH w/o B-fields -> X-ray emission of this zone
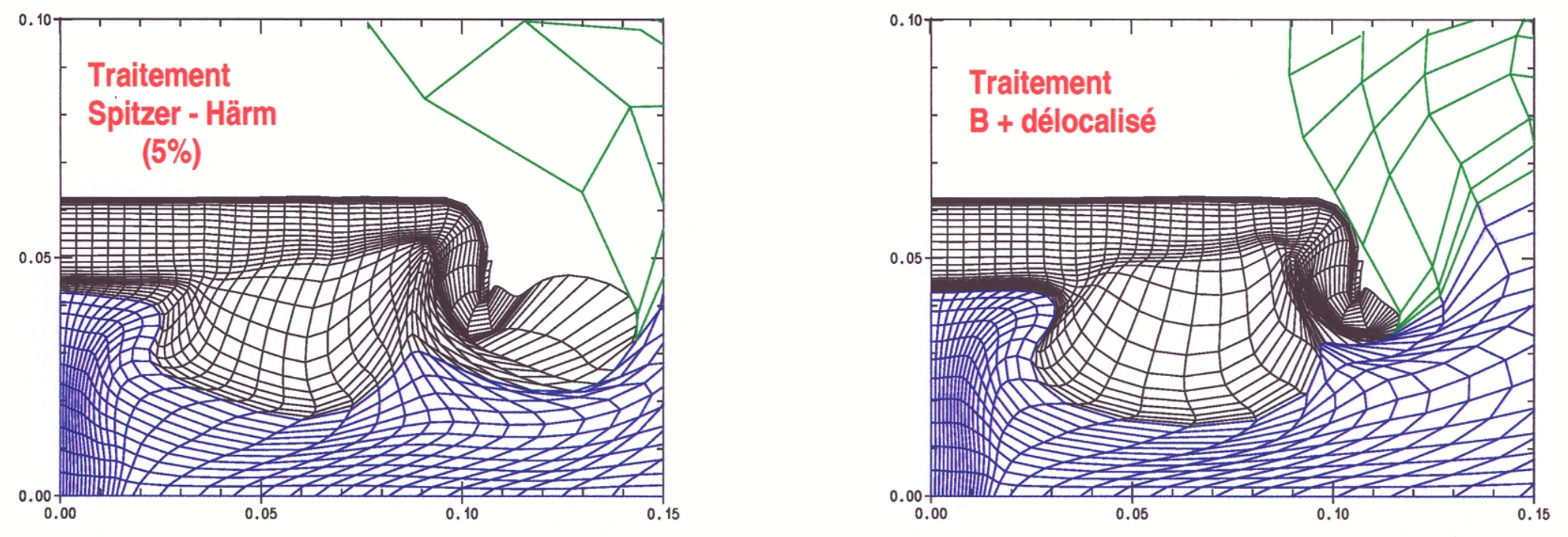

DAM - lle de France 


\section{Hydrodynamic effect on X-ray emission}
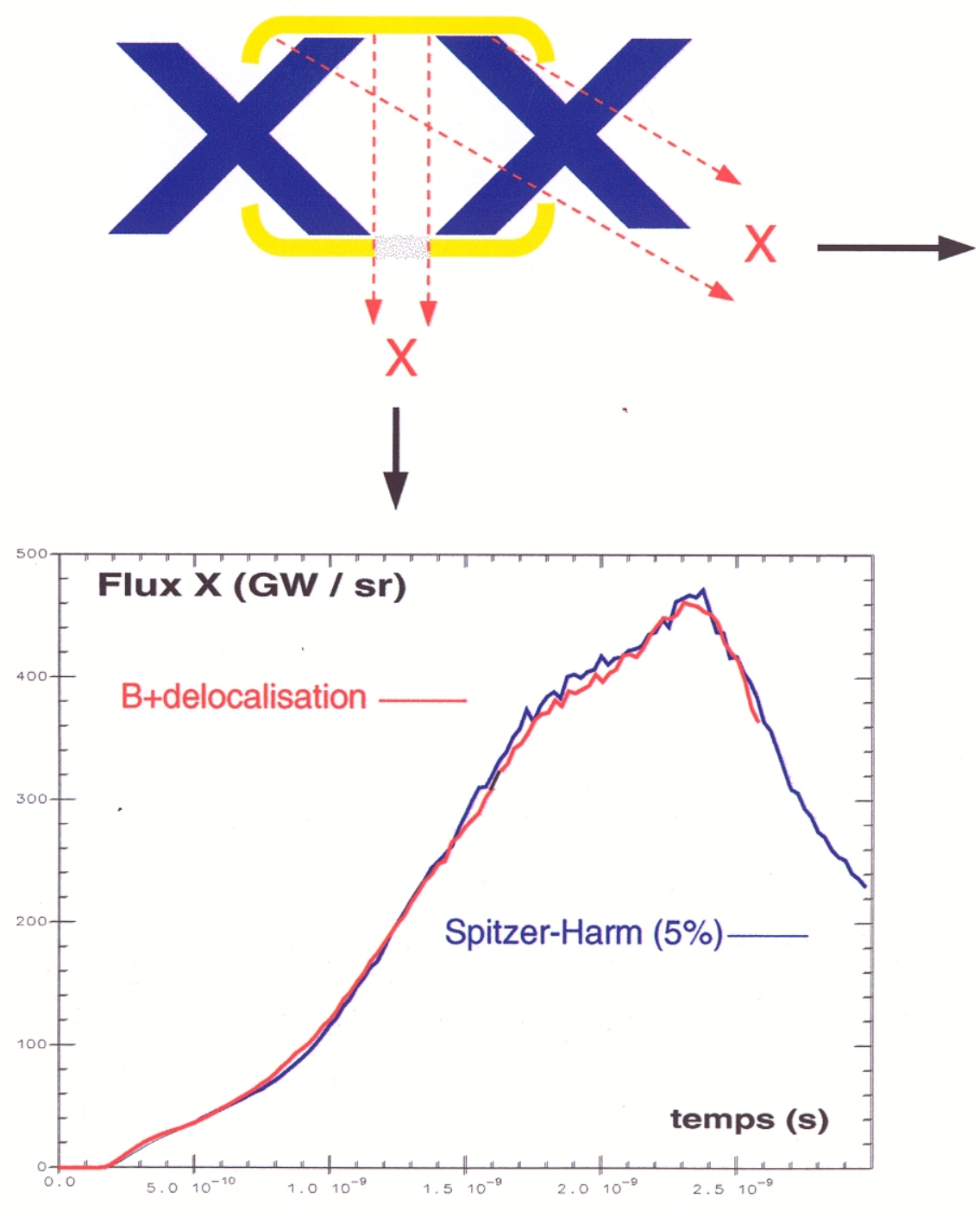

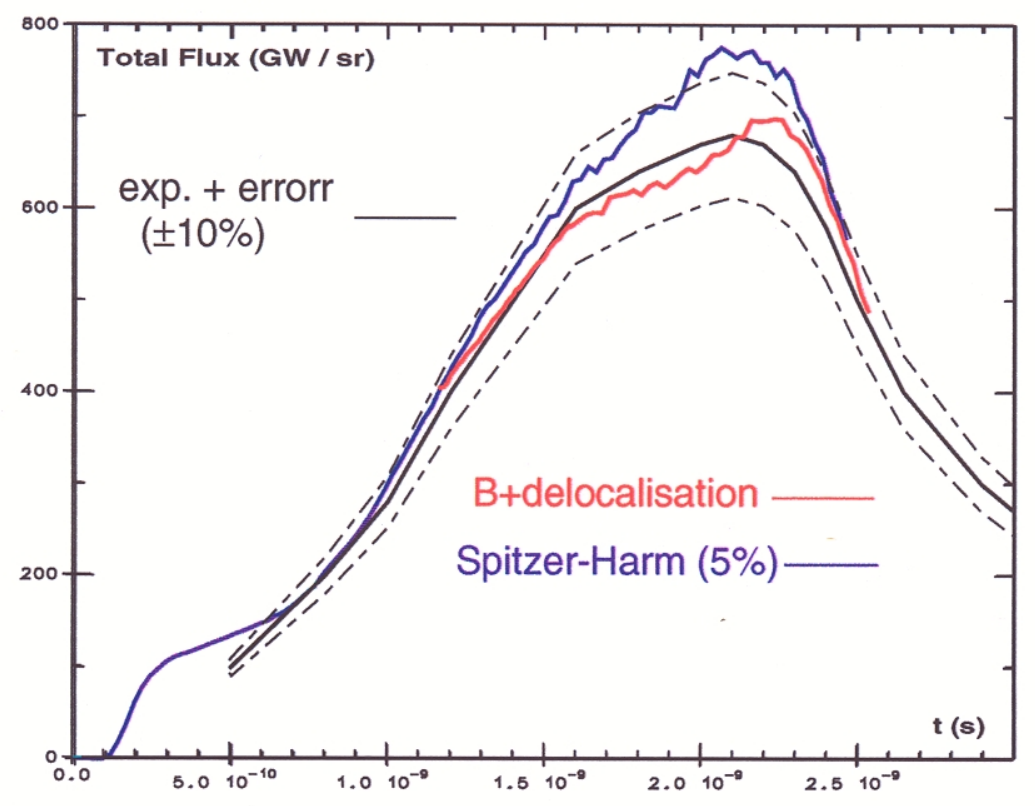

$\checkmark$ For Dante, both heat flux models give similar results

$\checkmark$ B-fields reduce X-ray emission of 'LEH' and enable us to better reproduce exp. data

Both simulations are inside error bars 


\section{Effects of our model on LMJ cavity}

Max. laser power $=400 \mathrm{TW}(16 \mathrm{~ns}) ;$ Laser energy $=1,4 \mathrm{MJ}$

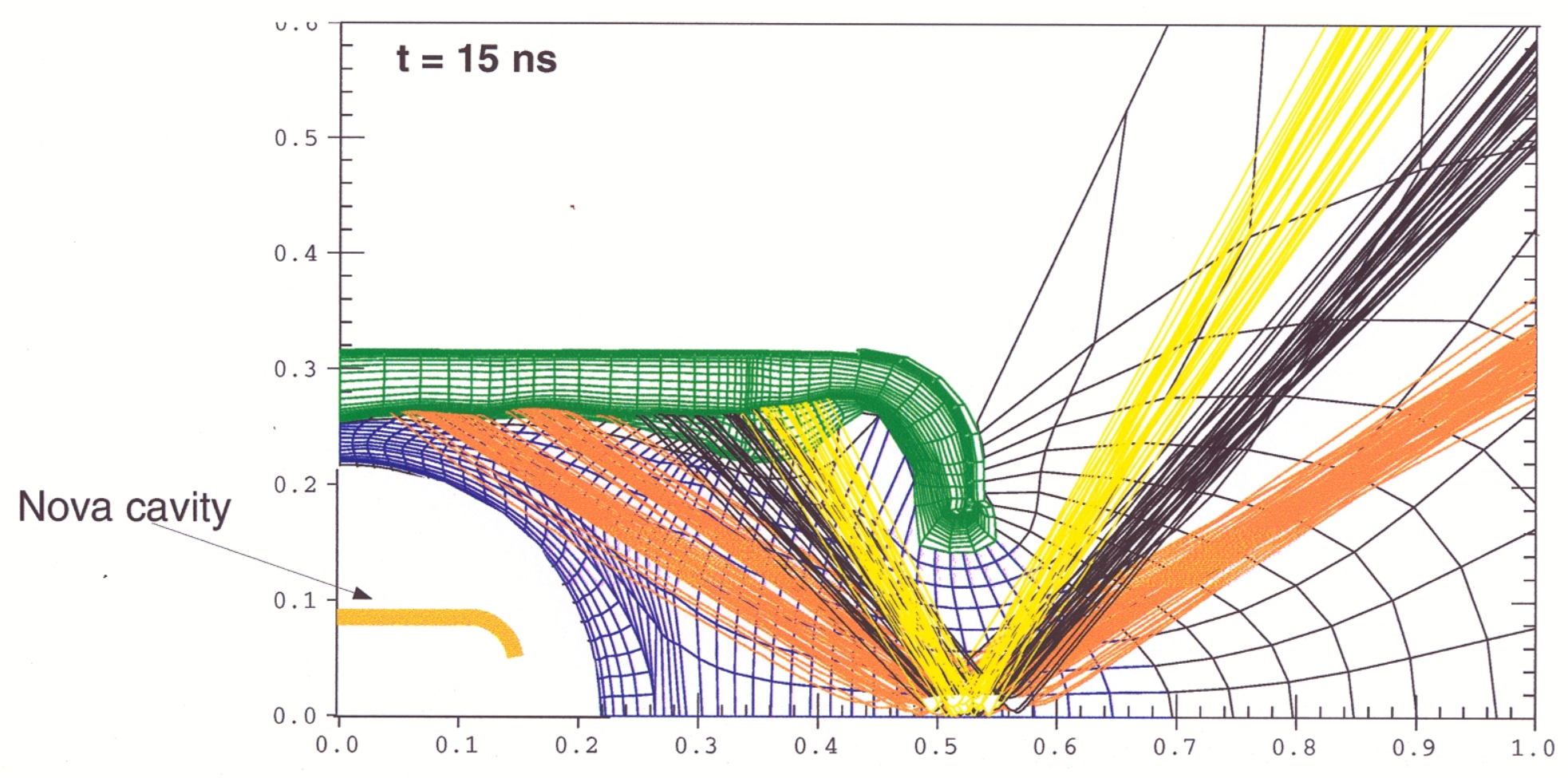




\section{Magnétic fields at 16 ns (max. laser power)}

B effects are important in a large part of the cavity
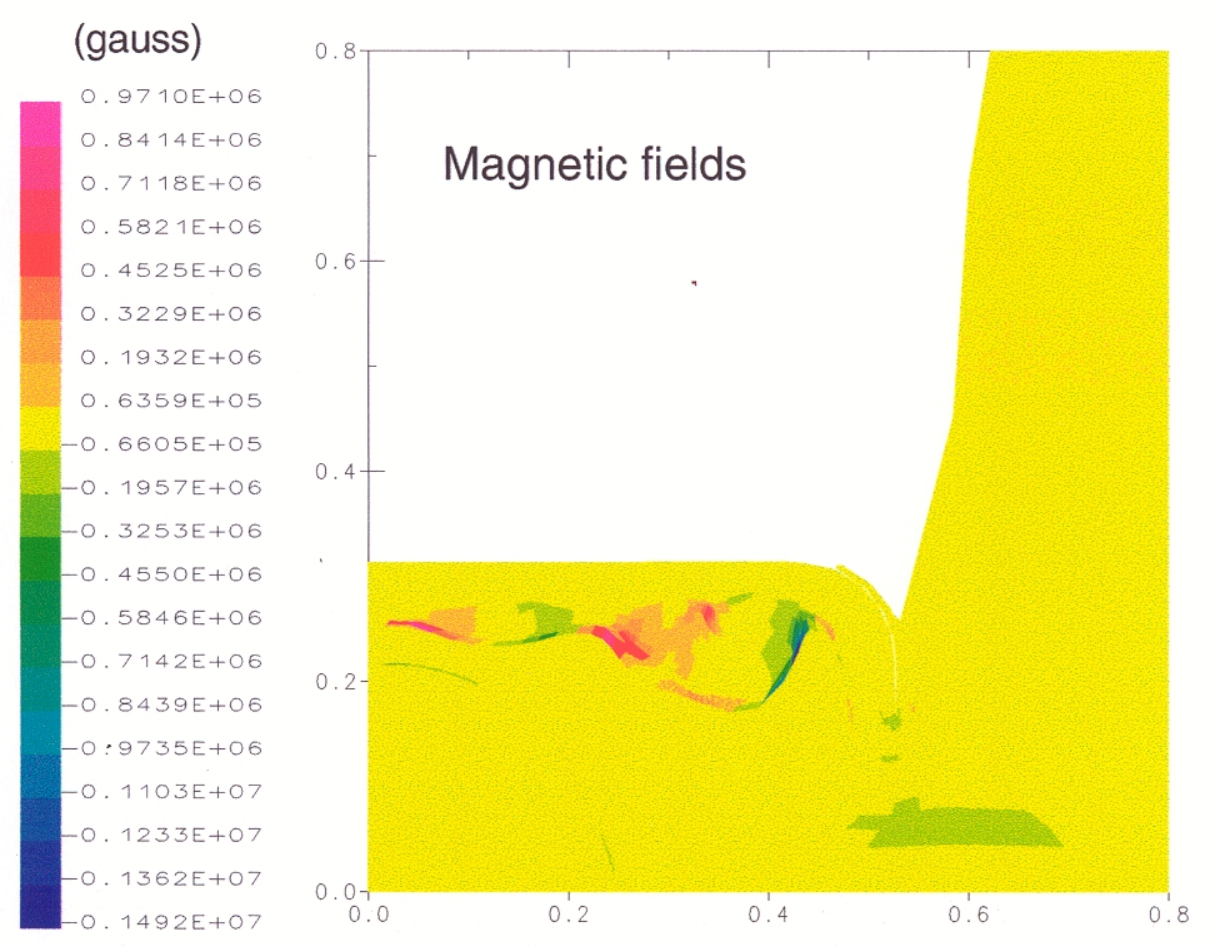

We expect some modifications for

$\checkmark$ electron température

$\checkmark$ ion température

$\checkmark$ density

Jionization

$\checkmark$ hydrodynamic speed

$\checkmark \ldots$ 


\section{Electron temperature color map at $t=16 \mathrm{~ns}$}

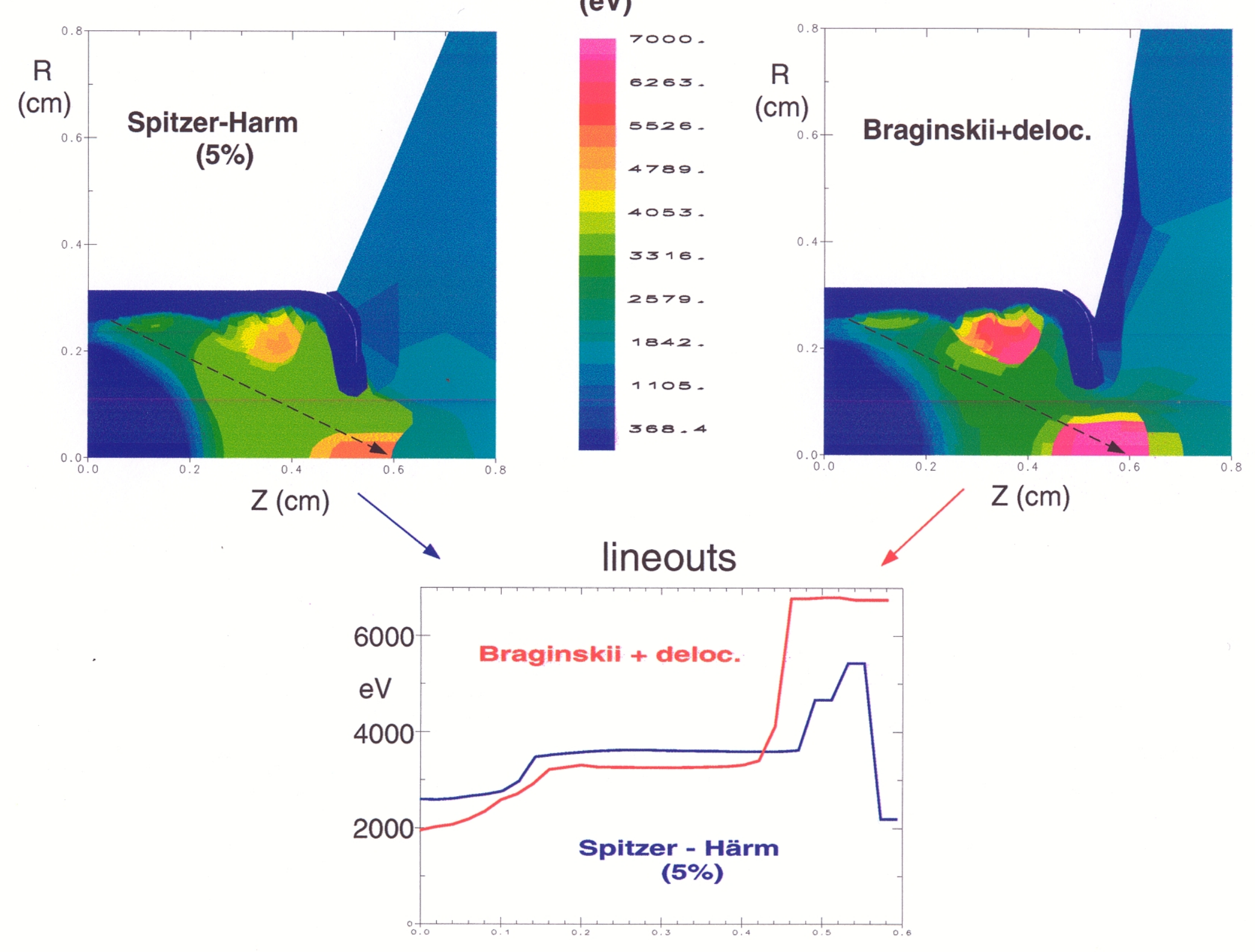

(eV) 


\section{Electron density map at $\mathrm{t}=16 \mathrm{~ns}$}

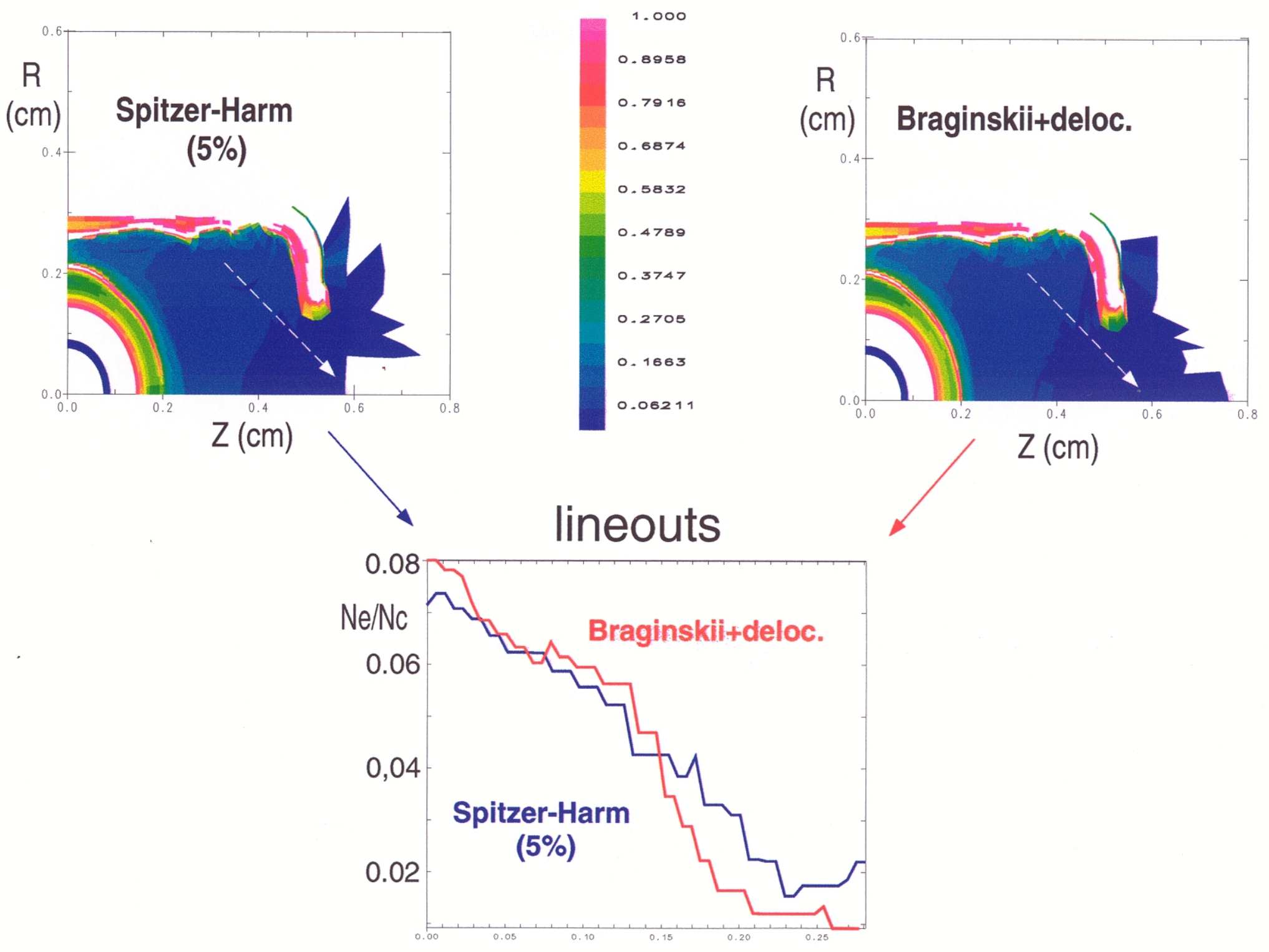




\section{X-rays non uniformities on the ablation front}

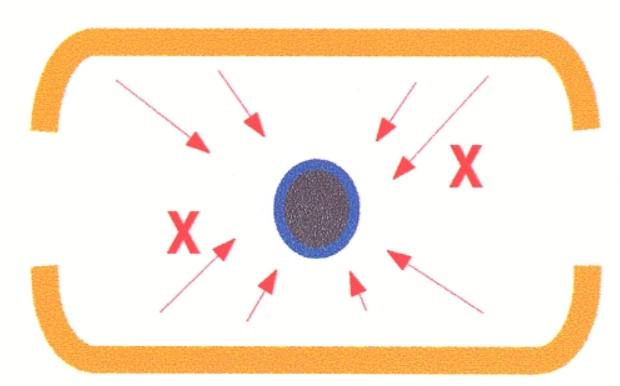

Expansion of the X-ray flux on a Legendre polymomial basis

mode 2

mode 4

mode 6
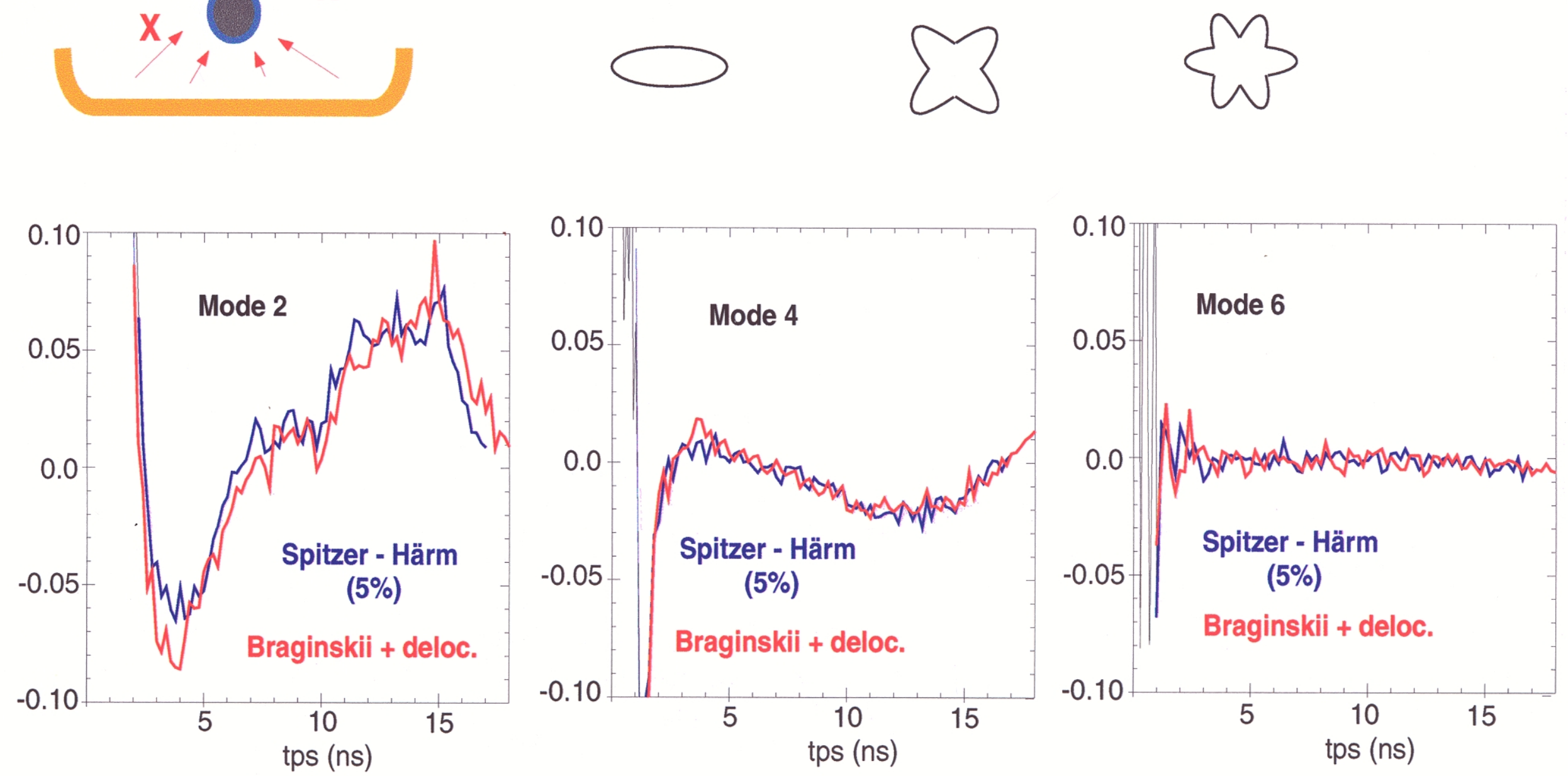

!neither beam-phasing nor pointing optimization ! 


\section{Cavity : summary}

$\square$ Simulations using magnetic fields and nonlocal fluxes match Thomson

scattering results unlike simulations with $\mathrm{S}-\mathrm{H}$ fluxes.

$\square$ The fields diffusion inside cavity reduce non-local effects.

$\Rightarrow$ Using Braginskii fluxes, simulation passes through expérimental error bars

$\square$ Hydrodynamics quantities $(\mathrm{Te}, \mathrm{Ti}, \mathrm{Ne}, \mathrm{V}, \ldots)$ can be hardly modified

by magnétics fields $\Rightarrow$ effects on others processes (Laser Plasma Interaction)

$\square$ The radiation temperature and the irradiation symetry of micro-ballon are not affected by our model $\Leftrightarrow$ limited Spitzer-Harm $(\mathfrak{f} \sim 5 \%)$ 


\section{Spherical target (Omega facility)}

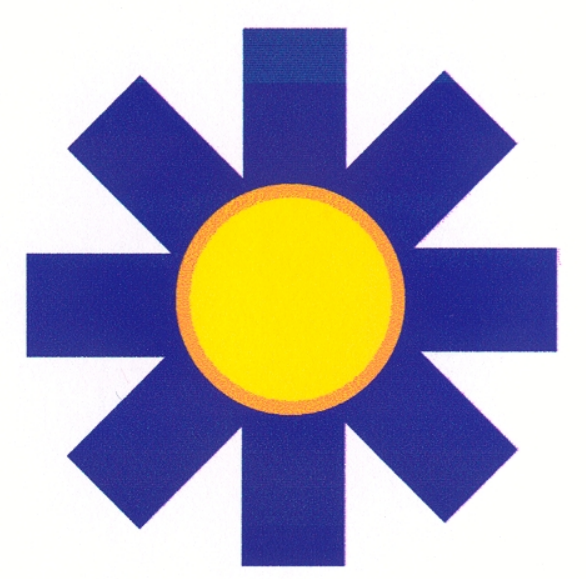

$\checkmark \mathrm{CH}$ targets $(950 \mu \mathrm{m})$ cover with gold $(2.5 \mu \mathrm{m})$

$\checkmark$ laser : $-3 \omega$

- intensities from 1 e13 to $1 \mathrm{e} 15 \mathrm{~W} / \mathrm{cm} 2$

- square pulses for from 1 to 4 ns

- $\mathrm{fwhm} \approx 500 \mu \mathrm{m}$
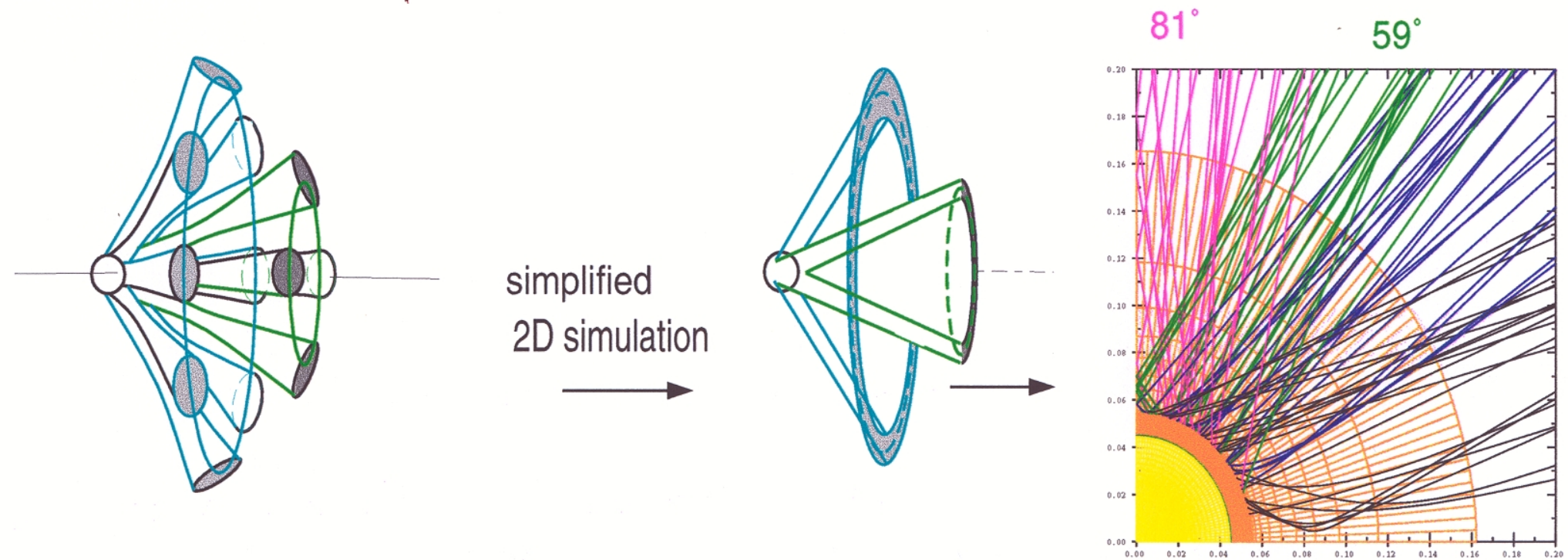

$\checkmark$ X-ray Diagnostics : spectrum, conversion efficiency, imaging with spectral resolution 


\section{Influence of self-generated magnetic fields}

$\boldsymbol{\sim}$ Crossed gradients Te-Ne can create B-fields

$\checkmark$ For this geometry, with an isotropic irradiation, gradients are collinear $->$ no B-fields

$\checkmark$ In experiment, we can have unbalanced laser power between beams (cones)

$$
\mathrm{I}=1.4 \mathrm{e} 14 \text {, at } 21^{\circ}(-14 \%) \text {, at } 42^{\circ}(+2 \%) \text { at } 58^{\circ}(-0.3 \%) \text { at } 81^{\circ}(+6 \%) \text { worst case }
$$
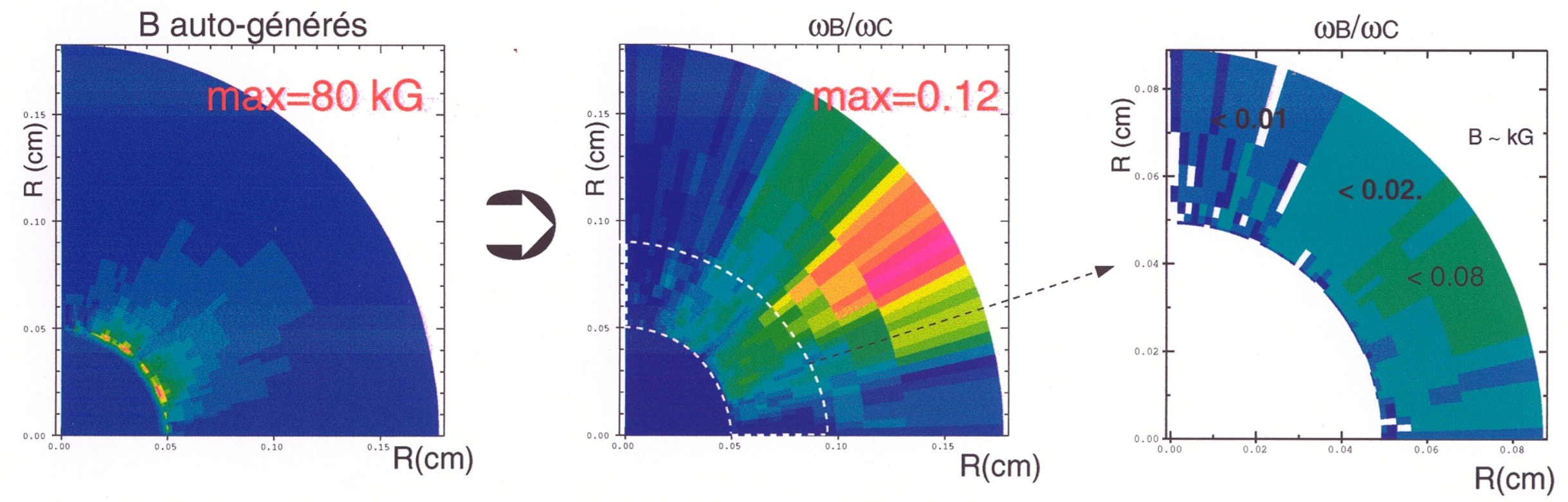

$\rightarrow$ No influence of magnetic fields (same res. w or w/o B)

$\Rightarrow$ Only nonlocal flux acts on plasma in these experiments 


\section{X-ray Conversion}

\section{X-ray Energy / Absorbed laser energy}

$\checkmark$ We test the influence of heat flux on X-ray conversion

$\checkmark$ Expérimental data come from several experiments

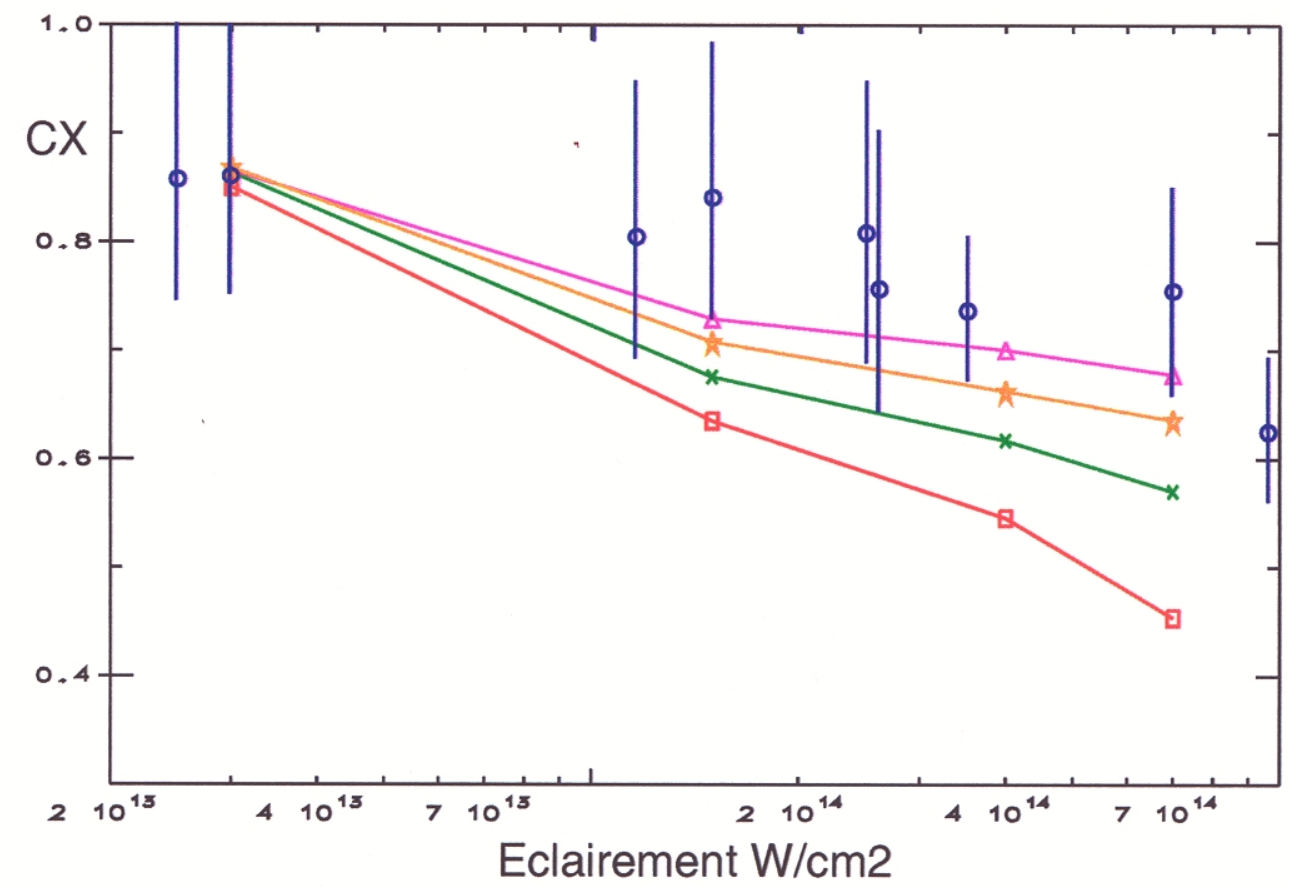

- Experiment

- Spitzer-Harm $f=0.6$

- Spitzer-Harm $\mathbf{f = 0 . 1}$

- Spitzer-Harm $\mathrm{f}=\mathbf{0 . 0 3}$

- non-local

$\checkmark$ The slightly limited $\mathrm{SH}$ flux and the nonlocal flux reproduce experimental data 


\section{Heat fluxes for high and low laser intensities}

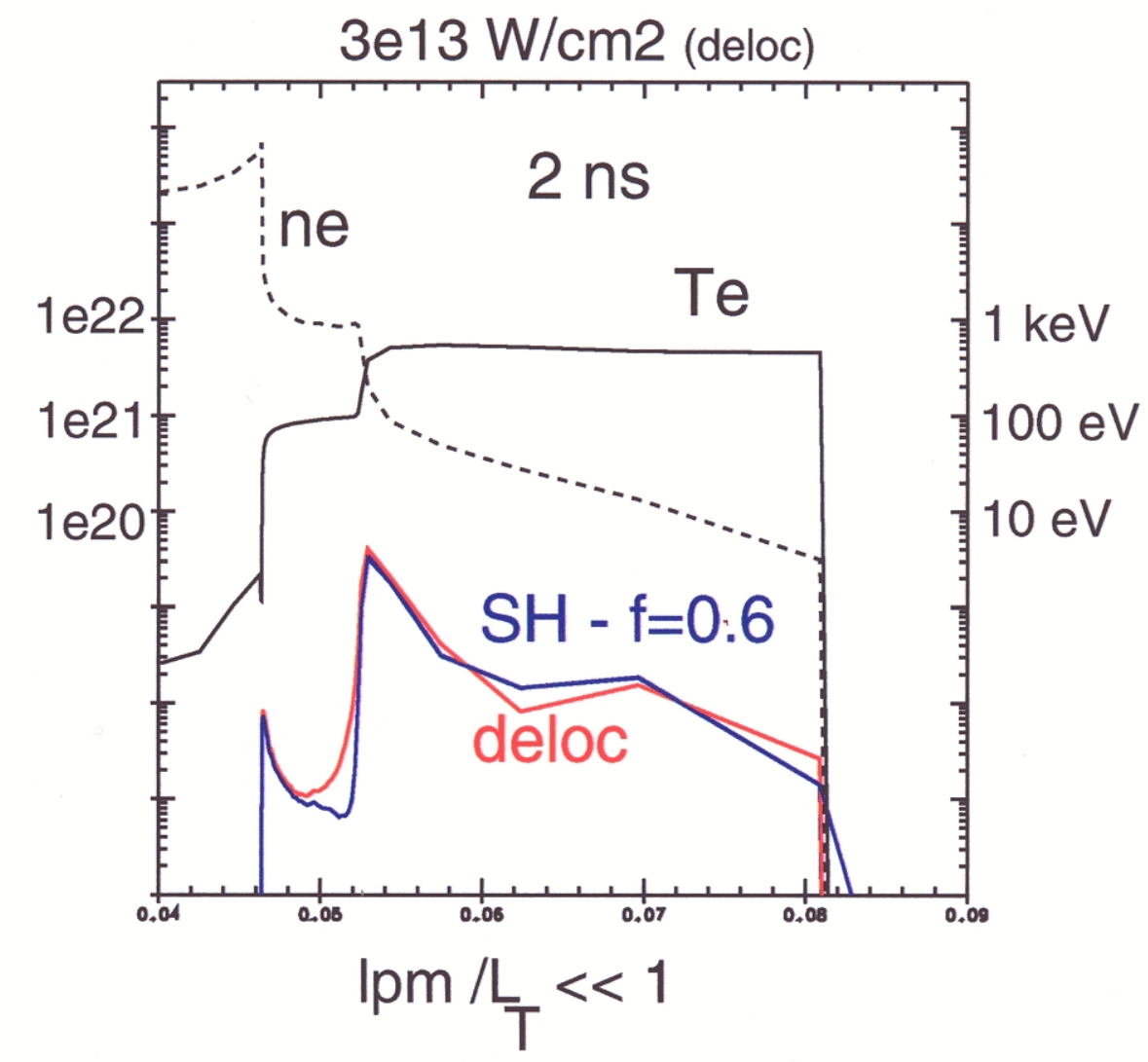

- low temperature, smooth gradient, short

e.m.f.p, the nonlocal flux tends towards

Spitzer-Harm flux.

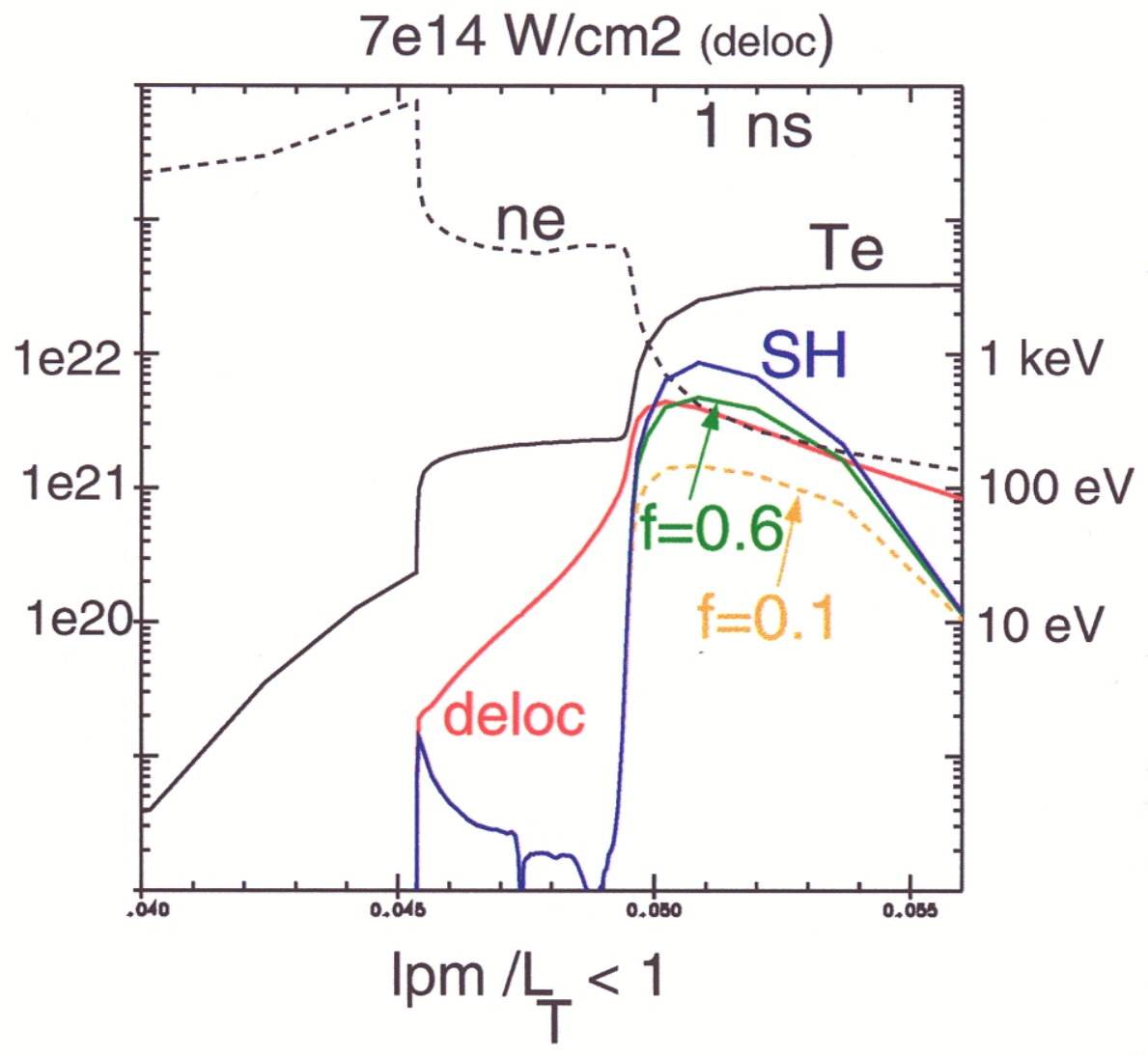

-high température, sharp gradient, long e.m.f.p., the flux is nonlocal and different from Spitzer-Harm flux. 


\section{Simulation of X-ray imaging}

\section{Characterize plasma expansion}

From 2D hydrodynamic computations, we can simulate diagnostic (post-process)

emission at $2 \mathrm{keV}$
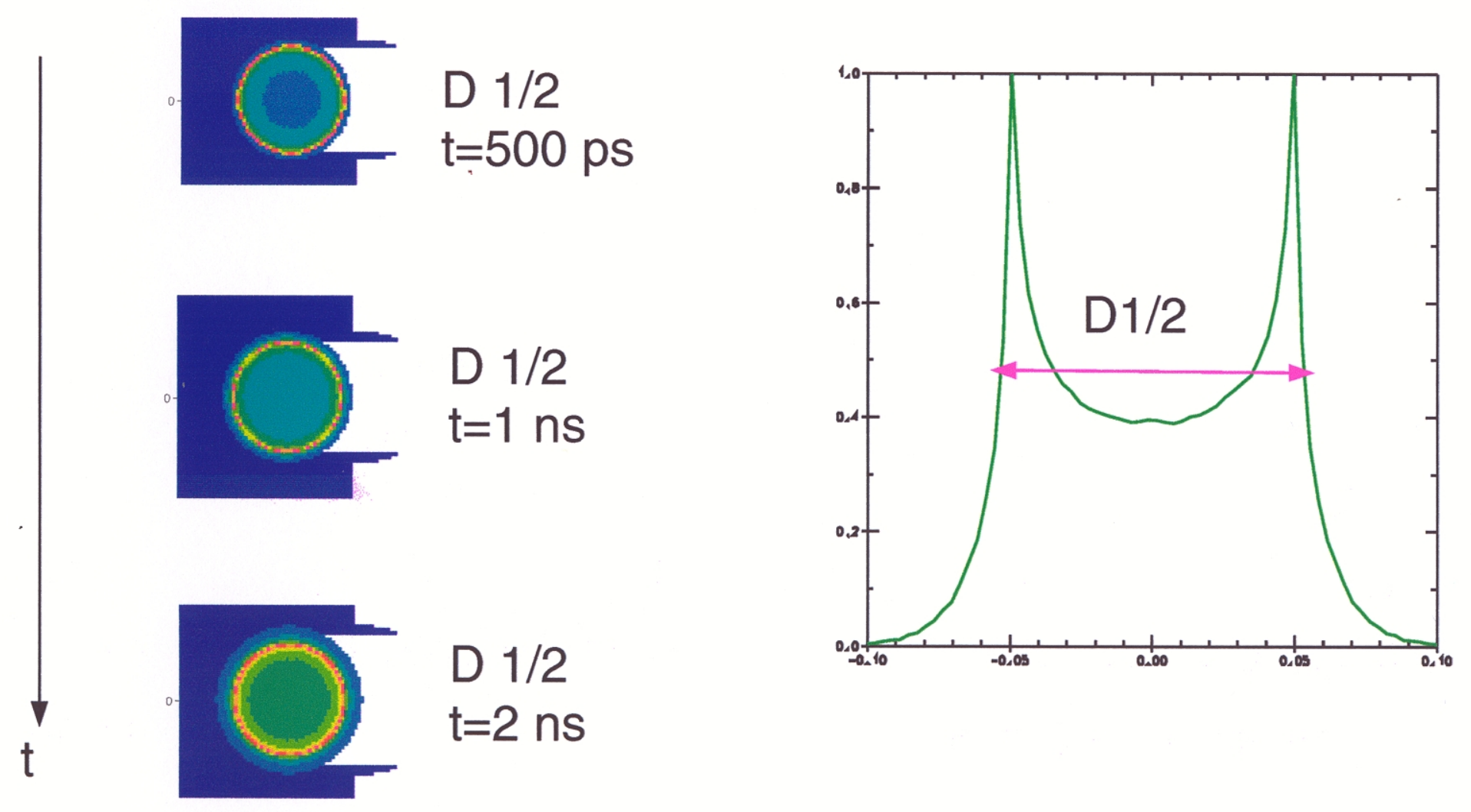


\section{Emitting zones movement for $\mathrm{I}=7 \mathrm{e} 14 \mathrm{~W} / \mathrm{cm} 2$}
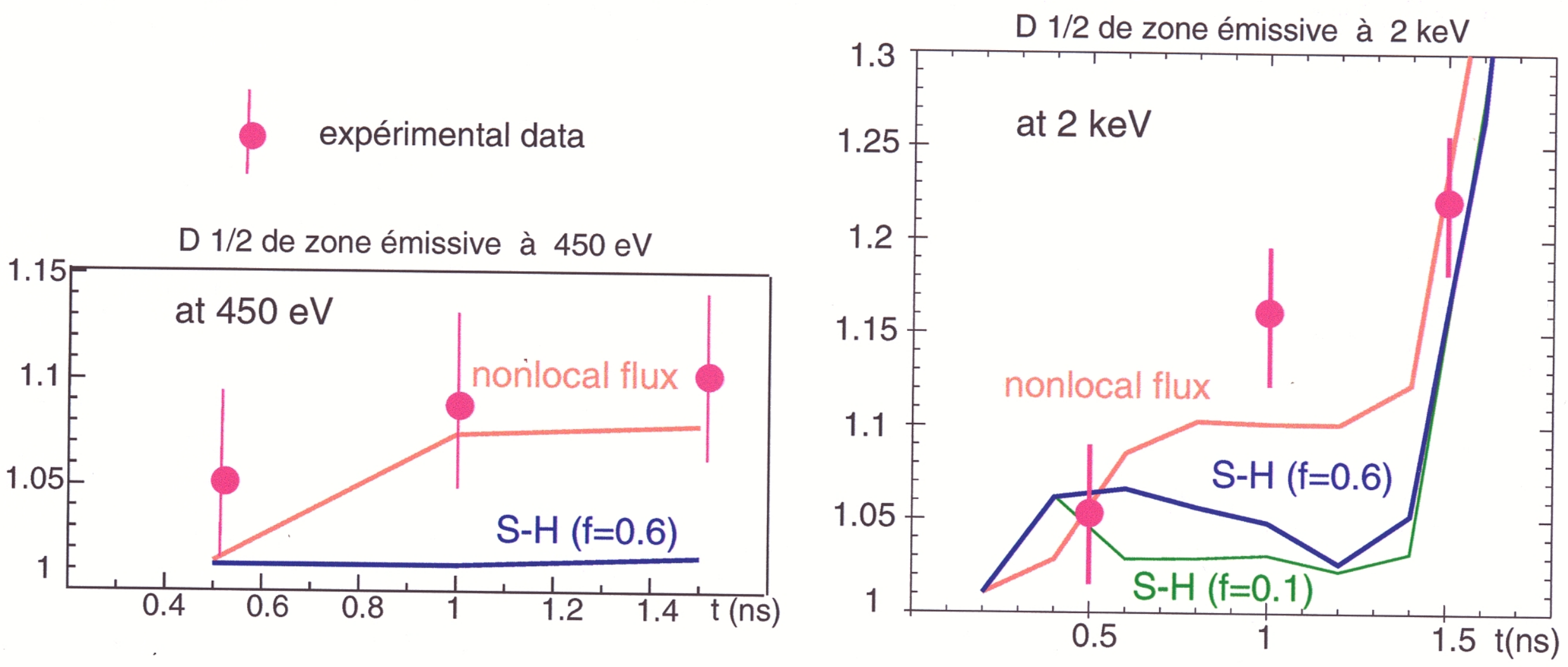

$\checkmark$ Spitzer-Harm fluxes do not be able to reproduce experiment

$\checkmark$ A flux limiter does not improve results

$\checkmark$ Using nonlocal fluxes, we get simuation closer to experimental data 


\section{Emitting zones movement for $\mathrm{I}=4 \mathrm{e} 14 \mathrm{~W} / \mathrm{cm} 2$}

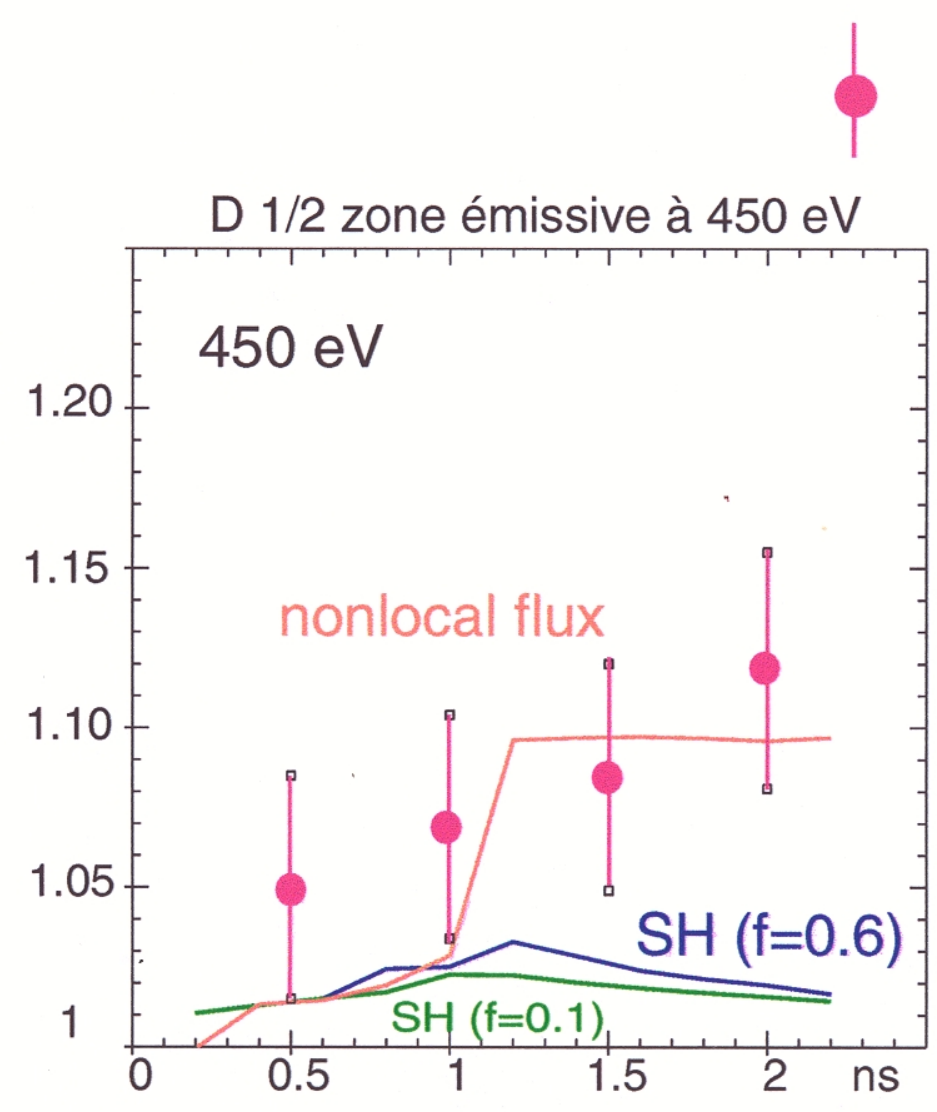

expérimental data

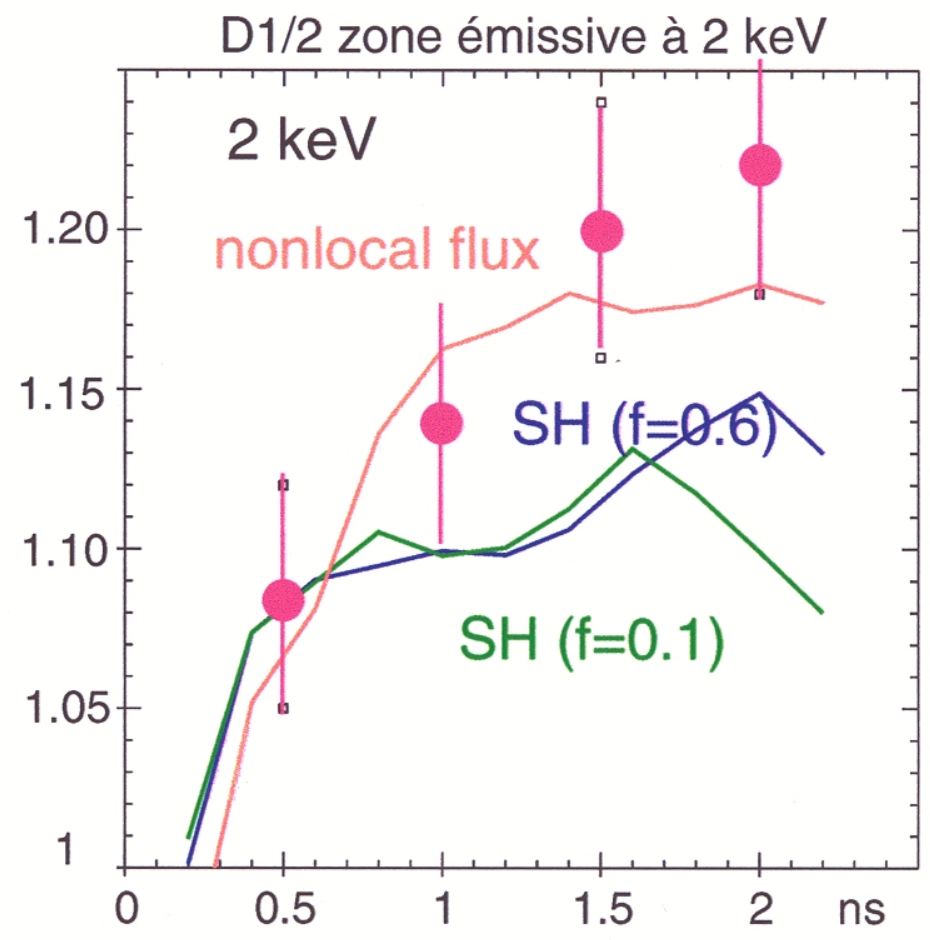

$\checkmark$ Discrepancies between S-H and nonlocal fluxes are reduced but only nonlocal simulation passes through experimental data. 


\section{Emitting zones movement for I = 1e14W/cm2}
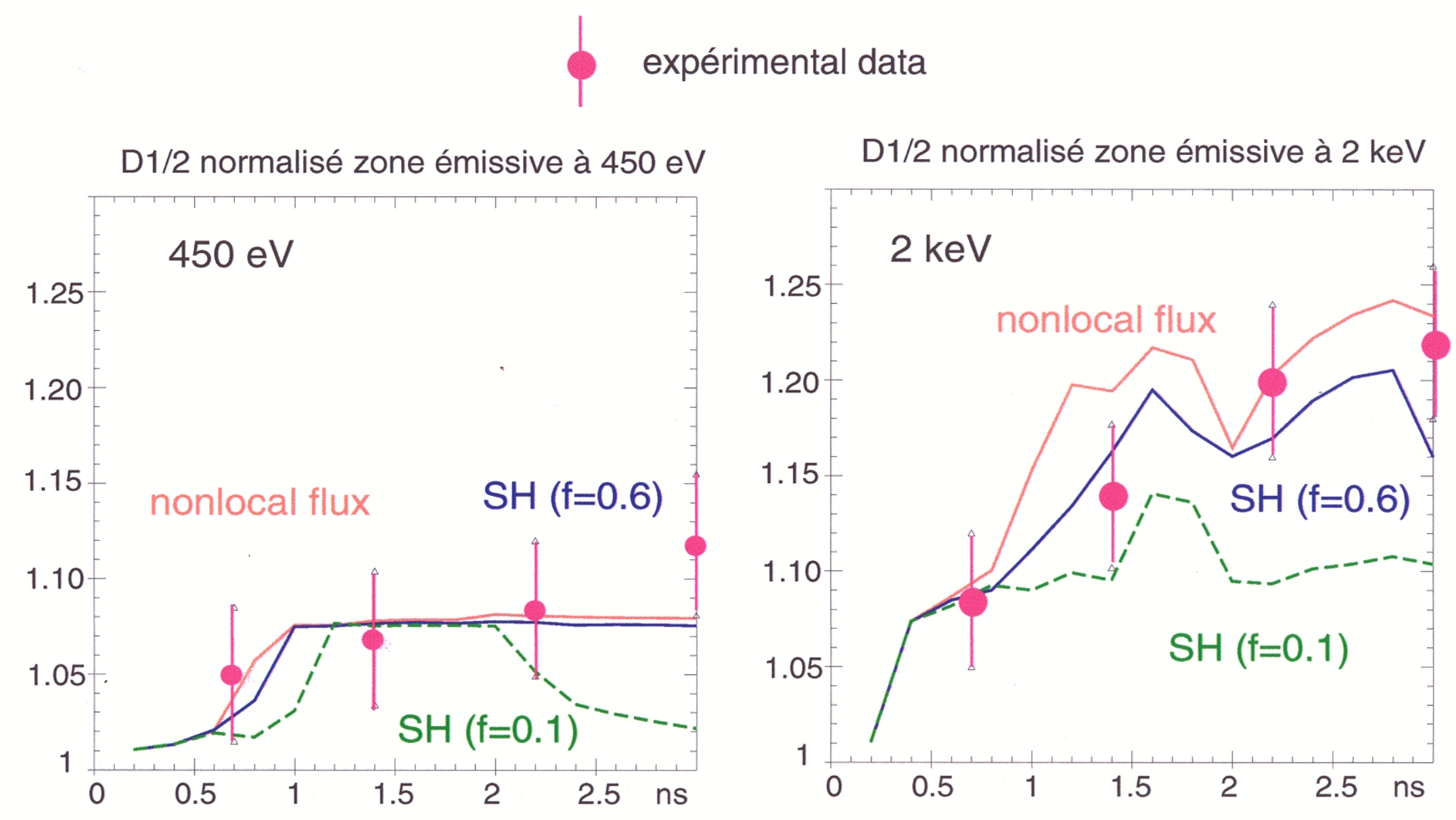

$\checkmark$ Non local flux tends towards $\mathrm{SH}$ flux for low intensity

$\checkmark$ Both models reproduce experiment 


\section{Spherical target : summary}

$\square$ Unlike cavity experiments, only nonlocal effetcs act on heat fluxes

$\square$ If some results like X-ray conversion efficiency can be explain by the use of Spitzer-Harm fluxes, only the nonlocal fluxes reproduce the movement of emitting zones.

$\square$ The variation of laser intensity in experiment allows us to test the convergence of our model to Spitzer-Harm model (low flux). 


\section{Conclusion}

$\square$ The use of Spitzer-Harm fluxes, limited or not, does not allow us to reproduce some experimental results.

$\square$ From one experiment to another, and even from one diagnostic to another, the flux limiter value can be different : interpretation $\Leftarrow \Rightarrow$ prevision

$\square$ Nonlocal fluxes combined with magnetic fields improve simulations and so our understanding of laser plasma experiments (up to now...) 


\title{
Modelling electron heat conduction in $\mathrm{FCl} 2$
}

\author{
Guy Schurtz \\ Philippe Nicolai \\ Michel Busquet
}

$C E A / D A M / D I F$

G.Schurtz, Ph.Nicolai, M.Busquet - Phys.of Plasmas 7, 10 Oct 2000

G.Schurtz- APS - DPP conference - Montreal (2000)

Ph.Nicolai, M.Vandenboomgaerde, B.Canaud \& F.Chaigneau Phys.of Plasmas 7, 10 Oct 2000 


\section{Hydro simulations : scale lengths}

- Spatial scales : micron $<\mathbf{k} \mathbf{c m}$

Time scales : ps $<\tau<$ ns

$==1$ fluid, 2 températures

Local Thermal Equilibrium

NLTE radiation transport

- Euler Equations coupled to

- rad. \& fast ions transport

- laser light propagation

- Transport coefficients

- LTE : EOS, ionisation, material strength, conductivities,opacities)

- NLTE : approximated models (e.g. : Radiom)

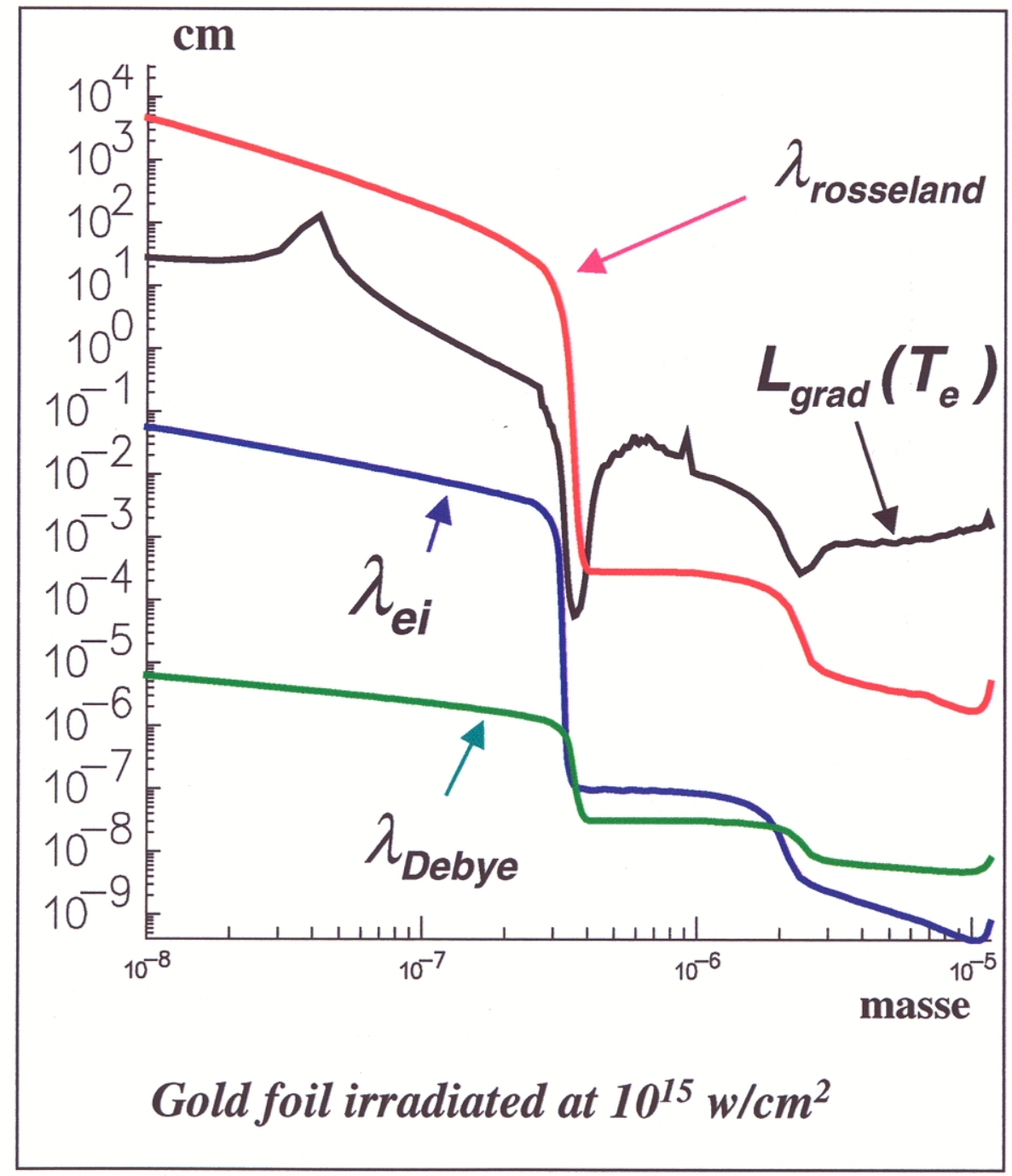




\section{Energy flow in a laser irradiated gold target}
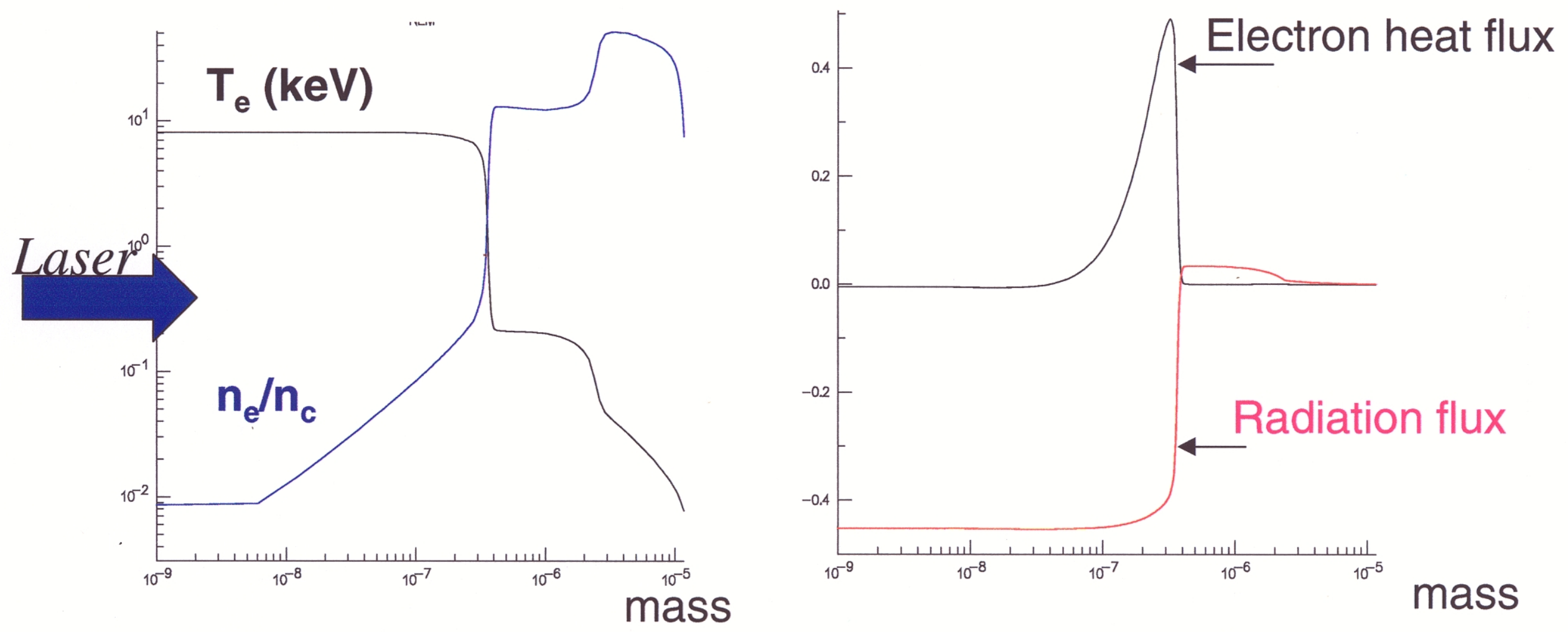

- Energy is transported to the emissive zone by the heat flux

- Electron heat conduction determines the X-ray conversion efficiency of the target 


\section{FCl2}

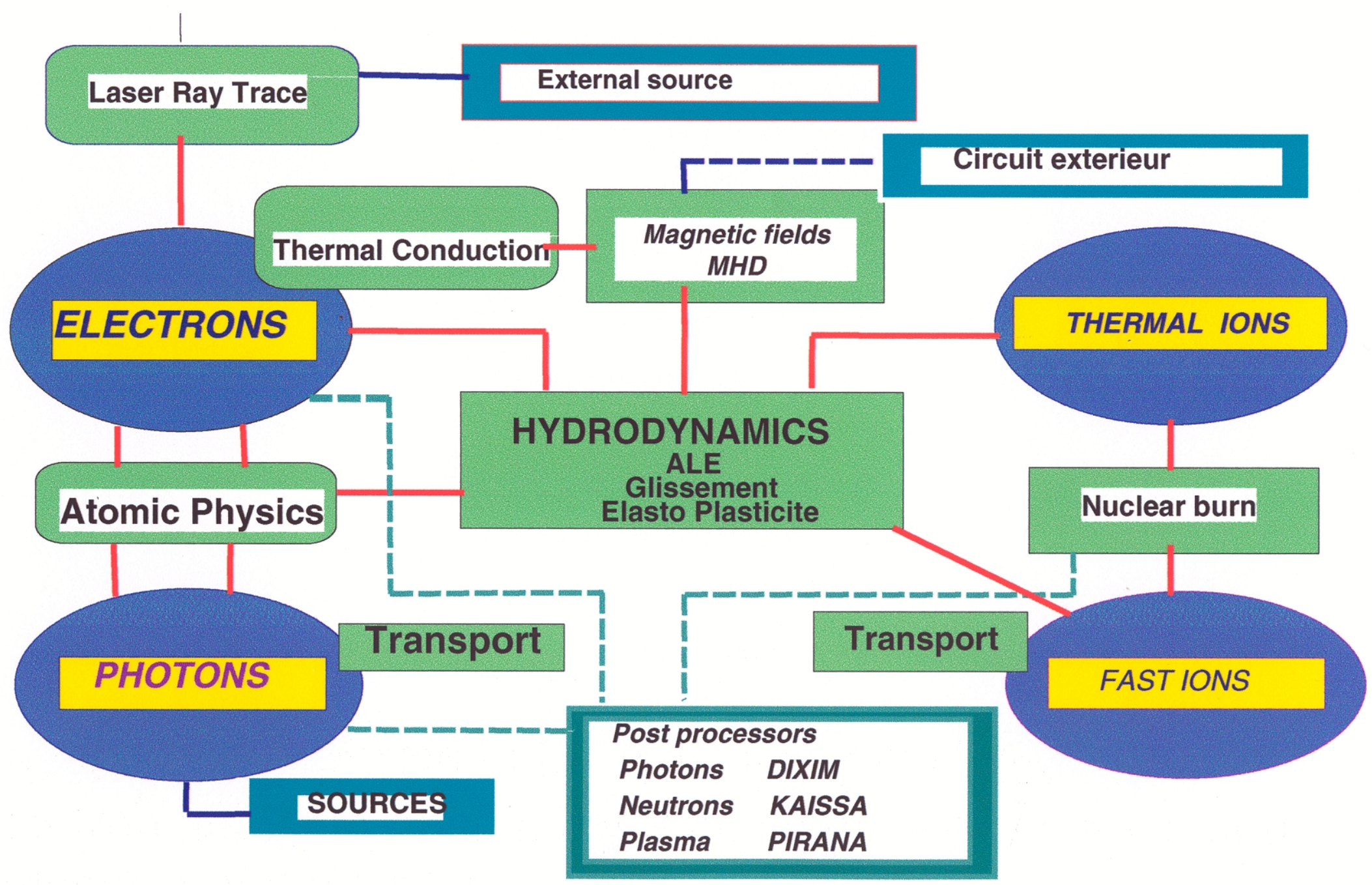




\section{A « venerable old kludge » (L. Suter dixit): the flux limited Spitzer Harm heat conduction}

- The classical Spitzer Harm theory fails at restituting observed data

- In order to reproduce experiments one limits the heat flux to some fraction $f$ of the free streaming limit

$$
Q_{f s}=\frac{3}{2} n_{e} k T_{e} v_{t h}
$$

- $f$ is the main adjustable parameter of numerical simulations

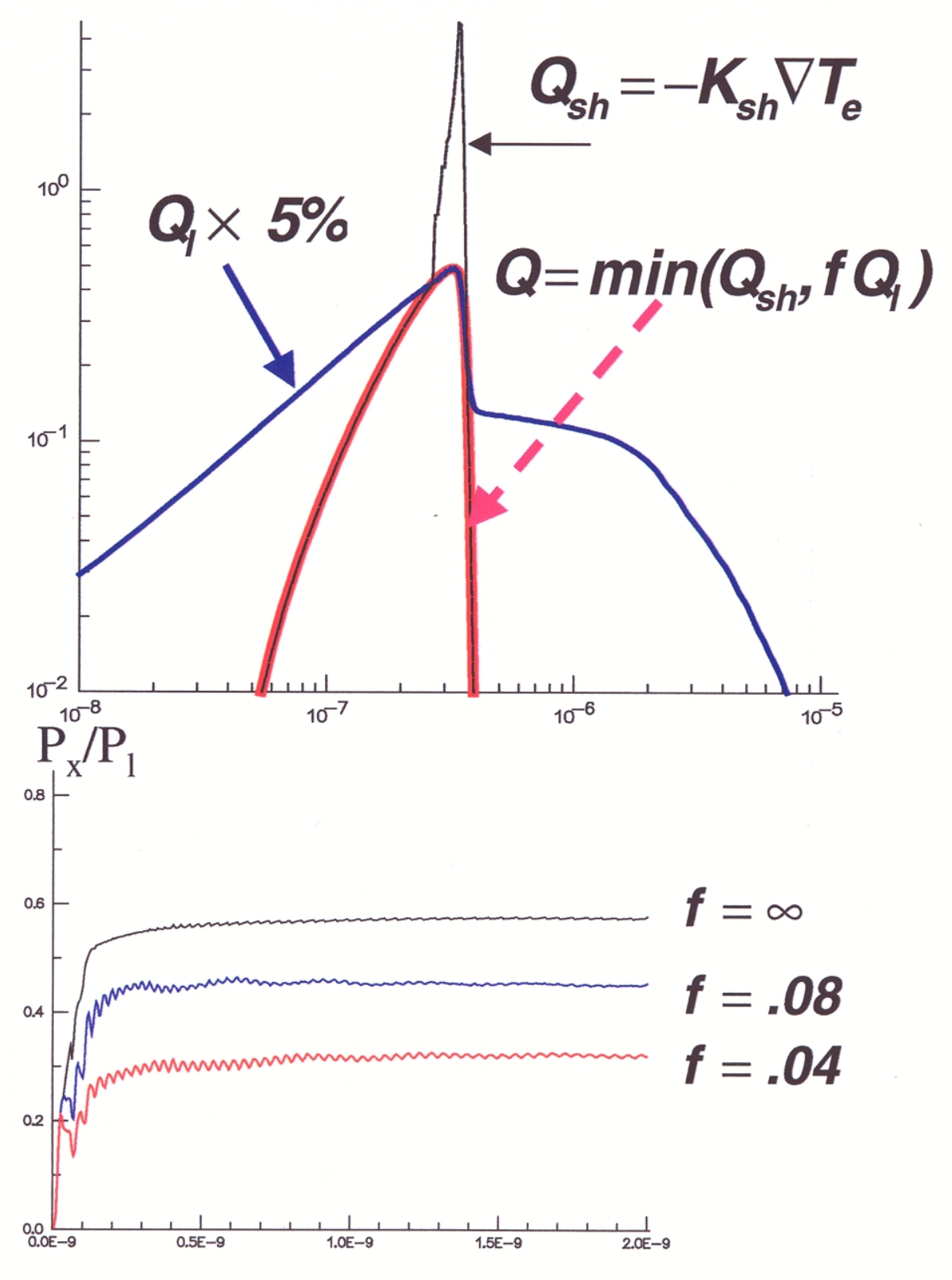




\section{A few good reasons why the heat flux should be inhibited}

- Electrons cannot move freely between collisions.

- Self generated magnetic fields

》 measurements inidicate $B$ in the range $[0.1,1] \mathrm{MG}$

- Collisions are enhanced

- Ion acoustic turbulence

» no real experimental evidence

» no general agreement among theoreticians

- Free electrons depart significantly from LTE

" non maxwellian d.f. predicted by Fokker Planck codes

»Non Local heat flux theories 


\section{Validity of Spitzer linear theory}

- the perturbation calculation

$$
f(v, \Omega)=f_{0}^{m b}(v)+\Omega . f_{1}(v)
$$

is valid as long as $\left|f_{1}\right|<<f_{o}$

- As $\lambda_{e}(v) \approx\left(v / v_{t h}\right)^{4} \lambda_{e i}$

$$
\lambda_{e}<<T / \nabla T \mid
$$

this condition cannot be fullfilled for all velocities

- major contributions to the heat flux occur at $\sim 3,7 \mathrm{~V}_{\text {th }}$

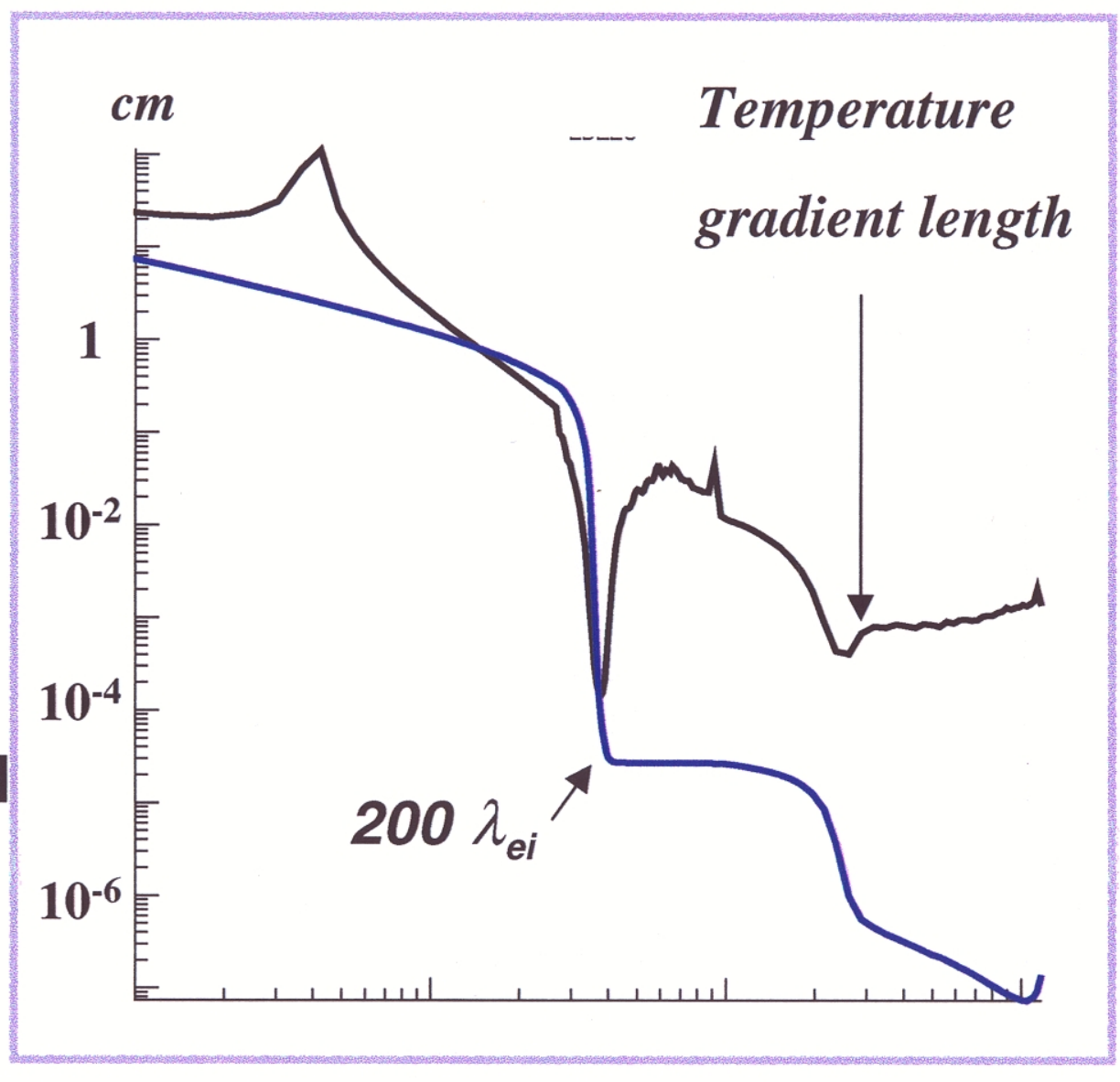

- $\mathrm{f}_{0}$ cannot be maxwellian any more in case of sharp gradients 


\section{Non local heat flux}

- 1D non local practical formula

$$
\begin{aligned}
& Q_{n l}(x)=\beta_{1 d} \int_{-\infty}^{\infty} Q_{s h}\left(x^{\prime}\right) W\left(x, x^{\prime}\right) \frac{d x^{\prime}}{a \lambda\left(x^{\prime}\right)} \\
& L M V: W\left(x, x^{\prime}\right)=e^{-\tau\left(x, x^{\prime}\right)} \text { avec } \tau(x, y)=\frac{\left|\int_{x}^{y} n_{e}(z) d z\right|}{a n_{e}(y) \lambda_{e}(y)}
\end{aligned}
$$

- Difficulties

- Formulation limited to 1D slab geometry

- Boundary conditions

- Simple rationale for an heuristic extension to other geometries

- fluxes are vectors

- kernels become tensors
$\boldsymbol{Q} \rightarrow \overrightarrow{\boldsymbol{Q}}$

$W \rightarrow \vec{\Omega} \boldsymbol{W} \vec{\Omega}$ 
$\checkmark$ Computation of non local flux at $r$ requires summation over all contributing points $r$

In spherical coordinates :

$$
\vec{Q}_{n l}(r)=\int_{4 \pi} \vec{\Omega} d^{2} \Omega \int_{0}^{\infty} \text { contribution }(r+\delta \Omega) W(r, r+s \Omega) \frac{d s}{\lambda^{*}\left(\overrightarrow{r_{0}}+\vec{s} \vec{\Omega}\right)}
$$

$\checkmark$ If we assume that the distribution funces on has the azimuthal symmetry around the direction of grad T (i.e. P1 approx tmation), contribution in direction $\Omega$ is $Q_{h} . \Omega$

$$
\vec{Q}_{n 1}(\vec{r})=\int_{4 \pi} \vec{\Omega} \vec{\Omega} d^{2} \Omega \int_{0}^{\infty} \overrightarrow{Q_{s H}}(\vec{r}+s \vec{\Omega}) W(\vec{r}, \vec{r}+s \vec{\Omega}) \frac{d s}{\lambda^{*}\left(\vec{r}_{0}+s \vec{\Omega}\right)}
$$

$\checkmark$ Comparesto classical 1D results

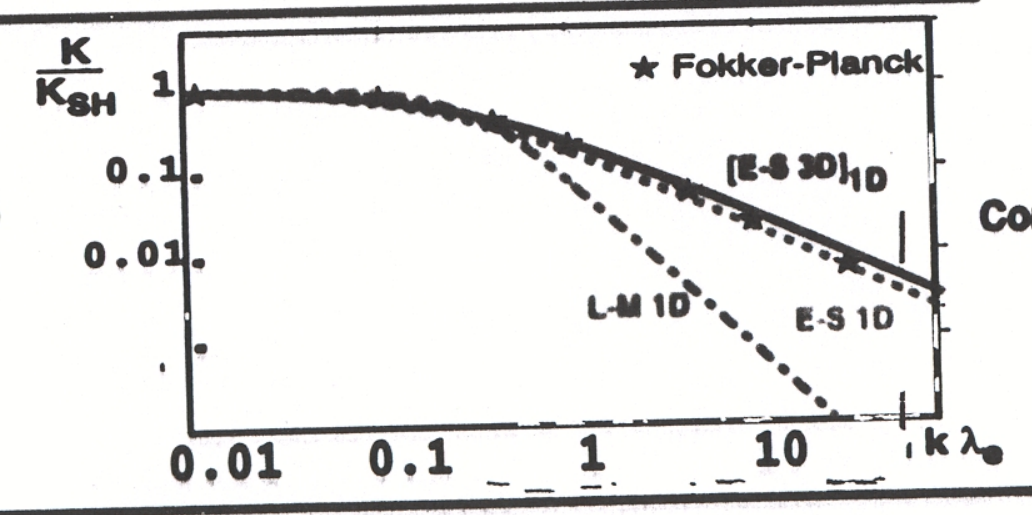




\section{Introduction in a 2D code}

- 2 approches :

- 1 : Direct calculation of $Q$ on a given mesh point $i$ from contributions of all other mesh points $\mathrm{j}$

- drawbacks :

- high computationnal cost, ray effects

- reflective boundary conditions difficult to handle

- advantages :

- accept différent kernels

- 2 : «adjoint » method : start from « source points » and reformulate $Q_{n l}$ as the integral solution of a transport problem.

- advantages :

- Numerical Analysis provide standard tools

- clear link to kinetic equations (Fokker Planck)

- drawbacks

- requires a symetrical kernel

- complex but for an exponentiel kernel (LMV-like) 


\section{Equivalent transport equation for the exponential kernel}

- Introduce the scalar $q(r, \Omega)$ solution de $\vec{\Omega} \cdot \vec{\nabla} q(r, \Omega)=\frac{1}{\lambda(r)}\left(\frac{3}{4 \pi} \vec{\Omega} \cdot \vec{Q}_{s h}-q(r, \Omega)\right)$ and let $Q$ be its 1 st order angular moment $\vec{Q}=\int_{4 \pi} q \vec{\Omega} d^{2} \Omega$

- We have $\overrightarrow{\boldsymbol{Q}}=\frac{3}{4 \pi} \int_{4 \pi} \vec{\Omega} d^{2} \Omega \int_{-\infty}^{\infty} \vec{Q}_{s h}(r+s \Omega) \vec{\Omega} \quad \exp -\left|\int_{r}^{r+s \Omega} \frac{d r^{\prime}}{\lambda\left(r^{\prime}\right)}\right| \frac{d s}{\lambda(r+s \Omega)}$

- Approximated Sn quadratures

$-1 D$ slab : the S2 $\left(\mu \pm= \pm \frac{1}{\sqrt{3}}\right)$ solution is the non local LMV flux

- more generally, $\mathrm{S} 2$ is equivalent to $\mathrm{P} 1$ :

$$
\begin{aligned}
& \boldsymbol{H}=\int_{4 \pi} q(\Omega) d^{2} \Omega \\
& \overrightarrow{\boldsymbol{Q}}=\int_{4 \pi} q(\Omega) \vec{\Omega} d^{2} \Omega
\end{aligned}
$$

$$
\begin{aligned}
& \left(\frac{1}{\lambda}-\vec{\nabla} \cdot \frac{\lambda}{3} \vec{\nabla}\right) \boldsymbol{H}=-\vec{\nabla} \cdot \overrightarrow{\boldsymbol{Q}}_{s h} \\
& \overrightarrow{\boldsymbol{Q}}=\overrightarrow{\boldsymbol{Q}}_{s h}-\frac{\lambda}{3} \vec{\nabla} \boldsymbol{H}
\end{aligned}
$$




\section{The 3D n.l. flux as the solution of a transport equation}

$\checkmark$ Introduce the scalar q solution of the steady state transporation

$$
\vec{\Omega} \cdot \vec{\nabla} \mathrm{q}(r, \vec{\Omega})=\frac{1}{\lambda(r)}\left\{\frac{3}{4 \pi} \vec{\Omega} \cdot \vec{Q}_{S H}(r)-q(r, \vec{\Omega})\right\}
$$

$\checkmark$ Define the optical depth as : $\tau\left(r, r^{\prime}\right)=\int d r^{\prime \prime} / \lambda\left(r^{\prime \prime}\right)$

$\checkmark$ The solution is $q(r, \Omega)=\frac{3}{2 \pi} \int \frac{\exp [-\tau(r-s \Omega, r)] \Omega \cdot Q \operatorname{sh}(r-s \Omega) d s}{\lambda(r-s \Omega)}$

$$
\text { (D) } 0
$$

Form the firstorder angular momemtum of $q: \vec{Q}=\int_{4 \pi} q(r, \vec{\Omega}) \vec{\Omega} d^{2} \Omega$

$$
\vec{Q}=\frac{3}{4 \pi} \int_{4 \pi} \vec{\Omega} \vec{\Omega} d^{2} \Omega \int_{0}^{\infty} \frac{W(r, r+s \Omega) Q s h(r+s \Omega) d s}{\lambda(r+s \Omega)}
$$


C $\triangle$ The LMV flux is the S2 solution of the transport equtiotion

$\checkmark$ Solve the $1 \mathrm{~d}$ transport equation along directions $\mu= \pm 1 / \sqrt{3}$

$$
Q(x)=1 / 2 \int_{-\infty}^{\infty} \frac{\exp \left[-\sqrt{3} \tau\left(x, x^{\prime}\right)\right] \operatorname{Qsh}\left(x^{\prime}\right) d x^{\prime}}{\lambda\left(x^{\prime}\right) / \sqrt{3}}=\text { LN }{ }^{\prime} \text { formula with } \lambda=\sqrt{3} \lambda_{\mathrm{Imv}}
$$

$\checkmark$ if $S 2$ is correct, $P 1$ is o.k. : $q(r, \Omega)=H(r) / 4 \pi+3 \Omega . Q / 4 \pi$

$$
H=\int_{4 \pi} q(r, \Omega) d^{2} \Omega
$$

$\checkmark H$ and $Q$ are sqlutions of the momentequations

$$
\begin{aligned}
& \left(\frac{1}{\lambda}-\nabla \frac{\lambda}{3} \nabla\right) H=-\nabla \cdot Q_{s h} \\
& Q_{n 1}=Q_{s h}-\frac{\lambda}{3} \nabla H
\end{aligned}
$$




\section{Comparison to LMV : multigroup diffusion}

$\checkmark$ The transport kernel is symmetric whereas the LMV kernellis non symmetric

$$
W_{I m v}(x, y)=\exp [-\tau(x, y)] \quad \tau_{i m v}(x, y)=\int_{D}^{y} \text { is }(n \theta(s), T(y))
$$

$===>$ electrons are transported forny to $x$ at the hot thermal velocity

$\checkmark$ the transport equation must be solved for different energy groups : $\lambda_{g}=2\left(\frac{\mathrm{Eg}}{\mathrm{kT}}\right)^{2} \lambda_{\theta}$

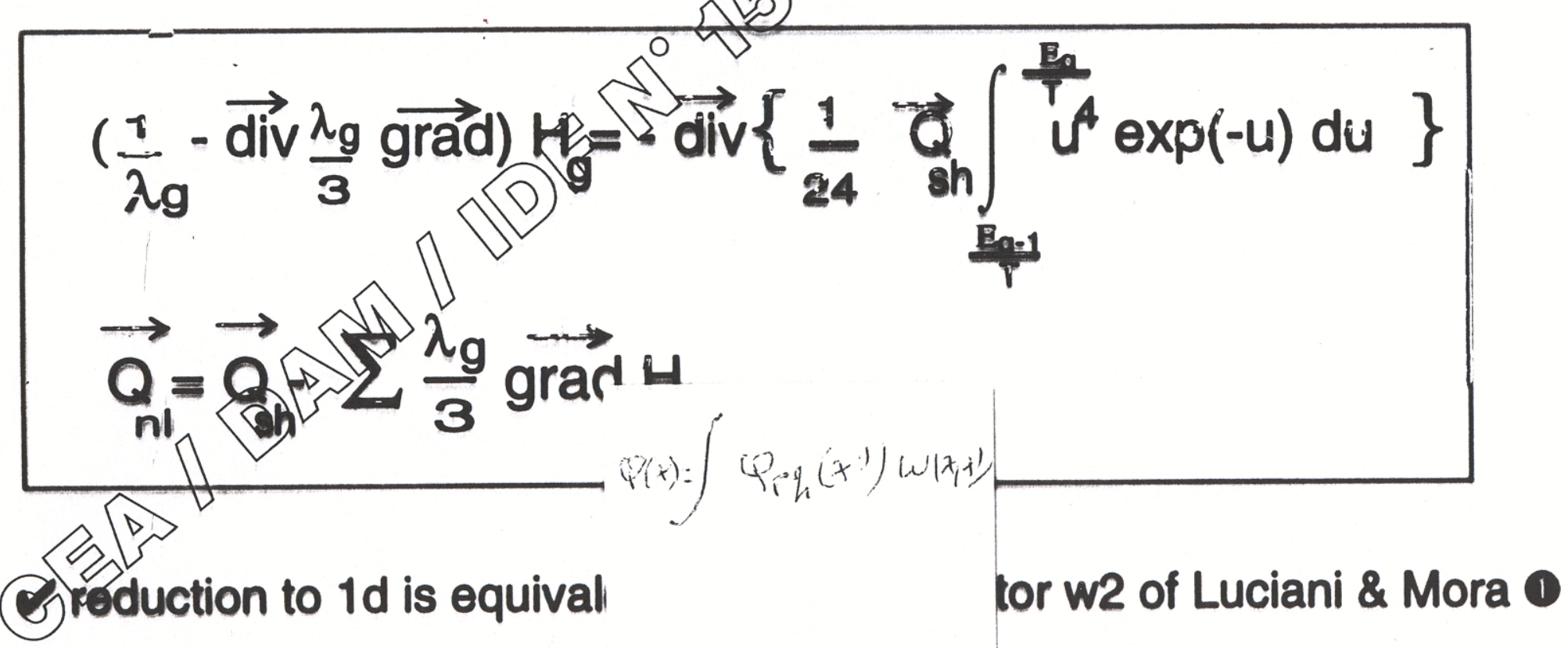

- J.F. Luciani , P.Mora in Lase \& part. beams 12, 387 (1994) 


\section{$\mathrm{H}$ is the departure from the maxwellian d.f.}

- Fokker Planck

$-\mathrm{P1}$

- steady state

- Lorentz model pour C1

$$
\begin{aligned}
& v \nabla . f_{1}-\frac{e E}{m_{e} v^{2}} \frac{\partial\left(v^{2} f_{1}\right)}{\partial v}=C^{o} \\
& \frac{v}{3} \nabla f_{0}-\frac{e E}{3 m_{e}} \frac{\partial f_{0}}{\partial v}=-v_{e i}^{\prime} f_{1}
\end{aligned}
$$

- Solve (1) near the maxwellian :

$$
f_{0}=f_{0}^{m}+\Delta f_{0} ; \quad f_{1}=f_{1}^{m}+\Delta f_{1}
$$

- from definition $Q_{s h}=\int f_{1}^{m} v^{5} d v$, et $C^{0}\left(f_{0}^{m}\right)=0$

- let

$$
\lambda(v)=\sqrt{\lambda_{e i}(v) \lambda_{e e}(v)} \approx \sqrt{Z} \lambda_{e i}(v), \text { et } C^{0}\left(\Delta f_{0}\right)=-\frac{v \Delta f_{o}}{\lambda_{e e}(v)}
$$

- Multiply System (1) by $m_{e} v^{4} / 2$ and eliminate $\Delta f 1$ :

$$
\begin{aligned}
& {\left[\frac{1}{\lambda}-\nabla \cdot \frac{\lambda}{3} \nabla\right] \frac{m_{e} \sqrt{Z}}{2} v^{5} \Delta f_{o}=-\nabla \cdot\left(g(v) Q_{s h}\right)} \\
& Q=Q_{s h}-\frac{m_{e} \sqrt{Z}}{2} \int \frac{\lambda}{3} \nabla\left(\Delta f_{o}\right) v^{5} d v
\end{aligned}
$$

Par identification,

$$
H=\frac{m_{e} \sqrt{Z}}{2} v^{5} \Delta f_{0}
$$




\section{Electric fields}

$\checkmark$ Electric fields

slow down fast electrons (limit the range of delocalization propagator)

accelerate low energy electrons ( return current) Already included in SH theory)

$\checkmark$ LMV \& Bendid solution : multiply the kem ${ }^{0}$ by $\exp \left(-\theta\left|\Phi(X)-\Phi\left(X^{\prime}\right)\right| / k T\right)$

wo the electrical potential is given by Spitzer : $\theta \mathcal{E}=k T[\operatorname{grad}(\operatorname{logn})+\gamma \operatorname{grad}(\log T)]$

(shown to be reasonably accurate, even in sharp gradients)

taking the spatial derivative gives the the equivalent transport mean free path $\lambda^{\prime}$

$$
\frac{1}{\lambda^{\prime}} \frac{\mid e \mathcal{E}}{\mathrm{k} T}=\begin{aligned}
& \text { Harmonic mean of collision mean free path } \\
& \text { and stopping length at energy } k T
\end{aligned}
$$

At group energy Eg, the stopping length should be taken as $\frac{E_{g}}{\mid \boxminus \mathcal{E}}$

th 


\section{Effects of a non local heat flux}

- Reduces the maximum heat flux

- Preheat of dense target

- Non isothermal corona and counter streaming fluxes

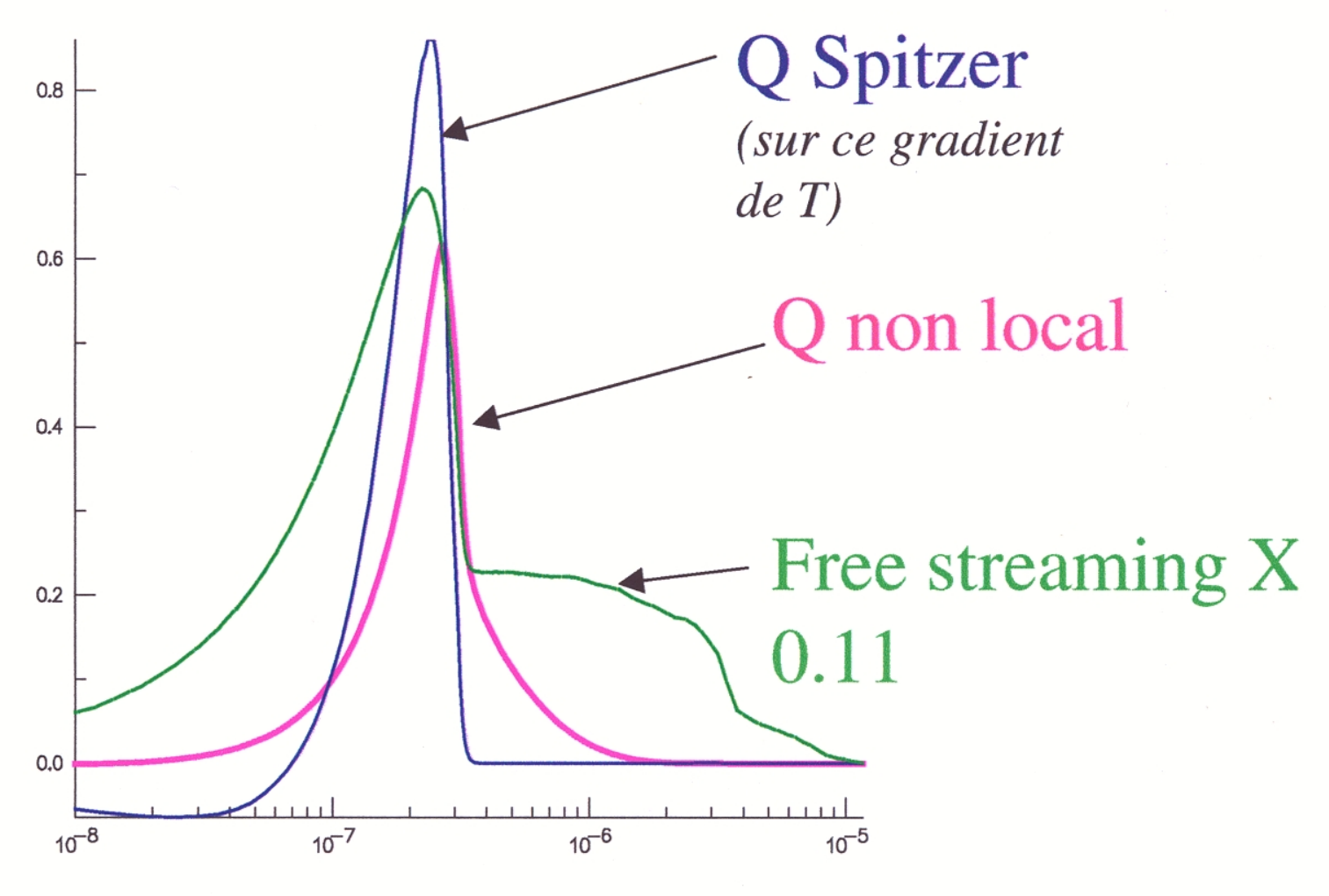

- All effects cannot be reproduced with a single flux limiter 


\section{Summary of multigroup equations}

- Define energy dependant transport coefficients :

$$
\begin{aligned}
& \lambda_{e i}^{g}=\lambda_{e i}\left(\frac{u_{g}}{k T}\right)^{2}, \frac{1}{\lambda_{n l}^{g}}=\frac{1}{\lambda_{e i}^{g}}+\frac{e|E|}{u_{g}} \\
& \lambda_{e e}^{g}=Z * \lambda_{e i}^{g}, \quad \gamma_{g}=\frac{1}{24} \int_{u_{g-1 / 2} / k T}^{u_{g+1 / 2} / k T} \beta^{4} e^{-\beta} d \beta
\end{aligned}
$$

- Get the local flux $Q_{\mathrm{loc}}$ from linear theory (Spitzer, Braginskii,..)

- Solve for all groups

$$
\left[\frac{1}{\lambda_{e e}^{g}}-\nabla \cdot \frac{\lambda_{n l}^{g}}{3} \nabla\right] \boldsymbol{H}^{g}=-\nabla \cdot\left(\gamma^{g} \boldsymbol{Q}_{l o c}\right)
$$

- Compute the heat flux from

$$
\boldsymbol{Q}_{n l}=\boldsymbol{Q}_{l o c}-\sum_{g} \frac{\lambda_{n l}^{g}}{3} \nabla \boldsymbol{H}^{g}
$$

- Compute distribution functions from

$$
f^{0} v^{5}=f_{m b}^{0} v^{5}-H(v)
$$


How accurate NL distribution functions are ? Comparison to FP results

- Test problem : $1 D, Z=4, n e=10^{21}$, initial temperature gradient problem run with $1 D$ Fokker Planck code C2M2

Fluid code FCi2 $+n l$ model

- Numerical results at $0.5 \mathrm{ps}$ :
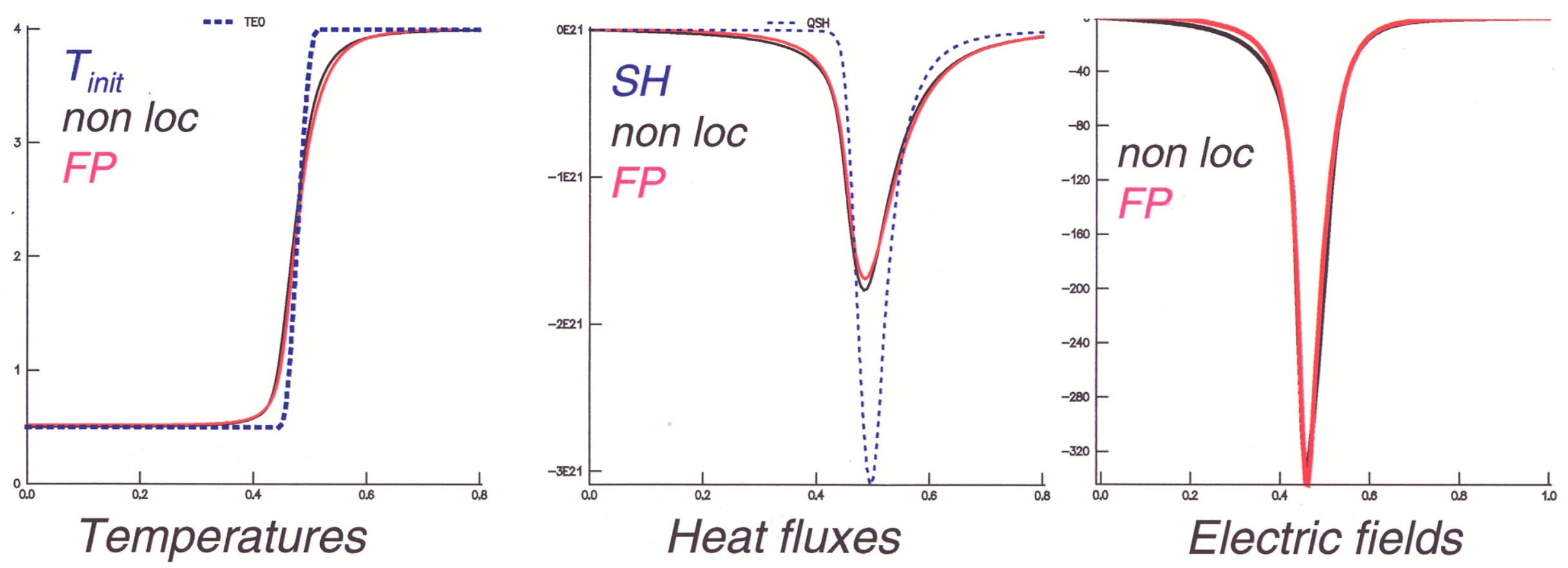


\section{Distribution functions}

- Distribution function predicted by the non local model agree reasonably well with Fokker Planck calculations

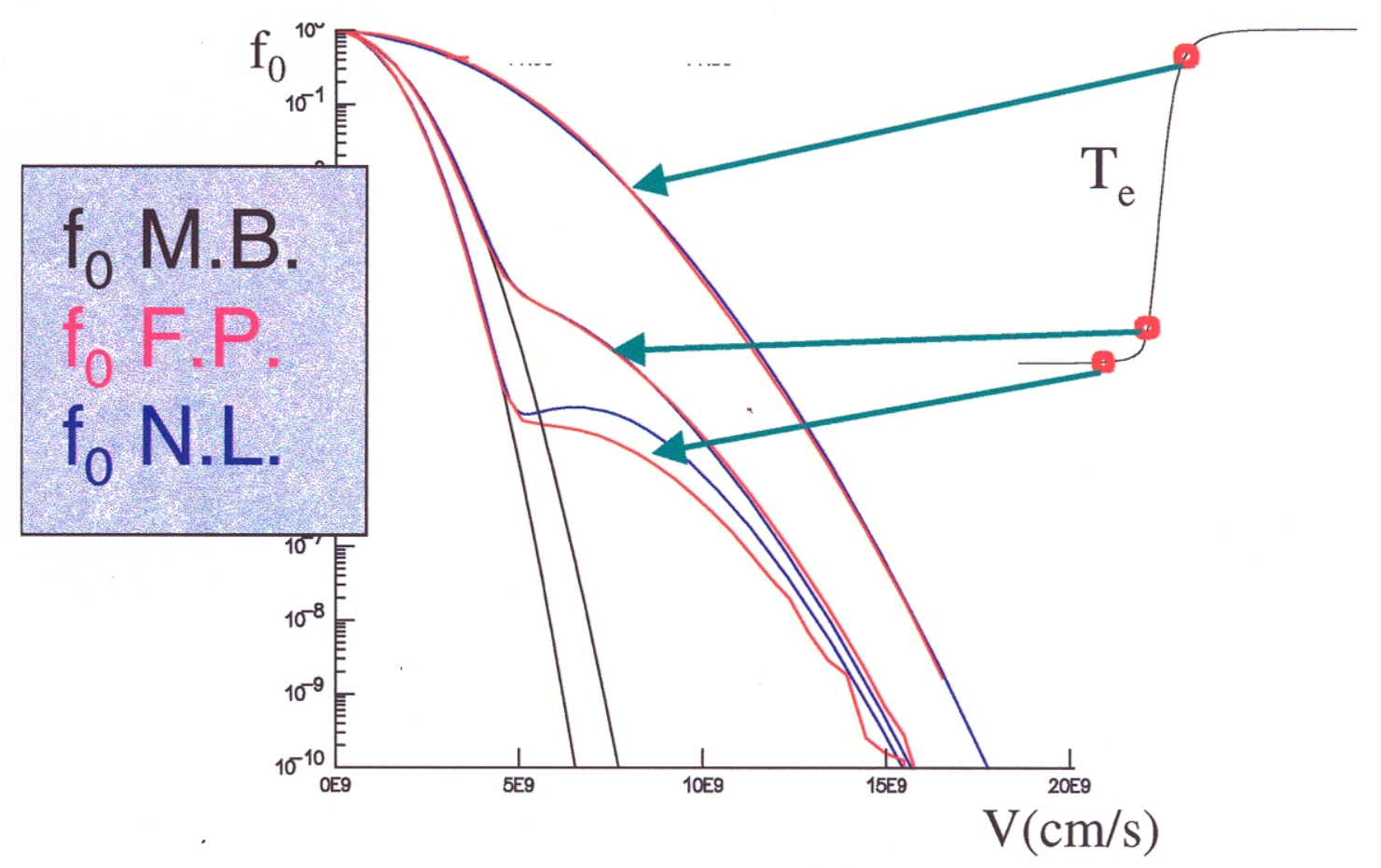

- Computing moments of f0 exhibit other non local effects (e.g. Non Local Efields)

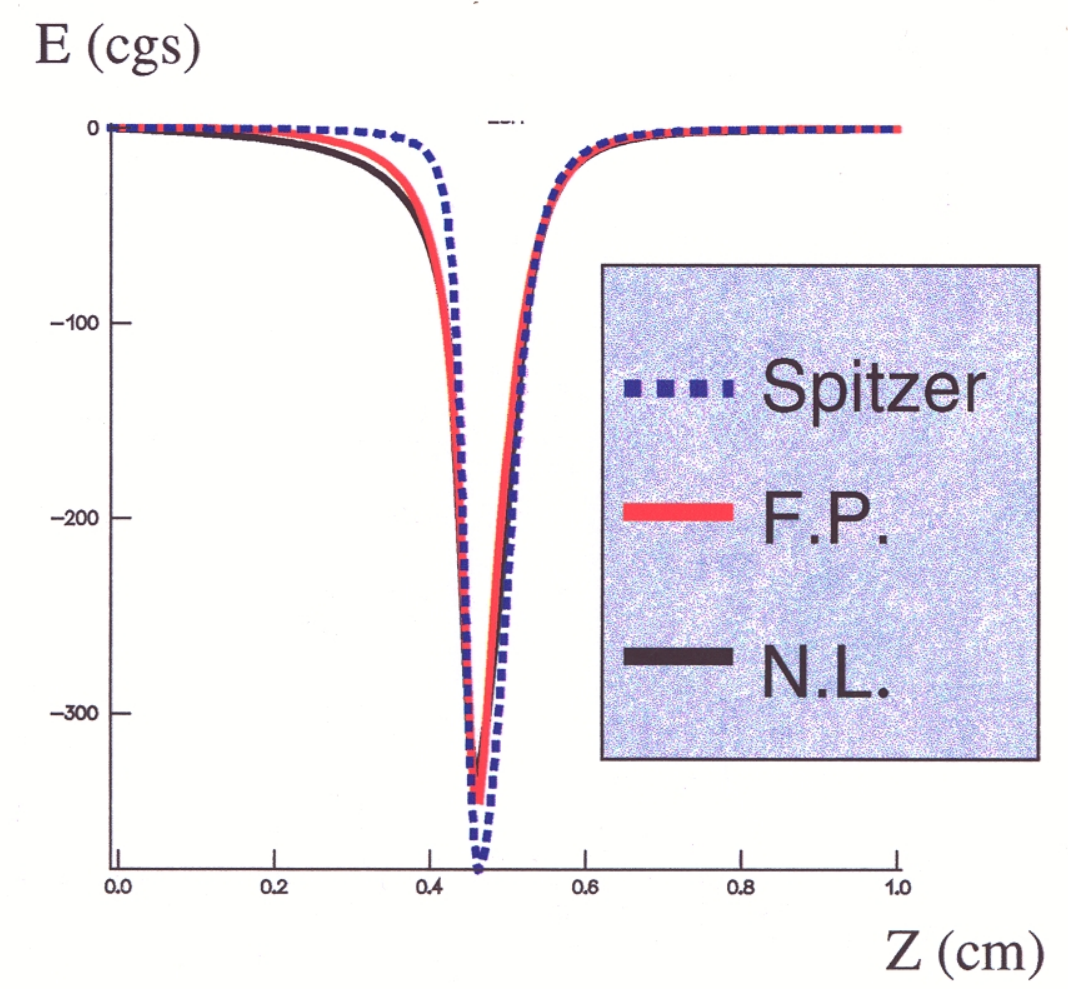


Non local heat equation in Fourier variables: eigen values of $\rho \mathbf{C}_{\mathbf{v}} \mathbf{d T} / \mathbf{d t}=$ - div. $\mathbf{Q}$

- Conditions

- uniform density, harmonic temperature perturbation of wave number $\mathrm{k}$

- 1 group diffusion, $a=32$

- Spitzer Harm

$$
\omega \approx v_{t h} \lambda k^{2} \Rightarrow 10^{-15}<\tau(s)<10^{-13}
$$

- Non local, no E field

$$
\frac{Q_{n l}}{Q_{s h}} \approx \frac{1}{1+(a \lambda k)^{2}} \quad \lim _{\lambda k \rightarrow \infty}(\omega) \propto v_{e i}^{0.01}
$$

$\mathbf{Q} / \mathbf{Q}_{\mathrm{sh}}$

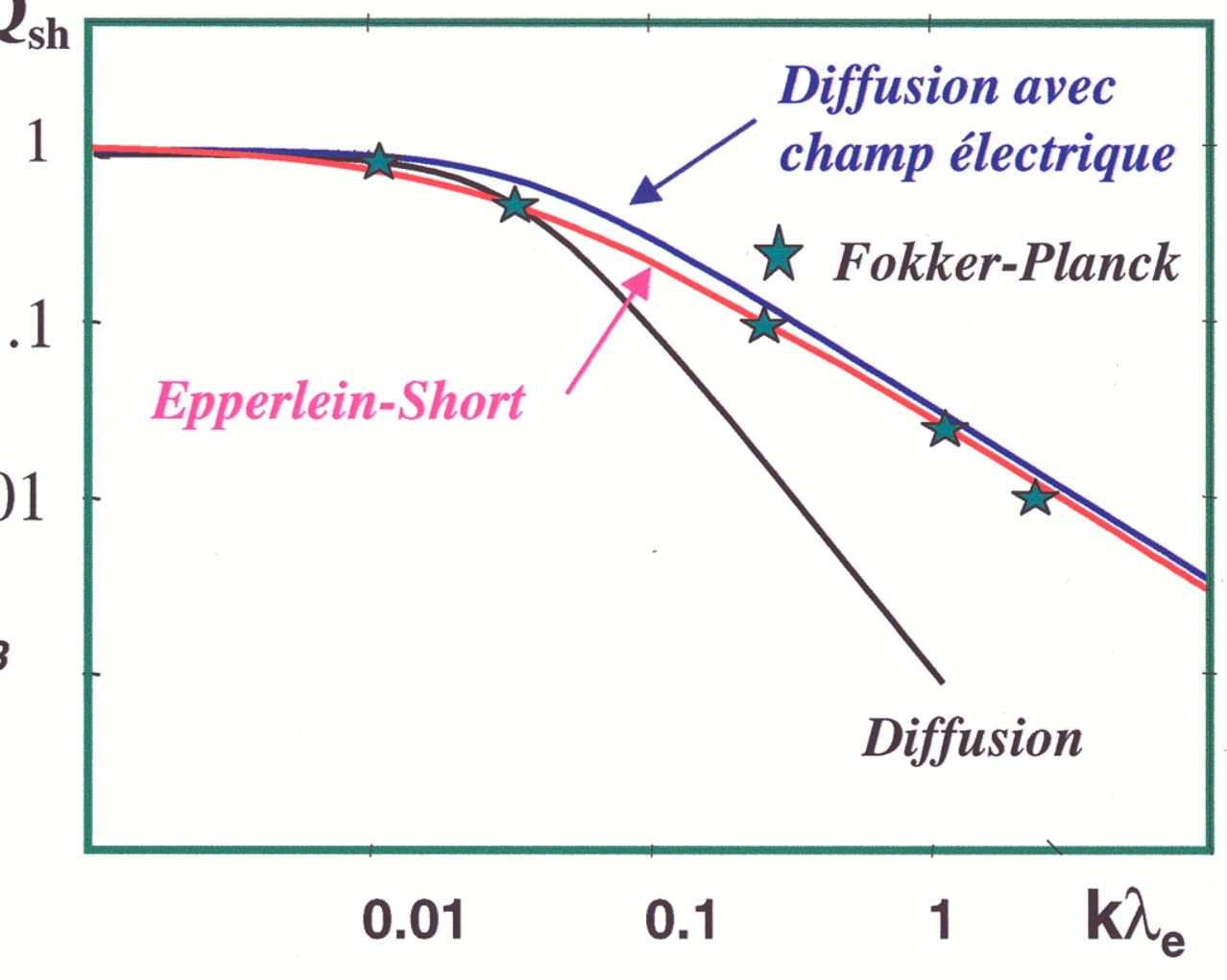

- Including E-field « a la Bendid-Luciani »

$$
\frac{Q_{n l}}{Q_{s h}} \approx \frac{1+b \lambda k}{1+b \lambda k+(a \lambda k)^{2}}
$$

$$
\lim _{\lambda k \rightarrow \infty}(\omega) \approx k v_{t h}
$$




\section{Planned improvements}

- Probably needed

- Introduce the Nernst term in Faraday equation

$$
\left(\frac{\partial B}{\partial t}\right)_{\text {Nernst }}=\nabla \times\left(u_{\text {nernst }} \times B\right), \text { with } u_{\text {nernst }} \propto \frac{Q}{n_{e} T_{e}}
$$

- Second order space differencing

- Achievable with a yet unknown (accuracy/cost) ratio

- improve treatment of collisions (e.g. $\frac{1}{\lambda} v \partial_{v} H$ instead of $\frac{H}{\lambda}$ )

- Improve introduction of $E$ and $B$ fields ( $E \partial_{v} f^{0}$ and $B \times f^{1}$ instead of $\left[\frac{1}{L_{E}}+\frac{1}{L_{B}}\right] f^{1}$ )

- full P1 steady state FP equations for H? 
Numerical Implementation of the model 


\section{Spitzer \& Harm : Conduction matrix}

- energy balance of a cell $\rho c_{v} \frac{d T}{d t}=-\nabla \cdot Q \Rightarrow M_{i} c_{v} \frac{d T_{i}}{d t}=-\sum_{j} A_{i j} Q_{i j}$
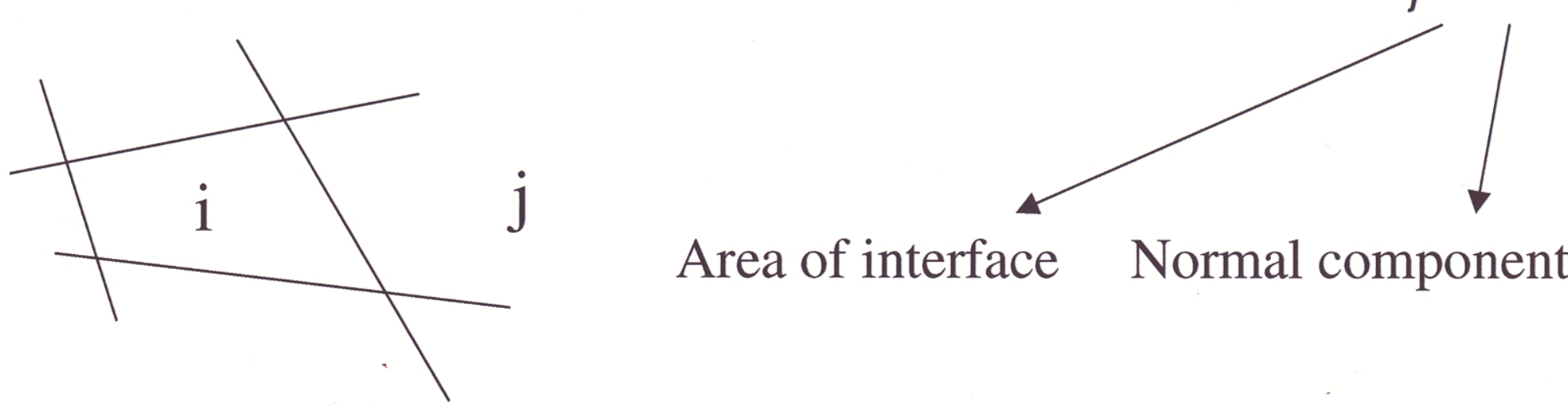

Area of interface Normal component of Q

- SH fluxes $\boldsymbol{Q}_{s h}=-\boldsymbol{K} \nabla \boldsymbol{T} \Rightarrow \boldsymbol{Q}_{i j}=G_{i j}\left(T_{i}-T_{j}\right)$

- space differenced heat flux equation $\left[M c_{v}\right] \frac{d \tilde{T}}{d t}=[D] \tilde{T}$

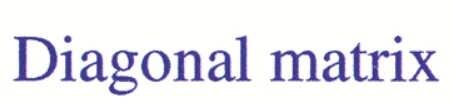

Symmetric

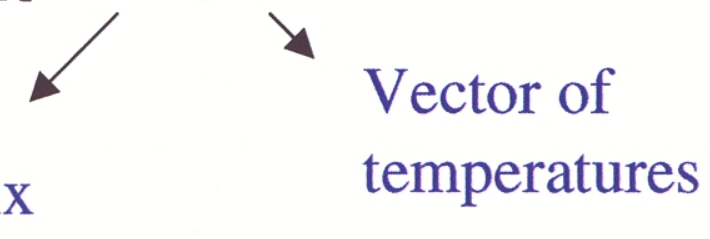

- Only $1^{\text {st }}$ order accurate . May be extended to $2^{\text {nd }}$ order (9 diagonals) 


\section{Time differencing}

- eigen values of the $\mathrm{SH}$ heat flux equation

- let $\mathrm{k}$ be the wave number of an harmonic temperature spatial mode

- this mode is damped with the characteristic time

$$
\tau=\frac{1}{v_{t h} \lambda k^{2}}
$$

- The largest wave number one can sample on a mesh of width $\Delta x$ is $k_{\max } \sim 1 / \Delta x$

- if we integrate the heat equation as $\left[\boldsymbol{M} \boldsymbol{c}_{v}\right] \frac{\tilde{\boldsymbol{T}}^{n+1}-\tilde{\boldsymbol{T}}^{n}}{\Delta \boldsymbol{t}}=[D] \tilde{\boldsymbol{T}}^{n}$ (explicit scheme), the code becomes unstable unless we satisfy the very restrictive criterion $\Delta t<\tau_{\min }$

- Implicit time differencing : $\left[\boldsymbol{M} \boldsymbol{c}_{v}\right] \frac{\tilde{\boldsymbol{T}}^{n+1}-\tilde{\boldsymbol{T}}^{n}}{\Delta \boldsymbol{t}}=[\boldsymbol{D}] \tilde{\boldsymbol{T}}^{n+1}$
is unconditionnally stable 


\section{The non local conduction matrix}

- Matrices

- Let $\left[D_{s h}\right]$ be the finite difference analog of div. $K_{s h} \mathrm{grad}$

- and [ $\Delta]$ the finite difference analog of $\lambda$ div. $\lambda / 3 \mathrm{grad}$

- The 1 group non local heat flux verifies
( V=matrix of volumes) $\quad\left\{\begin{array}{c}(V-[\Delta]) \tilde{H}=-\lambda\left[D_{s h}\right] \tilde{T} \\ Q_{n l}=Q_{s h}-\frac{\lambda}{3} \nabla H\end{array}\right.$ - the non local heat flux equation $\left[\boldsymbol{M} \boldsymbol{c}_{\boldsymbol{v}}\right] \frac{\tilde{\boldsymbol{T}}^{n+}-\tilde{\boldsymbol{T}}^{n}}{\Delta \boldsymbol{t}}=\left(\boldsymbol{I}-\Delta[\boldsymbol{V}-\Delta]^{-1}\right)\left[\boldsymbol{D}_{s h}\right] \tilde{\boldsymbol{T}}^{n+1}$
thus writes

$$
=\left[D_{n l}\right] \tilde{T}^{n+1}
$$

- Usual diffusion matrices are sparse (+other desirable properties as M-matrices) whereas $D_{n l}$ is a full matrix

- eigen values of $D_{\mathrm{nl}}$ look like $\frac{v_{t h} \lambda_{e i} k^{2}}{1+\left(a \lambda_{e i} k\right)^{2}} \quad$ (bounded for large $\lambda k$ !) 


\section{practical solution}

- Look for a numerical solution in the form $\left[M c_{v}\right] \frac{\tilde{T}^{n+1}-\tilde{T}^{n}}{\Delta t}=[D] \tilde{T}^{n+1}+\tilde{S}$ where $D$ is a diffusion-like matrix

(5 or 9 diagonals $+I-D$ is a symmetric M-matrix)

- For each cell boundary (ij), compute $\left[\boldsymbol{D}_{s h}\right], \boldsymbol{Q}_{s h}^{i, j}, \boldsymbol{Q}_{n l}^{i, j}$ from the temperatures at the beginning of the time step $T^{\mathrm{n}}$, and set $S$ to zero

- Compute D and S as follows :

- Whenever $\boldsymbol{Q}_{s h}^{i, j} \cdot \boldsymbol{Q}_{n l}^{i, j}>\mathbf{0}$, set $\boldsymbol{D}^{i, j}=\boldsymbol{D}^{j, i}=\boldsymbol{D}_{s h}^{i, j} \frac{\boldsymbol{Q}_{n l}^{i, j}}{\boldsymbol{Q}_{s h}^{i, j}}$

(this multiplier must be bounded in order to avoid excentric values)

- Otherwise, set $D^{i, j}=D^{j, i}=0, \quad S_{i}=S_{i}-Q_{n l}^{i j}, \quad S_{j}=S_{j}+Q_{n l}^{i j}$ 


\section{Non Local Heat Conduction}

- Substitution of a simplified Fokker Planck equation to the classical concept of delocalization kernel allows the extension of non local theory to 2 or 3D flows. This model is implemented in $\mathrm{FCl} 2$.

- Heat flow predictions agree qualitatively and quantitatively with Fokker Planck in both 1D and 2D

- reduction of the heat flow at maximum

- preheat of dense cold zones

- counter streaming fluxes in the corona

- Electric fields are accounted for at two steps of the model

- the computation of linear fluxes that are sources for delocalization include E fields

- The delocalization operator is modified ( mfp limited to stopping length)

- This modification ensures a correct asymptotic behavior of eigen values.

- Distribution functions are a by product of the model

- comparison to F.P. indicate the NL distr. funct. are reasonnably accurate.

- Allow calculation of other non local moments (e.g. E and B fields) 


\section{Self Generated Magnetic Fields in FCl2}

- A- MHD model implemented in $\mathrm{FCl} 2$

- B- Coupling Bfields to non local transport 


\section{Magnetohydrodynamics : équations of motion}

- lons

$$
\begin{aligned}
& \left(\frac{\partial}{\partial t}+u_{i} \nabla\right) u_{i}-\frac{Z e}{m_{i}}\left(E+\frac{u_{i}}{c} \times B\right)+\frac{1}{m_{i} n_{i}} \nabla P_{i}=\frac{R_{i e}}{m_{i} n_{i}} \\
& \left(\frac{\partial}{\partial t}+u_{e} \nabla\right) u_{e}+\frac{e}{m_{e}}\left(E+\frac{u_{e}}{c} \times B\right)+\frac{1}{m_{e} n_{e}} \nabla P_{e}=\frac{R_{e i}}{m_{e} n_{e}}
\end{aligned}
$$

- Électrons

- Hypotheses

- momentum conservation $\mathbf{R}_{\mathrm{ei}}=-\mathbf{R}_{\mathrm{ie}}$

- plasma neutrality : $n_{e}=Z^{*} n_{i}$

- $m_{e} \ll<m i: j=e n_{e}\left(u_{i}-u_{e}\right)$ est steady

- linear perturbation calculations $\square>\frac{\vec{R}_{e i}}{e n_{e}}=\overline{\bar{\alpha}} \cdot \vec{j}-\frac{\overline{\bar{\beta}}}{e} \cdot \vec{\nabla} T_{e}$

- 2 transport coefficients

$\alpha=$ electrical resistivity tensor

$\beta=$ thermo-electric tensor 


\section{Ohm 's law}

- Sum : momentum balance

$$
\rho \frac{d u}{d t}+\nabla\left(P_{e}+P_{i}\right)=\frac{1}{c} j \times B
$$

- Différence : Ohm 's law

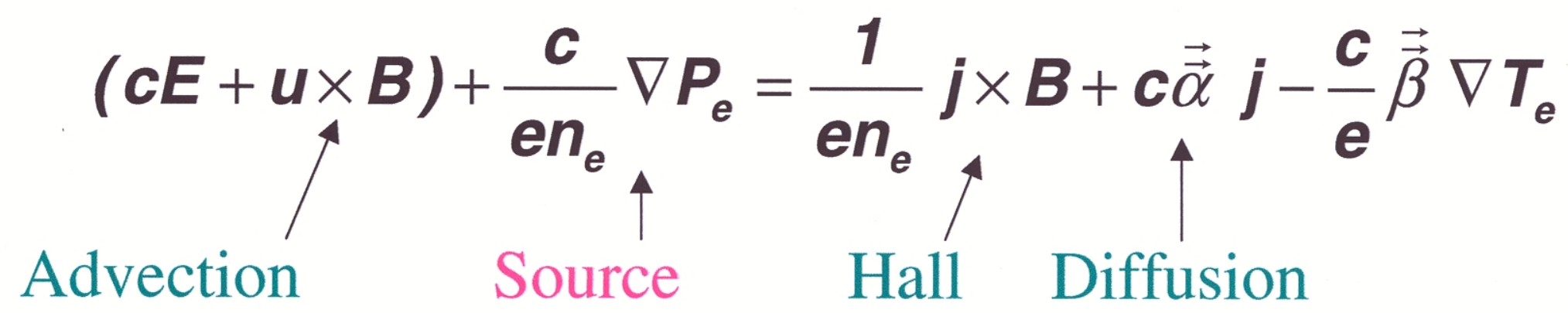

- Simplified equation used in $\mathrm{FCl} 2$

$$
(c E+u \times B)+\frac{c}{e n_{e}} \nabla P_{e}=c \alpha_{\perp} j
$$




\section{Induction equation}

- Ohm 's law

$$
\begin{aligned}
& c E=-u \times B-\frac{c}{e n_{e}} \nabla P_{e}+c \alpha j \\
& j=\frac{c}{4 \pi} \nabla \times B-\frac{1}{4 \pi} \frac{\partial E}{\partial t} \\
& \frac{\partial B}{\partial t}=-c \nabla \times E
\end{aligned}
$$

- Ampère

- Faraday

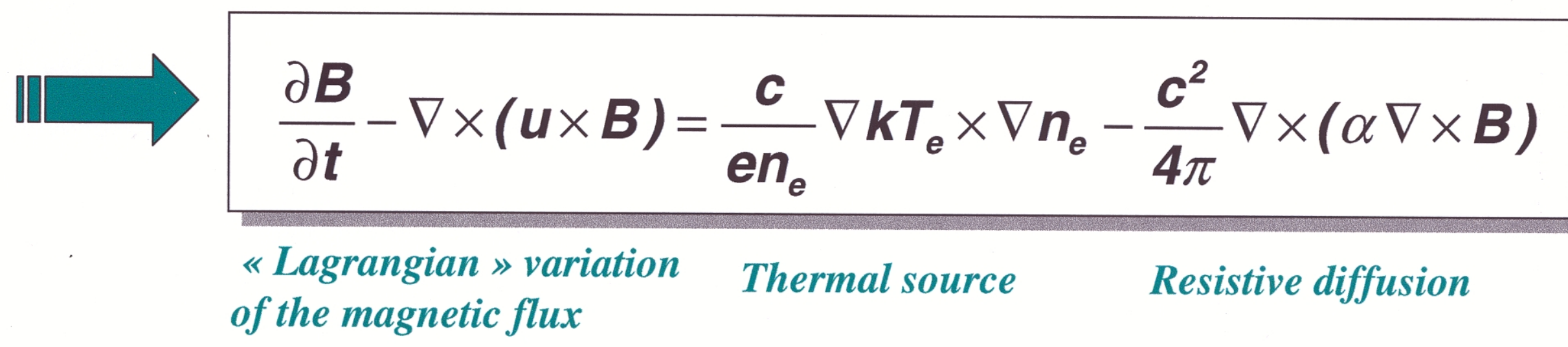

- Boundary conditions : $B=0$ ( sauf $Z p$ ) 


\section{Effects of magnetic fields}

- Laplace force is most often negligible:

- magnetic pressure : $\quad P_{(\text {Mbar })} \approx 0.04 B_{(\text {Mgauss })}^{2}$

- B $\sim 1 \mathrm{Mg} \square \mathrm{P}_{\text {mag }} \sim 40 \mathrm{~kb}$ to compare to $\mathrm{P}_{\mathrm{e}}$ (Mb-Gb)

- The heat flux is strongly affected

- electrons rotate aroung field lines and cannot participate to a heat flux any more

- appearance of a heat flux orthogonal to the temperature gradient (Righi Leduc)

- Bfield effects are characterized by the dimensionless number

$$
\omega \tau=\frac{\lambda_{e i}}{R_{l}}=\frac{\omega_{c}}{v_{e i}}
$$




\section{Model implemented in FCl2}

- Resistive MHD, scalar electrical conductivity (Spitzer)

- in 2D axisymmetric geometry $\quad B \equiv B_{\theta}$

- Braginskii heat fluxes including modified heat conductivities according to Epperlein, Haines, Nicolai

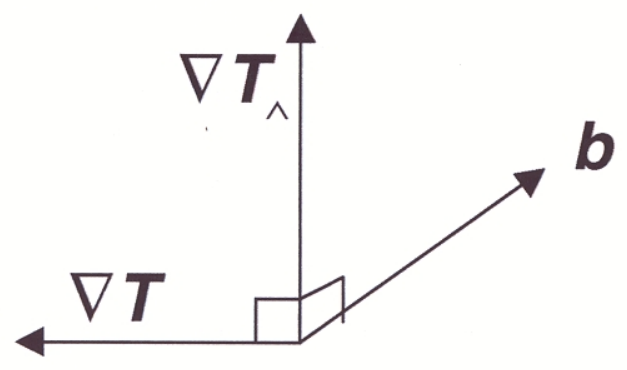

$$
\begin{aligned}
\boldsymbol{Q}= & -\chi_{\perp} \nabla \boldsymbol{T}_{e}-\chi_{\wedge} \boldsymbol{b} \times \nabla \boldsymbol{T}_{e} \\
& -\frac{\beta_{\perp} \boldsymbol{T}_{e}}{\boldsymbol{e} \boldsymbol{n}_{e}} \boldsymbol{j}-\frac{\beta_{\wedge} \boldsymbol{T}_{e}}{\boldsymbol{e} \boldsymbol{n}_{e}} \boldsymbol{b} \times \boldsymbol{j}
\end{aligned}
$$

- For $\mathrm{B}=0, \quad j=0, \chi_{\wedge}=0, \chi_{\perp}=K_{s h}$

- Absence of the Nernst term (though probably necessary because $\mathrm{v}_{\text {nernst }} \sim 0.1 \mathrm{v}_{\text {th }}$ ) 


\section{Coupling the non local model to magnetic fields ...}

- Bfields effects : the relevant parameter is the Hall number $\Omega=\omega \tau$

- at reduced velocity w, the number to consider is $\Omega w^{3}$

- perturbation terms like $w^{4}\left(w^{2}-4\right)$ become $\quad w^{4}\left(w^{2}-..\right) /\left(1+\Omega^{2} w^{6}\right)$

» the perturbation calculus is valid again, even in sharp gradient conditions

» non local effects are cancelled by B fields

- Heuristic used in $\mathrm{FCl} 2$

- use Braginskii instead of SH fluxes as delocalization source

- Limit the delocalisation mfp to the Larmor radius $r_{1}=m v / e B$

» for small $\Omega$, smooth gradients : $Q$---> Spitzer Harm

》 for small $\Omega$, sharp gradients : $Q$---> non local flux

» for large $\Omega \quad:$ Q---> Braginskii « relocalized » 


\section{Delocalisation $\mathbf{m f p}$ in presence of magnetic fields}

- Delocalization mfp are strongly reduced in presence of magnetic fields

- A priori reduction factors are $\quad c_{\perp}=\frac{1}{1+\Omega^{2} w^{6}} \quad c_{\wedge}=\frac{\Omega w^{3}}{1+\Omega^{2} w^{6}}$

- Our non local model only uses a single scalar non local mfp

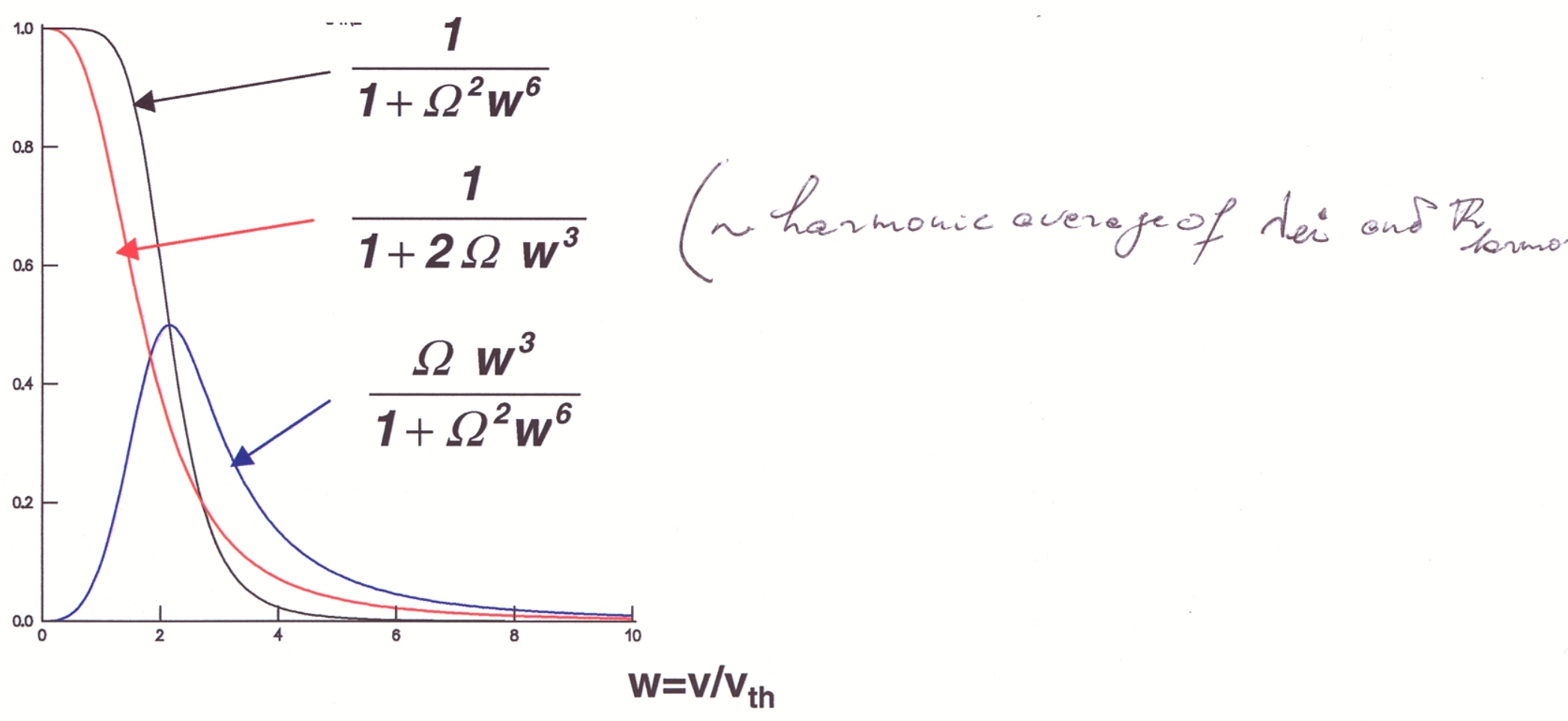




\section{Cas test d 'Epperlein et Rickard}
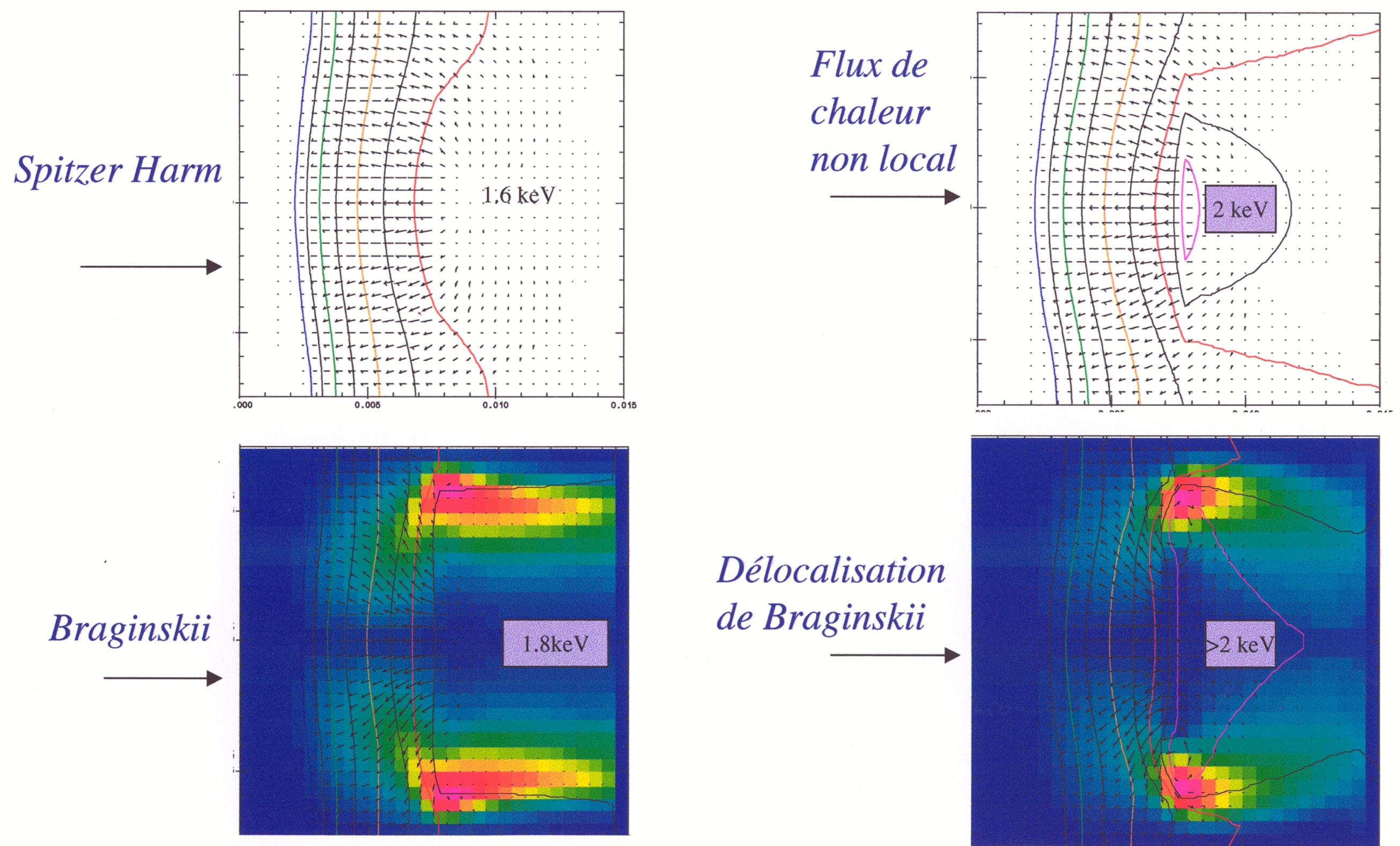

Délocalisation de Braginskii

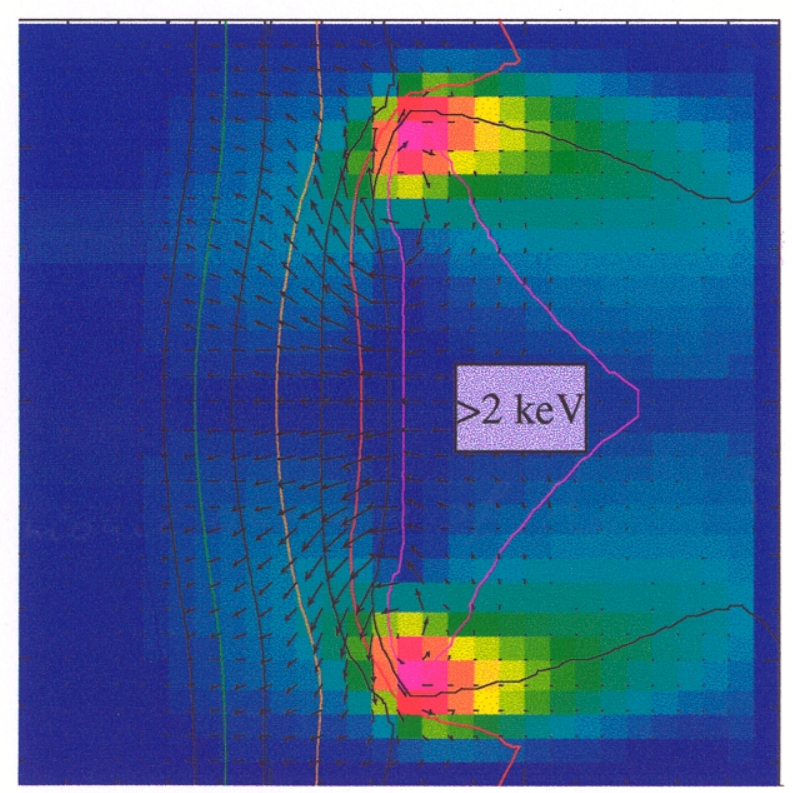


- « zero current » electric fields

- may be computed from the non local distribution function $\boldsymbol{f}_{0}^{n l}$

$$
E_{n l}^{o}=-\frac{m_{e}}{6 e} \frac{\nabla \int f_{0} v^{7} d v}{\int f_{0} v^{5} d v}
$$

- degenerates to the Spitzer null current electric field for $\boldsymbol{f}_{o}=\boldsymbol{f}_{o}^{m b}$

$$
E_{s h}^{o}=-\frac{k T_{e}}{e}\left(\nabla \ln \left(n_{e}\right)+\frac{5}{2} \nabla \ln \left(T_{e}\right)\right)
$$

- Magnetic fields sources : $\left(\frac{\partial B}{\partial t}\right)_{s}=-c \nabla \times E^{0}$

- degenerates to the classical thermal source $-\frac{c}{\boldsymbol{e} n_{e}} \nabla n_{e} \times \nabla k T_{e}$

- non local effects may appear (e.g.: Kingham \& Bell PRL 88-2002) 
Non local effects cause kinetic sources to depart significantly from thermal sources

- Sources for B fields $\left(\frac{\partial B}{\partial t}\right)_{s}^{k i n}=\frac{c m_{e}}{6 e} \nabla \times \frac{\nabla \int f_{0} v^{7} d v}{\int f_{0} v^{5} d v} \rightarrow-\frac{c}{e n_{e}} \nabla n_{e} \times \nabla T_{e}$

- Epperlein \& Rickard

$$
B_{\theta} \text { at } 120 \text { ps }
$$

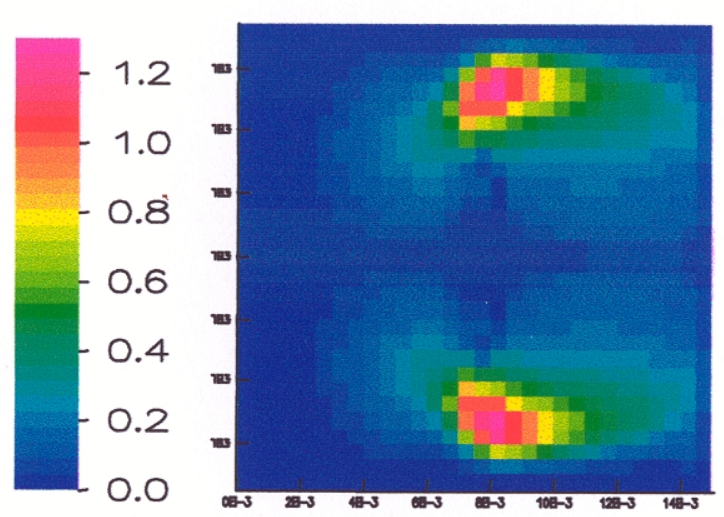

Thermal source

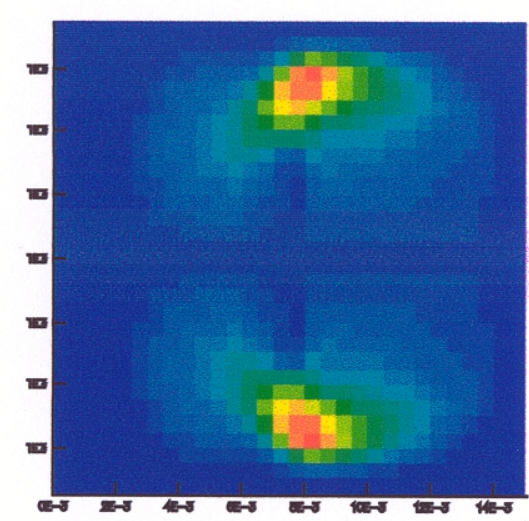

Non local source

- Kingham \& Bellh.002

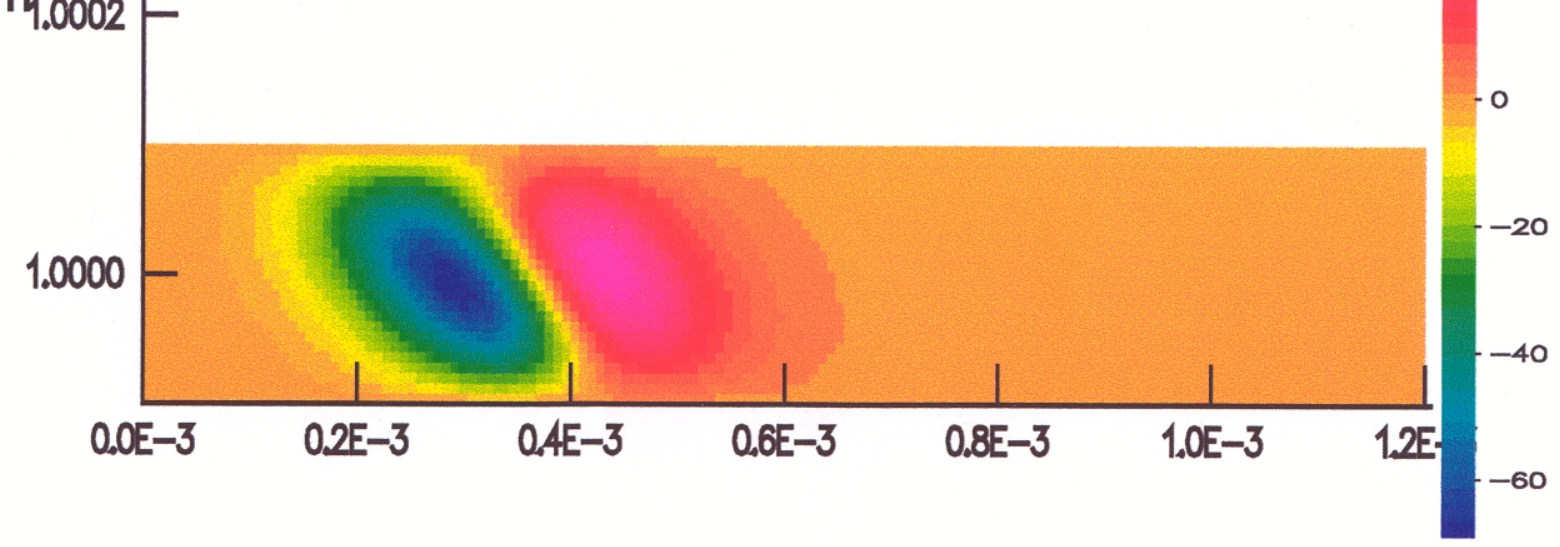




\section{Missing terms in the induction equation}

- Epperlein problem

B1
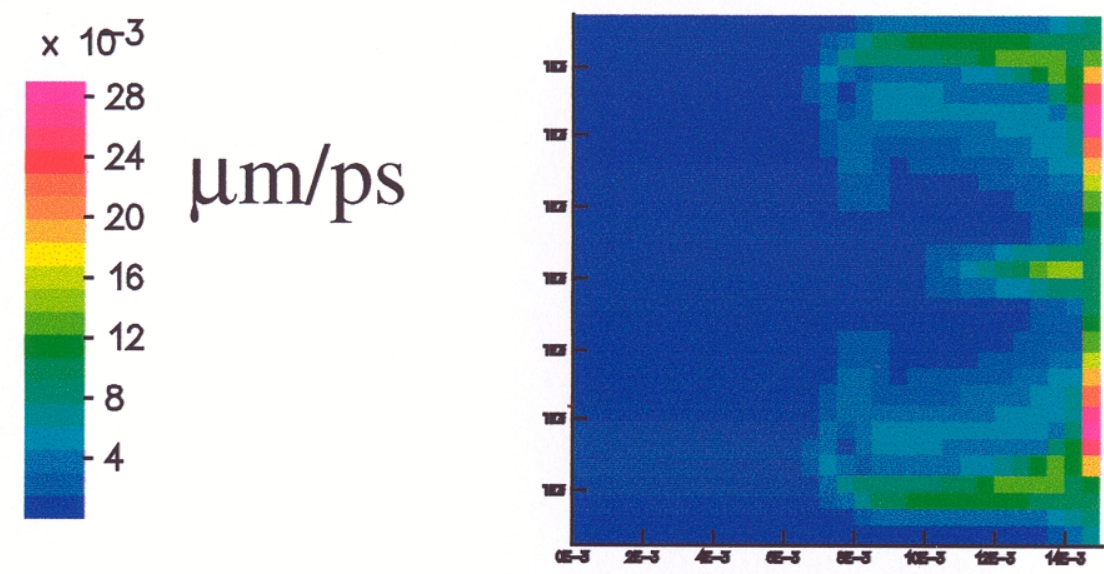

The Hall term is negligible

Hall velocity $\mathrm{j} / \mathrm{en}_{\mathrm{e}}$
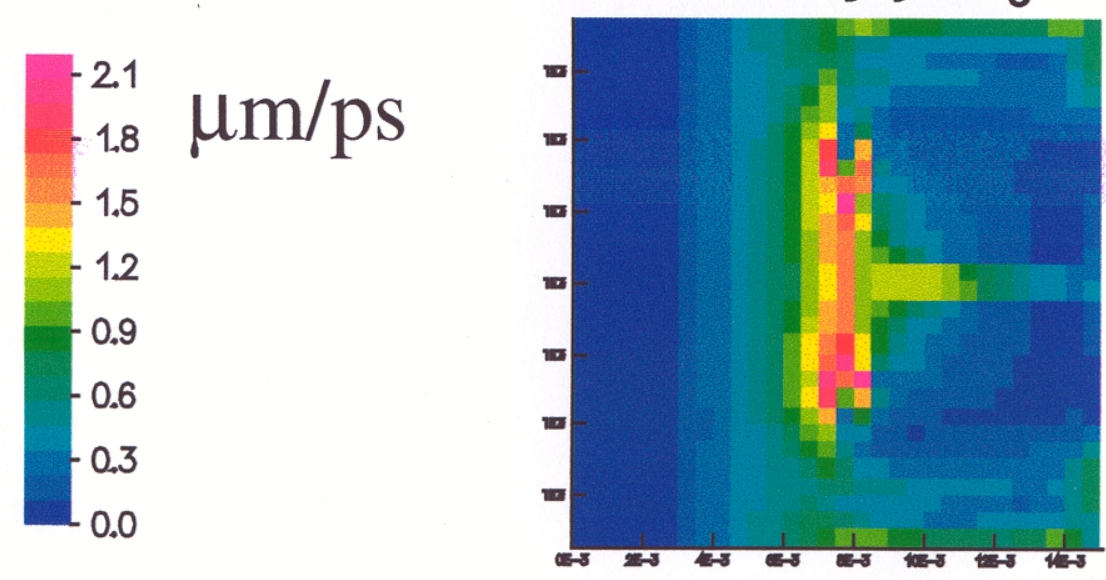

Nernst velocity $\sim 0.07$ thermal velocity

$==>$ Nernst advection time scale is shorter than growth time scale

Nernst velocity $0.72 Q / n_{e} T_{e}$ 


\section{Coupling non local model to magnetic fields}

- We delocalize Braginskil fluxes with a modified diffusion operator

- Respects limit regimes

- for $\mathrm{B}=\mathbf{0}, \mathrm{SH}$ or non local fluxes according to gradient lengths

- At moderate $\omega \tau$, non local effects are cancelled and we find Braginskii

- Interpolation in intermediate situations is unclear : needs to be validated with 2D FP simulations including B fields.

- Calculating sources with non local distribution functions exhibit new effects.

- Non local sources may be reduced because $f_{0} v^{7}$ has smoother gradients (e.g. Epperlein problem).

- Non local sources may be enhanced because $\operatorname{grad}\left(f_{0} v^{7}\right)$ and $\operatorname{grad}\left(\mathrm{f}_{0} \mathrm{v}^{5}\right)$ have larger angles (eg.: Kingham \& Bell)

- The coupled model needs being improved

- Introduction of Nernst in the induction equation

- Further theoretical investigation and numerical validation are 


\section{Numerical Investigation of Recent Laser Absorption and Drive Experiments of $\mathrm{CH}$ Spherical Shells on the OMEGA Laser}

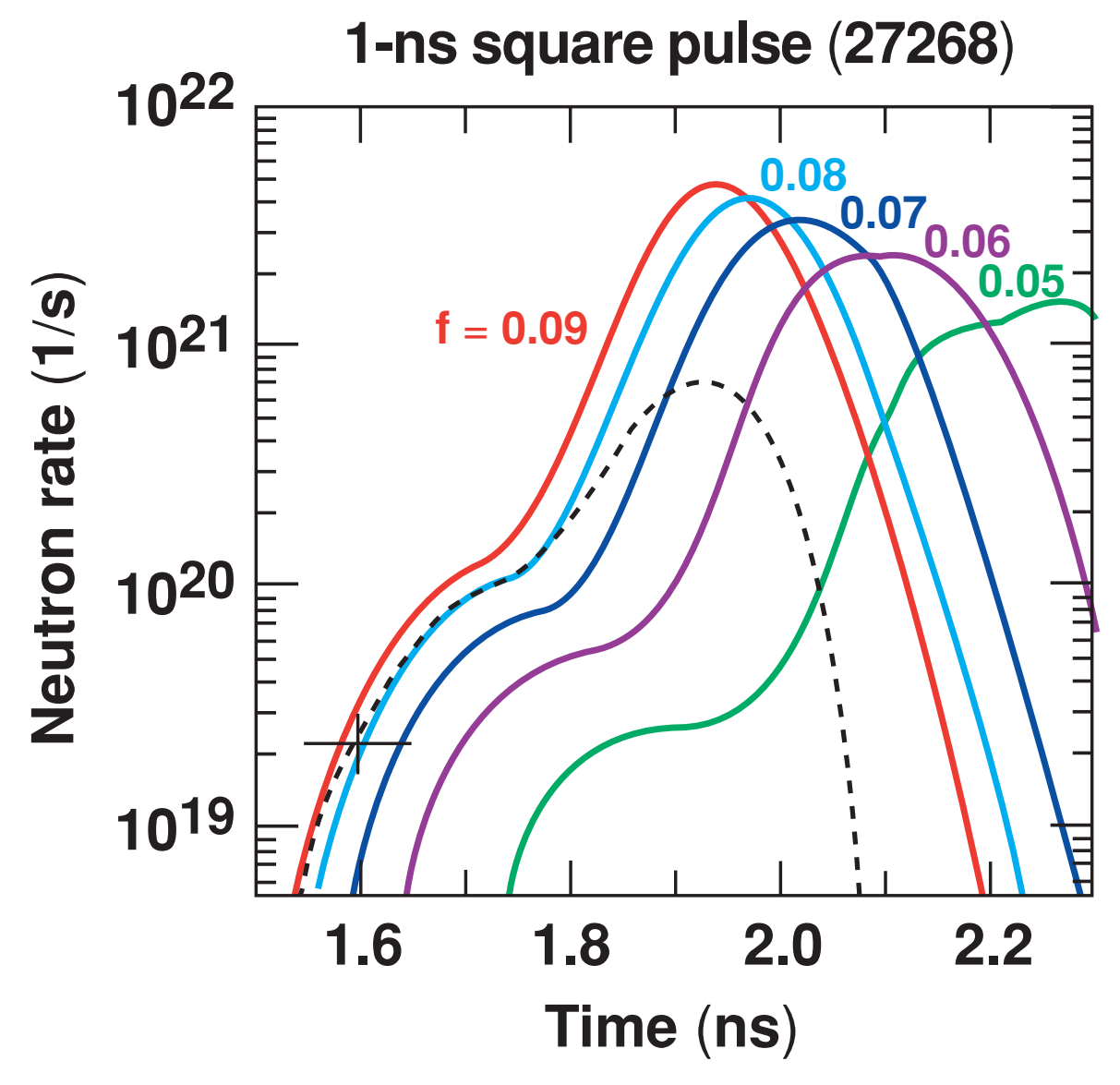

J. A. Delettrez

Laboratory for Laser Energetics University of Rochester 32nd Anomalous Absorption Conference Oahu, Hawaii 21-26 July 2002 


\section{Contributors}

J. P. Knauer, W. Seka, P. Jaanimagi, and C. StoeckI

Laboratory for Laser Energetics

University of Rochester 


\section{Summary \\ Dedicated experiments on the OMEGA laser have measured absorption fraction and implosion timing}

- Neutron temporal diagnostics (NTD), shell trajectory, and temporal x-ray emission measured the drive efficiency.

- Laser absorption was measured with improved diagnostics.

- The timing and the level of both the shock yield and the onset of the compression yield are sensitive to the flux limiter.

- Absorption measurements require a flux limiter value below 0.06 (harmonic).

- A flux limiter between 0.07 and 0.08 gives general agreement with implosion timing.

- Work is ongoing to reconcile the two results. 


\section{The flux limiter affects independently the drive and the laser absorption fraction}

- The flux limiter controls the flow of the absorbed energy into the target and affects

- the drive though the mass ablation rate and

- the absorption fraction through the electron temperature in the corona.

- It is active at and inside the critical surface.

- Two methods are used to compute the thermal flux:

- the sharp cutoff: $Q=\max \left(Q_{\mathrm{SH}}, Q_{\mathrm{FS}}\right)$

- the harmonic mean: $\mathbf{Q}=\left(\mathbf{Q}_{\mathbf{S H}} \mathbf{Q}_{\mathrm{FS}}\right) /\left(\mathbf{Q}_{\mathrm{SH}}+\mathbf{Q}_{\mathrm{FS}}\right)$ 


\section{The absorbed energy was measured with two independent diagnostics}

- Two differential plasma calorimeters measure the plasma and scattered light reaching the tank wall (time integrated).

- Two full-aperture backscatter stations (FABS, $f / 6$ ) measure the scattered and refracted light through two focusing lenses (time integrated and time resolved).

- Two subsidiary scattered light diagnostics measure the scattered/ refracted light between the lenses (time integrated and time resolved).

- The signals from all six calorimeters are very consistent with overall errors estimated at $2 \%$ (absolute) from shot to shot. 


\section{The drive timing was obtained from $x$-ray and neutron diagnostics}

- The shell trajectory was measured with an imaging streak camera and a framing camera.

- The onset of stagnation was via the shock yield measured with the neutron temporal diagnostic (NTD).

- The temporal x-ray emission was obtained from a diamond detector. 


\section{The neutron burn history shows details of the shock arrival and the stagnation phase of the implosion}
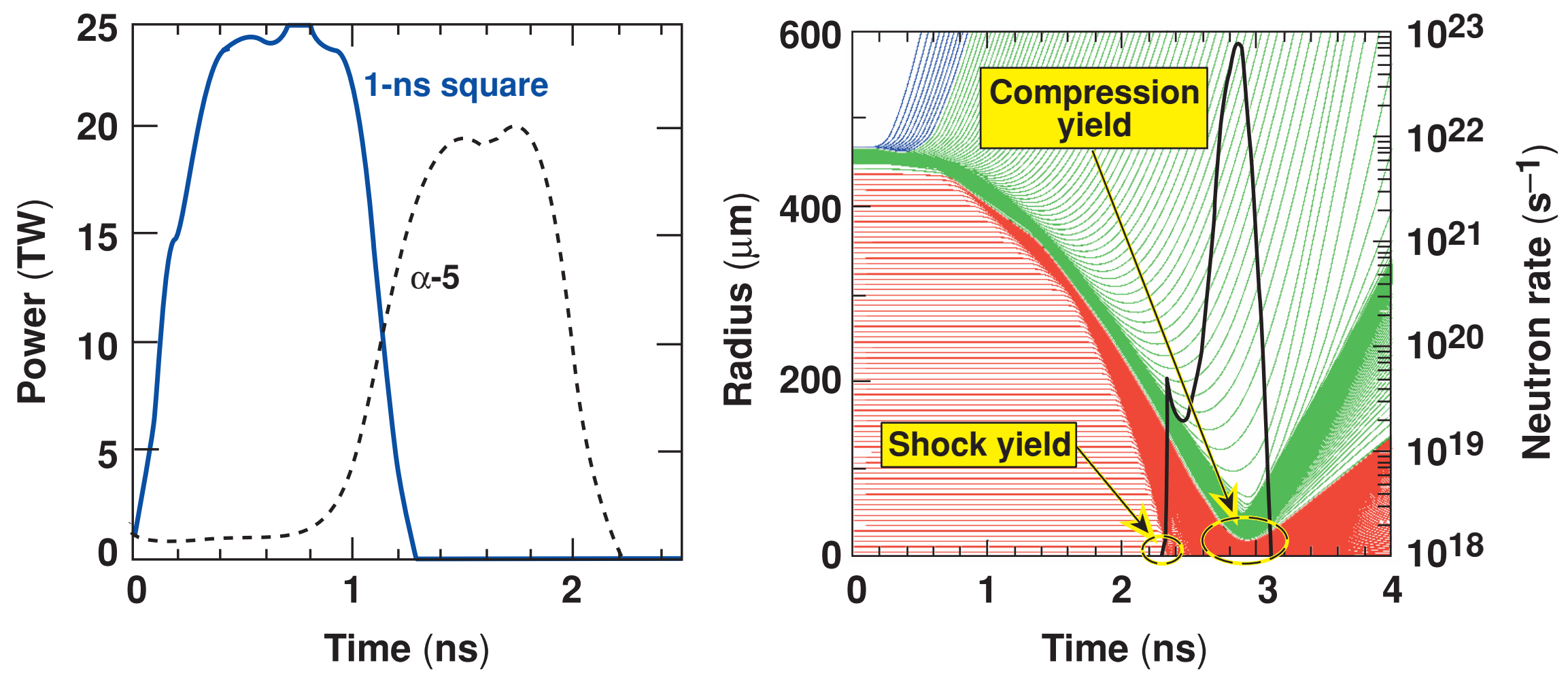

Targets are $15 \mu \mathrm{m} \mathrm{CH}$ or CHSi shells filled with 15 atm $\mathrm{D}_{2}$, $\mathrm{D}_{2} / \mathrm{Ar}$, or $\mathrm{D}_{2}{ }^{3} \mathrm{He}$, and diameters $930 \mu \mathrm{m}$ and $1100 \mu \mathrm{m}$. 
The laser absorption is modeled in LILAC with 2-D ray tracing and classical inverse bremsstrahlung

- The ray trace uses the measured DPP spatial distribution, including the effect of SSD and PS.

- The absorption model includes the Langdon effect.

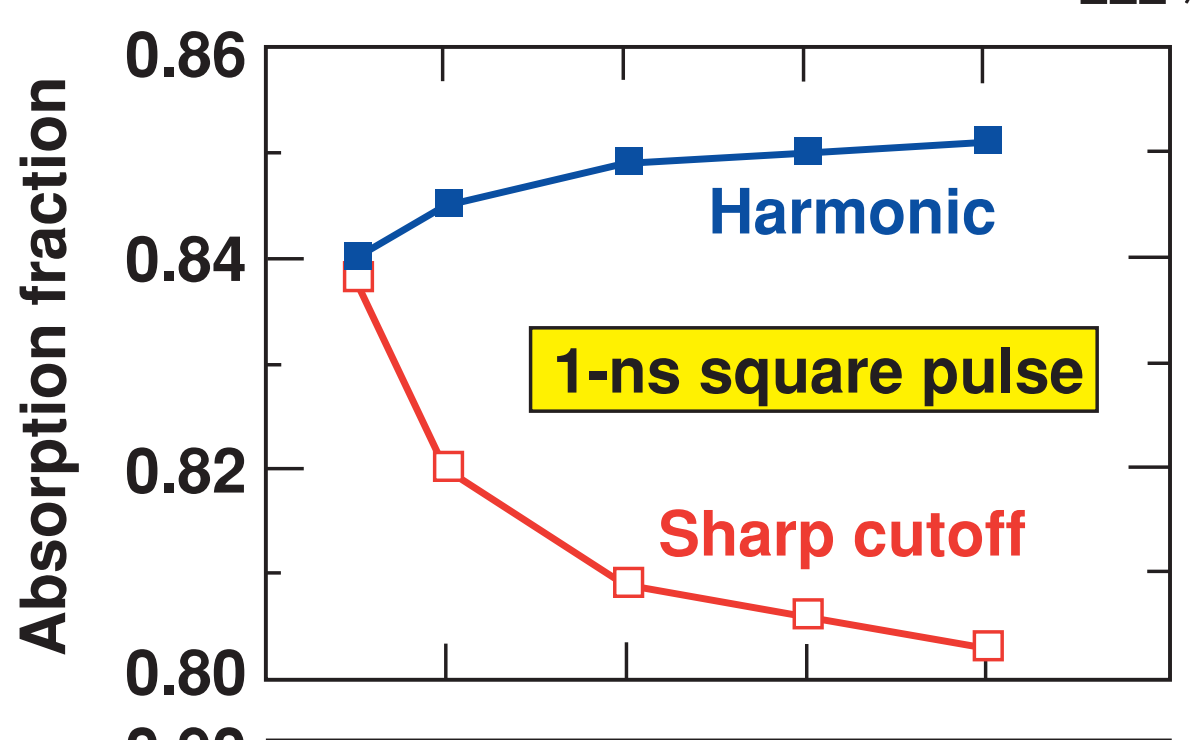

- The density profile at and below the critical surface is zoning dependent.

- The harmonic mean method is less sensitive to zoning than the sharpcutoff method.

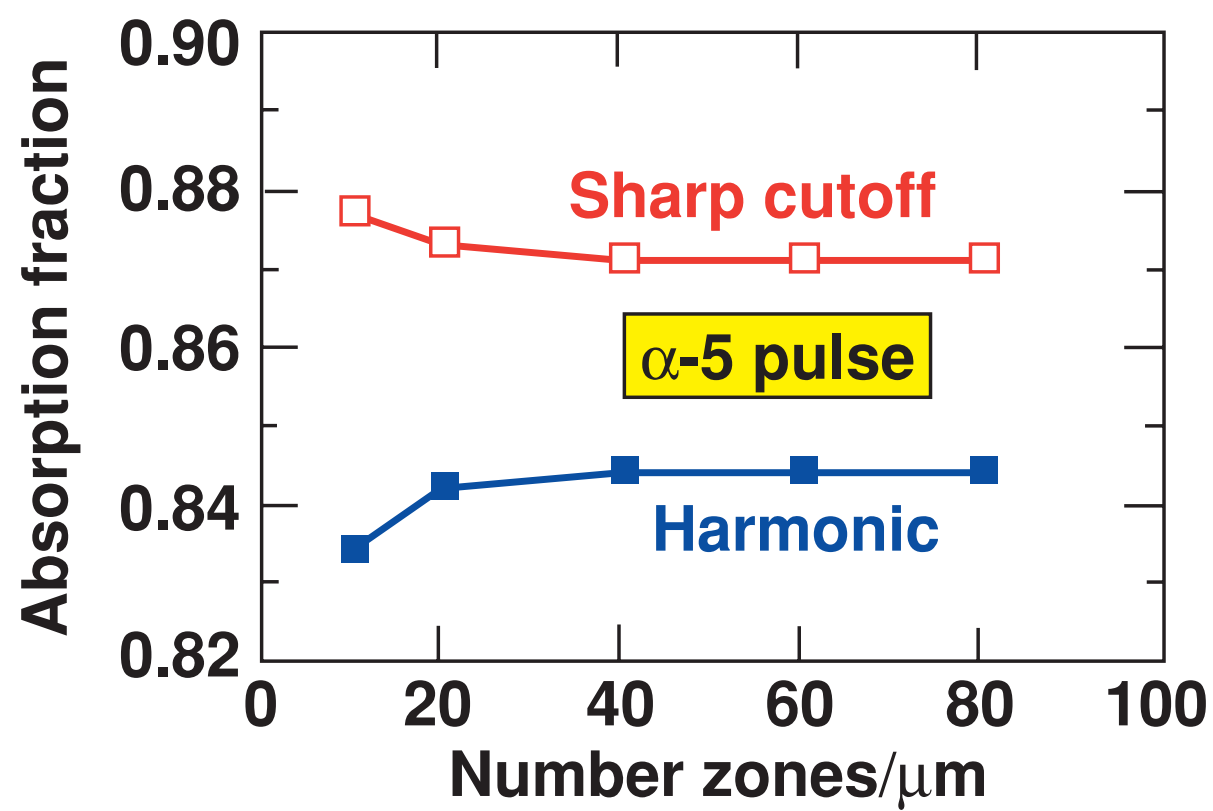


The measured and simulated absorption fractions show the same trend over a wide range of experimental conditions

Scattered light absorption

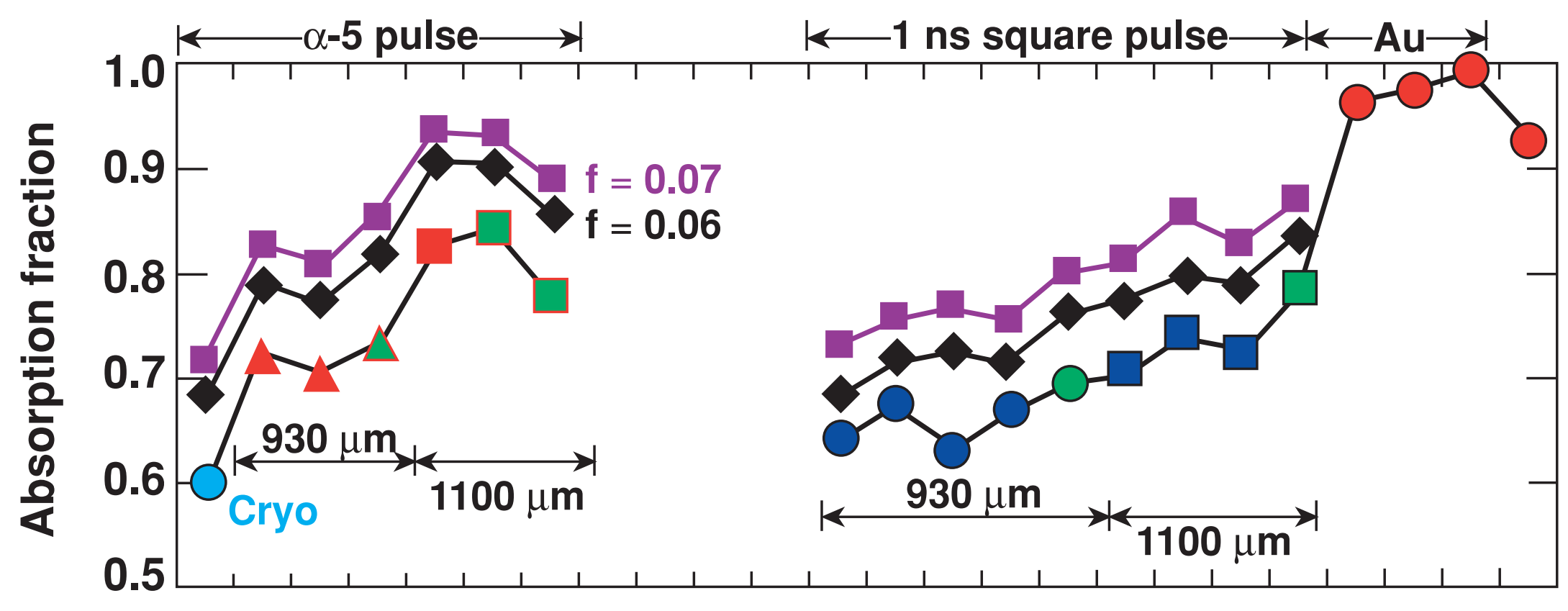

Green fill: CHSi shells Experimental error bars are size of symbols 
For $\mathrm{CH}$ shells and generic conditions LILAC needs a low value of flux limiter to match the experimental measurements
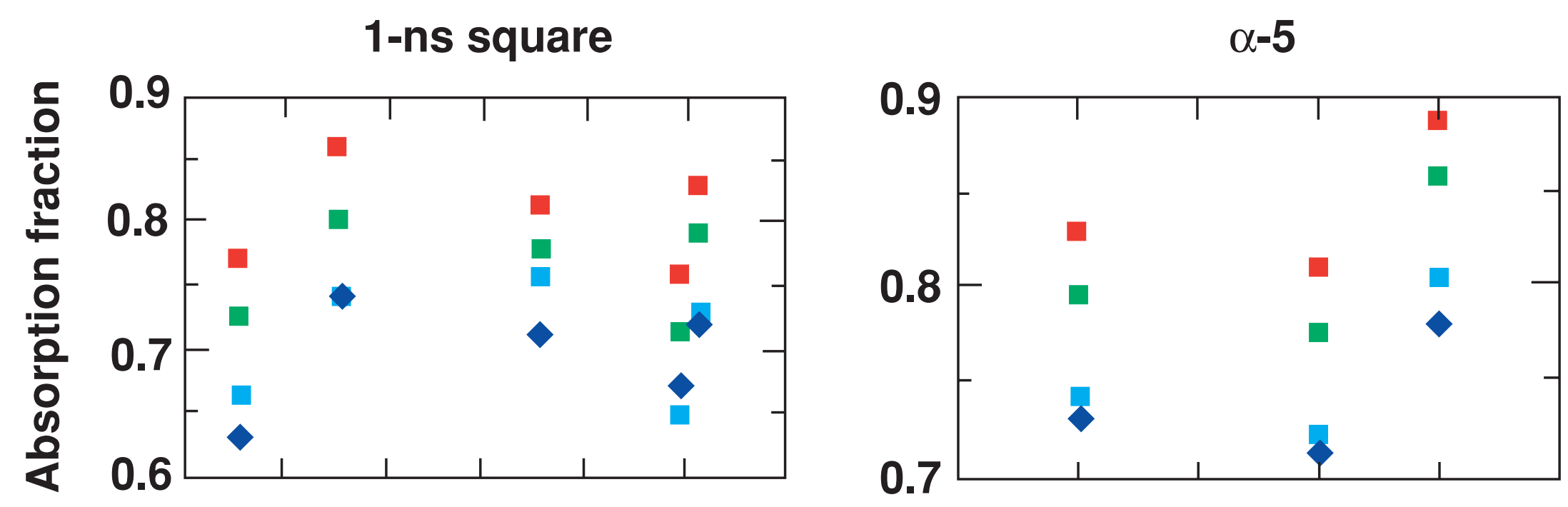

$$
\begin{array}{ll}
\square f=0.07 & \square f=0.05 \\
\square f=0.06 & \forall x p .
\end{array}
$$


The NTD timing is best matched by a flux limiter between 0.07 and 0.08 harmonic
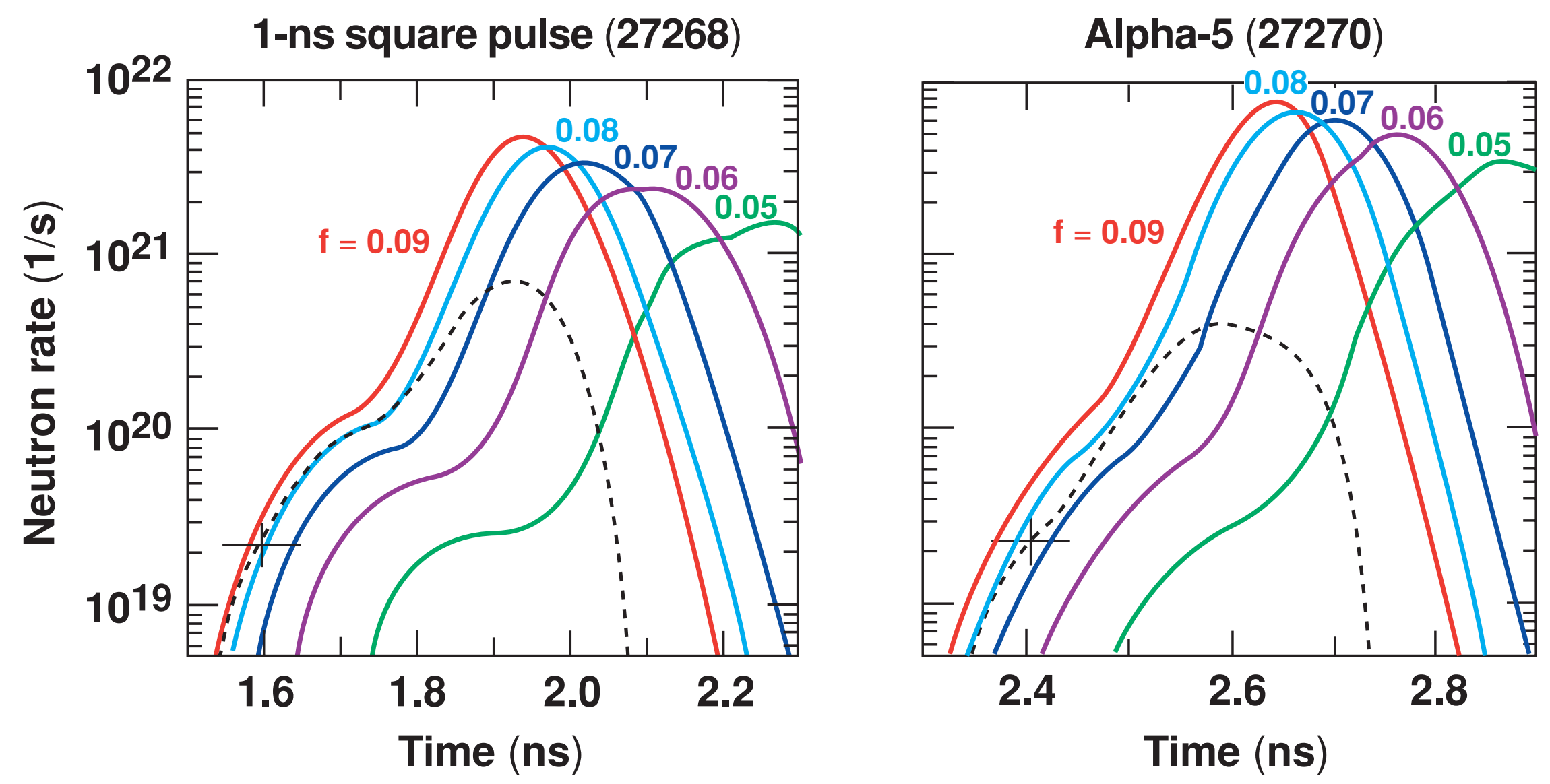


\section{The shell trajectories confirm the results of NTD}

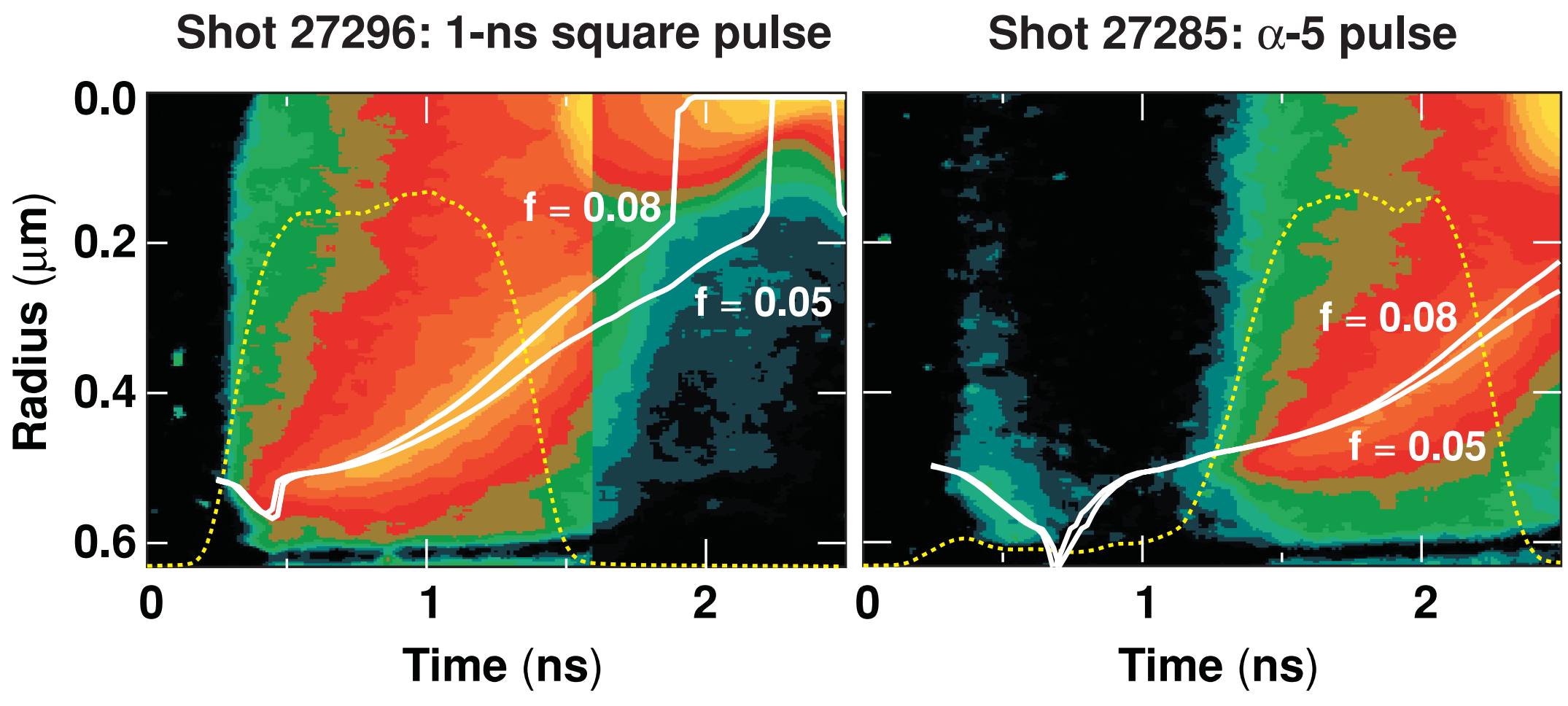




\section{Reconciliation between the results of the absorption and implosion timing is difficult}

- Flux-limiter values between 0.07 and 0.08 are supported by

- NTD and x-ray timing in the experiments reported here,

- Ar emission timing in doped-core mix experiments, ${ }^{1}$ and

- Fokker-Plank calculations of the thermal flux.2, 3

- Absorption measurements agree with a flux limiter below 0.06 .

- Time-dependent flux limiter ${ }^{3}$ goes the wrong way.

- Many considered scenarios failed because of the coupling between absorbed energy and drive efficiency through the flux limiter. 
Summary/Conclusions

Dedicated experiments on the OMEGA laser have measured absorption fraction and implosion timing

- Neutron temporal diagnostics (NTD), shell trajectory, and temporal x-ray emission measured the drive efficiency.

- Laser absorption was measured with improved diagnostics.

- The timing and the level of both the shock yield and the onset of the compression yield are sensitive to the flux limiter.

- Absorption measurements require a flux limiter value below 0.06 (harmonic).

- A flux limiter between 0.07 and 0.08 gives general agreement with implosion timing.

- Work is ongoing to reconcile the two results. 


\title{
A brief review of 2D Fokker-Planck codes without B-fields
}

\author{
Richard P J Town
}

LLNL 


\section{Most 2D FP codes have used the diffusive approximation in the high $\mathrm{Z}$ limit ${ }^{1}$}

- SPARK keeps only $\mathrm{f}_{0}$ and $\mathrm{f}_{1}$ :

$$
\begin{aligned}
& \frac{\partial f_{0}}{\partial t}+\frac{v}{3} \underline{\nabla} \cdot \underline{f_{1}}= \\
& \frac{1}{v^{2}} \frac{\partial}{\partial v}\left[\frac{v^{2}}{3} \underline{a} \cdot \underline{f_{1}}+Y\left(C f_{0}+D \frac{\partial f_{0}}{\partial v}\right)+\frac{Y n Z}{6 v} v_{0}^{2} \frac{\partial f_{0}}{\partial v}\right] \\
& \underline{f_{1}}=\tau\left(v \nabla f_{0}-\underline{a} \frac{\partial f_{0}}{\partial v}\right)
\end{aligned}
$$

where:

$$
\begin{aligned}
& \left.\underline{a}=e \underline{E} / m, \tau=v^{3} /(Z+1) n Y\right], \\
& Y=4 \pi\left(e^{2} / m\right)^{2} \ln \Lambda, C=I_{0}^{0} f_{0}, D=\frac{1}{3} v\left(I_{2}^{0}+J_{-1}^{0}\right)
\end{aligned}
$$

1P. Shkarofsky et al, "The Particle Kinetics of Plasmas" (1966). 
The Japanese have developed a 2-D FP code called KEICO'

- The code expands to $f_{0}$ and $f_{1}$ only and retains the electron inertia term $\left(d_{1} / d t\right)$.

- Preheating due to nonlocal electron thermal transport suppresses the Rayleigh-Taylor growth rate:

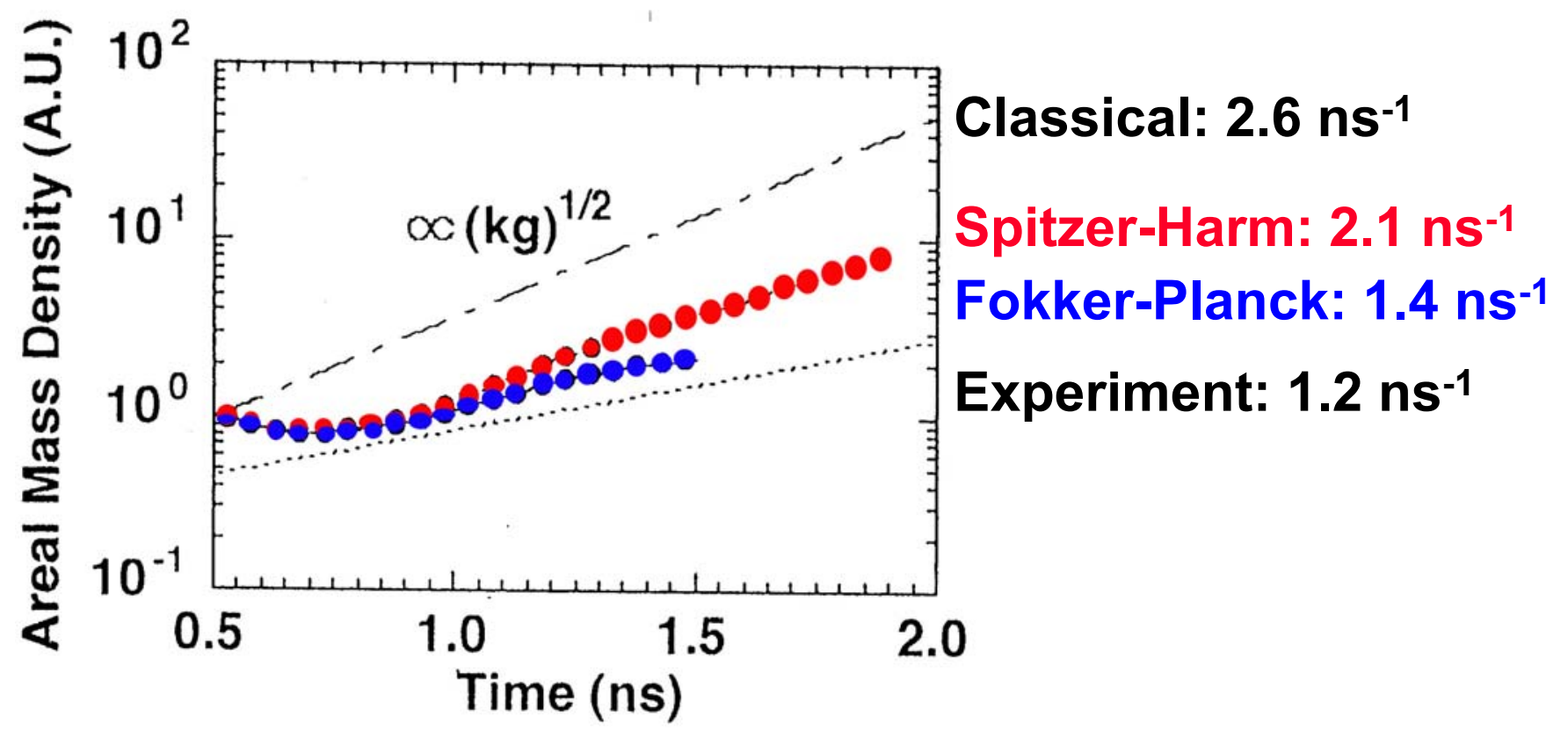


The SPARK code uses the ADI scheme to invert the Fokker-Planck equation ${ }^{1}$

$$
\begin{aligned}
& \frac{\partial f_{0}}{\partial t}=\underline{\nabla} \cdot\left[\chi\left(\nabla f_{0}-\underline{a} \alpha \frac{\partial f_{0}}{\partial v}\right)\right]+ \\
& \frac{1}{v^{2}} \frac{\partial}{\partial v}\left[\chi\left(\underline{a} \cdot \underline{a} \frac{\partial f_{0}}{\partial v}+v \underline{\beta} \cdot \underline{a} f_{0}\right)+Y\left(C f_{0}+\left(D+\frac{Y n Z v_{0}^{2}}{6 v}\right) \frac{\partial f_{0}}{\partial v}\right)\right]
\end{aligned}
$$

- Finite difference the above using Chang-Cooper weighting.

- Two alternatives for calculating the electric field were tried:

- Implicit moment method (curl E = 0)

- Total current equals zero.

- When $\mathrm{J}=0$ there was a deterioration in quasineutrality. 


\section{SPARK modeled the interaction of a short pulse laser with a solid target}

- Thermal smoothing becomes less effective in smoothing small scale $\left(<80 \lambda_{\text {mfp }}\right)$ temperature modulations when the electron transport is modeled by Fokker-Planck.

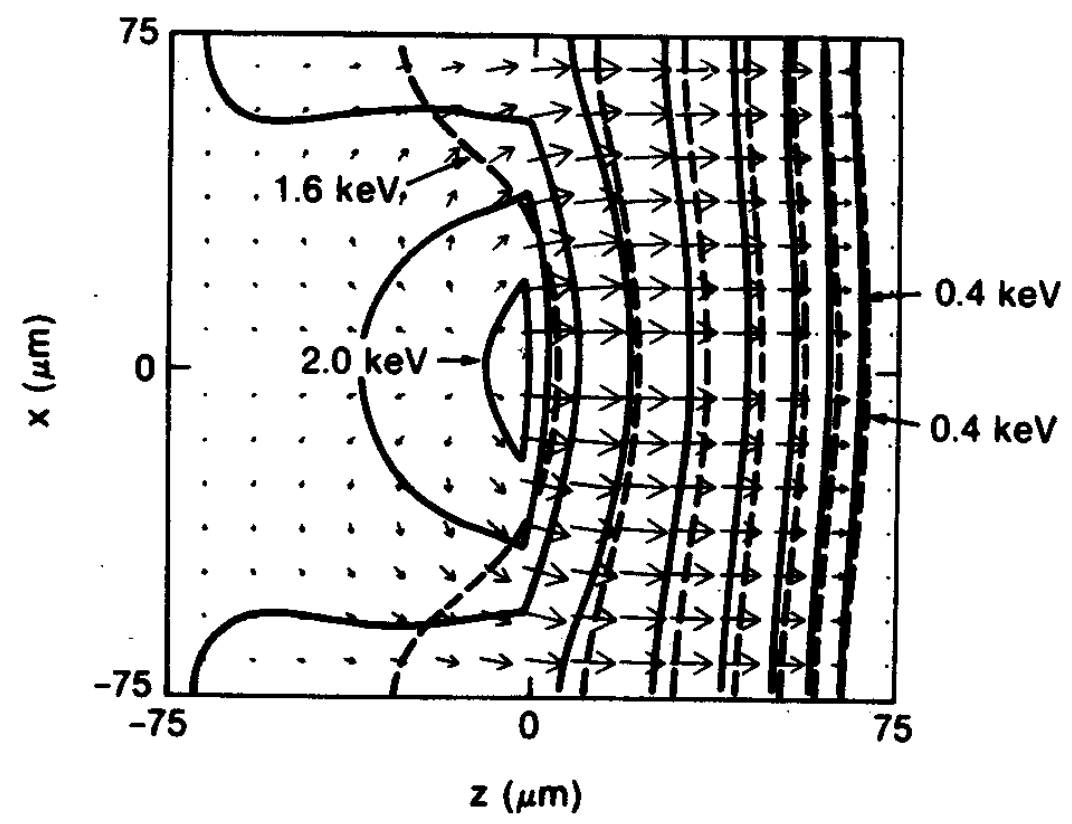

${ }^{1}$ E. M. Epperlein et al, Phys. Rev. Lett. 61, P2453 (1988) 


\section{SPARK simulations showed the heat flow was preferentially directed into the target}

- The heat flow into the target did not exceed $0.1 \mathrm{q}_{\mathrm{fs}}$

- The heat flow laterally was much less than $0.1 q_{\mathrm{fs}}$

- Large angle between q and grad T were found
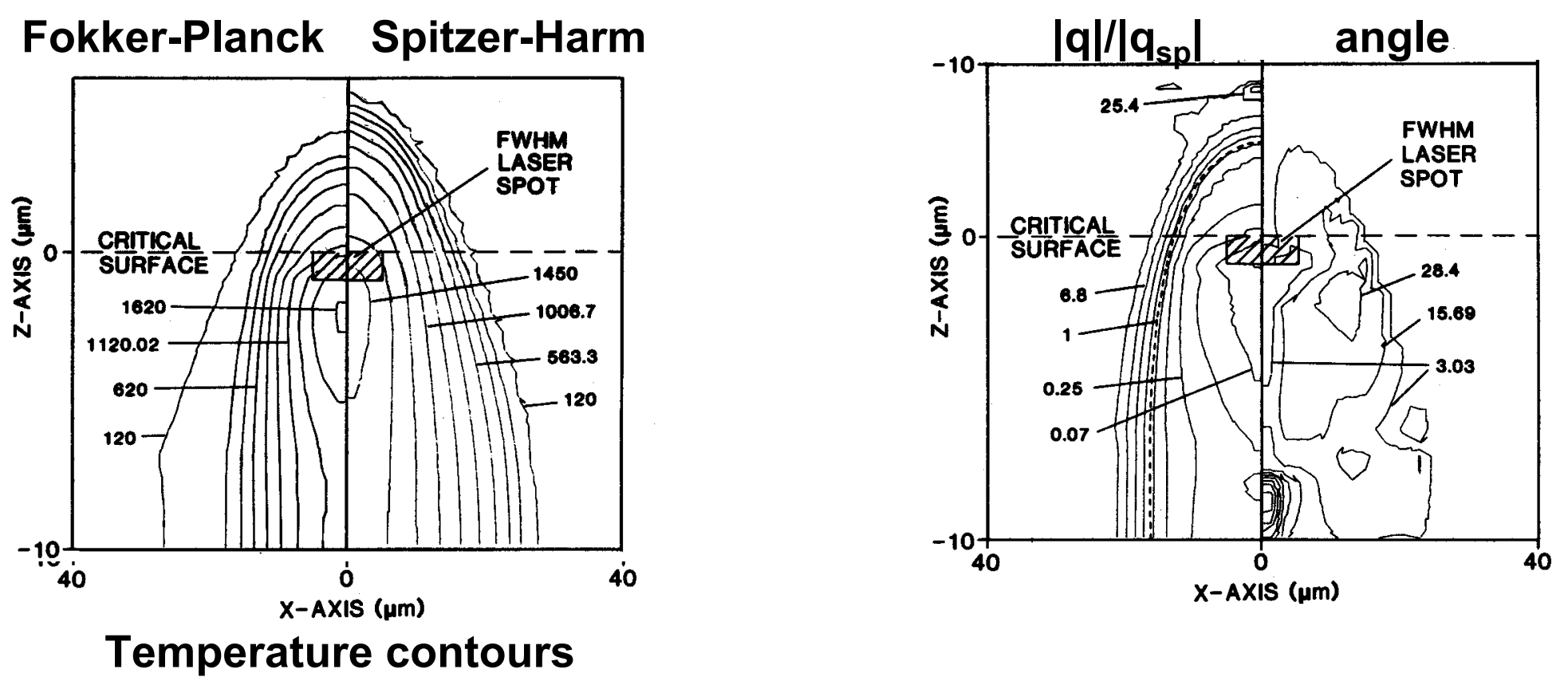

1G. J. Rickard et al, Phys. Rev. Lett. 62, P2687 (1989) 


\section{SPARK has been used to model filamentation}

- Nonlocal heat flow was found to enhance the laser filamentation rate.

- Enhanced levels of self focusing, with filaments following the ray trajectories, was found when an $\mathrm{f} / 2$ lens was modeled.

(a)

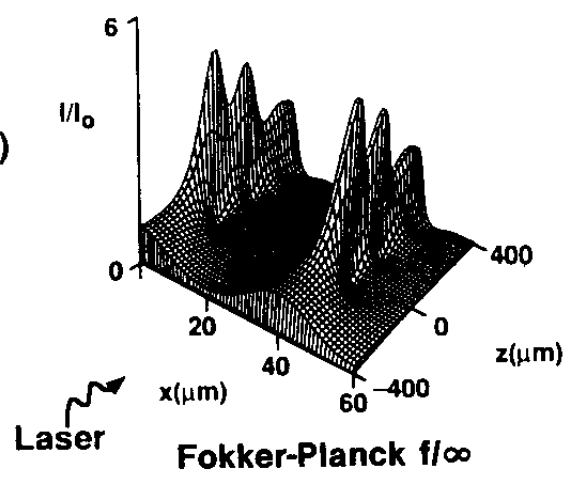

(c)

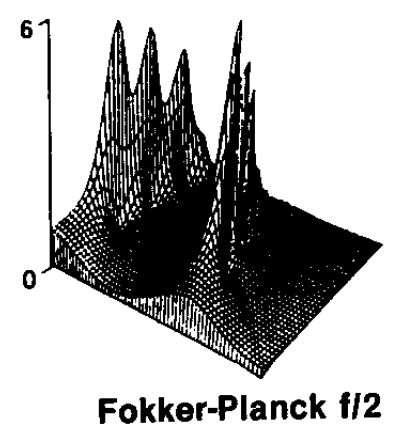

(b)

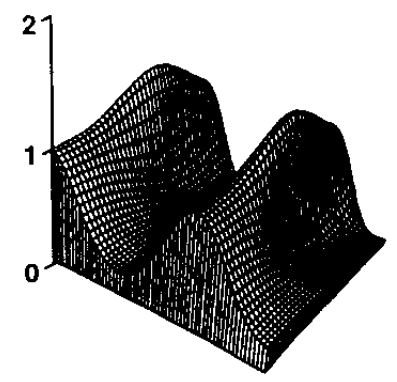

Spitzer-Härm f/o

(d)

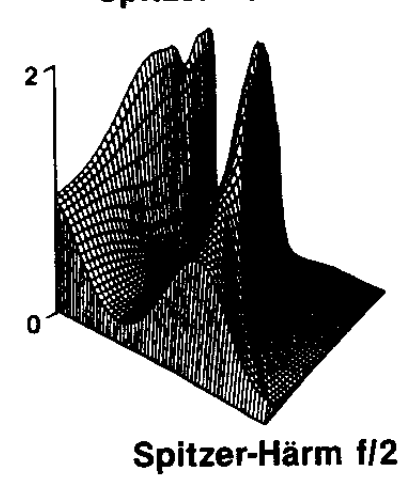

${ }^{1}$ E. M. Epperlein, Phys. Rev. Lett. 65, P2145 (1990) 
One-dimensional simulations have been performed to assess how the foam alters the behavior of the target

UR

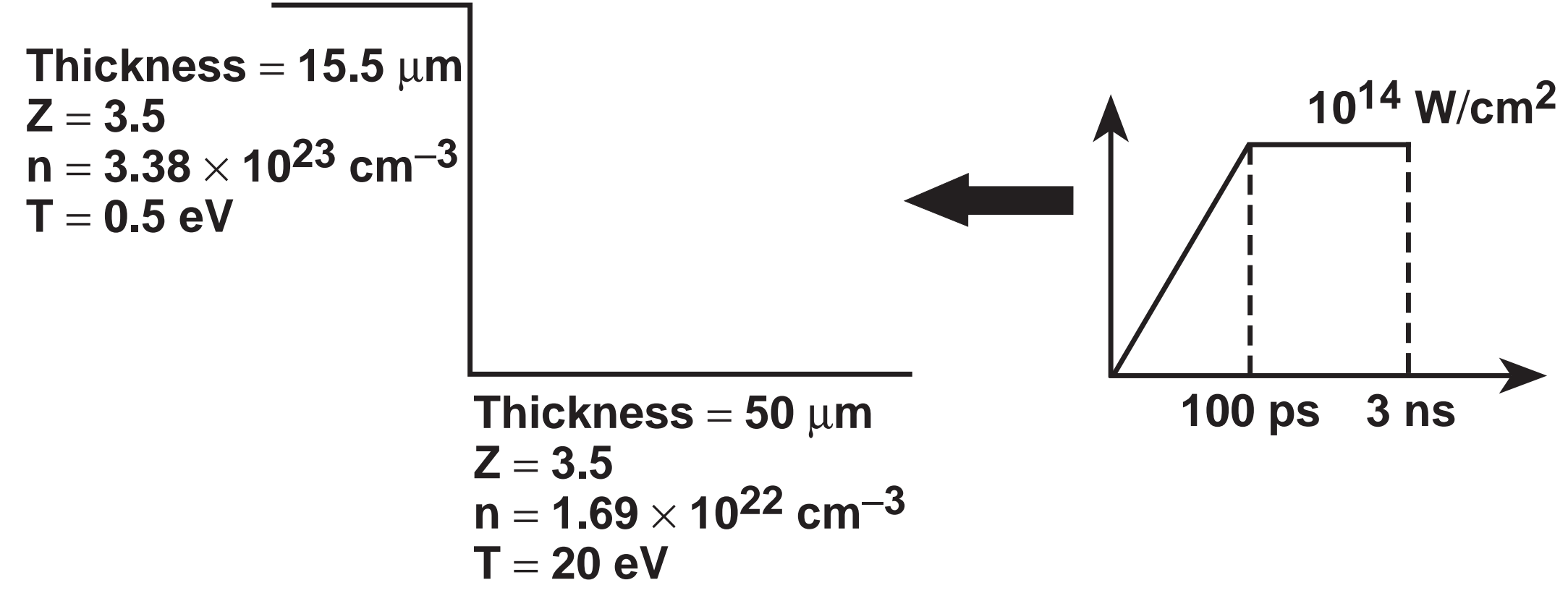




\section{The Fokker-Planck simulations have higher ablation velocities than equivalent Spitzer simulations}

Fokker-Planck simulations show:

- larger preheat of foam and bare targets

- lower peak densities

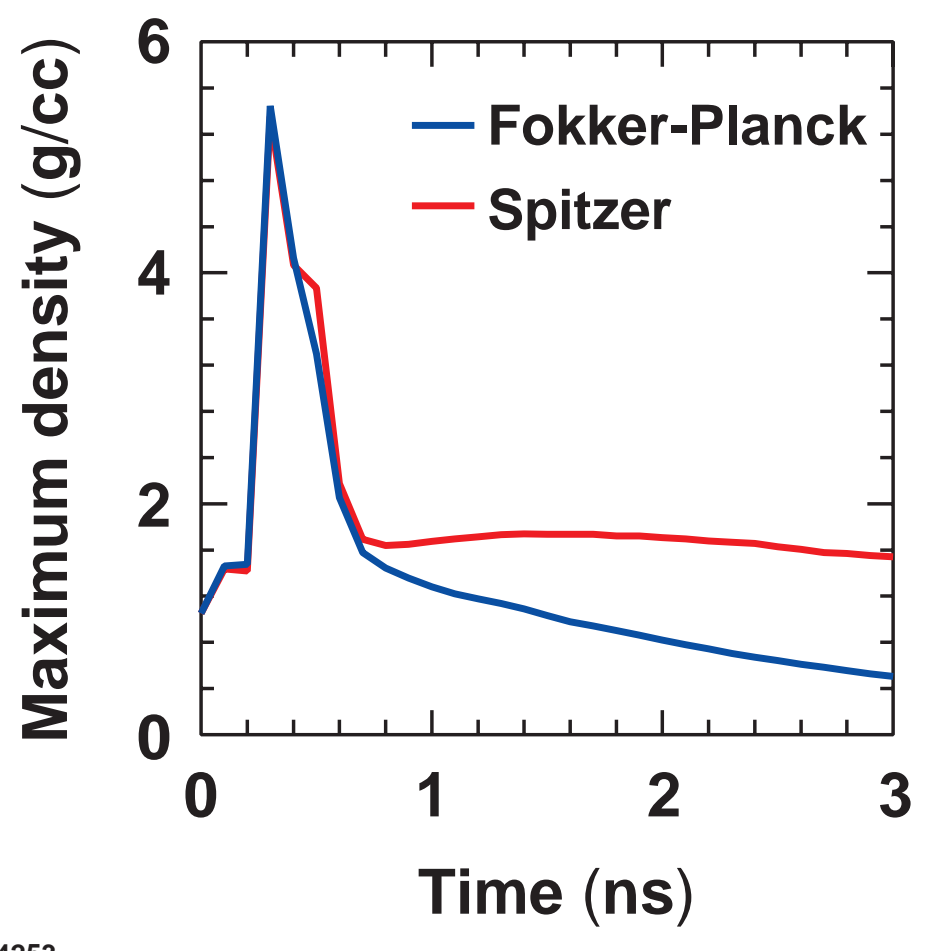

- higher ablation velocities

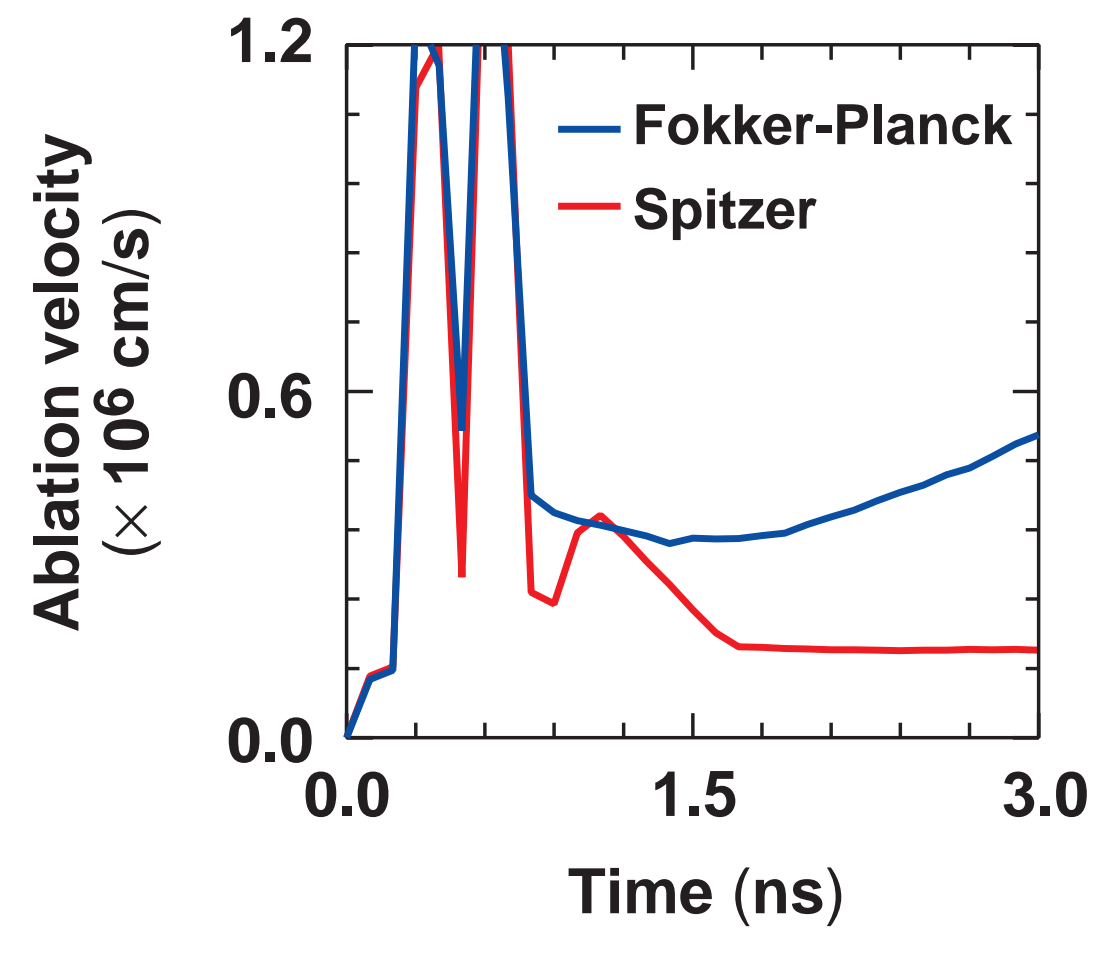


Temperature contour plots show enhanced heat front penetration into the foam for the Fokker-Planck simulation

- The Fokker-Planck temperature contours are less smooth where the energy is being absorbed.

- The Spitzer temperature contours are less smooth at the heat front.

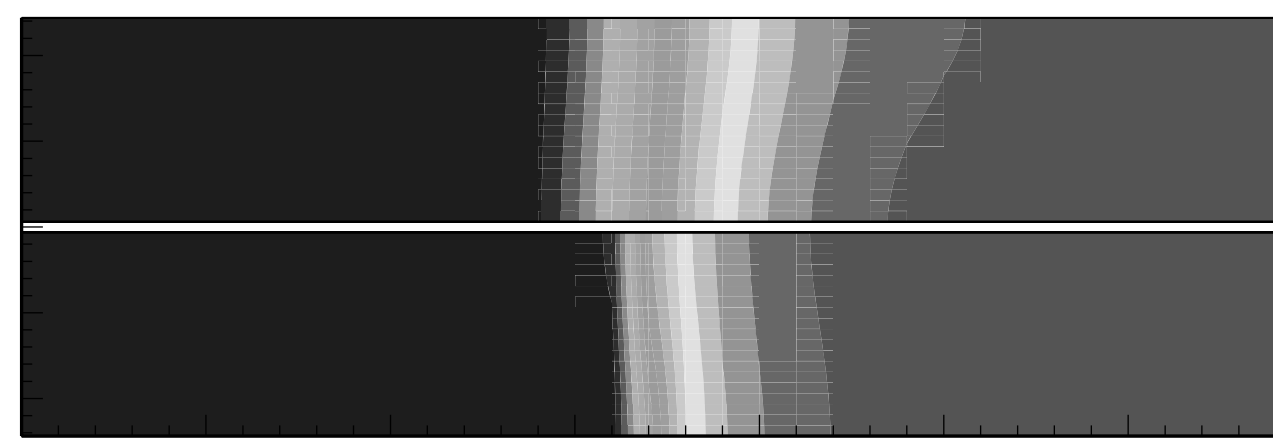

Fokker-Planck

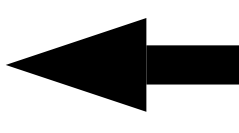

Spitzer

$\begin{array}{llllllll}55 & 114 & 174 & 234 & 293 & 353 & 413 & 472\end{array}$ 


\section{$\sigma_{\mathrm{rms}}$ is a measure of the nonuniformity at a particular distance in the foam}

UR

Defining:

$$
\sigma_{\mathrm{rms}}^{2}=\frac{\int(\mathbf{T}-\langle\mathbf{T}\rangle)^{2} \mathrm{dx}}{\int\langle\mathbf{T}\rangle^{2} \mathrm{dx}}
$$

- The Fokker-Planck is less smooth in the energy-absorbing region but is more smooth than Spitzer in the main body of the foam.

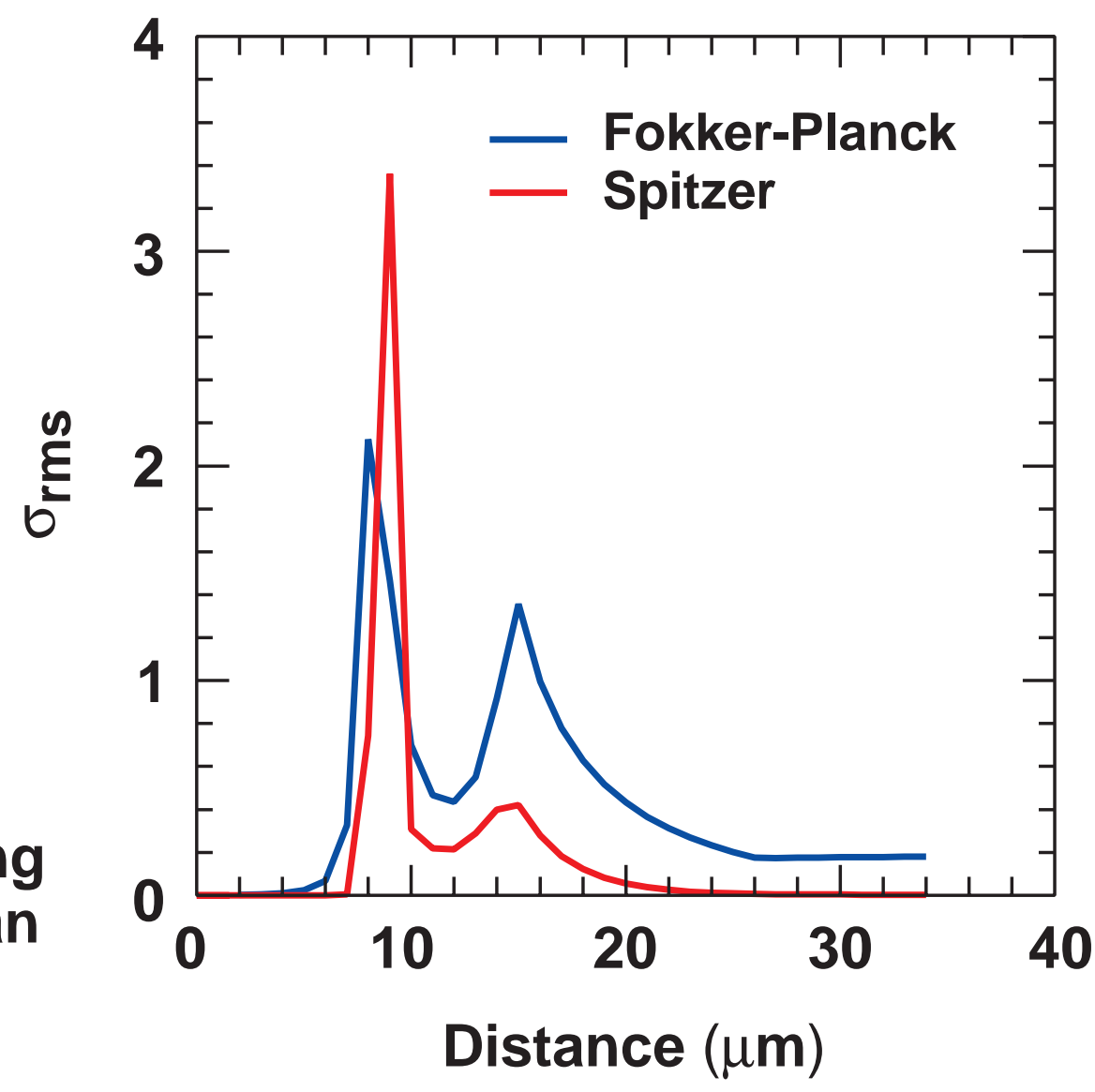




\section{Two-dimensional Fokker-Planck calculations of an idealized hohlraum were performed}

- All boundaries were reflective, apart from the outer radial wall, which was kept at a fixed temperature.

- The laser propagated radially outward from $200 \mu \mathrm{m}$ from the axis and escaped from the outer radial wall.

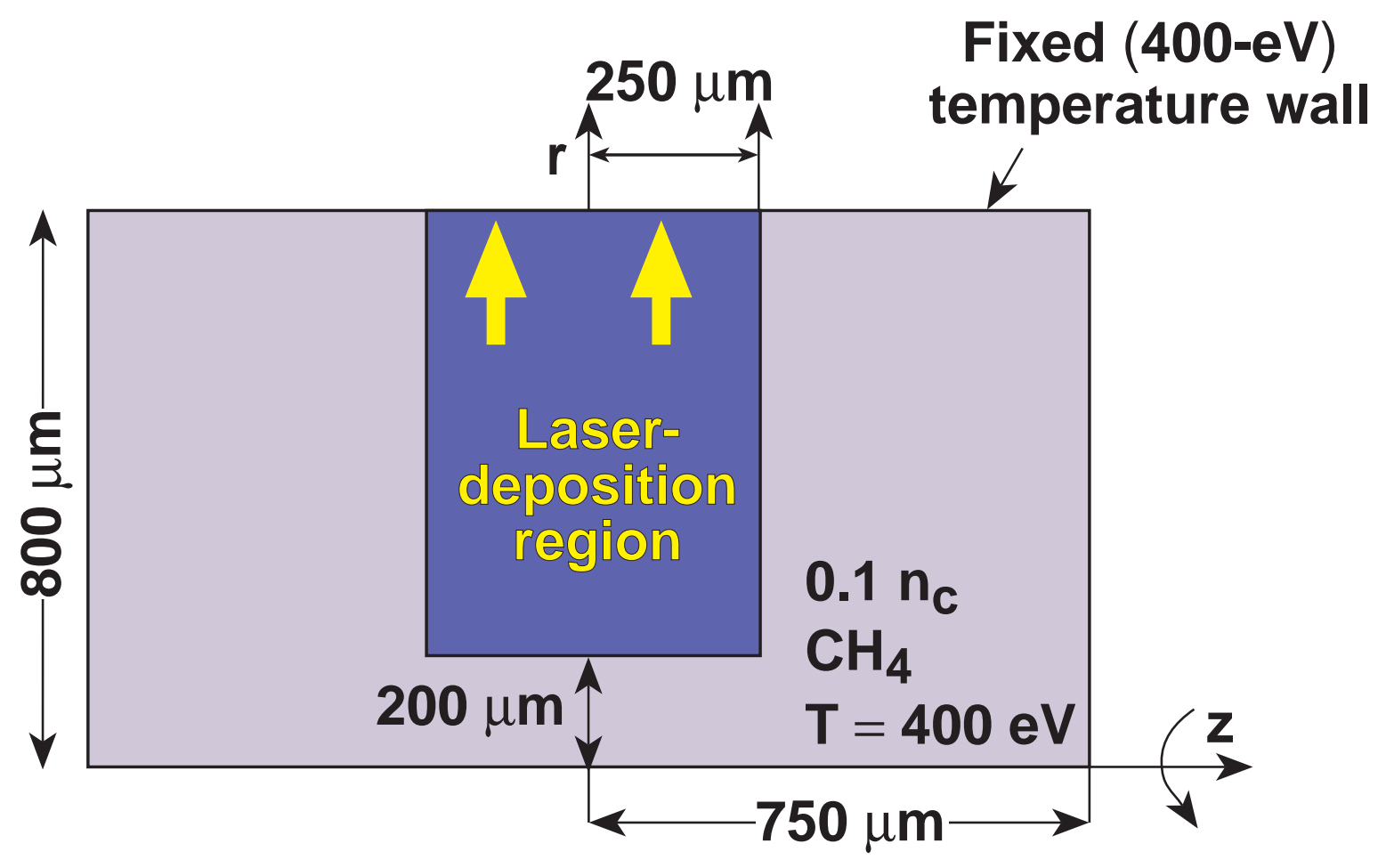


Fokker-Planck calculations show a cooler, but more dispersed heated region than $f=0.01$ Spitzer calculations

- Contour plot of electron temperature after 200 ps shows the $f=0.01$ Spitzer calculations bottles up the absorbed laser energy.

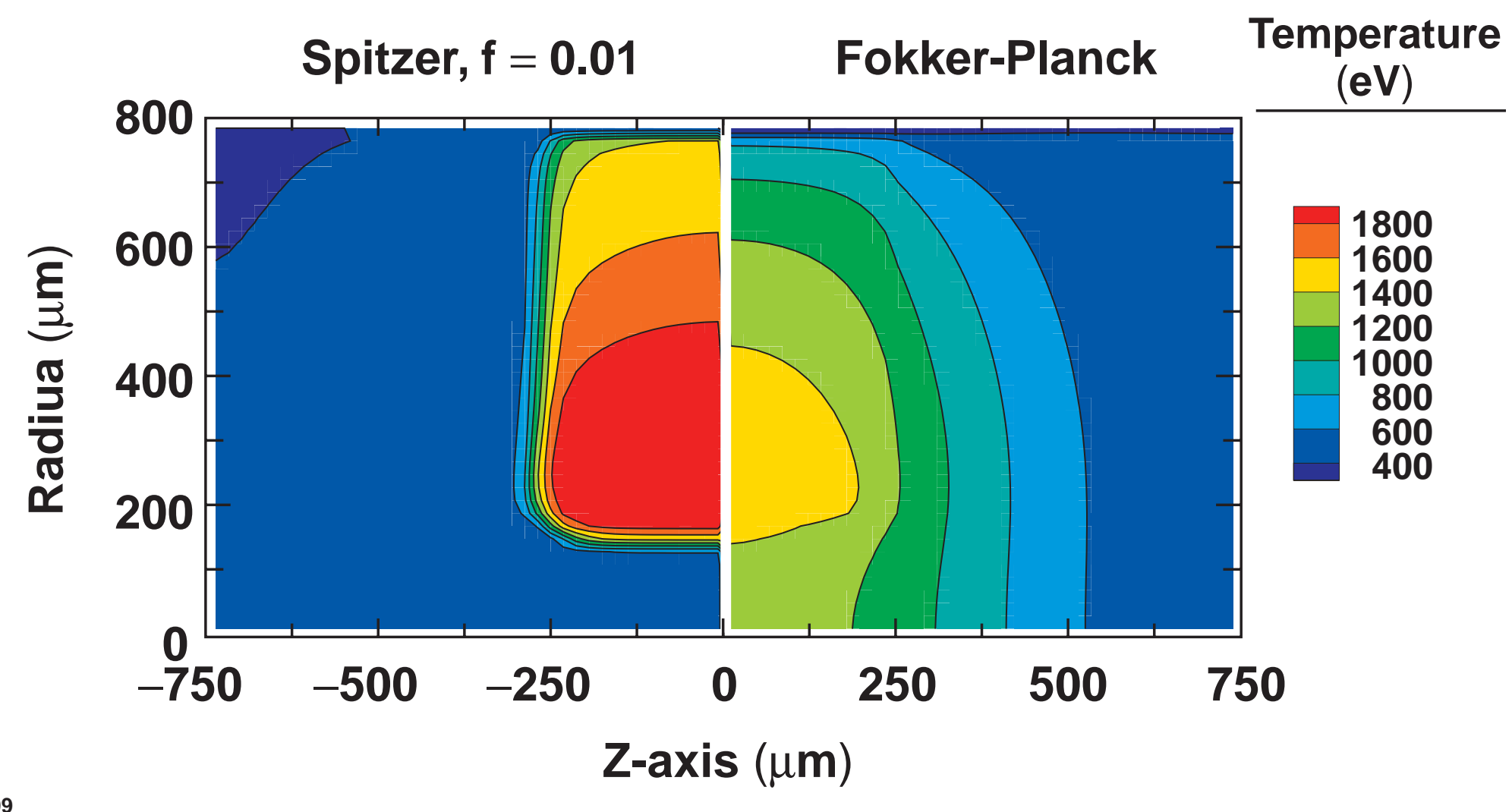




\section{A depletion of low-velocity electrons leads to a higher inferred electron temperature}

- The electron distribution is non-Maxwellian and can be approximated by the DLM formula:

$$
\mathbf{f}(\mathbf{v})=K_{\mathbf{n}} \exp \left(-\mathbf{v}^{\mathbf{n}} / \mathbf{v}_{\mathbf{n}}^{\mathbf{n}}\right) .
$$

- The flat-top electron distribution reduces the number of electrons at low velocity, which leads to an overestimate of the temperature:

$$
\mathrm{C}_{\mathbf{S}}^{\mathrm{FP}}=A \mathrm{C}_{\mathbf{S}}^{\text {Maxwellian }}
$$

- Afeyan ${ }^{1}$ calculated the overestimate to depend on the

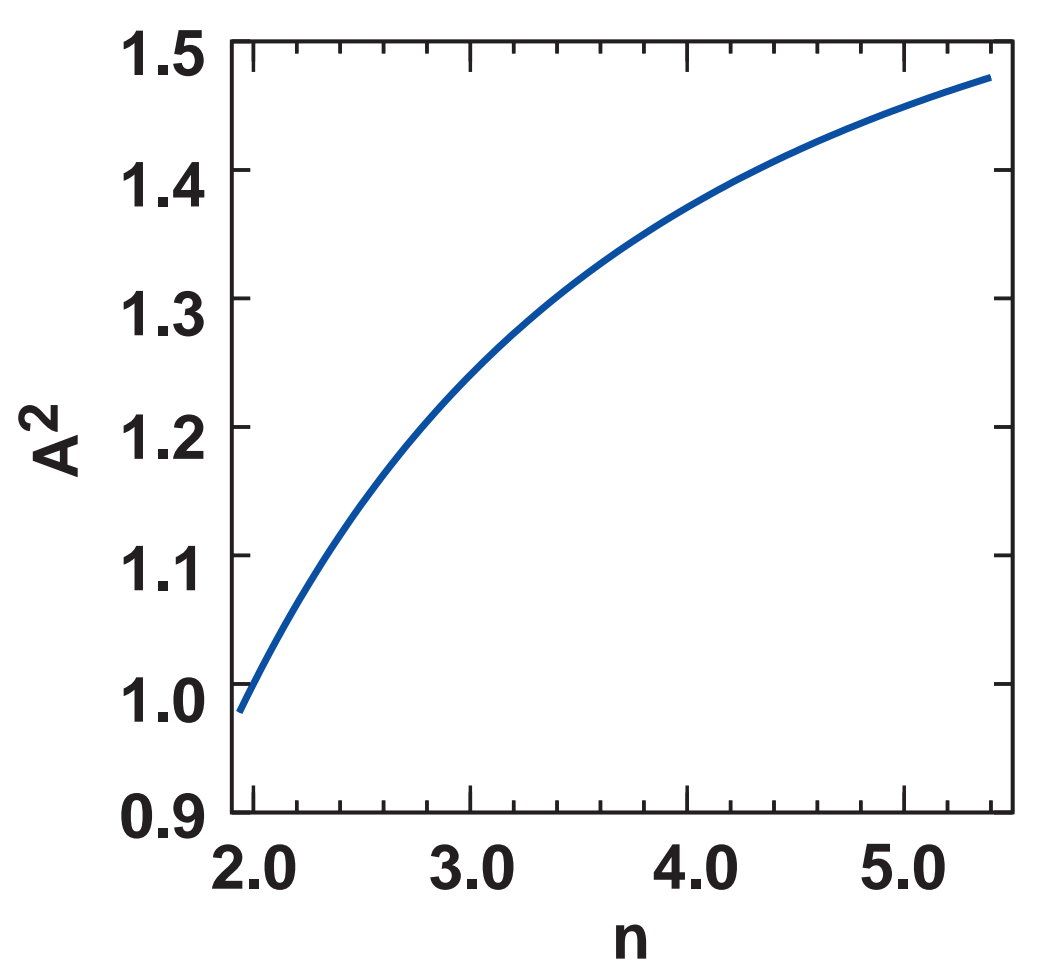
parameter $\mathbf{n}$ :

$$
A^{2}=\frac{3 \Gamma^{2}(3 / n)}{\Gamma(1 / n) \Gamma(5 / n)}
$$




\section{The distribution function is non-Maxwellian throughout the hohlraum}

- In the energy-absorption region there is a deficit of low- and high-velocity electrons.

- In the thermal-conduction region there is an excess of high-velocity electrons.

- The "n" has a peak value of 2.8.
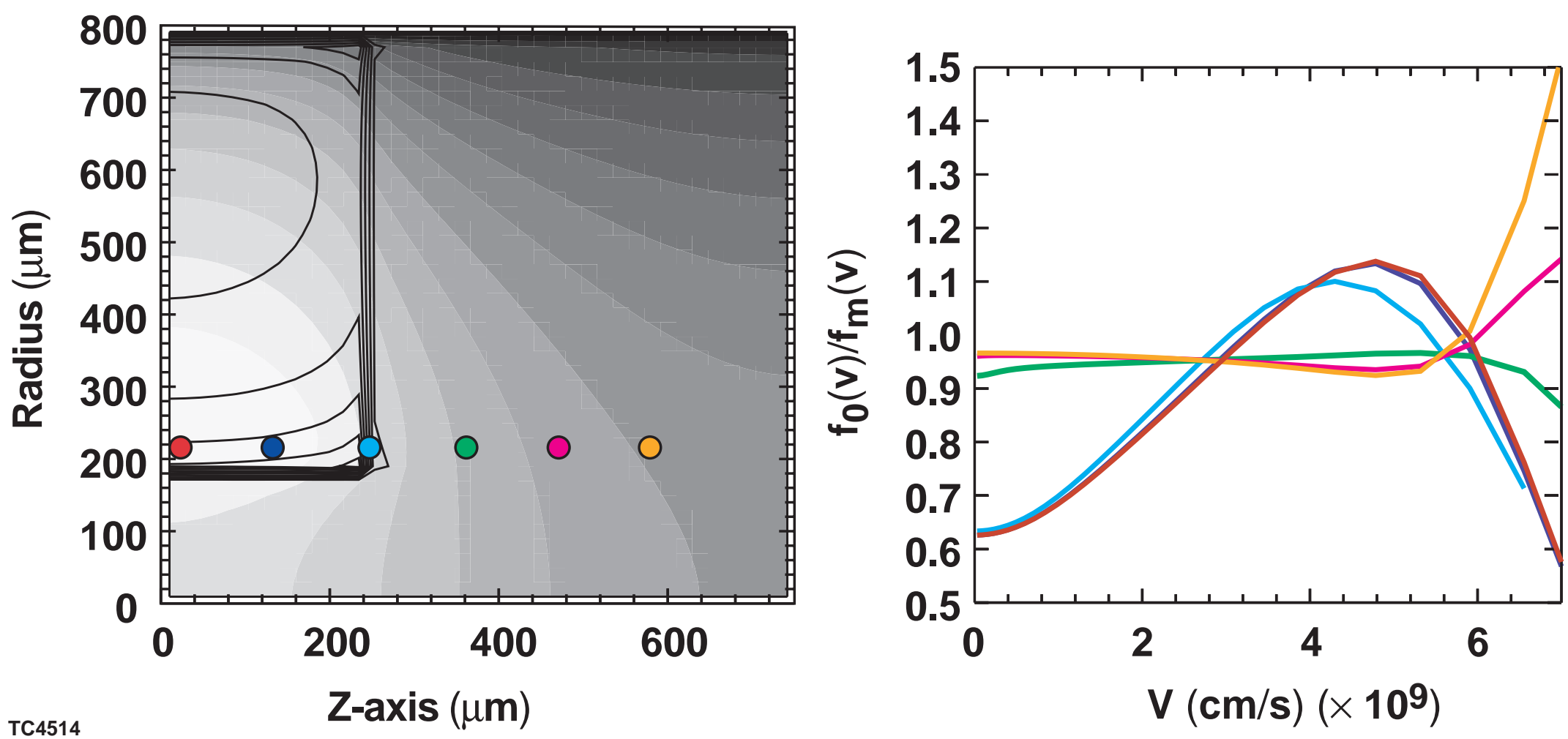


\section{Fokker-Planck Calculation of ICF Implosions}

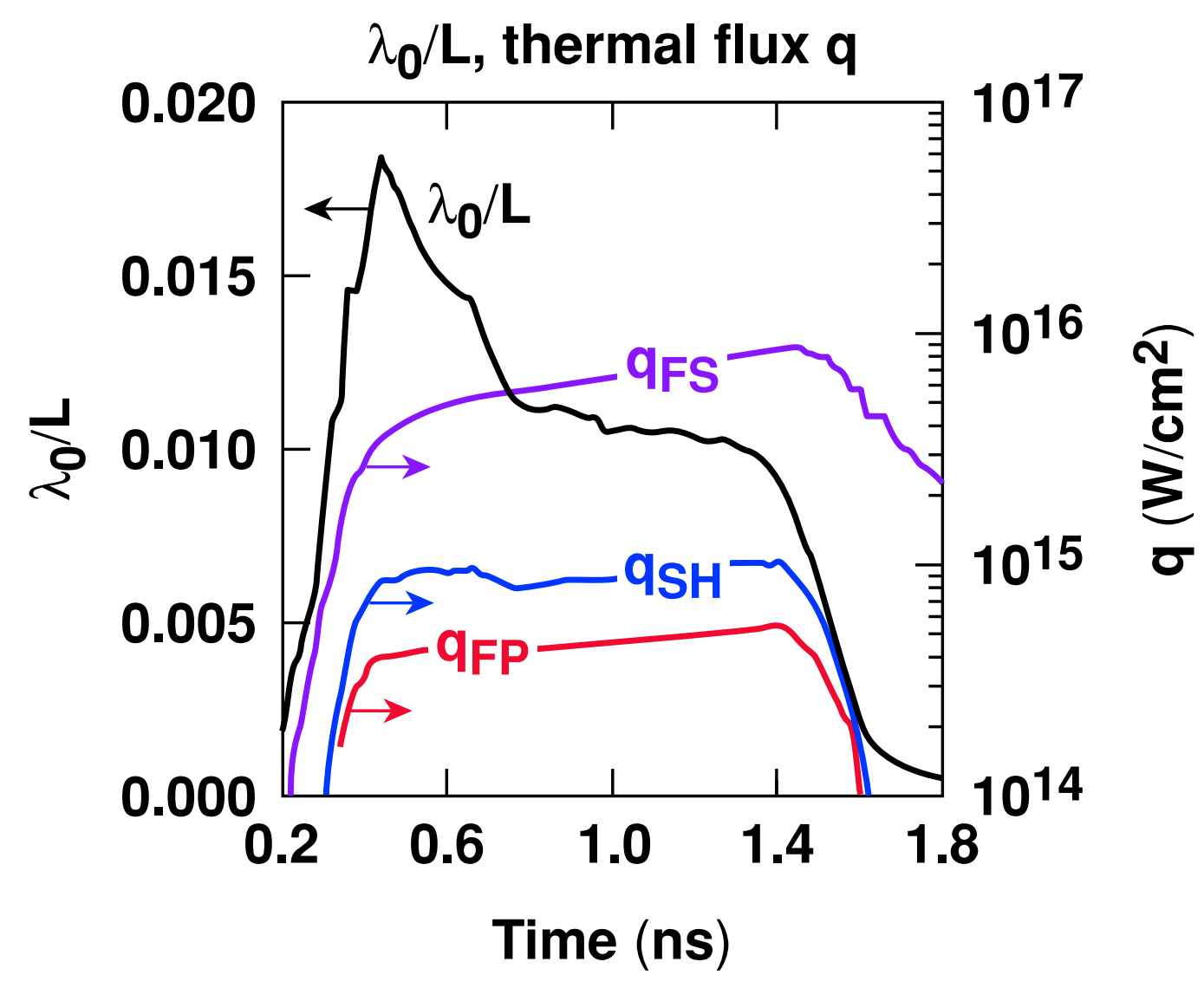

A. Sunahara, J. A. Delettrez, R. W. Short, and S. Skupsky University of Rochester Laboratory for Laser Energetics 43rd Annual Meeting of the American Physical Society Division of Plasma Physics

Long Beach, CA 29 October-2 November 2001 


\section{Summary \\ We have developed a 1-D Fokker-Planck Code and combined it with the 1-D hydrodynamic code LILAC}

- For CH implosions, comparison of Fokker-Planck (FP) with flux-limited Spitzer-Härm (SH) diffusions shows that

- the flux inhibition factor is time dependent

- with FP, the laser absorption is higher than with SH due to a longer density scale length at the critical surface

- in the acceleration phase, FP gives a density-scale length at the ablation surface $50 \%$ longer than $\mathrm{SH}$

- FP gives good agreement with the experimental bang time. 


\section{FP Code Equations}

The distribution function is expanded in Legendre modes to second-order

- $f(z, \vec{v}, t)=f_{0}+f_{1} \cos \left(\theta_{z}\right)+f_{2}\left\{3 \cos ^{2}\left(\theta_{z}\right)-1\right\} / 2$

- The Fokker-Planck equations for $f_{0}, f_{1}$, and $f_{2}$ are calculated with e-i and e-e collisions.

- For closure, a simplified $f_{3}$ equation is used.

- The electric field is calculated based on the current free condition.

- $\Delta \mathbf{T}_{\mathrm{e}}$ and $\Delta \mathrm{n}_{\mathrm{e}}$ are calculated from the hydrodynamics equations without $\nabla \cdot q_{e}$

- $\mathrm{T}_{\text {eff }}=\frac{4 \pi \mathrm{m}_{\mathbf{e}}}{3 \mathrm{n}_{\mathbf{e}}} \int_{0}^{\infty} v^{4} \mathrm{f}_{0} \mathrm{~d} v$ is computed from FP using $\Delta \mathbf{T}_{\mathbf{e}}$ and $\Delta \mathbf{n}_{\mathbf{e}}$ as source terms. 


\section{In the FP calculation the flux inhibition factor $\left(\mathfrak{f}=q_{\mathrm{FP}} / \mathbf{q}_{\mathrm{FS}}\right)$ is time dependent}
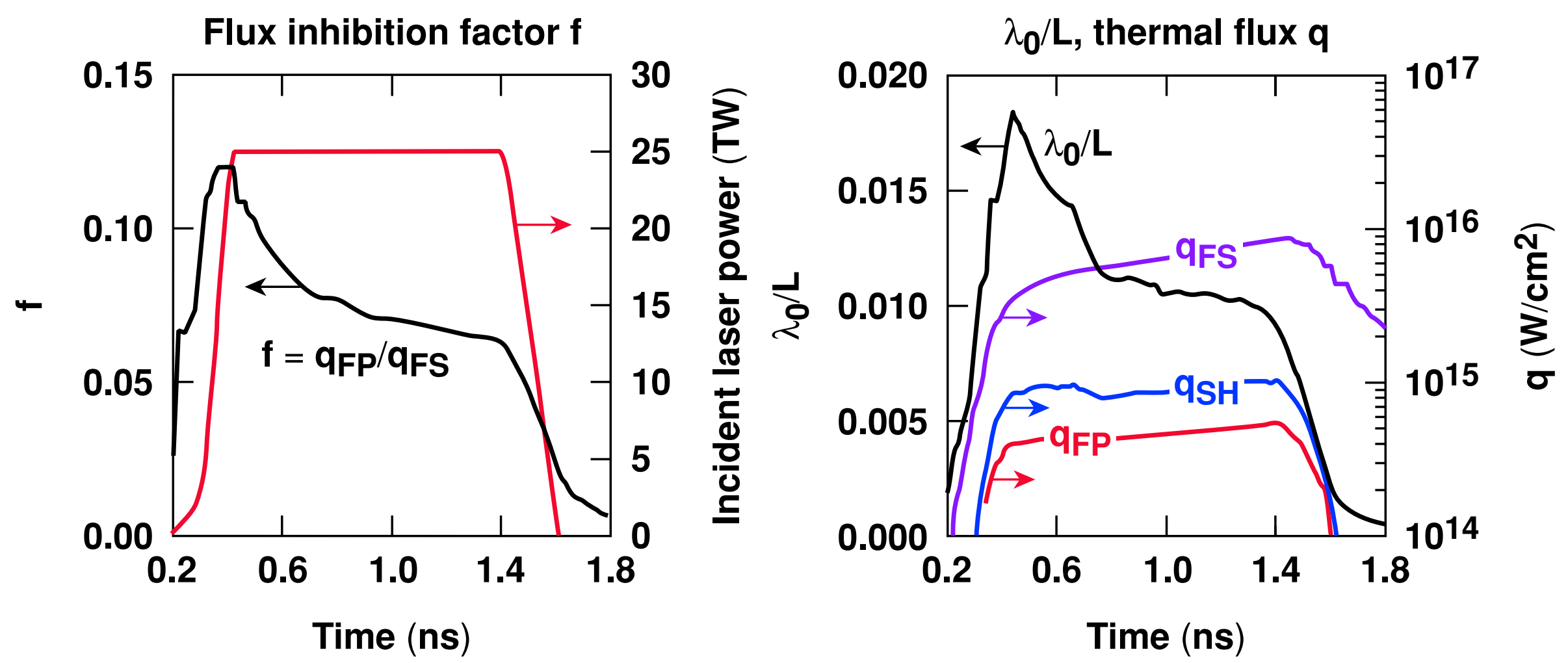

- Quantities measured at the critical surface

$-\lambda_{0}$ : electron mean free path for $90^{\circ}$ collision scattering

- $L$ : electron temperature scale length $L=L_{T e}=T_{e} / \frac{\partial T_{e}}{\partial x}$ 


\section{To match the flux-limited SH flux with FP, the flux limiter should be changed in time}

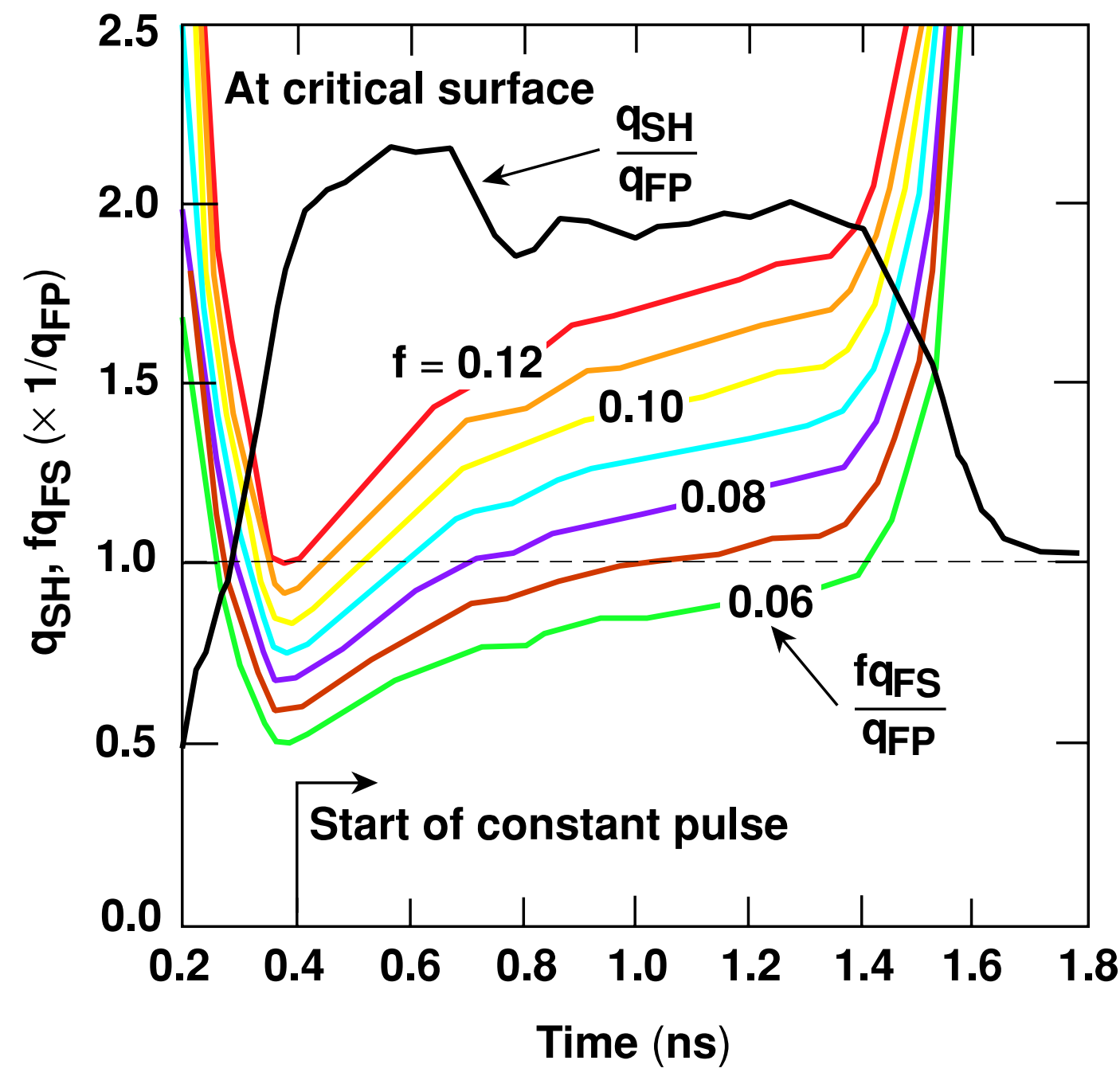

$q_{F P}=\min \left(f_{F S}, q_{S H}\right)$

$f$ : flux-inhibition factor

$\mathrm{q}_{\mathrm{FS}}$ : Free-streaming flux

$$
\begin{aligned}
& q_{F S}=n_{e} T_{e} v_{\text {th }} \\
& v_{\text {th }}=\left(\frac{T_{e}}{m_{e}}\right)^{\frac{1}{2}}
\end{aligned}
$$

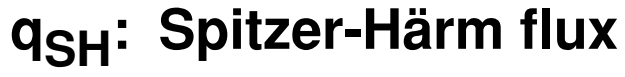

Absorbed laser poweraveraged flux limiter

$\langle f\rangle=\frac{\int \mathrm{fI}_{\mathrm{A}} \mathrm{dt}}{\int \mathrm{I}_{\mathrm{A}} \mathrm{dt}}=\mathbf{0 . 0 7 5}$

$I_{A}:$ Absorbed laser power 


\section{Early in the pulse, FP gives a large density scale length at the critical surface than $\mathrm{SH}$}
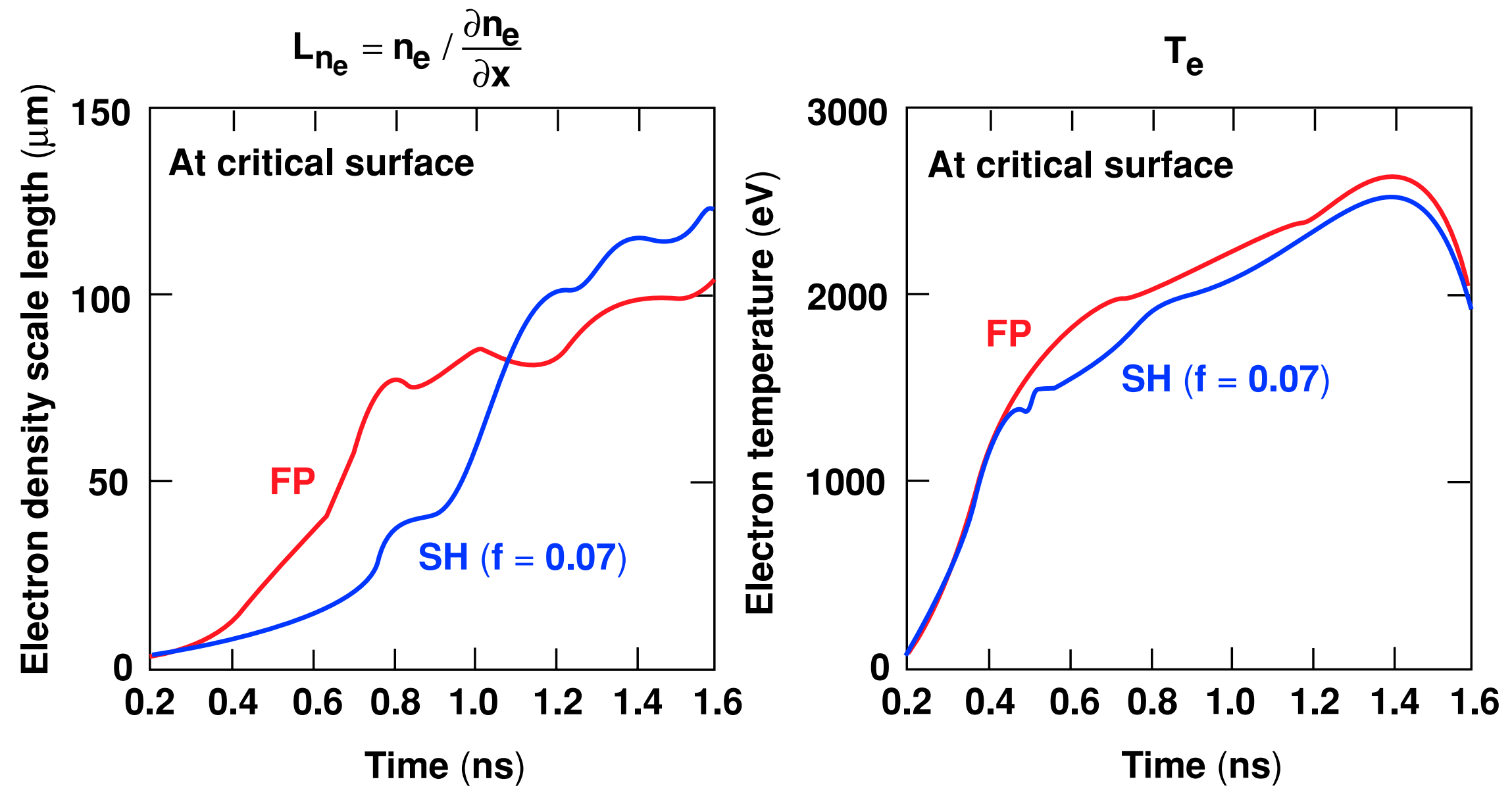

to a larger absorption fraction than in the $\mathrm{SH}$ case. 
FP gives a large laser absorption early in the pulse and results in an increase of the total laser absorption fraction

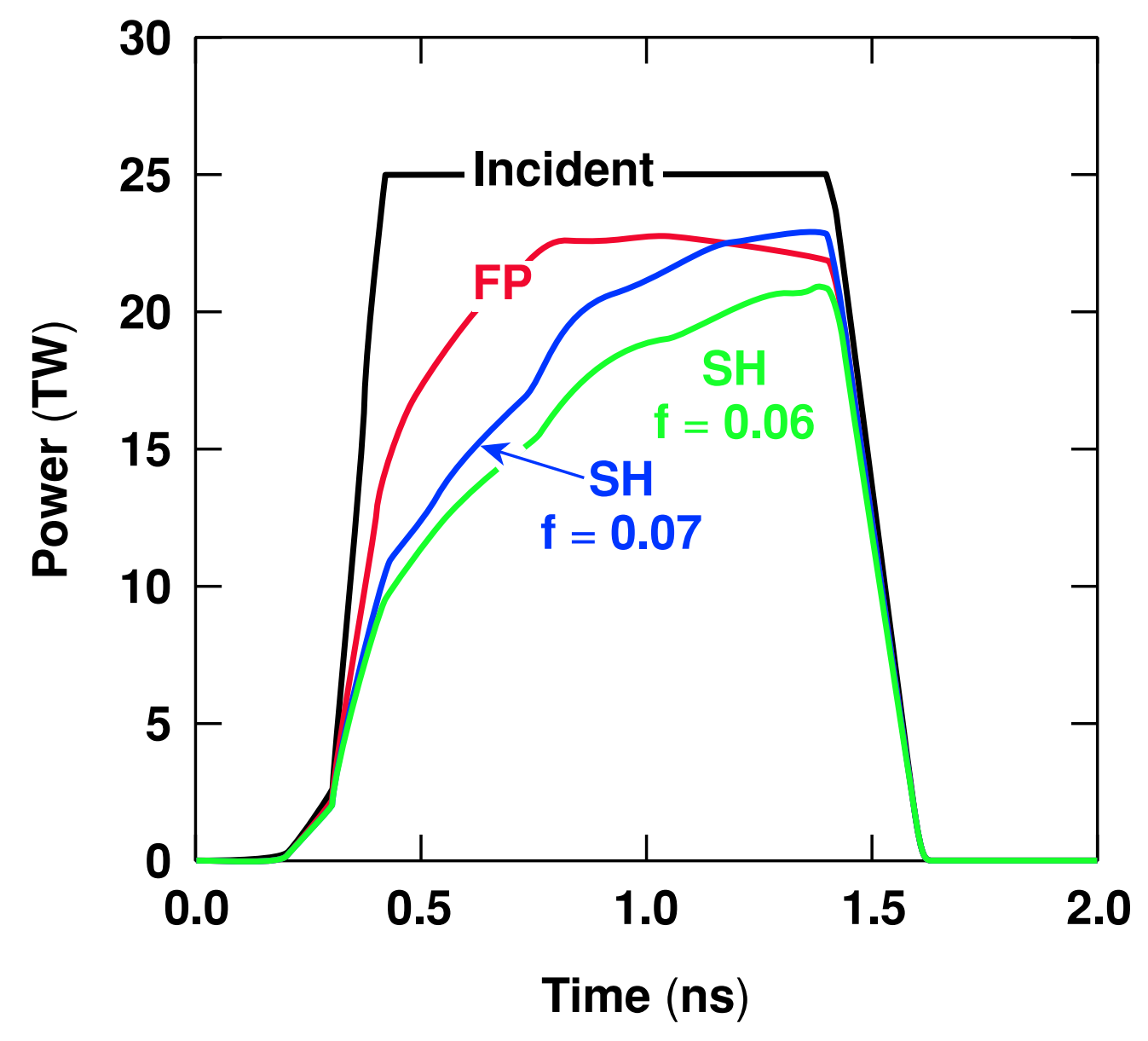

Total absorption fraction

FP: 0.83

$\mathrm{SH} f=0.07: 0.76$

$\mathrm{SH} f=0.06: 0.68$ 


\section{During the acceleration phase, FP gives a relatively low value for the mass ablation rate}

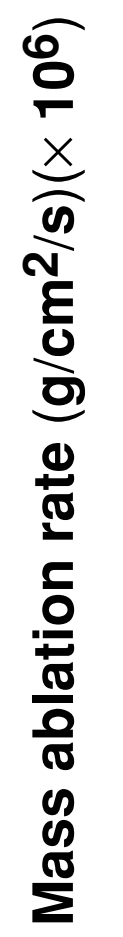

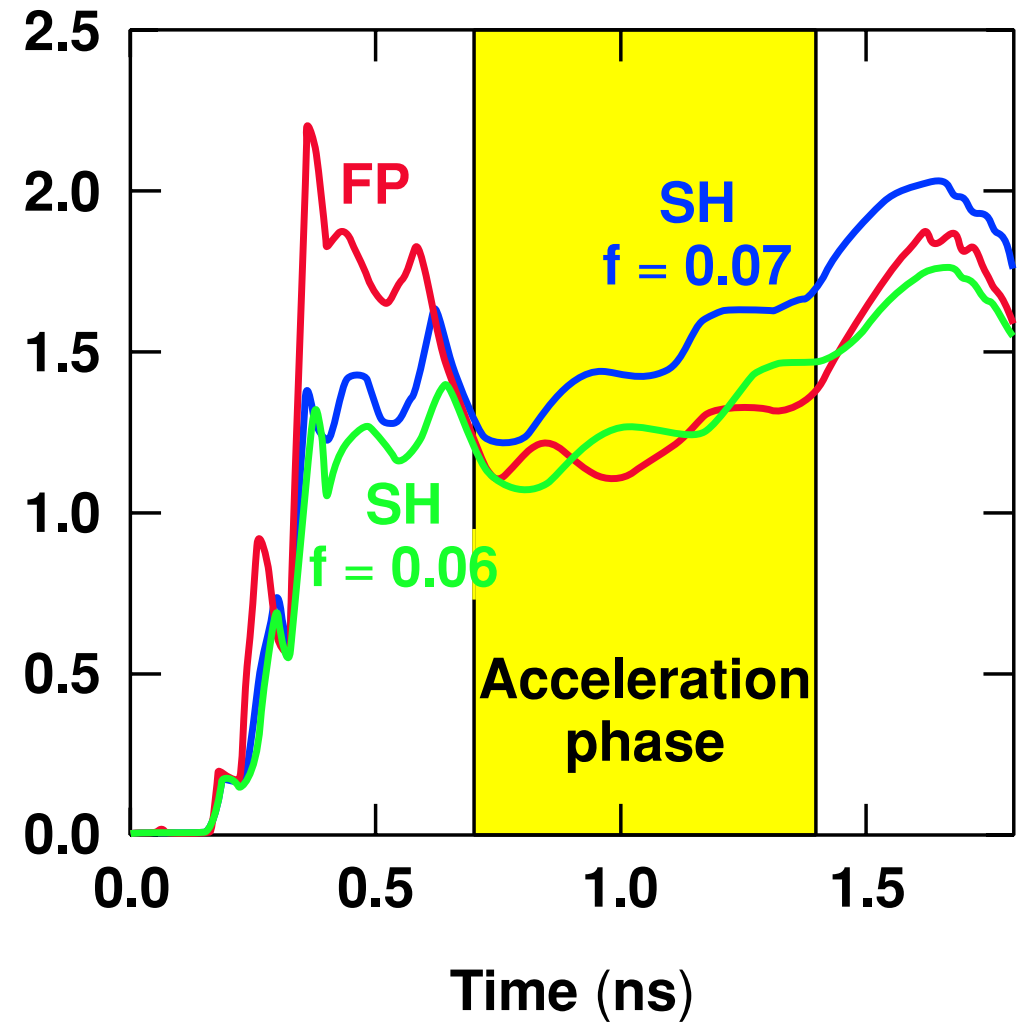

Time-averaged values over the acceleration phase

\begin{tabular}{|l|c|c|}
\cline { 2 - 3 } \multicolumn{1}{c|}{} & FP & SH \\
\hline $\begin{array}{l}\text { Ablation density } \\
<\rho_{\mathrm{a}}>\left(\mathrm{g} / \mathrm{cm}^{3}\right)\end{array}$ & 3.06 & 3.77 \\
\hline $\begin{array}{l}\text { Ablation velocity } 10^{5} \\
<\mathrm{V}_{\mathrm{a}}>(\mathrm{cm} / \mathrm{s})\end{array}$ & 4.01 & 3.99 \\
\hline $\begin{array}{l}\text { Minimum density gradient } \\
\text { scale length }<\mathrm{L}_{\mathrm{m}}>(\mu \mathrm{m})\end{array}$ & 1.31 & 0.83 \\
\hline
\end{tabular}

The early large mass ablation rate causes the large scale length in the FP case. 
For the 1-ns square pulse, both the $\mathrm{SH} f=0.07$ and FP show good agreement with experimental results
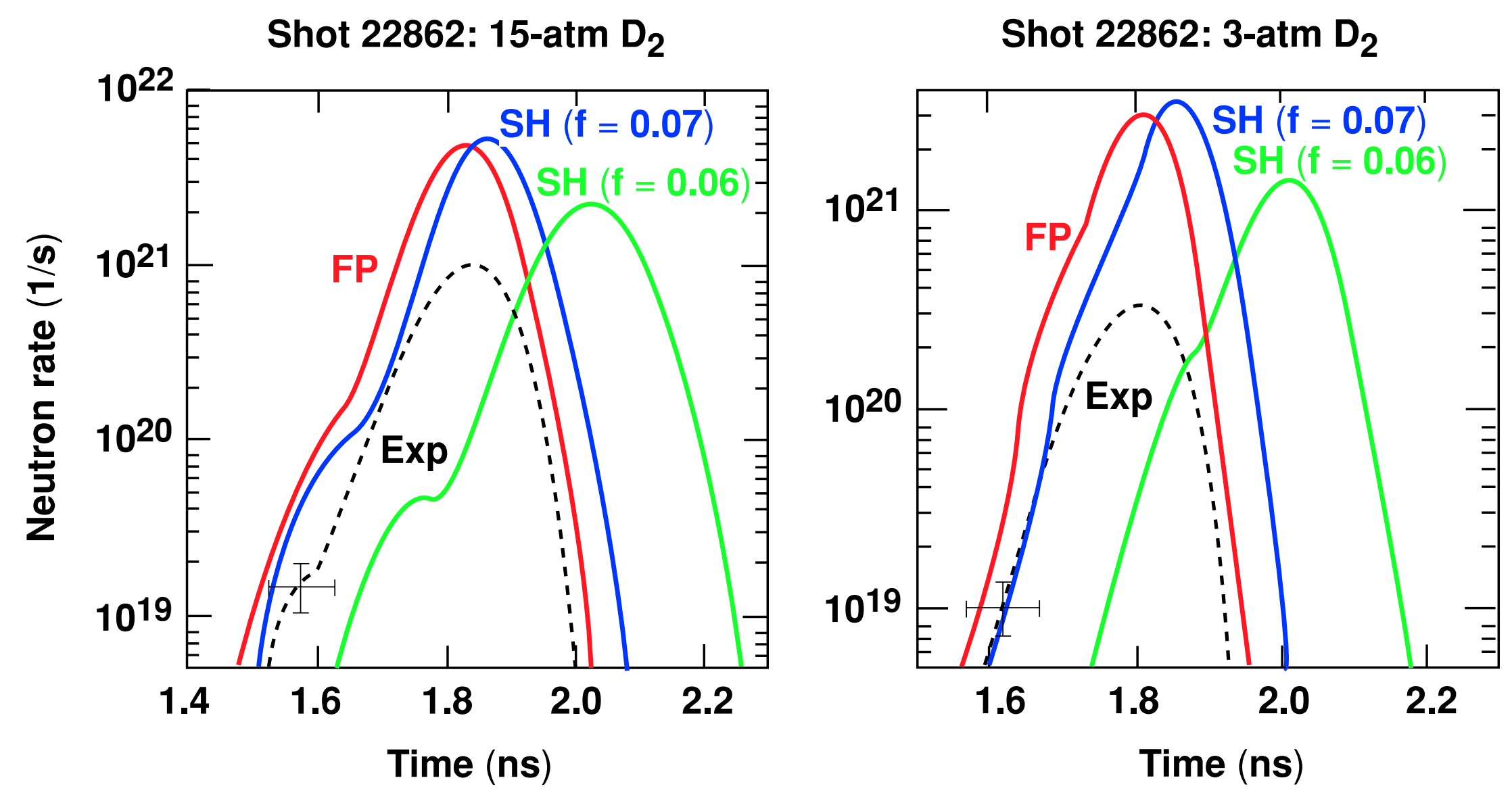

From FP, $\langle\mathbf{f}\rangle=0.075$ 
For the 400-ps square pulse, the FP bang time coincides with $\mathrm{SH} f=0.09$ case, confirming that a larger flux limiter is needed for the short pulse

UR

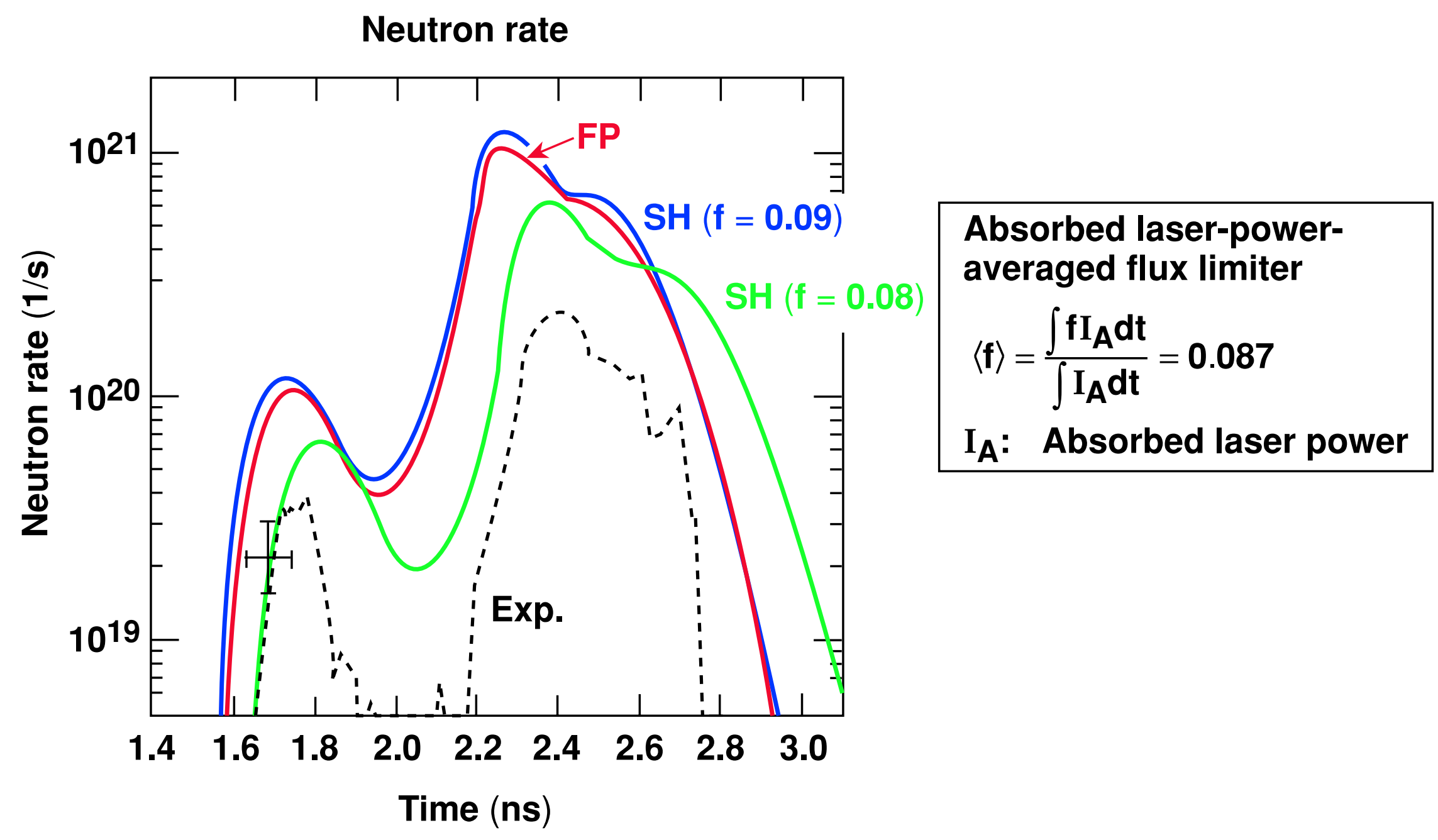




\section{Conclusions}

We have developed a 1-D Fokker-Planck code and combined it with the 1-D hydrodynamic code LILAC

- For CH implosions, comparison of FP with the flux-limited SH model

- The flux inhibition factor is time dependent.

- With FP, the laser absorption is higher than with SH due to a longer density scale length at the critical surface.

- In the acceleration phase, FP gives a density-scale length at the ablation surface $\mathbf{5 0} \%$ longer than $\mathbf{S H}$.

- FP gives good agreement with the experimental bang time.

- Calculations for cryogenic targets with shaped pulses are planned. 
The Collisional Delta-f Method

Stephan Brunner, John Krommes and Ernest Valeo presented at

LLNL Electron Transport Workshop

Sept. 9-11, 2002 


\section{Summary}

- Objective: Development of low noise MC techniques for transport applications:

$$
f=f_{0}+\delta f, \quad \frac{\delta f}{f_{0}} \ll 1
$$

where $f_{0}$ satisfies simplified equations and $\delta f$ is solved by MC techniques.

- First applied to microturbulence problems in MFE: Kotchenreuther, Lee, Lin, Dimitz, Cohen, where $f_{0}$ was taken fixed.

- Our contribution:

- Evolution of $f_{0}$, which enables transport time scale simulations.

- Algorithm for computation of quasineutral $\vec{E}$.

- Introduction of noise reductions techniques. 


\section{Combined Fluid-Kinetic Equations. [Barnes (91)]}

Fokker-Planck Equation for Electrons:

$\frac{\partial}{\partial t} \boldsymbol{f}+\overrightarrow{\boldsymbol{v}} \cdot \frac{\partial}{\partial \vec{x}} \boldsymbol{f}+\frac{(-e)}{m} \overrightarrow{\boldsymbol{E}} \cdot \frac{\partial}{\partial \vec{v}} f=-\left\{C_{e e}[f, f]+C_{e i} f\right\}$.

Decomposition of the distribution uniquely determined by:

$$
\begin{aligned}
& f(\vec{x}, \vec{v} ; t)=f_{\mathrm{SM}}(\vec{x}, \vec{v} ; t)+\delta f(\vec{x}, \vec{v} ; t), \\
& f_{\mathrm{SM}}=\frac{N / \bar{N}}{[2 \pi T / m]^{3 / 2}} \exp \left(-\frac{1}{2} \frac{[\vec{v}-\vec{u}]^{2}}{T / m}\right) .
\end{aligned}
$$

Constraints: $\quad \int \delta f \vec{v}^{j} d \vec{v}=0, \quad j=0,1,2$.

1. Taking the first three velocity moments of the F-P equation $\Longrightarrow$

Fluid Equations for Background Parameters $[N(\vec{x} ; t), \vec{u}(\vec{x} ; t), T(\vec{x} ; t)]$.

2. Rewriting the F-P equation $\Longrightarrow$ Effective equation for $\delta f$ :

$$
\begin{gathered}
\frac{D}{D t} \delta f=-\left\{\frac{D}{D t} f_{\mathrm{SM}}+C_{e e}\left[\delta f, f_{\mathrm{SM}}\right]\right\}, \\
\frac{D}{D t} \doteq \frac{\partial}{\partial t}+\vec{v} \cdot \frac{\partial}{\partial \vec{x}}+\frac{(-e)}{m} \vec{E} \cdot \frac{\partial}{\partial \vec{v}}+C_{e e}\left[f_{\mathrm{SM}},\right]+C_{e i},
\end{gathered}
$$

Approximation : "Linearizing" the self-collision operator:

$$
C_{\mathrm{ee}}[f, f]=\underbrace{C_{\mathrm{ee}}\left[f_{\mathrm{SM}}, f_{\mathrm{SM}}\right]}_{=0}+\underbrace{C_{\mathrm{ee}}[\delta f, \delta f]}_{\text {neglect }}+C_{\mathrm{ee}}\left[f_{\mathrm{SM}}, \delta f\right]+C_{\mathrm{ee}}\left[\delta f, f_{\mathrm{SM}}\right]
$$




\section{Fluid-Kinetic Equations Cont'd}

Fluid Equations for Background Parameters

- $j=0 \Longrightarrow$ Continuity equation:

$$
\frac{\partial N}{\partial t}+\frac{\partial}{\partial \vec{x}} \cdot(N \vec{u})=0 .
$$

- $j=1 \Longrightarrow$ Momentum equation:

$$
\begin{aligned}
m N\left(\frac{\partial \vec{u}}{\partial t}+\vec{u} \cdot \frac{\partial \vec{u}}{\partial \vec{x}}\right)=-\frac{\partial}{\partial \vec{x}}(N T)-\frac{\partial}{\partial \vec{x}} \cdot \Pi(\delta f) \\
+(-e) N \vec{E}+\vec{R}_{e i}\left(f_{\mathrm{SM}}\right)+\vec{R}_{e i}(\delta f) .
\end{aligned}
$$

- $j=2 \Longrightarrow$ Heat equation:

$$
\begin{aligned}
\frac{\partial}{\partial t}\left(\frac{3}{2} N T+\frac{1}{2} m N u^{2}\right)+ & \frac{\partial}{\partial \vec{x}} \cdot\left[\frac{5}{2} N T \vec{u}+\frac{1}{2} m N u^{2} \vec{u}+\Pi \cdot \vec{u}+\vec{q}(\delta f)\right] \\
& =(-e) N \vec{u} \cdot \vec{E} .
\end{aligned}
$$

CLOSURE to the fluid equations from moments of $\delta f$ :

Stress tensor:

Drag of $\delta f$ on ions:

Heat flux in $\delta f$ :

$$
\Pi(\delta f)=m \overline{\boldsymbol{N}} \int \overrightarrow{\boldsymbol{v}} \overrightarrow{\boldsymbol{v}} \delta f d \vec{v}
$$

$$
\vec{R}_{e i}(\delta f)=-m \bar{N} \int \vec{v} C_{e i} \delta f d \vec{v}
$$

$$
\vec{q}(\delta f)=\frac{m}{2} \bar{N} \int(\vec{v}-\vec{u})^{2} \vec{v} \delta f d \vec{v}
$$


Fluid-Kinetic Equations Cont'd

Representing $\delta f$ using the Collisional $\delta f$ method

$$
\begin{gathered}
\frac{D}{D t} \delta f=-\left\{\frac{D}{D t} f_{\mathrm{SM}}+C_{e e}\left[\delta f, f_{\mathrm{SM}}\right]\right\} \\
\frac{D}{D t} \doteq \frac{\partial}{\partial t}+\vec{v} \cdot \frac{\partial}{\partial \vec{x}}+\frac{(-e)}{m} \vec{E} \cdot \frac{\partial}{\partial \vec{v}}+C_{e e}\left[f_{\mathrm{SM}},\right]+C_{e i}
\end{gathered}
$$

Representation of $\delta f$ with Marker Particles:

$$
\delta f(\vec{x}, \vec{v} ; t) \simeq \sum_{i=1}^{n_{p}} w_{i}(t) \delta\left(\vec{x}-\vec{x}_{i}(t)\right) \delta\left(\vec{v}-\vec{v}_{i}(t)\right)
$$

Marker Distribution: $g(\vec{x}, \vec{v} ; t) \simeq \sum_{i} \delta\left(\vec{x}-\vec{x}_{i}(t)\right) \delta\left(\vec{v}-\vec{v}_{i}(t)\right)$.

MARKER EQUATIONS:

$$
\begin{aligned}
\frac{d \vec{x}}{d t} & =\vec{v} \\
\frac{d \vec{v}}{d t} & =\frac{(-e)}{m} \vec{E}+\frac{\delta \vec{v}_{e e}}{\delta t}+\frac{\delta \vec{v}_{e i}}{\delta t} \\
\dot{w} \doteq \frac{d w}{d t} & =-\frac{1}{g}\left\{\frac{D}{D t} f_{\mathrm{SM}}+C_{e e}\left[\delta f, f_{\mathrm{SM}}\right]\right\}
\end{aligned}
$$

- Random Increments $\delta \vec{v}_{e e}$ and $\delta \vec{v}_{e i}$ reproduce $C_{e e}\left[f_{\mathrm{SM}}, \delta f\right]$ and $C_{e i} \delta f . \Longrightarrow$ Monte Carlo Simulation.

- Collisions in $\mathrm{PIC}$ and $\delta f$, including approximations on $C\left[\delta f, f_{\mathrm{SM}}\right]$ : Takizuka \& Abe (77); Xu \& Rosenbluth (91); Dimits \& Cohen (94); Lin et.al(97); Chen \& White (97).

- Two weighted $\delta f$ scheme [Hu \& Krommes (94)] $\Longrightarrow$ Avoids evaluating $g$. 


\section{Study Case: Relaxation Through Self-Collisions.}

DISCARDING terms in the weight equation:

MOTIVATION: They require evaluating partial derivatives in velocity space of $g$ and $\delta f$, which is costly in computation time and demanding statistically.

CONSEQUENCE: Different markers, having undergone different stochastic trajectories, can end up at the same point in phase space with different weights $w_{i}$. $\Longrightarrow$

- The initial definition $w_{i}(t)=W\left[\vec{v}_{i}(t), t\right]$ is violated.

- SPREADING $\Delta w$ OF MARKER WEIGHTS

$\Longrightarrow$ INCREASING NUMERICAL NOISE.

JUSTIFICATION: By reinterpreting the weight field $W$ at a given point in phase space as the average over all particle weights in the vicinity of that point, it can be proven that the system of marker equations REMAINS EXACT [ Chen \& White (97)]. 


\section{ELECTRIC FIELD RUNAWAY FIELD}

Electric field: $E=5 \cdot 10^{-2} m \bar{\nu}_{\mathrm{ee}}(0) v_{\mathrm{th}}(0) / e$,

Runaway field: $E_{c}=0.11 m \bar{\nu}_{\mathrm{ei}} v_{\mathrm{th}} / e$,

$$
Z=1, \quad n_{p}=10^{4}, \quad \Delta t=10^{-2} \bar{\nu}_{\mathrm{ee}}(0)^{-1} .
$$

TEMPERATURE

(Ohmic heating)

\section{AVERAGE VELOCITY \\ $\sim$ CURRENT}
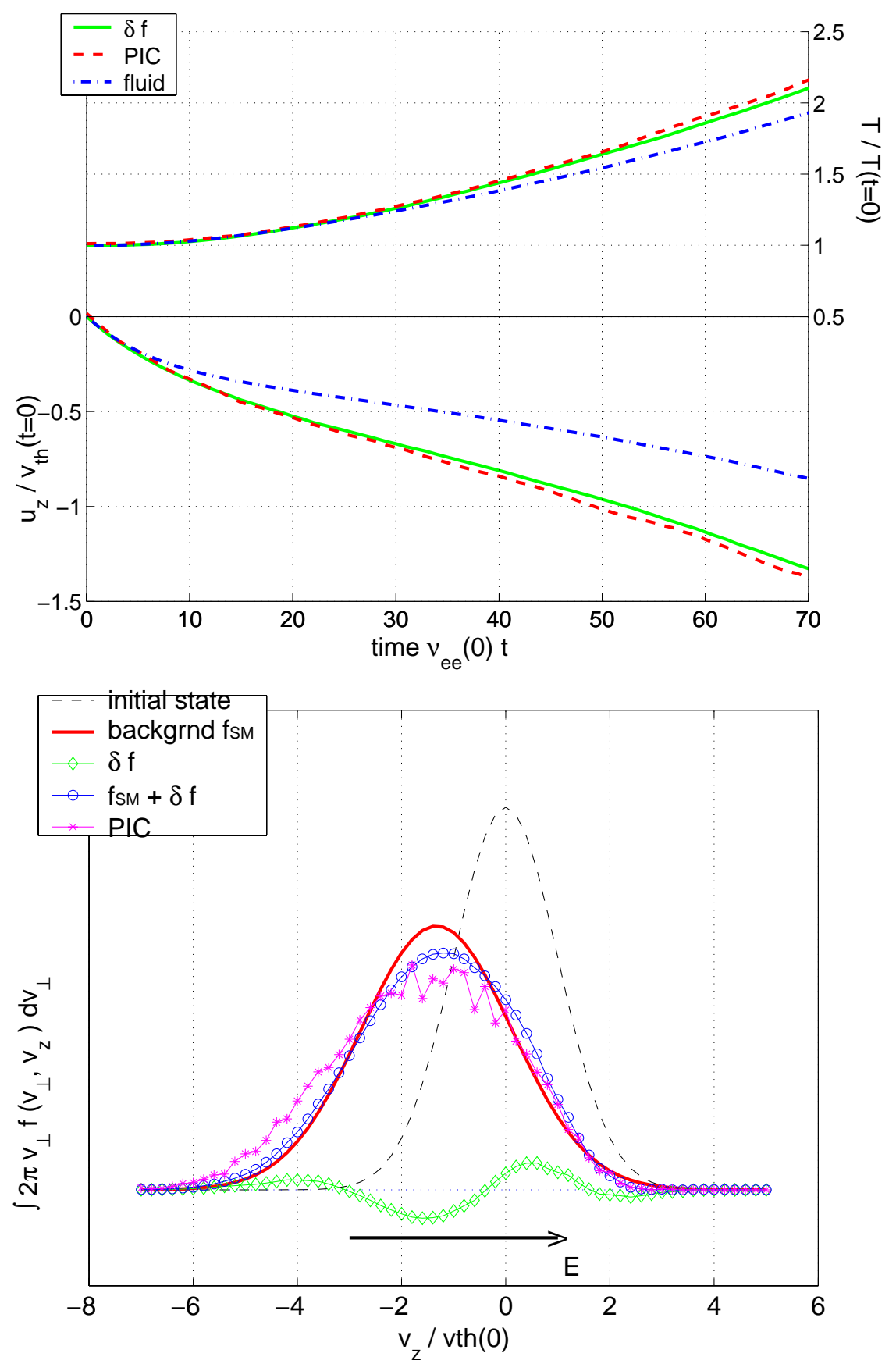


\section{ELECTRIC FIELD « RUNAWAY FIELD}

Electric field: $E=10^{-4} m \bar{\nu}_{\mathrm{ee}}(0) v_{\mathrm{th}}(0) / e$,

\section{AVERAGE VELOCITY $\sim$ CURRENT}

SOURCES/SINKS $\Longrightarrow$ GOOD RESOLUTION MAINTAINED AT $|\vec{v}| \gg v_{\text {th }}$
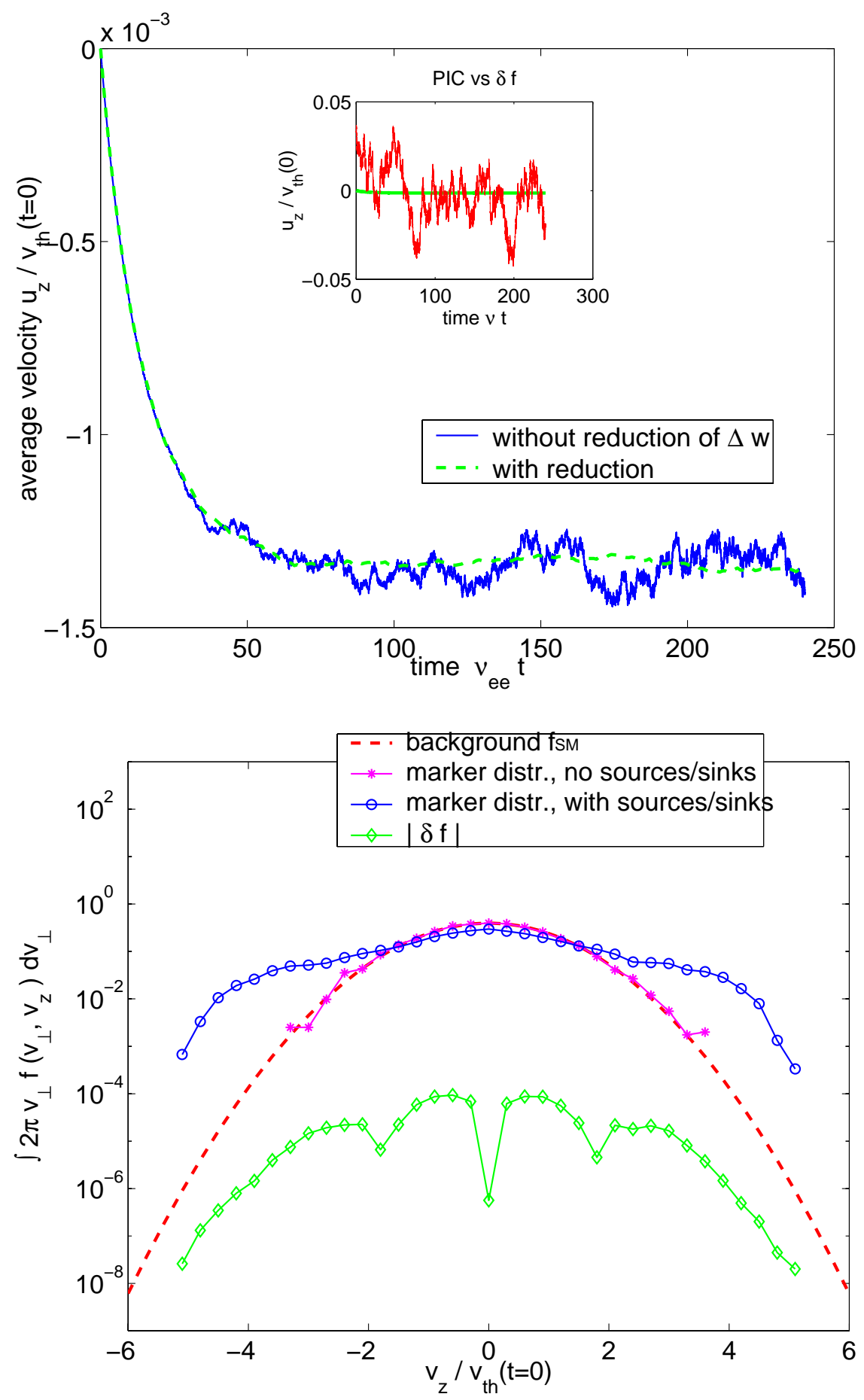


\section{ELECTRIC FIELD « RUNAWAY FIELD, Cont'd.}

\section{LINEAR REGIME}

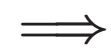

\section{SPITZER CONDUCTIVITY}

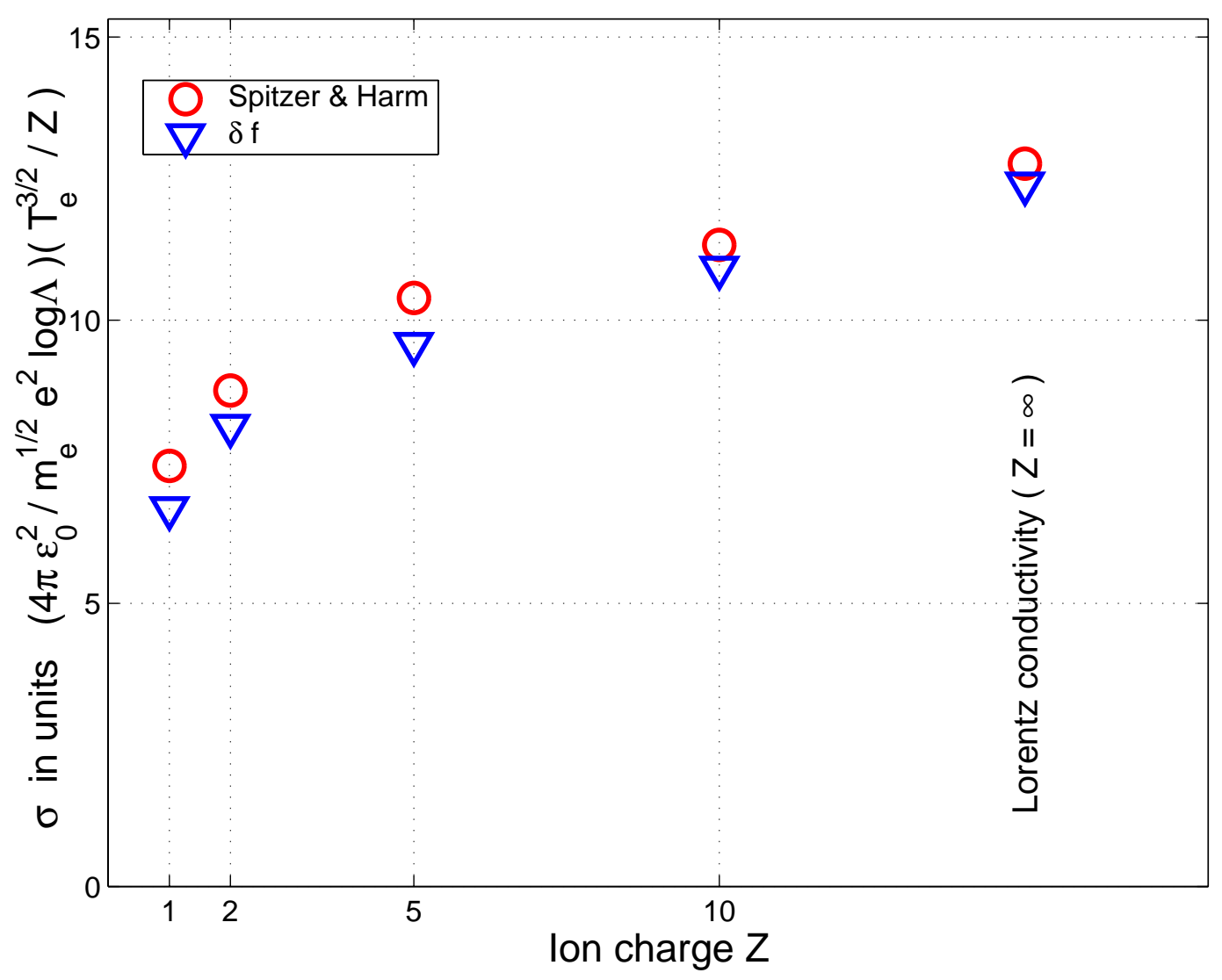




\section{Illustration 2: Linear, NonLocal Electron Heat Transport}

Assuming small amplitude, 1-Dim perturbations of the electron dist.:

$$
f(x, \vec{v} ; t)=f_{\mathrm{M}}(|\vec{v}|)+\delta f(x, \vec{v} ; t), \quad \delta f / f \ll 1 .
$$

- Only the linearized F-P equation is solved.

- No evolution of the background required.

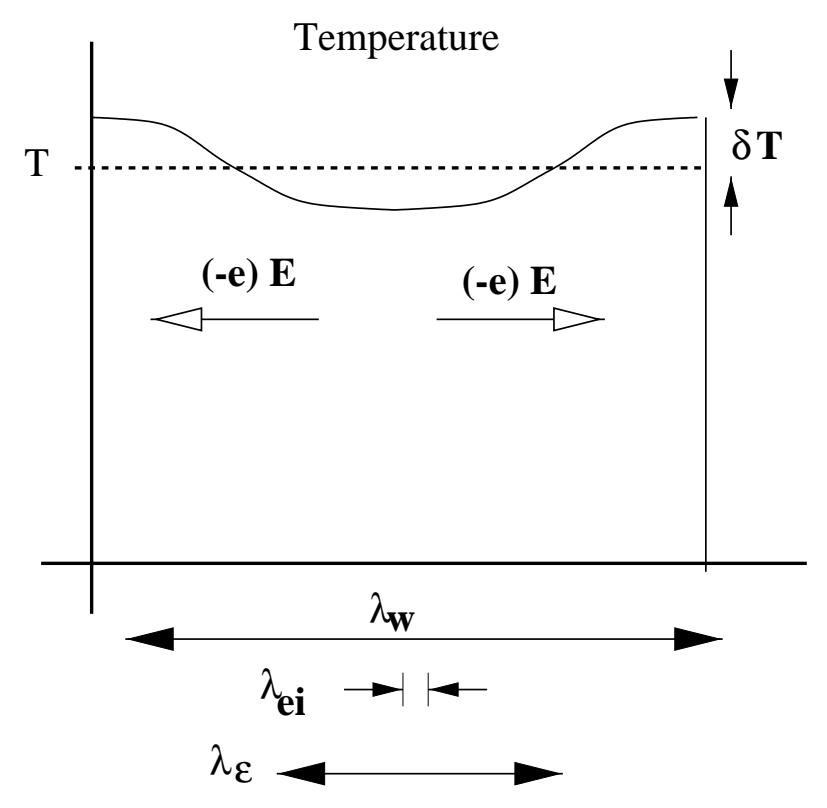

Initial Condition:

$\delta f(t=0)=\delta T \frac{\partial}{\partial T} f_{\mathrm{M}}$,

$$
\delta T=\cos \frac{2 \pi}{\lambda_{w}} x
$$

Periodic boundary conditions.

Assuming high $\mathrm{Z}$ plasma, $Z \gg 1 \Longrightarrow$ Varaiables $(x, v)$.

- $\bar{\lambda}_{e i} / \lambda_{\text {wave }} \ll 1 \Longrightarrow \delta f=\delta f_{0}(x,|\vec{v}|)+\delta f_{1}(x,|\vec{v}|) \frac{v_{x}}{|\vec{v}|}+\ldots$

- Stopping Length: $\bar{\lambda}_{\epsilon}=\sqrt{\frac{\tau_{e e}}{\tau_{e i}}} \bar{\lambda}_{e i}=\sqrt{Z} \bar{\lambda}_{e i} \Longrightarrow \bar{\lambda}_{\epsilon} / \lambda_{w} \sim 1$. 


\section{Linear, NonLocal Electron Heat Transport. Cont'd}

The linearized F-P equation:

$$
\begin{gathered}
\frac{\partial \delta f}{\partial t}+v_{x} \cdot \frac{\partial \delta f}{\partial x}+\frac{(-e)}{m} E \cdot \frac{\partial f_{\mathrm{M}}}{\partial v_{x}}=-\left\{C_{e e}\left[f_{\mathrm{M}}, \delta f\right]+C_{e e}\left[\delta f, f_{\mathrm{M}}\right]+C_{e i} \delta f\right\} \\
\text { is expanded in the small parameter } \bar{\lambda}_{e i} / \lambda_{\text {wave }} .
\end{gathered}
$$

The lowest order anisotropy: $\delta f_{1}=-\frac{\lambda_{e i}(v)}{2}\left(\frac{\partial \delta f_{0}}{\partial x}+\frac{e E}{T} f_{\mathrm{M}}\right)$, is used for obtaining an effective equation for $\delta f_{0}(x,|\vec{v}|)$ :

$$
\begin{aligned}
\frac{\partial \delta f_{0}}{\partial t}-\frac{\partial^{2}}{\partial x^{2}}\left(\frac{v \lambda_{e i}(v)}{6} \delta f_{0}\right) & +C_{e e}\left[f_{\mathrm{M}}, \delta f_{0}\right] \\
= & \frac{v \lambda_{e i}(v)}{6} \frac{e}{T} \frac{\partial E}{\partial x} f_{\mathrm{M}}-C_{e e}\left[\delta f_{0}, f_{\mathrm{M}}\right] .
\end{aligned}
$$

This equation is then solved using the collisional $\delta f$ scheme. 


\section{Computing the Self-Consistent Electric Field}

Continuity Eq.: $\frac{\partial}{\partial t} \delta N+\frac{\partial}{\partial x} \Gamma=0 \quad+$ Quasineutrality $\Longrightarrow \frac{\partial \Gamma}{\partial x}=0$ $\Gamma=\frac{1}{3} \int_{0}^{\infty}\left(4 \pi v^{2}\right) d v v \delta f_{1}=-\int_{0}^{\infty}\left(4 \pi v^{2}\right) d v \frac{v \lambda_{e i}(v)}{6}\left(\frac{\partial \delta f_{0}}{\partial x}+\frac{e E}{T} f_{\mathrm{M}}\right)$. METHOD 1: $\quad \frac{\partial E}{\partial x}=-\frac{T}{e} \frac{\sqrt{2 \pi}}{96} \frac{\partial^{2}}{\partial x^{2}}\left[\int_{0}^{\infty} d v\left(4 \pi v^{2}\right)\left(\frac{v}{v_{\mathrm{th}}}\right)^{5} \delta f_{0}\right]$.

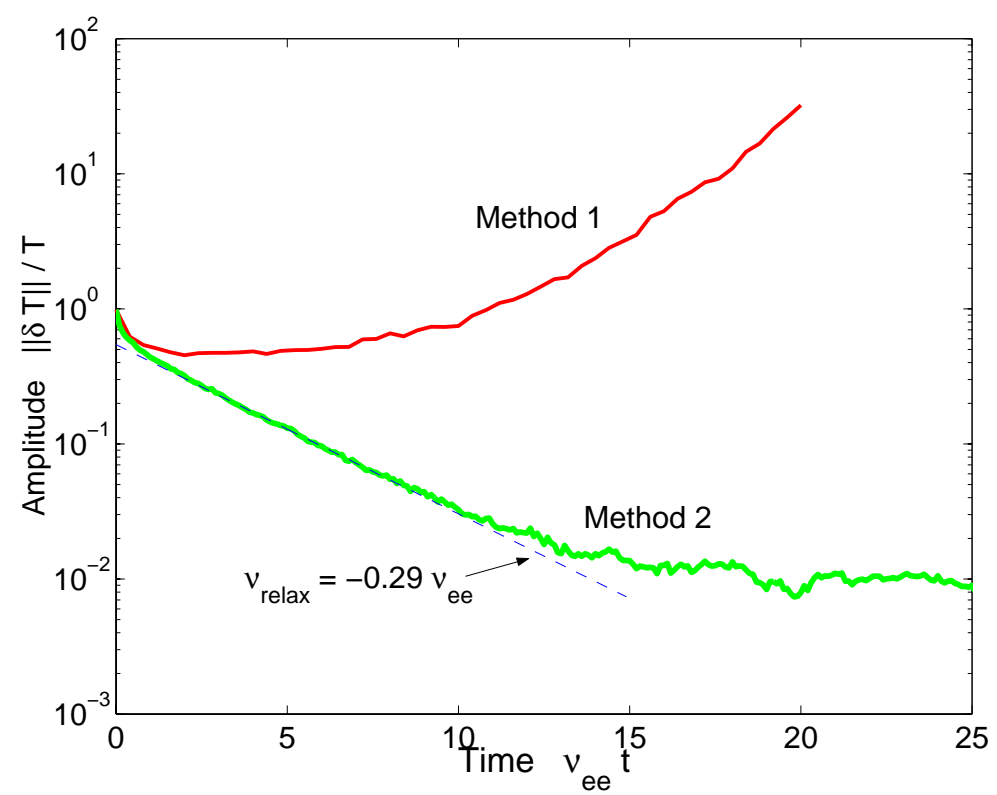

$\bar{\lambda}_{\epsilon} / \lambda_{w}=0.1$

Solution to Numerical Instability:

Impose Numerical Invariance of Density on the Spatial Grid $\left\{X_{k}\right\}$ :

$$
\begin{aligned}
& \delta N_{k}^{j+1}=\sum_{i=1}^{n_{p}} w_{i}^{j+1} S\left(X_{k}-x_{i}^{j+1}\right)=\sum_{i=1}^{n_{p}} w_{i}^{j} S\left(X_{k}-x_{i}^{j}\right)=\delta N_{k}^{j} . \\
& \frac{d w_{i}}{d t} \simeq \frac{w_{i}^{j+1}-w_{i}^{j}}{\Delta t}=\frac{1}{g}\left\{\frac{v \lambda_{e i}(v)}{6} f_{\mathrm{M}} \frac{e}{T} \sum_{l=1}^{n_{x}}\left(\frac{\partial E}{\partial x}\right)_{l}^{j+1 / 2} S\left(X_{l}-x_{i}\right)-C_{e e}\left[\delta f, f_{\mathrm{M}}\right]\right\} \cdot \\
& \text { METHOD } 2=\text { Linear System: } \quad \sum_{l=1}^{n_{x}} M_{k l}\left(\frac{\partial E}{\partial x}\right)_{l}^{j+1 / 2}=A_{k} .
\end{aligned}
$$




\section{Comparison with NonLocal Hydrodynamic Approach}

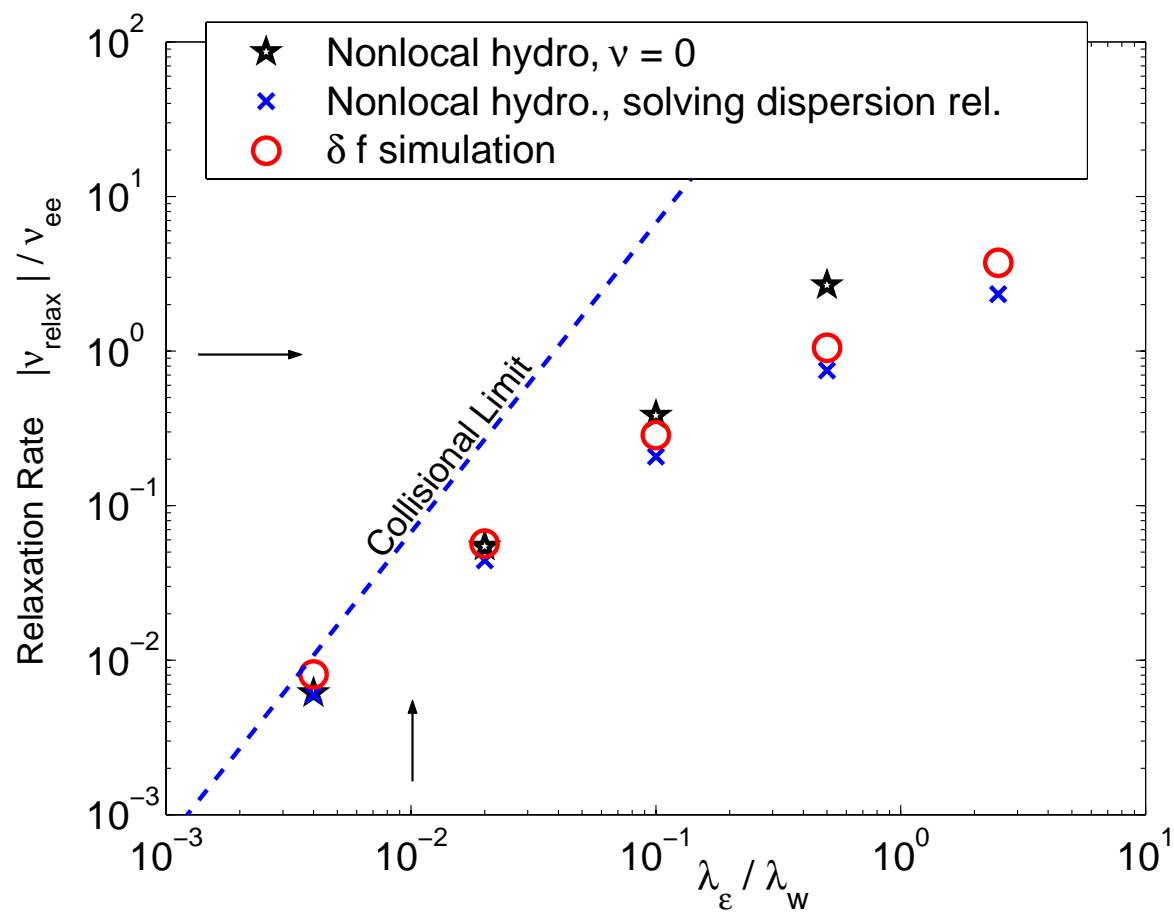

Collisional Limit OK for:

$$
\bar{\lambda}_{\epsilon} / \lambda_{w} \stackrel{\sim}{<} 10^{-2}
$$

Transport is nonlocal both in space and in time.

NONLOCAL HYDRODYNAMIC APPROACH: [Bychenkov et.al (95)]

Solves the same linearized F-P equation for $\delta f_{0}$ as in $\delta f$ simulations, however using a generalized Laguerre polynomial decomposition. The solution is then applied for deriving closure relations to the fluid Eqs.,valid for all regimes of collisionality.

$$
\text { In reciprocal space }(k, \nu) \text { : }
$$

$$
\begin{aligned}
j_{x}=(-e) \Gamma_{x} & =\sigma(k, \nu) E^{\star}+\alpha(k, \nu) i k \delta T, \\
q_{x} & =-\alpha(k, \nu) T E^{\star}-\chi(k, \nu) i k \delta T,
\end{aligned}
$$

Heat Eq.: $\frac{3}{2} N \frac{\partial}{\partial t} \delta T+\frac{\partial}{\partial x}\left(q_{x}+T \Gamma_{x}\right)=0 \Longrightarrow \nu_{\text {relax }}=-\frac{2}{3} \frac{k^{2}}{N}\left(\chi-T \frac{\alpha^{2}}{\sigma}\right)$. 
Illustration 3: NonLinear, NonLocal Electron Heat Transport

Considering large amplitude, 1-Dim temperature perturbations of the electron distribution:

$$
f(x, \vec{v} ; t)=f_{\mathrm{M}}\left[v \mid N_{e}(x), T(x, t)\right]+\delta f(x, \vec{v} ; t) .
$$

Requires:

- Solving the full nonlinear F-P equation.

- Evolving the background when applying the $\delta f$ method.

- Enforcing quasineutrality $\Longrightarrow$ Equation for $\vec{E}$

(Similar algorithm as in linear code).

HERE: No assumption on $Z \Longrightarrow$ Variables $(x, v, \mu=\cos \theta)$. 
NonLinear, NonLocal Electron Heat Transport. Cont'd

Computing Background Temperature

\section{TWO ALTERNATIVES:}

1. "Stiff" constraint on kinetic energy $\operatorname{Kin}(\delta f)$ :

$$
\operatorname{Kin}(\delta f)=\frac{m}{2} \int \delta f v^{2} d \vec{v}=0 .
$$

$\Longrightarrow$ Heat Eq. for background temperature:

$$
\frac{3}{2} N_{e}(x) \frac{\partial}{\partial t} T(x, t)+\frac{\partial}{\partial x} q_{x}(\delta f)=0 .
$$

2. "Soft" constraint on kinetic energy $\operatorname{Kin}(\delta f)$ :

$$
0 \simeq \operatorname{Kin}(\delta f) \ll \operatorname{Kin}\left(f_{\mathrm{M}}\right)
$$

“Numerical feed-back" of $\operatorname{Kin}(\delta f)$ back into $f_{\mathrm{M}}$ :

$$
\frac{3}{2} N_{e} \frac{\partial}{\partial t} T(x, t)=\alpha_{\text {relax }} \operatorname{Kin}(\delta f) .
$$




\section{BENCHMARKING NonLinear Code with Linear NonLocal Hydrodynamic Approach}

Relaxation of Small Amplitude Sinusoidal Perturbations in the Background Temperature: $\delta T / T=0.1$.

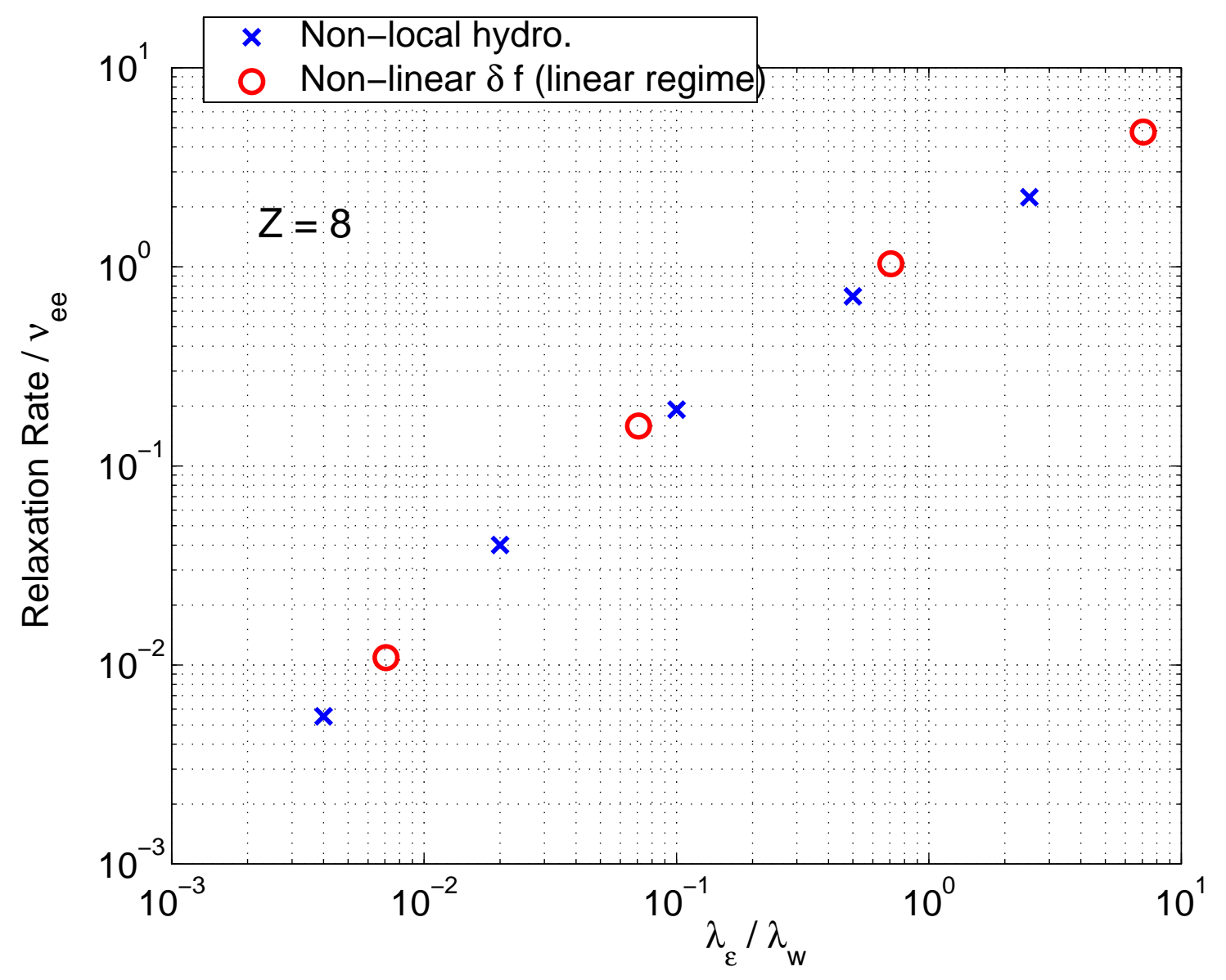




\section{Relaxation of Gaussian Temperature Peak}

\section{TEMPERATURE:}

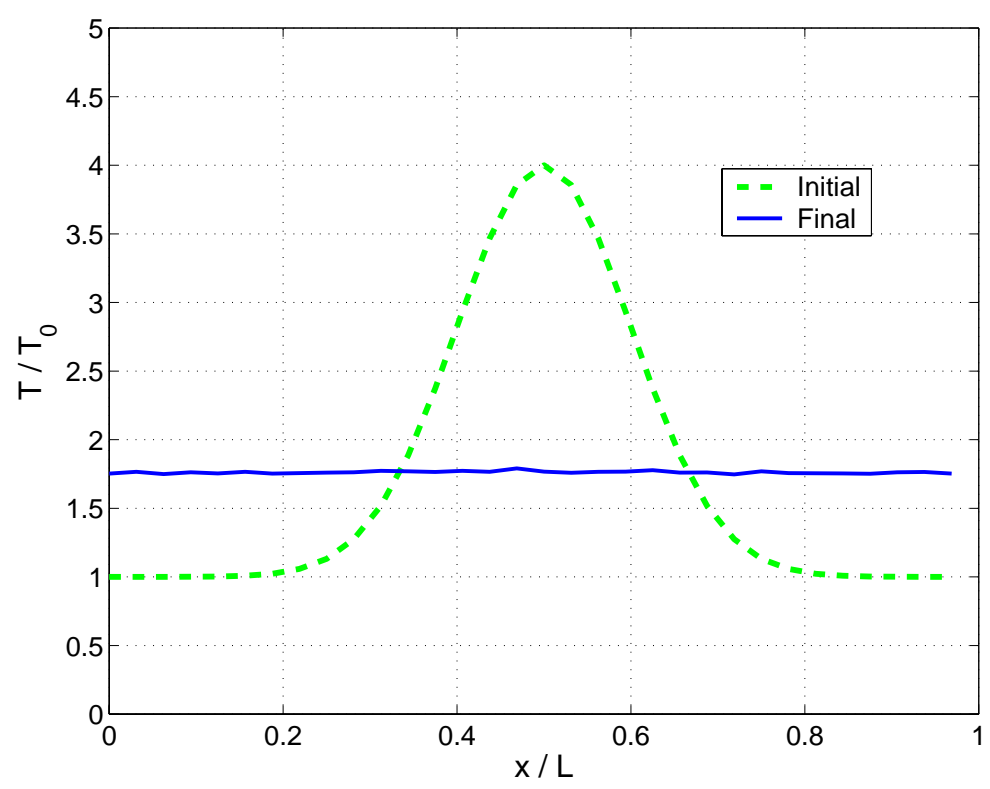

INITIALLY:

$$
\begin{gathered}
T(x, t=0)=T_{0}+\delta T \exp \left[-\frac{1}{2}\left(\frac{x-0.5 L}{\Delta x}\right)^{2}\right], \\
\delta f \equiv 0 .
\end{gathered}
$$

Parameters:

$\lambda_{e e}(0) / L=10^{-1}$ (Evaluated at base of peak)

$$
Z=3
$$

$\Delta x / L=10^{-1}, \delta T / T_{0}=3$

Uniform density: $N \equiv N_{0}$ 


\section{COMPARING $f_{\mathrm{M}}$ AND $\delta f$ AT TIME $t=5.0 \nu_{e e}(0)$}

\section{Hot Peak}

$$
X / L=0.5
$$
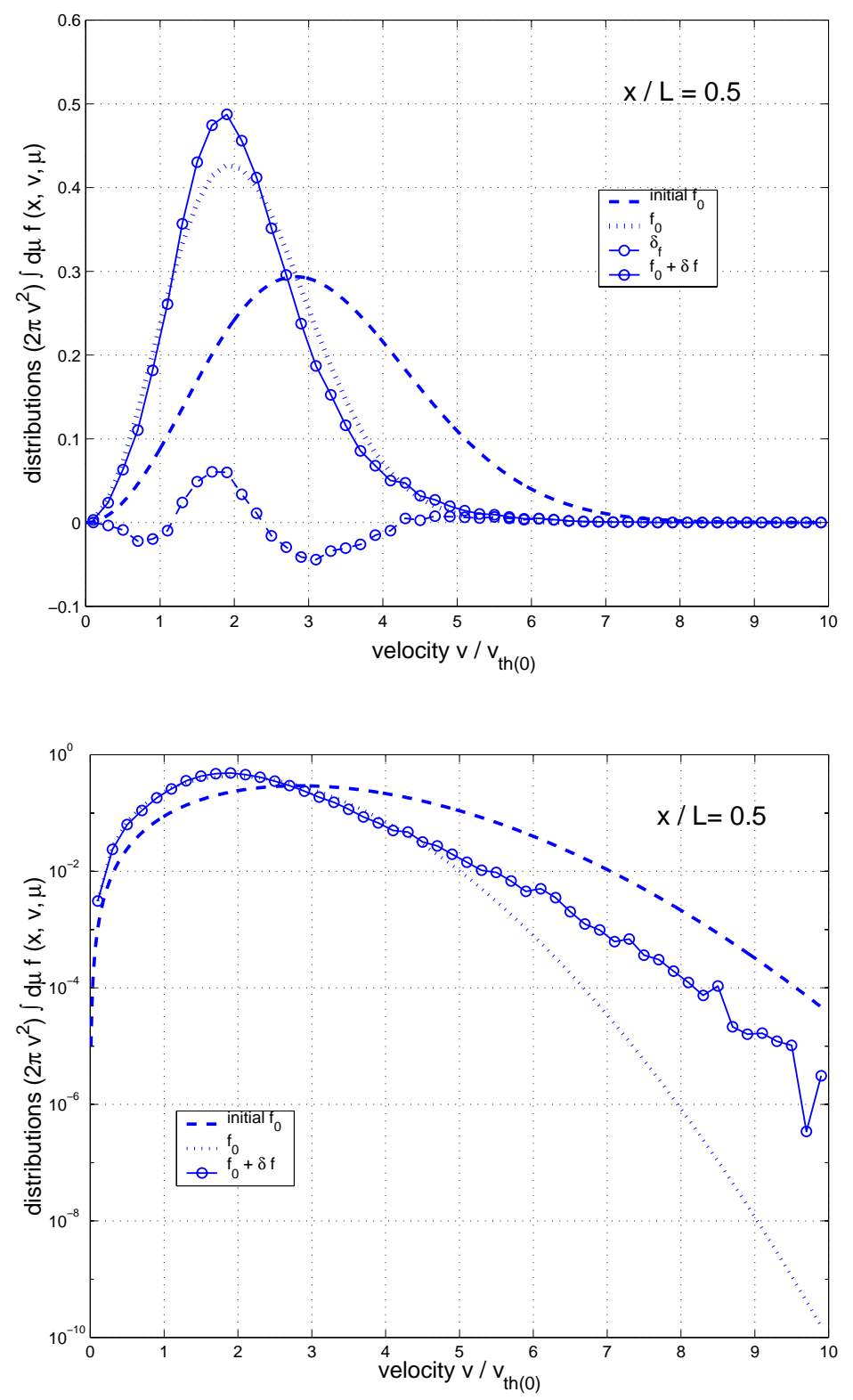

Cold Boundary

$$
X / L=0.0
$$
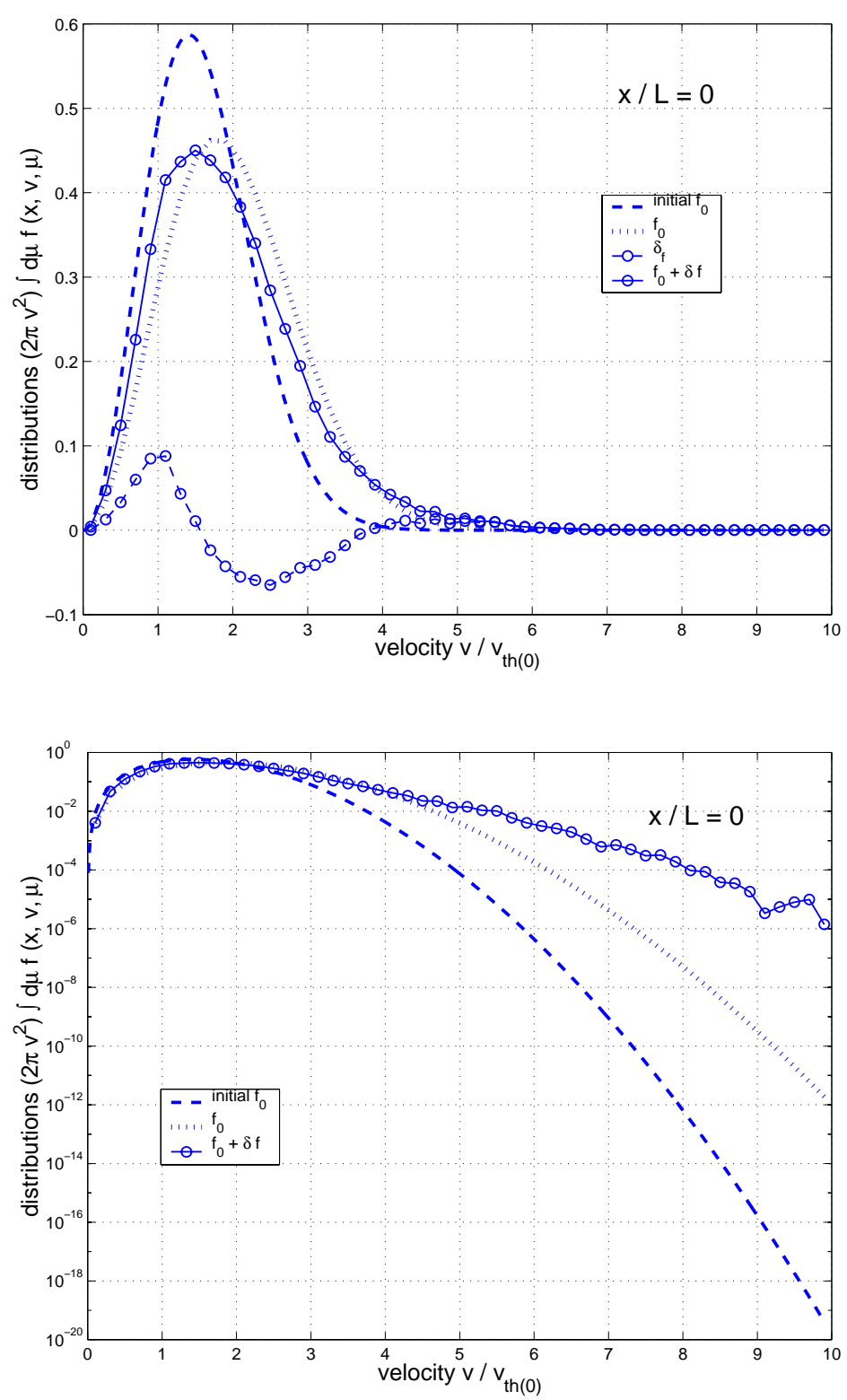


\section{SCALING ON PARALLEL COMPUTER}

- Code is Parallelized using MPI.

- Running on Origin 2000 and Linux Cluster.

- Implements a high-quality, parallel pseudo-random number generator (C.Karney).

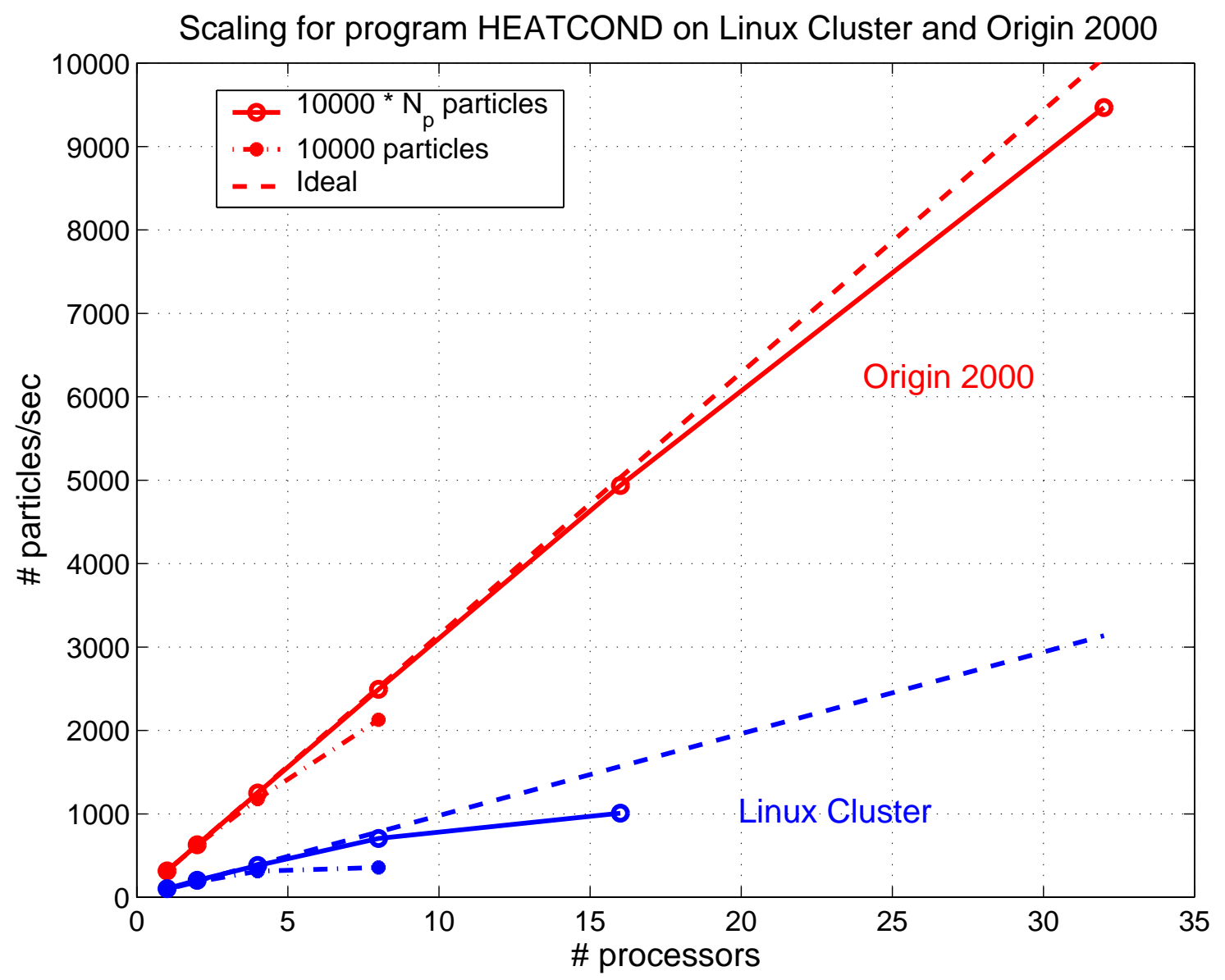




\section{REFERENCES:}

Xu \& Rosenbluth (91); Dimits \& Cohen (94); Lin et al.(97)

S. Brunner, E. Valeo, and J. A. Krommes, Physics of Plasmas 6, 4504 (1999); 7, 2810 (2000). 